

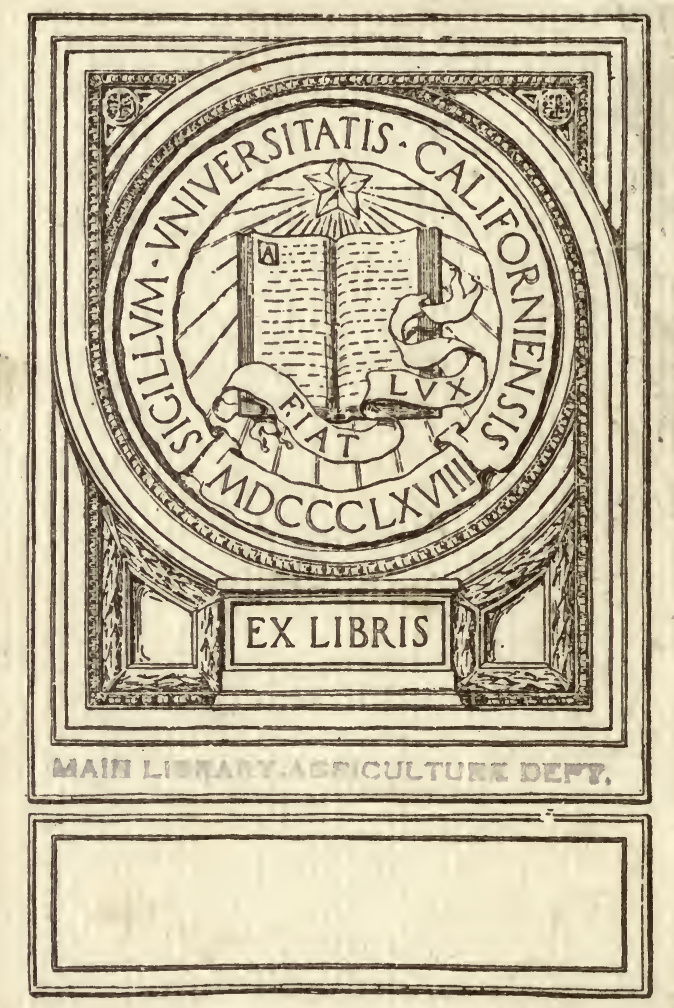







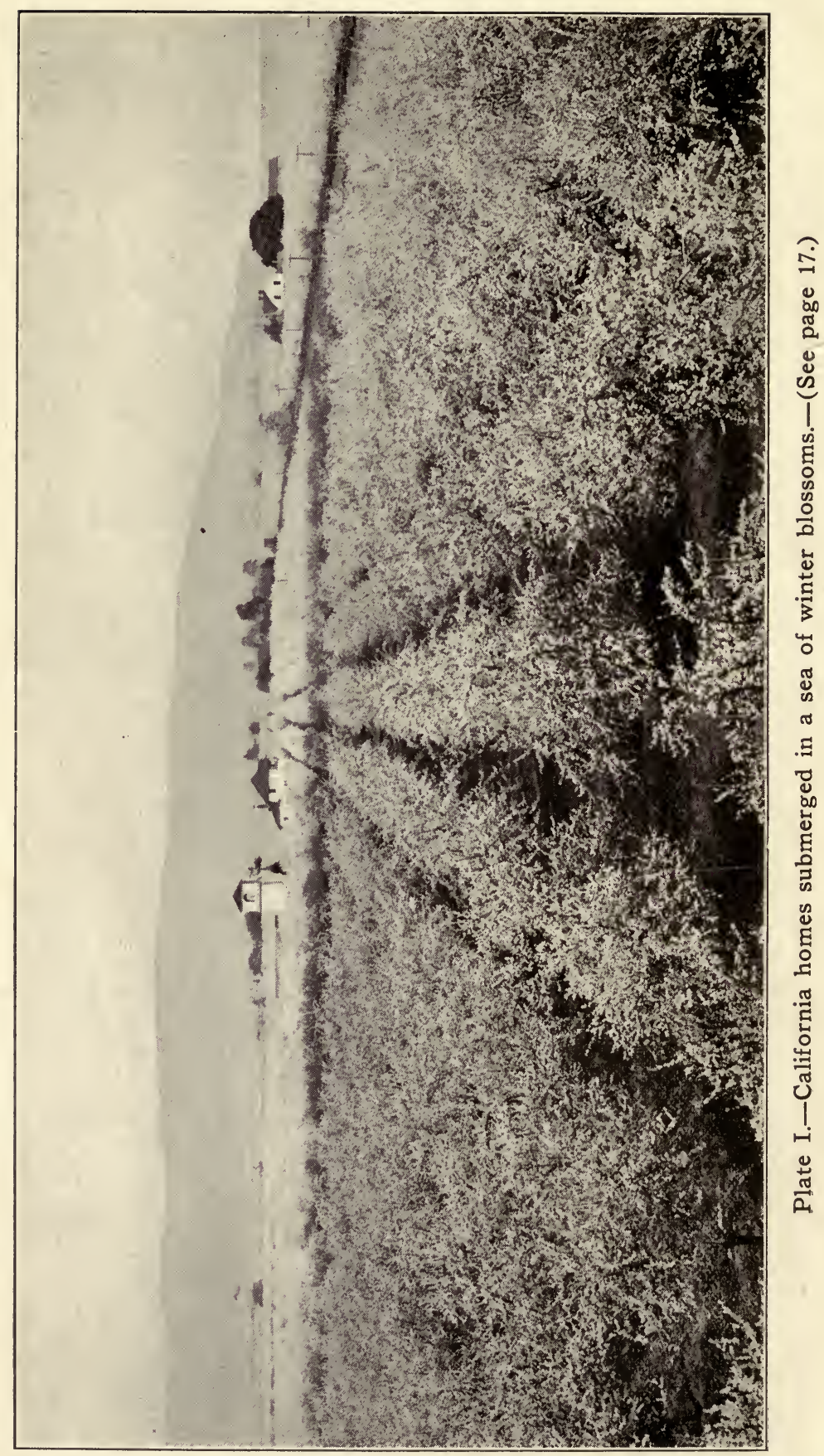




\title{
THE
}

\section{CALIFORNiA FRUITS. AND}

\section{HOW TO GROW THEM}

\author{
A MANUAL OF METHODS WHICH HAVE \\ YIELDED GREATEST SUCCESS, WITH THE \\ LISTS OF VARIETIES BEST ADAPTED TO \\ THE DIFFERENT DISTRICTS OF THE STATE
}

\section{BY \\ EDWARD J. WICKSON, A. M.}

Professor of Horticulture in the College of Agriculture of the University of California: Horticulturist of the Agricultural Experiment Station: Author of "California Vegetables in Garden and Field" and "One Thousand Questions in California Agriculture Answered": Editor of the Pacific Rural Press: Member of the National Council of Horticulture. Etc.

The branch here bends beneath the weighty pear.

And verdant olives flourish round the year;

The balmy Spirit of the Western gale

Eternal breathes on fruits untaught to fail;

Each dropping pear a following pear supplies,

On apples, apples; figs on figs arise.

The same mild season gives the blooms to blow.

The buds to harden, and the fruits to grow.

-Pope's Hom. Odys. Bk. VII.

SEVENTH EDITION-Fully Revised

SAN FRANCISCO. CAL.

PACIFIC RURAL PRESS

Nineteen Hundred Fourteen 


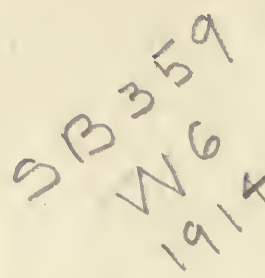

Copyright, I914.

By E. J. Wickson and Pacific Rural Press, Published June, 1914.

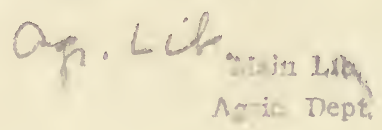

THE HICKS-JUDD COMPANY

Printers AND Bookbinders

51-65 First Street, San Francisco 


\section{PREFACE}

The Sixth Edition of this treatise has shared the good fortune of its immediate predecessors in meeting a public demand which covered the available supply much sooner than anticipated. Because of the desirability of keeping the work abreast of the progress of a constantly changing and developing industry, the publishers have assumed the burden of resetting the type for each edition, resisting the temptation of greater profit which would attend reprinting from plates with minimum revision. Therefore this edition is wholly set anew-the seventh opportunity for free revision which the writer has enjoyed during the publication of the work, which has reached a total of nineteen thousand copies since the appearance of the first edition in 1889 .

Of the quality of the book, it does not become the writer to speak, but he may express his satisfaction at its popularity. Its circulation may be cited as a testimonial of its suitability for service in the building up of the fruit industries, and the demand for it may be regarded as rather unique, when it is remembered that the book deals exclusively with the fruit growing of a single State which is only one, although it be the greatest, of the agricultural interests of that State. The demand for the book is an exponent of the continued activity in California fruit planting, and its sale abroad indicates the fact that the outside world is watching California's fruit development, and desires to know the methods by which such a great product as $\$ 100$,000,000 worth in a year is secured.

The writer repeats the request which he has made in earlier editions that all readers whose observation and work teach them any better way than he has described in this book shall share with him the advantages of such greater wisdom.

$$
\text { EDWARD J. WICKSON. }
$$

University of California, Berkeley, May, 1914. 



\title{
CONTENTS
}

\author{
PART ONE: GENERAL.
}

Chapter.

I The Climate of California and Its Modifications.

II Why the California Climate Favors the Growth of Fruit.

III The Fruit Soils of California.

IV The Wild Fruits of California.

V The California Mission Fruits.

VI Introduction of New Fruit Varieties.

PART TWO: CULTURAL.

VII Clearing the Land for Fruit.

VIII The Nursery.

IX Budding and Grafting.

$\mathrm{X}$ Preparation for Planting.

XI Planting of Trees.

XII Pruning Trees and Thinning Fruit.

XIII Cultivation.

XIV Fertilizers for Trees and Vines.

$\mathrm{XV}$ Irrigation of Fruit Trees and Vines.

PART THREE: ORCHARD FRUITS.

XVI Commercial Fruit Varieties.

XVII The Apple.

XVIII The Apricot

XIX The Cherry.

XX The Peach.

XXI The Nectarine.

XXII The Pear.

XXIII Plums and Prunes.

XXIV The Quince. 


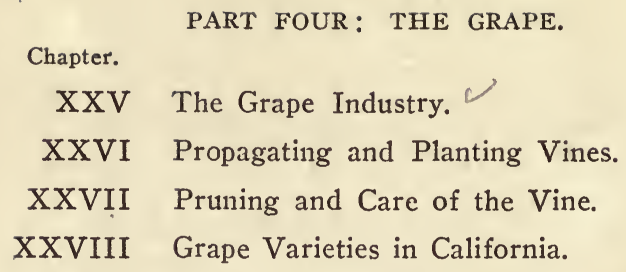

PART FIVE: SEMI-TROPICAL FRUITS.

XXIX The Date.

XXX The Fig.

XXXI The Olive.

XXXII The Orange.

XXXIII The Pomelo or Grape Fruit.

XXXIV The Lemon."

XXXV Minor Semi-Tropical Fruits.

PART SIX: SMALL FRUITS.

XXXVI Berries and Currants.

PART SEVEN: NUTS.

XXXVII Nut Growing: Almond, Walnut, etc.

PART EIGHT: FRUI'T PRESERVATION.

XXXVIII Fruit Canning, Crystalizing and Preserving.

PART NINE: FRUIT PROTECTION.

XXXIX Injurious Insects.

$\mathrm{XL}$ Diseases of Trees and Vines.

XLI Suppression of Injurious Animals and Birds.

XLII Protection from Wind and Frost.

PART TEN : MISCELLANEOUS.

XLIII Utilization of Fruit Wastes. Topical Index. 


\section{CALIFORNIA FrUits}

PART ONE : GENERAL

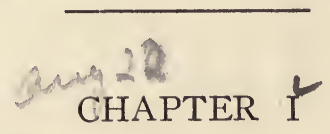

\section{THE CLIMATE OF CALIFORNIA AND ITS LOCAL MODIFICATIONS}

In climatic conditions affecting horticulture we have in California almost an epitome of all other parts of the United States, with added climatic characters which no other part possesses. We have high mountain valleys with wintry temperature-conditions, where only hardy northern fruits can be grown; we have hot valleys where the date palm confidently lifts its head to the fiery sunshine, while its feet are deeply planted in moist substrata beneath the sandy surface; but we can not claim tropical conditions, because our dry air denies us many strictly tropical growths, although we have frostless sites for them. Intermediate between the cold and snow of the mountains and the heat and sand of the desert, we have every describable modification and gradation, and, naturally, it is between these extremes that our richest inheritance of horticultural adaptation lies.

When the breadth and scope of our horticultural adaptations are realized, it becomes apparent that an enumeration of the fruits we can grow successfully would be, in fact, a catalogue of the known fruits of the world, except those which are strictly tropical. Wherever there is a northern or southern departure from the equator sufficient to bring energy to mankind, or where the same is accomplished by elevation upon tropical mountainside or plateau, there also are fruits which find a welcoming home in California, and are improved by the intelligent cultivation and selection which here prevail. On the other hand, it has been abundantly demonstrated, during recent years, by official awards at great exhibitions 
and by the sharp criteria of the markets as well, that the fruits of wintry regions are quite as much benefited by transfer to proper locations in California as are the people who come to grow them. From north and south alike, then, California makes grand acquisitions and includes within her area the adaptations of the whole country, with some which no other State possesses.

But while this horticulutral scope is claimed for the State as a whole, it is necessary to add that local adaptations within the State must be very narrowly drawn. Our greatest failures have followed ill choice of location for the purpose intended. Whenever certain California fruits have been ill spoken of, they have been produced in the wrong places, or by ill-advised methods. It is possible, then, to produce both poor and perfect fruit of a given kind. It may be said this can be done anywhere by the extremes of culture and neglect, but to this proposition it must be added that in California equally excellent methods and care may produce perfection in one place and the opposite in another. One who seeks to know California well must undertake to master both its horticultural greatness and littleness; and so closely are these associated, and so narrow the belts of special adaptations, that there are many counties which have a range of products nearly as great as the State itself.

It is hard for the stranger to realize this. It is difficult for him to believe that the terms "northern" and "southern" have almost no horticultural significance in California; that northern fruits reach perfection, under proper conditions, at the south, and vice versa; that some regions of greatest rainfall have to irrigate most frequently; that some of greatest heat have sharpest valley frosts; that some fruits can be successfullly grown through a north and south distance of 300 miles, but can not be successfully carried a few hundred feet of either less or greater elevation; that on the same parallel of latitude within a hundred miles of distance, from coast to mountainside, one can continuously gather marketable Bartlett pears for three monthsnot to mention the second crop, from belated blossoms, which is often of account on the same trees in the same season.

Through the multitude of local observations, which seem perplexing and almost contradictory, it is possible to clearly discern certain general conditions, of both nature and culture, which may be briefly advanced as characteristically and distinctively Californian.

The climate of the Pacific Coast is described by the meteorologist as "insular or moderate," as contrasted with the "continental or excessive" climate of the regions east of the Sierra Nevada. The west coast of Europe is also insular in its climate. The northern limit of an annual mean temperature of 50 degrees Fahr. is 50 degrees and 47 degrees of north latitude on western coasts of Europe and America respectively. But though there is this similarity in mean annual temperature, there is a decided advantage pertaining to our climate over that of west Europe in that our range of temperature is less; that is, extremes of heat and cold are nearer together, and 
changes are therefore much less excessive. This characteristic of our local climates is due in the main to two great agencies, one active, bringing heat, the other passive, shielding us from arctic influences.

First: Our proximity to the Pacific Ocean. Professor Alexander G. McAdie, for twenty years in charge of the San Francisco office of the United States Weather Bureau, and now Professor of Meteorology at Harvard University, says of the mildness of the California climate: "The Pacific Ocean is a great natural conservator of heat, the mean annual temperature of which near the California Coast ranges from 50 degrees to 60 degrees $F$. The strength of the westerly winds which prevail on the California Coast for more than half the days of the year is due to the fact that the whole drift of the atmosphere is prevailingly from the west to east. The climate of west coasts is consequently less severe than the climate of east coasts."*

Second: Another agency contributing to the mild climate of the Pacific Coast consists in the the mountain barriers upon our northern and eastern boundaries. It was Guyot who first called attention to the fact that the Sierra Nevada and the Cascade Mountains reach the coast of Alaska and bend like a great arm around its western and southern shore, thus shutting off or deflecting the polar winds that otherwise would flow down over the Pacific Coast States, while California has her own additional protection from the north in the mountain arch which has its keystone in Mount Shasta.

\section{CHIEF TOPOGRAPHICAL AND CLIMATIC DIVISIONS OF CALIFORNIA}

California is usually divided into three main areas and climates, each distinct in typical conditions and yet separated by regions, more or less wide, in which these conditions merge and influence each other. Dr. Robertson says : $\dagger$

Isothermal lines which normally run east and west are, as they near the Pacific, deflected north and south, and define three distinct climatic belts. These may be named coast, valley and mountain; and while they resemble each other in having only two seasons, they are dissimilar in other respects. These differences depend upon the topography of the country, and are of degree rather than of kind; altitude, distance from the ocean, and situation with reference to mountain chains, giving to each region its characteristic climate.

How similar are the conditions which prevail in these belts may be learned from the data shown in the following table, which includes points separated by nearly the whole length of the State, the difference in latitude of the extreme north and south points being seven or eight degrees. Thus, through a north or south distance great as that which separates the States of Georgia and New York, similar climatic conditions prevail in California. In the following table the averages are deduced from observations by the United States Weather Bureau observers for a long series of years:

*"The Rainfall of California," University of California Publications in Geography, 1914. †Report of State Agricultural Society, 1886, page 322. 
Seasonable and extreme temperatures and average rainfall in various California regions from the records of the United States Weather Bureau to the close of 1913.

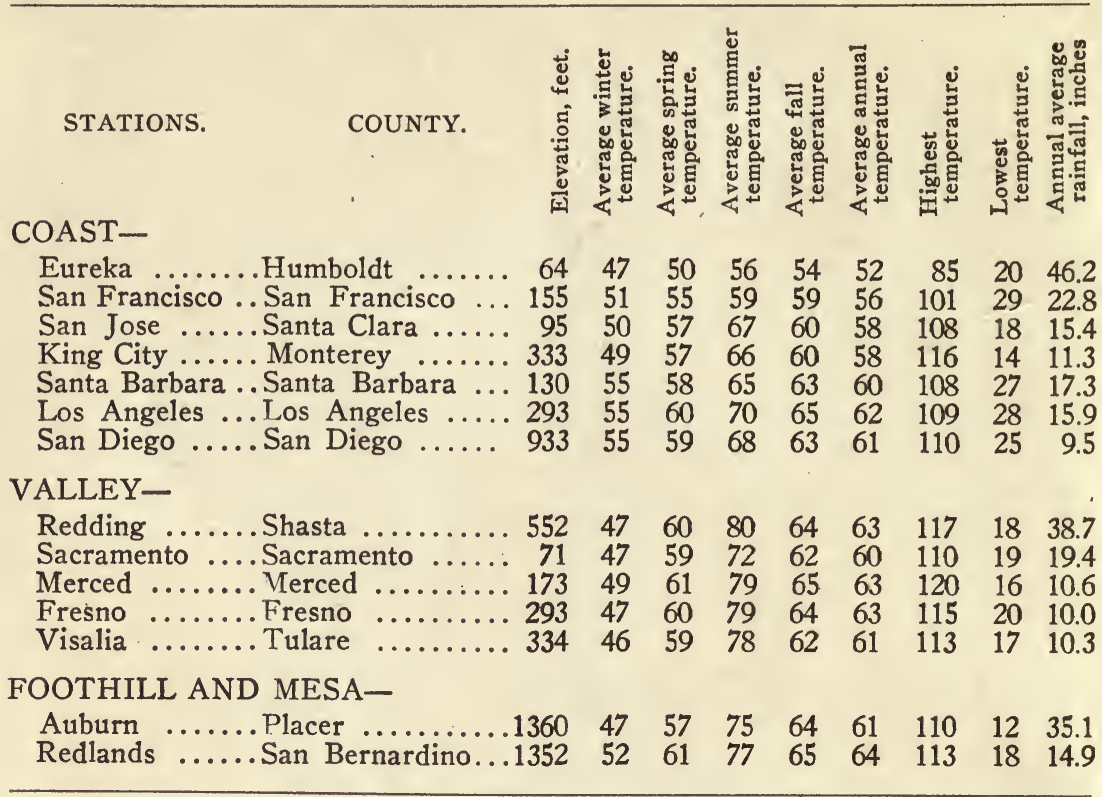

\section{LOCATIONS FOR THE GROWTH OF DIFFERENT FRUITS}

It is intended to describe as definitely as possible the locations suitable for the growth of different fruits in the special chapters given to those fruits, but there are a few general conditions which should be outlined.

In discussing the choice of location for an orchard it is not intended to speak geographically. As has already been intimated. latitude, which is a prime factor in geography, is of exceedingly small account as an indication of horticultural adaptations in California. The fact becomes strikingly apparent when it is known that the apple and the orange, fruit kings whose kingdoms lie at opposite borders of the temperate zone, so far distant that one may be called semifrigid and the other semi-tropical, have in California utter disregard for the parallels of latitude, which set metes and bounds upon them in other hands, and flourishes side by side, in suitable localities, from San Diego to Shasta. Impressive as this truth may be, it is not so startling as another fact, viz., that fruits, in suitable interior situations, ripen earlier at the north than in coast valleys at the south.

"That almost any extreme of weather," says Professor McAdie in his publication just cited," can be found within the limits of California is readily comprehensible when it is considered that the state is 800 miles in length, contains a hundred million acres, and 
varies from deserts as much as 280 feet below the sea to Shasta, 14,380 feet, or the High Sierra, where 66 peaks exceed 10,000 feet in height, while 40 exceed 13,000 feet, and Mount Whitney rises to 14,502 feet."

It is apparent then, in view of such diversity, that the selection of locations for orchards must be made with a knowledge of special conditions governing the distribution of equal temperatures and other natural agencies contributing to the development of fruit. This distribution, as has been intimated, is not by parallels of latitude, great circles which appear as straight lines on a map, but by curves, which proceed in various directions, governed chiefly by topography. These are curves of temperature, of rainfall, of elevation, of soil formation and deposit. Geography must be interpreted by topography and climatography.

Let these ruling conditions be reviewed, then, briefly: First, as to general areas; second, with reference to special situations and locations.

\section{COAST CLIMATE}

The chief characteristics of the coast are equable temperature, increasing southward; summers cool and winters warm, as compared with the interior; abundant rainfall, decreasing considerably southward; a somewhat humid atmosphere, as compared with the interior; frequent fogs or overcast skies; prevailing westerly winds.

The extension of coast influence toward the interior is governed by local topography. Coast valleys open to ocean winds are cooler and moister and demand hardier fruits than valleys sheltered by intervening ranges. Gaps and passes in the ranges are subject to winds of considerable force and low temperature, and are not generally favorable for fruit; on the other hand, situations sheltered on the north and west favor growth of fruits even though quite near the coast. Sometimes a distance of a few miles, sometimes a wind-break or natural forest, or of planted trees, so modifies coast influences that fruits do well. Elevation on the sides of coast valleys secures similar results. For example, the floor of the Pajaro Valley is well suited for apples, late pears, cherries, plums, prunes, and berries (except gooseberries), while on adjacent hillsides peaches do well.

In southern California, coast winds are warmer than in the upper half of the State, but coast influences intrude further, as a rule, because the hills near the coast in Southern California are low; the high ranges, answering to the Coast Range of the upper part of the State, trending far into the interior. On the coast side of these ranges fruits ripen later than in sheltered interior points in the upper part of the State, but eastward of the mountains, where soil and moisture favor, or irrigation is practised, extra early locations have been found and are now being rapidly developed-in the Imperial and adjacent valleys, for instance.

Some of the horticultural effects of the conditions prevailing on the coast may be described as follows:

Late Ripening of Fruits.-The late ripening of fruits west of the mountains in southern California has just been mentioned. Intrusion of coast influences has the same effect in more marked degree 
at the north because the ocean winds are colder. Directly on the coast, at Pescadero, San Mateo County, for example, fruits ripen about a month later than in Santa Clara Valley, which is just across the Coast Range. Napa Valley, though about forty miles inland and sheltered by ranges of hills, still is sufficiently affected by the coast influences to mature fruits considerably later than Vaca Valley, ten or fifteen miles further east, beyond a higher range, which completely bars out these influences. Similar local effects are found in southern California. For instance, in Ventura County in a canyon sixteen miles from the ocean, and at an elevation of sixteen hundred feet, fruits ripen three weeks earlier than on the coast or in the valleys opening thereon.

Failure of Certain Fruits.- Though killing frosts are few directly on the coast, the deficiency in summer heat and sunshine renders some fruits unsatisfactory. This is especially the case in the upper coast region. Grapes and figs ripen imperfectly, while but a short distance back from the coast, in situations, sheltered by ridges parallel to the coast, they do well. Elevation sometimes produces corresponding effects.

Pests and Diseases.-Certain blights are more prevalent under coast conditions. The scab blight of the apple, the curl-leaf of the peach, and some other blights, are prevalent on the coast and in coast valleys, on the river bottoms in the interior, and on the mountains, and less serious, or wholly absent, in the hot interior valleys. Some insects prefer the coast but thrive also in the interior, as hot dry wind is excluded by dense growth of the tree and the included air becomes moister abave irrigatèd soil. "A notable instance is the black scale, which, with the black smut which attends it, has long been a grievous pest of growers of olives and citrus fruits, and has recently become prevalent on deciduous fruit trees in some regions. On the other hand, in the face of ocean winds the codlin moth seems to have less chance to fill pears and apples with her destructive offspring, than she enjoys in interior valleys and mountain regions. Directly under coast influences, moss and lichens gather quickly and should be removed. Spraying with alkaline washes not only kills insects but cleans the bark from parasitic vegetable growth. Although fruit trees on the coast are not so subject to sunburn as in the interior, there is especial value in low heading to withstand winds; there should also be plenty of room given the trees, that sunshine may have free access to warm the ground all around the tree, which may be undesirable in the interior.

\section{VALLEY CLIMATE}

The characteristics of the interior valley climate are higher summer and lower winter temperatures than on the coast, the range of temperature being nearly the same both north and south; rainfall abundant in the north and decreasing rapidly southward, so that as a rule the interior valleys in the south half of the State require irrigation; very dry air and almost constant sunshine, freedom from fogs 
and from dew in summertime; winds occasionally strong, hot, and desiccating in summer and cold in winter.

Local Modifications.-The term "valley climate" is broad, and includes everything, away from the coast to a certain elevation on the slope of the mountains. Certain small valleys protected from cold northerly winds and from fog-bearing westerly winds and open to the spring sunshine, have a forcing climate which produces the earliest maturing fruit of the season; earlier not only than the coast and the mountain, as has been stated, but also somewhat earlier than adjacent locations in the broad, open valley. Slight elevation, even on the sides of small valleys, frequently secures freedom from winter frosts and ministers to early ripening. Elevation above sea-level on the rims of great valleys also secures similar results and gives rise to thermal belts in which semi-tropical fruits are successfully growing even as far north as Shasta County. On the floors of great valleys moderating influences are secured on the lee side of wide rivers and by planting on the river bank or on slightly elevated swells rather than on the level, open plain. The river bottom lands of the great valleys, though subject to severe frosts, are freer from the effects of desiccating winds than the open plains; they are, however, more favorable to the spread of certain blights than the plains.

Some of the horticultural effects of valley conditions are as follows: Early ripening and perfection of summer and autumn fruits, owing to continual sunshine and dry air; forced maturity of certain fruits, as apples for instance, which destroys character and keeping quality; injury from sunburn and hot winds in summer, which seriously affect both fruit and foliage of some varieties; occasional injury to tender fruits (semi-tropicals) and to young trees of hardy fruits, which have been kept growing late in the season, from low temperature, which sometimes is reached suddenly on the floor of the valleys; freedom from some blights and insects which are prevalent on the coast, but not from others. Many of these minor troubles are, however, counterbalanced by the earliness, size, beauty, and quality of certain fruits, and by the most rapid and successful open-air drying of fruits, owing to high autumn temperature, the freedom from summer fog, dew and generally from rain during the drying season.

\section{FOOTHILL CLIMATE}

Foothill climate is usually considered as a modification of valley climate. It has been shown that up to about two thousand five hundred feet, on the western slope of the Sierra Nevada, the seasonal temperatures are quite like those of the valley, but the rainfall increases about one inch for each hundred feet of elevation. There are, however, in the foothillls, places where early spring heat and freedom from frost give very early ripening fruits, and other places at the same elevation where winter temperature drops below the valley minimum, and where late frosts also prevail. This is governed by local topography. In many of the small valleys among the foothills, both of the Sierra Nevada and the Coast Ranges, frosts 
are more severe than on the hills adjacent or in the broader valleys to which they are tributary. These small "protected valleys". are apparently. warm and cozy for early blooming deciduous and citrus fruits, but they are really very dangerous. They frequently have such narrow and obstructed openings that cold air is dammed up over their lower lands and frosts are more severe and later than in valleys which have ample and free outlets and seem less protected.

Of course the disposition of cold air to settle in low places and to flow down canyons and creek-beds while the warm air rises and bathes the adjacent hillsides, has much to do with frost in the hollow and the freedom from it on the hills. The constant motion of the air on the slopes is also a preventive of frost, providing the general temperature is not too low. It is not uncommon to find in deeper valleys, protected against the western wind, flakes of snow and a wintry chill, with dormant vegetation, while one thousand feet higher up the foliage is fast developing.

\section{MOUNTAIN CLIMATE}

Above an elevation of two thousand five hundred to three thousand feet, conditions gradually intrude which 'resemble those of wintry climates. The tender fruits, the apricots, peach, etc., become liable to winter injury and give irregular returns, or as greater elevation is attained, become wholly untrustworthy. Early blooming of these fruits during warm spells which are followed by severe frosts, renders the trees unfruitful. At four thousand to four thousand five hundred feet the hardy apple and pear flourish, ripening late, and winter varieties possessing excellent keeping qualities. Here, however, winter killing of trees begins and locations even for hardy fruits have to be choosen with circumspection.

There are elevated tracts of large extent among the Sierras where the common wild plum, choke-cherry, gooseberry, and California chestnut are produced abundantly. April frosts have killed the fruit of those same plums, transplanted to lower ground, while those left in their natural situation were quite unharmed. It has been observed that these plum trees with other fruits and nuts in their original positions, invariably occupy the broad tops of the great ridges instead of the sides and bottoms of ravines or narrow, pent-up valleys. Follow nature in the choice of orchard sites (with due regard to a supply of moisture in the soil, either natural or artificial) and little hazard attends the culture of the hardier fruits of our latitude among, the highlands of the State than is incident to other seemingly more favored localities. The beauty and quality of these mountain fruits are proverbial.

\section{A RULE OF GENERAL APPLICATION}

What has been thus suggested of the great variation of temperature conditions within narrow limits should lead to the conclusion that not only must the kind of fruit to plant be determined by local 
observation and experience, but often varieties of these fruits must be chosen with reference to adaptation to local environment. For this reason it is impossible to compile tables of varieties suited for wide areas-and yet it is true that some varieties have shown themselves hardy and satisfactory under all conditions. These facts will be shown by the discussion which will be given to each of the different fruits.

\section{REST AND ACTIVITY OF FRUIT TREES}

Indication has already been made of regions adapted to the growth of early and of late fruits. There is, of course, difference in time of rest and of returning activity in blooming. On the mountains under wintry conditions the trees leaf out and bloom late, following more or less the habit of Eastern trees. In the foothills, the valleys, and the coast, there is less difference in time of rest and of leaf and bloom. Even in regions where there may be a month's difference in ripening of fruit, as, for example, in the Vacaville district, fifty miles inland, and in Berkeley, two miles from the bay shore, trees bloom almost at the same date. The difference in ripening is due to the higher temperature and fuller sunshine of the interior situation, which have a forcing effect, while the low temperature and dull skies of the summer on the coast retard maturity.

The rest of the tree, in all save the mountain district, is not dependent upon the touch of frost. It comes rather from thirst than from cold. The immense weight of fruit, the vigorous growth of wood, and the exhaustion of moisture from the soil by the draught of the roots to compass this growth, are the chief causes which bring the sere and yellow leaf in California. It is not frost, for the petunias may be blooming and the tomato vines still green in the fields. But the time has come for a rest. The trees sleep; but merely as a nap at midday; the early rains wake them soon. The roots are active first, then the buds swell, and the blossoms burst forthsometimes as early as January-the almond first heralding the advent of California's springtime.

Sometimes this season of rest is too short for the good of the tree or vine. The early rains, when followed by a spring-like temperature, as sometimes happens, induce activity in the top as well as the root, and the tree is not in condition to withstand cold weather, which may follow. It is probable that such stimulated activity, suddenly checked, is responsible for more ills to tree and vine than are usually attributed to it. Both to insure hardiness and fruitfulness it is important that a tree shall have summer moisture enough to finish its late summer and early autumn growth-processes. Reference to this important matter is made in the chapter on irrigation. 


\section{CHAPTER II}

\section{WHY THE CALIFORNIA CLIMATE FAVORS THE GROWTH OF FRUIT}

It is pointed out by the earliest students of meteorology, as related to horticulture, that perfect development of fruits depends upon certain atmospheric conditions, which are included in the term climate: First, temperature; second, light; third, humidity or atmospheric moisture,-considered wholly apart from soil moisture. It was also shown that temperature and humidity should be equable, or as free as possible from excessive extremes or rapid changes.

Obviously, the chief characteristics of the California climate are: First, freedom from extremes of low temperature; second, an abundance of sunshine; and third, and atmosphere with a low percentage of humidity. It will be interesting to introduce enough statistics to demonstrate these claims, and to cite reasons why these conditions are of special value to the fruit grower.

\section{THE OFFICE OF HEAT IN FRUIT PRODUCTION}

Temperature conditions may preclude the success of a fruit tree either by destroying it outright, by dwarfing it, or by preventing it from setting or ripening its fruit. Extremes of temperature accomplish the death of plants, and insufficient or excessive mean temperatures may prevent fruition without killing the plant. The first quality of the California climate to arrest the attention of fruit growers in the states east of the Rocky Mountains is the freedom from the effects of the extremely low winter temperatures, to which is due the deplorable failure, in the eastern and western states, of many of the fruit varieties from the west of Europe, and to escape which such zealous efforts now being so successfully put forth to secure hardy varieties of native and foreign origin.

How slight is the injury from low temperatures in all parts of the State where fruit is largely grown may be seen from the following compilation of extreme low temperatures at different points approximately at the same latitude on the coast, in the interior valleys, and on the foot-hills.

Lowest Temperatures at Several California Points.

\begin{tabular}{|c|c|c|c|c|}
\hline $\begin{array}{l}\text { Coast and Coast } \\
\text { Valleys- }\end{array}$ & $\begin{array}{l}\text { Deg. above } \\
\text { zero. }\end{array}$ & Interior Valleys- & $\begin{array}{l}\text { Deg. above } \\
\text { zero. }\end{array}$ & $\begin{array}{l}\text { Deg. above } \\
\text { zero. }\end{array}$ \\
\hline Eureka .... & .. 20 & Redding & $\ldots \ldots 18$ & \\
\hline & 12 & luff & 18 & \\
\hline erdale & 17 & $\mathrm{O}$ & 15 & Nevada City \\
\hline Napa & 18 & ille & 19 & Colfax ........ \\
\hline San Fra & 28 & to & 19 & ... 12 \\
\hline Jos & 18 & $\mathrm{M}$ & 16 & Jackson \\
\hline Gilroy & 20 & Fresno & .. 17 & \\
\hline S & & Tulare City & .. 18 & ille.. \\
\hline Los Angeles & 28 & Colton & . 19 & Redlands ..... \\
\hline San Diego & & Imperial & 20 & ・ \\
\hline
\end{tabular}


These records will show any one familiar with winter killing of the leading orchard fruits that such disasters are not to be feared in the chief fruit regions of California. Local temperature is largely controlled by local conditions, as has been already pointed out, and in the districts named in the table there are special locations where the lowest temperature probably differed a few degrees from the figures given.

\section{NECESSITY OF ADEQUATE SUMMER HEAT}

Passing beyond the freedom from winter killing, it may be remarked that the influences of certain degrees of heat upon the growth of the plant and the perfection of its fruit, has been the subject of much close observation. Boussingault conducted careful experiments, and showed that a temperature above a certain minimum of heat is found necessary for germination, another for chemical modification, and a third for flowering, a fourth for the ripening of seeds, a fifth for the elaboration of the saccharine juices, and a sixth for the development of aroma or bouquet.

Originally the mean annual temperature was alone observed, and the polar limits of plants, it was presumed, could be thereby determined. More recently it was taught that the mean temperature of seasons is of more importance than that of the year, and it is believed that to the relative distribution of heat over the seasons rather than to the absolute amount received during the year, we are to attribute the fitness or unfitness of a region for the growth of certain kinds of vegetation.

It is held in Europe that the mean heat of the cycle of vegetation of the vine must be at least 59 degrees Fahr., and that of the summer from 65 degrees to 66 degrees Fahr. It is stated to be impossible, for instance, to cultivate the vine upon the temperate tablelands of South America, where they enjoy a mean temperature of 62.6 degrees to 66.2 degrees Fahr., because these climates are characterized by a constancy of temperature, never rising to the higher heats necessary to the process of sugar forming, and the vine grows and flourishes, but the grapes never become thoroughly ripe. Boussingault shows that, in addition to a summer and autumn sufficiently hot, it is indispensable that at a given period-that which follows the appearance of seeds-there should be a month the mean temperature of which does not fall below 66.2 degrees Fahr. As will appear presently, this temperature test should not be taken alone, but it will serve as a standard to show one feature of the horticultural adaptation of the California climate. Boussingault claims the need of 66.2 degrees Fahr. for a single month. To be sure to include this, the accompanying table gives the average summer temperature at the leading fruit-growing centers named. 


\section{Average Summer Temperature at Various California Points}

\begin{tabular}{|c|c|c|c|}
\hline $\begin{array}{l}\text { Coast and Coast } \\
\text { Valleys- }\end{array}$ & Interior Valleys- Deg. F. & Foothills- & $\begin{array}{l}\text { Eleva- } \\
\text { tion. }\end{array}$ \\
\hline Upper Lake & Redding & Auburn & $.1,363$ \\
\hline Napa $\ldots . \ldots \ldots 665$ & Oroville & Colfax & $.2,241$ \\
\hline Livermore ..... 69 & Marysville ..... 78 & Georgetown & $.2,500$ \\
\hline San Jose .... & Sacramento ...... 72 & Caliente & $.1,290$ \\
\hline Hollister & Merced ........ 79 & Fall Brook & . 700 \\
\hline Santa Barbara & Fresno & Redlands .. & $.1,352$ \\
\hline Los Angeles .... 70 & .... 78 & .......... & .... \\
\hline San Diego ..... 68 & Riverside .......73 & 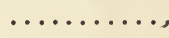 & \\
\hline
\end{tabular}

These points are selected because the European varieties of the grape reach perfection in their vicinity. The excess of heat above that required, as is found at all the interior points mentioned in the table, results in a very high sugar percentage in the grapes, and contributes to the ripening of a second and third crop, as will be noted presently. The superior length of the growing season in California, of course, is an important agency toward the same end.

\section{DIRECT SUNLIGHT ALSO A REQUISITE}

Count de Gasparin was first to point out that not alone sufficient heat, but abundance of continuous sunshine is a requisite of perfection in fruit growth and ripening, and on his authority may be based a claim of exceptional value to the fruit grower in the months of cloudless skies which are characteristic of the California summer.

"The solar rays," says Gasparin, "do not only produce heat, but brings us light, and the effects of the heat and light rays differ in a very pronounced manner. Without light there is no fructification; it is not necessary that the want of light should be complete that there should be a failure of fruits. In fact, diffused light alone does not suffice for the greater number of plants; cultivated plants will not ripen their seed without the direct rays of sun, and the longer they are deprived of it the smaller the quantity which they will mature."*

Again referring to the grape, for in connection with the growth of this fruit the most careful researches have been made, Humboldt wrote: "If to give a potable wine the vine shuns the islands and nearly all seacoasts, even those of the West, the cause is not only in the moderate heat of summer upon the seashore, but it exists more in the difference which there is between direct and diffused light ; between a clear sky, and one veiled with clouds." $\dagger$

*Cours d'Agriculture, t. II, p. 96.

tCosmos, t. I, p. 349. 


\section{Normal Cloudiness at California and Eastern Points}

California-

Red Bluff ........4.4

Sacramento .........3.9

San Francisco .....4.8

Fresno ..............

San Diego ..........4.8

Eastern-

Rochester, N. Y. ...6.6

New York, N. Y. . .5.5

Philadelphia, $\mathrm{Pa}$...5.6

Baltimore, Md. .....5.4

Cleveland, Ohio ...6.6

Grand Haven, Mich.6.2

Jacksonville, Fla. ...4.2

New Orleans, La. ...4.8

Mar. April. May. June July.

4.2

3.5

4.3

2.9

4.4

5.4

5.3

5.4

5.1

5.3

5.4

4.1

4.8

$\begin{array}{lll}3.6 & 1.9 & 1.0 \\ 2.7 & 1.5 & 0.5 \\ 4.2 & 3.8 & 4.3 \\ 2.7 & 1.5 & 0.7 \\ 5.3 & 4.8 & 4.3\end{array}$

\section{2}

5.2

5.1

5.1

4.9

4.8

4.1

4.3

Aug. Sept. Oct. Nov. ${ }^{\text {Avg. for }}$ mo.

It is noticeable that at the California coast points the average cloudiness is almost twice that of the interior valleys, while at the East the interior fruit regions of western New York, Ohio, and Michigan, have a greater. average cloudiness than the Hudson River, New Jersey, and Delaware regions near the Atlantic seaboard. The average cloudiness in the Eastern fruit regions is rather more than twice as great as in the regions of California, where most fruit is grown.

This excess of advantage, as it may be termed, in connection with the high and protracted heat already mentioned, takes practical form in the successful ripening of a second and sometimes a third crop of these grapes in a season, from later bloom on younger cane growth. This behavior is of more value as a demonstration of climatic conditions than otherwise, for it is generally better to produce the main crop alone than to undertake later ones.

Another indication of excess of advantage in the interior valley is found in the development of high sugar contents, which is of direct value in raisin production. The same tendency, though perhaps of less commercial value, is seen in the fact that some grapes which yield a good claret wine nearer the coast develop too much alcohol when grown in the interior.

The advantage of California over Eastern and Southern fruit regions in the abundance of clear sunshine is shown in the table on the preceding page. Cloudiness is rated from 0 to 10 , three observations daily, and the figures in the table are the averages from these daily observations for a series of years, compiled from the records of the U. S. Weather Bureau.

\section{RELATION OF ATMOSPHERIC HUMIDITY TO THE GROWTH OF TREES}

Here is another important condition of the climate of California which is intimately related to those which have been considered, and which is to be credited with no small influence in the perfection 
of our fruits, and that is the low percentage of humidity which our atmosphere contains. In California the percentage of humidity is high in the winter and low in the summer; in the East the condition is just reversed. For this reason summer heat is far more oppressive in the East than in California, and for the same reason certain serious fungoid diseases which prevail at the East, though found here in less injurious degree directly on the coast, may be wholly unknown in the interior where the air is drier. The dry air also favors the access and action of light and heat, for Tyndall says that a sheet of vapor acts as a screen to the earth, being in a great measure impervious to heat.

It is not necessary, then, that there should be clouds to lessen the chemical effects of sun heat in fruit ripening. Not only do clouds intercept sunshine, but water vapor in the air-when to the eve the sun is bright as ever-can absorb a large quantity of the effective. sun rays, and so retard fruit ripening. Hence an apparently sunny country which has much invisible water vapor in the air, may prove defective in fruit-ripening qualities.

It is true that air free from humidity allows rapid escape of heat by radiation as well as free access of it, and in dry air frost is more severe, but at the time of the greatest fruit growth, from June to October, radiation down to a frost point is prevented by other natural agencies. In the early spring and late autumn the humidity percentage rises again and checks radiation just at the time of the year when it is most desirable to have it checked.

The accompanying table, compiled from the records of the United States Weather Bureau, shows the prevailing relative humidity in the East and South and in California.

\section{Normal Relative Humidity at Eastern and California Points}

\begin{tabular}{|c|c|c|c|c|c|c|c|c|}
\hline Eastern Stations- April & May & June & July & Aug. & Sept. & Oct. & Nov. & $\begin{array}{l}\text { vg. for } \\
\text { 8. mo. }\end{array}$ \\
\hline Jacksonville, Fla. ....72.3 & 73.0 & 77.6 & 78.6 & 80.9 & 82.9 & 79.8 & 82.2 & 78.3 \\
\hline Philadelphia, $\mathrm{Pa} . \ldots .63 .4$ & 68.9 & 69.2 & 68.6 & 71.2 & 75.2 & 70.7 & 73.6 & 70.1 \\
\hline Rochester, N. Y. . . . 667.8 & 68.6 & 69.1 & 67.3 & 70.7 & 75.2 & 75.6 & 76.2 & 71.3 \\
\hline Grand Haven, Mich...70.9 & 71.4 & 73.7 & 69.0 & 73.1 & 75.0 & 75.7 & 79.1 & 73.5 \\
\hline St. Louis, Mo. . . . . . 63.7 & 67.8 & 68.8 & 66.3 & 67.3 & 70.2 & 65.9 & 71.1 & 67.6 \\
\hline New Orleans, La. . . . . .76.0 & 74.2 & 78.1 & 78.3 & 78.8 & 77.3 & 74.0 & 79.4 & 78.0 \\
\hline $\begin{array}{c}\text { Galveston, Tex. ......88.6 } \\
\text { California Stations - }\end{array}$ & 78.0 & 79.3 & 77.4 & 78.1 & 77.2 & 75.6 & 80.4 & 78.5 \\
\hline Los Angeles .......73.1 & 75.2 & 73.0 & 75.4 & 76.2 & 72.9 & 74.3 & 66.6 & 73.3 \\
\hline Fresno ........ & 52.7 & 42.4 & 34.7 & 34.7 & 43.6 & 55.1 & 64.1 & 48.3 \\
\hline Sacramento .. & 67.6 & 66.1 & 59.8 & 59.8 & 59.0 & 62.4 & 66.8 & 63.6 \\
\hline Red Bluff & 56.8 & 43.7 & 35.6 & 35.3 & 43.6 & 51.6 & 60.7 & 48.8 \\
\hline
\end{tabular}

\section{THE THREE POINTS COMBINED}

The three great advantages of the California climate-abundant heat, continuous sunshine, and dry air-taken in connection with the fitness of the soil and the great length of the growing season, insure the characteristic excellence of California fruit, and the early 
maturity, great growth, and abundant fruitage of our trees and vines. Heat, sunshine, dry air, and a rainless summer also minister directly to the curing of fruits in the open air. All things considered, it is doubtful whether any area in the world excels California in possession of natural adaptation to fruit production and preservation.

\section{A RECAPITULATION OF CALIFORNIA'S CLIMATIC ENDOWMENT}

Through the multitude of local observations, which seem perplexing and almost contradictory, it is possible to clearly discern certain general conditions of both nature and culture, which may be briefly advanced as characteristically and distinctively Californian. Of these, perhaps the most striking is the length of the growing season.

Take, for instance, the peach in a good peach region. The bloom may appear in February, followed by the grand foliage expanding to a leaf-size, marvelous to one unused to such peach leaves. The shoots of new growth rush out with vigor promised by such leaf; and yet the fruit below expands as though it would burst its skin in rapid enlargement-and still it grows. The new shoot, apparently weary of its several feet of extension, stops for a rest, and then, reviving, starts out its laterals-while still below the peach is growing. The laterals push out a foot or more-all carrying large, fresh leaves. While these are in full vigor, the fruit ripens, after having a full year's joint work of root and foliage, if it is a late variety. Is it any wonder it weighs a pound? But still the tree is active. It forms its terminal buds, and then all along the new main shoots and their laterals are formed the leaf and blossom buds for the following year. Still the foliage holds green and active, if the moisture below be adequate, and the leaves seem loath to fall in the ninth month from time of blooming. Is it any wonder California peaches are large and the trees require pruning and thinning to enable them to carry the weight produced in such a season of growth? And what has been said of the peach is true of other trees, according to their nature and habits. The trees themselves are more eloquent of California's conditions for growth than descriptions or statistical tables can be made.

But the quality of the light and heat, if the term is admissible, is a factor as well as their duration. The air, free, not alone from clouds, but from the insensible aqueous vapor which weakens sunshine in its effort to serve vegetation in a humid climate, has a clearness and brilliance from its aridity which makes each day of the long, growing season more than a day in other climates, and thus adds to the calendar length of the growing season. The surplus light and heat also act directly in the chemistry which proceeds in the tissues of the plant, and we have not only size, but quality, color, aroma - everything which makes the perfect fruit precious and beautiful beyond words. 
It is true that for commercial purposes it is not possible to allow this process to go too far, for its later effects are higher sweetness, accompanied by such juiciness that the fruit can not endure transportation. But go to the tree to apply the only test which can fairly be put to a juicy fruit, and the demonstration of the service of clear, unobstructed sunshine through an adequate period is complete. But if this can not be done, place the judgment upon the mature peach carefully sun-dried and intelligently cooked, or upon the ripe peach skilfully canned, and the distinctive adaptations of California for fruit production will display themselves.

But there are other agencies involved in the perfection of fruit than intensity and duration of light and heat. Without adequate moisture in the soil, the air which we have credited with such benign power in carrying heat and light for perfection of fruit would transmit the same as agencies for the destruction of the tree which bears it. If this moisture comes from rainfall, it descends at the time of the year when the tree is least active, consequently is least retarded by a clouded sky and moisture-laden air, and least affected by atmospheric disturbances. Strong storm winds find the tree with reefed sails, and able to endure pressure which would tear it to pieces if they came upon its grand spread of foliage on branches heavy with fruit. It is a priceless horticultural endowment that no tornado can pierce our protecting mountain-barriers, and that it is exceedingly rare that our local winds disturb the confident swaying of the branches and leaf movement beyond the activity which ministers to the sap flow. And if the adequate moisture is not from rainstorm, but by irrigation, the same facts remain, for the water reaches the tree without interrupting its aerial activity. Temperature is maintained, light is unobstructed, and the tree is refreshed with moisture without the chill and darkness which favor fungoid parasites. Of all the ways by which moisture could come to soils supporting fruit tree or vine, the natural by its time, and the artificial by its method, endow California with the best.

The characteristics of the California climate which have been especially pointed out in this sketch are not propitious to fruit culture when they exist to excessive degree, as in some interior or continental climates. Local conditions of altitude, distance from the sea, and exposure to the sweep of arctic winds, induce sudden and great weather changes which are serious in their effects. Excessively low percentage of atmospheric humidity, in connection with desiccating wind, often produce greater evaporation from the leaves than the roots can supply. Excessively dry air admits a parching sun heat at one time, and at another facilitates radiation of heat, until the rapid decline in temperature makes killing frosts frequent. It is evident that California has these agencies constantly held in check by her insular situation and protecting environment, and owes her wonderful adaptation to growth of tree and perfection of fruit not more to the possession of certain conditions than to the fact of their existence in moderation. 


\section{CHAPTER III}

\section{THE FRUIT SOILS OF CALIFORNIA}

The favoring characteristics of the California climates, which have been described, find their fitting complement in the adaptation of the California soils to the perfect development of fruit-bearing tree and vine. In their wonderful variety and consequent great range of special adaptations within narrow limits of area our soils also resemble our climates. As a man may sometimes find within the boundaries of an ordinary-sized farm such a difference of atmospheric conditions that the same fruit will thrive in one spot and not in another, so he may find differences in soil which will tend to produce the same results. For this reason the precise spot in which to plant any given fruit must be chosen with regard to both soil and exposure. In the chapters devoted to the several fruits, there will be an attempt made to describe the soil requirements of each, so that the inexperienced planter may not err seriously in choosing the location for each kind of fruit he desires to grow. While this is true, it will also appear in these special chapters that the choice of roots upon which to bud or graft, gives the planter a certain latitude and independence. This is of greatest value in the planting of home orchards, or orchards for local markets, in regions where the soil is not what is usually preferred for fruit production. With proper choice of stocks and wisdom and diligence in cultivation, one need hardly despair of growing good fruit on soil which will support any laudable plant growth. And yet in commercial orcharding, the secret of which is producing most abundantly and cheaply, too great attention can not be paid to choice of specially adapted soils.

It is an interesting fact that more complete and exact knowledge exists of the soils of California than of any other State of the Union, and for this knowledge the public is indebted to E. W. Hilgard, Professor Emeritus of Agriculture, and Director of the Agricultural Experiment Stations of the University of California from 1875 to 1905. For the last forty years he has given all the time he could spare from many other and pressing duties, to the examination, and, when needed, the analysis, of representative soil specimens, and to practical expositions of their nature, adaptations, and requirements in the event of exhaustion from too long cropping, and he has published a general treatise entitled, "Soils; Their Formation, Properties, Composition and Relations to Climate and Plant Growth in the Humid and Arid Regions," which includes the results of his long study of California soils and climates.

Since the honorable retirement of Professor Hilgard and of Professor R. H. Loughridge, who was for many years closely associated with him in soil work, the University instruction and research in soils have been in charge of Professor C. B. Lipman, whose original work, especially in soil bacteriology, is making notable contributions to a 
fuller understanding of the distinctive character of California soils and their intelligent ultilization.

Veny extensive studies of California soils have been recently made under the direction of Dr. Milton Whitney, Chief of the Bureau of Soils of the United States Department of Agriculture, and a new classification and nomenclature of them, from his points of view, with extended maps of their occurrence, are to be found in the special reports of the Bureau.

In 1913 a systematic and comprehensive study of California soils preliminary to their mapping and an exposition of their suitabilities was begun by Professor C. F. Shaw of the University of California.

\section{DISTINCTIVE CHARACTERS OF CALIFORNIA SOILS}

One of the most interesting and important recent achievements in soil investigation consists in demonstrating distinctive differences between soils formed under arid and under humid climatic conditions. In the development of this subject certain distinctive characters of California soils clearly appear, and they are of direct relation to the thrift, productiveness, treatment and longevity of fruit trees. These characters are: (a) lightness and consequent permeability and ease of cultivation; (b) depth, admitting exceptional root extension and penetration; and (c) richness, containing some kinds of plant food in considerably greater amounts than are found in the soils of humid regions. These characteristics, as demonstrated by Prof. Hilgard, may be outlined in this way with special reference to their relations to fruit growing.

Lightness.-California soils predominately exhibit the sandy, silty or pulverulent nature of all soils formed under arid conditions, save in case of pre-existing clay formations of former geological epochs, as well as slack-water deposts of the present epoch, all of which are substantially independent of climate. While "sand" in the humid regions means virtually quartz grains only, in the arid country it means very largely grains and powder of the other soil-forming minerals as well. While, therefore, in the humid region sandy land as a rule means poor land, in the arid, on the contrary, sandy lands are at least as desirable as heavier ones, both on the score of high productiveness, durability, and ease of cultivation, together with ready resistance to drouth.

Depth.-Another point of great importance is that the difference between soil and subsoil, which is so striking and important in regions of abundant rainfall, is largely obliterated in arid climates. Very commonly hardly a perceptible change of tint or texture is found for depths of several feet; and what is more important, material from such depths, when thrown on the surface oftentimes subserves the agricultural uses of a soil nearly or quite as well as the original surface soil. The unconcern with which irrigators proceed to level or otherwise grade their land, even though this may involve covering up large areas of surface soil with subsoil from several feet depth; the rapidity 
with which the red loam of the placer mines of the Sierra Nevada foothills is re-covered with the natural forest growth of the region, etc., are examples familiar to the residents but surprising to newcomers, who are accustomed to dread the upturning of the subsoil as likely to deprive them of remunerative crops for several years, until the "raw" subsoil has had time to be "vitalized" by the fallowing effect of the atmosphere, and to acquire the needful amounts of humus or vegetable mold. Thus the surface soil, which in the humid regions supplies the bulk of the nourishment, becomes here of minor importance, serving chiefly as a mulch to prevent waste of moisture; while the active process of nutrition occurs in the deeper portion of the soil stratum, whose composition, as well as condition of disintegration and aeration, is substantially the same as above. The second foot is rarely found to differ materially from the first, even as to humus content; for the latter, being almost exclusively derived from the humification of roots, the leaves and herbage on the surface being mostly oxidized away under the intense heat and abundant aeration of summer; it not uncommonly happens in very porous soils that the first six inches of surface soil are poorer in humus than the second foot.

Practical Results of Lightness and Depth.-The "lightness" and previousness of the prevailing soils of the arid region permit of the penetration of roots to depths which in the humid region are inaccessible to them on account of the dense subsoils, which prevent the needful access of air. This deep penetration enables even annual plants to avail themselves directly of the stores of moisture in the substrdta, at depths which in the humid region are scarcely reached save by the tap-roots of some perennials and trees; while the latter themselves reach depth never approached by them in the region of summer rains. Professor Hilgard has personally found the ends of the roots of grapevines at a depth of twenty-three feet, in a gravelly clay-loam; from ten to fifteen feet are ordinary depths reached by the root system of fruit trees. Even the roots of cereals have been found to penetrate to a depth of twelve feet in California sandy alluvial soils and to fourteen feet in loams. Such depth of rooting, when conservation of moisture is secured by proper surface cultivation, enables deciduous fruit trees to grow thrifty and bear fine fruit through six months of drouth while as many weeks of drouth may bring distress and loss of fruit to surface-rooting trees on the shallow soils of the humid region. Recent investigations at the California Agricultural Experiment Station have also disclosed that the good physical and chemical conditions of the deeper layers of our soils. have also made possible the penetration from the surface layers, of various forms of micro organisms upon which we are dependent not only for a solution of the insoluble plant food, but for the addition of nitrogen to the soil from the atmosphere.

Richness.-The foregoing conditions are rendered the more significant and effective through the third characteristic of soils formed in arid climates. The average aggregate amounts of plant-food ingredients are markedly greater in the arid than in the humid soils, wherever their derivation is at all generalized. Among the agriculturally important ingredients contained in larger average amounts in the arid soils 
than in the humid, lime stands foremost; its percentage in soils not derived from calcareous formations being from twelve to fourteen times greater in the arid than in the humid soils. Magnesia follows lime in this respect, but the average difference is only about half as great. The average content of potash in the arid soils exceeds that in the humid in about the proportion of one to three or four. But no such constant difference exists in respect to phosphoric acid. As regards humus, and the nitrogen of which it is the carrier and reservoir, its amount is usually considerably less than in the humid soils; but the total nitrogen percentage does not differ widely, because the humus of arid soils contains, on the average, from three to five times as much nitrogen as is found in the humus of humid soils, and therefore, the supply of soil nitrogen is very nearly the same in both regions, while from several causes, the humus-nitrogen of arid soils is more available to plants.

Practical Lessons from the Constitution of Our Soils.-The extraordinary depth of our soils, which reveals a favorable physical, chemical and biological nature, teaches the importance and essential nature of: (1) deep tillage; (2) deep incorporation of manures and fertilizers; (3) deep irrigation. It is clear that if we can make conditions just as congenial for the roots of plants in the lower layers of the soil as in the upper, there is but one course left open to us, namely: taking advantage of the opportunities afforded us by nature, if we would maintain the fertility of our soils. To do this we must encourage the deep rooting of our trees, and nothing in the line of soil management can accomplish the desired end so well as making available to the roots in the deeper soil layers, air, plant food and water by the methods above enumerated.

\section{CLASSIFICATION OF CALIFORNIA SOILS}

Any attempt to classify the soils of California upon scientific lines or even to describe them in their wonderful variety, according to their geographical occurrence, would lead beyond the limitations of a treatise upon the practice of fruit growing. Rather let an attempt be made to designate certain grades of soil with brief characterization of their leading features as they are related to the growth of fruits. By such a course it may be made to appear that though the soils of the State are predominantly light, deep and rich and thus eminently fitted for fruit growing, there are many degrees in the possession of these characters or any of them, in local soils, and upon this individual manifestation they rate all the way from perfection to defectiveness. Let a classification proceed then upon a descending scale.

Light, Deep Loams. - Admixture of clay with enough coarse materials to secure permeability to air and water, ease in cultivation, deep root penetration and free drainage of surplus water, produces soil of the highest adaptability to the growth of fruit trees and vines. These soils rae popularly known as loams. They are designated as sandy loams, medium loams and clay loams, according to the proportion of clay commingled with the sand or coarse materials. 
Professor Hilgard has devised the following nomenclature of soils based upon their content of clay; sandy soils, less than 5 per cent of clay; sandy loams, from 5 to 10 per cent; ordinary or medium loams, from 10 to 15 per cent; clay loams, from 15 to 20 per cent; clay soils, from 20 to 50 per cent of clay.

The coarse materials are sand grains of various sizes or rock particles in various degrees of disintegration. The fine materials are clay and rock powder, commonly designated as fine silt. Loam soils may result from deposits by flowing water or may consist of debris but little removed from local rock disintegration. They include a wide variety of materials but agree in the possession of striking adaptability to fruit culture. Some of the leading instances of such soils may be cited.

Loams of the Valley Plains.-On the east side of the Sacramento Valley low ridges and swales at right angles to the river's course come in from the foothills, forming a gently undulating plain with a fall of from fifteen to twenty feet per mile, sometimes right up to the river channels. Nearly all the soils of the east side have a reddish tinge, showing the admixture of the red foothill soil and demonstrating, by the way, that all these lands are well drained. In cuts ten to twelve feet deep, made by the sloughs, the reddish plains loam is seen to reach from six to ten feet in depth, being then underlaid by gravelly substrata. The width of this class of profusely fertile valley land, east and west, varies considerably, according to the meanderings of the rivers. Away from the water courses, the higher lands of the valleys are largely red or yellow loams, sometimes clayey and difficult of cultivation unless taken just in the right condition, sometimes gravelly and apt to dry out unless the natural water supply is supplemented by irrigation, but mostly a free-working, fairly retentive, light loam, very satisfactory for some kinds of fruit.

The soils of the San Joaquin Valley have, as a rule, a much greater admixture of sand than those of the Sacramento Valley; there is also a more distinct subdivision of the valley lands into upland or "bench" lands, and lowland or alluvial lands proper.

Upon the upland or plains soils, especially of Fresno and Tulare counties, wonderful progress in fruit-growing by irrigation has been made during the last few years. Though its summer aspect is most forbidding and almost desert-like in lack of vegetation, the application of water has shown exceptional quickness of growth, early bearing, and lavish productiveness of tree and vine. These plains loams vary in appearance, and are from this fact locally named, "reddish loam," "white ash," and "sand hill." All are distinctly calcareous. Even in the case of the latter, which is the lightest and made of almost 90 per cent of inert sand, it is so deep and has its plant food in such highly available condition that it is producing very large crops of fruits where there is no rise of the bottom water to prevent root penetration. In the foothills of the Sierra Nevada there are some loose loams of light color resulting from the decomposition of granite, but they are as a rule inferior to the red foothill soils, which are more clayey, and will be mentioned among the clay loams later. 
The soils prevailing in the valley of Southern California, from Redlands at its head to Los Angeles at it opening out toward the sea, consist chiefly of granitic sand, which at some points on the slopes forms the soils exclusively, but everywhere constitutes a prominent ingredient of the valley and mesa lands. These mesa lands are conspicuous for their orange-red tint, and the red sandy loam of which they are composed, to depths varying from ten to as much as eighty feet, is evidently the choice soil for orange culture. It is manifest that at some remote epoch it filled the entire valley. Of the middle portion much has been washed away, but islands of it form red-land tracts of greater or less extent all over the region, traversed by and more or less commingled with, the gigantic wash from the valleys and canyons of the Sierra Madre. The latter frequently consists largely of gravel, and were it not for the luxuriant natural vegetation borne by these gravel beds, few would have thought of devoting them to the costly experiment of orange planting, which, nevertheless, has proved eminently successful even on these unpromising-looking masses of debris. In the upper valley (San Bernardino Valley proper) the red loam is conspicuous, and gives its name to the flourishing city and citrus district of Redlands, on the terminal slope; but the heavy flow of water from the upper canyons, notably from that of the Santa Ana River, has scoured it out of the valley itself, and left there, at least on the northern portion, gray and blackish granitic loams of great depth and productiveness, underlaid, and therefore underdrained, by the enormous gravel beds that hold the artesian water of this favored region.

The reddish mesa soils prevail through the smaller Southern California valleys as well, and are similar in character, as they are derived from similar geological formations.

Where the surface descends gradually to the seashore, and not in bluffs, there are as in Los Angeles and Orange counties, coast flats several miles in width, where the soil is a dark-colored sandy loam, glistening with scales of mica, and more or less affected with alkali in the lower portions. Similar soils are found in tracts of greater or less extent up the coast as far as Santa Barbara at least. As a rule, these seashore lands are very productive, but fruits for them must be chosen with reference to their low level and exposure to coast influences.

The light loams of the so-called desert region of Southern California are not inferior in productive capacity to some of the best soils of the great valley, which they greatly resemble, save in the scarcity of humus, or vegetable matter. Only a detailed survey, however, can determine the tracts having an arable soil, as against those overrun by arid sand. The soil of the Colorado River bottom is highly productive, easily worked, being quite light. It is a highly calcareous soil, and now, as the water of the Colorado River has been made available for irrigation, is yielding rich returns for cultivation.

The valleys of the seaward slope of the Coast Range have mostly gray, light, and silty, rather than sandy soils, quite similar in appearance from Ventura to Humboldt county, though differing considerably 
in composition, those of the southern region being more calcareous, and apparently richer in phosphoric acid; as the coast region consists for the most part of low ranges with intervening valleys, the valleys are, as a rule, small, though a few show considerable area. In such a country the soil surface shows wide diversity with its smaller areas than on the vast stretches of the great interior valley; consequently, so far as soil goes, the coast farms are often suited to a wider range of fruits than the interior valley farms of similar size.

\section{ALLUVIAL OR SEDIMENTARY LOAMS}

These soils have been considered from the earliest plantings by Americans as par excellence the fruit soils of the great valley of central and northern California. They occur along the courses of existing streams, and extend back to variable distances, until they merge into the valley loams, or adobes. These deposits are considerably higher than the present beds of the streams, and are sometimes described as "next to river bottom." They consist of fine alluvium, with seldom any admixture of coarse materials. These river soils are usually very deep and they are naturally well drained.

These deposits cross the valley in somewhat irregular courses; they are of greater or less width according to the drainage area whence they have come. They vary also in depth, and taper down on eithe: side to the level of the red loam or adobe upon which they have been deposited. Such strips are first chosen by the fruit planters of the district in which they occur. In the valleys of the rivers crossing the eastern side of the San Joaquin Valley, there are, bordering the streams as well as Tulare Lake, considerable areas of brown to blackish loam varying from heavy to light, but for the most part easily tilled and exceedingly rich. Considerable fruit has been grown for years on these situations, and some kinds do well on these bottoms which do not show adaptation to the plains. Some even of the higher lying portions of these "black lands" support thrifty orchards without irrigation. The wider stretches of alluvial soils in the upper part of the valley, as in the Mussel Slough country and the Visalia region, for instance, are notably well adapted to fruit growing. The occasional intrusion of alkali, which must be carelly avoided, is the chief obstacle to the general approval of these alluvial lands for fruit purposes.

Soil of similar character is found in some small valleys consisting of an alluvial wash from the bordering hills which in some places reaches a depth of thirty feet or more without notable change in character. Such soils have proved very fertile and durable.

The rich river bottom, adjacent to the beds of the main rivers and sloughs of the valley, has usually a dark, rich, and moist soil, easily tilled and not subject to baking and cracking. It is largely used for the growth of vegetables and alfalfa, but considerable areas have been planted with fruit trees, especially with pears, which do not suffer from submergence of their roots for considerable time.

In the coast valleys of the State there are also very extensive areas of alluvial soils which are largely used in fruit production, as well 
as upland loams formed in place by the disintegration of local rock formations. The famous fruit region extending from Oakland southward nearly one hundred miles, including the Alameda and Santa Clara Valleys, has very large areas of alluvial soil, ranging from deep, rich blackish loams used for vegetables and small fruits to lighter loams resulting from intermixture of sediment brought by streams from adjacent hillsides with the clay of the valley bottom. It is to these deep, rich alluvial deposits that the region owes its great reputation in fruit lines.

\section{CLAY LOAMS}

Of loams containing sufficient clay to render them somewhat heavy and tenacious, there is also a great variety in California. Their suitability for different fruits depends upon selection of roots adapted to their character and upon the depth and degree of retentiveness of the soils themselves. They are more difficult of tillage than the free loams, but offer some compensation therefor in their richness and durability.

Clay Loams of the Foothills and Valley Border.-The soils of the foothills of the Sierra Nevada, throughout its courses along the great valley, vary from a moderately clayey loam to a heavy, though not uncommonly gravelly, often orange-red clay. This character seems to be sensibly the same, whether the soil be derived from the decomposition of the ancient slate bed-rock or directly from the dark-colored granites, thus creating a presumption that the two rocks are closely related. The soils are highly charged with iron to the extent of from seven to over twelve per cent, which being finely divided, imparts to them the intense orange-red tint. The soils of the foothills agree with the soils of the valley in having a good percentage of lime, while the supply of potash and phosphates, as well as of organic matter, is smaller, and sometimes low, though never apparently inadequate for present productiveness, in the presence of so much lime.

Along the base of the foothills of the Sierra there is in Fresno, Tulare, and part of Kern county, a narrow belt, irregular in width, of partly red and partly black clay or adobe, so highly calcareous as to break up, when dry, into small fragments, producing a condition that has received the name "dry bog." It is upon this that many of the citrus orchards of the Porterville and Mt. Campbell districts are chiefly grown. A white, calcareous marl sometimes occurs beneath this soil at varying depths, inducing chlorosis or yellowing of citrus leaves, owing to its impervious nature which does not allow of good drainage and therefore kills the roots through suffocation and acid production. Westward of this "dry bog" land there is a belt of reddish or brown loam soils, corresponding to those similarly located in the Sacramento Valley, but generally more clayey, and hence frequently designated as adobe by contrast with the very sandy soils of the valley at large, although properly they should be classed simply as clayey loams. This belt is eight to ten miles wide in middle Tulare county and narrows to the north and south. Here these lands have a gentle slope of ten to twenty feet per mile from the base of the foothills, and appear, to be underlaid at a depth of twelve to fifteen feet by water-bearing gravel. 
The soil is a reddish, more or less sandy, loam, changing little in its aspect for several feet. Its adaptation to fruit is shown by the products of the Lindsay region.

\section{CLAY SOILS}

Thus far a very small area of true adobe* soil has been employed in horticulture. There is a great difference in the character of what is known as adobe in different localities. Its color varies, as the popular terms "black waxy," "black," "brown," and "gray" adobe indicate. Its physical condition and chemical composition also vary greatly. The black adobe of the east side of the Sacramento Valley is easily tilled as compared with the gray adobe on the west side, which is very refractory and often largely impregnated with alkali. To render soil of adobe character useful for fruit growing, this tendency to dry out and crack, thus allowing evaporation from below as well as from the surface, must be overcome. The discussion of this point belongs to the chapter on cultivation. Adobe soils are, as a rule, rich and durable and therefore promise long fruitfulness to trees and vines with roots adapted to heavy soils, but difficulty of cultivation, excessive retention of water, and other evils are always present. Some suggestions on the treatment of such soils will be given in the chapter on fertilization.

\section{DEFECTIVE SOILS}

Although California soils are predominantly of the depth, lightness and richness best suited to the growth and bearing of fruit trees and vines, it should always be borne in mind that there are marked exceptions, and failure to observe this fact has resulted in considerable disappointment and loss. There is in California much land which is bad from a horticultural point of view and it is apt to occur even in the vicinity of lands of the highest excellence. It is, therefore, necessary to advise that the closest examination to be made before investment be made in the planting of fruits.

Although there are instances of deficiency in plant food in California soils and considerable areas of land sterile through excéss of saline and alkaline salts, these are usually indicated by the local reputation of the tracts, if the newcomer will take pains to make inquiry. It is rather the more obscure, subsoil conditions which lead to loss or failure, and they may be unknown even to men who have owned or farmed the land for years for ordinary field crops. These defects are, in the main, three:

Leachy Subsoils.-While it is best in all cases to choose deep soils for cropping purposes, it is frequently profitable to grow fruit on soils with defective subsoils. Among these defective subsoils there is frequently encountered, underlying good alluvial loams, a very pervious sand or gravel which allows of a too rapid escape of moisture and plant food. This may result in starving the tree or killing it for

*This name has been erroneously applied to the loam commonly used in the construction of adobe houses. Agriculturally, it means "a heavy clay soil," such as could not be used in building. 
want of water. Under such circumstances it is possible with three or four feet of good loam above the gravel to maintain profitably the shallower rooted trees by practicing heavy green manuring and constant summer cultivation to conserve what moisture the loam retains and by recourse to irrigation, when required.

Hardpan.- It has ferquently been observed that even where hardpan has been blasted prior to tree planting, the evil effects of waterlogging in a rather retentive overlying soil have either killed trees or made them unprofitable. This condition occurs not only on level land, but also on rather steep hillsides where on a priori considerations it would never be expected. In such cases drainage gives immediate relief and the maintenance of a good humus supply in the scil with constant summer cultivation, will usually insure good moisture conditions.

"Plow Sole" or "Plow Pan."-Constant plowing to the same depth or a failure to break up thoroughly the silty deposits at the bottom of irrigation furrows may in a few seasons produce a hardened and difficulty permeable layer of soil known, according to its origin, as plow sole or "irrigation hardpan." 'This may also be aggravated through the treading of stock and fruit pickers. Such hardene 1 soil layers interfere with root development and make for poor asration and water supply. They must be broken up by plowing or subsciling.

Rise of Ground Water.-The rise of the water table mainly due to excessive irrigation or the impermeability of one of the unclerlying soil layers is a question of the most serious significance and one which the prospective purchaser of land or the owner of cropped land must not lose sight of. For this reason precautions taken in the examination of land for hardpan, irrigation with necessary, but not superfluous, amounts of water and adequate provision for drainage wiil not only go far toward making land profitable at the time cropping is commenced, but will prevent troubles for the future through the accumulation of alkali and other baneful physiological effects on plants of a high water table.

Alkali.-The term "alkali" denotes an accumulation of salts, in a limited depth of soil, which may be of such nature and quantity as to render the soil partially or totally unfit for profitable cropping. The term has no necessary reference to the reaction of the soil, as is commonly supposed, therefore, a misnomer, and should not be confused with the term "alkaline," as referred to soil, since the latter denotes merely a "sweet" or favorable condition for the development of most of our crop plants.

The "Alkali" salts may include common salt, Glauber salt, carbonate of soda, Epsom salt, the chlorides of calcium and magnesium and more rarely some others, but for practical purposes we may take the ordinary classification namely that of "black" and "white" alkali as being sufficient for the needs of soil management. By the "black" alkali which is by far the most harmful of the sodium of salts mentioned, we mean carbonate of soda. It is so called because it dissolves out the humus and forms a black slimy layer on the surface. Very 
small quantities of this salt are sufficient to make clay soils unworkable because of the puddling effect it has on clay, and similarly very small quantities of it have the power of stopping the important process of nitrification. Since poorly aerated soils in the arid regions are favor-, able to the reactions which produce "black" alkali, especially where carbonates are plentiful or where excessive amounts of nitrate of soda are used for fertilizing, no pains should be spared to insure to soils, as nearly as possible, perfect aeration through drainage and tillage; great care should be exercised in the use of irrigation water, and nitrate of soda should be employed sparingly.

The term "white alkali" is usually applied to either common salt or Glauber salt or both in soils. Where present in sufficient quantity white alkali salts may, through evaporation of water, be deposited on the surface of the soil as a white efferescence, such as that seen in exaggerated form on the Nevada desert, on some soils of the San Joaquin Valley and on some of the Coachella Valley soils. Of the "white" alkali salts common salt is by far the more harmful and most fruit trees are not very resistant to it. They can, however, be successfully grown in very considerable quantities of Glauber salt.

The actual tolerance of fruit trees for the various salts mentioned, varies greatly with the different trees for the different salts, and it is always best, when planting on alkali land is contemplated, to consult the revised reprint of Bulletins 128 and 133 of the California Experiment Station, and after having determinations for alkali made on the soil, to be guided by the information therein contained. The same publication also contains much valuable information with reference to the reclamation of alkali land and describes conditions under which it may and may not be feasible to reclaim such lands so as to preclude the possibility of useless expenditures.

Prospecting for Soil Defects.-For subterranean prospecting, Professor Hilgard commends a steel rod not less than a quarter of an inch in diameter (round or square, preferably the latter), well pointed at one end, and provided at the other with a stout iron ring for the reception of a stout cross-handle, such as is used for post-hole augers. With such a prod, or sounding rod, not less than five feet in length, the exploration of the subsoil for hard-pan or dense clay layers becomes a matter of a few minutes. It is easy also to detect thus the presence of underlying layers of quicksand, gravel, or other loose materials through which irrigation water would waste, or which would prevent the rise of bottom water within the reach of plant roots, by the large interspaces between their grains. Any remaining doubts as to the nature of such underlying materials at particular points can then quickly be solved by the use of a post-hole auger or by digging, for thorough inspection of each foot of depth which may be found desirable. Subsoil prospecting or sampling can alsu be very easily done by boring with an ordinary carpenter's auger, $11 / 2$ inches in diameter-the shank being lengthened by welding-in a piece of steel, three or four feet in length, according to the depth it is thought desirable to penetrate. Separate samples for each foot of depth are thus easily taken. 


\section{CHAPTER IV}

\section{THE WILD FRUITS OF CALIFORNIA}

The wild fruits of California are numerous, and for the most part peculiar to the region, being either of local genera or local species of more widely distributed genera. Very few are identical with the wild fruits common to great areas of the continent. For this reason our wild fruits constitute a very interesting subject for botanical study, and they are now, perhaps, more widely than ever before, attracting the attention of botanical promologists. Viewed from the standpoint of practical pomology or ${ }^{\circ}$ horticulture, our wild fruits can not be claimed, on the whole, to have attained any very great importance.

A few fruits, as will be noted further on, have demonstrated their culinary or household value, and are locally sought for, but none have any notable commercial value. This may be due to the fact that some of our most delicious wild fruits are very exacting in their choice of conditions, and can not be moved far, even within the limits of our own State.

Another reason why we have made little of our own wild species is found in the fact that our climate favors the superior growth of the best improved fruits of nearly all parts of the world. Therefore, we have little occasion for recourse to the improvement of local wild fruits, because of superior hardiness and adaptation, as has been done in other parts of the country.

The distribution of our wild fruits is determined by limitations of areas of similar climatic conditions. In a general way it may be said that fruits are most abundant in foothill and mountain regions, and that our great valleys have always been practically destitute of them, except along streams borders. These fruits are most abundant in the northern portion of the State, but some exist throughout the State, usually thriving at higher elevations as they proceed southward.

Oregon Crabapple (Pirus rivularis).-This fruit, though more abundant in the more northerly regions of the coast, as its name indicates, is found in the northwest counties of this State. It chooses a moist situation, becomes a tree fifteen to twenty-five feet high, shows white bloom, and red or yellow oblong fruit, about half an inch long. The flavor is rather acid, but the fruit is eaten by the Indians, and was sometimes used for jelly-making by early settlers.

Wild Plum (Prunus subcordata).-This must be regarded as one of the most useful of our wild fruits. Even now, when the plum varieties of all the world have been introduced, residents in some of the Sierra regions, where an excellent variety (Kelloggii) abounds, prefer it to the cultivated fruit, both for eating and preserving and jelly-making. The typical species is widely distributed over the mountainous regions of the State, and is a low shrub with white bloom and fruit three-quarters of an inch long, of red color and inferior pulp. 
The better variety has a narrower range, forms a larger shrub, and bears a yellow fruit, larger and better than the typical species. Some attempts have been made to improve this variety by cultivation and selection of seedlings, and the results are promising, as fruit has been shown at our fairs notably better than the wild gatherings. The roots have also been used to some extent as stocks, but seem to possess no marked advantage. The late Mr. Felix Gillet, of Nevada City, reported that grafting an improved plum on the wild stock seems to cause the root to grow to much greater size than natural to it. Observation upon grafted and non-grafted seedlings in the same nursery row convinced him of this behavior. Other experimenters have condemned the stock because of dwarfing and suckering. In the early days the wild plums in the mining regions of the mountains were largely made use of and are highly praised by pioneers.

Oso Berry (Osmaronia cerasiformis).-This fruit is sometimes called the "California false plum." It has a plum-like form, onehalf inch long, and is of a rich, blue-black color, but is bitter, though not disagreeable to birds and animals, which feed upon it. The white bloom of the shrub has an almond odor. Used as a stock, the plum varieties grafted upon it have been dwarfed.

Wild Cherries (Prunus sp.).-Quite a group of wild fruits come under this generic grouping, and they have marked and widely different characteristics. The western Choke-cherry (Prunus demissa) closely resembles the Eastern choke-cherry, and bears its round, red, or dark purple fruit on a racme. It is used for marmalade by housewives in the mountain districts. This species has proved of some utility both for its fruit and as a stock for grafting in early days when better cherry stock was not available. Another species, Islay (Prunus ilicifolia), has evergreen foliage, and is a useful hedge plant.

Of species bearing fruits in umbels, or true cherry style, we have the Bitter Cherry (Prunus emarginata), which makes a handsome tree, sometimes thirty feet high, but its oval, dark red fruit is quite bitter and astringent. The bush form bearing bright red fruit intensely bitter, is the variety of Californica.

California Grape (Vitis Californica).-Along our streams the native grape-vine attains large size and fruits freely, the fruit resembling the "frost grape" of the East. The vine frequently covers and sometimes kills large trees with the density of its foliage. Some variation is reported in the species, but it is possible that some of the better kinds are seedlings from some imported species, bird planted. The species has attained something of a reputation as a phyloxeraresisting root for grafting, but it has proved exacting in its choice of soils and situations, and otherwise not desirable, and some Eastern species are now relied upon for this service.

Elderberry (Sambucus glauca).-The elderberry makes a fine tree in California, sometimes twenty feet or more in heigint, and with a trunk a foot and a half in diameter. The fruit is borne in large quantities and is used to some extent for preserves and pastry. 
Raspberries (Rubus sp.).-In the mountains of the eastern part of the State is a scarlet hemispherical berry of pleasant flavor. which is called "thimbleberry" (Rubus parviflorus.) It seems to have an advantage over a variety (velutinus) of the same species which is found near the coast and has a dry, insipid fruit. Another raspberry, which is found in all hilly and mountainous regions, both on tine coast and in the interior is Rubus leucodermis. It resembles the black-cap raspberry of the Atlantic slope, except that it has yellowish-re 1 fruit. This fruit is quite largely gathered for domestic uses, and some efforts have been made to cultivate the plants.

Salmon Berry (Rubus spectabilis).-The beauty, size, and delicious flavor of this fruit are highly commended by all who have enjoyed it in the upper coast counties of California and farther northward. The plant makes a strong bush, five to ten feet high, and delights in the woods and shady banks of streams. The praise of all who know the fruit has led to frequent attempts to introduce the plant to warmer and drier parts of the State, but such efforts have thus far uniformly failed.

Wild Blackberry (Rubus vitifolius).-This fruit should perhaps be called a "dewberry," as it has trailing, or, at most, but partially raised stems, which extend from five to twenty feet. The plant occurs abundantly on banks of streams and other sufficiently moist locations, both in the coast and interior regions of the State. Around the margin of Humboldt Bay, on land cleared by fire or axe, blackberries spring up abundantly on the denuded land. Tons of the fruit are saicl to remain after the local housewives have done their utmost in preserving and jelly-making. In the low-land region around Stockton considerable quantities are sometimes gathered for sale. The fruit, which has been held in high repute ever since pioneer days, is ob!cng, black, and sweet. The species is variable, and the anomaly, a white blackberry, has been reported from Del Norte county. The native wild blackberry is one of the parents of the Loganberry and of some of Mr. Burbank's hybrids which are widely know.

Wild Strawberries (Fragaria sp.).-We have in California two Eastern species: Fragaria vesca and F. Virginiana. Thus far these have only been reported from localities in the Sierra mountain region. Another, the sand strawberry, has been found identical with a Sunth American species, Chilensis, and it occurs along the coast, where the fruit is esteemed, and is sometimes abundant enough to gathor in quantity. A fourth species, wood strawberry, is local, and is named Californica. It bears a small round fruit and is partial to the coast region. Recently some cultural attention has been given to the wild strawberries, and varieties worthy of propagation have been reported by growers resident in the Sierra region. Mr. Albert F. Etter of Briceland, Humboldt county, has secured notable results in crossing with the wild strawberry, as will be noted in the chapter on that iruit.

Wild Gooseberries and Currants (Ribes sp.).-Some of our currant species are achieving quite a reputation abroad as ornamental shrubs, but they bear insipid fruit. The fruit of Ribes tenuiflorum is, 
however, more agreeable, and is esteemed for jellies, etc., by dwellers in its region, which is the mountain region of the extreme north of the State. We also have a species (bracteosum) which has something of the black currant flavor and a fair-sized fruit, black with whitish bloom, and very sweet.

There are also several species of Ribes which are classed with the gooseberries, but only three bear edible fruit. One of these (Ribes divaricatum) is peculiar to this coast; another (Ribes oxyacanthoides) occurs at an elevation in the Sierra Nevada and thence extends eastward beyond the Rocky Mountains. The berries are small to medium, of pleasant flavor, and well armed with spines. Another species (Ribes leptanthum), common in San Luis Obispo and Kern counties, resembles the flavor of the cultivated gooseberry, and is free from spines.

Cranberries and Huckleberries (Vaccinium sp.).-We have several species belonging to the same botanical genus as the Eastern cranberry, but quite different from it both in growth of the plant and character of fruit. The fruit of two species is reddish, but insipid. Other species ( $V$. ovatum, etc.) have dark blue or purple fruit. Some of these are locally esteemed, and the argument drawn from them is that the cranberry of commerce would succeed. It should be stated, however, that the situations in which these plants thrive are not at all according to the requirements of the bog cranberry. A huckleberry (Vaccinium ovatum) is largely gathered in the redwood region of Northern California, for canning and pie-making. The berries are juicy and delicious, and the preserved fruit has a very agreeable fivor. In one year as many as two thousand boxes were profitably gathered on the hills of western Sonoma county.

Other Berries.-There are many small, wild fruits commonly designated as berries, which are of considerable botanical interest. The fruit, too, may be said to be edible, judging by the taste of Indians, birds, and wild beasts, but not likely to be much more than ornamental in the eyes of white people. They may be briefly enumerated:

The "manzanita" (various species of Arctostaphylos, especially $A$. manzanita) the "little apple" of the Spaniards, bears a rather dry but sub-acid fruit.

The "bear berry" (Arctostaphylos uvaursi) is esteemed by the Indians both as food and medicine.

"The western buffalo berry" (Shepherdia argentia) has small acid edible fruits.

The "salal" (Gaultheria shallon), small fruit, either red or purple, is also a favorite of the aborigines.

Of "barberries" we have three species of Berberis. One, aquifolium, is called the "Oregon grape," chiefly notable for its handsome bloom, which has been chosen the State flower of Oregon. Thi fruit is dark blue and the root is said to be a febrifuge. Another species (nervosa) has a larger fruit which is esteemed in cockery; and a third species (pinnata) bears a small, pleasant-flavored fruit. It is the Lena amarilla of the Spanish Californians.

Our "service berry" (Amelanchier alnifolia) is from a quarter to a third of an inch in diameter and of a purple color. 
The "lemon berry" is a fruit of Rhus integrifolia, and is coated with an acid exudation which is said to dissolve in water and make a pleasant drink. The fruit of Rhus trilobata is said to have both a sweet and an acid coating.

The berries of the "toyon" or "tollon" (Heteromeles arbutifolia)" or "California holly," are said to be eaten by Indians, but they serve the white people a better purpose in Christmas decorations.

The "jujubs" of commerce (Zizyphus jujuba) has a local relative in $Z i z y p h u s$ parryi, which is, however, dry, and mealy, rather than juicy.

The "beach strawberry," or "sea fig," is the fruit of Mcsembrianthemum aequilaterale, a relative of the ice-plant. The good-sized fruit is gathered along the seashore, and remotely suggests a strawberry.

Wild Olive (Forestieria Neo-mexicana.) - This is a tall willowlike shrub, found in springy places on the borders of the Mojave Desert. It also grows in the Salinas Valley. It bears an abundance of small fruits which, from their botanical relationship to the olive, have attracted some attention. Experiments to determine its standing as a possible root for the olive have been suggested.

Wild Nuts of California.-The wild nuts of California are of very little commercial importance. The wild almond (Prunus Andersonii) of the eastern slope of the Sierra Nevadas is chiefly of botanical interest, although some experiments are in progress in its use as a grafting stock for the sweet almond. The California filbert (Corylus Californica) has none of the quality of the improved filberts nor even of the wild hazelnut. Our native chestnut, the giant Chinquapin (Castanopsis chrysophylla) has a sweet kernel, but a hard shell, almost like a hazelnut; its near relative, Castanopsis sempervirens or Bush Chinquapin of the Sierra Nevada and dry Coast Range, is said to have a bitter flavored kernel. The nuts of both of these species are very difficult to obtain because the fruit sets sparingly and the squirrels harvest the crop early. Our native walnut (Juglans Californica) is better in flavor than the Eastern black walnut, but its hard shell makes it of little commercial account in competition with better, cultivated nuts. The root is widely used as a stock for the English walnut.

The one native nut which is regularly sold in the local market is the "pinenut"-seeds of several species of Pacific Coast pines, particularly the "Nevada Nut Pine" or "One-leaf Pinion." Their flavor is somewhat resinous, but is agreeable.

The seeds of two species of palms, Washingtonia filifera and the Lower California Erythea armata, are sought for by the Indians, who also eat the sweetish fruit of the Yucca Mojavensis, which somewhat resembles in shape the banana, and in flavor the fig, and is called the "wild date."

The Indians also use the acorns of several species of California oaks as food, extracting the bitterness by soaking in water, and then making a rude bread of the acorn meal.

The "jajoba," or "goat-nut" (Simmondsia Californica), is a low shrub, the fresh fruits of which, deprived of their seed-coats, are eaten 
like almonds, and when dried by fire and ground they are used as a beverage, in the form of tablets made up with sugar, or as a simple infusion. Fire-dried seeds contain 48.30 per cent of fatty matter; the oil is suitable for foods and of good quality, and is said not to turn rancid. In lower California it is prepared by boiling with water.

The nuts of the California laurel were roasted by the native tribes and esteemed a great delicacy.

Cactus.-The common cactus (Opuntia Engelmanni) bears a sweet edible fruit which the Indians dry in large quantities for winter use. By long boiling they make a sauce, which, after slight fermentation, they consider especially nutritious and stimulating. The local species has been used by Mr. Burbank in crossing to secure improved spineless fruits on plants of greater productiveness. 


\section{CHAPTER V}

\section{CALIFORNIA MISSION FRUITS}

Cultivated fruits were first brought into California from the south. Mission work among the Indians of Lower California was actually begun by the establishment of the mission at Loreto by Salvatierra, October 19, 1697. The following years horses and cattle were brought from Mexico, and from this introduction came ultimately the vast herds which roamed the hills and plains of California. Probably the first seeds and plants of cultivated vegetables and fruits came about the same time, for there was a small garden and a few fruit trees at Loreto in 1701. But Loreto was not fitted for horticulture, and in the same year an expedition in charge of Father Ugarte, who is called the founder of agriculture in Lower California, crossed over the mountains to a more suitable location at the mission of Vigge Biaundo, which had been destroyed some time before by hostile Indians. Ugarte restored the mission, made irrigating ditches, and planted fruit trees and vines. This effort was successful from a horticultural point of view, for in 1707 Ugarte made more wine than would suffice for mission use, and sent some to Mexico in exchange for other goods. Thus began the export trade in California wine.

The Jesuits continued their establishment of missions in Lower California until there were fiftecn missions, at five of which there were vineyards, and presumably as many or more which had gardens with fruit trees.

The variety of fruits grown, in Lower California was small. They had figs, oranges, citrons, pomegranates, plantains, and some olives and dates. There were no North European fruits, with the exception of a few peaches, which, however, did not appear to thrive.

The Jesuits were supplanted in Lower California, in 1768, by the Franciscans. The Franciscans, led by Junipero Serra, at once pressed northward, and entered the territory which is now the State of California. Their first establishment was at San Diego, in 1769. Thence they proceeded northward, braving many perils, and undergoing great hardships, establishing missions through the coast region of the State. Credit is given to the secular head of the expedition to San Diego, Don Joseph de Galvez, representing the king of Spain, for ordering the carrying of seeds of fruits, grains, vegetables, and flowers into the new territory, and from the planting at San Diego the same varieties were taken to the twenty missions afterwards established.

Kinds of Fruit at the Missions.-It is of no little interest to ascertain how great a variety of fruits was grown in these mission orchards. Vancouver, in 1792, found a fine orchard at Santa Clara, with apple, peach, pear, apricot, and fig trees, all thrifty and promising. 
$\mathrm{He}$ also describes at the mission of San Buena Ventura apples, pears, plums, figs, oranges, grapes, and peaches and pomegranates. Robinson described the orchards connected with the Mission of San Gabriel as very extensive, having among their trees oranges, citrons, limes, apples, pears, peaches, pomegranates, and figs. There were also grapes in abundance. Edwin Bryant noticed at San Luis Obispo Mission the orange, fig, palm, olive, and grape. At the Mission San Jose he found an inclosure of fifteen or twenty acres, the whole of which was planted with trees and grape-vines. There were six hundred pear trees and a large number of apple and peach trees, all bearing fruit in great abundance and in full perfection. The quality of the pears he found excellent, but the apples and peaches indifferent. E. S. Capron, in a general enumeration of the fruits grown at the missions, includes cherries.

Early Planting by Others than the Padres.-Though the earlier Spanish population had the example of successful horticulture before them for half a century at the missions, they did not seem inclined to emulate the efforts of the padres upon their own grounds, except in occasional instances. General Vallejo planted fruit trees in Sonoma Valley as early as 1830, and of his place it is said: "It is an old and well-cultivated place, well known in all the northern portions of California while this State was still Mexican territory." Exceptions there were, also at the south. The old fruit garden on the Camulos Rancho, in Ventura county, has become famous. Freemont, writing of his observations in 1846, says that among the arid, brush-covered hills south of San Diego he found little valleys converted by a single spring into crowded gardens, where pears, peaches, quinces, pomegranates, grapes, olives, and other fruits grew luxuriantly together.

Scarcely had six years elapsed sibsequent to the settlement of the pueblo of San Jose on its present site, before the inhabitants were enjoying the benefits of luxurious fruits. Before 1805 more was grown than could be disposed of in its natural state.

Decline of the Mission Orchards.-The decline of most of the mission orchards and gardens followed the secularization of the establishments in 1834. There were a few exceptions, where the mission lands fell into enterprising Spanish or American hands. During the years of neglect, the more tender trees died, and the more hardy survived. The pear and the olive vied with the vine in withstanding drouth and the trampling and browsing of the cattle that roamed unmolested through the deserted gardens. These pears, as will be described presently, were turned to good account by the early American settlers; the olive and the vine furnished cuttings for most of the plantations made during the first twenty years or more of American occupation.

But it seems that not all the mission orchards were permitted to fall into decay after secularization. In 1846 Bryant found at the Mission San Jose two gardens inclosed by high adobe walls. The area was from fifteen to twenty acres, all of which was planted with fruit trees and vines. There were about six hundred pear trees and 
a large number of apple aand peach trees, all bearing fruit in great abundance, the quality of the pears being excellent, the apples and neaches indifferent. Other visitors to some of the mission orchards between the events of secularization and American occupation speak of being regaled with pears and milk, a dish which seemed to them ambrosial after the weary journeys overland across the deserts, or after months of ship fare.

Planting of Mission Fruits by Early Settlers.-There were quite considerable plantations, chiefly of mission grapes and oranges, by early settlers in the neighborhood of Los Angeles. General Bidwell saw in Los Angeles in 1845 the largest vineyard that he had seen in California, and the vines were the most thrifty. Wine was also abundant-even the Angelica. Los Angeles had orchards, also, mostly of oranges. The largest orange orchards at that time were those of Wolfskill, Carpenter, and Louis Vigne. During recent years the modern city of Los Angeles has been built over and beyond them.

Among the early planters of mission fruits in the northern part of the State was Yount, who planted vines in Napa Valley in 1838, and other fruits later. John Wolfskill, of Winters, saw grapes and peaches at Yount's in 1841, and J. M. Pleasant took peach pits from Yount's over into Pleasant's valley, Solano county in 1851. Dr. Marsh, on his place at the base of Mount Diablo, had, in 1842, a mission grape vineyard more than an acre in extent, and in good bearing. The vines were planted about 1838. Mr. Wolfskill planted a few vines on Putah Creek in 1842.

Partial Revival of the Mission Fruit Gardens.-After the incoming of Americans in 1849 some of the old Mission trees were secured by enterprising men, and made to renew their youth by pruning, cultivation, and irrigation, that they might minister to the great demand for fruit which sprang up among the gold seekers. The trees richly reciprocated the care and attention given them, and there still exists at the San Gabriel Mission old pear trees grafted over with improved varieties by W. M. Stockton in 1854. The first fruits offered for sale in San Francisco markets were from the pear trees of Santa Clara and San Jose Missions, and from the mission grapevines of the same localities, and of Los Angeles county. These grapes, packed in sawdust, came up the coast by steamer, and were then re-shipped to the mining camps, arriving for the most part in good condition, and were very popular. It is recorded that one thousand five hundred tons of these grapes were sent from Los Angeles county to San Francisco and the mines in 1852. Another instance in which thrift followed neglect is seen in the fact that, in 1858, Don Andres Pico, who succeeded to possession of the orchard at the San Fernando Mission, did a considerable business in drying pears and other fruits, using the labor of the Indians.

At the present time vestiges of the old mission orchards still remain, the pears and olives still bearing, and in some cases the old date palms guarding the desolate scenes, or standing as reminders of the old regime, while the new life of California is surging up around them. 


\section{RUSSIAN FRUITS}

The second introduction of cultivated fruits to California was by the Russians. The exact date of their planting at Fort Ross on the ocean side in Mendocino county, is not known, but is believed to have been as early as 1812. The survivors of the original Russian planting look "very old and mossy, and are not very thrifty, but still bear some fruit every year." They were planted too closely, and have undergone periods of neglect, no doubt, The trees are apple for the most part, but there were also cherries, and some of both fruits survive. The trees are all believed to have been grown from seed, and if this be true some fortunate results were obtained, for there is still grown in Green Valley, Sonoma county, a medium-sized, bell-shaped apple, lightly striped with red, which is called the Fort Ross or Russian apple, and was probably propagated by grafts from the Fort Ross orchard. Seeds were also secured from this source for propagation of apple trees in early days in that section of the State. 


\section{INTRODUCTION OF NEW VARIETIES}

The first cultivated fruits of old era came to California with the padres. The first fruits of the new era came with the American pioneers. Though not a little inquiry has been made, it is not yet possible to declare definitely who brought the first budded or grafted trees upon California soil. It is a tradition in the family of Martin Lelong, who came to California as a member of Stevenson's regiment in 1846, that he brought with him a small lot of trees of French varieties of apples growing in a box, and that they were planted in Los Angeles.

In the fall of 1849, W. H. Nash joined with R. L. Kilburn in ordering from a nursery in western New York a small box of thirtysix fruit trees, which packed in moss, well survived the journey around the Horn, arriving and being planted in Napa Valley in the spring of 1850. The shipment included Rhode Island Greening, Roxbury Russet, Winesap, Red Romanite, Esopus Spitzenburg apples; Bartlett and Seckel pears, Black Tartarian and Napoleon Bigarreau cherries.

Before this introduction of grafted fruit trees, and, indeed, for several years afterwards, there, were many shipments, of fruit-tree seeds from the eastern States to California. Mr. Barnett planted Kentucky seed as early as 1847 in Napa county. T. K. Stewart brought to California with him, in 1848, about two hundred pounds of vegetable and fruit seeds, the latter including peach, pear and apple, all of which were planted on the American River, within the present limits of Sacramento, in the spring of 1849. At the same time he planted figs and olives, and, in 1851, seeds of oranges. From all these he secured bearing trees.

But these early efforts at improvement of California fruits were but faint forerunners of the zeal and enterprise which followed the great invasion by gold seekers. As soon as the first thought-to get gold directly from the soil-would admit the second-to get it indirectly, by agricultural and horticultural arts-there came a demand for something better than the wild fruits of the mountains, better and more abundant than the fruits from the mission orchards. At first everything in the line of fruit-tree seed which could be obtained was planted. Thus the immediate vicinity of the mines soon began to show growing fruit trees. But seedlings of any kind would not satisfy the planters, and effort was put forth in every direction after grafted trees of the best varieties. Oregon had a few years the start of California as an inviting field for immigration and the advantage also of winning the attention of those who went out, not as gold seekers, but as agricultural producers. Oregon had grafted trees in bearing, and nursery stock as well, about the time the demand sprang up for it in California. Its introduction was then, however, of very recent date. Up to 1847 
the cultivated fruit of Oregon consisted of seedlings introduced by the Hudson Bay Company, in 1824, and by the early settlers from the Mississippi Valley. In that year occurred the first considerable, if not the very first, introduction of grafted fruit upon the Pacific coast. The story of that venture has been so often wrongly told that it is well to record its interesting incidents in the words of one quite near to the event, if not actually participating in it. Seth Lewelling, of Milwaukee, Oregon, writes:

In 1847 my brother, Henderson Lewelling, crossed the plains from Henry county, Iowa, to Oregon, bringing with him a pretty general variety of grafted fruits. He fitted up a wagon for the purpose, selected small plants, and planted them in soil in the boxes and watered them to keep them alive. He told me that in some places he had to carry the water a mile up the mountains to save his trees. When he arrived in Oregon, late in the fall, he had something over three hundred plants alive. The same fall William Meek arrived in Oregon with a few varieties of fruit trees. He and my brother put their steck together, and commenced the first nursery of grafted fruits on the Pacific coast. It was situated five miles south of Portland, just below Milwaukee, on the east bank of the Willamette river. For want of seedling stock, they could not increase their nursery much until, in 1850, my brother John and I crossed the plains, bringing with us some apple seed, which we planted that winter. We also found a gentleman named Pugh, in Washington county, Oregon, who had planted some apple seed in the spring of 1850 , which had grown well, and we bought his stock. During the winter of 1850-51 we put in aoout twenty thousand grafts. In March, 1851, I went to Sacramento, taking with me a box of grafts of apple, pear, peach, plum and cherry, and sold them in Sacramento. I believe I have the honor of being the first to distribute grafted fruit in California.

Other Early Introductions.-The introduction of grafted trees, for sale by Mr. Lewelling in the spring of 1851, was quickly followed by other commercial importations, and by shipments by planters for their own use, so that the plantings of 1851-52 were quite large. Still there was great doubt as to the success of the trees. The late G. G. Briggs, after his great melon profits of 1851 , went back to New York State for his family, and, returning to California, brought with him, as he says, "with no idea that they would succeed, but as a reminder of home," fifty peach and a few apple and pear trees. To his surprise, the trees grew well in 1852 , and the next year blossomed and bore some of the best peaches he ever saw. The pears also bore some fine fruit the same year.

Besides the introduction of grafted trees which have been mentioned, there were others in 1852, for, at a fair held in San Francisco in 1853, there were several kinds of apples, grown by Isaac A. Morgan, of Bolinas, on trees planted the previous year. Apples were also shown from Napa. David Spence, of Monterey, showed the first almonds grown in California. During the winter of 1852-53 the distribution of grafted trees must have extended widely over the State. Five dollars for a small tree was frequently paid at the nursery of Meek and Lewelling, in Milwaukee, Oregon, and the trees were carried overland into the mining districts of California, as well as brought to San Francisco for distribution through the valleys.

Fruit Gardens, not Orchards.-It is interesting to note that much of the pioneer effort was expended upon fruit gardens rather 
than fruit orchards. Two ideas, at least, led in this direction. One was the popular thought, which, however, was very early found to be erroneous, that frequent and copious irrigation was essential to the growth of fruit in this dry climate. Another was the ambition, which was correct, both from a horticultural and commercial point of view, to secure the fruit just as soon as possible, for the double purpose of determining what was adapted to the novel conditions, and to secure the magnificent prices which fruit commanded in the market. For these ends dwarfing stocks naturally suggested themselves, and were employed to an extent which seems wonderful when it is remembered that now hardly a fruit tree in the State is worked upon a dwarfing stock. Very early, say from '52 to '58, at San Jose, Oakland, Stockton and Sacramento, small areas, which would now only be considered respectable house lots, were turned to great profit with dwarf pear and apple trees. The place of Mr. Fountain, near Oakland, was called, in 1857, "The finest orcliard of dwarf trees in the State." It consisted of three acres set with one thousand six hundred apple and pear trees, all dwarf from root grafts, two years old, and four feet high, and most of them in good bearing. He started the branches from the ground, pruning severely, and heading in during the winter. $\mathrm{He}$ claimed that dwarfing gave him better and larger fruit, and from two to three years sooner than with standard trees. He did not irrigate, but plowed frequently, four inches deep, up to the first of June.

But though these dwarf-tree gardens were formaily declared "to be the fashion," and though the list of stock of one Sacramento nurseryman, in 1858, included ninety-five standard and eight thousand and sixty-eight dwarf pear trees for sale, the foundations of the greater orchards were early laid upon the basis of standard trees. Thus the Briggs' orchard, of one thousand acres on the moist land of the Yuba, was planted with trees sixteen feet apart each way, and Mr. Lewelling, and other early planters on the rich lands of central Alameda county, adopted about the same distance.

Quite in contrast, too, with the prevalence of dwarf trees, and contemporaneous with it, was the grand plan upon which the pioneer of pioneers, General Sutter, laid out his orchard on Hock Farm, on the west bank of the Feather River, eight miles from its junction with the Yuba, of which the following description was written about the time the trees were coming into bearing:

Several acres were set apart for an ornamental fruit orchard, the trees and shrubs being so arranged as to present a unique landscape garden, nearly every article in which is productive of fruit. The arrangement of the fruit trees is peculiar, a large portion of them being set on either side of the broad avenues opening through the extensive grounds in various directions, imparting to the whole an air of picturesque beauty seldom seen.

But neither the narrow dwarf-tree garden plan nor the broad landscape-garden plan has survived. Neither of them harmonized with the commercial idea of orcharding-large production and economy of cultivation, and both are now but curiosities of the early horticulture of California. 
Irrigation Abandoned.-The early abandonment of dwarf trees suggests also the early abandonment of irrigation in the valleys of Northern California-as early as 1856 . Facilities which had been secured for irrigation of orchards were allowed to go unused, because it was thought better not to use them. One case is reported in Napa county where means to furnish the orchard with thirty thousand gallons of water per day were allowed to lie idle. The substitution of cultivation for water, of course, attended this reform. The announcement of a practice, in 1856, "to plow deep, dig wide and deep holes for planting, and work the ground from February to July, allowing no grass or weeds to grow among the trees," shows that the thorough and clean culture, for which California is famous, is not a recent idea in our practice. Even the abandonment of the plow, and almost weekly use of the cultivator, was the practice of some growers in the San Jose district before 1860. In fact, the descriptions of orchard management in that day include nearly the whole variety of methods which now prevail. Later experience has, however, shown that irrigation facilities are more valuable even for deciduous fruits than was once thought possible. This proposition will be discussed in the chapter on irrigation.

Early Wisdom and Enterprise.-It is evident to anyone who studies the records, that California was very fortunate in numbering among the early settlers so many men with horticultural tastes, skill, and experience. The rapidity with which fruit trees were multiplied, and the confidence with which these early comers entered upon the nursery business, shows their training. Although there were many trees brought here from the East and from Europe, they constituted only a very small percentage of the plantings of the first few years, but the orchards, with the exception of a very small number of trees introduced to furnish grafting and budding stock, were the product of the soil. When this is borne in mind, it becomes all the more wonderful how so much could be done in a new country, in a distant part of the world, in so very short a time. It was an observation which was put upon record as early as 1856, that "some varieties of fruit are much improved by change to this State, and some are not benefited." The test seems to have been that if a variety was not better than at the East, it should be discarded.

The First Oversupply.-The wonderful stimulus given to the fruit interest by the results obtained in growth and in marketing, soon induced larger plantings than the demand warranted. In 1857 it was publicly stated that "there are single farms in this State, containing each over half a million fruit trees in orchard and nurseryone person owning enough trees, when fully matured, to produce as much fruit, other than grapes, as will be sold this year throughout our State. The day is not far distant when fruit will be an important crop for raising and fattening swine." This was, to a certain extent, a statement of a croaker, for plantations continued, rare varieties were brought from the East, the South, and from Europe; the growth of some fruits continued to be very profitable and the nursery business, 
confined to fewer hands, was profitable also. The idea that quality rather than size should be striven for, led to more discrimination in propagation and better treatment of trees.

The decade from 1858 to 1868 was one of quiet in the fruit interest of California. Many of the too hastily and carelessly planted trees died from lack of proper cultivation and pruning, and the borer wrought sad havoc. In 1860 and 1861 there was serious depression. It is recorded that peaches were worth but one cent a pound, and many were allowed to go to waste as not worth gathering. The flood of 1862 destroyed many trees along the Sacramento River, and replanting was slow until prices began to improve, as they did soon afterward. The rapid development of the mining interest in Nevada, and the construction of roads across the Sierras, opened the way for the disposition of much fruit growth in the foothills and in the region around Sacramento.

The imports of dried and canned fruits were large, and growers were exhorted to take steps to secure this trade for themselves. Something was done in this direction, for by 1867 the local product of canned fruit was equal to the demand. Drying did not advance so fast; for two years later there were imports of six thousand barrels of dried apples, while the hundreds of thousands of bushels of the fruit were rotting under the trees in our orchards.

The decade under review was also notable for the first appearance of cured raisins and prunes at the State Fair of 1863. The raisins were from the Muscat of Alexandria grape, and the report states that so-called raisins exhibited previous to that time were merely dried grapes. Dr. J. Strentzel, of Martinez, was the first exhibitor of Muscat raisins, and he exhibited also dried grapes of four varieties to show the contrast between a raisin and a dried grape. J. R. Nickerson, of Placer county, exhibited the dried prunes, which were of the German variety.

Though this decade was one of uncertainty and doubt, there were rich lessons of experience learned, and the foundations for coming greatness were well laid. Many of our leading lines of production trace their beginnings to this period, and their later developments have been beyond any anticipations then cherished.

The New Era.-Another era in California may be marked as beginning with the year 1869 , because then the first fresh fruits were sent East over the newly-opened overland line. The first season's shipments amounted to thirty-three tons of pears, apples, grapes, and plums; in 1870 seventy carloads, or about seven hundred tons, were sent.

The Eastern shipment of fresh fruits began its new era with the year 1886, when the first full train load of fifteen cars of fresh fruit from deciduous trees went overland. Shipping train loads of oranges from Southern California began at an earlier date.

During the present decade shipments of fruit and fruit-products have increased until a very large aggregate in weight and value has 


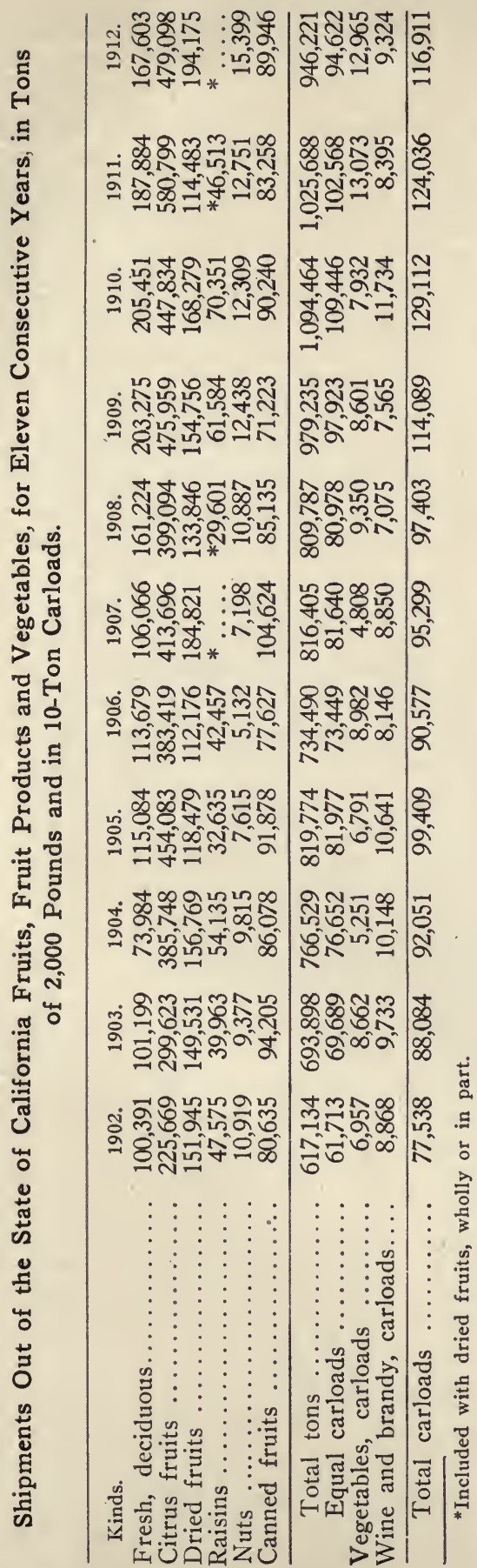

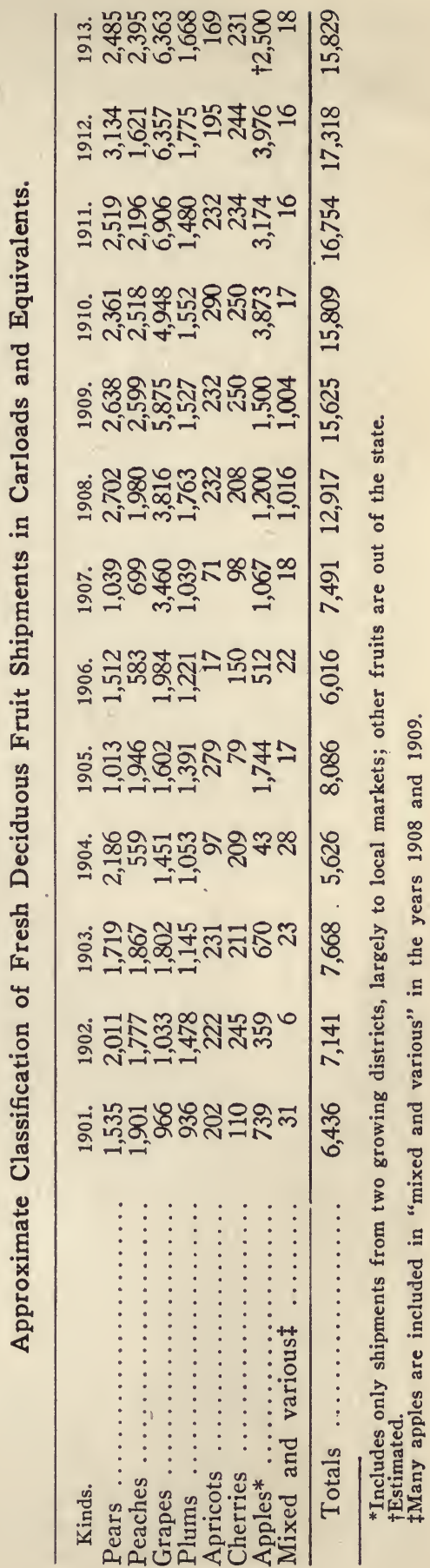


been attained. The volume of shipments beyond State lines is shown by the statement on the next page compiled from the records of the California Development Board.

A full statement of citrus fruit production and shipment will be found in Chapter XXXII.

The Fruit Interest of 1914.- The fruit interests of California now constitute the greatest single industry of California, and the fruit output of California is far greater than that of any other State in the Union. Notable progress has been secured in planting, in the growth, preparation and marketing of the product, in the contest with injurious insects and plant diseases, and, in fact, in all things which contribute to success. It is true that there are problems still unsolved, and there have been grievous losses to individuals who have proceeded upon too great expectations or have erred in location for various fruits. Such mishaps will be less frequent in the future. At present there is a disposition to proceed more cautiously and to profit by the lessons which have been learned, many of which will be mentioned in their proper places in later chapters.

Some dimensions of the present fruit interests may be suggested by the following statistics showing numbers of trees and vines, which have been carefully compiled from reports of the assessors of the counties submitted to the State Board of Equalization. As the enumerations were made as a basis of taxation they are not likely to be exaggerated. Though imperfect, they are the best available.

Number and Acreage of Fruit Trees and Vines in California, 1912.

\begin{tabular}{|c|c|c|c|c|}
\hline Fruit. & Bearing. & Non-bearing. & Total. & Acreage. \\
\hline Apple & 2,3 & $1,523,5$ & $3,876,409$ & 51 , \\
\hline Apricot & $2,877,5$ & 11 & 3,37 & 44,944 \\
\hline$\ldots \ldots \ldots \ldots \ldots \ldots$ & 505,123 & 231,087 & 736,210 & 8,180 \\
\hline Pear ... & $1,398,741$ & 620,344 & $2,019,085$ & 25,238 \\
\hline Peach & $8,341,036$ & $2,104,707$ & $10,445,744$ & 04.459 \\
\hline Nectarine & 58,0 & 5,739 & 63,773 & 637 \\
\hline $\ldots \ldots \ldots \ldots \ldots \ldots \ldots \ldots \ldots$ & 1,178 & 317,960 & $1,496,396$ & 14.964 \\
\hline $\operatorname{Pr}$ & 7,92 & 1,123 , & 9,044 & 90.441 \\
\hline Quince......... & 20 & 3,324 & 23,705 & 220 \\
\hline Fig $\ldots \ldots \ldots \ldots \ldots$ & 330 & 219 & 549 & 21,995 \\
\hline Olive & 1,056 & 17 & 1,22 & 19.951 \\
\hline Lemon & 1,81 & 1,471 & 3,286 & 43,979 \\
\hline Or & 9,022 & 4,146 & 13,168 & 146.317 \\
\hline Alr & 1,38 & 678 & 2,063 & 27.512 \\
\hline Walnut $\ldots \ldots \ldots \ldots \ldots \ldots \ldots \ldots$ & 786 & 839 & $1,626,072$ & 40.652 \\
\hline Grapes, acres $\ldots \ldots \ldots \ldots \ldots \ldots \ldots$ & 263 & $82, \varepsilon$ & ......., & 346,673 \\
\hline Berries, acres $\ldots \ldots \ldots \ldots \ldots \ldots$ & & 2,019 & $\ldots \ldots \ldots$ & 14.677 \\
\hline Totals & & $* 14,037,582$ & $53,001,129$ & $1,002,523$ \\
\hline
\end{tabular}

*Acreage of grapes, acres and berries omitted.

It is interesting to estimate the total value of the annual products of California trees and vines, using the best data and judgment available. 
Rank of California in the United States in the Production of Fruits-From the Last U. S. Census.

\begin{tabular}{lcr} 
Kind of fruit. & Rank among states. & Value of product 1909. \\
Almond & First & 700,304 \\
Apple & Ninth & $2,901,622$ \\
Apricot & First & $2,768,921$ \\
Cherry & First & 951,624 \\
Fig & First & 260,153 \\
Grape & First & $10,846,812$ \\
Lemon & First & $2,976,571$ \\
Olive & First & 401,277 \\
Orange & First & $12,951,505$ \\
Peach & First & $8,563,427$ \\
Pear & First & $1,660,963$ \\
Plum and prune & First & $5,473,539$ \\
Walnut, English & First & $2,247,193$ \\
Berries & Third & $1,789,214$ \\
Total value* & First & $50,704,834$ \\
\hline
\end{tabular}

*Including minor fruits not listed.

Thus it appears that California leads the other states in every fruit except two. California also leads in the total value of all fruits produced in all states-producing in fact about one-fourth of all the fruit grown in the United States.

Four crops have been gathered since the census year, 1909, and the California fruit interests have notably advanced. Including this increase and using the commercial value of the fruits as they reach the markets instead of "farm value" which the census gives, a total value of these products in 1914 is estimated to be about one hundred millions of dollars.

\section{INFLUENCE OF THE FRUIT INDUSTRIES UPON CALIFORNIA DEVELOPMENT}

Enlistment in California fruit growing has proved exceedingly satisfactory to tens of thousands of people in the various ways along which they have approached it. The fruit districts are full of cottage homes sheltering families of those who have begun with small investments and have made a good livelihood, and often considerably more, from a few acres of fruits grown largely without expenditure for hired labor. The study of the needs of the tree or vine and ministering to them by personal effort has brought new health and new incentive to the worn and weary who have taken up outdoor life and activity in California fruit growing with a wise choice of location, land and fruits, for obviously in all investments one must be wise as well as willing.

In large operations hundreds have notably succeeded by purchasing good land in large tracts at low rates and making ample investment for its development and improvement. Some of the most delightful of our towns and villages have arisen as a direct result of such employment of capital. Well established communities, well churched and schooled, well provided for in local trade and transportation, have followed investment and devoted effort in colony enterprises. 
Hundreds, also, have purchased large tracts of wild land and have developed fine estates for their own personal gratification, with thriving orchards of all kinds of fruits, rich pastures tenanted with improved livestock, parks, gradens and buildings comparable with the estates of the European nobility, except that California conditions favor freedom and variety in outdoor effort unknown in Europe, and command proportional interest and enthusiasm. Estates for winter residences in California are exceptionally desirable, not only because of natural advantages and greater possibilities of development, but because of the advanced standing of the State financially and socially.

All of these lines of effort, then-home-making in a small way, colony enterprise and private estate development-have yielded on the whole great satisfaction and success. Fruit growing has been the central idea in nearly all of them, but it is obvious that activity in any productive line begets opportunity for other lines, and so all branches of agriculture have advanced and the diversification is highly desirable. Opportunities in manufacture, trade and professional effort of all kinds have been quickly seized and developed with much originality and success. Fruit growing has created them all and has in turn been advanced by all, for every accumulation of capital promotes it. Successful toilers in all lines become planters. The ancestral delight of the race, to sit beneath one's own vine or fig tree, is nowhere more enthusiastically manifested than in California, and nowhere else does the emotion of comfort in ownership yield such profound and:protracted satisfaction.

\section{THE OUTLOOK OF THE INDUSTRY}

The outlook for Cálifornia fruits and fruit products involves considerations of much economic interest. Though the volume is already large and there may be experienced now and then temporary dullness or depression in this line or that, the business is on the whole brisk and profitable. There is such a wide range in the fruits grown and the products made from them, and such changes in local conditions in the many purchasing States and foreign countries with which Californians deal, that there must be some fluctuations in the values of some of the supplies offered in distant market. The result is that first one fruit and then another one seems to be more or less profitable. The fact, however, that all are increasing in volume and the total traffic brings each year more money to the State, is a demonstration of the standing of the collective output. Each year new markets are found, both at home and abroad, and the capacity of old centers of distribution is shown to be greater than anticipated. There is every reason to expect that the products can be profitably multiplied. There have been secured, largely through co-operative efforts of growers, so many improvements in handling and transportation that distant shipment has become more safe and profitable and distribution far wider. It is reasonable to believe that further improvement in movement and reduction of cost will be realized and the per capita consumption in the populous parts of our own country proportionally advanced. In spite of all that wintry States can do for local supplies, California can find open mar- 
kets before and after the short ripening season of the Eastern States for her early and late fruits, and can use her own midseason fruits in the drying and canning industries, though it is a fact that in the height of the Eastern fruit season a considerable quantity of California, fruit will command the highest prices because of its exceptional size, beauty and keeping qualities. The citrus fruits, so long as they are allowed to remain under the favoring tariff which now exists, will continue to supply an American product of exceptional quality and freshness, while prunes, nuts, raisins and wines will not only do this, but will push forward into the trade of Europe, as they are now beginning to do in a most vigorous manner. A very significant report was made by one of the United States Consuls in France recently that our canned and dried fruits were appearing on the shelves of all the provision shops of the smaller French towns and were being freely sold without reducing the prices of the locally grown fruit. Practically the same thing could be said of points in Germany and other European countries. The fact is that European countries can not grow fruit enough to supply their own people and fruit has been largely a luxury. California dried fruits are being welcomed by the great middle classes and are likely to become a staple of their diet. This explains the ultimate disposition of the large amounts now going direct from California to Europe.

California's exports of high-class food supplies to European countries are likely to reach values like those of the wheat and barley which we are now sending to that part of the world. The development of adjacent territory on the American continent and other Pacific countries may shape the future of California as a fruit producing State in a way which can at present only be dreamed about. It should be remembered that California has a unique character from a horticultural point of view. Not only does the State have a monopoly of semi-tropical conditions of the United States (excepting small parts of the Gulf States and Arizona), but California has command of the whole of northwest America and the whole of northeast Asia, not only in the supply of semi-tropical fruits, but in early ripening of hardy fruits as well.

California does not grow tropical fruits, as has already been conceded in Chapter I. They must come from the islands and the tropical south coast countries. Semi-tropical fruits are, however, vastly more important in commerce than tropical, and a region which successfully combines northern orchard fruits with the whole semi-tropical class commands the fruit trade of all accessible populous regions which have limited fruit capabilities. There are now four such regions with the kind of population which makes for industrial advancement-Southern Europe, South Africa, parts of Australia and California. As already shown, we are competing successfully with South Europe in the capacious markets of North Europe. South Africa and Australia are unfortunate in lying in the southern hemisphere, which is mostly ocean wastes, and they are handicapped by tropic crossing in their northern shipments, although the fact of opposite seasons may help them, and also us, in avoiding competition of trade which both desire. California will soon be less than half as far by sea from European and Atlantic coast ports as at present, but California in the future will have less 
occasion for such distant recourses. Prophets, far-seeing in world courses, declare that the Pacific ocean is to be the arena for commerce greater than the world has yet seen, and the Pacific coast countries are to contain the greater part of the world's population. This greatest quartosphere with its superlative opportunities and activities will have California as its treasure house of fruits and fruit products. During the long winter the citrus fruits will afford tonic and refreshment, and before hardy fruits bloom in northern climes the same fruits will appear from the early ripening districts of California. In this traffic California will not only be practically without a competitor, but, sitting beside the sea, there will also be every advantage of water transportation and the sustaining ocean temperatures of the fruits in transit. California dried and canned fruits will render acceptable diet even though the most Arctic stretches along which development may advance in North America and North Asia, while a succession of fresh fruits will flow to all Pacific ports throughout the year. California, too, will be the winter residence for all the North Pacific millionaires and the haven of rest and recuperation for all who are worn by Arctic cold or tropic heat throughout the great circle of the Pacific ocean. Here the arts will flourish, education attain its highest achievements and culture prevail. Then fruit growing both as a commercial enterprise and as a home delight will attain value, volume and perfection, of which present achievements are but a faint foreshadowing. 


\section{PART TWO: CULTURAL}

\section{CHAPTER VII}

\section{CLEARING LAND FOR FRUIT}

The greater part of the orchard and vineyard area of this State was naturally almost clear for planting. The removal of large trees, which paid the cost of the work in firewood, or the grubbing out of willows on some especially rich bottom land, was about the extent of clearing which our earlier planters had to undertake, and many of them perhaps never had to lift an axe. Still there has always been some clearing done, here and there, even since the earliest days, especially upon hill lands, the peculiar value of which for some fruits is generally recognized.

The lands which need clearing are in the main foothill slopes of the Coast Range and the Sierra Nevada. In the south there is besides, sometimes, the debris of the desert flora to clear away when water is secured and the rich wilderness is subdued. This work is, however, so easily accomplished that it hardly rises to the dignity of "clearing," as understood by the Eastern mind.

It is not possible in this connection to enumerate all of the great variety of shrubs and trees which the settler lays low in his clearing. The grand trees which figure most largely in lumbering operations are not met with as a rule in foothill clearings. The trees which the settler encounters are rather the degraded valley growths, which, though assuming grand proportions in the valleys, become "scrubs" amid the harsher environment of the hillsides. This is notably true of the oaks and some other trees.

Chamisal and Chaparral.-Of true shrubs to be removed, it will only be possible to name a few of the most abunant. The common manzanita (Arctostaphylos manzanita) occurs on dry ridges everywhere, both on the coast and at great elevations, sometimes only growing a few inches from the ground, sometimes rising eight or ten feet. Next to this, perhaps, the two terms which the land clearer has most to use are "chaparral" and "chamisal." To distinguish between them it may be said, however, that the term chamisal properly applies to the shrub Adenostoma fasciculatum var. obtusifolium, which is abundant on dry soils in the Coast Ranges and more rarely in the foothills of the Sierra Nevada, often covering extensive areas with dense and almost impenetrable growth, producing an effect on the landscape like that of the heaths of the Old World. Another species, A. sparsifolium, with narrow, scattered leaves, is sometimes abundant on the mountains east of San Diego.

By chaparral is generally meant shrubs of several species of Ceanothus, forming dense thickets and giving its name to certain soils on which it most abounds, both in the Sierra foothills and the hillsides 
of the Coast Range, where it is known as California lilac. The genus includes the "flat brushes," as they are called, from their trailing on the ground, or low, horizontal shoots; also the "white thorn" of more upright growth.

Other Small Growths.-Shrubs of frequent occurrence also are the poison oak (Rhus diversiloba), chiefly on the north sides of hills in all parts of the State, but most abundant in the Coast Ranges, and other species of Rhus which are not poisonous; the hazel nut (Corylus rostrata), which has been mentioned in the chapter on wild fruits; the buckthorns, several species of rhamnus, well distributed on the hillsides and mountains of the State.

In some parts of the State there are also large areas of sagebrush or wormwood make up of several species of Artemisia, sage or chia, two species of Salvia, and the famous white and black sages of the bee-keepers, which are species of Audibertia, occurring chiefly on the mountains of Southern California. Add to these the spireas, the azaleas, the rhododendrons, the sweet-scented shrubs (Calycanthus), etc., and include nearly all the wild fruit trees, brushes and vines mentioned in a previous chapter, and one will gain the idea that though California is widely considered a bare State, the land clearer has a host of plants confronting him and disputing his right to the soil.

Cost of Clearing.-The cost of clearing on the foothill slopes of the Sierra Nevada and the Coast Ranges is too variable to admit of estimates except such as may be made on the spot by experienced persons. The cost varies, of course, according to the density of the growth of trees and underbrush, and the rate of wages to be paid. Though in some cases higher cost is reached, probably as a rule the expense of clearing will be from $\$ 5.00$ to $\$ 30.00$ per acre, less whatever the firewood might be worth. In exceptional cases, where there is a large growth and a good wood market near by, the wood may pay the expense or more; even the roots of chaparral sometimes sell in our cities at $\$ 3.00$ or $\$ 4.00$ per cord. It sometimes happens that charcoal can be produced to advantage; in fact, there are now orchards upon land which was secured in the first instance for the charcoal to be made upon it. Usually, however, the clearing is an item of expense and must be reduced as much as possible by working in the most economical and effective way.

Though in most cases of clearing by the actual settler himself the problem is merely one of muscle and persistence, some few hints may be given from the experience of others which may be useful. Spare time during the summer and fall can often be used to advantage with a sharp axe in trimming up the smaller trees, which are large enough to yield fencing material, and getting out posts from the redwoods and oaks, and rails and pickets from the pines. By thus using the waste material the settler can often get out enough fencing material to inclose his land and thus save considerable expense. Brush, too, which can not be made use of, can be lopped off-in short, all the sharp axe work can be done in a dry time. The actual clearing, however, should be done in winter, when the ground is wet and soft, and digging is easy or "snaking out" is possible. 
Partial and Thorough Clearings.-Orchards are planted on both partially and thoroughly cleared land. By the former practice clearing enough is done to give space for the tree holes, the debris is burned up, and the trees planted. In this kind of work the stumps are left to be taken out at a convenient season, the object being to get fruit trees to growing as soon as possible. Where one is working with little more than his own muscle, and has no capital, this sort of planting is better, perhaps, than not planting at all, but it must be borne in mind that all subsequent work will be done at a great disadvantage, and as cultivation is likely to be very imperfect, it would be a question whether in the end anything would be gained by such a plan. The encumbered character of the ground will, of course, prevent the use of the horse in cultivation until most of the stumps are removed. Aside from this decaying stumps and roots in the soil often kill the young trees; especially is this the case with old oak stumps.

Clearing land for orchard or vineyard is a very different thing from clearing for pasture, as is done in the redwood region of the northwest Coast Ranges of the State, where the stumps are untouched; the trees not taken by the lumberman are girdled and left a prey to decay and storms, and the brush slashed and burned every few years to prevent it from completely taking possession of the land. Clearing for fruit should be thorough, everything which will interfere with good cultivation removed; roots grubbed so that as little shooting up as possible is secured; the ground evened up to obviate standing water, and, where needed, arrangements made for irrigation and drainage, as will be considered later.

Removal of Trees.-The first operation in clearing will be the removal of the trees. This can be partly done in the dry season if one has unemployed time. In such case the tree is felled and worked up into fire-wood and the stump left for subsequent treatment when the. ground is moist. Unless there is idle time to employ, the whole work can, however, be better done in the winter, for then the top of the tree may be made to help pull out its own roots. This done sometimes by digging out the soil and cutting off the main lateral roots below the depth to which the plow will reach. By thus reducing its anchorage the tree will topple over, or may be pulled over with a team and tackle, and it will usually lift out its stump quite effectively.

A Steam Puller.-An arrangement for tearing out trees without digging has been used to some extent in Santa Cruz county, which is said to handle redwood trees up to four feet in diameter successfuliy. it consists of a portable engine and a "puller," which is a windlass operated by steam, from which a wire cable is carried to the tree which is to be pulled down. A strong chain is put around the tree at a distance above the ground proportioned to its diameter in such a way as to give necessary leverage. The immensely strong hook at the end of the cable is attached to this chain and the cable is slowly wound upon the reel. The coil begins to grow taut, a dull creak and strain are heard as the roots begin to be torn from the earth. Two chains are used, a second tree being prepared while the first is falling, that no time may be lost. The cable is detached from the falling tree, and a horse 
draws it from amid the debris of fallen foliage to the next victim. The extraction of roots by this method of pulling is said to be very complete, and the earth is loosened to a considerable depth.

Powerful traction engines, manufactured for hauling combined harvesters and steam plows, have also been very successfully used for the removal of large trees in land clearing.

Horse-Power Stump Pullers.-The use of horse-power devices for tree felling and stump extraction has increased considerably of late. The one which has achieved good results is a local invention called a "California Stump Puller." It is simply a specially designed capstan worked by one horse, with a wire cable five-eighths of an inch in diameter, an improved snatch lock, chains, and a drafthook to unite the cable with the chains. Power is applied to the capstan with a sweep. It is calculated that with this device, properly adjusted, one horse is enabled to produce an effect equal to the capacity of 60 horses without it, and that a 1,200-pound horse which can move a dead weight of one and a half tons for a short distance can move a dead weight of 90 tons with the devices employed in the machine: It is so rapidly adjustable that on one trial in Napa county eighteen stumps were pulled in eighteen minutes, long roots coming clear out of the ground with each stump.

The Use of Powder.-Another means for the removal both of stumps and of growing trees which has come into quite wide use during the last few years, is high explosives, which have vastly cheapened the clearing of lands, where either large trees or stumps have to be removed. Full instructions for the use of powder are furnished by the agents in San Francisco, and they often send an expert to start the work and give instruction if there is much to be done. It has been estimated that the cost of handling trees and stumps with explosives is less than one-fifth that by hand grubbing, and the ratio of saving increases as the trees are larger, as powder is cheaper than muscle.

Removing Shrubs and Brush.-In the case of removing shrubs of a somewhat tall growth, the top is made to help out the roots. This is done either with a good strong rope or a chain. To do this requires two men and a pair of horses, and two chains, each ten or twelve feet long. A chain should be placed around the bush some distance above the ground, to give leverage. If the bush is not removed at the first pull, start the horses in the opposite direction. While the driver is unfastening the chain from the chapparral, the second man can place the other chain around another bush, and the one who gets through his work first should at once assist the other. In this way the horses are kept in constant employment, and neither of the men need lose a moment's time. This work should be done when the ground is thoroughly wet.

Another rig to snake brush and small trees is contrived in this way: Use single and double block or shives with 1-in. diameter rope. On the block you pull from (the double block) use two $10-\mathrm{ft}$. chains with ring in one end and hook on other. Secure this to a good anchor bush or to three or five of them, enough to hold. Fasten them right 
down close to the ground so there is no leverage on them. As soon as you have pulled all you can reach in a circle around the anchor trees, take one anchor chain off, or start with one anchor chain, then hook on other, take in big circle on single block end. One to four chains can be used; take two half hitches as high as possible, make first chain taut; 2nd chain $2 \mathrm{ft}$. slack; $3 \mathrm{rd}$ chain $3 \mathrm{ft}$. slack, and so on-so your horses are pulling one bush at a time. Chains do not tie in hard knots when horses are pulling on them, as ropes do.

A Vine Puller.-What is called a "vine puller," because it is used to clear off old vineyard, is also available for shrub pulling. Use two wagan wheels, long pole $4 \times 6 \times 12$ pine with large strong iron hook on one end. Bolt the hook on, allow the end with the hook on to project over the axle 18 in. Bolt the pole down to axle. This gives you great leverage. Roll right along on the short end over the axle. Use a good five-eighths chain. Take double half-hitch around bush; raise the pole into air, take a short tie on the bush; start the horse and out comes the bush.

Roller and Plow.-Where manzanita grows upright, as on hills north of the bay, the same methods of extraction can be employed with it, first slashing off enough to allow adjusting the rope or chain a few feet above the ground. Where it grows lower, as, for example, on the hills of Santa Clara, the manzanita brush is gone over with a roller so as to break it down, and then the land is burned over. The roller should be rigged with a tiller (header fashion) so that the horses can push the roller and walk over the flattened brush. The only object of the rolling is to smash the brush down so that it will burn readily. When the brush is got rid of in this way, the plow is trusted to get rid of the roots. The plow should be of the pattern known as "prairie breaker," without coulter. Horses should be shod with a plate of sheet iron between the shoe and hoof to prevent snagging, and not less than four of them used. Much of the Santa Clara county vine belt was cleared in that way. Of course this method only answers for the lighter-rooted growths; tough-rooted chaparral, oak, holly, etc., must be grubbed out, unless the roots are snaked out by the tops, as has been described.

Marketable Products of Clearing.-Whether any money can be made from the results of clearing depends altogether upon local markets for wood and charcoal, and the cost of transportation to them. From clearings near large towns enough can be sometimes had to pay for the work and hauling, and along railways wood can often be shipped with profit. This can only be learned by local inquiries.

Charcoal Burning.-Charcoal can usually be sold to advantage, and wood can sometimes be profitably disposed of in this way when it cannot be marketed for fuel. A considerable acreage of unprofitable fruit trees has been disposed of in this way recently. Charcoal is made from most kinds of wood, and sometimes stumps and large roots are charred. A simple process of charcoal burning is given by an experienced burner, as follows:

To burn a pit of charcoal, the prime necessity is to perform the process of combustion with the least possible contact with air. Select a suitable place not 
too far from the dwelling, because the operation must be watched from time to time by night as well as by day. It is not necessary to dig much of a "pit" in the ground. Choose hard limbs of pine, spruce or whatever wood is most available of that kind. Dry, dead limbs, if not decayed, take for choice. Set them up wigwam fashion, close together, fitting them as well as they will allow, the apex forming the chimney. Be careful to keep the chimney free, because the fire should be there applied to brisk "kindling" as far down as possible. Build round and round, taking the precaution to lay three or four straight pieces, three or four inches in diameter, along the ground from the outside to the center. These may have to be withdrawn to promote the draught.

The wood all being in place it is now required to cover it thoroughly. In the absence of turf or sods, it must be thatched with leafy green boughs, or anything that will prevent the earth or dirt that is now heaped on from running through. Pack this soil covering carefully, exclude air as far as possible, except when the port-holes referred to near the ground are needed. The direction of the wind will determine which ones are to be opened. When the fire-after a few hours, more or less, according to the materials-has got a good hold, close also the chimney. Visit the pit regularly night and day; lessen or increase the draught as may seem needed; and in a week or ten days the two or three cords of wood should be turned into good hard coal. When uncovered, water or dirt should be thrown upon coal that is too lively when spread out on the ground.

Cutting to Kill Brush.-Just when to cut to kill depends upon the character of the growth and of the season. One conclusion seems to be that with deciduous growths the best time to cut is when they have just made their most vigorous growth, and this is in the summer -but the month to be chosen for the work will depend upon the location, though August is generally selected as the best time.

In the case of evergreens, the cutting should be just before the coldest weather, in which they are the nearest dormant. Evergreens, however, differ much in tenacity of life, for while most kinds are easily killed, the California redwood will endure almost any abuse with the axe or fire and still spring up repeatedly and persistently for years.

The Use of Sheep and Goats on Sprouts.-On sprouting brush, there is, perhaps, no cheaper or more effective means of repression than sheep and goats. They are used after the top growth is cleared away instead of grubbing, if one can wait, for by the persistent cutting down of growth, many small stumps and roots will decay enough in a year or two to be plowed out with a strong team and plow.

Burning of the Debris.-However the trees and underbrush may be wrenched from the soil, fire is the final cleaner. Where trees are to be worked up into fire-wood, it should be done as soon as they are felled, for the work is much less than after they become dry and hard. If it is not designed to break the land the first winter, the wood is left to season and it becomes lighter and easier to handle. The brush and roots, if no use is to be made of them, can be left to lie on the clearing to dry out during the following summer, and after the first rains of the following fall the whole area can be burned over. Such stumps as do not burn with the brush must be gathered in piles and re-fired. Burning before the first rain should not be attempted, unless it be in exceptional situations, because of the danger of communicating fire to the surrounding country, which is a standing danger in our dry climate. Under the present law it becomes necessary to secure permis- 
sion from the State Forester at Sacramento before starting field fires in the dry season. After the rain, clean up the ground perfectly.

First Crop on a Clearing.-It is the opinion of some cleurers in the redwood region that the soil is not fit for fruit trees the first year after the original growth is removed, and they grow a field crop the first year. They claim that peas are the best corrective of "redwood poisoning," and fortunately in the upper redwood district they have a climate well suited to the pea. Whether their theory is right or not, their practice is of advantage, because they get a better cultivation and aeration of the soil, and kill out much of the sprouting from the old roots, which is usually quite persistent in the moister parts of the State.

Surface Leveling and Draining.-There is often occasion to clear the land of stone and rocks. The latter should be blasted out of the way so that the land may be clear for the plow and cultivator. Once in a while one will come upon a stone wall inclosing an orchard in this State, as trim and true a wall as the most thrifty New England farmer can boast, but walls are not common. Our valley orchard lands are, as a rule, naturally as free from stone as they are from underbrush, but on the hills it is different. Probably the best wav to dispose of much of the stone is to dig trenches in the natural water runs, put in stone, cover with small brush, and then with soil deep enough so the plow will not reach the brush. This disposes of the stone for all time, and at the same time helps to drain the soil. Concerning other treatment of the land after the rubbish is removed, P. W. Butler writes as follows:

When water runs are wide, lateral ditches should be cut extending entirely through the moist areas. If during the rainy season a sun is likely to have more water than can be conveyed properly through a covered trench, it should be left open and graded, so that a team ian cross it, and for fifteen feet on each side sow to alfalfa, which will take the place of unsightly weeds, that would otherwise grow at the point that cannot be cultivated.

To distribute the work more evenly through the first year buildings can be erected, a well dug, and the trenches done in the dry season, while all the grubbing, leveling, plowing and planting must be done the following season, as soon as the ground is sufficiently moistened. All depressions where water would stand should be filled, and all flat places should be graded until water will readily flow off, and not be retained so near the surface of the ground as to cause it to become soured. This leveling can be best done by one man and a pair of horses. Plow the adjacent elevated land and scrape into the places to be filled. The land is now ready for plowing, and should be done thoroughly, subsoiling to as great a depth as the removal of the stumps will allow. It is now well to go over the ground again with the scraper and level all the most elevated points so they can be readily reached by water in irrigating. Then cross-plow as deeply as possible without again subsoiling, harrow and drag, and the ground will be ready to plant.

Mr. Butler writes with reference to the foothills of the Sierra Nevada, where irrigation must be practiced. Where irrigation is not used, leveling, or rather grading, may be unnecessary, but it is often quite desirable that there may be no depressions to retain surplus water. The life of the trees and ease of cultivation may demand this unless the soil should be light and deep enough to allow free drainage. 


\section{CHAPTER VIII}

\section{NURSERY OPERATIONS}

California nursery stock is unrivaled in growth, health and vigor. This is the verdict of all the visiting horticulturists, and has been formally declared by the victories of California tree growers at the World's Fairs held in this country, where the highest premiums were awarded to Californians in nearly all classes in which they exhibited since 1885 .

The quality of the trees which can be purchased at our nurseries, and the very reasonable rates at which they are sold, make it little worth while for the orchard planter to try to grow his own trees. In fact, the investment called for to purchase a good assortment of wellgrown trees will be one of the best which the orchard planter can make. The professional grower, if he is honest and enterprising, can give the purchaser the advantage of his experience and skill in the choice of stocks suited to his soil, varieties of fruit adapted to his situation, and be of assistance to him in other ways connected with his enterprise; and such helps to an inexperienced planter or to a newcomer are very valuable. There may be, however, some reader who is distant from established nurseries, or possessed of limited means, who may like to use his spare time in growing his own trees, and to such suggestions are offered. There will, however, be very much which can be learned only by actual experience.

In the selection of a location for a commercial nursery there are matters involved which it is not proposed to discuss. Attention will be paid rather to matters connected with what may be called a farm nursery. The first point will be the selection of a small piece of ground, which offers proper soil, exposure, and, in some parts of the State, facilities for irrigation.

Proper Soil for Nursery.-The soil should be a mellow loain, easy of cultivation and not disposed to crust and crack. In all respects what one would choose as a rich, kind garden soil will answer well for the nursery. The soil should be moist, but thoroughly drained, either naturally or artificially, for time and labor will be largely wasted on a water-logged soil. In this respect a soil which might yield fair crops of some shallow-rooted vegetables would not always be suitable for young trees, which, to do well, must have favorable conditions to send the roots to considerable depth. Good spots are often found in the rich loam along the banks of creeks, as in such situations one finds generally a deep alluvium, well drained by the creek. But such situations, if liable to overflow, should be rejected because standing water is not good for trees, and because the soil will be apt to be soaked with water and inaccessible just at the time when the trees should be lifted for transplanting to orchard.

It is not always possible to find an ideal nursery spot on every ranch, but still trees may be well grown on less favorable places if 
attention is given to correcting natural defects. For example, if the soil be naturally heavy, it may be improved somewhat by repeated plowing and cultivation, during the year before starting the trees. If it be an adobe, its mechanical condition may be greatly improved by the application of a top dressing of lime at the rate of six hundred to one thousand pounds of lime to the acre. For this purpose "lime waste," which contains both lime and wood ashes, can be had cheaply at the kilns. Old plaster which may have been left from house repairs is excellent. Even builders' lime would not be very expensive, for but little would be required for so small a plot of land as a farm nursery would need to cover. The lime will increase the amount of plant food in a heavy soil as well as render it more friable. Another way in which a small area of heavy soil may be improved is by the addition of sand. A few loads of sand, if it can be had near by, will remove the tendency to crack, and will act as mulch to prevent evaporation of moisture. If the soil be very loose and subject to too rapid drying.out, the remedy will be moderate irrigation during the summer, but it should cease early enough to allow the young trees to ripen their wood before the frosts of autumn. Mulches of various light, fine materials, rotted straw and the like, may be used to advantage among the young seedlings in preventing drying out of the soil, if the plot is to be hand-worked, but such materials are apt to be in the way of neat, thorough work with the horse. A mulch of sand, if available, is not open to this objection

In choosing soil for a nursery, a piece of land which has been in cultivation for garden or field crops is to be preferred over a newlycleared piece. It is often the case that soil from which old stumps or shoots have recently been removed has become soured from the process of decay in the dead wood. Although the deposits of humus from decay of woody fiber tends to enrich the soil, afterwards certain acids are formed if the land lies without cultivation. These are not favorable to the growth of young roots, and a crop to which as much time is given as a crop of young trees, should not be placed upon it. This evil quality in the soil is removed by cultivation and aeration, or may be corrected by the application of lime. This state of soil is most complained of in connection with old stumps and roots of oak trees.

Situation and Exposure.-Warmth in the soil is necessary to a good growth, and a good year's growth is essential to the production of a satisfactory tree. Drainage contributes notably to the warmth of the soil. Exposure is also of importance. Plenty of sunshine and protection from cold winds are to be secured. Sometimes a little elevation is desirable. It would be a serious mistake to seek moist, low land if the piece lies at the bottom of a little valley or depression where the cold air settles during the night and frosts are frequent. In such cases choose higher ground. Of course, in broad, open valleys there is not this objection, for such seasonable frosts as may be expected there are not injurious to deciduous nursery stock. The greatest nurseries in the State are in the open valleys, not on the lowest ground, however, in all cases, but on what would be called good, rich valley land. There are, however, situations in the thermal belts in which the temperature does not fall low enough to check growth of deciduous trees and cause 
the leaves to drop. In such cases it has been found desirable to select lower and colder ground for the nursery of deciduous trees.

Preparation for Nursery Ground.-The best preparation for nursery ground is the growth, the previous season, of a cultivated or hoed crop. This will secure frequent working of the soil, thorough pulverization of the clods, etc. The produce of the hoed crop should thus pay the cost of putting the land in good condition, at least. Where the retention of moisture is an object, as it really is in some parts of the State where the annual rainfall is sometimes small and no facilities for irrigation provided, it will perhaps pay better in the end to keep the land in bare fallow during the previous summer; but there must be frequent and thorough cultivation, keeping the surface always mellow, more moisture will be lost by evaporation than a hoed crop would require for its growth. Properly cultivated fallow soil will have a moisture within a few inches of the surface, while unworked soil adjoining will be baked hard and dry to a depth of several feet. During the winter immediately preceding planting, the green stuff should be allowed to grow for a time, but should be plowed under before it gets high enough to interfere with perfect turning of smooth furrows. The decay of this green crop is of advantage to the soil. Another plowing in the spring, and a thorough harrowing, will leave the ground in good condition to receive the pits or root grafts, as the case may be. In this plowing for nursery there should be deep work done and subsoiling, as will be more fully set forth under the head of preparing land for orchard, to which the reader is referred.

Growth of Seedlings for the Nursery.-The two chief ways of producing fruit trees are, from seedlings grown on the spot; second, from buds and root grafts upon stock imported from the East or from abroad. First, as to the growth of seedlings :

It is usual to take seeds from sources where they can be collected with the least trouble. Apple seeds are washed out from the pomace of the cider press; apples and pears from the coring and peelings of canneries and drying establishments; pits of the stone fruits are derived from the same source. Supplies can usually be purchased from such establishments at a moderate cost. The trouble is that from such supplies one is apt to get seeds and pits from all varieties, possessing different degrees of health and vigor. There is just as much to be gained from selecting the seed from which to grow good strong stocks for fruit trees as there is in selecting good garden or field seed. One can generally get good peach pits, for it is easy to have the order filled. when the cannery is running on strong-growing yellow varieties, for these are believed to be most vigorous, and yet some claim much preference for pits from vigorous seedling trees, and made extra efforts to secure them. Wherever it is possible, and if one is only to produce a small lot of trees, it is practicable to select from the fruit the seeds for planting. Not only is there great difference in the strength of different varieties, but individual trees vary greatly. If one is taking seeds from an old orchard to start his nursery with, he can take pains to get his seed from his strongest trees, and thus secure also that which is probably best adapted to his locality. 
Apple and Pear Seedlings.-For a small lot of apple and pear trees the seed can be best sown in boxes. Select plump pips and keep in moist sand, from the time they are taken from the fruit until sowing. Fill the boxes, which should be three or four inches deep, with good garden mold, cover the seed about half an inch, and then cover the soil lightly with chaff or fine straw to prevent the surface from drying out. Be sure that the boxes have cracks or holes in the bottom for drainage, and the whole is kept moist, but not wet. When the seedlings have grown to the height of three inches they can be set out in the nursery rows as one would set out cabbage plants.

Cherry Seedlings.-There are different ways of handling pits of stone fruits to prepare them for seeting out in the open ground, which will be described. The cherry is grown from pits of two wild varieties; one is commonly called the "Black Mazzard." It is the common wild cherry of the East, and is the original type of what are known as the Heart and Bigarreau types of cherries. The other is the "Mahaleb," a European wild species, which is used in the East, where it thrives better than the Mazzard, as it is hardier stock. In this State the Mahaleb does not seem to have much dwarfing effect, as trees on that stock in this State over twenty-five years old are twenty-five inches in diameter of trunk. The Mahaleb, however, ripens its wood earlier, and for this reason may be valuable in the colder parts of the State. It is also freer from root trouble by extremes of wetness and drouth in the soil, and is largely used on low lands. The Mazzard is, however, chiefly used in California. Cherry stones are sometimes taken from the fullyripened fruit, dried for two or three days, the stones cracked carefully and planted at once in good soil and kept properly moist. They will germinate soon and make a growth of a foot or so the first season. Such stocks are taken up for grafting in the winter and set out in nursery row the next spring. A better way of treating cherry is that given by W. W. Smith of Vacaville;

The fruit of the Mazzard should be allowed to get perfectly ripe on the tree, then gathered and let lie in a heap for three or four days, so that they may be partially or wholly freed from the pulp by wasining them in water. They should then be spread out in the shade and stirred frequently for about twenty-four hours. This will give the outside of the pit time to dry sufficiently to prevent molding, while the kernel itself will remain fresh and green. They should then be placed in moist (not wet) sand and kept so until the rains set in in the fall, when they can be planted in drills, in good, rich, mellow soil prepared the previous spring and kept clean of weeds through the summer, ready for the purpose. They should never be allowed to get perfectly dry; and the reason for it is that we have but little or no freezing and thawing weather in this country to cause the pits to open; but if they are kept constantly moist it answers the same purpose as freezing. The seeds of the Mahaleb cherry will sprout with less difficulty, but the same rules for keeping the Mazzards will apply to them. fruit.

Other stock for the cherry will be discussed in the chapter on that

Citrus Fruits.-The propagation of citrus fruits will be described in detail in Chapters XXXII, XXXIII, and XXXIV.

The Larger Stone Fruits.-In handling pits of the larger stone fruits, apricot, peach, plum, etc., the chief requisite is to prevent 
drying and great hardening of the pit. Some plant in the fall and trust to natural conditions to start the seedling in the spring, but this interferes with the cultivation of the ground, and leaves the seedling to grow in soil which has perhaps been puddled by heavy winter rains. There must also be much hand work done to clear the rows from weeds. It is much better to keep the pits from drying by covering with sand moderately moist, hasten the sprouting by appropriate treatment toward spring, and then plant out in thoroughly prepared soil, and they will make a satisfactory growth. The following method, by D. J. Parmele, of Vacaville, has given good results:

Keep the pits out of the sun until the rains commence in the fall, then put them into a box about a foot deep with openings at the bottom for drainage, and scatter sand or fine earth through them, putting about two inches on top, and place them under the eaves of a building on the south side, where they will get well soaked every time it rains. If there should be a long dry spell during the winter, water them a little. About March they will open and sprout. Then take a plow and open a deep furrow in loose, mellow ground, and, with a hoe, pull about two-thirds of the dirt back into the furrow, breaking the clods, and making it fine, the same as you would if you expected to plant onion seed there. Drop the sprouted pits in straight line, and cover two inches. On account of the extra work in preparing the ground, the trees will be large enough to bud in July.

Another way is to spread out the pits on a smooth piece of ground and cover with sacks, and over these a layer of straw three or four inches thick to retain moisture. The pits may be planted out as soon as they crack open, although no harm will be done if they are allowed to lie until the sprouts are well out.

Another method which has been especially recommended for treatment for almonds is the following: Lay boards upon the ground and cover them with an inch of sand; spread on this a layer of almonds and then another inch of sand, and so on. Keep the pile wet, and in three weeks of warm weather they will burst open. Plant in drills one inch deep and put over them a light coat of rotten straw.

If from any cause the pits have become quite dry, they should be soaked in water two or three days before planting.

Nut Tree Seedlings.-In growing nut-tree seedlings, much the same methods are followed as with pits of stone fruits. There are methods described in detail by California growers which should be given. As has been said, the nuts may be planted at any time after ripening, in the milder parts of the State, if the grower will undertake the greater care and cultivation. On some light soils where the rainfall is not excessive, this is not much trouble. Felix Gillet, of Nevada City, gives this as his method:

The nuts may be planted as soon as gathered, though in Nevada City it is too cold to plant them in the fall, for the frost in winter would surely lift the nuts right out of the ground. For keeping and sprouting walnuts, I throw into the bottom of a box one inch deep of sand, then a layer of nuts; put in another inch of sand, and another layer of nuts, and so on to one or two inches from the top. Then water well with a sprinkler and water again during the winter whenever the sand gets too dry. The sand has to be pretty well saturated with water, especiallv from the first of January down to planting time, which is in February, March or April, according to localities. The latter part of March or first week in April is best for Nevada City. The nuts are planted in drills and covered to a depth of two to three inches. 
In propagating chestnuts it is always better to select for seed the largest, finest and healthiest nuts; in the fall or beginning of winter the nuts have to be planted in a box of damp sand, by layers, the box being kept in a cellar. The nuts may be stored in a hole in the open ground, a layer of chestnut leaves being first thrown in the bottom of the hole, on top of that a layer of nuts, then another layer of leaves, and so on to the top, which has to be properly covered with two or three inches of earth so as to prevent the frost injuring the nuts. In February or March, according to location, the nuts are taken out and planted in drills to a depth of three to four inches; less for smaller seed like American chestnuts.

In growing seedlings of English walnuts, Mr. J. Luther Bowers, of Santa Clara, has shown that water-soaking of nuts may make it unnecessary to undertake storage in damp sand, if the nuts are of the last crop. He describes the method as follows:

The nuts should be large and thin shelled and should be of last year's crop. To ascertain this, break a few and split the kernel open at the germ end, or the point where the root starts. If the meat of the kernel shows a clear color they are of last year's crop, but if the flesh shows any discoloration they are old and will not germinate. I have often got hold of a lot that were mixed, old and new together. Never risk a lot of this kind, for failure will follow. After the nuts have been selected place them in some kind of a tin vessel; a five-gallon oil can, with the top removed, is just the thing. Then cover them with hot water at not over 110 degrees $F$. Let them remain in this water for 24 hours and plant at once, keeping them in the water all the time. Do not let them become the least bit dry, and be sure the soil is moist, and put every nut in with the sharp point exactly straight down. The root starts from this point and will go straight down, and if not molested wili the first year be about three times the length of the top; that is, if the top grows one foot, the straight tap root will be three feet or more long, and will be from three-fourths to an inch thick where it grew out of the nut, tapering both up and down.

Tribble Brothers, of Elk Grove, give the following as their practice with native black walnuts:

Gather the nuts as soon as most of them have dropped from the trees, and put them in a trench. Cover with leaves, and on the leaves put a thin layer of earth, leaving them until sprouted and ready to plant in rows. When ready to plant, assort the nuts according to the growth of the sprouts, and as we find the longest sprouts make the most rapid-growing trees, and by selecting we can get even growth in the nursery rows. In our soil we plant the nuts about five inches deep.

Imported Seedlings.-A very large proportion of some kinds of the cherry, pear and apple trees produced in this State are worked upon imported seedling stocks. These stocks are cheap, convenient to handle, and are therefore popular. It is easy enough to grow peach, almond, apricot, and Myrobalan seedlings, but small seeds, like apples and pear, often do not show up well in the spring, especially if the soil is of a kind that crusts over withl rain and sunshine. Therefore our nurserymen import these seedlings in the winter, plant them out, as has already been described, and bud in the following summer, grafting the next spring where the buds fail. If the seedlings are large when received, they are often root-grafted at once, and then one summer in the nursery gives a tree suitable for planting out. These stocks are of betten budding size during their first summer than California seedlings, which are apt to overgrow.

Myrobalan plum seedlings were formerly imported to a large extent, but are now chiefly home-grown, and seedlings are used instead 
of cuttings, which formerly were employed largely. This stock has secured great favor for plums and prunes, and in some situations, for the apricot, as it is hardier against extremes of drouth and moisture.

Prof. Newton B. Pierce, of Santa Ana, has discovered in California upon imported seedlings a serious root-fungus which kills all kinds of orchard trees in Europe, and he advises the use of home-grown seedlings to escape this danger.

Fruit Trees from Cuttings.-It is feasible to grow a number of kinds of fruit trees from cuttings, but it is not desirable in many cases to do it. Trees grown from a graft or bud in a seedling root are much better. The root system of a seedling is naturally stronger and more symmetrical. The roots from a cutting start out at the bottom and spread out horizontally and irregularly. This style of a root system is expressively named "duck-foot roots," and they do not give the tree a deep, strong hold on the soil. Trees can, however, be multiplied very fast from cuttings. Notable instances of this are the Myrobalan plum and the Leconte pear. Cuttings of deciduous trees should be taken from well-matured wood of the previous season's growth, and planted in rows and in well-prepared soil, as has already been described for the sowing of fruit tree seeds. The cuttings should be taken before the sap begins running in the winter. A cutting about ten inches long, four-fifths of its length buried in the ground, will answer. Be sure that the ground is firmed well at the base of the cutting, but keep the surface loose. Small wood is better than large, though, of course, the extreme ends of twigs should be rejected usually. Cultivation of cuttings is the same as that of seedlings, and budding, when the cuttings are to be used as stocks, is also governed by the same rules.

The orange and lemon can be grown from cuttings, but the work is done during the summer while the ground is warm. Cut from wood one or two years old; set in the open ground with partial shade and give plenty of water (dry ground is death to their tender roots), but be sure that there is free escape for surplus water. Cuttings started in the warm weather and given partial shade and plenty of irrigation are very apt to succeed. This method of growing these fruits is not, however, in wide use or favor.

The propagation of the grape, olive and fig from cuttings will be considered in the chapters on those fruits.

Planting Out in Nursery.-For planting out in nursery, the term "spring" is given as the proper time, but in California it must be remembered that spring is not any definite division of the year. "Spring weather" comes from the first of February to the first of May, according to the latitude or elevation or exposure resulting from local topography. Cherries may be ripe in Vaca Valley before fruit trees put out leaves in Modoc county; and between these extremes there are advents of spring in other places according to the situation. These facts are more fully set forth in the chapter on climate. Spring must be detected in the behavior of vegetation and not by the calendar. When the tree buds swell and the leaves appear, spring has come for 
that locality. But whether one can plant his nursery then or not will depend upon the character of the soil and the condition of the rainfall for that season. This varies much from year to year. As a rule, however, in most parts where fruit is grown at present in large quantities, the heavy cold rains will be over by the first of February, and then nursery operations can commence if the soil is in good condition. If not, the planter must wait until the soil is dry enough to work nicely. There will, of course, be heavy rains after the first of February; but they will only necessitate cultivation to loosen the soil, if the nursery ground is well situated for drainage, and if it is not it should not be used for this purpose.

Supposing the ground has been deeply plowed and thoroughly harrowed, as has been already described, the laying out of the ground is the next operation. Everything should be done with a view to the use of the horse in cultivation. The rows should be laid out as straight as possible. Some use a plow furrow; some an arrangement like a corn-marker, with two cultivator teeth set four feet apart; some stretch a line, to get the pits or root grafts as true to it as possible, and some trust to the furrow for straightness. No rule can be laid down for means to be employed; the result must depend upon the eye and skill of the individual. Some people can hardly shoot a straight line with a gun. Each must do the best he can in this respect.

There is a difference in practice as to distance between the rows in nursery. The usual distance is four feet, but others claim that it is better to make the rows six feet apart, especially where no irrigation is practised, as this gives the young trees more room, and if the ground is kept thoroughly cultivated, as it should be, it 'gives the roots a greater supply of moisture to draw upon. In growing a small lot of trees, where there is plenty of land, it is, of course, desirable to give them every advantage in the way of facilities for growth, but on the other hand, an overgrown tree is not desirable. Thrift and strength must be sought rather than size.

At the ends of the rows spaces of about twelve feet should be left as turning-ground for the horse when cultivating, and as a roadway. The length of nursery rows depends upon the taste of the grower. It is convenient to have alleys wide enough for a horse and cart at intervals of one hundred to three hundred feet, but in small nurseries the head-lands would probably give all the access required.

The depth for planting seeds and pits must be regulated by the size of the seed and the character of the soil, as is always laid down by the authorities, and in this State another condition must be made, and that is the climate or weather conditions prevailing in the locality. Where the rainfall is generally light and the soil loose, seed must be planted deeper than where good spring showers are to be expected. In fine soils seeds must be planted shallower than in coarse, even with the same rainfall. Judgment and experience must dictate in this matter, and if a man has no experience, he is pretty apt to get it.

During the spring months the cultivator must be used as often as may be required to keep the weeds from getting, too high, or the soil from becoming too densely packed by heavy rains, but the ground should 
never be worked when too wet. It requires some watchfulness and promptitude to use the cultivator just at the right time.

Nursery Irrigation.- - In parts of the State where the rainfall is adequate, cultivation thorough, the soil sufficiently retentive, and atmospheric conditions favorable, the seedling will make its growth without irrigation, and many nurseries are on ground not provided at all with irrigation facilities. In other parts of the State irrigation is necessary. Water should be applied sparingly, and yet enough to keep the seedlings in healthy growing condition. This is shown by the leaves, which should not droop or curl. Excessive irrigation should be guarded against, because a soft, excessive growth is very undesirable. Water is a good thing, and in some cases a very necessary thing, but the use of it should be wisely regulated. At budding it is necessary that the sap should be free and the bark slip easily. To foster this condition it is sometimes desirable to give a watering a few days before budding commences. Water should be applied by running it through shallow furrows between the rows, and the cultivator should follow as soon as the ground is dry enough to work freely. 


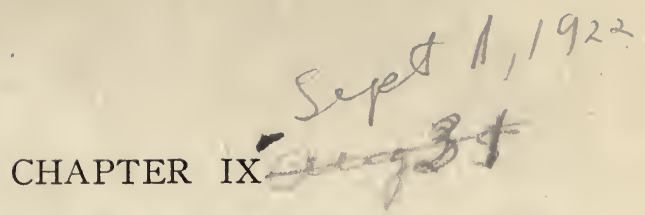

\section{BUDDING AND GRAFTING}

If the nursery ground has been well worked and the seed properly handled, the growth of the seedling will be strong and rapid. If an early start was had and other conditions favorable, some kinds will be ready for budding in June, and the production of what are called "June buds," as will be described presently. In ordinary practice, however, budding will come later, and the budding season extends from July to October. The weight of the budding of deciduous trees is generally done in August and September.

\section{BUDDING}

The process of budding, as employed on all the common fruit trees, is very simple. It consists in lifting the bark and inserting a bud from another tree in such a way that the inner bark of the bud shall come in contact with the layer of growing wood in the stock, and then it will be quickly knit to it by the new cell-growth if the bark is closed around the inserted bud closely enough to prevent the air from drying the two surfaces at the point of contact. In the engraving 1 is the cutting or "bud stick" from the tree of the kind into which it is desired to transform the seedling. This cutting is usually made from the growth of the present season, which has well-formed buds at the axils of the leaves, although in some cases older dormant buds may be used, as will appear in the discussion of the different fruits. If buds are desired to mature early, pinch off the ends of the growing shoots from which they are to be taken. Suckers and so-called "water-sprouts" should not be used, but rather well-formed wood from the branches of the tree. It is requisite that the buds be taken from a vigorous healthy tree of the variety desired. But sticks can be carried or sent considerable distances if packed in damp moss or other material to prevent drying, but care must be taken not to enclose too much water or decay will be promoted. Fresh shoots in tight tin boxes without wet packing are safer and carry very long distances. Sealing the ends with grafting wax is also a good precaution against drying out.

Budding knives can be bought at all seed stores and cutlery establishments: They have a thin, round-ended blade at one end of the handle, and at the other end the bone is thinned down, or a bone blade inserted. The former is for cutting and the latter for lifting the bark of the stock into which the bud is to be placed. Armed with a bud stick and such a knife, the "budder" starts in upon a row of seedlings. Bending the seedling over a little and holding it between his left arm and his left leg, he reaches down for a smooth place on the bark as near the ground as convenient to work, and makes a horizontal cut, and from that a perpendicular cut downwards toward the roots, as shown 


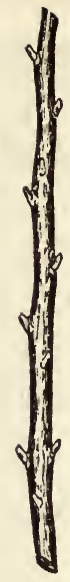

1 A stick of bud wood.

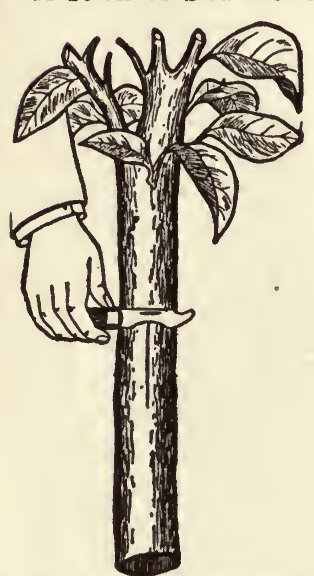

4 Transverse incision.

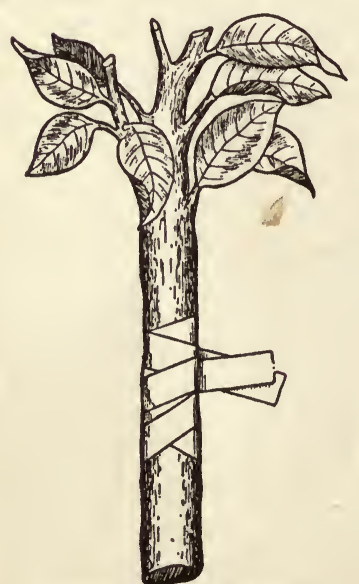

7 Waxed cloth ready for twisting.

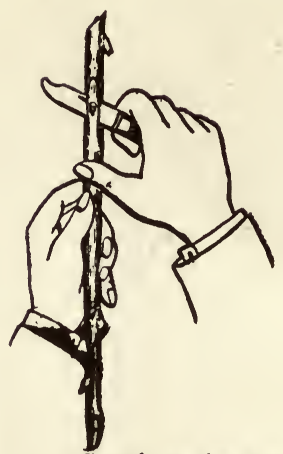

2 Cutting the bud.

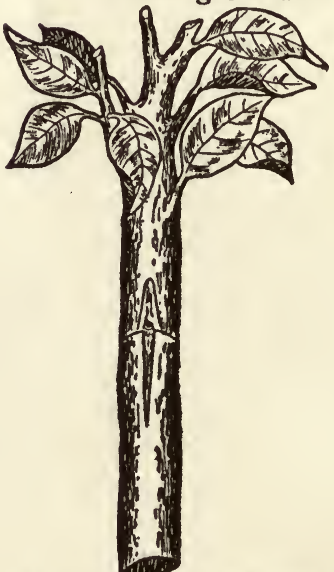

5 Inserting the bud.

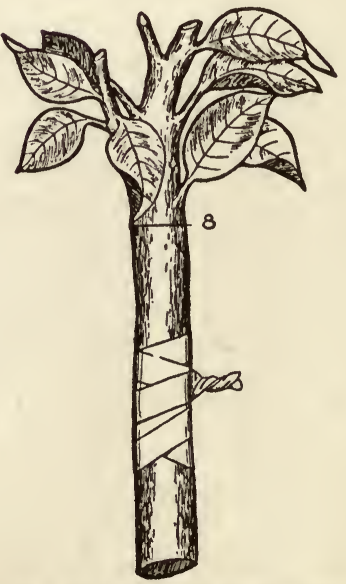

8 Waxed cloth tightly 9 Bud staked and tied. twisted.

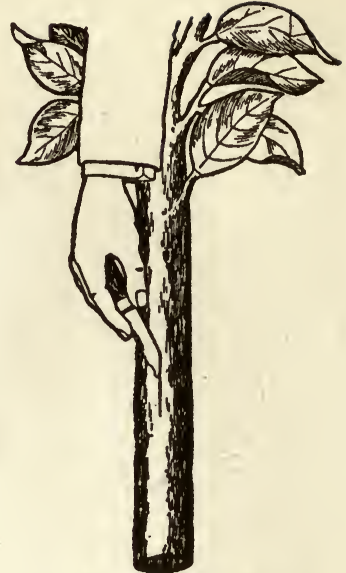

3 Vertical incision.

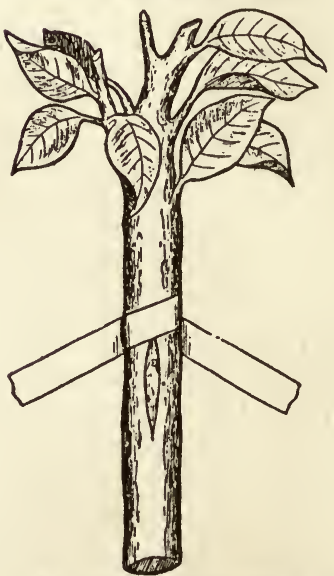

6 Inserted bud ready for tying.

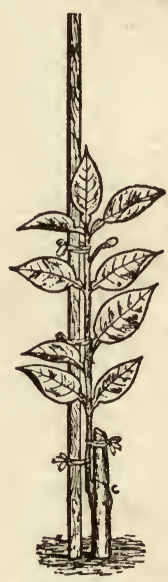


at 3 , in the engraving, with the bark slightly lifted and ready for the insertion of the bud. Next he cuts from his bud stick a bud, as shown at 2. This carries with it, on the back, a small portion of the wood of the bud stick as well as the bud and bark. It was once claimed that this wood should be carefully dug out, but in budding most kinds of trees it is not necessary; in fact, it may be better to leave it in; such at any rate is the general practice. The point of the bud is now inserted at the opening at the top of the slit in the bark of the stock and pushed down into place, as shown in figure 5. To handle the bud, the part of the leaf stem which is left on is of material assistance. Nothing remains now but to apply the ligature which is to hold down the bark around the bud.

There are various ways of tying in the bud. Any way will do which holds down the bark closely, but not too tightly. Different materials are also used, soft cotton twine, stocking yarn, strips of cotton cloth, candle wicking, etc. The last-named is perhaps the best material, on all accounts, although strips of cheap calico bear evenly upon the bark and do very good work. The use of twine is speedy, but the strands bearing upon a narrow surface, and not being elastic, they are apt to do injury by cutting into the bark unless carefully watched and loosened. The fiber from basswood bark was formerly largely used, but has given place to the other materials named, which are more handily obtained. The buds must be examined about a week or ten days after the insertion, and the ligature loosened, for otherwise it will cut into the rapidly-growing stock. Sometimes trees are badly injured by neglect in this particular.

In making June buds, where immediate growth of the bud is desired, some growers make a hard not with the cord around the stock, above the bud, and then use the loose ends to tie the bud. When the binding around the bud is loosened, the hard knot remains on the stock, girdles it, and forces the sap, into the bud. Thin wire, known to nurserymen as "label wire," is also used for this purpose.

In going through the nursery row, all seedlings, which are large enough are budded at once. In going through the row again to look to the bands, if the bud is seen to be fresh looking, it is considered to have "taken." In stocks where the first bud has dried up, another is inserted lower down. Sometimes seedlings which were too small to hold a bud at the first working over are given a bud later in the season, or left for taking up for root grafting in the winter.

In nursery practice the budder does not'stop to tie his buds, but is followed in the row by another man, who carries the tying material, and does this part of the work.

The common method of budding thus described is used on all common orchard fruits. Special styles of budding for!special fruits will be described in the chapters treating of those fruits.

Usually the budded trees are allowed to stand iin the nursery row with no other treatment that year than the insertion and care of the bud, the latter remaining dormant until the !next spring. Then, as soon as the sap begins to swell the buds on the stock, the top is cut off down to about two inches above the bud, and all growth is kept off 
except that of the inserted bud. When that has grown out about twelve inches, the stub is cut off to about three-quarters of an inch or less from the bud, and the wood is quickly grown over by the bark. As there are apt to be dormant buds on the stock below the inserted bud, the trees have to be examined from time to time, and all such suckers removed. This is the common practice with budded trees. Exceptions will be noticed presently in connection with definitions of different kinds of trees know to the trade.

Spring Budding.- What has been said in reference to budding applies to the use of dormant buds. It is also possible to work with what is called a "pushing bud." This process, as described by a distinguished French authority, consists of retarding the growth of the buds on the scions by burying them in the ground until the sap is starting well in the stock in the spring, and then putting them in, trimming off the top of the stock so as to force the bud into growth. In this way the grower of a rare variety may secure trees for planting out the following winter, or he may secure a stock of buds for fall budding, and thus multiply his stock of a desirable variety very rapidly. A modification of this method consists in taking buds in the spring when they have grown out even half an inch, and inserting them by the usual method of lifting the bark, when the sap is flowing well in the stock. Then cut off about half the stock, so as not to give the bud too much sap at first, and afterward, when it is seen to have taken well, the balance of the stock is cut off near the bud. This method gives a tree the first season and saves a year over dormant budding. Shade and protection from dry wind are desirable.

\section{GRAFTING}

The next process of propagation to be considered is that by grafting. lts success, as with budding, consists in bringing the growing wood (inner bark or alburnum) of the scion into contact with the same layer of the stock. It can be applied to any part of the tree, from the topmost branch to the lowest root, as is the case when new trees are made from scions and root fragments. Thus grafting pertains both to the production of young trees for planting out and to the transformation of old trees bearing worthless fruit into producers of choice varieties.

Grafting for the production of young trees is first in order. Instead of budding the seedling during the first summer of its growth, it may be allowed to complete its season's growth, and drop its leaves. When thus dormant the young trees are taken from the ground, the roots rinsed off with water if the ground is wet and sticky, or merely shaken free from clinging earth if in a dry time. Enough trees are dug at once to graft at a sitting. The grafting can be done at the work bench in the tool-house or barn, and if one is pressed with other daylight work, it may be done by lamplight at the kitchen table, if the housewife can be conciliated for the muss it will make.

Care of Scions.-The scions should be previously selected, and whether taken from trees on the place or brought from near or distant sources away from the farm, should have been placed as soon as pro- 

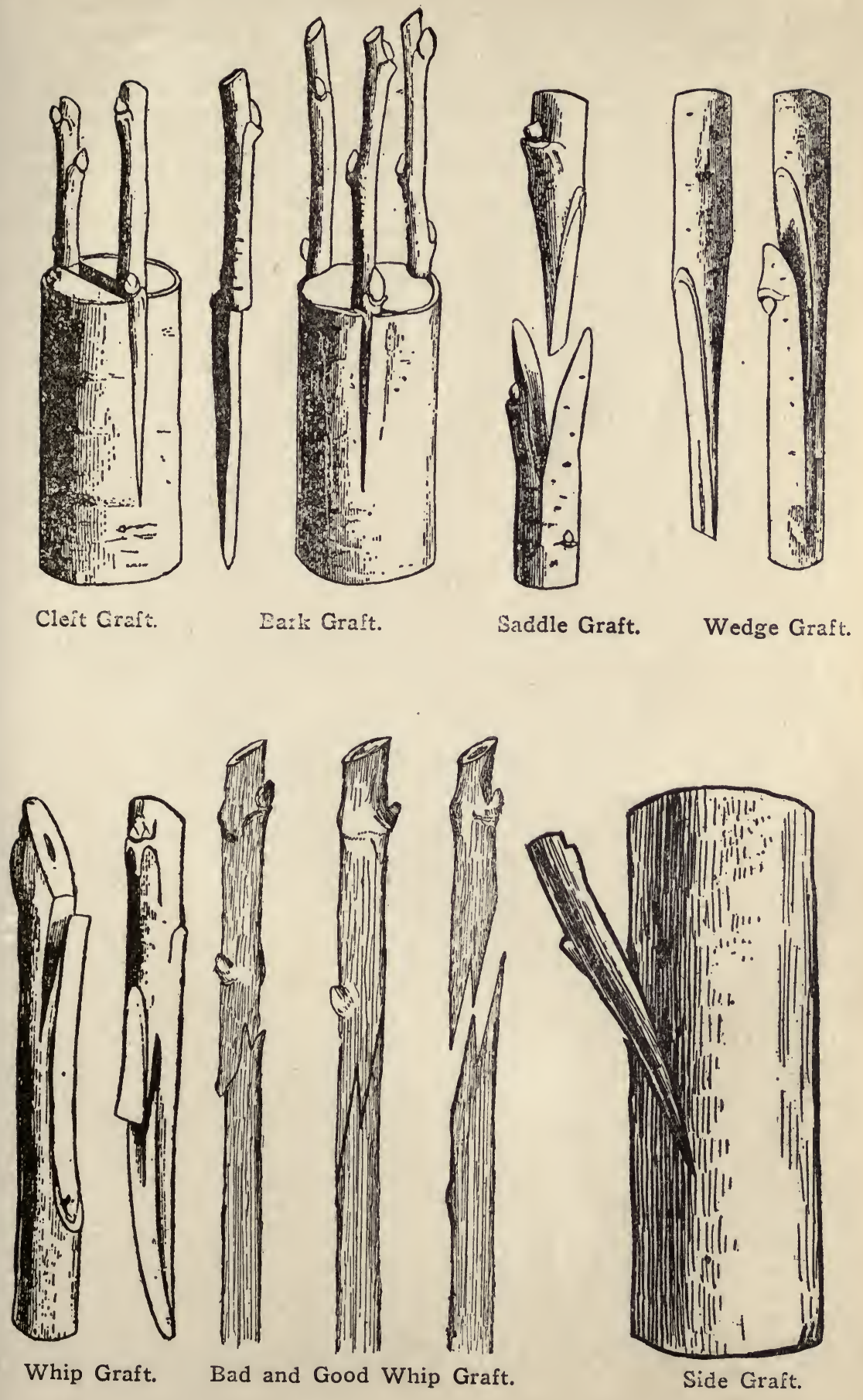
cured in moist earth on the north side of the house or other building, where they will keep cool and damp until one is ready to use them. At the East and other parts of this State where the ground is apt to freeze, it is necessary to keep scions in the cellar with their butts covered with moist sand, but over most of the area of the State nothing more is needed than to put down in the earth at the base of a tree or on the north side of a building, with, perhaps a box or barrel inverted over them to keep out mice and other intruders. Care must be taken not to let them dry up. If it is desirable for any reason to keep scions dormant long into the spring or summer, of course storage in a cool cellar is better, for in the open ground the scions will burst into leaf after a warm spell of spring weather.

In selecting wood for scions, as for bud sticks, never take water shoots or suckers that start from the body of the tree and push up through the older branches, but always give the preference to sound, fully-matured wood, at the ends of the lower or nearly horizontal branches. Careful experiments have shown that trees grown from such scions are more likely to take on a low, spreading habit than those from the central or upper branches. The scions should be tied in bundles with a stout cord; and a piece of a shingle, with the name of the variety written plainly and deeply thereon, should be tied in with each bundle.

Grafting Wax.-In grafting, a good grafting wax is requisite. The ingredients are mixed in different proportions by different growers. A few recipes which are known to give good results are as follows:

Two and one-fourth lbs. resin; $2 \mathrm{lbs}$. beeswax, 3/4 of a lb. tallow.

One $1 \mathrm{~b}$. mutton tallow; 2 lbs. beeswax; 4 lbs. resin.

Two lbs. resin; 2 lbs. beeswax; $1 / 4 \mathrm{lb}$. tallow, and a little linseed oil.

Two lbs. resin; $1 \mathrm{lb}$. beeswax, 1 quart linseed oil; 4 tablespoonfuls turpentine.

One lb. beeswax; 5 lbs. resin; 1 pint linseed oil; 1 oz. lampblack.

One lb. beeswax; 5 lbs. resin; 1 pint linseed oil; 1 pint flour - the flour stirred in after the other ingredients have boiled together and cooled somewhat.

All these mixtures are made with the aid of gentle heat, and during grafting the wax must be kept warm enough to apply easily with a small brush. To do this the wax dish may be kept on a hot brick, to be changed for a fresh one as it cools, or, better still, is to heat the wax in an old fruit can or something of that kind, inside another, which is partly full of warm water. A more capacious heater can be made by removing the top of a five-gallon oil can and making a hole for draft on one side near the bottom. A slow fire can be kept going to heat the wax pot which is suspended from a rod across the top. A wire handle makes this outfit portable. The wax should not be so hot as to run too easily, but just right to spread well.

Grafting is greatly facilitated by the use of strips of waxed cloth or waxed paper, the latter being quite good enough for grafts, which are low enough to be protected by a ground covering; also for root grafts. This waxed paper is made by spreading a thin coat of wax, with a brush, upon tough, thin wrapping paper, cutting up the paper, when cold, with a sharp knife, on a board, into strips about an inch 
wide. Waxed cloth is made by dipping cheap cotton cloth into hot wax, pulling the pieces between the edges of two boards to take out as much wax as possible, and when the cloth is cold, tearing it up into half-inch strips for small grafts or wider strips for larger grafts. When grafting is going on indoors, these strips hanging near the stove are kept in good, soft condition for use.

There are grafting preparations which do not require heating, but remain in a semi-fluid state, and then become very hard by contact witk the air. The following is a popular French preparation:

Melt one pound of resin over a gentle fire. Add to it one once of beef tallow, and stir it well. Take it from the fire, let it cool down a little, and then mix it with a tablespoonful of spirits of turpentine, and after that add about seven ounces of very strong alcohol. The alcohol cools it down so rapidly that it will be necessary to put it once more on the fire, stirring it constantly. Great care is necessary to avoid igniting the alcohol.

This wax is easily prepared, and when well corked will keep for six months. It is put on the wounded part of the tree, very thin, and soon becomes as hard as stone. Thus it is valuable not only for grafting, but for covering the scars caused by removing limbs in pruning. It is, however, rather an amateur's recourse, as it is rather expensive for large commercial operations.

As a substitute for all the old grafting-waxes, asphaltum, "Grade $\mathrm{D}$," has recently been widely and successfully used. It is kept a little warm to spread well. Resin may be melted in one part to four.

Cleft Grafting.-Where various-sized stocks are to be used, as will be the case with a bunch of home-grown seedlings, different styles of grafting must be used. Where the stock is much larger than the scion, as is apt to be the case with California seedlings, the cleft graft will be simplest. Cut off the top smoothly above the root crown and then split the top of the stock, as shown in the engraving. Then prepare the scion by whittling it to wedge-shape at the lower end. Open the slit in the stock with a little wedge and insert the scion so that its inner bark matches with the inner bark of the stock, something as shown in the second figure. It does not matter whether the outside of the scion is flush with the outside of the stock or not; the vital point is to get the growing layers, just inside the barks, in contact with each other, and, to be sure of this it may be well to give the scion a slight diagonal pitch, for if the barks cross each other, this desirable contact is sure to be made. It is well to make the side of the wedge of the scion which goes nearer to the center of the stock a little thinner than the outside.

A scion for a root graft is cut longer than for use in the top of the tree, for in planting, the point of grafting is placed a little way underground. Such scions are usually cut wtih four or five buds. After the scion is in place, it only remains to wrap it closely with a piece of waxed cloth or paper, in such a way that all the cut surfaces are covered, extending the wrapper a little below the split in the root. Paint over the wrapper with warm wax put on with a brush, put a little on the top of the scion, and the graft is complete.

Side Grafting.-Another method which prevents splitting the stock is the side graft, shown in the accompanying figure. It consists 
in bending the stock to one side and cutting in diagonally with a thinbladed, sharp knife, a little more than half way through the stock. Into this open cut insert the scion so that the inner barks touch; then allowing the stock to straighten up, holds the scion firmly. Covering with a wax band drawn tight makes a good job, and such grafts make as good growth as the buds set the previous summer. This method can be used with stems or branches up to an inch in diameter, and is essentially the same, as will be mentioned later, as a side graft for working over old trees. In this style of grafting, a stub of three inches or more may be left above the graft, and to this the graft can be tied to prevent blowing out if it makes a strong growth. Afterward the stub is cut back with a sloping cut and waxed or painted to prevent checking.

Whip Grafting in the Stem.-Grafting above the root or in the stem of the stock when stock and scion are about the same size, is done by tongue or whip grafting. The accompanying sketch shows a whip graft in the stem of the stock. Grafts up to an inch in diameter can be made in this way, but it is generally used for smaller wood. Care must be taken to secure proper contacts of the inner barks at least on one side of the stock. After pushing the parts together, a wax band holds them firmly in place, or the joint may be simply tied and painted over with wax.

A Root Graft.-When the root stock and the scion are about the same size, the tongue graft is also used, as shown in the figure. In making this both the stock and scion are given a sloping cut of about the same length, and a secondary cut made in each. When the two are put together the wood "tongues in," or interlocks as shown in the engraving. The object of this is to make more points of contact for the inner barks of root and scion, and at the same time to interlock the two more firmly. In putting the two together, if the stock is slightly larger than the scion, be sure to put the scion so that the inner bark contact is made, and this will bring the scion a little to one side of the center. Bind with the wax band, and paint with wax as in the case of the former graft.

In large nursery practice expert grafters have come of late years to make this root graft without wax, merely tying in the graft. For amateur work at home it is much safer to use the wax.

Grafting in the root, where the root is much larger than the scion, may be done without splitting the root by cutting or sawing out a triangular piece on the side of the root, cutting the scion to fit and trusting to a strong band to hold it in place. This graft is illustrated in the chapter on propagating the grape. It also works well with root grafting the walnut, and is used by some in ordinary top grafting on other trees.

Planting out Root Grafts.-This root grafting can be done in the winter before it is time to plant out, and the grafts can be made a few at a time, as convenient. The grafts, then, as fast as prepared, should be bedded in moist sand in the cellar, and will make their contact firm, and even start to growing a little. In planting out in the nursery rows be sure the earth is firmed well around the root, otherwise many will be lost. Plant ten or twelve inches apart in the rows. Keep 
the weeds down and the soil well cultivated and loose on the surface, and the first season's growth will give a tree fit for planting out in orchard in the coming winter. For irrigation the rules already given for the growth of seedlings for budding will apply.

\section{PRUNING TREES IN NURSERY}

As for other treatment of the trees (either from bud or root graft) in nursery during the first year, there is some difference of opinion and practice. If the young tree will be content to make a straight switch with good buds in the axils of the leaves, but no laterals thrown out, it will be in the best possible shape for planting in the orchard, and gives the planter a chance to make the head at whatever height suits him, and to secure uniformity through the orchard. All trees will not, however, be content with this growth, but will push out laterals all along the stem. Even in this case some let the whole growth go for the planter to treat as he thinks best. Another plan is to go over the nursery when the young stock is about two feet high and pinch back the laterals part way, but retaining the leaves nearest the stem to shade the stem. This pinching back is done from the ground up to a height of one to one and a half feet, and above that the growth is left to take its natural course, to be cut as desired when the head of the tree is formed. Pinching back develops buds near the stem and gives the planter a better chance to head the tree lower if he likes. Another practice which prevails to some extent, is to pinch off the terminal bud when the young tree has reached a height of about two and a half or three feet in the nursery. This soon forces a growth of lateral branches, which are in turn pinched after they have grown out a couple of feet. The result is the formation of a head on a nursery tree the first year, and when such trees are planted in orchard they are merely cut back on the laterals, leaving the head as formed in the nursery. Such trees are difficult to handle in packing, and take much room in shipment. There may, however, be an advantage in such practice for the home grower if he is situated in parts of the State where the greatest season's growth is attained. Orchard planters generally, however, prefer a June bud or a yearling of moderate growth, without laterals.

\section{CLASSES OF NURSERY STOCKS}

The several classes of stock which are to be had from nurseries are as follows:

Root Grafts.-These are seedling roots, or pieces of them, on which scions of the desired variety have been grafted on the bench and the junction healed over in the cellar. No growth has yet started in the scion. If the tree planter wishes this kind of stock, he should plant it out in nursery row in the spring and remove the trees to orchard the following winter.

June Buds.-For multiplying varieties very fast, buds are kept dormant in a cool place; or, by pinching off the top shoots of the current year are forced to mature buds very early. These buds are put 
into seedling stocks as early in the season as it is possible to get new buds developed. This can be hastened by pinching tips of new shoots from which they are to be taken, which forces development of lateral buds. After budding, the top of the stock is girdled with knife or cord, or partly cut away, and growth is forced on the bud so as to give a small tree at the end of the first summer. This method of propagation is growing in popularity in this State, especially in the foothill districts, where small trees are preferred for transplanting.

Dormant Buds. - Trees are sold in dormant bud when they are lifted from the nursery and sent out before any growth has started on the inserted bud. The bud should be seen to be the color of healthy bark. Such trees should only be used when yearlings are not to be had and gain in time is very important. Care must be constantly taken that growth starts from the right bud, and that it be protected from breaking off by wind or animals. A considerable percentage of loss is usual, and extra dormant buds should be planted in nursery rows to fill vacancies.

Yearling Trees.-These are trees which have made one season's growth from the bud or graft. Two-year-olds have made two seasons' growth, and so on. The proper way to count the life of a tree is from the starting of growth in the bud or graft, for this point is really the birth of the tree.

\section{WORKING OVER OLD TREES}

Another operation which may be properly considered as a branch of propagation is the working over of old trees. There is much of this being done every year in this State. The old seedling fruits in the older settled parts of the State are being made to bear improved varieties; trees of varieties illy adapted to prevailing conditions are changed into strong growing and productive sorts; trees are changed from one fruit to another, when affinity permits. This will be mentioned in the discussion of the different fruits. Still another reason for working over is to secure more valuable and marketable varieties. Sometimes a mixed orchard is made to bear a straight line of one sort which is in demand, or when the grower finds he has too many trees of a single kind, which give him more fruit than he can conveniently handle when it all ripens at one time, he works in other varieties so as to get a succession of varieties adapted to his purpose, and thus secures a longer working season in which to dispose of them. This is especially the case in large orchards of apricots, peaches, and plums, when the grower depends upon drying his crop. Information concerning the successive ripening of varieties can be gained from the special chapters on the different fruits. For all of these reasons, and others which need not be enumerated, the work of the propagator is continually going on even in our large bearing orchards. As with young trees, so with old, transforming the character of the tree is done both by budding and grafting.

Budding Old Trees.-One way to prepare an old tree for budding is to cut back the branches severely during the latter part of the 
winter, which has the effect of forcing out new shoots around the head of the tree, and in these the buds of the desired variety are set in the summer, just as is done in budding nursery stock, except that the budding should be done rather earlier because the sap does not run as late. When the shoots are budded, those being selected which are situated so as to give the best symmetry to the new head, the shoots not budded are broken a foot or so from where they emerge from the old wood, and are allowed to hang until pruning time. At the winter pruning the budding branches are topped off a little above the bud, and when the new shoot starts it is often loosely tied to the stub of the old branch to prevent breaking out in the wind. When it gets strength, the stub is cut away smoothly to allow the wound to heal over.

Another way is to insert the buds in the old bark at points where it is desirable to have the new branches start. This is sometimes done by lifting the bark, as in ordinary budding, and slipping the bud under, sometimes by what is called shield or plate budding, which consists in removing a piece of the old bark entirely and putting in its place a piece of bark of the desired variety, having upon it a dormant bud. With plate budding it is necessary to be careful to have the inserted bark just the size of the bared spot, and to wrap it more closely than when the bud is slipped under the bark of the stock. In all cases in budding old trees, care must be taken to get fully-matured buds, and it is well to take them from large shoots, which have a thicker and firmer bark than may be used in budding nursery stock. It is also desirable to be very sure that the buds are taken not only from a tree of the desired variety, but from a healthy, vigorous tree of that variety.

In selecting buds, also, one must be sure that he gets leaf buds, and not fruit buds only. In taking buds from some kinds of bearing trees, of course, he may sometimes, to get well-ripened buds, be obliged to take both fruit and leaf buds together. This will work well if care is taken not to rub off the leaf bud. It is rather easier, however, to work with buds from young trees not yet in bearing if one can be sure that these trees are of the desired variety.

Grafting Old Trees.-Old trees are also renewed by grafting. This is most generally done by the old process of "top grafting." The main stem or the larger branches are cut square off, and the scions, usually two, but four or more if in the trunk, are shaped and set into clefts in the stock as shown in the engraving. It is better to use limbs above the main fork, or head of the tree, than to graft in the trunk, if the old trees are of good size. The following description, which the writer borrows in part from some unknown source, will serve to guide novices in the matter:

The outfit necessary for doing the work consists of a small, fine saw, a regular grafting knife, or a pocket-knife with a long, straight sharp blade, wax, light mallet, and a hard-wood narrow wedge. After selecting the limb to be grafted, saw it off-your own judgment will guide you as to best point, but before the saw gets quite through the limb, cut the bark on the under side of the limb to prevent the liability of peeling down.

Next split the stub with knife and mallet and insert the wedge in the center of the cleft to hold it open. It is usual to cut the scion with two buds, but sometimes better results are had by using scions with but a single bud. Whittle 
the scion wedge shape, so that it fits nicely down into the cleft. To do this, hold it in the left hand with the bud at the ball of the thumb, then cut the side toward you; as will be natural, turn it over, and cut the opposite side in the same way, making the wedge a very little thinner on the edge opposite the bud than the other. This will insure a firm pressure at the points where the bark of scion and stock meet.

When set, the bud of the scion will be on line with the outer long portion of the graft. The point to be closely observed in adjustment is to have the inner or sap bark of the scion connected with the same of the stock. If a trifle too far in, or too far out, the work will be a failure. Some people set the graft a little out at the top and a little in at the bottom, so as to be sure of a connection at the crossing point, but there will be firmer hold if there is a union the whole length. Our rule has been to have the wood of the scion come exactly parallel with the surface of the stock, and we seldom fail in getting firm adhesions and solid limbs after years of growth.

After the scions are set, and two should be put into one limb if large, carefully withdraw the wedge and apply the wax, so that every part of the wood and bark cut and split is well coated. In doing this use extreme care not to move the scions at all from their sittings. If the pinch of the stock is seen to be severe, a small wedge may be left in the center to save the scions from crushing. If there is a large cleft, it may be filled with damp clay before waxing over.

Most grafting over of old trees is done by this method, using one or another of the wax preparations described upon a preceding page. If the cut surface of the stock and the split is thoroughly waxed over as low as the bark is split, there is usually little trouble with the growth of the scion and the healing over of the stock. In the warmer valleys in the interior, the sun is often hot enough to melt the wax and cause it to run and bare the wood surfaces. This is prevented by dusting the wax thoroughly with brick dust well powdered; but, by a little experimenting with the recipes already given, one can secure a wax which will stand any heat likely to be encountered.

For grafting over trees by working upon the limbs, the neatest and surest work can be done by methods of grafting which do not require the splitting of the stock. There are various ways of doing this. One method is shown in the engraving on the next page, and consists in cutting the scion as shown, and inserting it beneath the raised bark and then binding well with waxed bands, the preparation of which has already been described.

Another method is an application of what the French call oblique side grafting. It consists of making an oblique cut downward through the bark of the stock and for a distance into the wood, using a chisel and mallet or even a strong knife. A saw and knife are also used for making this cut, as will be described in the chapter on the peach. A small form of side graft has already been shown earlier in this chapter. In it the scion is held in with a wax band. Some growers remove the top of the stock with a sloping cut about half an inch above the scion, as shown in the engraving, and wrap the waxed band well around and over all the exposed surfaces. Others do not remove the whole of the limb until the scion has started well into growth, and then they cut down and pare the stock and cover with a band or with a wax that will not run in the sun.

Several ingenious devices have been patented by Californians for securing uniformity in the incision in the stock and in shaping the scion, but it is so easy to succeed with ordinary tools that such inven- 
tions have never come into wide use. Machines for the bench grafting of vines have, however, been successfully employed to a certain extent, but are not generally used.

\section{TIMES FOR GRAFTING IN CALIFORNIA}

There is nothing particularly new about the methods or means employed for grafting deciduous fruit trees in California, but the time at which the operation can be successfully done, and the condition of the scion, are different from those held to be necessary in other climates. It is not at all requisite that the scions should be carefully stored away to keep them in a dormant condition, nor that the grafter should haste to do his work in just such a state of sap-flow in the spring time. It was early discovered that grafting could be successfully done with growing scions, and that scions could be cut from one tree and set in another nearly at any time the grafter desired. Grafting is therefore possible much later in the season than is prescribed elsewhere, and it is also possible to begin earlier. In one of the largest apple and pear orchards in the State it is common to graft in December. The absence of freezing weather saves the graft from injury. As our trees start their flow of sap early, and often when the ground is too wet for comfortable orchard work, it is the practice of many to get their grafting and pruning done before the heavy midwinter rains begin. The practice of most growers is, however, to conform somewhat nearly to traditional methods, to do most of the grafting in the spring months, and to use dormant scions, the growth of which is retarded by heeling them in on the north side of a building, or keeping them in sand in the cellar, as the grower chooses. Of course it should be understood that there are parts of the State where the winter conditions are more nearly like those at the East, and practice has to conform to them.

As to whether it is better to remove the whole top of the tree and graft all the limbs in one year, there is some difference of opinion. The prevailing practice is to graft over part of the limbs one year and the balance the following year; or else to leave part of the top to shade the bark and take part of the sap flow until the grafts start out well, and then cut it away. When a large amount of grafting is to be done, the limbs may be cut off during the weeks preceding grafting. In this case, the cut should be made a foot or two above the grafting point and a second cut be made at this point, when ready to put in the scions.

Whenever old bark is exposed by cutting back for grafting, thorough protection against sunburn must be provided. The simplest way to do this is to cover the exposed bark with good whitewash. By using thirty pounds of lime, four pounds tallow, and five pounds of salt with enough water to make it flow well, a tenacious whitewash can be secured.

What has been said thus far relates especially to the working over of old trees of common deciduous fruits. Though much the same method will succeed with some of the semi-tropical fruits and with nut trees, the discussion of their propagation and grafting over will be deferred to the chapters devoted to them, and this will also give opportunity to describe methods especially adapted to these fruits. 


\section{PREPARATION FOR ORCHARD PLANTING}

The two essentials in preparing land for trees or vines are deep and thorough cultivation, and provision for drainage, unless the situation is naturally well drained. Drainage will be considered in connection with irrigation in another chapter. In this place, however, by way of emphasis, it may be remarked that high land is not necessarily well drained, although the general feature of the surface may be an incline, nor is low land necessarily wet, although the surface may be apparently level. For horticultural purposes the drainage of the land must be considered on the hillside as well as in the valley, for reasons which will be more fully set forth in the chapter on drainage.

The preparation of land for fruit planting should begin with grading. In irrigated orchards this is essential for the equal distribution of water. Even where irrigation is not anticipated, it is of decided advantage to smooth down hummocks and fill sags which are likely to collect water in the rainy season. As has been shown in Chapter III, this can be done on most California soils without danger of uncovering a sterile subsoil. Some intimation of the method of grading is given at the close of Chapter VII. In preparation for the irrigated orchard, and irrigation is now widely employed even in regions where formerly rainfall was the sole reliance, it is important that accurate grading should be done and the use of the surveyor's level and grade stakes will be found very desirable. All moving of soil should precede the general plowing.

For the planting of orchard or vineyard the land must be put in as good tilth as possible, and extra expenditure to secure this will be amply repaid in the after-growth of the trees and vines. If practicable, it will be all the better to have the process of preparation begin a year before the trees or vines are to be set. This is true either with newlycleared land, as has been described, or with old grain or pasture land which is to be used, leaving the surface rough during the winter, facilitates the access of air to the lower layers of the soil, and in a certain sense may be said to sweeten and enliven it. Following in the furrow with a subsoil plow is very desirable, either at the first plowing or later. Such treatment of old grain land breaks up the old hardpan,* which has probably been formed by years of shallow culture. The preparation should continue during the following summer, and can often be made both thorough and profitable by the growth of a summer "hoed crop," the culture of which will kill out many weeds and secure good pulverization of the soil. If no summer crop is grown, the land should be kept in cultivation by plowing the weeds under as long as the surface soil retains moisture enough to start them. A special advantage of such summer-fallow in regions where the rainfall is apt

*In this connection the term means "plow-sole." Treatment of true hardpan will be described in the next chapter. 
to be short is that a prevention of evaporation, the trees or vines set the following winter will have a good part of the rainfall of two seasons to grow with, and the result will often be very noticeable. If there are supplies of manure available, as is often found in old corrals on our grain or stock farms, it is better to gather and apply this the winter before the planting of the trees. If this is not done, it should be left until after the trees are planted, and then be spread upon the surface during the winter, and plowed it after it has been in part leached into the soil by the rains. Application should be made evenly all over the surface, and not massed around the roots of the trees, unless it is to be applied as a mulch to the surface after the spring cultivation is over, as will be considered later.

If it is thought desirable to plant the land immediately after breaking up, put in the plows as early in the fall as it is possible to do deep work, that is, to plow to a depth of ten or twelve inches, or more. Harrow thoroughly. If it is still early, cross-plow. Thorough and deep breaking up as soon as practicable to plow in the fall, and following in the furrow with the subsoil plow, working to a depth of fourteen inches or more, is a good preparation for fruit planting. For this kind of work good teams are needed and the plow should be sharp and bright. If the work is hard for the team,' set the plow so as to take less land, but do not sacrifice the depth. Harrow again thoroughly, and the land is ready for the trees or vines.

Avoiding Dead Furrows.-Unless dead furrows can be used to advantage for surface drainage in case of heavy rain-storms, it will be of decided convenience in laying off to have the field free from them. This can, of course, be secured by beginning the final plowing at a line in the center of the field, turning all furrows inwards. In this case, too, if a right-hand plow is used, the team will always turn on unplowed land, and thus avoid trampling upon and packing the loose soil. The slight ridge in the center of the field formed by the first two furrows can be easily leveled by a couple of back furrows, and when properly harrowed the field will be found smooth as a floor for staking out for planting.

\section{LAYING OUT FOR PLANTING IN SQUARES}

It is very desirable, both for convenience in cultivation and for the beauty of the orchard, that the trees should stand in straight lines, and care should be taken to attain that end. Most orchards and vineyards in this State are laid out in squares; that is, the rows of trees or vines are all at right angles to each other, as shown in the accompanying sketch. This is the simplest arrangement; and by some of our largest planters is held to be the best. It is true that the trees are not equidistant from each other in all directions, and that, theoretically at least, there is a portion of the ground unused-supposing that the roots occupy a circle, as do the branches. Practically, however, it may be doubted whether the hungry roots of well-grown trees or vines leave any portion of the soil unvisited. 
There are also forms of double squares and alternating squares available for planting at long distances, with growths between, which are ultimately to be cut out, or for vines between fruit trees. Such mixed planting is, however, but little practiced in California.

\section{VARIOUS WAYS OF MARKING FOR SQUARES}

Marking With a Plow.-This method was used in laying off some large orchards in the Sacramento Valley. A common two-horse turning plow is rigged with a "marker"-a light wooden bar extending at right angles from the beam, the bar being as long as the desired distance between the rows of trees. On the end of this bar a crosspiece is fastened perpendicularly, so that it scratches along on the surface of the ground. The line of the first furrow has to be designated by a flag stake, to which the plowman proceeds. When this is done, the team is turned and sent back along the next row, the location of which has been fixed by the marker, and so on for the length of the field, the marker being turned each time to indicate the next furrow. Following the same course the other way of the field leaves the trees to be planted at the intersection of the furrows.

Measure and Sight.-Another method which is quite commonly used and answers a good purpose in small plantings is the combination of measure and sight. The sighting stakes are usually plasterers' laths pointed at one end and whitewashed to make them more visible to the eye. In the use of these it is necessary to measure the distances and locate the laths to mark the ends of the rows all around the field. Then locate a line of laths across the field each wayl through the center, these laths occupying places which the trees of these two central rows will fill. After these are in place, measurement can be dispensed with, and the job can be finished by sighting through. The man on the ends of the rows has three laths to sight by in each row, and the stake driver places the stakes as directed by the sighter. Good location can be done this way if a man has a good eye and patience enough.

Marking Off With a Wire.-A measuring wire or chain is, perhaps, the best means for getting accurate location of trees or vines. It is used either for setting in squares or in other arrangement, as will be described presently. Measuring wires are made of annealed steel wire about one-eighth of an inch in diameter. The length varies according to the wishes of the user. If it is desired to lay off the plantation in blocks of one acre, the wire should be two hundred and eight feet nine inches long, for that is approximately the length of one side of a square inclosing an acre of ground. But some use a wire as long as three hundred feet, when the acre measure is of no consequence; and others, in smaller plantings, make the wire just the length of the piece they have in hand. At each end of the wire is fixed a strong iron ring about one and a half inches in diameter, to be slipped over stakes; some use a larger ring, say three inches in diameter, because it is easier to handle in pulling taut. Along this wire, patches of solder are placed exactly at the distances desired between the rows of trees or vines, and to these places pieces of red cloth are sometimes fastened so that the points may be easily seen. Another style of measuring wire is made of 
small wire cable about a quarter of an inch in diameter, made of several strands of small wire. It is more flexible and less likely to become kinked than the large wire, and can be easily marked off to represent the distances, at which rows of different kinds of trees should be placed, by separating the strands a little at the desired points and inserting a little piece of red cloth, pressing the wires together again and tying firmly with a waxed thread to prevent slipping. In this way the same wire can be easily arranged for planting vines or for the trees requiring the greatest distance between the rows. Another advantage of the cable is that any stretching can be taken up by retwisting, which can not be done with the stretching of a single wire. Another good style of planting wire is made of 2, 4 or 6-foot links of No. 12 steel wire (including the diameters or the small rings turned at each end of the link pieces). As all planting will probably be at multiple distances of these link-lengths, the cloth tags can be changed and the chain thus be marked for any desired distances.

Finding a True Corner.-To use the measuring wire for laying out trees on the square, it is necessary first to get one corner true, and then a field of any size can be marked out accurately. Select the side of the field which is to serve as the base of the square and stretch the wire along that, say fifteen feet from the fence, which will give room enough to turn with the team in cultivation or to drive along in picking-time. When the wire is thus stretched parallel with the boundary of the field, place a stake at each of the distance tags on the wire, and these stakes will represent the first row of trees or vines. To find a square corner, begin at the starting point and measure off sixty feet long this row with a tape line, and put a temporary stake, then from the starting point measure off eighty feet as nearly at a right angle with the first line as can be judged with the eye, and run diagonally from this point the temporary sixty-foot stake. If the distance between these stakes is one hundred feet, then the corner is a right angle. Now, having the outside lines started at right angles to each other, one can proceed with the measuring wire and lay off as large an area as he desires, if care is taken to have each line drawn parallel with the last, and all stakes accurately placed with the tags on the wire-providing the land is nearly level or on a uniform grade. In locating trees over uneven ground, the measurements will have to be made from tree to tree, with the tape line held as nearly to a level as possible.

Rows on Hillsides.-Laying off orchard or vineyard on hillside too steep to plow both ways, there is advantage sometimes in placing the rows up and down the hill nearly twice as far apart as the rows along the face of the hill: In planting trees thus the advantage to be gained is by enabling you to keep the team well up the hill; thereby you are able to plow or: cultivate the trees close on the lower side of the rows. There is no difficulty in cultivating the upper side of the rows, for the plow or harrow is always below the team. If trees are planted as recommended, the team can be guided up the hill a little between the rows, then allowed to drop downhill one step, and thus one can cultivate the trees close on the lower side. The same rule will apply to vines. 


\section{QUINCUNX PLANTING}

There is much confusion in the use of this term in this State. It is, in fact, made to cover almost every kind of arrangement which is not on the square. Webster defines the term to mean "the arrangement of things, especially of trees, by five in a square, one being placed in the middle of a square." Trees set in quincunx would stand as shown in the accompanying diagram. To locate them in this form it is only necessary to proceed as already described for planting in squares, by fixing upon the base line and locating two side lines to it at right angles. Place the stakes on these two lines just half the distance desired between the trees, and have the measuring wire long enough to reach across from one line to the other. Near one end of the wire place another mark just half way between the end and the first tree mark; that is, if the trees are to be twenty-four feet apart in the squares, this additional mark should be twelve feet from the end of the wire. Now set the first row with the end of the wire at the corner stake, and set stakes at each twenty-four foot mark.

Proceed now to the first half-way stake, and instead of putting the end of the wire at this stake, put the twelve-foot mark there. Put stakes now at each twenty-four foot mark again to locate the trees in that row. In the next row put the end of the wire at the first stake and proceed as in the first row. Thereafter using the end of the wire and the twelve-foot marks alternately, the stakes will be set in quincunx all over the field. If the midway stakes are now pulled out along the two side lines, the remaining stakes show where the trees are to be placed. This way of planting locates about 78 per cent more trees upon any given area, but it brings the trees at irregular distances from each other, and except in furnishing a way to arrange an orchard with permanent and temporary trees, there does not seem to be any advantage in it.

\section{PLANTING IN EQUILATERAL TRIANGLES}

This is the arrangement generally implied when the term "quincunx" is wrongly employed. By it the trees are all equally distant from each other, and thus the ground divided as equally as possible. The arrangement admits 15 per cent more trees to the acre than the setting in squares, and the ground can be worked in three different directions. This arrangement also gives better facilities for irrigation. Objections are urged to it, however, in that it does not admit of thinning trees by removal of alternate rows, as is sometimes desirable, and that one has to take a zigzag course in driving through the orchard. sketch.

Hexagonal planting places the trees as shown in the accompanying

It is termed hexagonal because, as the figure consists of six trees inclosing a seventh, a line drawn through the encompassing trees make a hexagon. It is also called septuple planting, because seven trees enter into its figure.

An orchard can be laid out in hexagonals by using the measuring wire as described for quincunx planting with the distance and half- 
distance marks, except that the guide stakes in the side rows must be placed at different distances apart. The following table, showing the distance for side stakes to reach desired distance between the trees, and the method of calculating the number of trees to the acre by the square and hexagonal or sextuple arrangement:

Trees set Septuple

10 feet apart.

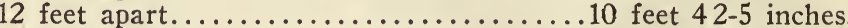

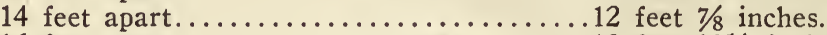

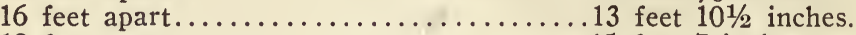

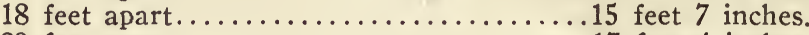

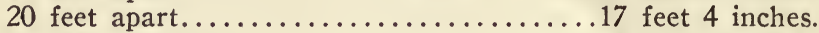

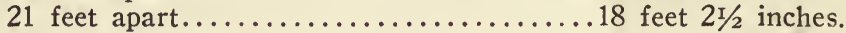

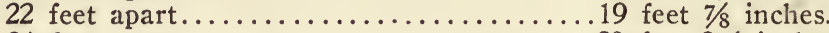

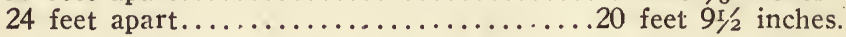

After the field is staked, each alternate stake in the check rows should be removed. The following table will show the number of trees to the acre by the square and septuple system:

\begin{tabular}{|c|c|c|}
\hline & & \\
\hline rat & 435 & 500 \\
\hline 21 & ...302. & \\
\hline 4 feet apart & .222 & .255 \\
\hline 5 feet apart. & .170 & .195 \\
\hline 8 feet apart & .134. & .154 \\
\hline 0 feet apart. & ..109. & .125 \\
\hline 1 feet apart. & & .114 \\
\hline 22 feet apart....... & $\cdots 90$. & $\begin{array}{r}.103 \\
86\end{array}$ \\
\hline
\end{tabular}

For any distance not given in the above table, calculate the number of trees to the acre by the square system, and add fifteen per cent. This will give the number if planted septuple.

Laying Out Hexagonals With a Triangle.-It is possible to lay out an orchard in hexagonal form by working from stake to stake with an equilateral triangle of dimensions equaling the distance required between the trees.

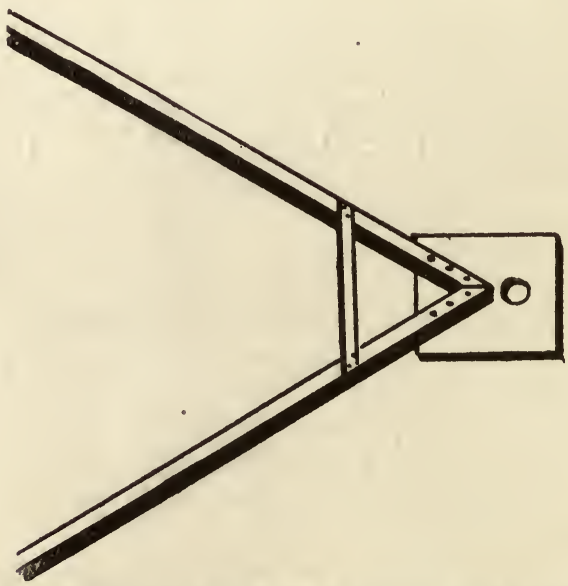

One corner of triangle-all being made alike. 
Take three strips of one-by-two-inch dry pine or redwood, and as long as you wish the distance between the trees. Cut the strips the same length, and fasten the corners of the triangle firmly together by nailing two pieces of pine board six by six inches.

If the long strips are set un edgewise, the triangle will be much stiffer and better to carry. Through the corner boards bore an inch hole, making sure that the three sides of the triangle measure exactly the same. If they do, the triangle must necessarily be perfect. Then brace it a little by nailing a lath across each corner, and it is readv for use.

Now split out some three-quarter-inch pins, one foot long, from good, straightgrained redwood. Make one hundred pins for each acre you have to lay off.

Three persons must now carry the triangle, beginning on one side of the field, say eight feet from the fence, and guided the first time through by a line of stakes. Carry the triangle with its side to the line of guide stakes and its point in. The head man and the inside man will stick pins, while the rear man will slip his corner each time upon the pin set by the head man.

After the first time across, the man at the inside point of the triangle alone will set pins, while the other two fit their corners upon the pins in the last row set. Thus one row of pins only is set each time you go across the field.

If the triangle is exact, and the first row of pins is set perfectly straight, and the pins are always set perpendicularly, everything will now work like a charm and the job will be perfect; and it is so simple and easy that a man and two small boys can lay off from five to ten acres in one day. Remember that no guide stakes are used anywhere after the first time through.

The Triangle on Hillside.-The use of the triangle requires a little nicety in "leveling up" where the piece is hilly. By using a plumb-line at two corners of the triangle, the third corner resting on one of the stakes, leveling the triangle and bringing one of the plumblines over another stake already set, the position of the other line would determine the position of the next stake. This method has worked fairly well, even in places where the slope was sufficient to give a fall of six feet between the trees, which were set twenty feet apart.

Locating in Triangles With a Chain.-Instead of a wooden triangle, a chain has been used in this way:

First stretch a chain along one side of the ground, setting by it the first row of stakes. This forms the base line. Have a piece of chain just twice the length of the established distance between trees, with ample rings on the ends and a joint in the middle. Put one of the rings over the first stake and the other over the second stake. Then take the joint in the middle of the chain and stretch it out reasonably tight. The wire forms a letter $\mathrm{V}$, at the focus of which stick a stake. The point is indicated with precision by the joint in the middle of the chain. Then take the ring off the first stake and put it over the third stake, leaving the one on the second stake where it is. Tighten the chain again, and another point is fixed. Thus continue all the base line, shifting the rings alternately, turning over the chain as one turns a pair of draughtsman's compasses in his hand when spacing off a line. The second row of stakes being set, set the. third row, and so on through the ground.

The suggestions given in this chapter should indicate ways enough to lay off orchard and vineyard ground to answer all needs, though there are other good ways not mentioned. It is hoped that the instructions will not be regarded as to explicit. They are intended for the guidance of the inexperienced planter, and will naturally seem laden with detail to those who have become familiar with the operations by repeated practice. 


\section{CHAPTER XI}

\section{PLANTING THE TREES}

After the field has been graded, thoroughly tilled and carefully laid off as has been described, the next step is digging the holes for the trees. "How large shall the holes be?" He was a wise fruit grower who, when asked this question, replied, "As large as the field." That is to say, it is much better to work the whole ground over deeply than to trust to deep holes and shallow working elsewhere. Where this is done, the tree holes need only be large enough and deep enough to receive the roots without folding them in or cramping them up. In a loose, deep soil, however, one can dig extra deep and broad holes if he desires, and will be repaid by extra growth of the tree; but in a close, tenacious soil a deep hole is not only undesirable, but often positively a danger to the tree unless drainage of the holes is provided artificially. Such holes hold water like a tub, and the loosening of the soil deeply facilitates its gathering in the hole. Many have found their trees in such places dwindling and. dying because their roots were soaking in water.

Planting on Some Shallow Soils.-As a rule, trees should have a deep soil, and for these deep, free loams, California is famous, but there are situations where very satisfactory growth and production can be had, even when the hardpan is near the surface and the soil would be called shallow. In such places it is the character of the subsoil which warrants the tree and vine planter in making use of them. Sometimes the hardpan is so thin and near the surface that it can be broken through with a pick in digging the tree hole. Otherwise boring is done. It is about forty years since Mr. James Rutter, of Florin (on the "bedrock" lands near Sacramento), first noticed that there were vines here and there which grew exceptionally well and bore large crops of fine fruit. He found by investigation that under these vines there were crevices in the bedrock, and from this he took the hint to bore through this hardpan in the bottom of the hole where he placed the tree, and in this way he gained access for the roots to the subsoil and egress for the water through the permeable substratum. He bored a hole two inches in diameter into or through the bedrock and rammed well into it one and a half pounds of black blasting powder. After exploding this, he sometimes bored a three-inch hole about four feet below the blast. Instead of blasting in the whole where the tree is to be planted, some bore and blast in the hardpan midway between the rows, placing the holes at "quincunx" with the trees. The shattering of the hardpan between the trees is said to be practicable after the trees are growing, and may in certain soils relieve trees which are suffering for lack of drainage. A half-pound cartridge of dynamite has been successfully used for subsoil blasting, and some have reported in favor of exploding a quarterpound cartridge quite near to a tree suffering from standing water, putting in the charge during the dry season. 
There are many situations where such practice would be beneficial, and in some cases mere digging or boring through the impervious stratum avails much. There are spots where "lava crusts" overlie gravel, and trees have been well grown by cutting holes through the lava to the gravel, filling with good soil and planting the trees in these holes. Their roots penetrate to the gravel stratum and obtain abundant moisture and nutriment. In certain situations where a shallow layer of soil overlies a heavy clay, trees have been blown over, but when a cut has been made through the clay, the trees have rooted deeply and have withstood the winds.

Shooting Holes for Trees.-Even where there are continuous depths of dense strata, and not shallow hardpan underlaid by free soils, trees can often be successfully grown by the use of high explosives. In his book on "Soils," Professor Hilgard gives these suggestions:

A permanent loosening of dense sub-strata is best accomplished by moderate charges $(1 / 2$ to $3 / 4 \mathrm{lb}$.) of No. 2 dynamite at a sufficient depth (3 to $5 \mathrm{ft}$.). The shattering effect of the explosure will be sensible to a depth of eight feet or more, and will fissure the clay or hardpan to a corresponding extent sidewise. If properlv proportioned the charge will hardly disturb the surface; or will throw out sufficient earth to plant the tree without further digging. Where labor is high priced this proves the cheapest as well as the best way to prepare such ground for tree planting, and it has often been found that in course of time the loosening begun by the powder has extended through the mass of the land so as to permit the roots to utilize it fully and even to permit, in after years, of the planting of field crops where formerly they would not succeed.

Upon the basis of these pioneer demonstrations, California claims priority in the now widely prevalent "dynamite farming." The most minute descriptions of approved methods are now available in the publications of the powder manufacturers and their traveling demonstrators give field instructions in materials and appliances to those who ask for them.

It is still a question, however, how for commercial plantings of trees and vines on such defective soils should be undertaken while there is plenty of good, deep land to be had.

Digging the Holes.-Holes for tree planting may be dug at a leisure time after the laying off of the field, even though it is not designed to plant the trees immediately, but our largest planters do not approve the practice. In such cases the sides of the holes should always be freshly pared off before the trees are put in, because the rain and sunshine are apt to cement the sides. In digging holes the surface earth should always be thrown to one side and the lower soil on another. The object of this is to have the top soil to place in direct contact with the roots when the tree is planted, the lower soil being used to fill up the hole with.

\section{TREE SETTERS}

No matter how carefully the stakes are placed in laying off the orchard, the trees will not easily come in line unless some handy device is used for bringing the stem just in the place occupied by the stake which was thrown out in digging. These devices are called 
"tree-setters," and there are a number of designs. Two are given, either of which will give good results. Take a piece of board one inch thick, four inches wide, and five feet long; bore an inch hole in the center, and one at each end at equal distance from the center; then cut a piece from one side of the board, marked by a square, the corner resting in the middle of the center hole. Make two stakes, each one foot long, that will easily pass through the end holes. Place the center of this board against the stake, where the tree is to be

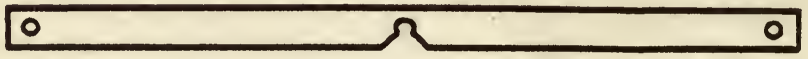

\section{Bar for Tree Setting}

planted; push the stakes into the ground through the holes in the ends, then lift the board from position and proceed to dig the hole. When dug, replace the board over the end stakes in its former position, then plant the tree with its trunk resting against the center notch in the board, and you have it in just the right place.

Another setter is in the form of a triangle: Take three pieces of plain one-inch stuff three to four inches wide and four feet long, and nail them together, forming a three-cornered frame, letting the ends project sufficiently to form a corner, as shown in the drawing. Next make a couple of smooth, hard stakes, well sharpened, and about a foot or sixteen inches in length. When you are ready to set your trees, place the frame flat upon the ground with one corner firmly and fairly against the stake which marks the place where the

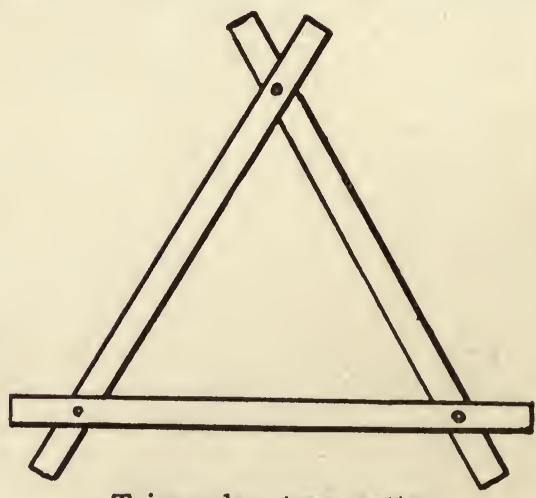

Triangular tree setter.

tree is to stand. Now in the other two corners stick the stakes already prepared for the purpose. This done, pull up the stake against which the frame was first placed-the one indicating a place for a tree-remove the frame, being careful in doing so not to move the other two stakes, which must be left to be used while setting the tree. After the hole is dug and everything ready for setting the tree, again place the frame against the two standing stakes, let the tree drop into the other corner, which will help support it while the dirt 
is being placed about the roots; and this will bring the tree exactly where the stake was originally. If the stakes are properly put in line, so will be the trees.

These setters are described as they are used when the hole is dug and the tree set at the same time. Such is the ordinary practice in planting. If one wishes to dig the holes beforehand, it is necessary to furnish more stakes, as two have to be left beside each hole to mark the position of the setter when the planting is done. Besides its use in bringing a tree into line, the tree setter enables one to judge of the depth of setting as compared with the surface of the surrounding ground. It is not easy to determine this with the eye if the hole be a large one. Where the measuring wire is used to set the stakes, it is sometimes stretched across again after the holes are dug, the tags on the wire thus indicating the places for the trees of the whole row. The trouble with this practice is the bother of having the wire in the way while filling and tramping the earth around the roots.

\section{SELECTING TREES}

In the purchase of trees it is well to patronize nurserymen in your own district, providing they are honest and intelligent men, who keep themselves informed as they should about their business. The advice of such a local nurseryman is often of great value to the newcomer, for he will know by his experience and observation much about the adaptations of fruits and varieties thereof to the region. If, for any reason, local nurseries do not meet your needs, seek some well-established nursery at a distance. It is much safer to deal directly with the grower of the trees than to patronize'traveling agents. Where, however, these agents are the accredited representatives of well-known establishments, they may save the planter time and trouble by taking his order for him. So-called "tree-peddlers," who are jobbers in trees and in too many cases send you refuse trees which they pick up cheap wherever they can, and label them to suit, without respect to truth or honesty, should be resolutely avoided, no matter what inducements or blandishments they may offer.

The California legislature of 1907 passed a law making it unlawful to sell fruit trees representing them to be a certain kind and afterwards to deliver trees of a different kind. To do this is a misdemeanor punishable by fine and imprisonment. Action may be begun at any time within seven years after the date of delivery of such trees.*

It is desirable, if possible, to visit the nursery and see the stock which is to be furnished. The trees should have a good healthy look, with clean bark, and of size enough to indicate a good, free growth. The matter of size is not the only point to consider, for size of the top is not so desirable as well-matured wood and plenty of roots. Stunted trees are not, as a rule, worth planting. There are cases, however, in which, by extra cultivation in good soil, fne trees have been grown even from "culls" from the nursery. The best rule is to

*Statutes and amendments to the Codes, 1907; chapter 229. 
select trees of good medium size, straight and healthy. During the digging season, ask to see samples of the roots as well as the tops, and do not purchase trees unless the roots are healthy looking and free from knots or excrescences. Gnarly and knotty roots in the young tree are a sure sign of insect pests or of unhealthy growth, and planting such trees has occasioned immense loss. Many have been led into purchasing poor trees because they may be had cheap. A tree selected merely because it is cheap may prove the most expensive thing a man can put in the ground.

Guarding Against Insects.-The top of the tree should be carefully examined to discover scale insects if there be any. For this purpose a hand-magnifier should be used. Such a glass should always be in the fruit grower's pocket. One can be bought at any optician's for a dollar or two, which will fold into its case so as to be carried without scratching. Our nurserymen, by forsaking old infested locations and obtaining new ground, now sell much cleaner trees than they did years ago. But still it is well to be always on the watch for pests. Disinfection of nursery stock is now officially provided. Details of treatment will be given in the chapter on injurious insects.

\section{TAKING TREES FROM THE NURSERY}

Trees should be carefully taken from the nursery rows, so as to obtain a good amount of small branching roots. In lifting from the home nursery, digging with well-sharpened spades, which will sever the long roots cleanly, is perhaps the best method. In the large nurseries tree diggers are generally used. They have two revolving coulters which cut through the surface soil each side of the trees, and a sharp, curved blade, which is drawn through the ground under the trees, loosening the soil and severing the long roots cleanly. The tree is then easily lifted, and has generally a much better root system than by the old style of "plowing out," which broke off so many of the small roots and lacerated the larger ones. Whether the tap root should be retained or not is not worth discussing on theoretical grounds. As a matter of fact and practice, the tap root cuts no figure at all in California orchard planting, although the discussion of the question was formerly very warm in this State, and is still occasionally heard. It is important, however, that the planter should have as many small lateral roots as he can get. The small fibrous roots are usually of little account, as they seldom survive transplanting, and it is better to clip them away, if the time can be afforded, as they often prevent the proper close contact of the soil with the larger roots. Cutting back all roots to short stubs at the base of the stem has succeeded in some instances in California on moist lowlands, but longer roots are far: safer in the deep drying of the surface layer which is to be expected in this State.

The roots, after lifting, should not be permitted to dry. Hence, in hauling from the nursery to the farm, the trees should be well covered with wet straw and old sacks, or, if shipped from the nursery to distant points, should be well packed. The best way to pack trees 
is, undoubtedly, to box them in with wet straw, but it costs less and they usually carry well considerable distances if carefully bundled with tules (dry reed stems), the roots packed in wet straw, and the packing and covering bound down tight with ropes to prevent drying out.

Attention should be paid to hauling away trees from the railway stations as soon as possible after arrival. It is not uncommon for shipments to lie on the station platforms for days, often when a desiccating north wind is blowing. Such treatment soon takes the life out of the trees, and often, no doubt, the nurseryman is blamed for failure of trees/ which have suffered some such neglect as this, either from transportation comparies or from the purchasers.

Heeling In.-On arrival at the farms, trees should be "heeled in" as soon as possible; even if it is the intention to plant at once, heel them in just the same, for delays arise often in the most unexpected manner. To heel in, dig a trench or plow a deep furrow, or a double furrow, in light, moist, but well-drained soil; put in the trees singly side by side, removing all the packing material carefully from the roots, laying the tops all one way, and then shovel the earth over the roots until they are well covered with loose soil, and be sure that the soil sifts down well between the roots. Ordinarily this treatment will hold the trees in good condition for a considerable time if need be. If, however, they have become dry before arrival, the bundles should be thoroughly drenched with water before heeling in. In extreme cases, where the top shows drying by shrinking and shriveling of the bark, the trees should be drenched, and then they should be covered root and top with earth for two or three days, when, if the trouble has not gone too far, the bark will recover its smoothness and plumpness. It should be very, seldom, however, that a lot of trees is allowed to get into such condition by neglect. In heeling in it will be found a great convenience and a safeguard against possible confusion by loss of labels, if each variety as taken from the packing is placed by itself in the trench. Nurserymen generally attach a label to each small bundle, if the trees are of several varieties, and the novice is apt to lose all track of his sorts when heeling in the trench, unless he heels in each kind by itself, leaving the nurseryman's label to mark the whole lot of each kind.

If the planter has his own ideas of after-treatment of his trees, or if he is a beginner and desires to adopt the suggestions which will be laid down in this book, he should insist that the nurseryman shall not trim up or cut back the trees before packing. Have the trees packed just as they are lifted from the ground. The work towards the shaping of the tree should be done after it is planted in the orchard.

\section{PLANTING THE ORCHARD}

The young deciduous tree should be dormant before being removed from the nursery row, and if its leaves have fallen it is good evidence of its dormancy. Such, however, is the effect of the climate 
of California, more apparent in some years than others, and with some kinds of fruit than others, and the young tree retains a small part of its activity very late, and in such cases it is not practicable to wait for the complete falling of the leaves. Sometimes for convenience of work, the trees have to be lifted before this takes place, and in such case it is desirable to remove the leaves to lessen evaporation. It is probably better to transplant in this condition for the sake of early setting in its new position than to wait for all the leaves to drop. This statement is not intended to include nursery stock which is kept growing late in the season by late irrigation. Such trees are not always desirable.

Time to Plant.- The best time to plant deciduous trees in California is soon after the early rains have deeply moistened the ground. It is not desirable, however, to have a stratum of dry earth below. This can be removed by irrigation when available. Early planting of common orchard fruits is of advantage for several reasons. First, an early-planted tree gets the full benefit of the season's rainfall, whatever it may be, and a late-planted tree, in a year with short rainfall, is apt to suffer during its first season's growth, unless it can be irrigated. The two main things to observe are the dormancy of the tree and the proper condition of the soil, and both of these are most apt to coincide in many parts of California about the first of January, but in heavy loams in region of large rainfall, the soil may then be too cold and wet. There will, however, be some variation from year to year, and different parts of the State disagree as to the date. Hence, the general rule must be based on conditions, that of the tree and that of the soil. If the novice can not judge these for himself, he must get the advice of some one of experience in the locality.

The dormant period of a tree in California, as has been stated in another connection, is very short. As many cold-climate annual plants become perennial here, so our deciduous trees, in comparatively frostless portions of the State, evince a tendency to become evergreen. The period of dormancy in the root is also shorter than the inactivity of the top. Trees transplanted early are found to have their root wounds calloused over and new rootlets considerably advanced before the buds swell. Therefore, by early planting the tree begins soon to take hold upon the soil, the latter being well settled around it by rains, which often follow early planting, and the high winds, which are apt to come in the spring in some parts of the State, find the tree well anchored and ready to maintain itself.

Again, the proper condition of soil, if not seized at its first coming, may not recur until after the great storms of the winter are over, say in February or March (in most parts of the State), and then often the buds are bursting into bloom and leaf. Planting when the soil is water-soaked and cold is very undesirable, for in such condition it cannot be properly disposed about the roots, the inactive roots may begin to decay, and trees moved at this period are apt to show their dislike of the treatment. If the work has been delayed unavoidably, so that early planting cannot be done, it is better to keep the trees 
neeled in until the proper soil condition returns, even if it be rather late, for a little extra attention to cultivation for retention of moisure will pull through a late-planted tree.

These remarks are of very wide application in this State, but there are exceptions. In our high altitudes, where the climate approaches Eastern conditions in cold and snowfall, practice in planting will also approximate Eastern methods. In regions of very heavy rainfall and on the upper coast where the rainy season and moisture from fogs are prolonged late in the spring, late planting is safer and surer than in the warmer, drier parts of the State.

Another consideration, too, is the slope of the land to be planted. Our hillside fruit growers in regions of heavy winter storms sometimes plant slopes, which, if plowed deep in the fall, are apt to wash badly during the heavy winter rains. On such slopes it is better to plow late in the winter, after the heavy storms are over, and plant when the soil has become warm and mellow.

\section{THE OPERATION OF PLANTING}

Tree planting should be carefully and well done, but it need not necessarily be slowly done. With a kind soil deeply worked and just in the right condition for planting, trees may be put in well and rapidly. Two men work together at a decided advantage. Using the straight "tree-setter," which has already been described, one takes each end, and as soon as the center notch encloses the tree stake, the setter stakes are pushed into the soil, the "setter" is laid aside, and the two men, taking up their shovels or spades, begin first around the outside of the hole, throwing all the surface dirt on the same side of the hole and leaving the tree stake to be thrown out last, because its remaining serves to center the hole. The lower soil is now thrown to the other side of the hole, and when depth enough is reached, the soil at the bottom of the hole is loosened up to the depth of a shovel thrust, without removing it from the hole. A shovelful or two of the surface soil is thrown into the center of the hole, being allowed to remain higher in the center, because this generally furnishes a cushion about the natural shape of the under surface of the root system of the tree. Now replace the tree-setter upon its end pegs, let one man hold the tree with its stem in the central notch in the setter, and while the other man shovels in the surface earth rather slowly at first, the man who holds the tree with one hand will spread out the roots, pulverize and pack the earth around them, being sure that no cavities are left under any of the roots, but that their surfaces everywhere come into contact with the soil, and that they spread out as widely as possible. The earth is being continuously put in by the shoveler, and when the roots are covered the planter steps in the hole and carefully firms the soil down upon the roots by tramping (especially at the cut ends of the roots around the outer side of the hole), at the same time judging of the perpendicularity of the tree with his eye. When this is done, both men use their shovels and fill up the hole with the earth taken from below, being sure to leave 
the last few inches at the surface pulverized, but untramped, unless the soil be very light so that tramping will not overpack it. Some one said long ago that one should not plant a tree as he does a post, ramming down the earth to the very top of the hole. Many trees are doubtless ruined by over zeal in this respect.

The shovel has been mentioned frequently as the tool to be used in planting. Where the soil is deeply plowed, well worked, and free from stone, the shovel is the most rapid tool. Under other conditions the long-handled spade, and in some cases the long-handled spading fork, serves admirably in loosening the soil at the bottom of the holes and in breaking up lumps while filling in. One man with a shovel or spade, and the other with the fork, make a good combination in this respect.

Planting in a Furrow.-A practice which has been largely followed in the Sacramento Valley and which attains greatest speed and cheapness consists in laying off as described in the preceding chapter, and then proceeding with a heavy listing plow, followed by a subsoil plow in the same furrow. The trees are then rapidly set with the least digging. This is all done before the field is plowed. Plowing immediately follows planting. The advantages of this method are ease of work on firm ground instead of a plowed surface, and escape of injury to this surface by men and teams in planting after plowing. It does not, however, prepare the land so well as deep working before laying out.

\section{RANDOM SUGGESTIONS}

The roots of every tree should be examined before planting. All large root ends should have a fresh, clean cut with a sharp knife or shears. Make a slanting cut with the cut surface on the underside of the root. Where a root is mangled or bruised, it should in most cases be cut back to a sound place.

The wound made by the cutting away of the seedling stock above the bud should be at the north or northeast, in order that this weak point may be shaded as much as possible from the afternoon sun.

If the roots of young trees grow more to one side than the other, place the strongest roots toward the prevailing wind.

The use of water to settle the earth around the roots is sometimes desirable in sections where the rainfall is light or uncertain. Pour in four or five gallons from the water wagon after the hand work in spreading the roots and in pressing the soil under and around them has been done and the hole partly filled. When the water has soaked away, fill the hole with fine earth without tramping. In irrigated districts leading the water along the line of trees in a furrow to settle the ground at planting is a good practice. It is almost essential in the planting of evergreen trees which are best moved during the dry season. In early planting in parts of the State where the rainfall is abundant, there may be no need of water-settling; puddling the roots, or dipping them in thin mud and planting them with this mud adher- ing, is governed by much the same conditions as water-settling; it may 
insure growth of the tree when otherwise it might be seriously injured by drouth. With puddled roots especial care should also be taken to leave the surface loose to prevent evaporation. In making puddle, use loamy soil and never adobe, for in dry time the latter will bake around the roots and may kill the tree.

The Use of Manure.-Never put manure in the hole with the $r$ tree. Sometimes the injunction is, Never put anything but well rotted manure in the hole. It is better to put in none of any kind. Manure should be spread upon the ground after planting. The rains then leach it out and it may be turned under in the spring plowing. There are; however, light soils in the drier parts of the State where turning under manure in the spring is a disadvantage, as it makes the soil too porous and facilitates evaporation. On such soils, extra care should be taken to have the manure thoroughly decomposed by composting, as will be described in the chapter on fertilizers, and all applications should bex made either late in the spring to act as a mulch in the summer, or if a mulch is not thought desirable, apply the manure in the fall before the first rains, so that it may be turned under at the first plowing and have the whole winter for disintegration. In this dry climate there is often misapprehension, especially among newcomers, as to what is wellrotted manure. They take the scrapings of the corral, which have been trampled and pulverized, but which, having been kept dry, have never rotted. When this is put in the holes with the tree and then moistened by rainfall or irrigation, it will burn the tree, the first sign of injury being the drying up of the leaves. It is, on the whole, safest and best to put nothing but well-pulverized surface soil around the roots of the young tree.

Depth of Planting.-The depth to which trees should be set has always been a matter of discord among the planters. The safest rule under ordinary circumstances is to get the tree as nearly as possible the same depth it stood in the nursery row; that is, so as to have it stand that way when the ground has settled, or the surface returned by cultivation to its normal level. In planting in loose soil in the drier parts of the State, it is often desirable to plant rather low, because several inches depth of the surface soil become dry, and the roots should be well in the moist layer. But if irrigation is to be practised, it must be remembered that the water level will rise when the soil is saturated and deep-planted trees are apt to suffer. The experience of recent $~$ years is decidedly against deep planting, which used to be advised because of our dry climate. Thousands of trees have been ruined by planting too deep except in light sandy soil.

Speed in Planting.-On good soil, well prepared, trees can be put in rapidly and the job still be well done. It is reported that on one occasion, in planting almonds, twenty men finished sixty-four acres from Friday noon to Wednesday night, placing the stakes, digging the holes, and planting the trees. This would be almost three-quarters of an acre per day per man. In planting peaches and apricots an average of one hundred trees per day to the man has been attained. On the mellow loam, in another case, the average was one hundred and twenty- 
five trees to the man, digging holes two feet square in land which had been plowed twelve inches deep. Such work is only possible on good soil, well prepared, and by men who work well together.

Mapping and Labeling.-Where mixed varieties of fruit are planted, the orchard should be mapped as soon as the trees are set. A good durable map is made of the glazed muslin, such as carpenters and architects use for their drawings. The map can be easily drawn to a scale by using a fraction of an inch to represent a foot. After the map is made, it can be rolled on a broom stick and easily preserved. With such a record, the grower need not care what becomes of the labels, as he can locate a variety at any time by its row and tree number. If, however, one desires labels, let them be made in this way: Take a piece of common sheet zinc five inches wide. Across this, cut pieces three-quarters of an inch wide at one end and tapering to a point at the other. Near the wider end write plainly with a common lead-pencil the name of the variety: This will get brighter by exposure to the weather. The small end may be coiled around the branch of the tree; it will yield as the tree grows and will do no injury. Such labels will last for a long time. Labels attached by a cord or wire should be removed as soon as the trees are set, for they are apt to be forgotten and the tree seriously injured by the cutting in of the ligature. Even when labels are used the map is the only surety, because any kind of a label is apt to be lost by accident or through malice or mischief of intruders.

Mulching.-Although early planted trees on deep soils in regions of sufficient rainfall need only good cultivation, after planting, there are cases in which mulching is desirable. Various light materials may be used for a mulch, but nothing is better than well-rotted straw, in which fermentation has killed all weed seed. Apply it to a distance of two feet around the tree, and to a depth of not less than six inches. It is best done as soon as the tree is planted, and is to be especially recommended when late planting is practiced. Even in localities of light rainfall, if the trees are well mulched early in the winter, irrigation may be unnecessary for the young deciduous tree. Trees planted very late in the spring may, by using great care and mulching well, make as great a growth as those set out early in winter. This should not be an excuse for late planting, but where late planting is necessary, mulching will help the trees to pull through. It is a far easier way of keeping the ground moist than by irrigating, but is not a substitute for it where systematic irrigation is necessary, though irrigation may often be lessened, and in some cases obviated, by extra cultivation or mulching, at least until the trees come into bearing.

Guarding Against Sunburn.-Newly-set trees should be protected against sunburn. This can be easily done by winding strips of burlap from old grain sacks spirally around the stems beginning just below the ground surface to the points where the young shoots will appear. When these start the strip can be loosened and rewound so as to protect the bark between the shoots. The top can be fastened with a stitch or two with a twine needle. Manufactured "tree protectors" of paper or rushes which are readily adjusted around the trees 


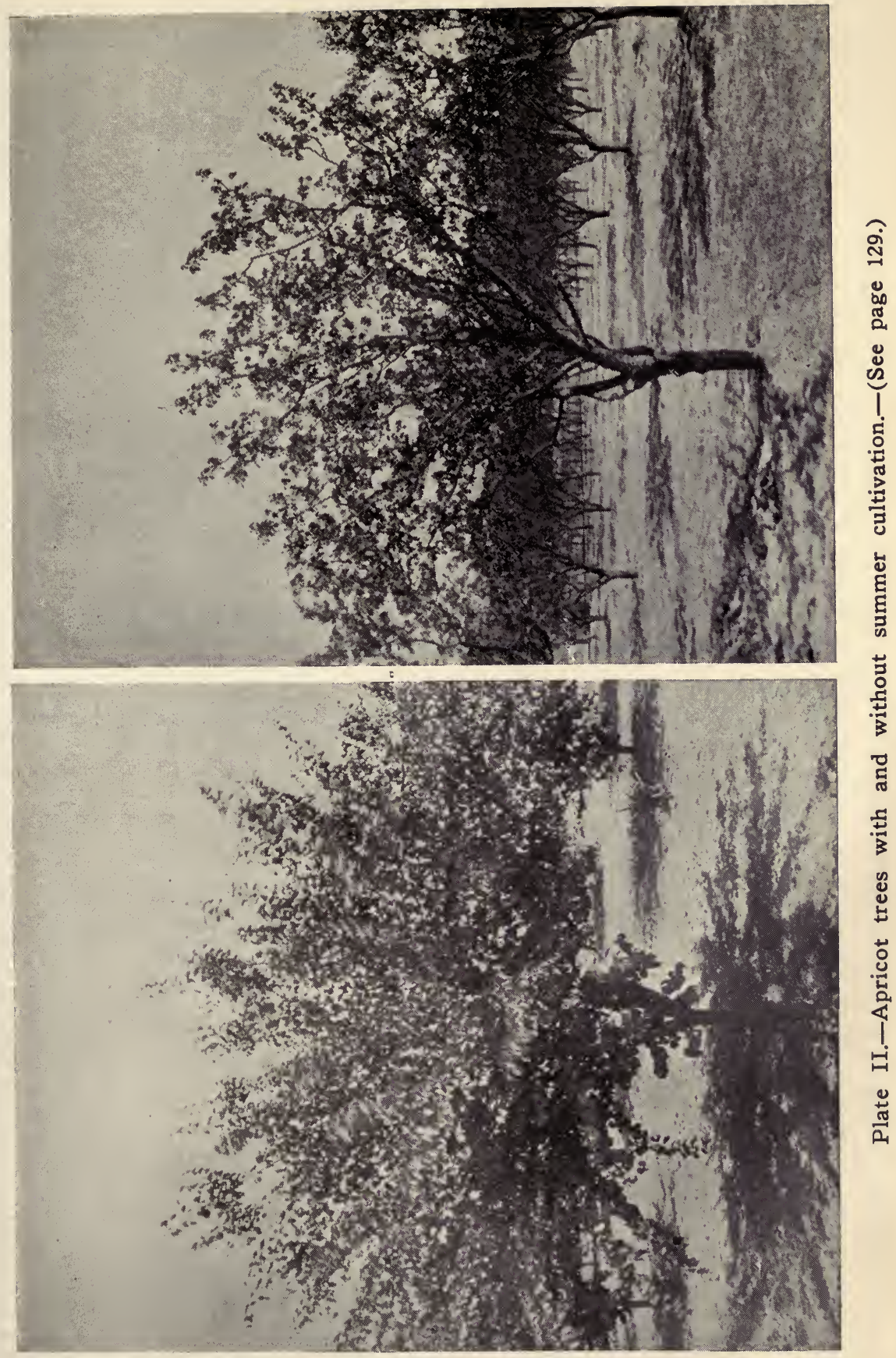



are now largely sold. Whitewash made according to the formula at the close of Chapter IX is a good protection from sunburn. For young trees, however, it should be made with air-slacked lime, which has lost some of its causticity. Another whitewash, which has been largely used for young trees, consists of four ounces of whale oil soap dissolved in each gallon of water, Spanish whiting being stirred in to give the solution a paint-like consistency. Millions of trees have perished in this State, and as many more been condemned to sickly lives, because of sunburn, and borers which seek the injured bark for entrance. Pruning has much to do with saving trees from this evil, as will be shown in the proper connection, but in the hotter parts of the State, the first precaution should be to shade the bark of the young tree with some artificial protection. The use of white lead and oil paint has, however, seriously injured young bark.

Cutting Back at Planting.-Whatever idea the grower may have as to shaping his tree, it must be cut back when planted. Lifting from the nursery has removed a considerable part of the root system of the young tree and the top must be reduced accordingly. The planter who dislikes to sacrifice the fine top will sacrifice future growth and vigor by retaining it. The tree may struggle through and regain strength, but it will for years be smaller than if it had been properly cut back at planting. If the moisture supply should be short the tree may die the first summer which would have survived if differently treated at planting. The manner of cutting back depends somewhat upon the style of pruning to be followed afterward, as will be considered in the next chapter. 


\section{PRUNING TREES AND THINNING FRUIT}

It is not intended to enter into a discussion of the general theories of pruning. The reader desiring to pursue them is referred to the abundant literature on the subject in Eastern and European treatises. The effort to approve or condemn these theories by considering them in the light of California experience and observation might lead to interesting conclusions, but it has no place in a work aiming merely at an exposition of what appears to be the most satisfactory practice in California fruit growing. It will be found that this practice varies somewhat in the different regions of California, sometimes in degree, sometimes in kind, because of different local conditions, and it might be found that nearly all reasonable theories of pruning could be verified in California experience.

Pruning in California is at present almost exclusively a shaping process. Our fruit trees are naturally so prone to bear fruit that pruning to produce fruitfulness is seldom thought of, and still more rarely practiced, while pruninng to reduce bearing wood, and thus decrease the burden of the tree, is quite widely done, to take the place, in part, of thinning out the fruit. Pruning to restore vigor to the tree, as in cutting it back to induce a new wood growth, is also rather a rare proceeding, but probably could be much more widely employed to advantage. We prune, then, for shape and for the many practical advantages which adhere in the form now prevailing in California orchards. Some of these advantages are peculiar to our climate; others we share with those who advocate a similar form elsewhere.

Our best orchards of the same fruits in adjacent localities are almost identical in form and general appearance of the trees, and those more distant differ chiefly in the extent to which the same principles are applied. And this is not because the trees are allowed to follow their natural inclination, which should secure resemblance but because their natural bent is resolutely conquered by agreement of growers that they know what is good for the tree; and this substantial unanimity is the result of the experience of the last fifty-five years. People possessed of the art temperament sometimes complain of the depressing uhiformity and artificiality of orchard-tree shapes in California. They are apt to lament the fact that systematic orcharding destroys the picturesqueness of tree-growth. They should understand that such conception of a fruit tree has no place in commercial fruit growing. The producing tree is the result of the conception of an agency to serve certain purposes. The orchardist does not pursue uniformity merely for its own sake, but rather for the purpose it serves, and the fact that many thinking men have practically agreed upon a certain form as an ideal of producing ability is demonstration that such form is, at least, approximately correct. There is an industrial conception of a tree, 
which is necessarily and essentially different from a conception of picturesqueness based upon the feral type. The wild tree is rude and crude from a cultural point of view.

\section{PRACTICAL PURPOSES OF PRUNING}

One of the first things for a beginner to undertake as he approaches the practice of pruning trees and vines is to form a good idea of the purposes to be served. Imitation is not the foundation of intelligent pruning, though it yields many valuable suggestions. Satisfactory work rests upon a correct understanding of the reasons for each act and to the attainment of this, all study, observation and experience should tend. Possessing this, one can proceed capably, modifying method to meet condition, and producing desirable results, Receive all suggestions and then go quietly to the tree and study your problem in its shade. The tree is the best revelator of its needs. Some of the best pruners in California are men who were untrained to horticulture before they entered upon their orchard work. Reading, discussion, systematic instruction are all valuable. They save much time and many errors, but recourse to the tree affords the sovereign test of attainment.

These may be counted among the practical purposes to be attained by pruning in California: (a) Convenience of the grower; (b) health and strength of the tree; $(c)$ regulation of heat and light; $(d)$ attainment of strong bearing wood; $(e)$ attainment of size in fruit; $(f)$ promotion of regular bearing. Examine trees with reference to their embodiment of these characters and one can hardly fail to secure rays of light upon the subject of pruning which seem dark to so many.

Convenience.-Trees which branch near the ground are most quickly and cheaply handled in all the operations of pruning, spraying, fruit-thinning and picking. Low trees with obliquely-rising branches are more easily cultivated than any form with horizontal branches, unless the head is carried so high that the animals pass easily under the tree. To do this sacrifices all the other conveniences and economies which actually determine profit, and is really out of the question from a commercial point of view. Sometimes it does not pay to pick some fruits at a certain distance above the ground, when picking at half that distance yields a profit.

Health and Strength.-It is imperative in most parts of this State that the sunshine be not allowed to touch the bark during the heat of the day. This protection is secured even for young trees by low branching and encouragement of small, low laterals. The low tree with properly spaced branches attains superior strength by virtue of thick, strongly knit, short growth between branches, and by its strong, stiff, obliquely-rising growth sustains weight which brings horizontal branches to the ground, and thus even high-headed trees are liable to continually increasing interference with cultivation, and the desperate grower has to raise the head of his tree higher into the air and farther above the profit line, while at the same time he renders it more liable to sunburn, to bark-binding and to unthrift by forcing the sap to flow 
an unnecessary distance and through wood and bark which impede its movement. Besides a low tree escapes stress by strong winds which a high tree invites and at the same time is less able to withstand. Pruning for health and strength of tree also includes the removal of unthrifty or diseased parts, which are not only an incumbrance to the tree but may communicate to other parts the causes of their ill condition.

Heat and Light.-The maintenance of strong bearing wood in the lower part of the tree is conditioned upon the proper pruning of the top of the tree. How far the upper levels or the shade-layer of the tree can be safely opened, depends upon the local climate in each fruit region. The rule must be the higher the summer heat the denser the tree; the lower the heat the thinner the tree; but everywhere the proper condition of openness must be constantly in view in pruning. Not alone must this be done to maintain thrifty growth below, but it is also essential to the best growth and ripening of the fruit in the lower and interior parts of the tree. Fruit inferior in size, color and quality results, in part, from lack of pruning to regulate the admission of light and heat, sometimes one, sometimes both, to the shaded portion of the tree.

Bearing Wood.-Good fruit develops on good bearing wood and good bearing wood is the product of proper degrees of light and heat, as has just been urged. But bearing wood in the case of some fruits is new wood; and reduction of old wood for the purpose of forcing the growth of new wood must be constantly in mind. Renewal is more or less a consideration with all trees, and especially the securing of strong new wood. This is a point upon which close study of the bearing tree will yield most satisfactory suggestions.

Size of Fruit.-The size of fruit, providing the tree is healthy and vigorous, depends upon the character and amount of bearing wood which the tree is allowed to carry. Removal of part of the fruit burden is done by thinning after it is well set, but this labor should always be minimized by antecedent pruning, which aims to retain more or less bearing wood according to the vigor, size and bearing habit of the tree. Thinning out of bearing shoots and spurs, when either are clearly seen to be in excess, should be the constant study of the pruner.

Regular Bearing.-This point is largely involved in the preceding and affords an additional incentive. Regulating the amount of fruit borne in one year may involve the profit of two years, because a tree may not be able to produce an excessive amount of fruit and perfect good fruit buds for the following year. It may generally make buds which will bloom, but not always that. If it does make the bloom, it is no guaranty that the bloom will be strong and effective for bearing. Consequently, pruning for reasonable amount of bearing should always be borne in view and should be practiced at the close of the year of nonbearing with particular diligence, if the alternate year bearing habit is to be broken up.

The foregoing are among the practical purposes to be served in pruning. There are others, but these will suffice to emphasize a single point, and that is, that pruning can not be compressed into a single formula, nor can one learn it by a recipe. There are various ends to 
attain; they may be attained in different ways, although it is not strange that substantial agreement in methods does largely prevail. It is better to try to understand the purposes than to memorize the formulæ. Get the tree and its interest clearly in the mind; have an ideal toward which to work; be more interested in why a neighbor prunes in a certain way than how he does it. Learn constantly by all available means, and at the same time study the visible forms and aim to understand their fullest significance.

\section{FORMS OF TREE BEST SUITED TO CALIFORNIA CONDITIONS}

The form of deciduous fruit tree which prevails with singular uniformity all over the State is the "vase," or "goblet," or "wine-glass", form, all these terms signifying a similar shape. There are different ways in which this form is secured and maintained in different parts of the State, and with different fruits, which will be especially noted in the chapters devoted to these fruits.

The mainspring of success in California is to grow low trees. Low is a term admitting of degrees, it is true, any may imply a trunk six inches up to one or two feet, in the clear. In addition to the general advantages of low-trained trees which have been described, there are special reasons for this form in California. Hundreds of thousands of trees have been destroyed by the exposure of a long, bare trunk to the rays of the afternoon sun. The sun-burned sides have given the conditions desired by borers, and destruction has quickly followed. - Some times young trees have not survived their first season in the orchard, because of burned bark, or this, with the added injury by the borers. It is also found by California experience that growth is more vigorous in the branches when they emerge near the ground. Even where actual burning may not occur the travel of the sap through the longer distance of trunk is undesirable. It is believed, also, that benefit results from shading of the ground at the base of the trees, by reducing evaporation, and by maintaining, a temperature of soil better suited to vigorous root-growth.

But whatever may be the reasons, the fact is indisputable, the higher the prevailing summer temperature, and the greater the aridity, the lower should the trees be headed. Trees which will do well in the central and upper coast region and adjacent to the bay of San Francisco, with twenty-four to thirty-six inches of clear trunk, would dwindle and probably perish in the heated valleys in all parts of the State. In such situations, both north and south, the best practice is to head the tree fifteen, twelve, and even some hold as low as six inches from the ground. There will always be some difference of opinion as to detail, but the necessity of making the trunk short enough to be effectually shaded by the foliage is admitted by all growers.

Variations of the Vase Form.-There are several variations of the vase form to be found in California orchards. The prevalent is a vase form with short-jointed leaders which will be described in detail 
as the "common vase form." Another, which is gaining in popularity, aims to grow leaders trained along in directions first chosen for them, and this will be described as the "vase form with continuous leaders." The latter method aims to secure more open centers and to produce fruit nearer to the leaders or, as one may call them, the ribs of the tree-the figure having a rough resemblance to the ribs of an inverted umbrella rather than the ribs of a man.

Characteristic of the Common Vase Form.-This vase form is a product of French ingenuity in the training of dwarf trees, but it has undergone very marked modification in California, losing much of the accuracy of its outline and gaining vastly in speed of work and in bearing capacity of tree without sacrificing any practical value which adheres in the design.

This vase form dispenses with the central stem or trunk at a certain short distance above the ground, but this is not done for the purpose of securing a hollow or open-center tree, which is a leading characteristic of the old European vase form. The few branches which are desired to grow from the short stem are pruned when the tree is young to induce successive branching with short interspaces. At each cutting the aim is to get two branches from one, and nearly as posible of equal vigor, so the California tree does not, except, of course, in occasional instances, show the outline of a leader from the bottom to the top, but there is a succession of branchings, turned this way or that by the skillful pruner, occupying available air space, distributing the weight so it comes more nearly over the center of gravity and at the same time knitting the fibers of the branch so that the weight of the fruit is well sustained. This idea, however, is not allowed to go so far as to wholly close the
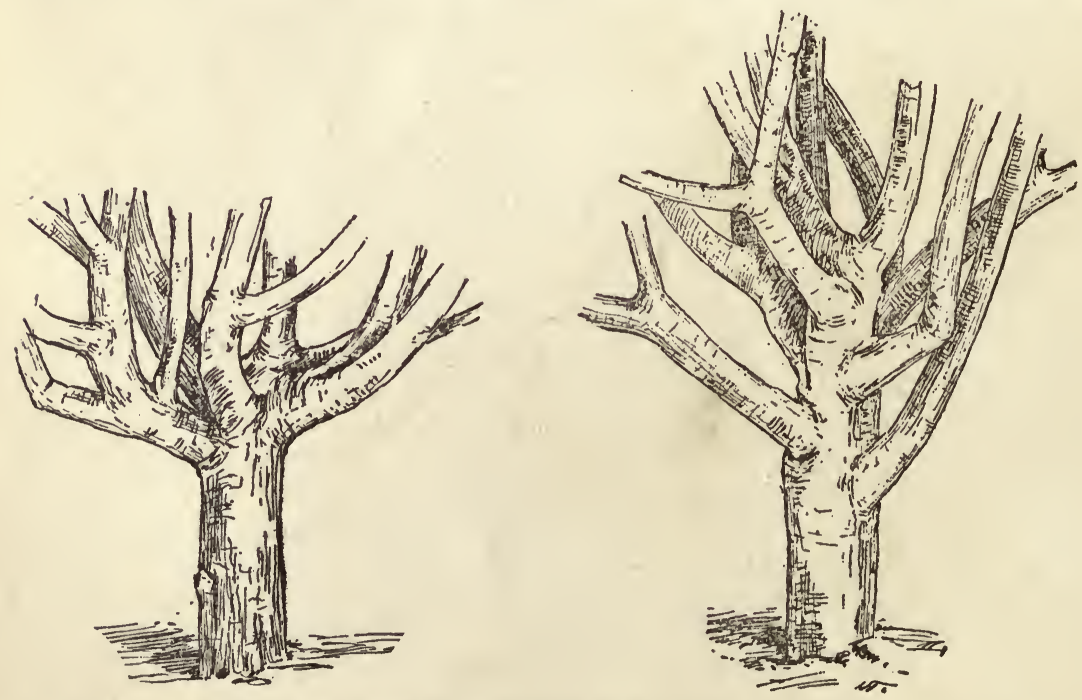

Forms of head resulting from cutting bsck.

Twelve-year-old apple tree in the writer's garden in Berkeley, showing forms of head resulting from cutting back for greater and less spacing of main branches at planting. 

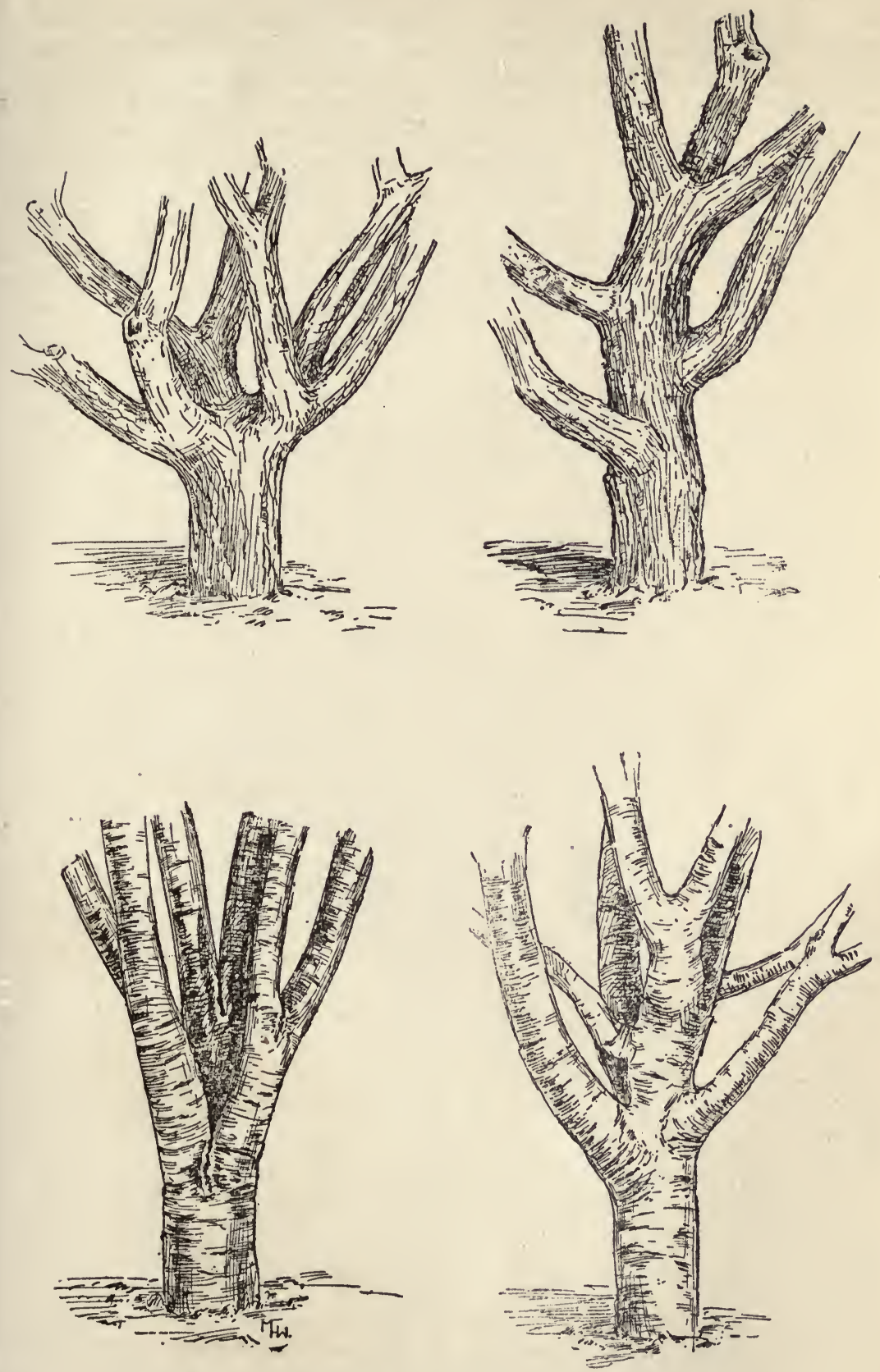

Results of cutting back to longer and shorter stems.

Apricot and cherry trees twelve years old, showing results of cutting back to longer and shorter stems at planting, as an experiment in head form. 
interior of the tree, but to retain such degree of open interior as is found desirable. When the tree is laden with fruit, the weight naturally expands the top quite enough to admit the sunlight without exposing either the fruit or the branches to danger of burning. Thus it appears that instead of the true vase of wine-glass, with hollow interior and thin walls, we have the general exterior outline of this model, but give a good part of the central area of the figure to bearing shoots, and thus secure a large bearing surface with well-strengthened supports.

It has been found that this many-branching form, developed upon a few main branches well placed upon the trunk, gives a stronger tree than can be had by growing a considerable number of leaders, all starting from near the point where the tree was headed at planting. Such leaders crowd each other at the point of emergence from the stem, and when laden with fruit, sway outward and break out at this point. A vastly stronger tree is secured by starting but four or five branches from the low trunk and letting them emerge from different sides of the stem, and at different levels. Thus teach main attachment to the stem has abundant room, and the wood enlarges symmetrically and solidly. The expansion of the top is attained by the branching which follows the cutting back of succeeding years. Starting branches from nearly the same level on the stem has been the occasion of great losses of overladen trees, and quite a considerable recourse to strengthening up weak trees by running bolts through from side to side at the points where experience shows breakage is likely to occur. In this respect it is now clearly shown that the practice which was widely adopted a few years ago of beginning with a very short stem and using the three or four adjacent buds nearest the point to which the tree was cut back at planting is defective. It is much better not to cut back so far at planting, but to leave a longer trunk, keep a greater distance between the main branches and still have the lowest branch as near the ground as before, thus securing a tree which is practically as low as that secured by the old method of starting. This point will be enforced by illustrations.

\section{HOW TO SECURE THE COMMON VASE FORM}

For the benefit of the inexperienced reader, it will be well to illustrate the steps by which the form of tree found so generally desirable is to be attained.

Cutting Back at Planting.-This has been already mentioned as essential to strong growth of the transplanted tree. It is also the prime act in securing a tree with a low head and strong branches. Formerly trees were cut back farther than desirable and the branches allowed to crowd each other, as has just been stated. It is better to retain twenty-four inches of stem than twelve inches-providing care is taken during the first summer to prevent, by pinching, the growth of too many branches near together. Allow those to grow which are more distant from each other on the stem and pinch the intervening shoots. In this way one can have the lowest branch at six inches from the ground in the hot valleys if desired, or twelve inches in the const 
valleys, and the highest branch at eighteen or twenty-four inches. This gives about twice the distance between the main branches which was formerly allowed, and it is of vast advantage to the strength of the tree. The illustrations of this fact are from trees planted by the writer in 1887 to test this matter. At this date they are large trees and show the forms of heads resulting from different spacing of branches on the young trees during the first summer's growth.

First, then, cut back the tree just after planting, as shown in the engraving, deciding first at which height you wish trees to form heads, and cut them all back as uniformly as possible and still secure a good bud just below the point of cutting. To preserve these buds the trees should be handled carefully while removing from the nursery and during planting.

If the tree has already grown laterals in the nursery where the head is desired, three or four of these properly' placed on the stem may be selected to form the main branches, shortened in to the sound bud nearest the stem, and other laterals, not desired to form the head, removed. This treatment is shown in the engraving of a young peach tree well branched in the nursery. If all the laterals on the young treehave started out above where the head is desired, as is sometimes the case, it may be necessary to remove the whole top, and usually others will start below afterwards. If there are no buds visible on the stem at the place where the head is desired, the choice must be made between heading the tree higher up, where the buds are, or cutting back without regard to buds, trusting to the development of latent buds at the right place, or to the growth of a shoot from below, which can be cut back to form a head the following year. It is for this reason, among others, that planters prefer a yearling tree which has not branched, but has good buds all along the stem. Peaches and apricots usually branch in the nursery, but usually have dormant buds at the bases of such branches which can be employed in making new growth where it is desired.

After cutting back at planting, the shoots desired to form the head are allowed to make their full growth without interference. All shoots not desired for branches are pinched off after growing out two or three inches, leaving a bunch of leaves to shade the trunk and contribute to its stouter growth. Constant watchfulness is necessary to pinch off undesirable branches all the first summer.

First Pruning.- In the winter following planting, the shoots of the previous season's growth are cut back to about ten or twelve inches from their junction with the stem. Some prefer to cut shorter, but this is apt to huddle the branches too close together when they get old and stout. Growers, however, do not agree on the exact length which these future main branches should be left at first pruning.

If, during the first summer's growth, all shoots except the number desired to form the head have been pinched back, the first winter pruning consists only in cutting back the main branches. If laterals have grown on the parts of these branches which are to be left on the tree, they should be cut back to a bud or two. Some growers practice cutting away all such laterals cleanly because they are too young to bear fruit, but it is better to shorten and retain at least a part of them and, when 
growth starts, pinch the tips after throwing out'a few leaves to shade and thicken the branches, just as the short growths left the previous summer serve the main stem.

Second Pruning.-During the second summer it is usual to allow two branches to grow from each of the main branches left at the previous winter pruning, and to pinch off all others, as described. These branches are allowed to run out their full growth, except where excessive growth is made, and then it is repressed by summer pruning. This is done with the apricot in the warmer parts of the State, as will be considered at length in the chapter on that fruit. Usually, however, the main branches are untouched during the second summer's growth unless some are running out so far as to make the tree lop-sided.' During the following winter the main branches are cut back from one-half to two-thirds of the growth they have made, and if too many strong laterals have grown below this point, some are shortened, others are removed entirely where they are apt to cross or crowd each other or to interfere with cultivation. It is not desirable, however, that all small growth should be cleanly removed. Some of these small shoots will bear a little fruit and the leaf action is in any case desirable as a contributor to the strength of the larger branches to which they are attached. Besides, they serve to shade the bark from sunburn.

Third Pruning.-When the tree reaches its third winter pruning, its form is well outlined, and early-bearing trees like the peach, apricot, almond, Japanese plum, etc., will give the grower a respectable
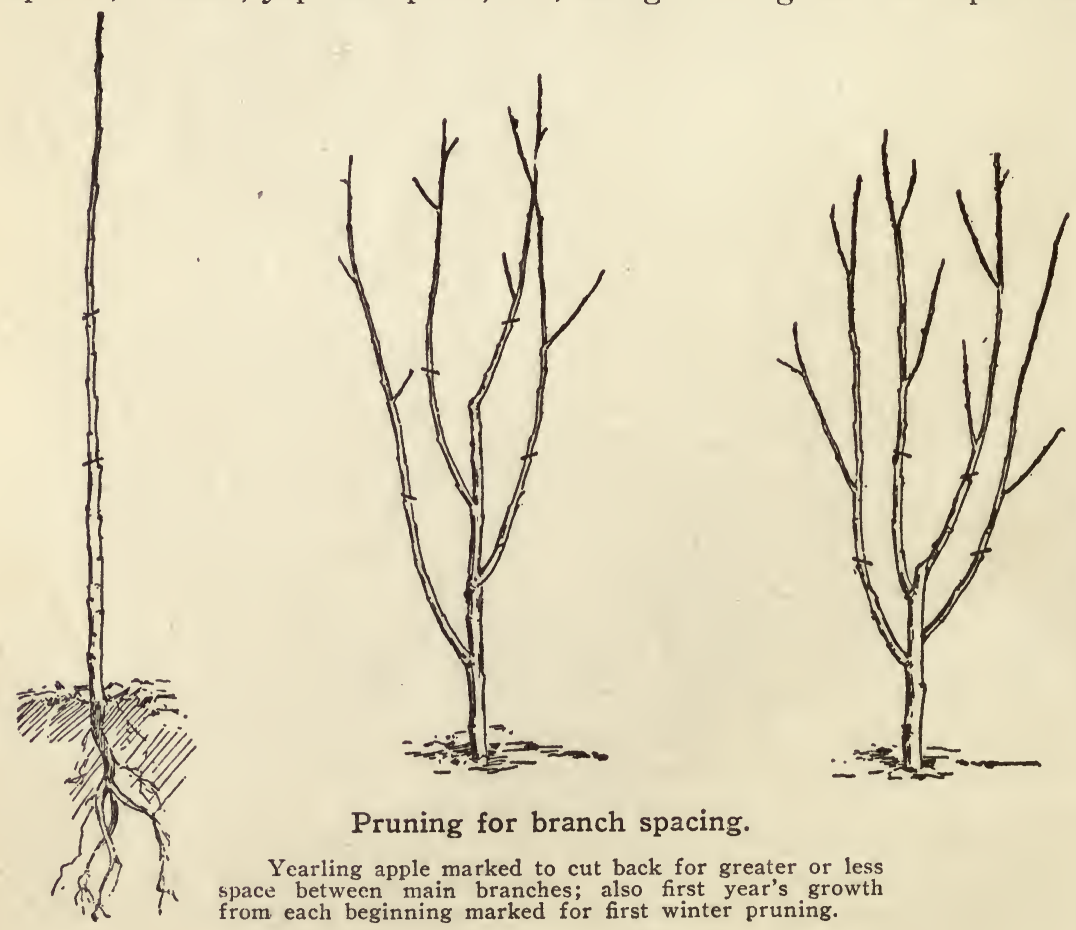

Pruning for branch spacing.

Yearling apple marked to cut back for greater or less space between main branches; also first year's growth from each beginning marked for first winter pruning. 
crop the next season. To bear this crop greater care should be taken at the third winter pruning to leave the small laterals low down on the main branches, for on them, clustered close in the head of the tree, most of the first crop will be found. Though some trees, as stated, do bear earlier than the third summer, the fruit is not usually considered of commercial account until the third summer. An engraving is given of a peach tree just after its second winter pruning. It is a very good representative of the common vase-form of a tree as grown in California. It has four main branches, each issuing from a different point on the stem, each permitted to carry two main branches, which are not arranged around the circumference, but some of them tending toward the center. At the third pruning more shoots have been left than are required by the rule, for, starting with four main branches, there are usually sixteen left at the third pruning.

\section{VASE FORM WITH CONTINUOUS LEADERS}

This form has the same prototype as the common vase form, viz.: the French garden tree and it adheres more closely to the motives and characters of its prototype. As practiced in the Winters region on the west side of the Sacramento Valley, it is aptly described by D. J. Whitney as follows:

"It is called the open-top system of pruning. Hold in mind a wide, shallow bowl with long legs bent inward and joining at the bottom coming down rather regularly from rim to base and you know what a typical open-top apricot tree looks like. The idea seems to have leaders to come up along the outside of the tree, none in the center at all, to have the center of the tree open, or occupied only by fruit wood and not much of it. In the yearly pruning the development is all outward. There is an effort to have fruit wood along the leaders down to the crotch, but often without success. Looking over an orchard from above, there can be seen a lot of flat green disks set regularly twenty to thirty feet or so apart."

This variation of the vase form is quite a departure from the common vase form, both in its purposes and methods. It is used in the Winters' region for apricots, peaches and plums. It is coming into the upper San Joaquin Valley for peaches, the leaders, however, being grown nearer to upright because of the weaker wood of the peach. As used for that fruit it will be discussed in detail in Chapter XX. It is also used for the lemon, as exemplified by the flat, saucer-shaped trees which one will see in some lemon orchards. As something of a departure, both in principles and practices, from the common vase form which has widely prevailed for the last forty years, it has still to widely demonstrate its claims to superiority.

\section{PRUNING BEARING TREES}

Three winter prunings of deciduous trees usually establish their permanent form, and subsequent pruning is chiefly directed toward the retention of that form; for strength of branch and stem; for renewal 
of bearing wood; for regulation of amount of bearing wood; for relative light and shade, and for convenience in cultivation and other orchard work. Naturally, these ends are sought according to the needs and habits of different fruits, and the methods of attaining them will be discussed in the chapters treating of these fruits. There are, however, certain general considerations which are proper in this connection:

Pruning during the dormancy of the tree induces greater growth of wood during the following summer ; pruning during the active period reduces wood growth and promotes fruit-bearing. The amount of wood removed during the dormant period will make the summer growth

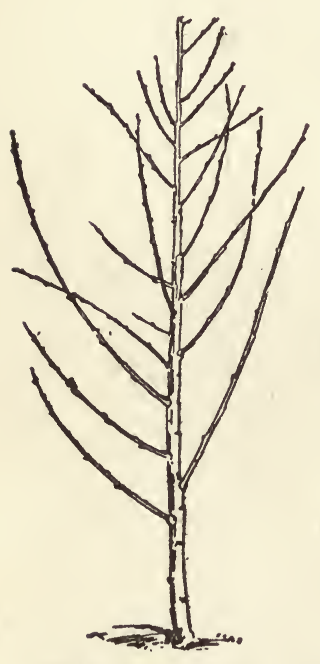

Yearling peach. Cut back at planting.

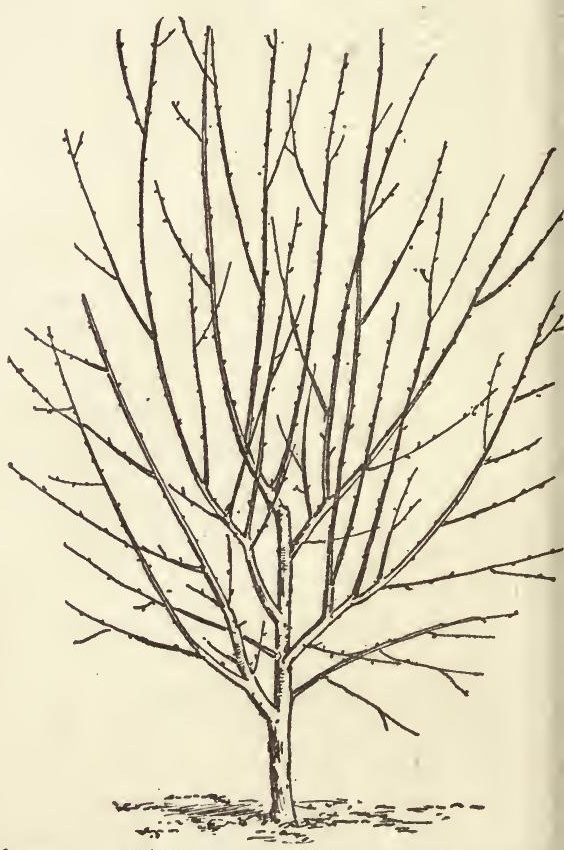

First summer's growth in the orchard.

These sketches, and those en pages 118 and 119 , represent the progress of the peach tree from a branched yearling to bearing form entering the third summer.

of wood proportionately stronger. Whether the total weight of wood growth would be greater may be questioned, but the effective wood growth is certainly greater. Whether the feet of new wood grown on a peach tree cut back to stumps in the winter would be greater in weight than all the inches of growth which would be scattered all over the surface of the tree if not cut back, may be doubted, but the new growth secured by cutting back will be of immense vigor, and the following year will bear large fruit, while the new growth on the tree not cut back will be thin and short and the fruit absent or indifferent. The weaker the tree or the branch or the twig of the tree, the greater part of it to be removed when dormant to get the stronger new growth. 
to a certain definite height is wrong. Trees shorn across at a certain line become thick as a brush with top shoots which require extensive thinning, or the bearing wood will soon be all at that level through failure of the densely shaded bearing wood below. Cut to the nearest lateral below the line you wish to approximate, and shorten the lateral, if desirable, and the result will be fewer and stronger shoots than from a stub-cut.

In the treatment of bearing trees the main effort should generally be toward thinning or reducing the number of bearing shoots. This is related to the important work of thinning the fruit to reduce the burden of the tree, and will be mentioned again in that connection. The work

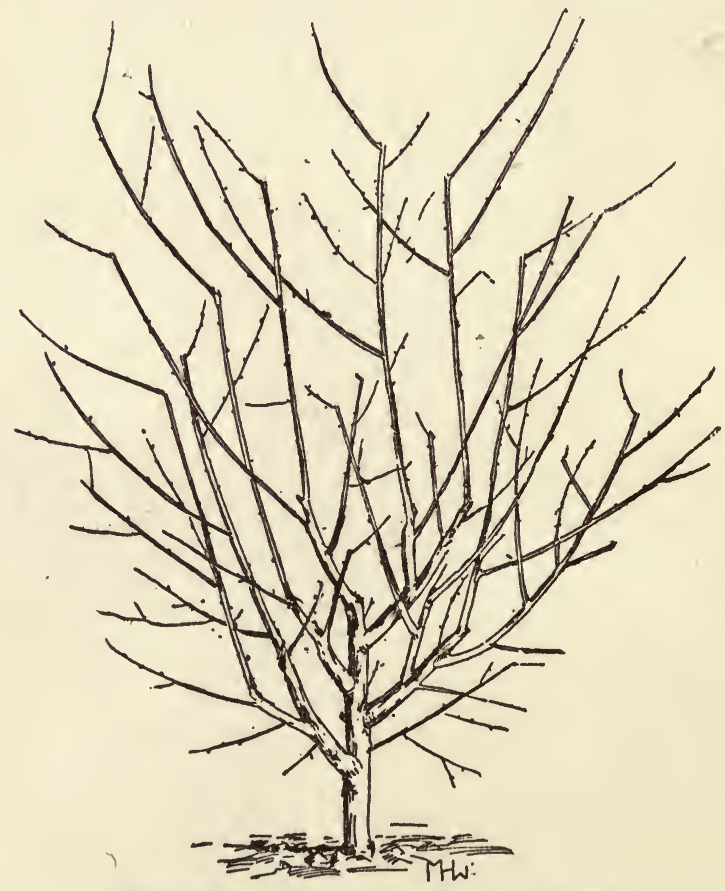

Second winter pruning in orchard.

has, however, a bearing beyond the size of the individual fruit specimens. It involves the whole future of the tree as a profitable affair. An unthinned tree becomes a thicket of small, weak, and dying laterals and spurs. An attempt to cure this afterwards by sawing out many large branches is only partially successful, though perhaps the best thing that can be done after such condition has been allowed to exist. The only way to keep the interior of the tree full enough of strong, bearing wood is to resolutely and regularly thin out surplus shoots as the tree advances in age and size. This work is as important with trees which are not regularly cut back, as with those which are thus treated. It is one of the most vital as well as the most generally neglected item in orchard practice. 
In thinning out lateral bearing shoots seldom leave more than one at any point; select the strongest; remove the rest close to the branch. When a new shoot springs out at the base of an older one remove the, older one; when a new shoot breaks out on the side of an older one cut the older one back to that point. In thinning always reject the older, weaker laterals or spurs. This does not apply to the outbreak of strong suckers or water sprouts below the main branches; they should usually be cleanly cut away unless a new main branch is desirable.

Pruning of bearing trees should always have regard to the removal of branches which have become decrepit through sunburn, blight or disease of any kind, frost injury, or in any form die-back from whatever cause. Such wood is not only of lessened value, but there is also danger of extension of the trouble. Removing such wood and training new wood to take its place should always be in mind.

Where cutting of large branches is demanded for any reason it should be remembered that the wounds are most quickly healed and least injury to the tree is to be apprehended if the cutting is done near the beginning of the growing season, and not at the beginning of the dormant period.

\section{TIMES FOR PRUNING}

Some changes of view have lately prevailed as to the times, within the dormant period, during which winter-pruning can be done to the best advantage. Formerly it was thought to be a vital matter that no cutting should be done until the leaves had fallen, and this is still the prevailing practice, and may prove to be on all accounts the best. Recently, however, pruning in autumn has been quite widely practiced.

Fall Pruning.- There is a time near the end of the active season in California when the foliage changes its aspect. There is no marked change in color, perhaps, but there is a certain limpness and drooping which betokens decided decline in activity. It comes first to the early fruits, the cherries and apricots, for instance, and upon old trees earlier than young ones. The buds are well formed; the season's growth apparently complete. There are no frosts to hasten the fall of the leaf and it remains in place. Does it render any important service? On the conclusion that'does not, many growers begin the winter pruning while the days are longer and the ground dry and firm, rather than delay pruning until the short, dark days and rain-soaked soil of December and January render pruning expensive and disagreeable. Those trees are first pruned which first assume the appearance described, and the work proceeds with other varieties afterwards until the winter pruning may be finished by December 1-about the time when it commonly began under the old practice. Not only is more thus accomplished in the same number of days' work, but the orchard is earlier in shape for the winter spraying and cultivation, and the grower is ahead of his work and not behind it all the season if the season is unusually rainy. Several years' practice of this method discloses no bad results except in the one item of increasing danger from frost. Vines and trees pruned early in the dormant period have a tendency to start growth earlier than those pruned late in the dormant period. In places, then, where 
early bloom and fruit-setting are particularly threatened by frost, this practice may be undesirable.

Spring Pruning.--Resting largely upon this matter of retarding growth, the practice of pruning very late in the dormant period, or, in fact, at the beginning of the growing season, is also gaining wider adoption where frost injury is especially feared. It is not actual freezing, but a drop of two or three degrees below the freezing point which is feared, and during recent years such a temperature has wrought havoc with some fruits, in early valley regions particularly. Later pruning, even after the bloom and foliage have appeared, has worked no injury to the trees, but it is less conveniently done than when the trees are free of foliage.
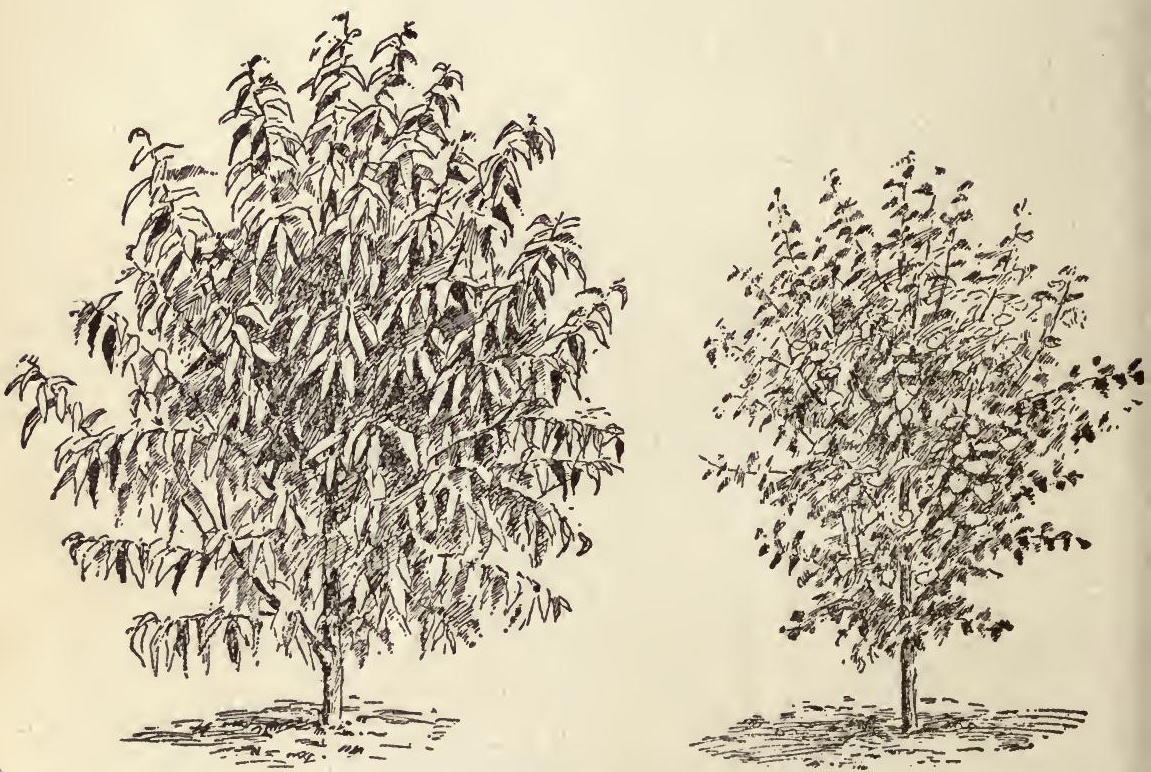

Young peach and apple trees, showing branches well spaced on the stems.

Summer Pruning.--Summer pruning, to induce bearing, is, as has been previously intimated, but little employed in this State, for the constant tendency of our trees is to bear early and to overbear. Enough has, however, been done in individual cases to show that fruit-bearing is promoted by pruning after the chief growth of the season has been attained. If the pruning results in forcing out laterals late in the season it has been done too early. What is desirable is the strengthening or development of fruit buds, and this will be accomplished after the energy has been too far dissipated to make new wood growth.

Summer pruning to check the too exuberant wood growth of some kinds of trees is employed to some extent, chiefly in the warmer parts of the State, where the vegetative process in some trees seems fairly to run riot, and unless checked is apt to ruin the tree by breaking to 

pieces when the wind and weight of fruit test its strength. The methods of summer pruning employed in different parts of the State for different fruits will be considered in connection with the special chapters on these fruits.

Summer pruning to preserve form is another matter, and relates in the main to pinching in, to check undesirable extension and to direct the sap toward shoots in which growth is desired. This practice is approved by most of our orchardists, and is employed by them to a greater or less extent. More people believe in it than practice it, however, because the summer months, with their long succession of fruits to be gathered and shipped or dried, and the additional consideration that there is always a scarcity of labor at this time, give the orchardist so much work to do that he is more apt to confine his "pinching" to a little that he may do now and then when he has a few moments' leisure than to do the work thoroughly and systematically. The result is that the regular winter pruning is the main operation for tree shaping in this State.

There is such a great difference in opinion about summer pruning that it will be very difficult to make any assertions about it which will not be disputed. Much of this difference comes, of course, from differ-

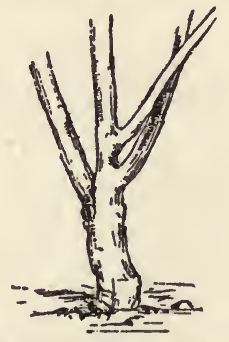

Weak tree from ill-spaced branches.

ent conditions prevailing in different trees and in different parts of the State, and some of these will be met, as already promised, in following chapters. Leaving these wholly out of consideration at this time, it is safe to advise those who wish to secure symmetry or any particular form in any kind of a tree, that they can resort to summer pinching with advantage, and can sometimes to advantage remove wood too large for the thumb and finger to sever.

Constant watchfulness should be maintained for adventitious shoots starting out on stem or limb at points where branches are not desired. Wherever they start out strongly, they should be pinched, or entirely removed, according to the best judgment to be formed in each case. They should not be allowed to divert the sap from the fruiting wood to make the generally coarse and sterile wood which is characteristic of them. Suckers which properly, according to Downing, are "shoots sent up from the root or from parts of the stem below the surface of the soil," should be removed whenever discovered. In common California parlance the term "sucker" is used as a synonym for "watersprout" and signifies undesirable shooting from any part of the tree or vine. 


\section{RENEWING OLD TREES}

Improving and renewing trees by cutting back and grafting has already been considered under the head of propagation. It is often desirable to renew trees of a satisfactory variety, and this is done simply by cutting back when the tree is dormant. Cutting back was formerly done early in the winter, before the rise of the sap begins, but more recently it has been seen that the exposure of large cut surfaces for weeks or months before growth begins, results in drying and shrinkage of the bark and checking of the wood, both of which are avoided by amputation later in the dormant period or during the early part of the growing season. In cutting. back, of course, those stumps should be left to support new branches which will secure the best balance and symmetry in the new head. When the new growth starts there generally appear many more shoots than are desirable, and selection of the best-placed and most vigorous should be chosen, the others either being rubbed off in the bud or pinched back when a few leaves are put out. In cutting back trees, the exposed trunk and branch stumps should be wrapped in old sacking, or carefully whitewashed as protection from sunburn.

In removing large limbs it is desirable that the cut should be made in the right place so as to secure quick covering of the scar with new growth. Cutting so as to leave a long stub results in an unsightly piece of dead wood on the tree, and this, in decaying, carries the decay deep into the center of the trunk or branch. Cutting too close prevents covering with the new bark, and also results in a hole in the branch. Cutting just to the right mark, which is the outer edge of the little collar or swelling which will be found at the base of all branches, enables the wound to grow over quickly, and if the wound is properly treated when cut, there will be no decay, and the wound will soon be obliterated.

In amputating large branches, an undercut with the saw should be made first so that the bark shall not be torn as the branch falls. Another good way is to saw off first at a distance from the final cut, and then saw off smoothly at the right place when the weight is removed.

Trees often become "hide-bound," as it is called. Especially in this dry climate the bark gets dry and tough, therefore can not expand in proportion to the growth of the tree, or supply the amount of sap necessary for the demand. Slitting such trees here and there up and down the trunk and main limbs with a sharp knife seems to have good effect, for often in three months the cut opens half an inch, and a fine, clear bark, with an increase of growth, results. On old trees, too, there is often a growth of moss and lichens which should be removed. This can be done by scraping off the rough, loose bark and spraying with an alkaline wash, composed of one pound of caustic soda or potash to six gallons of water. If scale insects are present, the lime, salt, and sulphur spray should be used, as will be described in the chapter on injurious insects. This will remove the parasites, give the trees a clean, bright bark and contribute to their vigor. 


\section{PRUNING TOOLS}

There is some difference of opinion as to the comparative value of the pruning knife and the pruning shears. The knife, if sharp, and well used, makes a smooth cut, with no bruising of the bark, and such a wound heals over perfectly. The shears, if of good pattern and sharp, also make a very good cut, but there is always some little injury to the bark on the side opposite the entry of the blade. On small cuts, say three-quarters of an inch or less, if the blade is kept very sharp, the resistance does not make sufficient injury to the bark to seriously consider, and the speed with which the shears can be used renders them the main reliance for all the smaller pruning. Nearly all styles of hand shears are used in this State.

There are, also, two-hand shears, which are very powerful, and enable one to work very quickly. When kept well sharpened they are very effective tools. There are a number of styles in use, both homemade and imported.

Still another arrangement of shears is mounted on a pole, the cutting blade being operated by a cord, and having a spring to throw the blade back. The pole is jointed, so that one or more lengths can be used. With this device one can stand on the ground and shorten in the top shoots of a tree very handily.

For larger cuts than can be made with the pruning knife or onehand shears, there are pruning saws of different styles, of which two styles are chiefly used. One has a frame made of the best spring steel, constructed somewhat on the plan of a butcher's saw, except that the saw blade is much narrower; and instead of being stationary, it revolves so that the pruner is enabled to adjust the blade to cut at any angle, as is often necessary to do when cutting where limbs grow close together, and where it would be impossible to use an ordinary saw of a wider blade. The blade is only one-fourth to one-half inch wide, and therefore not liable to get pinched in the cut. Strength is imparted by a tension screw under the handle, which tightens the blade. The blade is easily detached by slackening the tension screw, and lifting the blade out of the slot in the clutches at each end. The blade can be thus reversed and made to cut with a push or a pull, as may be desired.

Another popular saw is the curved pruning saw, with twelve and fourteen-inch blades, which cuts with a pull.

During recent years it has been possible to find quite full assortments of pruning tools at the hardware and general merchandise stores in all our fruit districts where these devices can be compared and selection made according to individual preference, for there can be no best tools for all men and all uses.

\section{CUTTING TO A BUD}

Whatever may be used to make the cut, it is important to sever the twig or shoot at that distance from a wood bud which gives that bud the best chance to grow well, and at the same time facilitates the healing and complete obliteration of the scar. Cutting too far from the bud leaves a stub which dies back, and is likely to carry decay into the 
pith and thence down into the limb. Cutting too close to the bud or carrying the slope down too far behind it, does not give it enough live wood to carry it, and it makes a weak growth.

Cutting to inside buds with trees of spreading habit, and to outside buds with upright growers, or to a side bud when lateral extension is desired, should always be remembered as a means of throwing new growth in the direction demanded by symmetery and equal occupation of the space allotted to the tree. This is one respect in which study of the habit of the tree suggests proper practice.

\section{COVERING WOUNDS}

Whenever wood is cut with so great diameter that it will not grow over in one season, the wound should be coated with something to keep the wood from checking and decaying. It has been amply demonstrated by California experience that smooth-paring of the cut made by shears or saw is a waste of time. Large wounds should, however, be covered to prevent checking of the wood and drying back of bark edges. Nothing is better or cheaper for this covering than lead and oil paint, a little thicker than for ordinary use, and applied sparingly, so that it will not run down the bark. Asphaltum, "Grade D," applied warm is used in the same way with satisfaction.

\section{GATHERING UP PRUNINGS}

Gathering up prunings for burning is tedious and expensive, and several efforts have been made to substitute machinery for hand labor. Anderson's Brush Rake, invented by W. C. Anderson, of San Jose, has been used to some extent. It readily gathers all kinds of tree and vine brush, compresses it considerably and is easily discharged of its load by a slight lift while still going forward. It is said to save about one-half the cost of hand raking, Brush is often gathered into windrows by the use of horse rakes borrowed from the hay field.

Baling Prunings.-There is a fuel value in prunings which has become more clear since pumping for irrigation is so widely practiced, but loose prunings are too expensive in handling. T. G. Rogers, of Winters, has contrived a "brush baler." It is a large strong saw-horse inverted, to which is bolted a long, heavy lever. Attached to a cross piece on the lever are four heavy tines bent in a semi-circle. The sawhorse is filled with brush, the lever is then pulled down and fastened by a ratchet brake, the brush is forced into a small, compact bundle, and when bound with wire makes a bundle easily handled by the fireman.

Prunings for Fertilizing.-Although many propositions for returning prunings to the soil and several machines for cutting have been used, such practice has never widely prevailed because of cost of labor involved. At the Limoneira lemon orchard in Ventura county, a feed cutter run by a gasoline engine, and both mounted on a wagon-bed, is run through the orchard after pruning. Two men pick up prunings and feed them into the cutter as the wagon slowly proceeds. This waste from an evergreen tree seems to decay very readily in the soil as it is covered-in by cultivation. 


\section{THINNING FRUIT}

Intimately connected with the pruning of bearing trees, is the thinning of the fruit or proper spacing of the individual fruits so that each shall have space and sap to allow its attainment of satisfactory marketable size. It has been fully demonstrated that no demand is profitable which will be content with the undersized fruit from an overladen tree. The superior price for good-sized fruit for all uses, not excluding drying, is unquestionable; the total weight secured may be variable as between thinned and unthinned trees, but it can be accepted as an indisputable fact that any increase of weight there may be upon an unthinned tree will not be nearly an equivalent for the loss in value. It is the conclusion of our largest and most successful growers that, large as is the expenditure required for careful and systematic thinning of fruit, it is the most directly profitable outlay which they have to make for orchard maintenance.

Objects in View in Fruit Thinning.-But thinning fruit has objects beyond the value of the visible crop which it makes profitable. No overburdened tree can discharge the two-fold summer duty of every cultivated fruit-bearing tree, which is to perfect this season's fruit and lay a good strong foundation for next year's bearing. If the tree, after fruit gathering, has not the strong, vigorous foliage to complete the formation of fruit buds for the following year, there will either be a lack of bloom or a show of bloom unfit to set, and the tree will work for itself next year, and not for you, because this year you would not work for it. In this particular, thinning fruit coincides in purpose with pruning to limit the amount of bearing wood, which has already been considered.

Other objects there are also which are related directly to the profit of orcharding and should command respect from the most careless. The following is an emphatic statement of the case:

There are at least six ways in which growers are repaid for thinning peaches, nectarines or apricots designed for drying:

First: You can thin off half the fruit when small quicker than you could pick it when large, and when mature the time required to fill a basket depends mainly upon the number of peaches it holds.

Second: It takes just as long to cut and spread on a drying tray a small peach as a large one. It takes longer to cut eight peaches that will weigh a pound than to cut three and pick five off when they are little.

Third: If peaches run six to the pound the weight of pits will not vary much from that of the cured fruit. If they run three to the pound, they will weigh not much over half. A ton of large peaches is as likely to yield 400 pounds of dried as a ton of small fruit of the same variety to yield 300 pounds. It means a difference of about $\$ 8.00$ per ton in the value of the fresh fruit to the dryer. It will cost over $\$ 1.00$ per ton to thin a heavily laden peach orchard in a way to make that difference.

Fourth: Granted that you leave fruit to reach the same weight as maturity, still you leave it along the body and in places on the limbs where the weight has no breaking leverage and take it off the ends where it may get sun-burned and is almost sure to break the tree.

Fifth: Vitality drawn from the plant and certain elements of fertility from the soil, are in proportion to the number of seeds matured. The pulp cuts little figure except in aerial substances and water. 
Sixth: Suppose that fruit dried from peaches that weigh three to the pound only brings one cent a pound more than that from peaches half that size. Two cents would more accurately measure the difference in value. Still, the smaller figure is enough to meet the whole cost of picking and hauling or of cutting and drying in any well-managed establishment.

When to Thin Fruit.-Thinning of fruit should begin with the winter pruning of bearing trees, as has been already urged in connection with regulating the amount of bearing wood allotted to each tree. After this is carefully done, there is the thinning of bloom, which is urged on the ground of least possible loss of energy by the tree in the partial development of fruit to be subsequently removed. Handthinning of individual blooms is impracticable on a commercial scale, but the removal of spurs or twigs, or shortening of them with shears, is feasible enough. The objection must lie in the fact that profusion of bloom does not necessarily indicate an excessive set of fruit, and any severe reduction of bloom is, therefore, venturesome unless one is fully assured by local experience of the habit of the variety under treatment. Reduction of the amount of fruit itself is, therefore, the only safe proceeding, and this should not, as a rule, be undertaken until the first drop, through lack of pollination, has taken place. Even at greater theoretical loss of energy to the tree, it is better to err on the side of thinning a little too late than too early in order to secure the fullest assurance possible of the permanent burden which the tree assumes. Where spring frosts are likely to occur they afford additional reason for delay. If surety of the local conditions comes before the pits harden in the young fruit it is fortunate for the tree, but even after that it is still a greater saving to the tree and assurance of profit to the grower to reduce the fruit to a proper amount than to permit over-bearing.

The Practice of Thinning.-If the tree has not been sufficiently relieved of an excess of bearing wood during the winter pruning and has made a very heavy set of fruit, thinning with the shears by cutting out whole spurs or short bearing shoots, or even shortening in longer limbs, cutting always to a lateral when possible, is of no appreciable injury to the tree. After all the shear-work possible is done, the spacing of the fruits on the twigs and branches must be provided for. This was done in early days by beating the tree with a pole, and some still maintain that they can use the pole to advantage. The almost universal practice, however, is to use the hand in plucking or pushing off the small fruit. This is done very quickly by experienced workmen. If the trees are low, as they should be, most of the work can be done from the ground. It is best to work in vertical spaces and take all that can be reached from top to bottom without changing position; then move a step or two and take another vertical strip, and so on.

The distance which should be left between specimens depends upon conditions. It is as unsatisfactory to him by rule of inches as it is to prune by such a rule. The space to each fruit depends upon the kind, the age, vigor and strength of the tree, the size and thrift of the lateral or spur which carries the fruit, the moisture supply, the richness of the soil, etc. It also depends upon what use is to be made of the fruit, because it is possible to have some fruit which is too large for certain 
demands, though this objection does not often arise. The strength of the shoot is perhaps the most easily appreciable factor. With peaches, for instance, a shortened lateral one-eighth of an inch in diameter should only carry one peach, while one one-quarter of an inch in diameter might mature four good large fruits. It would evidently be wrong to work for an arbitrary inch-distance on all sorts of shoots, and it will be seen to be just as irrational if it be applied without regard to the other.conditions of the tree. If, however, a rule must be had, let it be this, that the distance between the fruit shall be two and one-half times the diameter desired in the fruit. This would fix an arbitrary distance, then of four to six inches for apricots and six to eight inches for peaches-with other fruits according to their respective sizes, and the late varieties with greater distance than early.

Any such standard, however, considers only the size of the fruit, not the strength of the tree, and therefore stops short of one of the important ends of thinning, to conserve the strength of the tree for next season's fruiting. Fruits might be thus spaced and still the tree be overladen, because it may be carrying too many bearing shoots. Calculate the burden of the tree in this way, for instance: Peaches which weigh three to the pound are of fair marketable size; sixty such peaches will fill an ordinary peach box of twenty pqunds; ten to twelve such boxes is fruit enough for a good bearing tree six to ten years of age. Now count the little peaches you have left on one main branch and its laterals, which ought to be about one-tenth of the tree, and thin down to about sixty. By doing a few trees in this way and thinking of the relation of the bearing wood to the fruit, one will soon get a conception of the proper degree of thinning, and proceed to realize it as rapidly as the fingers can fly along the branch.

It is seldom desirable to divide doubles in peaches; pull both off or leave both on, as they may be needed or not to make the load of the tree. Clusters of apples or pears should often be reduced to singles, except where size is apt to be too great.

All kinds of fruit are clearly subject to increase of size by thinning, but it is with only the larger fruits that the practice prevails at present. The dividing line seems to lie upon the prune. With this fruit thinning is only done by pruning the tree for the reduction of the number of bearing branches, while with some shipping plums hand thinning is practiced. Growers are still striving for a prune naturally of larger size rather than to have recourse to thinning.

The practice of thinning partially at first, trusting to further removal of fruit later if too much of it survives the natural drop and various accidents, is followed by some growers, but the rule is to finish at one operation.

The size of oranges on over-burdened trees can be increased by thinning, just as other fruits are enlarged, but it is not systematically undertaken, because it is not so necessary and because it is perhaps easier to get oranges too large and to be discounted for over-large and coarse fruit. Removing part of the fruit from young trees is often done-for the good of the tree, not for the good of the fruit. 


\section{CHAPTER XIII}

\section{CULTIVATION}

It was demonstrated very early in California experience in fruit growing, that "clean culture" is generally the proper treatment for trees and vines during the growing season, at least. Though the frequent stirring of the soil and eradication of grass and weeds have been advocated by certain horticulturalists for generations and have recently been demonstrated to be desirable by careful comparative experiments it has nowhere secured such wide adherence as in California. It may even be held to be an essential to successful growth of tree and vine in most soils and situations in California, and the several advantages of clean culture are intensified under our conditions.

Chief of these advantages is the maintenance of the soil in a condition favoring root growth, and the main feature of this condition is the retention of the moisture, though regulation of summer temperature in the soil is also involved. Where moisture-retention is not the chief concern, because of ample irrigation facilities, and the moderation of soil temperature of greater moment, a summer-growing cover crop may be of benefit to the trees. In irrigated districts of excessive heat and dry air this policy may prevail, but it will be only the exception to the rule of clean culture.

Retaining Moisture by Cultivation.-It is a familiar fact that water will rise in a tube of exceeding small diameter very much higher than the surface of the body of water in which the tube is held upright. The water rises by capillary attraction. A compact soil has extending through it, minute spaces, formed by the partial contact of its particles, which facilitate the rise of water from moist layers below, in accordance with the same principle which causes the water to rise in the capillary tube. This movement is constantly going on in firm soil, and as fast as the top layer is robbed of its moisture by evaporation, the water rises from below and it too is evaporated. During the long, dry summer, the water rises and is evaporated from a depth of several feet in some soils, and the earth, beneath the baking sun heat, becomes "dry as a brick."

When a soil is broken up by cultivation, capillarity is temporarily destroyed through the disturbed layer, because the particles are so separated that the mutual connection of the minute inter-spaces no longer exists. But if it can be roughly broken up, so that the disturbed layer takes the form of coarse clods, the air has free access to the upper surface of the firm soil beneath them, in which the capillary condition still exists, and evaporation proceeds in the same way, though in a somewhat less degree, as if there had been no cultivation. It becomes evident, then, that the pulverization of the disturbed layer must be so complete that the particles are separated and capillarity destroyed, and, farther, that the free access of air to the lower point, where capillarity 
exists, must be prevented. This is accomplished by the fine loose earth which acts as a mulch. When this is attained, only that moisture in the upper surface which comes in immediate contact with the air is evaporated, and the balance is retained for the use of the plant. Plants growing, then, in a well-cultivated soil, have the water in the lower soil held for their use, and as fast as they use it the supply is replaced through the firm soil below, which evaporation being stopped, remains moist and permeable by roots which extend freely, seeking the nourishment they need.

Such is a brief outline of the theory which explains the results gained by thorough cultivation of the soil, so far, at least, as retention of moisture is concerned. The practical demonstration is easy. Go into a well-cultivated orchard or vineyard, push aside the soil with the foot, and moisture will be found two or three inches from the surface, or even less in some soils, while on uncultivated land adjacent, digging to the depth of several feet will show nothing but hard earth, baked and arid. In such hard-baked earth, moreover, the sun heat is conveyed or conducted downward very rapidly during a hot day, so that in some cases the roots are seriously injured. When the surface is well tilled, it will act like a blanket, preventing a too rapid conveyance of heat downward, and thus also diminishing the intensity of evaporation.

Accurate demonstration of these facts has been secured as the result of many moisture determinations in cultivated and uncultivated soil by the University of California Agricultural Experiment Station.* Very striking exhibition of the condition of trees with and without cultivation is found in the engravings which are reproduced herewith. Upon the demonstration, the practice in the uncultivated orchard was radically changed. The exact determination of moisture present at various depths of the soil beneath these contrasted orchards in the month of July is as follows:

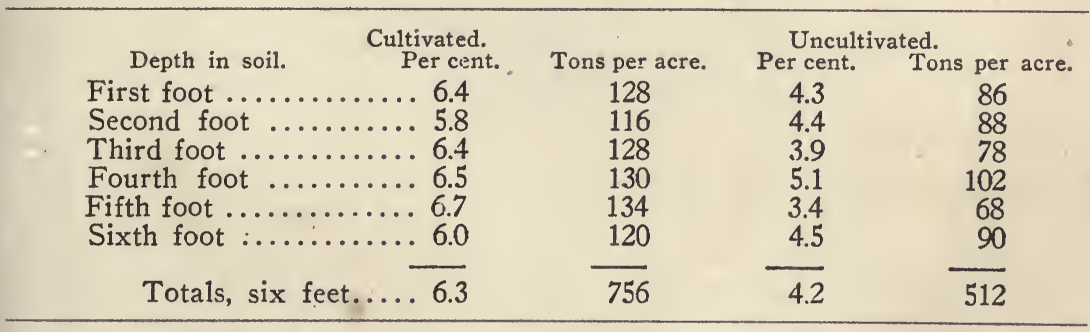
tion.

This shows a gain of nearly fifty per cent of soil moisture by cultiva-

Necessity of Adequate Cultivation.-It has been very fully demonstrated by California experience that adequate depth of tilth must be attained. The depth of cultivation, or the thickness of the dustmulch, as some like to call it, must be sufficient to prevent the access of the dry air to the firm soil below. At the East, where they have a moister air a thin mulch may answer, but in California, with a thirsty air for such a protracted period, there must be deeper tilth. Two or

*Bulletin 121. 
three inches of dust spread over a hardpan layer formed in some soils by cultivation, will not retain moisture well in California. The cultivator should go twice that depth, ordinarily, and then the result will be accomplished if it is done frequently enough to prevent the re-firming of the surface by atmospheric moisture or by the rise of moisture from below. The exact significance of depth in the loose, surface layer has also been demonstrated by moisture determination in the subsoil at different points by the California Experiment Station, as follows:

Percentage of Moisture in Cultivated Loam Soil.

\begin{tabular}{|c|c|c|c|}
\hline $\begin{array}{l}\text { Depth. } \\
\text { Three inches } \\
\text { Six inches .. }\end{array}$ & $\begin{array}{l}\text { Niles. } \\
\ldots 5.4 \\
\ldots \quad 6.3\end{array}$ & $\begin{array}{c}\text { Santa Maria. } \\
5.3 \\
8.5\end{array}$ & $\begin{array}{c}\text { Venturia. } \\
8.3 \\
9.3\end{array}$ \\
\hline
\end{tabular}

These may be accepted, probably, as average results: Variation may occur in soils of different characters. The capillarity in a heavy soil is vastly greater than in a light soil. The difficulty of securing a pulverized surface layer is also greater in the heavy soil. The poorer the pulverization, the deeper the layer must be. Naturally, then, growers' practice will vary. The rule will remain that there must be depth enough to secure effective protection of the firm soil beneath from agencies promoting evaporation.

Loss of Moisture by Weed Growth.-One of the most active agencies for the exhaustion of moisture from the subsoil is the growth of weeds. To cultivate the soil in winter and spring, and then to allow a summer growth of weeds to "shade the soil" is a great error. Although under cover of rank weeds moisture many appear even at the surface and convey the impression of moisture-saving, the fact is, as fully demonstrated by experience and actual experiment, the moisture in the lower layers of the soil is reduced and trees are thus robbed of their supply. Weed growth must be resolutely suppressed during the dry season, if one has to operate by rainfall or desires to make best use of irrigation water.

Moisture Storage in the Soil.-Conservation of moisture in the soil is not only the surety of the current season's growth and fruitfulness, but is the safeguard against injury from the years of deficient rainfall which occur now and then in California. The moisture supply is equalized by this storage of the soil, and a surplus from the liberal rainfall of one year is held over to supply the lack of the next. Of course, the well-cultivated surface is also well calculated to catch water. While from a hard surface much of a heavy rainfall flows off quickly to a lower level before it can penetrate, a loose soil, if sufficiently deep, retains all that falls upon it, except the excess, which disappears by drainage.

It has sometimes been held by California orchardists that planting some tall-growing crop, like corn, so as to shade the young tree and the ground around it, is an advantage. This is a great mistake. Though some rich, moist soils may afford moisture enough to grow both the tree and the corn, it is a fact that in most cases the growth of the corn 
is made at the expense of the tree, and sometimes almost costs its life and thrift. It has been amply shown by investigation that though shading ground by a leafy growth may make the surface layer of the soil moister, the lower layers are invariably made drier, and it is in these lower layers that the tree seeks its sustenance. The young tree should be shaded as has been described in the chapter on planting, and not by a growing plant.

\section{GROWING CROPS BETWEEN TREES AND VINES}

The possible advantage of a cover growth of clover in regions of high heat and ample moisture has been noted at the opening of this chapter. The rule, however, must be: Grow nothing whatever between the trees if you desire the full success of the latter. As with all rules, this one may admit of exceptions.

Inter-cultures in orchard or vineyard may be allowed under certain conditions of the soil and the purse of the grower. If the soil is deep and moist and rich, the cost of planting and cultivation, and sometimes more, may be made by growing a crop among your trees. Of course, , if irrigation is available, much more can be done in this direction than if dependent upon natural supplies of water.

There is much difference as to crops in amount of injury they may do to the trees. Growing alfalfa, without irrigation, has been known to kill out an orchard, and yet alfalfa growing in an orchard under conditions may be a great advantage, as described in the next chapter. Grain is less dangerous, but still is objectionable, both because of exhaustion of soil and moisture, and because of danger to trees from heat deflected from strow and stubble. The crops least injurious, because of their requirements, and because of the constant cultivation of them, checks the loss of moisture by evaporation are corn, beans, potatoes, beets, carrots, etc., squashes, and other members of the melon family, onions, and other shallow-rooting vegetables. In the growth of these, however, there should be a width of several feet of well-cultured soil on all sides of the tree, unoccupied.

In soils exceptionally rich and deep, and where rainfall is abundant, inter-cultures of small fruits or vegetables may be carried on for a long series of years with profit both from the trees and the inter-culture. In similar deep, rich soils, with irrigation, immense crops of small fruits and vegetables, even as high as twelve to twenty-four tons of tomatoes per acre have been taken from between orchard rows, and one hundred and fifty sacks of onions per acre from between the rows of a strawberry plantation. In Ventura county some fields of lima beans, in favorable years, have paid over $\$ 70$ per acre-grown between young trees. In other parts of the State considerable amounts of peas for sale to canners are grown between the rows in young orchards. This crop is especially desirable when good sale is assured, because the plant is hardy and can make a good part of its growth during the rainy season and the ground be cleaned up and well cultivated early in the summer. As beans and peas are legumes, their roots enrich the soil,as will be noted in the chapter on fertilization. 
How Exhaustion by Inter-Culture May Be Avoided.-But all inter-cultures are a loan made by the trees to the orchardist. The term may be very long and the rate of interest very small in some cases, but sooner or later the trees will need restitution to the soil of the plant food removed by inter-cropping. This may be accomplished by the use of fertilizers. Still the rule that the trees or vines should have all the ground is generally true. It is also true that on merely ordinary soils, trusting to rainfall, or on shallow soils, trusting in part in irrigation, the trees or vines should have the full strength of the land and all the help which can be given them in the shape of thorough cultivation.

\section{METHODS OF TILLAGE}

In general terms the main objects of tillage of orchard and vineyard are two: Winter cultivation for moisture reception, and summer cultivation for moisture retention.

Wherever early winter plowing can be done without too great danger of soil washing, it affords the best available means of admitting water to the great reservoir in the lower levels a deep soil. Too frequently large volumes of rain water, enriched by air-washing as it falls and by fine soil-particles as it flows, are allowed to run off into the country drainage, with the double loss of fertility and moisture to the fruit grower. Deep penetration of winter rains should be, in all safe ways, promoted. Cultivation for retention has already been strongly urged and is quite generally recognized.

To serve these main purposes there are two main divisions of practice in this State, each of which has variations of greater or less importance.

First: Winter plowing followed by frequent use of cultivator and pulverizer in summer.

Second: Use of cultivator at intervals both winter and summer, following, if needed, with pulverizer in the summer.

The main features of each division of practice, and some of the claims by which each method is supported by its advocates, will be noted.

Plowing Orchard and Vineyard.-There is considerable variation in the practice of plowing orchard and vineyard, in the kinds of plows employed, and the times chosen for the work. Some plow but once, toward spring, whenever the ground is in suitable condition; and, if there is much growth of weeds and clovers, a looped chain is run from the plow to the end of the evener to aid in drawing under the tall growth. Sometimes, however, the growth gets so rank before the soil is in condition to plow that the weeds are mown before plowing. Where but one plowing is done, the soil is usually thrown away from the trees and afterwards is leveled back by harrowing or cultivating. If this practice is adopted, care should be taken that the soil is properly returned about the tree roots, for injury is sometimes done by bringing the roots too near the surface, which is soon afterwards intensely heated by the sunshine. 
It is undoubtedly better practice to plow earlier, when the green stuff gets a good start, but is still not too high to turn under handily. In this practice the weed stems are less woody, and they easily decay and act as a fertilizer. Where early plowing is practiced, it is usual to plow again when the second growth of weeds reaches the proper state in the spring. When two plowings are given, the earth is usually thrown away from the trees in the first plowing, and returned toward the trees in the second plowing. But this order is sometimes reversed in situations where rainfall is heavy and the soil retentive, for the dead furrow between the rows often acts as a surface drain to carry off surplus water, which is thus prevented from standing around the tree roots. In all modes of plowing it is desirable that before the summer heat comes, the surface be leveled as completely as possible.

Too much stress can not be laid upon the importance of plowing when the soil is in good condition and not otherwise. To disregard this is bad enough in all soils, but it is a grievous mistake to work any of the clayey soils when they are out of condition. If too wet, they are puddled by the plow and dry down in hard clods, impenetrable by air, and even resist water itself for a long time. When clods are thus formed, it may require long effort to bring the soil back to a good friable condition. The cultivation of adobe is one of the problems of California agriculture. The more refractory it is, the more particular care is needed to take it when it is in proper condition to work. To work it when perfectly dry is simply impossible, and if it is plowed when too wet and sticky, it becomes hard, lumpy, and altogether unmanageable. The condition which favors best results by tillage must be learned by experience.

Another mistake apt to be made where the orchard or vineyard is but one of the branches of a mixed farm, is to put aside the plowing until all the field work is done, and in some seasons the soil in the orchard has become so dry that it turns up in large clods which are afterwards partially reduced by the harrow, but never put in the fine tilth which should be secured for the retention of moisture and otherwise to encourage the growth and productiveness of the tree.

Breaking up Hardpan.-Those who advocate the use of the plow, claim several advantages for it. The chief is that more thorough tilth can be secured. In most, but not all soils, there is formed by cultivation an artificial hardpan at whatever depth the implement attains, if this depth can be kept the same for many successive cultivations. This hardpan, in some soils at least, becomes impervious to water and is otherwise an injury to the growth of the trees. It occurs in irrigated and unirrigated land alike, but probably is more quickly formed by irrigation. When continuous summer cultivation is practiced, the hardpan will be found at whatever depth the teeth uniformity reach. The remedy is to plow in winter just below this hardpan layer and thus break it up, and then by the action of the air and rains it is reduced, and cultivation may proceed as before. Where the hardpan is formed by the plow, the ground should be plowed shallow one year and deeply. the next, thus alternating from year to year. 
Green Manuring.-Another advantage in the use of the plow is, as has already been mentioned, the turning under of the growth of weeds, grass, and clover as a green manure. Many growers attach considerable importance to this, and some, who have orchards in which winter growth has been killed out by long cultivation, are seeking for a quickly-growing crop which they can sow with the first rains and secure growth enough to turn under with the winter plowing. This consideration will be further presented in the chapter on fertilization.

Plowing Hillside to Prevent Washing.-Where the slope of the land is sharp, there is much danger from washing during the rainy season, if the hillside is not terraced or furnished with ditches carefully laid out on contour lines to carry the water down on a gentle grade. The old plan of plowing furrows one above another around the hill to check the flow and let the water down easily, is often found treacherous unless one is able to strike good grades, because of the liability to collection of water at certain points and the subsequent breaking away and washing. Recently some of the foothill growers have adopted the plan of plowing furrows seven or eight feet apart straight down the hill in the direction of its deepest descent. The rainfall is thus distributed over the ground so that not much water is collected at any one place and the harm done by washing will not amount to much. Hillside work differs according to character of soil and of local rainfall and conference with experienced men in the region will usually afford the beginner the best suggestions of method. In some localities, the plowing of a few furrows at intervals to assist in penetration and the growth of a cover crop during the winter to assist in binding the soil, will be found better than any attempt at the early plowing, which may work admirably on level lands.

The Best Plow.-For plowing orchards and vineyards many kinds of plows are used, including the ordinary one- and two-horse walking plows, single and double sulky or riding plows, and gang plows of different kinds. Recently disk plows and harrows have become very popular. In several of the leading fruit districts there are plows made in the local shops which are patterned to meet the different soils prevailing. Which is the best plow is a question which can not be answered, it must be determined by local conditions, and the best way to get information is to consult the experienced cultivators of the locality.

Avoiding Injury to Trees and Vines.-The great problem is to use the plow so as not to injure the trees and vines. Injury to the roots is one ground on which those who advocate the banishment of the plow from the orchard and vineyard base their opposition, as will appear more fully presently. It is the usual practice to run the plow shallower when approaching the stem of the tree or vine, and this is easily done when using a riding plow or a two-horse walking plow between the rows and finishing up near the trees with a single-horse walking plow, which is a common practice. The injury by the plow, to which especial reference is now made, is that to the bark of the tree or to the vine stump. 
Makers of the special orchard and vineyard plows have recently made them adjustable so that the plow will work either side of the central line of draft, and these improved tools have rendered obsolete the early contrivances for accomplishing the result with common field plows.

Extensions of disks and of spring-tooth harrows are often made by attaching the parts to the ends of a central piece in such a way that the horses walk in the centers and the cultivators work under the low branches and very near to the stems of the trees. These are chiefly used with citrus trees whose foliage and fruits are permitted to grow very near to the soil surface.

Flat Hames and a Spreader.-Among the worst things for use among trees are the pointed iron hames which are found on most harnesses. They often seriously bark the branches under which the horse passes, and should be dispensed with. An arrangement widely used consists in having broad leather tugs and hames with only one long iron loop on the swell of the hame. The tug is passed around the hame and the end is brought through the iron loop from the under side so that the draft will hold the tug tight between the collar and the hame and the end between the iron staple and the pulling part of the trace. A spreader is put between the tugs; it is made of a hard-wood stick sixteen to eighteen inches long; a hole is bored in each end large enough for a two-inch screw, a hole punched in each trace about twelve inches from the rear end, and the tugs are screwed to the ends of the spreader, and the ends of the tugs attached to the plow clevis. This gives no iron or wooden surfaces at all, either on harness or whiffletree, to strike the bark.

Improved Singletrees.--Later than these came the orchard and vineyard singletrees, invented and patened by Californians, which are widely used and sold in all stores of the fruit growing districts.

Dispensing with Doubletrees.-Still other inventions which admit the use of two horses even close up to the trees, because they dispense entirely with whiffletrees and tugs, are known as the steel harness, Eastern inventions, which have secured the approval of some of our leading growers, for use in orchard and vineyard. The plow is attached to the steel yoke by a chain running between the horses. With them it is possible to work quite close to the trees and vines, and is especially desirable in the vineyard in working close to the vines when they have grown out about two feet, which is a difficult job with the old-style harness.

\section{SUMMER TREATMENT OF PLOWED ORCHARDS AND VINEYARD}

Where the orchard or vineyard is plowed twice during the winter, the land should remain after the first plowing as the plow leaves it. The moistening and aeration during the winter have good effect upon the soil both chemically and mechanically.

If but one plowing is done, when the chief rains are supposed to be over, there must be full effort put forth to reduce the soil to good tilth, 
and to level the surface as much as possible. This is done by harrowing with one of the several improved harrows which are now generally introduced and found very effective. They act in cultivating, clod crushing, and leveling, in a most satisfactory manner. They are too well known to need description. Each has its advocates and its adaptations to certain soils. As with plows, so with harrows and cultivators, the best for one soil may not be the best for another, and local inquiry among experienced fruit growers will be the best guide for the newcomer. In addition to the excellent implements brought from the Eastern States, there are others of California invention and manufacture which have very marked local adaptations, and almost every fruit region in California has some embodiment of local inventive genius in the form of implements of tillage.

The secret of success in handling the heavier soils in spring working is to secure as perfect surface pulverization as possible without compacting the soil. Light soils need a certain amount of firming after plowing, or else there is too free access of air and too great drying out. For these and other reasons, the grower has to study his soil and learn from observation the methods which succeed best with it. The practice which gave success under certain conditions might not be well adapted under other conditions. The use of the roller is a striking example of this fact. In some orchards the roller is a benefit, in others a decided injury. Its chief effect is compacting the surface layer, which is only desirable on very coarse open soils. The long-tooth harrow accomplishes a very marked compacting of the soil to the depth it reaches, and often settles the lower layer too closely and causes it to run together too solidly if rain follows. The modern cultivators, clod-crushers, diskharrows, etc., are superior in effect, each in the soil to which its action is most desirable.

After working down the soil after plowing, the cultivator is relied upon to kill the weeds, break up the crust which may form after spring rains or after irrigation, and to prevent the compacting of the surface layer of the soil from any causes.

\section{CULTIVATION WITHOUT PLOWING}

There are orchards in California which have not been plowed for years-in some cases the plow has not been used since the trees were planted. Instances of this kind are to be found both in irrigated and unirrigated land. It depends largely upon the mechanical condition and disposition of the soil whether the practice will give satisfactory results. It can not be trusted on land prone to develop hardpan, as has already been considered, and yet the term "cultivation" has taken such a wide range in this State, and the tools have reached such efficiency, that there is not as much difference as formerly between the plow and the cultivator, except that the former turns the soil and the latter stirs without turning. For some who oppose the use of the plow, use a chisel-tooth cultivator, cutting to a depth of eight inches in the spring, but at other times of the year they are not more than half as deep. This treatment would tend to dispose of hardpan. However this may 
be, and what the special nature of their soils, there are fruit growers, both in northern and southern California, who have for years trusted almost wholly to the cultivator, cutting to a depth of three or four inches, and keep their orchards throughout the year almost in the same state of tilth, never allowing a weed to grow. This practice is, however, becoming less prevalent, and for certain soils the question is practically settled in the minds of nearly all orchardists, while for other soils there is still doubt. For the heavier soils, which continuous shallow cultivation is apt to render too compact, it is necessary to have recourse to the plow to open the land for proper aeration and penetration of moisture which otherwise would be largely lost by surface run-off. The lighter soils do not require this and they seem to do well with continuous use of the cultivator. It is beginning to be clearly seen, however, that this treatment tends toward the decrease of the humus and the consequent impoverishment of the soil. Its water-holding capacity is also lessened. These facts have induced some growers to change their practice and to take up the plow during late winter or early spring to cover in the growth of green stuff which they allow to grow instead of frequently destroying it with the winter use of the cultivator. Either the fall and spring plowing, or both, followed by the summer use of the cultivator, the most rational and satisfactory practice for most of our deciduous orchards, though there are local conditions and circumstances under which different procedure is preferable.

\section{SUMMER CULTIVATION}

Whatever the winter policy may be, the essential point in summer cutivation is to preserve the surface layer of pulverized earth. It will not do to have a few inches of clogs, from the size of a pea to that of a goose egg, resting on a hard surface. The finer the pulverization the shallower can be the surface layer, and vice versa, and this is probably one reason why in practice the work of the plow is, in so many situations, found the best foundation upon which to rest the years' cultivation.

In order to secure this finely-pulverized layer, it is sometimes necessary to use what is called a "rubber," where there are many clods which are merely displaced by the harrow or cultivator. There are different styles, and they are generally home-made. The most common form is made of two-inch plank in lengths of three or four feet, bolted or spiked to pieces of four-by-four-inch scantling running crosswise, the edges of the planks lapped like the clapboards which are used for weather boarding. As these edges are drawn over the surface, the clods are rubbed into tilth if they are not too hard and dry.

But this rubbing may be very undesirable if it leaves the surface smooth and polished. It may reflect the sunheat even to tree-burning, and is apt to form an evaporating surface, which is most to be avoided. The best finish for the land is that produced by a light, fine-toothed harrow, and an attachment of this kind is provided with various clod crushers and cultivators. The result is a surface af loose earth, flat and fine, which approaches very closely an ideal condition. 
There is less difference than formerly in the use of the harrow or cultivator during the summer. Still some are content to use the cultivator only as a weed-killer, and after the weeds cease to grow and the spring showers are over, the cultivator is laid aside and the land left unstirred until the following winter. This, of course, refers to unirrigated ground, for wherever irrigation is practiced a cultivator must follow, except on hillsides where the surface is left undisturbed after the irrigation furrows are made until the beginning of the dry season. It is a fact, however, that even if no rain falls, the soil becomes compacted to a certain degree, and the best way to imprison the greatest possible amount of moisture below is to run the cultivator at intervals all through the dry season. It should run shallow and only stir the surface layer. The experience of the most successful growers is that frequent stirring without, however, bringing new soil to the air, is the best-paying practice.

\section{WHAT IS THOROUGH CULTIVATION}

As clean, thorough cultivation has been approved, it may be desirable to attempt to define the term. It can, however, only be approximately done, because of the great difference in individual views and practices. Some indication of the operations which are contemplated may be had in the following specifications upon which contracts have been let for care of orchard: First, plowing away from the trees, followed by harrowing; second, plowing toward the trees, followed by harrowing; ten summer workings with cultivator; three working with shallow cultivator or weed-cutter; five hand hoeings around the trees. The contract intends the most complete and perfect working of the soil and specifies the above merely that there may be no difference of opinion between owner and contractor.

In cases where the land is infected with morning-glory, weekly cutting with sharp, flat teeth beneath the surface is stipulated for in some cases, and this seems about the only way to cope with this formidable trespasser.

\section{CULTIVATION FOR WEED KILLING}

Cultivation for weed killing is a minor consideration in California, because cultivation for moisture conservation effectually disposes of most of them, and weeds do not start readily in the earth-mulch during the dry season. There are, however, a few most persistent pests which require heroic measures. Johnson grass and morning-glory are the most prominent of these. The only successful treatment consists in cutting constantly with a weed-cutter (a sharp horizontal knife), operated so as to pass under the whole surface and run so often that the plant is never allowed to show a shoot on the surface. It is of no use merely to cultivate or "weed-cut" as for other weeds. This spreads the pest more and more; but if the rising shoots are continually cut under the surface, and never allowed to get the light, it will kill the plant surely, but it may take two seasons to do it. Weed-cutting knives 
of this description are usually contrived by local smiths and are attached to sleds or fitted with plow handles, or used with a pair of thills and cultivator handles or other rigging as the operator may choose. The vital point is a blade of sheet steel, very sharp and rigged to run just under the surface. It must be used as often as once each week.

\section{MULCHING A SUBSTITUTE FOR CULTIVATION}

The use of a mulch or covering of the ground with a litter of light materials to prevent evaporation, is practised to a small extent in this State. Though mainly used for berries of different kinds, recourse has also been had to mulching by vineyardists. The materials used are various, such as partly-rotted straw, coarse manure, damaged hay, cornhusks, corn-stalks, vine prunings and leaves, and even fine brush from adjacent thickets. The practice has been found of greatest value on hillsides where cultivation is difficult, and danger of washing of loose soil is great. There are cases where vines have been grown several years in this way to the satisfaction of the owner. The danger of fire in our dry climate when the surface is covered to a depth of several inches with a dry mulch is considerable. As a rule, the mulch employed by the California grower is a perfect pulverization of the surface soil. 


\section{CHAPTER XIV}

\section{FERTILIZERS FOR TREES AND VINES}

It was a popular doctrine among early Californians that California soils would never need fertilization, and that there is something in our soil and climate which releases us forever from repaying anything to the ground for the wealth of produce which we take from it. Such a view is, of course, without foundation, and yet it is not difficult to see how it arose. Early attempts to enrich the soil by the turning under of coarse stable manure, as is done in other countries, was undertaken here on light soil in a region rather short of rainfall. The manure did not decompose, and its coarse materials made a soil, already too light to retain moisture well, so open and porous that its moisture was quickly carried away by evaporation, and crops did not grow so well as upon adjacent land which had not been manured. So the fiat went forth against manure. The corrals* became undisturbed guano deposits, and manure piles were fired in dry weather to get the soil poison out of the way. Innumerable tons of bones were gathered and ground in San Francisco and shipped away to countries which need fertilizers. Nature did much to foster the popular delusion, for field crops were gloriously large, and trees and vines grew rampantly and bore fruit the weight of which they were unable to sustain. How could there be more conclusive evidence that manure was a detriment to California soils?

A few decades of experience have swept away such fallacies and now California growers, especially those handling citrus fruits, are not only freely investing in commercial fertilizers but are buying and shipping considerable distances all available animal manures. They are also untiring students of the art of fertilization and the sciences underlying it. It was in response to their demand that the California Legislature of 1903 passed a fertilizer control law giving the University Agricultural Experiment Station regulation of the trade in fertilizing materials. All dealers are required to register and submit samples of their brands and there is constant inspection to detect departures. Semi-annual reports are published for public information and these, with special instructions for taking samples when purchasers desire analyses on their own account, can be had by application to the Experiment Station at Berkeley. The total amount of sales reported under the law for the year ending June 30,1912 , was 50,995 tons.

It is foreign to our purpose to discuss the general subject of the use of fertilizers in California, and the changes in belief and practice which have recently gained ground. Of course, the marked falling off in the yield of shallow-rooting cereals gave the first unmistakable intimation that there was nothing wrong about the old theory of the perpetual youth of California soils. The lands used for fruit are sometimes slow to show exhaustion, because trees are deep feeders, and the soils, as they are often the very best and deepest of the State, selected for fruit because of that very character, possess, in an eminent degree lasting

*Inclosures for livestock of any kind. 
properties, as is shown in the chapter on the fruit soils of California. But certain of these soils are already showing the need of refreshment, and intelligent growers are quick to minister to the lands which are giving them such generous returns, as they can well afford to do.

\section{WHEN IS FERTILIZATION NECESSARY?}

Though the use of fertilizers by our fruit growers is beginning, it should be plainly stated that at present, except perhaps with citrus fruit trees, or the oldest orchards of other fruits, it is not yet the rule that such applications are necessary. There are some soils which are really too rich for fruit. There is sometimes an over-rank growth of wood, which delays or prevents the formation of fruit buds, and there is a marvelous development of fruit which is inconsistent with the highest quality. For this reason the grower should not conclude, from the foregoing general remarks concerning the need of fertilization in California, that he must manure his soil whether it needs it or not. Especially is this the case with young trees, in which the wood growth is easily over-stimulated. As with irrigation, so in fertilization; the tree or vine itself will give the observing grower hints as to its needs, and if the growth of wood and color of foliage are such as obviously indicate health and vigor, it may be concluded that the plant needs nothing but good cultivation and intelligent pruning.

Usually cases of over-rich ground will cure themselves as the trees attain size and full bearing, and it is then that fertilization may be necessary. When the tree or vine which has been properly pruned and cultivated is not able to mature a good weight of well-developed fruit, and make a satisfactory wood growth, usually at the same time showing some degree of distress by the color of its foliage, it needs help; and if the grower is sure that the trouble is not from lack of moisture in the soil, he should bestir himself in the manuring of his orchard or vineyard. In examining the soil for moisture, one should dig deeply, for there have been cases of moisture near the surface, and drouth below.

\section{WHAT FERTILIZERS APPLY TO FRUIT TREES AND VINES}

A discussion of this subject from a chemist's point of view is beyond the scope of this volume. The results of research at the University Experiment Station at Berkeley are summarized in the treatise on soils by Dr. E. W. Hilgard, who maintains the position that the most intelligent and economical choice of fertilizers is to be made after ascertaining by analysis in what constituents the soil is deficient and in what it is well supplied. Some applications made in conformity with suggestions based upon analysis have proved very satisfactory. But as soils vary within narrow limits of area, there must be analysis for each soil in question.

Approaching the matter of choosing fertilizers without soil analysis, the method by local trial is open. In this recourse there is danger of error, as pointed out by Dr. Hilgard, arising from local differences in soil and subsoil, and must be checked by several check plots so interposed between the others as to not only check them by direct com- 
parison, and to prevent the washing of fertilizers from one fertilized plot to another, but they must also be compared, first of all, among themselves, to determine what is the normal product of the unfertilized land. It will frequently be found that these unfertilized check plots differ more widely between themselves than do the fertilized ones from them or from each other. Systematic experimentation, on plots arranged to avoid such errors, has been continued for several years by the University Experiment Station in the citrus district of Southern California. Results from this work, not yet available, can be secured, when ready, by correspondence with the Station at Berkeley. It is also hoped by this work to secure some knowledge of what specific effects on growth or fruiting are caused by the use of particular substances. Statements now freely made along this line are largely conjectural.

From these statements it must appear that the prescription of fertilizers is not an easy matter. Disappointments will naturally be encountered, but unquestionably the advantage is on the side of patient trial and wise investment in fertilizers honestly made and honestly sold. The observing grower must learn all that he can from experience. It is obviously the duty of the grower to constantly study fertilizer questions as presented in books and journals and to be alert for observation of the behavior of his own trees with the applications he may make. The publications of responsible fertilizer manufacturers and dealers, also convey important information when read discriminately.

Though the deficiencies of the soil, as learned by analysis, or by practical test, must be the basis of prescription of fertilizers, the analyses of fruits, as showing the special needs of the plants, are of the highest importance. The following analyses of the different fruits, containing in each case, skin, pulp, and seeds, are almost entirely from California-grown specimens, and are supposed to represent an average composition of the fruits named.

\section{Quantities of soil ingredients withdrawn by various fruits}

Compiled from analyses by G. E. Colby, University of California.

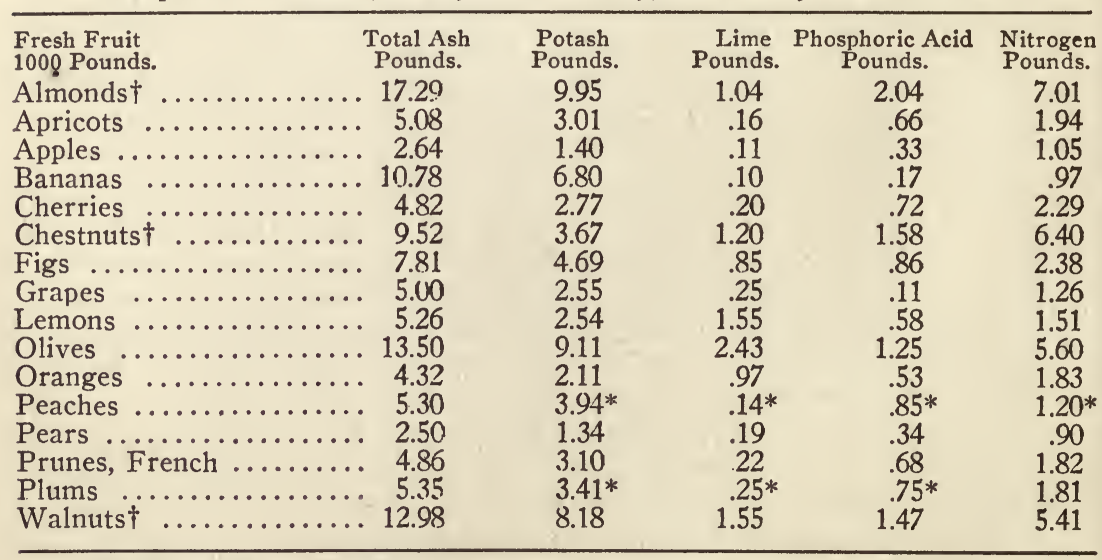




\section{FIRST AID TO THE INJURED SOIL}

Based upon the clear characteristics of California soils as already indicated in the chapter on that subject, Dr. Hilgard advises that any large-scale fertilization should begin with phosphates and nitrogen, and, should this not prove fully satisfactory, then with potash also, this being the order in which these substances are likely to become deficient in most of our soils under cultivation. In the course of time potash fertilization will become widely necessary in this State; in fact some California soils are naturally deficient in potash.

\section{AVAILABLE SUPHLIES OF PHOSPHATES}

Phosphatic manures which are clearly promotive of freer fruiting of trees and vines, are now being supplied to fruit growers by importers and manufacturers located in various California cities, and results attained by their use are such as to warrant continuance. They are bone and rock phosphates, which are transformed into superphosphates, and with nitrogenous matter added, serve as good applications both for growth and fruiting.

Home-Made Bone Manures.-Much good bone manure can be made by collecting bones, heads, horns, feet, etc., from butchers' shops or elsewhere. How to make such material available, by simple proceedings, is described by Prof. Hilgard as follows :

1. Bones put into a well-kept (moistened) manure pile will themselves gradually decay and disappear, enriching the manure to that extent.

2. Raw bones may be bodily buried in the soil around the trees; if placed at a sufficient depth, beyond the reach of the summer's heat and drouth and cultivating tools, the rootlets will cluster around each piece, and, in course of a few years, consume it entirely.

3. Bones may be packed in moist wood ashes, best mixed with a little quicklime, the mass kept moist but never dripping. In a few months the hardest bones vill be reduced to a fine mush, which is as effectual as super-phosphate. Concentrated lye and soil may be used instead of ashes. In this process the nitrogen of the bones is lost, going off in the form of ammonia, the odor of which is very perceptible in the tank used.

For neither of these processes should the bones be burned. The burning of bones, is an unqualified destriment to their effectiveness, which can only be undone bv the use of sulphuric acid.

4. Bones steamed for three or four hours in a boiler under a pressure of thirty-five to fifty pounds, can, after drying, be readily crushed in an ordinary barley-crushing mill, and thus be rendered more convenient for use. Practically, very little of the nitrogen (glue) of the bones need be thus lost.

\section{POTASH}

Though, as already stated, potash is commonly in good supply in California soils, it is very clear from experience that additions of potash, perhaps in more available form, are advisable. The fruit analyses already given show that the use of this substance by fruit trees and vines is very large. Recent experiments seem to indicate that potash ministers directly to the quality of the fruit in some cases. 
Ashes from wood fires are the most available source of potash, but it is a mistake to regard wood ashes as valuable only for their potash contents. Professor Storer has found by analysis of a number of samples of house ashes, that selected samples contain $81 / 2$ per cent of real potash, and 2 per cent of phosphoric acid; or say $4 \frac{1}{2}$ pounds of potash and one pound of phosphoric per bushel. Hence there is enough potash and phosphoric acid to make a bushel of ashes worth twenty or twenty-five cents, and besides that, some ten or fifteen cents additional may not be allowed for the "alkali power" of the ashes, i. e., the force of alkalinity which enables ashes to rot weeds and to ferment peat.

These facts suggest to the fruit grower that he should carefully preserve all home-made wood ashes and apply them to the soil at once, or, if stored for future application, be sure that they are kept dry. Leached ashes from the lye barrel, or ashes from open piles, leached by rains, are hardly worth handling. Coal ashes are almost devoid of fertilizing properties, though, if finely divided, as in the case of coals burning completely, their use is beneficial, mechanically, on clay soils, in the same way that fine sand would be.

The chief supplies of potash salts are now brought from Germany, but a strenuous effort is being made to develop a local supply from sea-weeds and by developing deposits in the arid regions of this country.

\section{NITROGEN}

Nitrogen ministers directly to the vegetative activity of the plant and this is a wonderful stimulant of wood growth and foliage. Supplies of this substance can be had from animal manures, which will be considered later. The effect of stable manure upon the soil and the plant is notably strengthening and restorative. For this reason money and effort are often well expended in securing it even beyond the cost of the equivalent of the plant food which it contains. Another natural form of nitrogen in cover crops or green manures will be discussed presently. Of commercial forms of nitrogen, tankage and dried blood are highly esteemed for orchard use, and there is large use also of Chile saltpeter, which contains about sixteen per cent of nitrogen, in immediately available form. From two to four hundred pounds per acre is the usual application, and it should be evenly distributed over the ground, not collected near the trees. Sulphate of ammonia is another available source of nitrogen obtainable in commerce; a good commercial article contains twenty per cent and over of nitrogen. It does not, however, act quite as rapidly as the Chile saltpeter. A suggestion of caution in the use of nitrogenous manures will be given presently.

\section{LIME, GYPSUM AND MARL}

Lime is another substance usually abundant in California soils, but still often desirable as an application. This is, notabıy, the case on our heavy clays or adobes, where, as has already been mentioned 
in another connection, the use of lime as a top dressing, at the rate of six hundred to one thousand pounds to the acre, not only makes the heavy soil more friable, but acts upon and makes available the large amount of organic matter which such soils usually contain. Lime also renders inorganic materials more available for plant food, corrects acidity, and may destroy insects and fungi. Application of lime is also desirable after applications of barnyard manure have been made for several years; and it is especially valuable wherever, in alluvial soils rich in vegetable matter, there is an excessive growth of wood and leaf. Usually light soils are not materially benefited by the use of lime.

Ground limestone is gaining standing as a fertilizer. It acts less vigorously and more slowly than burnt lime or hydrate of lime in improving heavy soils, but it serves a good purpose in overcoming sourness in loams, silts or sands. It should be used in double the amounts prescribed for other forms of lime.

Gypsum.-Gypsum, or land plaster (sulphate of lime), occurs in considerable quantities in this State and Nevada. It acts directly in correcting soils made alkaline by presence of carbonate of soda. Applied to soils not alkaline, gypsum sets free potash, magnesia, and ammonia, which may be present in insoluble form; and it also causes potash to be transferred from the upper to the lower layers of the soil, so that roots can everywhere find a store of it. Hence its special value when applied to deep-rooting plants. The reason why gypsum is so capricious in its action, which was long a mystery, is now held to be clear, because upon soils that are tolerably rich in fixed potash it will do good service, while upon soils poor in potash it will not. In any event gypsum is to be regarded as an excitant rather than as a form of plant food.

Of the several uses of gypsum, probably its chief value lies in its power as an absorbent. If added to manure in excess it delays fermentation, and it is, therefore, not a desirable addition to the compost heap. But for covering fermenting manures or scattering around moist places in horse and cow stables to absorb odors and fix volatile manurial substances it is of value. Gypsum does not correct acidity nor does it promote decay of organic matter as other forms of lime do.

Marls.-Marl is a calcareous earth, and is called shell marl, rock marl, earthy marls, etc., according to its origin and mechanical condition. A number of samples from different parts of the State have been analyzed and some of them commended for local application to soils needing lime, but they are usually not valuable enough to warrant hauling far.

\section{BARNYARD MANURE AND COMPOST}

Where fruit growing is carried on with stock growing, there are abundant supplies of manure available, but this combination is not characteristic of California, though prevailing to some extent, and likely to ise more prevalent as fruit planting extends farther from the centers which are wholly given to it. But even in the fruit centers 
there are certain amounts of material available from the animals that are kept for cultivation and hauling, or to be had, often, for the expense of hauling from adjacent towns.

As already stated, coarse, unrotted manure can seldom be used to advantage in this State, unless it be plowed under in the fall in heavy soils with ample rainfall, or on lighter soils, perhaps, if well irrigated. So great, however, is the demand for humus-enrichment of soils that all available supplies of stable,cleanings are readily sold in towns in the citrus districts to go considerable distances by rail to the orchards. Thus fresh manure is largely used, although either finely divided or well-rotted manure is superior. Corral scrapings, which are usually the first resource when the idea of manuring springs up in a neighborhood, are not always well decomposed, but they are finely divided, and therefore decompose readily as compared with coarse straw, which, it is said, has been found practically unchanged even after lying two years in a dry, loose soil. It is, therefore, of the greatest advantage to prepare barnyard manure with care for use in this State by some such method as will be described below, which includes composting, thereby turning to account nearly all organic material likely to be available. This advice is obviously for the use of the orchardist who keeps livestock rather than for the large commercial grower.

Clean up all the manure on hand just before the fall rains, putting the same on the land, and either cultivate it in or plow it under. What manure accumulates during the winter pile in a snug heap some five or six feet in depth, and throw it over some three or four times during the winter to keep it from burning, as well as to thoroughly $\mathrm{mix}$ it and thereby hasten decomposition. Put horse, cow, hog, chicken and every other kind of manure that can be had, all together. Never burn anything that will rot, but haul to the pile corn-stalks, roots, and all squash, melon, tomato, and potato vines, etc., as well as weeds of every description; in fact, anything and everything that will decay and make vegetable matter. Use fresh horse manure mostly to hasten the decomposition of said vines, weeds, etc., alternating as the heap is made. By so doing there will not be a weed seed left with vitality enough to germinate. It is well to have manure piles under a roof to avoid leaching during the longest and most excessive rains, but so situated that some of the rain falling on the barn can be easily conducted to the piles, giving them just the amount of water necessary to wet thoroughly without leaching, and no more.

Treatment of Manure Without Composting.-Even when composting all refuse vegetable matter with the manure is not thought worth the time and trouble, it is just as important to properly treat the manure when stored alone. This can be easily done by some such plan as is described below by an owner of a small fruit farm:

Collect the stable manure in a large bin and keep it wet enough to prevent burning or "fire-hanging." With a bin, say ten or twelve feet square and five or six feet high, built convenient to the barn, the manure can be placed therein and watered from time to time with much less trouble than it can be composted with other material. This, of course, presupposes the ability to run the water through a hose or by natural flow. Care must, of course, be taken that too much water be not supplied, causing the substance to be leached from the pile. But in my own experience I find the danger is at the other extreme, and when I open my pile I sometimes wish I had used more water. In filling the bin leave one end or side open as long as possible, for convenience of filling.

Barnyard manure and compost carefully prepared in some such way as described, and applied before the rains or early in the rainy 
season, to be turned under at the first plowing, will be in condition to be readily assimilated, and will not injure any soil. Where no composting is undertaken it is rational to apply the manure during the rainy season directly to the land if the rainfall is not large and the land fit to haul over. During the dry season the manure can be spread in the corral and tramped into dust by the stock because as long as it remains dry no losses by fermentation can occur. Near the end of the dry season, the corral should be scraped and all the material spread on the land. In this way the cost and trouble of manure piles can be avoided.

Sheep Manure.-The proximity of the orange orchards of Southern California to extensive sheep ranges led to large use of the manure from sheep corrals until supplies were practically exhausted. Afterwards large deposits in the San Joaquin Valley were opened and the material which has shown value by analysis in one case of above $\$ 14$ per ton, is finally ground and placed upon the market in a business way. One deposit mined for several years by George C. Roeding $\&$ Co., of Fresno, was several acres in extent, and at some points the material was ten feet deep. Such deposits can seldom be found now except in remote parts of the State.

Sheep manure is usually counted richer and quicker, though not so lasting in its effects, as stable manure. Being highly nitrogenous, too free use of sheep manure tends to excessive growth of wood, especially on young trees. Old bearing trees may be benefited by such a stimulant.

Poultry Manure.-Poultry keeping on fruit farms is a good combination providing the fowls are not confined on too small areas for this is apt to render the soil sour and foul and may cause trees to be unthrifty. Poultry manure can be used to advantage with fruit trees if applied sparingly, for it is a concentrated manure, as is sheep manure. On the average clear hen manure, free from dirt and trash, may, when fresh, average about 1 per cent nitrogen, 0.80 per cent phosphoric acid, and 0.40 per cent potash; in other words, 20 pounds nitrogen per ton, 16 pounds phosphoric acid, and 8 pounds potash. This contains 55 per cent moisture and when thoroughly dried might contain about half as much, which would make the amounts of plant foods just double what is stated above. At the values usually given for these plant foods, such manure might be worth about $\$ 10$ per ton.

\section{VARIOUS WASTE PRODUCTS}

The care advised in saving and treating barnyard manure, hen manure, bones, ashes, etc., should be extended to other waste products of the farm. Soapsuds should be allowed to run to adjacent trees unless used in the flower garden, but not too long upon the same trees, for it may kill them. Peelings and corings of fruit, cut for drying, should be fed to pigs, and the resulting manure secured. It is not wise to corral the swine in a dry run in the summer and allow the manure to be sluiced out by the winter rise of the stream. 
Prunings.-Prunings of the orchard and vineyard should be burned between the rows, in small piles, so as to distribute the ashes well. Danger to adjacent trees may be avoided by using the portable, home-made tin shields on the sides of the fires. It is not wise to carry all the prunings to the side of the highway and burn them there and allow the ashes to be lost. Vineyard prunings are sometimes cut up with an arrangement like a straw-cutter, which reduces them to bits about an inch in length. They are then scattered over the surface of the ground, turned under at the next plowing, and soon decay. The use of cut prunings on the lemon orchard has already been mentioned in the chapter on Pruning. Where, through lightness of soil and short rainfall, the woody fiber does not readily decay, burning upon an iron sled about ten feet long are practiced. At its front is a V-shaped iron rod, to which a horse can be hitched. On the sled are flaring sheet-iron sides and perforated bottom. This is filled with brush, a fire kindled, and as the horse moves forward fresh brush is added, while the ashes by its motion are sifted out very evenly all over the vineyard.

Refuse from Wineries.-The fermented husks, stems, and seeds, all containing valuable fertilizing properties, are often spread on the road and in holes, where it is of no account whatever. If scattered over the vineyard, much valuable substance would be returned to the soil. Professor J. L. Burd, of the University of California, estimates the contents and value of this somewhat variable substance as follows, value increasing as water percentage decreases:

\begin{tabular}{|c|c|c|c|}
\hline $\begin{array}{c}\text { Water } \\
\% .\end{array}$ & Potash. & $\begin{array}{l}\text { Phosphoric } \\
\text { Acid. }\end{array}$ & Nitrogen. \\
\hline Sweet pomace & 12.6 & 2.80 & 19.1 \\
\hline Fermented pomace (unpressed)... 70.59 & 7.4 & 3.98 & 9.6 \\
\hline Fermented pomace (pressed).... & 16.6 & 5.0 & 17.6 \\
\hline Mixed barnyard manure..... & 11.4 & 6.0 & 11.4 \\
\hline Grape pomace (old) ...... & 21.4 & 17.90 & 33.60 \\
\hline
\end{tabular}

The figures of potash, phosphoric acid and nitrogen are the numbers of pounds of each in a ton of pomace, and the value is that of a ton. The barnyard manure is, of course, inserted for comparison. Grape pomace, particularly when fresh, is likely to be acid, and it is desirable that the acidity be neutralized with freshly slaked lime. The use of large quantities of fresh grape pomace without this preliminary treatment might act unfavorably upon soil and plants.

Other Waste Products.-There are available from various manufactories different waste products which can not be specified. When any such material comes to the notice of the fruit grower, he should seek advice from the Agricultural Experiment Station, at Berkeley, as to the probable value of the material, and its special uses.

\section{CAUTION IN USE OF FERTILIZERS}

Besides the injunction already given against application of fertilizers when the soil is already quite rich enough to produce good fruit and plenty of it, it should be noted that manures unduly rich in animal matter should be used with caution, as they may over-stimulate the 
plant, delay or reduce fruiting, injure the quality of the fruit, and possibly engender disease in the tree or vine. Monstrous size and puffiness of oranges is clearly due in some cases to excess nitrogenous manures. Excessive use of soluble fertilizers like nitrate of soda may kill plants or trees outright.

The effect of excessive use of stable manures, or other manures very rich in nitrogen, upon the products of the vine has been frequently noted as destructive to bouquet and quality.

\section{METHODS OF APPLYING FERTILIZERS}

Suggestions concerning proper application of barnyard manures, both to young trees at planting and to bearing trees and vines, have already been given. The same conditions which cause slow decomposition of stable manures apply to any fertilizing material which is not readily soluble in water. All such material should be in a finely divided state. Surface applications of ground bone will, in the dry climate of California, lie practically unchanged for a long period. Ground bone should be plowed in as deeply as can be done without injury to the roots of trees and vines, and then, if the surface is kept cultivated, it will lie in moist strata and decompose, or be seized by the searching rootlets. On the other hand, super-phosphate, or other really soluble chemical fertilizers, will produce immediate results, and can be most economically used on light and easily permeable soils, on which falling water sinks and does not flow over the surface. In leachy soils a part of such fertilizers might be carried down beyond the reach of shallowrooting plants, but there is little danger of this in the case of trees and vines.

When superphosphate is used on irrigated ground, it is sometimes drilled in to prevent its being carried along with the running water. One way is to run a chisel-tooth cultivator ahead of the drill and to drill in the fertilizer as deep as feasible to do without injuring the roots. Spring application of fertilizers are conveniently made by spreading upon the vegetation which is plowed under at that season.

Manures with Irrigation Water.-Distribution of fertilizers by using the flow of irrigation water is described by A. S. Chapman, as follows:

We shovel sheep manure into the irrigating ditches, allowing each tree to receive about twenty-five pounds at each separate irrigation. Our basins cover the entire surface of the ground. We make no effort to choke such weeds as clover, alfilerilla, and the like; but the irrigator with his hoe destroys the obnoxious nightshade, hoarhound, and nettle.

In the fall of the year we follow with copious liming-about three barrels of unslacked lime to the acre-applied in the following manner at the head of our irrigating ditch: We plant a box (about three feet wide, six feet long, two feet deep) six inches under the surface of the running water. In it we place a barrel of the lime. It slacks and swells to twice its original bulk. A man stands on this with his hoe and sees that the water carries it off evenly. With an irrigating head such as we use, a man will run into the ditch four barrels a day, or about three barrels to the acre. We have a considerable fall, and the water runs very rapidly; but it takes up all the lime and the water runs white, like milk. 
We now leave the orange orchard till spring, when we plow under the weeds, manure and lime. We thus aim to supply our soil with nitrate of lime, potash and magnesia. Carbonic acid gas is absorbed by the water and attacks the inert plant food in the soil; hard-pan is prevented both by the mechanical effects and the vegetable matter and the lime.

The basin method of irrigation, to which allusion is made, will be more fully described in the following chapter.

\section{FERTILIZING MATTERS IN IRRIGATION WATER}

Water used for irrigation may carry in solution injurious substances, as, for example, alkali, as will be noted in the following chapter; or it may carry very valuable fertilizing properties. These facts can only be determined by analysis. Professor Hilgard has found that the water of one creek in Alameda county carries to the land it irrigates about half a grain of potash in each gallon, which means that if twelve inches of such water were used on the ground during the season, each acre would receive therefrom about twenty pounds of fully available potash. At Riverside a crop of oranges requires about forty-two pounds of potash per acre, of which the amount the irrigation water used in that case contained thirty-five pounds, beside other matters required by plants. These things have a definite cash value in the market; and this value the irrigator gets as a free gift in addition to the water. Even in the case of the Nile, the sediment is only part of the sum of fertility conveyed by the river.

\section{GREEN MANURING OR COVER CROPS}

Green manuring consists in plowing under a growth of weeds or a sown crop to secure by its decay a contribution of humus to the soil. Plants grown for this purpose are currently called "cover crops" because they cover the soil instead of allowing it to remain bare in "clean culture" of orchard or "bare fallow" of grain fields.

All plants by their decay in the soil add organic matter to it, and this matter is of nitrogenous character, but leguminous plants do this and a great deal more, through their exclusive ability to use atmospheric nitrogen gathered by the bacteria which cause nodules upon their roots. There is also special value in deep-rooting legume in soil amelioration. As has already been stated, where moisture is ample for both alfalfa and trees this plant is being used for a permanent cover of orchard ground as a substitute for clean culture. This is being successfully done to some extent with almonds and walnuts, as with other fruits also. In the San Jose districts apricot trees have been grown for several years in irrigated alfalfa, with a gain in the product of the trees. Alfalfa can be used for a certain time even when its permanent stand is not desirable, for it is not difficult to destroy alfalfa with a well-sharpened.plow, although the roots may have attained considerable thickness. Of course this practice depends upon moisture supply; where that is not abundant, clean culture for moisture conservation is unavoidable. But where moisture in excess of the 
needs of the trees is available it will be used in future indirectly for their benefit in ways we are only just beginning to discern, and one of these is likely to be the summer growth of legumes in the orchard. Cow peas on moist or irrigated lands may be used in this way.

A summer cover crop in California, however, except where irrigation water is cheap, may never be practicable. The wider problem is to secure the best leguminous plant which will make a heavy growth during the winter months, so that it can be plowed in early in spring and the ground put in shape for the thorough surface pulverization to prevent evaporation of moisture during our long, dry summer. For this reason we can not use many plants which are used for greenmanuring in humid climates. Crimson clover, cow peas, etc., do not make good winter growth unless the temperature is relatively high and frosts few and light. They make exuberant growth for a time in the spring when heat is adequate and moisture abundant, but at that time it is too late to grow crops for plowing under because the soil is too dry for their decay and their presence tends otherwise to the loss of moisture and makes it very difficult to secure a good surface tilth. The greatest care must be had not to allow a growth either of cover crop or of weeds to stand too long or its covering will do more harm than good. Hardy legumes are therefore the desideratum both for winter forage and green-manuring. The common "burr clover" (Medicago denticulata) is proving very satisfactory in some parts of the State, the "Canadian field pea," the winter vetch, the hairy vetch and fenugreek are coming into quite wide use in orchards in different parts of the State. Which plant is best in any locality must be determined by its local behavior. In some places native lupines make a good natural cover crop. If local conditions do not favor growth of legumes, a good winter cover of rye or other hardy grain may be grown. It will serve the same purposes, though, perhaps, to a less degree.

A Matter of Local Study.-The recourse to cover crops in the orchard or vineyard should be approached with a disposition to careful study and experiment. Unless it is done in the right way it is likely to be disappointing and the right way involves both the selection of the best legumes and the best ways to grow them. Scant growth, cloddy soil, loss of moisture and condemnation of the practice are likely to result from ill-considered methods. On the other hand, success with cover crops results in such numerous and important advantages that the wide introduction of them must be looked upon as one of the most important advances in California horticulture during the last decade.

Nothing more emphatic can be stated in support of green manuring under the proper conditions than a description of the role played by organic matter in soils. Professor C. B. Lipman, of the University of California, prepares such a description as follows:

1. By its binding action on the soil particles and by its sponge-like absorptive power, organic matter makes sandy soils more retentive of moisture. 
2. By its light, yet bulky mass, it prevents the extremely fine particles of clay soils from running together and becoming cemented, and improves the essential feature of aeration in heavy soils.

3. Organic matter contains most of the nitrogen found in soils and all plants except the legumes are absolutely dependent on it for their supply of that essential element.

The greater ease of tillage of soils enriched with organic matter is a matter of universal experience. Besides such a contribution, cover crops may be claimed to confer such specific benefits as these:

4. Organic matter is the source of energy and the laboratory in which the important beneficial soil bacteria manufacture available plant food and bring about other changes of importance to plants.

5. Through its decomposition by soil micro-organisms, organic matter acts as a source of carbonic acid and other weak acids which help to dissolve and make available the mineral matter necessary to the growth of plants.

While growing they probably assist in preventing the radiation of heat from the ground in time of a freeze. They do away with the cultivation of the ground during the winter months.

Each of these reasons suggests a chapter of discussion and explanation which can not be indulged in. Every reader should keep himself up to date in this progressive subject by thoughtful reading of our horticultural journals, proceedings of our horticultural assemblies, and the publications of the University of California Experiment Station. 


\section{CHAPTER XV}

\section{IRRIGATION OF FRUIT TREES AND VINES}

Whether fruit shall be grown with irrigation or not is a local and specific question, and it must be answered with due regard for several conditions, among which are: First, the minimum local rainfall; second, the depth and character of the soil and subsoil; third, the situation and environment of the ground on which the fruit is to be grown; fourth, the kind of fruit which it is desired to produce.

These conditions are all correlated, and a knowledge of them all is necessary to an intelligent decision as to correct practice in any given locality. For example, the amount of rainfall which is adequate in one locality, or in one situation, even, may be quite insufficient in another, because, first, one soil may be deep and fairly retentive, into which roots can penetrate and find abundant moisture; second, another soil may have sufficient depth, but be so porous as to lose its moisture by evaporation, or so leachy as to lose it by drainage; third, still another may be shallow, and quickly dried out under a fervid sun, or quickly drained by reason of a sloping substratum of rock or hardpan, while another similar soil, differently situated, may receive abundant moisture from the drainage of the slope above it; fourth, possibly in all the soils cited there might be adequate moisture for deciduous fruits, but citrus fruits would require irrigation; or enough for young, but not for bearing trees.

Thus it appears that even to decide whether a location has sufficient rainfall for the growth of fruit without irrigation, one must pass judgment upon all the conditions first mentioned. It is hardly worth while, then, to discuss such a topic upon theoretical grounds, or to attempt to answer the general question, Shall irrigation be employed in the growth of fruit? The true guide is enlightened local experience, and the true test is the growth of the tree and the excellence of its fruit. So long as the grower is able to secure every year a generous amount of good-sized and excellent fruit by natural rainfall, he need concern himself very little about irrigation; if his tree shows distress, and his fruit, even when properly thinned out, is not up to market standards every year, he may do well to provide himself with irrigation facilities, either for constant use or to supplement rainfall when it is occasionally deficient.

Of course it is not commended that the grower wait until the tree shows signs of distress before applying water. This is a very bad plan of proceeding, but the visible language of the tree is mentioned as indicating that the tree needs help, either at regular intervals or occasionally, and after such a warning the grower should be able to tell by examination of the soil and by study of the local rainfall record when this need will occur, and apply his water in advance of the need.

Recent experience has enabled fruit growers in all parts of California to arrive at a truer conception of the relation of irrigation to the 
growth of fruits. Many who long scouted the suggestion that irrigation was necessary for deciduous fruit trees in their districts, have found that water, in addition to the rainfall, was very profitable, either to enable large, bearing trees to produce larger fruit, or to maintain in full vigor their later summer growth and to make strong fruit buds, which insure the following year's production. It has also been widely demonstrated that a tree which is adequately supplied with water, no matter whether it be directly from the clouds or through the irrigating stream, yields fruit of better size, aroma, flavor and carrying quality than a tree which, from any cause, falls even a little short of an adequate supply. It is clear then that neither irrigation nor non-irrigation are in themselves principles, but are merely methods to be employed when conditions demand the one or the other.

Several claims against irrigated products may be stated and opposed in this way:

(1) The claim that nursery trees grown by irrigation are, from that mere fact, inferior is based upon experience in transplanting trees unduly forced by over-irrigation. Immense growth from the bud in a single season of an inch and a half in diameter and ten feet in height tempted buyers who wanted to get as much as possible for their money. The result of setting out such trees created a strong prejudice against irrigated nursery stock. It is now clearly seen that moderate, thrifty growth is the ideal in a young tree, and if the soil does not hold rainfall enough to secure this, water enough to secure it must be applied.

(2) The claim that irrigated fruit lacks aroma and flavor is based upon observation of monstrous, insipid fruit forced into such abnormal character by excessive irrigation. Growers who concluded therefrom that irrigated fruit was necessarily inferior, denied water to their trees and gathered small, tough, unmarketable fruit, because there was not enough rainfall to enable the trees to perform their proper function. As it is now conceded that the highest quality, including the delicate aromas and flavors, can be secured only by adequate moisture, it matters not how long since it fell from the clouds nor by what route it reaches the roots of the trees.

(3) The claim that irrigated fruit could not endure shipment was based upon the bruising and collapse of fruit which was unduly inflated by over-irrigation. The best fruit for shipping is the perfect fruit and that is secured as just stated. The fact that the greater part of the fresh fruit shipped across the continent from California has been more or less irrigated, according to the needs of different localities, has settled the point beyond further controversy.

(4) The claim that canners objected to irrigated fruit was based upon the early experience with over-irrigated fruit, which lacked quality and consistency. At present the canners encourage irrigation and all other arts of growing which bring the product up to the standards they insist upon.

(5) The claim that irrigated fruit is inferior for drying has the same foundation as the preceding claims and is just as clearly based upon misapprehension. Watery fruit is obviously inferior for drying but such fruit is the fault of the irrigator, not of irrigation. One of 
the plainest deductions from experience is that small, tough fruit makes unprofitable dried fruit, and that the best development of the fruit is essential to the best results from drying. Many comparative weighings have shown that the greatest yield in dried form has been secured from trees which have had water enough to produce good, large fruit. Even to bear fruit for drying, then, the tree must have moisture enough to develop size and quality. If lacking moisture, the tree serves its own purpose in developing pit and skin and reduces the pulp, in which lie the desirability and value of dried fruits.

Of course the water should be applied at proper times, in proper amount, and in a proper way.

\section{HOW MUCH WATER SHOULD BE USED?}

This is by its very nature an elusive question and any attempt to answer it by a definite prescription is more apt to produce folly than wisdom. For as it appears that whether irrigation is at all needed or not depends upon several conditions which must be ascertained in each place, so the amount of water, which is really an expression of the degree of that need, depends also upon local conditions of rainfall, of soil depth and retentiveness, of rate of waste by evaporation, of the particular thirst of each irrigated crop, etc. The result secured by the use of water is really the ultimate measure of the duty of water in each instance. In the case of fruit trees and vines, then, whatever amount of water secures thrifty and adequate wood growth and strong, goodcolored foliage, but not excessive or rank growth; and abundance of good-sized and rich, but not monstrous and watery fruit, is the proper amount for that place and that product,- -and to the ascertainment of that amount by local experience of himself and others, the grower should employ his most earnest thought and his keenest insight.

During recent years the writer has continually renewed his data of the irrigation practice of California fruit growers by systematic inquiry and has prepared four bulletins* which have been published by the Irrigation Investigations of the U. S. Department of Agriculture.

A study of local practice shows that infinite variety exists and in the nature of the case must exist, and that any definite prescription of the duty of water under various conditions is impossible. In some cases the amount of water at each irrigation must be small, and applications frequent because the soils are shallow, overlying bedrock, and a small amount saturates them. In other places an acre-foot of water is readily absorbed and retained in the deep soil. The annual rainfall also has little relation to the amount of irrigation, because neither fine shallow, nor deep coarse soils, can retain the volume of water which falls upon them during the rainy season. Then the varying rate of evaporation, the character of the tilth, etc., enter as factors and it becomes clear that he is fortunate who knows how much water to use on his own place.

*Farmers' Bulletin No. 116, “Irrigation in Fruit Growing;" Farmers' Bulletin No. 138, "Irrigation in Garden and Field;", Bulletin of Experiment Stations No. 108, "Irrigation Practice Among Fruit Growers of the Pacific Coast;" annual report of irrigation and drainage investigations, 1904, "Relation of Irrigation to Yield, Size, Quality, and Commercial Suitability of Fruits." 
It is interesting to note that results of close inquiry by the Irrigation Investigations of the U. S. Department of Agriculture to ascertain the amounts of water used by measurement of water running in main ditches and by estimate of the acreage to which the water is applied, do not agree closely with the growers' estimates of the amounts of water which they actually use. There are, of course, always issues between water-purveyors and water-buyers which can not be entered upon in this connection. A rough conclusion from data secured from the ditch flow, etc., is that from 12 to 30 acre-inches of water are used annually in irrigated orchards and vineyards, according to local conditions involved. It is quite clear that the amounts chiefly used would not be the average but would tend toward the lower figure. The details of these inquiries are found in the publications on irrigation of the Office of Experiment Station, U. S. Department of Agriculture.*

\section{RELATION OF RAINFALL TO IRRIGATION}

The amount of rain and the time it falls are clearly the most important factors in determining the necessity for irrigation. Absence of rainfall makes a desert of the richest soils at all elevations and at all exposures. Its only remedy is irrigation. But there are degrees of poverty in rainfall, and thorough tillage will often lessen the ill effects of a scanty supply, so that an oasis may be made to appear without water beyond that supplied from the clouds. This is the triumph of tillage in the arid region which is to be considered in another connection.

The line between adequate and insufficient rainfall can not be closely drawn. In the growth of common orchard fruits, irrigation is not resorted to at a number of points where the local rainfall sometimes is as low as 15 or 16 inches, but with less than that amount, unless the soil receive additional moisture by underflow, it is essential. Or the other hand, irrigation is regularly practiced in some localities where the rainfall sometimes rises to 45 inches. Under average conditions of soil depth and retentiveness, the amount of rainfall which may be considered adequate for deciduous orchard trees under good cultivation is about 20 inches. So definitely is this amount fixed in the minds of some California growers as meeting the needs of the tree for satisfactory growth and fruitage that, when rainfall for a season is less than that amount, irrigation is at once resorted to to supply the shortage.

But owing to local conditions of soil and climate, the rainfall, no matter how large, may not be relied upon to carry the trees through the dry season. The fact is that the soil is not capable either of receiving the heavy rainfall or of long retaining such portions as actually enter it. There is, then, a considerable part of the rainfall which is worse than worthless, because it does injury by soil washing and soil leaching, and places where extremely heavy rainfall occurs may be actually worse off than other places with less rainfall. Some localities of large rainfall lead in amounts of water supplied by irrigation. The

*Definite citation is not made because these publications are continually appearing with additional data on the effective use of water. The whole series should be examined. 
converse is also true, for some localities of light rainfall report success with deciduous fruit trees with a minimum amount of irrigation water.

Deciduous Fruits.-Without making too much of individual reports there appear instances enough to warrant the conclusion that the deciduous fruit tree can winter successfully with a small moisture supply and is, in fact, in less danger from lack of moisture than from over-supply at this time of the year. If there be enough moisture to prevent injury from evaporation, the tree will start good growth as the season advances and continue it if irrigation is given promptly and in sufficient quantity. There must always be a determination of what is an adequate supply by reference to local conditions, but as an estimate of necessary rainfall has been made at 20 inches, it is evident that adequate irrigation may be very much less than that. The rainfall of 20 inches is distributed through six or seven months. Some of it consists of light rains, with long, dry intervals, where there is slight penetration and quick evaporation. Some of it is lost by run off and by drainage. It is not surprising, then, that some growers having deep valley loams to render their irrigation effective, report success with deciduous trees with 8 or 10 inches of water applied just at the time of the tree's greatest needs and used, no doubt, with maximum efficiency. It seems to be a warranted deduction, from all data known to the writer, that 10 inches of water, applied at the right time to soils of good depth and fair retentiveness, and accompanied by good tillage for conservation, is an adequate supply for five months of growth and fruiting even when the rainfall is only about enough to prevent drying out during the winter season. Some growers report use of less than this. Certainly less will do for young trees under favorable conditions, and some of the least amounts are reported from the newly planted regions. As the trees advance in age and bearing, larger amounts will be required. Instances of greatest frequency of application may be taken as indicating soils lacking retentiveness, either through shallowness or coarseness, or either of these accompanied by extreme summer heat and aridity.

Citrus Fruits.-As these trees are evergreens, and as their habit is to make their chief fruit growth in the autumn after the work of the deciduous tree has been finished for the season, the irrigation season for them is much longer. As they are, in fact, almost always active and sustaining uninterrupted evaporation from their leaf surfaces, they must always be provided with moisture or ill will result to tree or fruit. They thus require more water than do deciduous trees. There is the same relation between irrigation and rainfall with citrus, as with deciduous fruit trees, but the degree of relation is different. Many trials have shown that it is practically impossible to grow satisfactory citrus fruits without irrigation, unless there be underflow, and this is attended by the usual difficulties of high ground water and undesirable. There is no combination of heavy rainfall, or winter irrigation, and soil retentiveness which will supply the summer and autumn thirst of the orange or lemon in California. Irrigation, too, must be maintained both summer and winter wherever the rainfall is not well distributed and adequate. In 
the chief citrus regions of the State rainfall is seldom adequate except during January and February, and not always then. Under such conditions an estimate of the average requirements of citrus fruit trees in bearing would be about 20 inches of irrigation, irrespective of rainfall, although there are localities of larger rainfall and more retentive soils where crops of these fruits can be made with 10 inches used at just the right time.

\section{RELATION OF SOIL TO IRRIGATION}

As already stated, the desirability of irrigation is unquestionably, in many cases, conditioned upon soil depth and character. This relation has received careful attention from soil physicists, and an understanding of it involves problems of plant growth and the movement of water in soils, the leading facts of which are available in popular form.*

Analysis of such phenomena can not be undertaken in this connection but a few striking contrasts in existing practice are very suggestive.

On the famous river-bank fruit land of the Sacramento Valley, with loams of great depth and good retentiveness, and with an average rainfall of approximately 20 inches, irrigation is resorted to only in years of minimum rainfall, when the precipitation is perhaps only about half the average. At nearly the same level, as already cited, where the soil is shallow and overlies hardpan, irregular irrigation is required. But still more marked contrast is found in the foothills within sight of these valley fruit lands, where with twice the average rainfall irrigation must begin early in the summer and continue until autumn is well advanced, because, first, the slope is so rapid that much rainfall is lost by run off; second, the soil is too shallow above bed rock to hold much water. Even here, however, there comes in a local variation of measurable effect. When the soil lies upon vertical plates of bed rock much water is retained between them, and is capable of being reached by tree roots, while soil lying upon flat plates of rock has no such subterranean reservoir. In the foothill region there also occurs exceptional exposure from slopes facing the midsummer sun in an atmosphere whose dryness is but slightly ameliorated by the influence of air currents from the coast.

In the valley and foothill contrast, just cited, the unirrigated valley looks up to the irrigated foothills. There are also places where unirrigated hillslopes look down upon irrigated valleys. The uplands of San Diego County are nearer the coast than those above the Sacramento Valley. They, too, have a rainfall usually ample for deciduous fruits suited to their elevation. Their rolling plateaus of deep soil, free from excessive heat and evaporation which occur on the highlands farther inland and 500 miles farther north, produce very successfully without irrigation. In this region, however, the

*Relations of soils to climate, U. S. Dept. Agr., Weather Bureau Bul. 3. Water as a factor in the growth of plants, Yearbook U. S. Dept. of Agr., 1894, p. 165. Some interesting soil problems, Yearbook U. S. Dept. Agr., 1897, p. 429. The movement and retention of water in soils, Yearbook U. S. Dept. Agr., 1898, p. 399. The mechanics of soil moisture, U. S. Dept. Agr., Division of Soils, Bul. 10. 
rainfall in the valleys below is often less than the needs of even the deciduous fruit trees, and waters flowing from mountain snows through a region of unirrigated uplands must be used to irrigate them.

Still another striking contrast, and one involving another and wholly different factor, is found in the San Joaquin Valley. Near Visalia 2 feet above river bottom and 4 feet above the surrounding plains, there is a large area of deep alluvial soil with much decayed vegetable matter. The land is moistened by underflow from the river, and, though the rainfall is but $7 \mathrm{I} / 2$ inches, deciduous fruits are grown without irrigation. In the same county, and only 18 miles distant, there are areas of rich loam mixed with granitic sand 16 to 18 feet deep. In this locality, though the rainfall is $11 \mathrm{I} / 4$ inches, irrigation is practiced freely, as the loss of moisture in summer is very great.

\section{RELATION OF TILLAGE TO IRRIGATION}

Tillage, particularly during the dry season of the year, under some conditions, directly determines the need of irrigation, and is to a certain extent, as the popular phrase goes, a substitute for irrigation. Under all conditions surface tillage by promoting conservation of soil moisture, is determinative of the actual duty of water, whether it be from rainfall or irrigation. The effect of frequent surface tillage has been accurately determined by investigation and experiment, both in humid and arid regions. These experiments fully support the view taught by the experience of about half a century in California, in accordance with which thorough tillage has been so widely practiced in the arid sections as an essential to successful fruit growing.

As already maintained in Chapter XIII, the relations of tillage to soil moisture include both reception and conservation. For the reception of moisture, deep work with the plow, and sometimes with the subsoiler also, is almost indispensable. To retain this moisture and to prevent, as far as possible, its escape into the thirsty air of the arid region by surface evaporation, less depth and more thorough surface pulverization are required. Recent practice has been tending toward deeper summer cultivation, so that, as previously claimed, 5 or 6 inches of loose, finely divided soil is now obtained where formerly half that depth was considered adequate. It has also been shown that frequent stirring of this fine surface layer checks evaporation, even when no water is applied to compact the surface or where no weeds grow to draw upon the soil moisture. In a word, the aim of tillage in the arid region, so far as it relates to moisture supply in the soil consists in opening the soil to rain, or to irrigation, and in subsequently closing it to evaporation. These are the principles which were recognized and applied in California half a century ago and are now enjoying somewhat sensational renaissance in the "dry farming" agitation in the interior of the United States.

A Negative Declaration.--It is interesting that current practice affords full demonstration of the foregoing claims both positively 
and negatively. The negative argument in favor of moisture conservation by clean summer cultivation is found in the fact that growers in regions of heaviest rainfall approve the growth of cover crops, like clover, after the trees reach bearing age, and also that others employ scant summer cultivation, or cultivation for a short period only. The idea of these growers is that such practices relieve the soil of excessive moisture, either by the growth of the cover crop or by facilitating surface evaporation, and so prevent the tree from being stimulated to too large wood growth, or maintaining growth so late in the season as to enter the frost period in too active a condition and with new wood not properly matured. Quite in contrast with this is the practice, which is gaining ground in the hottest parts of the irrigated region, of growing alfalfa as a cover crop for the purpose of shading the soil and thus reducing soil temperature and, perhaps, of avoiding the ill effects of the reflection of burning sun heat from a smooth surface of light-colored soil, or the ill effect of "burning out of humus" by clean summer culture. In such cases more irrigation is needed to supply enough water for the growth of both trees and cover crop. But at present these exceptions are of rare occurrence.

Cultivation Not Determined by Irrigation.-The adoption of a policy of clean cultivation in the dry season is not conditioned upon the amount of moisture available either by rainfall or irrigation. It is pursued both where irrigation is practiced and where it is not, and also where the rainfall is greatest and where it is least. It prevails in the humid region where rainfall may rise to 60 inches or more, and in the arid region where it may not exceed one-tenth as much. As a matter of fact, there does not appear to be a good fruit soil so deep and retentive that it can retain enough even of a very heavy rainfall to effect good tree growth and fruit bearing if it is forced to sustain the loss by evaporation from a compact surface during the long dry season following. There may be, it is true, soils weak in capillary, in which water can not rise from a great depth and in which deep-rooting plants may find ample water in the subsoil, providing it is held there by impervious underlying strata. There are many more instances where loss by natural drainage is added to loss by evaporation. But, disregarding exceptions, the loss of moisture by both drainage and evaporation during the dry season is so great that the soil to a depth of several feet loses practically all the water which is available for plant growth, and the trees fail or become unprofitable. Loss by drainage can not, practically, be prevented, but loss by evaporation can be so reduced that trees and vines will be adequately supplied in spite of the loss by drainage. Because, therefore, the soil can not retain enough water in its natural state, no matter how much it may receive, clean summer cultivation, involving quite complete and more or less frequent stirring to the surface to the depth of 5 or 6 inches, as discussed in Chapter XIII, is the almost universal practice, irrespective of local rainfall or of irrigation.

Cultivation, However, Determines Success of Irrigation.-The prevailing motive for cultivation in the dry-summer region is moisture retention. In this respect good surface tilth is so effective that, though 
enough moisture can not be retained without it, so much can be retained with it that, even where irrigation or rainfall is moderate in amount, it may serve all purposes of the tree or vine. Thus cultivation enters into the fruit-growers' practice in the region under consideration, not to make large rainfall effective as it does in some parts of the region, but to make moderate rainfall effective, or to make small irrigation effective, by increasing the duty of water which is applied. It becomes not only a ruling consideration in the effectiveness of a certain amount of rainfall, as has already, been suggested in another connection, but it also determines the success of irrigation and the amount of water required; for, although it was an early and crude practice to rely upon irrigation to support uncultivated fruit trees and to irrigate more and more frequently as the ground became harder from its use, this policy has now no standing in commercial fruit growing. Not only was it wasteful of water, but it was otherwise detrimental to the thrift of trees.

Cultivation and Irrigation Work for Soil Improvement.-Thorough cultivation, both in winter and summer, has other very important ends in view. It opens the soil and promotes aeration; it encourages deeper rooting and thus encourages the tree to take possession of a greater soil mass both for moisture and other plant food. It is part of the very valuable policy of increasing humus by plowing under the natural growth of weeds or specially sown legumes, which is discussed in Chapter XIV. This affords opportunity to use water, beyond the amount the trees require, for soil improvement.

\section{WHEN TO IRRIGATE}

When to irrigate is governed by local conditions and the needs of different fruits, and can not be stated in general rules. There are, however, some principles involved which may be hinted at.

Winter Irrigation.-On lands with sufficient depth of fairly retentive soil, the grower may artificially supplement a scanty rainfall by thoroughly soaking the land by winter irrigation and then by careful summer cultivation he will be able to conserve enough water in the soil to carry deciduous fruit trees or vines through bearing and autumn bud formation without further water supply. But there are other situations in which no amount of winter irrigation nor rainfall will suffice for these ends. There are foothill orchard areas in which the winter rainfall is two or three times as great as in the valley situations where fruit is successfully grown without irrigation, and yet water must be applied in summer on those foothills or the fruit would be unmarketable and the trees in distress. The forty or more inches of rainfall falling on a shallow soil underlaid by sloping bedrock in some cases nearly sluices the cultivated soil from its foothold, and yet the oversaturation in winter avails nothing for summer growth, because most diligent cultivation can not retain moisture enough in shallow soil thus situated to sustain bearing trees in good crops of full-sized fruit. The same is true of valley soils underlaid by hardpan. In such cases winter irrigation could add nothing but distress to the soil over-soaked by rainfall, and summer irrigation, well-timed and adequate, is the secret of 
success in the orchard. The same conclusion, although for very different reasons, must hold for soils underlaid by gravel or sand, and thus too rapidly dried by leaching.

But even this generalization must be accepted only for situations endowed with conditions which justify it. There may be sloping hills with shallow soil where winter rainfall does not amount to saturation. Then winter irrigation to supply such irrigation is desirable, and then too, summer irrigation in proper amount and at proper intervals, will also be demanded. Among the foothills, also, there may be localities with depth of retentive soil in which water enough can be applied in winter to carry trees through the year. Thus we come again to the only safe generalization which can be made, and that is, that everywhere water must be adequate to the demands of the tree at the time it is needed, and whether it can best be applied in summer or winter, or both, or whether it is not necessary to make any artificial application at all, depends upon existing conditions which the grower must ascertain, and to which his policy and practice must conform. It is a fact however, that all soils, which under good cultivation are fairly retentive, winter irrigation, when water is most abundant, and usually carries most sediment, can be made to go far toward making summer irrigation unnecessary for all deciduous fruits.

As to winter irrigation, practice varies, some relying upon a single heavy flooding by using checks on contour lines, by which, perhaps, a foot in depth or more of water is allowed to soak into the soil; others use the same method of application in winter as in summer, and, therefore, give a number of irrigations in winter. There is, of course, much less danger of injury by water to deciduous growths in winter, because they are dormant, though an eye should be kept on drainage for excessive irrigation as for excessive rainfall. The grape and the pear are known to endure long submergence, but some other fruits are sensitive about it.

Summer Irrigation.-When this shall begin and when end are to be locally determined. In some places even the earliest fruits can not reach satisfactory size and quality without irrigation. In others rainfall with winter irrigation will suffice for proper development of early fruits, but not for late. In both cases the fruit may be satisfactory, but the tree unable to hold its leaf vigor until the work of the growing season is properly completed. It is then apparent that local practice must vary in order to reach the universal fact, and that is that all through the active season the tree must have constant and adequate moisture supply. Many evils in lack of bearing, in dying back, in unseasonable activity and the like are due to inadequate, intermittent and, in some cases, to excessive moisture in the soil.

Cultivation and Irrigation.-Although the relations of irrigation and cultivation have been freely discussed, it must be remarked in this connection that with such an extension of irrigation practice as is now realized, there is danger that those who have previously trusted so fully upon good cultivation may swing to the other extreme and trust too much to the stream of water and too little to the plow and cultivator. There is a temptation this way when one finds that he can run 
water in large amounts very cheaply. Not only is there danger of overirrigation in the growth of tree and fruit, but the ill effects of water upon the soil, when unattended by good cultivation, are constantly threatened. The tree needs air as well as water; it needs a certain free condition of the soil for its best root action. These needs can be amply secured when adequate application of water is quickly followed by soil-stirring. Irrigated soil rightly treated is delightfully mellow and free and of condition to invite the fullest activity on the part of the tree. Irrigated ground not properly treated becomes compacted, fissured, cloddy and generally hateful, losing moisture rapidly, setting around the roots like cement and tearing them by its subsequent shrinkage. These conditions do not occur on the lighter soils, and yet even these are best when cultivated in a rational manner.

\section{METHODS OF IRRIGATION}

There are various methods employed in California for the conveyance and application of water to trees and vines. Some of the principal ones will be described.

As this writing does not pretend to be a treatise on irrigation engineering, no attempt will be made to describe the more ambitious undertakings, which should never be entered upon without the engagement of a qualified engineer. Nor is it possible to discuss the numerous devices which are covered by patents. Investment should always be preceded by visits to irrigation works now in operation, and procedure should be guided by observation. The hints presented herewith relate chiefly to things the irrigator can do for himself.

Free Flooding.-Flooding - that is, the free flow of water over the whole surface, or the flow between rows with furrows near the trees to retain the water in the interspaces-is only employed on some flat lands where winter irrigation is used to supplement rainfall when the latter is occasionally below normal. In such cases water is available in large quantities, and the lay of the land favors quite even distribution. Even under these conditions the experience of growers soon leads to the adoption of deep furrows or lateral ditches, or some simple check system, as superior to flooding. Summer flooding is done only by those who are unacquainted with better methods or who count their trees of too little account to warrant extra effort. It seems, therefore, a fair conclusion that flooding is only resorted to as a temporary expedient, and has little standing.

The Check System.-With soils of such character that vertical percolation is very rapid, flooding in checks, by which water is held upon a particular area until it sinks below the surface, is considered necessary. There is a tendency to change from this method to a furrow system wherever practicable, because the former requires more soil shifting, a larger head of water for economical operation, more labor to handle it, more working in water and mud, and more difficult cultivation to relevel the land and to reduce a puddled surface to satisfactory tilth. For these and other reasons, perhaps, on loams of medium fine- 
ness one may find two adjacent growers pursuing different methods, while on coarse porous loams the check system prevails, and on fine, retentive loams, the furrow system is without rival.

The check system can be seen on the most extensive scale in the upper part of the San Joaquin Valley, where the land is so level and water so abundant that the checks can be measured by acres or fractions of acres. In its most perfect form it is found in Orange County and some parts of Los Angeles County, where the checks are measured by feet, rarely by rods. Very large checks are chiefly used for field crops, although also employed for winter irrigation or vineyards and orchards of deciduous fruits. With fruits, however, even in the same district, the tendency is toward using smaller checks carefully leveled before planting. With the large-check system permanent levees, either in rectangular form or on the contour plan, are generally used. The small-check system is chiefly laid off with temporary levees, quickly made with special appliances and as quickly worked back to a level as soon as the ground dries sufficiently after irrigation, and the whole surface kept well cultivated until the time arrives for a restoration of the levees for the next irrigation. The latter is the leading horticultural mode. It is carefully described by Mr. Sydmer Ross, of Fullerton, Orange County, California, as follows:

The check system, as carried out in the best-handled orchards, entails much hard work, but after you are through with an irrigation you know that each and every tree has had its full supply of water or you know the reason why. The ground must be cultivated, say, about 5 inches deep, so as to have plenty of loose soil with which to throw up a high ridge. Then a four or six-horse "ridger" should be run once each way through the rows, if it is a citrus or deciduous orchard, or twice should the trees be walnuts, because these trees are grown about 40 feet apart. After this is done the ridger should be run entirely around the outside of the piece to be irrigated, so as to have as perfect a ridge as possible on the outside. One man will ridge about 15 acres in a day. The ridger should be built with a steel plate extending along the bottom on both sides, bolted to the inside and projecting about 2 inches, so as to take good hold of the ground. Then with one horse attached to what is locally known as a "jump scraper," one side of the checks should be closed up, for the ridger in making the cross ridges breaks down the first ridge at its intersection. These renairs were at first made with a shovel, but the jump scraper, also called locally the "horse shovel," closes up the gaps very quickly. The practice generally followed is to close up the high side of the checks if the land does not cut by running water, but if it cuts, close up the lower side.

After closing up the checks the ditches are plowed out and then the V-shaped "crowder" is run twice through them. On lands inclined to cut it is advisable that the length of the rows to be irrigated should not be over 250 feet, but in heavy land this distance can be considerably increased, if necessary, without danger of cutting the ridges bv too long a run of water.

If the checks have been closed up on the low side of the ridge, it is better to run the water to the ends of the ditch and water the last row first; but if the high side has been closed up, it is best to water first the row nearest the gate or the main ditch, as the case may be, as in each instance dry earth will thus be available, if necessary, to close up the checks. The water is run down the, row to the end tree, and as soon as the last check is filled it is closed up, and so on till all are filled and closed, when the water is turned down the next row.

To do good work it is usual to allow three men for every 50 inches of water, but in our own practice we have had much better results by dividing up our water and running from 35 to 40 inches to a ditch and allowing two men for 
such streams. In doing this we get better work and find it much easier for the men. If everything is well in hand, each man will irrigate about 30 acres in a day.

For turning the water from the ditches into the checks metal dams or tappoons are used. Some of these have a gate for the division of the water when the stream is too large and is divided, and two rows are watered at the same time. The gate is not a great success, as the water is apt soon to cut its way under the tappoon, but it may be much improved by having a shelf for the water to drop on after it passes through the opening. The common practice for dividing water is to throw a tappoon partly across the ditch, putting a gunny sack on the opposite side to prevent cutting by the water. This is, on the whole, fully as satisfactory as using the tappoon with a gate.

All who follow this system should get ready for the water before it comes. A great many seem to think that if they ridge up their land, close up the checks, and plow out their ditches, everything necessary has been done. Such is not the case, as ditches that are liable to cut should be fixed in the weak places with brush or burlaps. Old gunny sacks cut open and spread out are excellent for this purpose. Occasionally there are places where it is impossible to get a perfect ridge. These should be looked up and fixed with a shovel. The jump scraper will not entirely close up a check; it generally requires a shovelful or two to complete it. It is usual after the water is turned down one row to fix up the next one, but it is an excellent plan to have a few rows fixed up ahead, for there come times when breaks occur and there is not time to make the necessary repairs, and when water once gets the start there is apt to be much trouble and hard work before it can be put under control, besides doing poor work.

After the ground is dry enough to work, the ridges are split with a listing plow or furrower attached to a cultivator. Then the ground should be run over with a harrow, setting the teeth to go well in, so as to pulverize the surface thoroughly. By using the harrow the ground can be worked about one day earlier than with the cultivator, and it also prevents the ground from baking till such time as it can be worked with the latter implement, besides doing far better work than with the cultivator alone, especially when there is much land to go over, as some of it is certain to get too dry before it can be reached, and then it will not pulverize well. All trees should be worked around by hand with either a fork or hoe, as soon after irrigation as the ground becomes dry enough and before it becomes hard.

Specifications for Homemade Implements for the Check System. -The following implements, used in preparing the ground for irrigation by the check system, were made on the fruit ranch of J. B. Neff, Anaheim, California, with the tools ordinarily found on a ranch, and with but little help from the blacksmith:

The Ridger.-This has sides of 2 by 16-inch pine 7 feet long, standing 18 inches apart at the rear and 5 feet apart at the front end. The sides may be made of two 2 by 8 -inch pieces with 2 by 3 -inch battens bolted on securely. The front crossbar is of 2 by 4 -inch pine 6 feet 2 inches long, and is set 20 inches from the end. The rear crossbar is of 2 by 4 -inch pine 4 feet 4 inches long. It is set 7 inches from the end of the sides. The diagonal braces are 1 by 3 -inch pine 6 feet 10 inches long. The short side braces are 2 by 3 -inch pine 15 inches long. The lower inside edge should be protected by a strip of steel or iron $1 / 8$ by 2 inches extending to and around the front ends, which should be beveled to a sharp adge. The inside should also be lined with sheet iron 6 or 8 inches above the $1 / 8$ by 2 -inch piece, and should have sheet iron pieces extending 16 inches beyond the rear end of the sides, tapered and braced in the manner shown in the cut, for the purpose of making 
the ridger firmer at the top. Every part of the ridger should be firmly bolted with $3 / 8$-inch bolts, except the $1 / 8$ by 2 -inch iron, which should have 3-16-inch bolts, and the sheet-iron, which may be put on with nails. The hooks on sides for hitching draft chain are $3 / 8$ by $11 / 2$ inches, and the draft chain is $3 / 8$-inch cable chain.

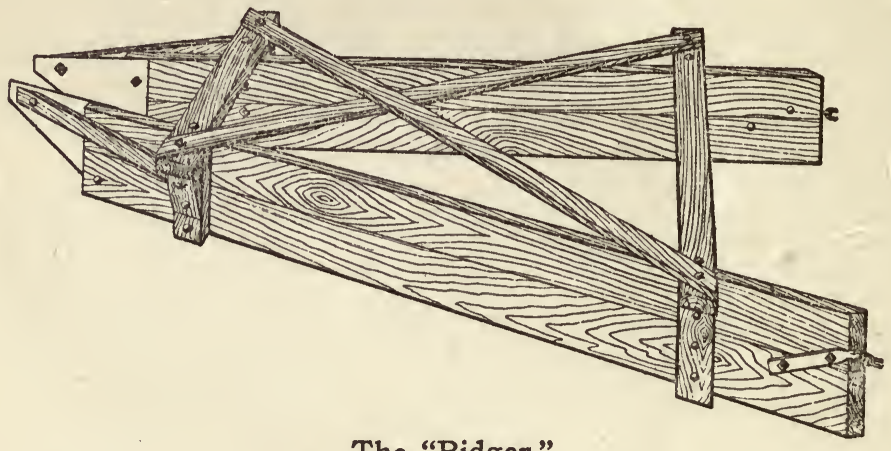

The "Ridger."

For levee making in the check system of irrigating trees and vines.

The $V$-shaped Crowder or Ditcher.-This has sides of 2 by 12 -inch pine and cross-brace of 2 by 9 -inch pine. The long side is 7 feet 8 inches long and short side 3 feet 6 inches long. This is also protected by a piece of steel or iron extending entirely around the ditcher and bolted with 3-16-inch bolts. The sides come together in a point and stand at an angle of 45 degrees. The brace is placed 2 feet 10 inches from the point on short side and 3 feet 10 inches from the point on long side. It also has two handles, as shown in cut, 3 feet long. These

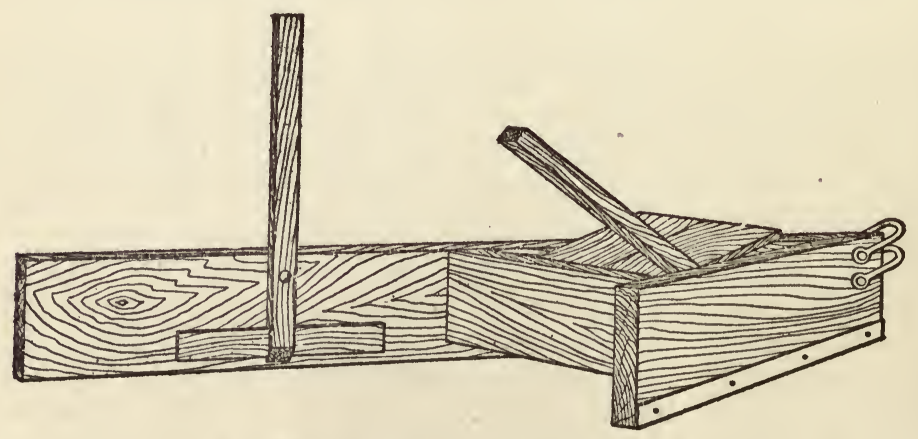

The "Crowder."

Used in the preparation and distribution of water in the check system.

are made of 2 by 3 -inch pine reduced so as to hold conveniently. The sloping handle is bolted to the short side. When in use this implement stands with the short side elevated at an angle of about 35 degrees, and a floor is placed in the triangular space so that it will be level when in use. An ordinary wide clevis is used for the draft and is placed as 
shown in the cut. A vertical hole may be made in front of the clevis pin and a small rod driven in to strengthen the hold of the clevis.

The Jump Scraper or Horse Shovel. - This is used for filling gaps in the ridges, and is the work of the blacksmith. The beams are $1 / 4$ by $1 \mathrm{x} / 4$ inches and 30 inches long from the draft ring to the bend downward. The shovel is of No. 16 sheet-iron, 24 inches long by 18 inches deep. The handles are those used on any cultivator. The beams are bent to stand 6 inches forward of a square placed on top of the beams. The braces are of $3 / 8$-inch round iron. The shovel is slightly cupped to make it hold more earth.

The Portable Gate or Tappoon.-These are for shutting ditches, and are made of No. 16 sheet-iron 2 feet wide and of any desired length, but usually 3 feet, 4 feet or 5 feet long. The corners are cut off to a circle, starting about 1 foot back of the corner. The handles are made

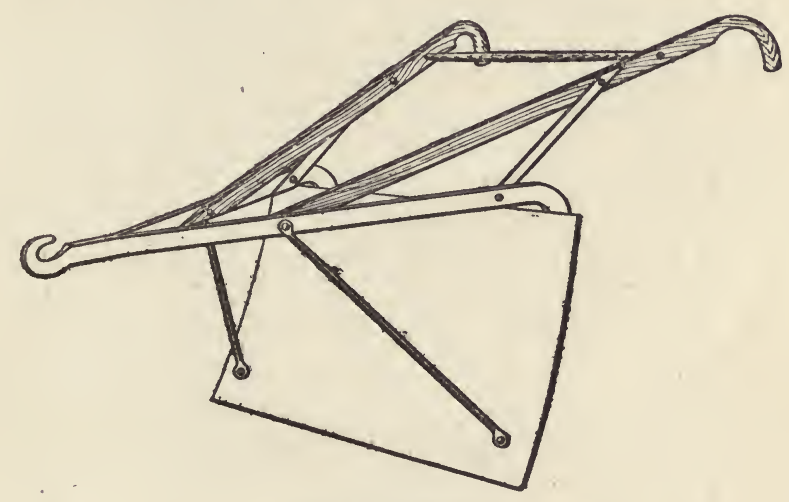

The "Jumper."

Used to complete levees by the "ridger" for the check system.

of 2 pieces of 1 by 3 -inch pine, 12 inches longer than the gate, and are placed one on each side of the sheet iron and secured by $1 / 4$-inch bolts.

The Combined Check and Furrow Method.-An effort to escape in some measure the puddling of the surface which results from allowing water to sink away upon finely pulverized soil, lies in the direction of breaking up the soil roughly in the bottoms of the checks, which facilitates the quick passage of the water into the subsoil. This is done by running a small plow or three large cultivator teeth attached to a single frame before the ridger is used to form the levees. Mr. A. D. Bishop of Orange County, California, uses a combined furrow and check system, as shown in the accompanying diagram. He furrows the land first with a three-tooth furrower at right angles to the direction in which the water is to flow, and then uses the ridger to make levees in line with the water, laying out the work so as to get the closest approximation to a level. When the levees are made, the jump scraper is used and the end of each third or fourth furrow bank is connected with the levees at alternating sides of the check made by the 
levees. This causes the water to flow through the furrows from side to side and distribute itself evenly over the whole ground. The number of furrows which can be passed before connecting with the bank depends upon the slope of the land-the nearer level the land the greater the distance that can be left between the connections, and vice versa. In this way the water is taken slowly down a grade where it would flow too rapidly were it admitted to furrows in the direction of its flow.

Another combination of the check and furrow system is found where the lowest spaces of a slope irrigated by furrows are laid off in checks to catch the overflow from the furrows and compel its percolation at a point which would otherwise receive too little water. The parts of a furrow system which lie farthest from the source of supply are

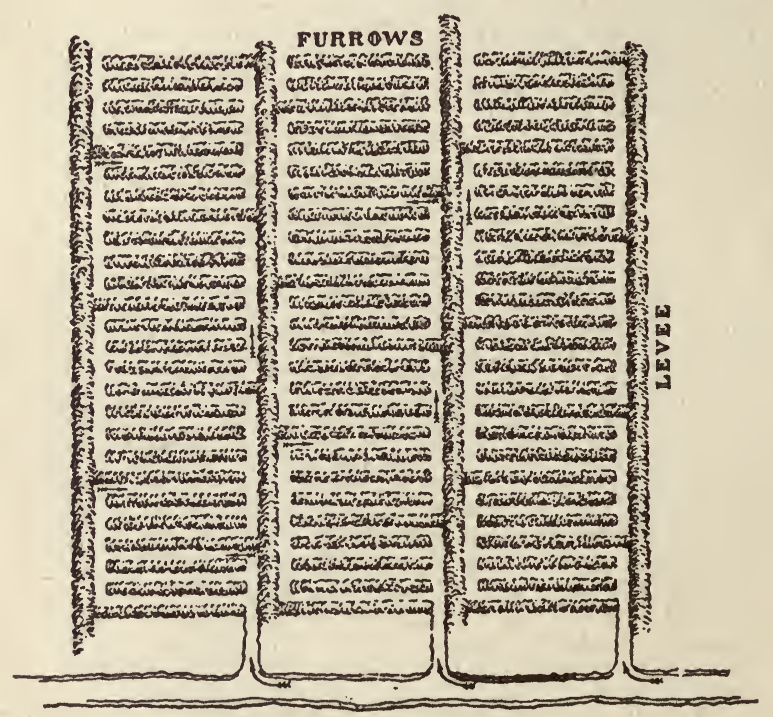

Combined check and furrow irrigation.

obviously least supplied, because long flow can not be maintained there without much loss from overflow. Holding the water in checks at the lower end-usually for two rows of trees-is quite a help toward even distribution.

The Basin System.-The term basin should be restricted to inclosures which do not aim at covering the whole surface, but only a small area immediately surrounding the tree. The check system is clearly a more rational and perfect method of flooding. When basins were used on ground capable of irrigation by the check or furrow systems, it was probably due to a misconception which has prevailed also in the practice of fertilization, that the tree derived its chief benefit from the soil immediately surrounding and beneath its bole, and that distant applications were likely to be wasted. Years ago it was held that the lateral root extension of a tree was equal to the spread of its branches, 


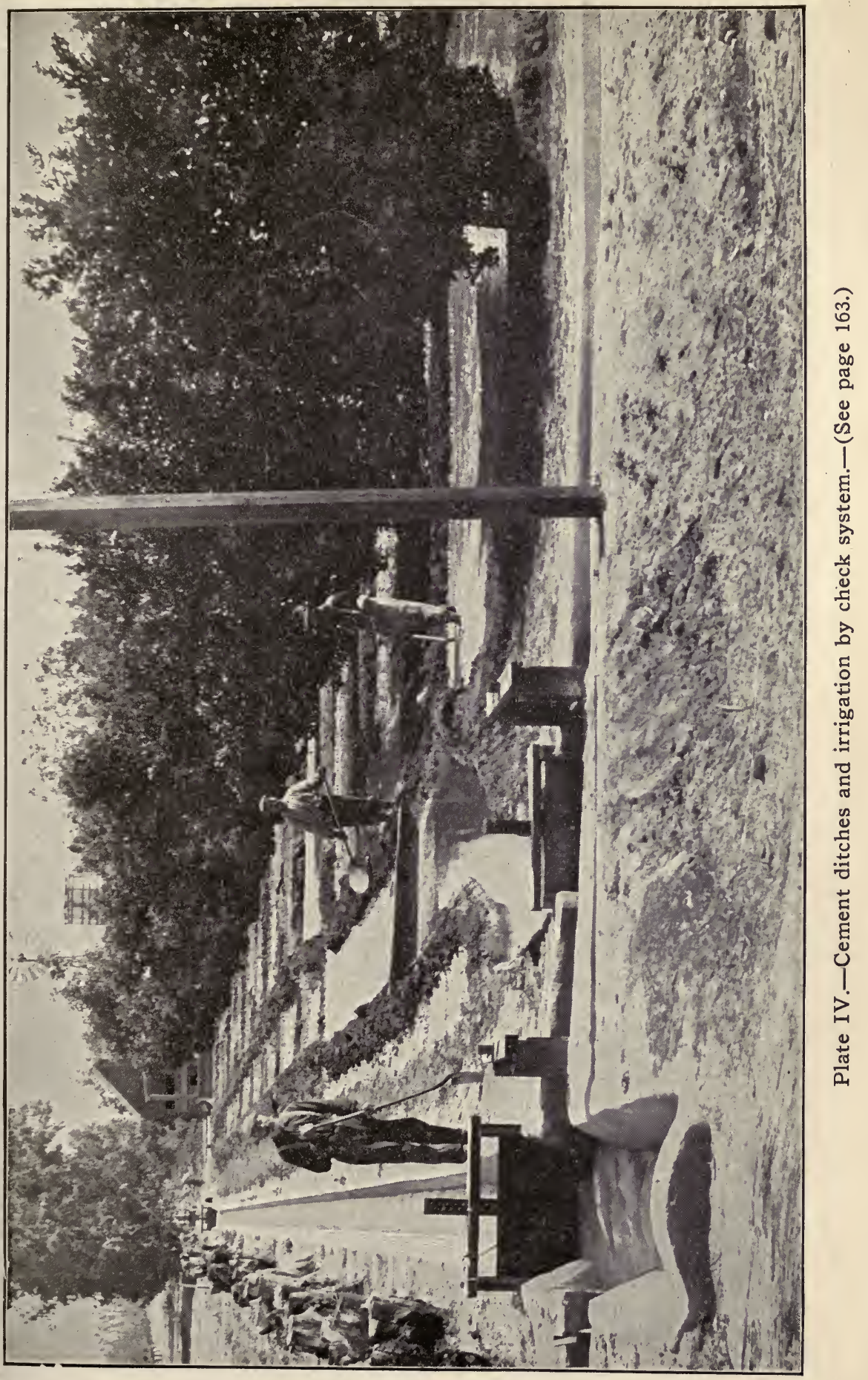



but recent investigations have shown that under favorable soil conditions the root extension is vastly greater. It is not reasonable then to restrict water or other plant food to the region chiefly occupied with the stay roots and not the feeding roots of the tree, and it is a frequent observation that basined trees do not do so well and that they show distress sooner than those under systems which secure more complete water distribution.

To the basin system may, however, be conceded these possibilities: (1) Trees may be grown on hillsides too steep for other means of irrigation unless the hillside be previously terraced; $(2)$ the basins afford an opportunity to use a very small stream of water by allowing it to run for a long time in each basin, thus making a miniature reservoir at the base of each tree; (3) for young trees a small amount of water may sustain growth, while with other methods the same amount of water would be almost wholly lost by evaporation or percolation, or both; (4) the expense of wider application of water and the necessary after-cultivation is obviated.

In planting on hillsides, terracing is the foundation of the basin system. Terraces are plowed and scraped out until they have width enough to accommodate a line of basins and a ditch at the foot of each bank to supply them. The terraces are given a little fall, alternating in direction so that the water, starting from the ridge above, is dropped through a box, or otherwise let down, from the low end of one terrace to the high end of the next, and so on until the stream reaches the bottom of the slope. As a basin is reached it is filled and closed and the water sent along to the next and so on. As these basins are usually small and shallow thev are filled two or three times in succession at each irrigation.

Wherever water can be handled in contour ditches or furrows, terracing should seldom be undertaken for commercial purposes. With slopes which do require terracing, basins on the steeper parts are largely made by hand labor, after plowing to loosen the whole surface, and the operation consists in moving the earth from the upper side of the tree so as to form a circular levee on the lower side, until the tree stands in a level, roundish pan as large as can be made without too much excavation and filling. As the slope becomes less the basins enlarge and reach a diameter, finally, where the sides can be made by turning a small horse or mule around the tree with a plow, the rim being further raised and shaped by hand so as to hold 3 inches or more of water without danger of breaking away.

The basins are filled with a small stream by ditch or hose or pipe line, according to the ground and notion of the irrigator. They are filled at such intervals as the water supply admits or the growth seems to need. The basin bottom is rarely disturbed. The cracking soil is finally given another dose of water to close up its wounds; meantime the frequent surface soaking puddles the soil and the conditions unfavorable to growth arrive sooner or later, according to the disposition of the soil to run together by water settling. Drying and cracking is lessened by filling the basin with manure or rotten straw or other light rubbish, or by a layer of coarse sand on the bottom. As the tree grows the foliage shades the basin and thus reduces evaporation. 
Where the surface is uneven or the soil too leachy to carry water well in a ditch, portable and adjustable carriers are used to advantage. Of these, slip-joint pipes of non-rusting metal or of wood, are most satisfactory and are coming to be largely used.

The Furrow System.-The furrow system is the prevailing method of irrigating fruit lands except with some soils which can be better handled with less water by the check system. The furrow system has, however, a very marked theoretical advantage in the escape from saturating the surface soil, which has to dry out again before it can be cultivated, and it is only with difficulty reduced to fine tilth after such puddling. Another advantage is in saving the water used

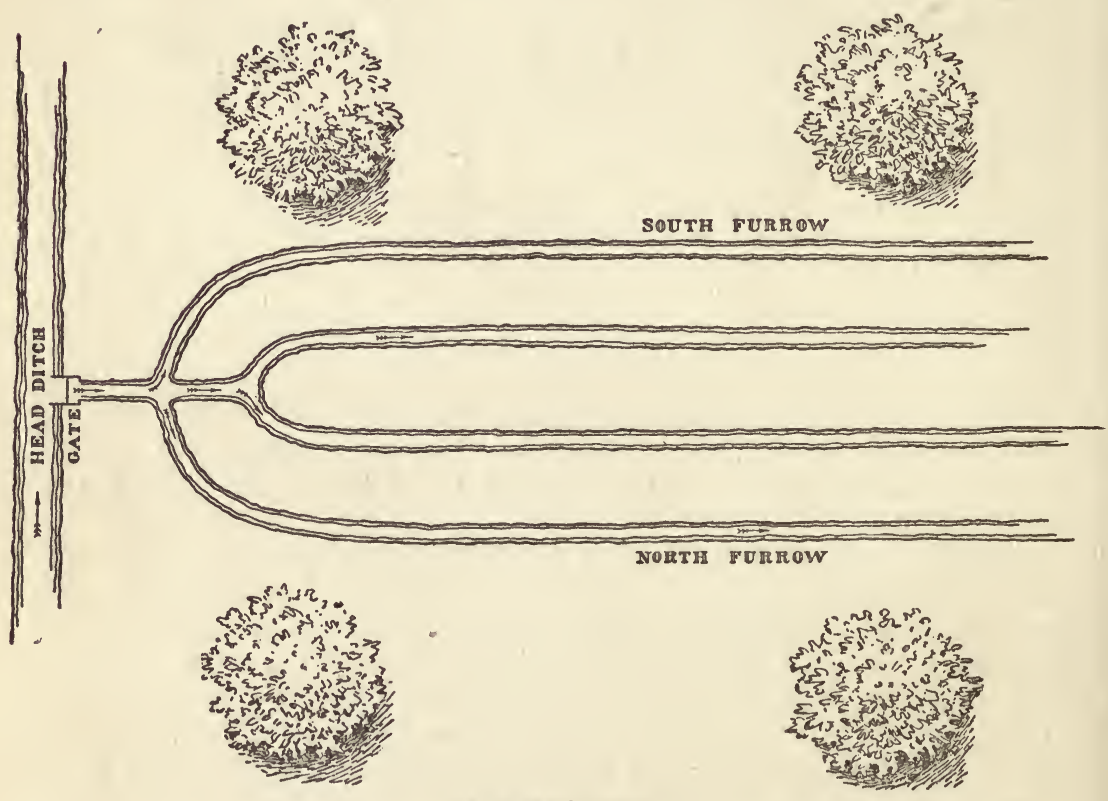

Large furrow.

Large furrow irrigation of orange trees at Palermo, Butte County, Cal.

in moistening soil which has to be dried by evaporation. Other theoretical advantages lie in the even distribution of the water with the least displacement of the soil and the introduction of the water to the subsoil, where deep-rooting plants should derive their chief sustenance. It is becoming quite clear that all these theoretical advantages have not been realized by the furrow system as generally practiced, and a number of modifications are now being introduced which promise their fuller realization. The changes now taking place tend toward reducing the difference between what are known as the "large-furrow" and the "small-furrow" methods, because the improvement lies chiefly in introducing the water more deeply in the soil, as will be shown later, and this is done by using fewer and deeper furrows. 
Irrigating by Large Furrows.-Where one to four furrows are used, these are large furrows, while the small-furrow system uses from five to eight or more between two rows of trees. Large furrows are made with the double-moldboard plow, or with a single plow followed by the "crowder," or by plowing out dead furrows between the rows, etc. Their number depends upon the size of the trees and the fitness of the soil for lateral seepage. They are wide enough and deep enough to carry or hold a large stream of water. This method is used chiefly for winter irrigation on land which is so nearly level that the water will flow slowly into the furrows and stand there until it disappears by percolation. It is also used where one or two summer irrigations is all that is required to carry the trees through. It is obviously adapted only to land of slight and uniform grade. Irrigation by a single furrow cut near to the row of trees is a widely prevalent method with young trees. When the trees are larger, or when inter-cultures are undertaken, the large furrows are multiplied. In this case the water is admitted to the furrows from a board flume. Large furrows are often used in a bearing orchard, the furrows being filled from a lateral ditch, this lateral being parallel to the main ditch. In this case the board dam is used to divert the lateral into one large furrow after another, and when the furrow is filled dirt is thrown in to prevent the reflow of the water into the lateral.

The great variety in large furrow practice is suggested in the foregoing. A systematic manner of proceeding is that of $\mathrm{Mr}$. A. Trost, of Palermo, California, as described by himself :

The soil is red, gravelly clay, the upper 12 inches without rocks; below this the gravel is more rock. At the depth of 3 or 4 feet the red clay changes into a whitish one and water enters it very slowly. My orchard is 12 acres1,120 feet long from north to south and 510 feet from east to west. The northeast corner is the highest. Here the water ditch enters, and I run my head ditch along the east side from north to south. There are 51 rows of trees in that direction, the north and south outside rows being olives. There are 23 orange trees in the row from east to west and 1 olive tree on the west end. All trees are 20 feet apart. I use 24 miner's inches per day for 5 days in the following manner: I use 4 furrows about 5 or 6 inches deep and about 3 feet apart between rows, leaving the rows nearest the trees from 5 to 6 feet from the trunks. The 4 lower rows on the west side I cross furrow with 2 furrows between the trees. I divide the 24 inches into 51 equal streamlets by using one gate for each 4 rows. First turn this amount into the furrow south nearest to tree. When the water has moved to the olive tree, I divide the water between the 4 furrows for the lower 6 trees and through the cross furrows. The next morning I divide the water at the tenth tree for the 4 furrows. On the third day I let only one-half the water go down in the furrow south of tree, the other in the one north nearest to tree. On the fourth day I turn part of it in the middle furrows near the head ditch, and by the fifth day I have my place equally wet from one end to the other, taking care that the top soil near the trunks of trees remains dry on the surface. I keep the soil around the trunks of the trees about 2 inches higher for a width of 3 feet. In this way I use all the water without running any off, and lose only the evaporation. The whole amount of water used is 120 inches, equal to 10 inches or 130,000 gallons per acre, or 4.5 acre-inches, or 1,200 gallons per tree.

I irrigate about every four weeks, running the water five days and turning it on again three weeks after it is taken off. I have irrigated as early as the 1 st of April and as late as the middle of October, depending on late rains in spring and early rains in fall; usually from five to six irrigations per year. After four or five days I cultivate 14 feet wide between the trees from 6 to 8 inches 
deep; for this I use a 7-foot cultivator and four horses. Near the trunk of the tree I work about two inches deep and a little farther away 4 inches deep, using the three-cornered orchard plow with a cultivator 4 feet wide and two horses.

Irrigation by Large Furrows Without Summer Cultivation.-An exception to the continuous cultivation of orchard ground which is prevalent in the irrigated regions of the Pacific Coast is found in the foothills of the Sierra Nevada in California, where furrows are made at the beginning of each irrigating season and used continuously during that summer. The ensuing winter plowing and early spring cultivation are relied upon to keep the soil in good condition. Although this constitutes an exception and the practice is widely followed for what seems to the growers of the region to be a good and sufficient reason, it does not militate against the truth of the continuous summer cultivation policy which elsewhere prevails, nor does it follow that this policy would not be better in some respects even in the region where it is abandoned. It is a district of very large water supply, and the arrangements of the water company are such that the grower must pay for a certain number of inches of water by the year, and is entitled to this amount of continuous flow. He has to use it or neglect it as it flows, and can not get more at one time by not using it at another. For this reason he has not the motive for close observation which prevails under other conditions, and to escape the cost of summer cultivation and fresh furrowing out he has recourse to frequent flows in the old furrows. The following interesting account of the prevailing method was prepared by Mr. W. R. Fountain, of Newcastle:

Water is supplied almost exclusively by one company, which has met requirements up to date, and seems fixed to supply in excess of demand. It is supplied by the miner's inch; price $\$ 45$ per inch per season for a constant supply. The inch is measured under 6-inch pressure.

Beginning May 1st, five months is called the irrigating season, but the purchaser can have the water twelve months per annum if he wants it. The water company collects monthly. The purchaser cannot start the season with little and increase at pleasure, except unon payment for the full season on the basis of the largest amount used at any time.

With this constant supply we use it constantly, piping to high points and moving it from place to place. When no fruit is ripening it is attempted to water a block of trees in twenty-four hours. The water is not checked back, but is run in ditches, mostly in one, but occasionally in two, along each row of trees or vines. When a variety of fruit is ripening more water is given the trees, while after a variety is picked and before any other is nearly ripe the effort is made to water each tree every ten or twelve days. Level land and low spots stand a good chance, as a rule, to get too much water, and a larger stream is used per row to force the water through quickly. Then it is taken off in a shorter time than it would be where the trees are on a side-hill and have a good drainage.

About 1 inch for each 8 acres is generally used. This is for deciduous fruits. The citrus fruits and berries require watering about once a week; if there is good drainage they would prosper if watered every three days. In such ground I have not heard of their getting either too much water or too much fertilizer. The general practice is to plow, cross plow, and then after each rain cultivate, with no cultivation whatever after beginning the use of water. I think an occasional cultivation after watering would help.

There is a tendency for the ditches to become packed after water has been flowing through them for some time, in which case but little water soaks into the ground. When this occurs I dig a pot hole in the ditch to allow the water to 
soak in, or else loosen the ground about the trees with a spade and carry the ditch through this loosened ground. I block out my ditches so that I can get my stream through the last tree in about sixteen hours. Where the water has not reached the end of some of the ditches, I turn the water into it from a stream that is flush, and by keeping a man with a hoe constantly with the water, I manage to get it over the field at about $4 \mathrm{p} . \mathrm{m}$. I wet about 350 trees in a block on hillsides; on a flat I wet less, using more water in each stream, and changing it about every twelve hours instead of every twenty-four hours. My trees grow about 130 to an acre.

Systematic Distribution of Water on Hillsides.-The Common method of carrying water in pipes to the various high points of several slopes or "irrigated faces" from which it can be admitted to large furrows crossing or descending those faces is open to some difficulties and disarrangements. P. W. Butler, of Penryn, has had in successful operation for several years a system of zigzag ditches for carrying and distributing and for catching outflow and redistributing on a lower face. This is also a system which makes ditches and furrows but once a year, and dispenses with summer cultivation. Mr. Butler's account, as illustrated by the accompanying diagram, is as follows:

The amount of water generally used in this section for the irrigation of deciduous fruit trees is 1 inch to 5 acres of orchard (miner's inch under 6-inch pressure), and is applied to each row of trees by one stream of water of sufficient quantity to just reach the end of the row. Much of the water is thus wasted because of inability to properly adjust its distribution. It is usually run twenty-four hours, then changed to other parts of the orchard until the whole is covered, which takes about three weeks' time, when the process is repeated, continuing throughout the summer, or from May 1 until October 1. There is no cultivation in the meantime, and at each irrigation the water is run in the same ditches. This system is followed in nearly all the orchards of Penryn and vicinity, some on quite steep hillsides, which suffer when the water is thus applied. I have never liked this method, and for many years have used a different system in irrigating all orchards over which I have had control. In my home orchard I have a reservoir on the highest land, from which water can be conveyed as desired to every part. My ditches are run on a grade with a fall from 2 to 3 inches to the rod and from 5 to 8 feet apart. At each irrigation the water is run about thirty-six hours before changing. The round of the orchard is made in ten to fourteen days. None of my small ditches exceeds 400 feet in length. When I begin to irrigate a section I turn on from the reservoir water sufficient to cover the section in a few hours, then lessen it until it just reaches the end of each row, but see that it reaches the end of each row if a little surplus passes over. This surplus I take up in a main ditch, to be again used on lower ground. This is continued until the lowest part of the orchard is reached, and very little water is ever wasted. By running on a grade that is so nearly level the water is applied uniformly, even on the driest parts of the hill slopes. I run the main distributing ditches in a zigzag manner, taking water from these ditches to cover the lower sections. I formerly used pipes to lead the water down the steepest grades, but this system I have abandoned and now use open zigzag ditches for mains. From the main zigzag ditches I do not take the water at the turning point, as there is more liability of breakage than if taken when running straight, or at whatever point is necessary to keep the distributing ditches on an average of 8 feet apart. The length of the zigzag ditches varies according to the slone of the hillside. When steep, the ditch, before turning, must be of greater length than where the ground is more level. (See diagram.) I use no gates, but bush the openings with coarse swale hay. I also bush the turning points of ditches as they are in permanent use throughout the season, and after the first few days' use require but little care to keep them in order. These 
ditches are torn up during the season of cultivation and have to be renewed every year.

I use a level set on a frame 8.25 feet long and about 2.5 feet high (one leg longer than the other) to make any grade desired. Then I drag its length on the ground after getting the level, and can mark the line of ditch nearly half as fast as a man can walk.

During the last ten years I have used many thousand feet of pipel in irrigating, but have found it too expensive to be practicable, and it frequently gets clogged, causing much trouble. 'The zigzag method of taking the water down hills on the dry ridges, distributing to right and left, picking it up again in zigzag ditches at the end of the rows or system, to be used again on lower ground, brings into use the largest quantity where it is most needed and utilizes it all without waste.

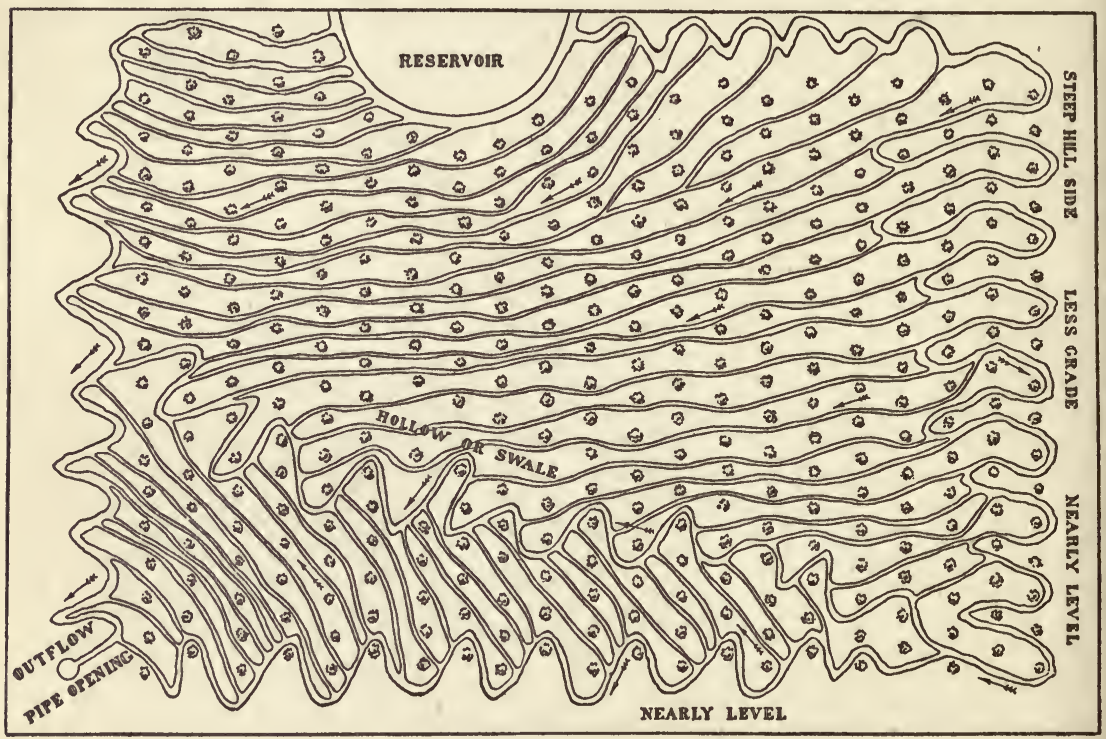

Zigzag ditches.

Large furrow system on hillsides with zigzag ditches for distribution, catchment, and redistribution.

Irrigating by Small Furrows.-It has already been suggested that recently the small furrow method of irrigation is undergoing certain modifications. The occasion for the change is that in certain of the heavier soils, particularly, the use of water in many shallow furrows followed by cultivation results in the formation of a compact layer, and this prevents the percolation of the water into the subsoil. This discovery led many Southern growers to resort to fewer and deeper furrows, and to new devices to enable the tree to get the benefit of the water. There has been wide use of the subsoil plow, with a wedgeshaped foot attached to a slim standard rising to the ordinary beam. The standard opposes its thin edge to the soil so as to cleave it with the least difficulty, and the foot, passing through or beneath the hardpan, lifts and breaks it. The result of the subsoiling is to open a way 
for the water to sink and spread below the hardpan. It is usual to run this plow once through the center of the interspace between the rows of trees, sometimes at right angles to the irrigation furrows. When this is done the water is admitted to the furrows as usual, but instead of flowing along smoothly it drops into the track of the subsoiler and runs there a long time before rising again to continue its course down the furrow. It is the experience of some growers that the water has taken five or six days to reach the lower end of the furrows, a distance which would have been covered in twenty-four hours if the subsoiler had not intervened. This has been shown to result in much water for the subsoil and a notable invigoration of trees which had been famishing, although shallow-furrow irrigation had proceeded regularly.

Recent changes in the furrow method at Riverside, California, are described by Mr. J. H. Reed as follows:

The handling of the water in the orchard has materially changed in recent years. Instead of flooding up, basining, or using shallow furrows, deep furrows, from 3 to 5 feet apart, are most generally used. In heavy adobe soils more furrows are used than in the more porous granite soils. The most usual length of furrows is 40 rods. Every precaution is taken to have, the surface wetted as little as possible.

The amount of water run at a time is materially lessened. Formerly the common practice was to run 3 inches per acre for twenty-four hours each thirty days. Now, 2 inches continuous run for seventy-two hours is found to serve a much better purpose, except on loose soils. The general practice in the valley is to irrigate once each thirty days. A few of the most careful orchardists had found that by intelligent and thorough manipulation of the soil they obtained as favorable results from the application of water every sixty days or more, using the same amount as they formerly did at intervals of half that time. The writer has watched with much interest an eight-year-old orchard that during the three years preceding the present received in all but ten irrigations, the usual amount of water being used only at each four irrigations the first year and three irrigations each the second and third years, with results comparing favorably with those on trees of the same age on the same soil in neighboring orchards that received the ordinary thirty-day irrigations. While there are few orchardists who have the skill and patience required to secure such results, they show the possibilities of improved cultivation in conserving moisture. So long as water is abundant and not expensive, more frequent irrigations will probably be generally practiced; but the advantage of running the water for a longer time, in furrows as deep as possible, covering the saturated bottoms as soon as practicable and keeping the surface perfectly pulverized and in loose condition, is being generally recognized.

The usual practice is now to have six deep furrows in 20 -foot spaces. The number varies according to the character of the soil, but is in any case less than in the small, shallow furrow system which formerly prevailed.

The recourse to deeper furrows and to the subsoil plowing has been made in several citrus fruit districts of Southern California. Its success depends upon conditions. There are cases in which too deep use of the subsoiler has admitted the water at a point too low for best results to the tree which grows on a leachy subsoil, and the cutting of roots by the subsoiler has in some cases brought shallow-rooting trees into temporary distress. The general conclusion, however, is that deeper introduction of water favors deeper rooting and is very economical of water by preventing the loss by evaporation from the surface, 
which, theoretically, is dry, but which actually, with shallow furrows over an irrigation hardpan, becomes too often saturated over nearly the whole space between the trees.

Cement Pipes and Flumes for the Furrow System.-The use of cement in the construction of flumes, pipes and outlets for distribution has advanced so rapidly during recent years that in nearly all districts local cement works can be found, at which irrigation structures and appliances of latest design can be studied. Other devices are first described and afterwards regularly advertised in our horticultural journals. Analysis of them is beyond the scope of this treatise, but they are none the less important and all who contemplate laying out irrigation systems, both large and small, should consider them carefully. It is the function of this work to deal chiefly with home-made appliances.

The Board Flume and the Furrow System.-Although in the older regions the cement flume is advancing in popularity, important service will always be rendered by the home-made board flume where suitable lumber is cheap. A detailed account of its construction and operation will be widely useful. The following is contributed by Mr. A. S. Bradford, of Orange county:

I consider the board flume best because it is in many places cheapest and because it will last fifteen or twenty years in California if made of good soft redwood. The common redwood lumber is generally so, but the so-called flume lumber is hard, generally, and will warp the flume out of shape. Even in the common redwood lumber hard pieces will be found, and these should be avoided. My first flume has been in use nine years and is apparently as good as ever.

The first thing to be considered is getting a flume put in properly, as this alone will cause much trouble if not done right. A flume should run nearly on a level. It should be placed about two-thirds in the ground at the commencement, and as soon as it comes out of the ground to about two-thirds of its height, there should be a drop made of 1,2, or 3 inches, if necessary, and then carried along as before, so as to keep the entire length of flume practically on a level.

Sixteen-foot lumber is better than longer, as it is lighter to handle. I prefer 8 -inch sides with 18 -inch bottom, or, in some cases, 10 -inch sides with 16 -inch bottom. The first section, however, should be about 2 feet wide, narrowed to the size of the flume, so as to control the stream. Collars should bei put around the flume everv 8 feet of distance; that is, one in the center and one to cover the joints at each end. These collars should be 2 by 3 inch stuff on th bottom and sides and 1 by three inches on top. This makes a strong, durable flume. The length of the flume should be divided, so that the stream will decrease as it goes along. The width should be decreased also, say from 16 inches to 14, 12, 10, and 8 inches, the sides being the same throughout or reduced so as to have 10 -inch sides on the 16-inch bottom and 8-inch sides on the rest, nailed to the side of the bottom, making 7 inches depth inside. Two-inch holes should be about 30 inches apart and 2-inch gates placed on the inside instead of outside, as they will collect less trash, the hole through the wood, if uncovered, making a lodgment for leaves, etc. In the narrow and flat flume it is much easier to fix the gates.

From 8 to 9 furrows for trees set 24 feet apart is sufficient. The streams should be run from one-eighth to one-half the capacity of the holes in the flume, according to the soil and fall of ground. I commence the stream small and increase it if necessary later on. 'The streams should be kept as near together as possible, and when the end is reached the gate should be nearly closed down, so as to allow the stream to just trickle to the end. In this manner the soil will become thoroughly wet from one end to the other. The streams should be run very slowly on most of our soils. A great many failures have been made on 
hard soils by running the stream too large and then reducing it. This seems to "slick" or cement the soil so that it will not take the water, and the consequence is a poor and unsatisfactory irrigation. On the other hand, if the streams are started small and allowed to soak the ground as they go along, it is simply astonishing how much water can be put in the ground. On sandy soils the streams should be larger. A little practice would give any one the desired information.

About three rows of trees at the lower end should be blocked up, provided one has no place where the overflow water could be used. This last provision is the better, however, as there would be only about 10 inches of water run over the last three or four hours, and a thorough job would be done from one end to the other.

I making furrows I have an extension made for my cultivator to bolt on each side and use four plows. With this extension I can wet the whole ground thoroughly. The furrows will extend under the limbs of the trees, and by making a slight curve around each tree the ground will become wet in the rows as well as between.

As compared with the check system, the furrow method, properly handled, makes the soil light and loose, while the check system is apt to pack the soil, rendering it lifeless and leaving it so thati it will not retain moisture long. $\mathrm{Be}-$ sides, the cost of ridging and extra labor in handling water in checks for one season will nearly pay for the flume, by which one man can do the irrigating. Two horses will furrow out 10 acres in half a day, and a little hand labor at the flume will connect the furrows. In the check system generally a disk is run first where the ridges are to be made, and then the ridger is run with four horses; then jump scraper is run to stop up one side of the blocks; then ditches must be made; then from 2 to 3 men are required to handle the water by shutting up the checks when Ifilled. Afterwards the ridges must be plowed down before the ground can be harrowed and got in condition to cultivate. At a glance one can see that it costs fully three times'as much to irrigate by the check system as by the furrow system, and with the latter the soil acts more as it does after a rain.

\section{OVERHEAD IRRIGATION}

Although Californians have always been strongly impelled by the desire to get irrigation water away from contact with the air and into the soil as directly as possible, to escape losses by evaporation and to maintain a loose soil-surface, sprinkling methods have recently become matters of considerable expenditure. Such systems are in operation in the orange orchards of Robert Baird of Porterville and R. D. Williams of Exeter. The former has overhead perforated pipes supported on redwood posts; the latter has underground pipes with a stand-pipe rising through the center of each fourth tree and surmounted with a revolving sprinkler. The cost of installation in each case is about $\$ 150$ per acre.* The desirability of such sprinkling methods is still to be demonstrated. In both cases the water is forced into the pipes by pumps.

\section{DEVELOPMENT AND STORAGE OF WATER}

It is, obviously, beyond the limitations of this work to attempt an extended review of irrigation enterprises and practices. The enterprises undertaken by capitalists, or by co-operation among settlers,

*Details are given in the Pacific Rural Press, July 13, 1912. 
require the services of competent engineers. All these matters are too great in extent and variety to be discussed in this work. As, however, it has been the aim of the writer to aid the inexperienced planter to help himself in small efforts, a little space will be given to suggestions as to how a planter may develop and use such small water supply as may be derived from spring, small creek or well, on his own land without employing an engineer.

Running Lines for Irrigating Ditches.-How far to go up a creek in order to bring water out upon a given piece of land is a question which frequently arises in individual practice. There is also doubt as to how much fall should be given to the ditch. The fall required by a ditch or canal depends upon the amount of water which it is desired that it shall discharge, and upon the width and depth with which it is intended that the water should flow. It may also be dependent upon the character of the soil in which the ditch is to be constructed, and upon the peculiarities of the water itself. A strong current in soft soil may cause mischievous erosions. Water carrying much sediment must never be allowed to move sluggishly, as clear water sometimes may. It is best to state the requirements to a competent engineer and act on his suggestion, or secure the counsel of a neighbor who has had experience with similar soil and water.

Having decided what fall to give the ditch, the nearest point of which water can be taken out of the creek to be brought to a certain piece of land is found by commencing with the point at which the water is to be delivered (generally the highest point of the land to be irrigated), and running up stream a line which has the inclination intended for the ditch.

To stake out this line when no special hindrances are in the way, use a home-made leveling instrument constructed as follows:

With sound, straight-edged lumber a triangle is made, as indicated in the sketch. The three pieces, $A B, 6$ feet long, $B C, 12$ feet long, and $C A, 4$ feet long, are made fast to each other at $A B$, and $C$. The board, $A D$, is fastened to the triangle at right angles to $B C$. Near $A$, on the board, $A D$, a plumb-line is made fast. The plumb, like a mason's plumb, hangs in a hole at $F$, so that when $A D$ is vertical, the string hangs very near the surface of the board, $A D$.

It will be seen that when $A D$ is exactly vertical, $B C$ is exactly horizontal, if the angles at $D$ are true right angles. An ordinary carpenter's square used in the construction of the apparatus will insure sufficient accuracy in the position of $A D$.

In marking on the board, $A D$, however, the line in which the string of the plumb will hang when $B C$ is exactly horizontal, more care is required. Two pegs are driven, as far apart as $B$ and $C$, for these points to rest on. The highest one is driven into the ground until the plumb-line follows about the center line of the board, $A D$. Having marked this nosition of the plumb-line, the triangle is reversed so that the end $B$ rests on the peg, where before we had the end $C$, and vice versa. Should the plumb-line be in a position at variance with the first one marked on the board, then the correct position for the $B C$ horizontal will be exactly in the middle between the two found by the aid of the two pegs.

It will frequently be found convenient to have a scale of feet marked off on $B C$. Holes in the pieces $A B$ and $C A$ at $E E$, or handles, will make the triangle convenient to carry. Only two men are necessary in using it. 
To use this instrument for locating the line of the ditch, calculate the amount which your line should rise between each two pegs. Drive a peg at the starting point with its top say six inches from the general surface of the ground. Hold one end of the leveling apparatus above this peg by exactly that amount which the line arises per each instrument length ( $\mathrm{B} C$ ), and swing the other end around into the direction from which the ditch is to come, until, when level, it is just six inches above the ground. Drive a peg here, which will, like the first, be six inches high, and proceed as before. Care should be taken to give the top of each peg exactly the correct elevation. The level must be horizontal when resting on any peg, and raised exactly that amount which the line rises per level length, above the preceding peg. It will

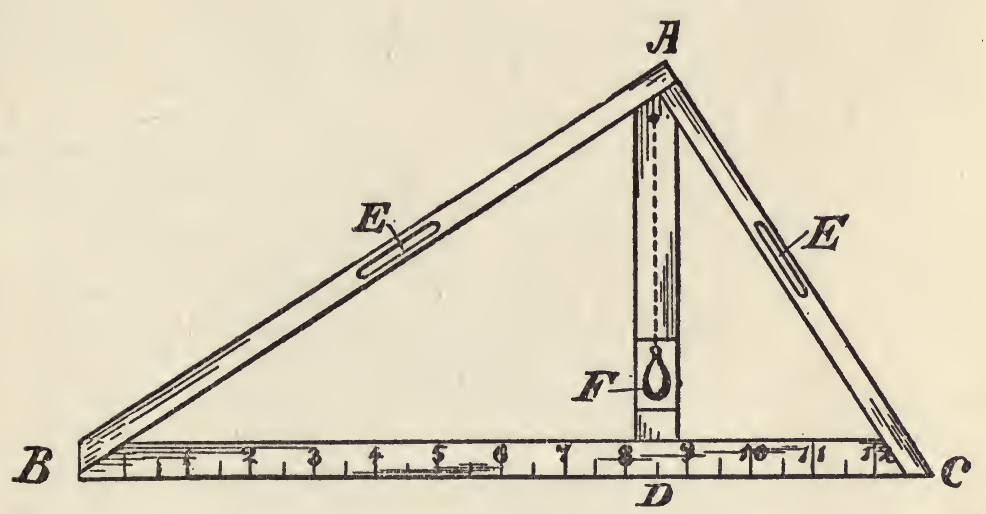

A home-made leveling instrument.

be found convenient to use a carefully prepared block to hold on the top of each stake at the rear end of the level instead of trusting to measurement each time.

Locating Contour Lines for Checks or for Distributing Ditches. -This work can be done with the aid of the level above described. For instance, to locate a contour (a line of equal elevation), as required in the construction of a check levee, drive a peg until its top has a convenient elevation from the ground, say one foot. Rest one end of the triangle on this peg and swing the other around until, when $B C$ is horizontal, this other end has exactly the same elevation from the ground as the top of the peg. At this point drive a second peg and proceed as before. If the tops of the pegs be chosen as the height of the levee, they may be retained as grade stakes as well as line stakes for the embankment.

Storing Water from Small Sources.-For individual uses quite a respectable water supply can sometimes be developed from apparently 
mean sources. This can be done by clearing out and opening up hillside springs, and often by tunneling into the hillside to intercept subterranean water-flows, or by pumping from a well. Even a small spring, yielding but two quarts per. second, would be sufficient to irrigate several acres in fruit trees. To derive the greatest benefit from small springs, however, a reservoir is necessary, in which the flow of twelve to twenty-four hours, or even a longer period, can be accumulated, and then discharged as required. It is by using water in driblets that many springs are wasted. A spring supplying even one and a half inches of water would be wholly swallowed up by a thirsty soil within two hundred feet of its source, when, by arresting the flow and accumulating it in a reservoir and discharging at intervals in a volume four times as large, it would more than cover eight times the surface. A spring flowing two quarts per second will discharge forty-three thousand two hundred gallons in twenty-four hours. This would require a reservoir forty by twenty feet, and seven feet deep, or double that width if the depth is decreased one-half. The shallower it can be made the better, for many reasons, but especially on account of the temperature of the water. That of springs is generally too low in summer for immediate use, and its value is greatly enchanced by being raised to an equal or greater temperature than that of the air. This is quickly done by exposure in a shallow pond. A reservoir can be constructed entirely in the ground where the slope will admit of it, and by lining the bottom and sides with clay well puddled, will answer for most purposes. Some are built of adobe, backed with earth and plastered on the inner side with hydraulic cement. Concrete of lime, sand, and broken stone, is however, the best material, where lime can be readily obtained, and any person with ordinary mechanical skill can construct them. The following hints on a dirt reservoir may be suggestive:

A reservoir should be built on the highest part of the tract sought to be irrigated by scraping the earth from the outside and from such a large area as not to affect the utility of the land from which it is taken. With a levee all around 5 feet high, 5 feet of water could be carried safely. The slopes ought to be two to one on the inside. A reservoir 20 feet square and 4 feet deep would hold 12,000 gallons. With the slopes as above the reservoir should be measured two feet from the bottom, or half way up the 4 feet of water; consequently to lay out a reservoir to hold 12,000 gallons, "put the stakes 12 feet square and build. For any other size one take 8 feet off the same as this: A reservoir 25 feet square will hold 18,750 gallons and would be 17 feet square at the bottom; one 30 feet square would hold 27,000 gallons and would be 22 feet at the bottom; one 35 feet square-27 at the bottom-will hold 36,000 'gallons; one 40 feet square-32 on the bottom-will hold 48,000 gallons. This spread upon the surface of an acre would be a little more than $13 / 4$ inches 'of rainfall.

Almost any loam or soil will hold water with a little puddling. The cheapest way to puddle is to build a pen the size of the intended reservoir, including at least a portion of that to be under the embankment, wet it very wet, put some hogs in the pen and keep feeding them barley, a little at a time, so as to make them not only walk around, but root for the barley. A half sack of barley fed to eight or ten hungry hogs in half a day will make a good puddle. If it did not work satisfactorily, the water could be taken off and the bottom covered about an inch deep with coarse sand mixed one part to five with Portland cement, put in dry, and let it be covered slowly. A barrel of cement may be counted at about 4 cubic feet and 'with the mixture above would cover the first-named 
reservoir about $13 / 4$ inches. This would make it tight. The supply pipe should come up from the bottom, so that the lift would never be more than the height of the surface.

Loss of Water by Seepage.-The great loss of Water by seepage during a long run has led to the cementing of ditches, and to the use of miles of large wooden, concrete and iron pipe by the irrigation companies of Southern California; also, where the slope is rapid, paving ditches with rock has been resorted to. Similar efforts naturally suggest themselves to the user of a small water supply to save his flow from loss. The lining of ditches to prevent seepage is being tested by the California Experiment Station at Berkeley, and publication of results is being made.* Where lumber is cheap the use of a board flume is an available means of saving water, when the soil is coarse and leachy.

Irrigation from Flowing Wells.-A considerable area of orchard is irrigated from flowing wells in different parts of the State. Nearly

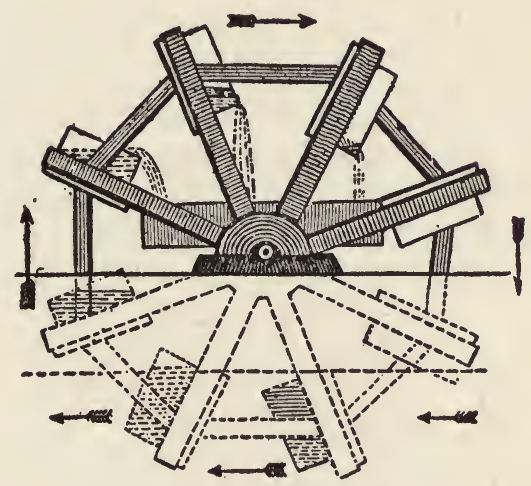

End view of irrigating wheel.

everywhere in the artesian districts there are local well-borers who have kept records of the strata traversed in their work, and can estimate closely the cost of securing water by this method.

Lifting Water from Flowing Ditch or Stream.-Where a stream has a rapidity of two miles or more per hour, and a lift to a height of six to sixteen feet will give head enough to distance the water over a considerable area, there is nothing cheaper than the current wheel which is largely used in this State. The engraving gives an end view of such a wheel. Eight pairs of arms, carrying flat buckets like those of a steamboat paddle-wheel, extend from a hub rotating on metal bearings. At either end, or both ends, of each bucket are fixed wooden or tin water boxes which fill themselves on entering the water, and on being brought to the highest point of rotation empty themselves into a receiving trough. This trough supplies the distributing ditches,

${ }^{*}$ Bulletin 188 and Circular 114, University of California Experiment Station, Berkeley. 
etc., and its inner end is so placed that it comes under the projecting buckets of the wheel without interference with the motion of the arms. The current of water in the channel underneath forces the buckets down stream, the latter delivering in the opposite direction at the top. By using a double set of boxes, one at each end of each bucket, the water may be delivered on both sides simultaneously. A little experimenting will indicate the proper size boxes, which depends upon the velocity and volume of water in the channel, as well as to the amount to be delivered.

At the Fancher Creek Nursery, in Fresno county, a wheel is used eighteen feet in diameter, and carries sixteen buckets, which empty into a trough sixteen feet above the ditch. The wheel lifts about one cubic foot in two seconds.

\section{PUMPING FOR IRRIGATION}

The use of pumps for irrigation is continually increasing. The capacity of pumps, their ease and cheapness of operation in this land of oil wells and of ponderous waterfalls whose power can be transformed into electric energy, warrant the conclusion that in many places water can be lifted from below more cheaply than it can be brought long distances by ditch; and that the supply is more constant and subject to the users' command and convenience. In all parts of the State well-boring and digging and pump construction have advanced very rapidly. Pumping plants of all capacities, from the greatest of the gasoline class, lifting five thousand gallons per minute from a depth of twenty-five feet, down to the plant with a throw of three hundred gallons per minute, all styles of motors and pumps are being constantly multiplied. These plants are being placed upon wells in the orchard or in the vicinity, or upon adjacent streams or ponds. Many new designs by California inventors are coming into use. It would require a volume to contain any adequate account California's recent progress in these lines. Economic pumping is governed by so many considerations that no general statement would be conclusive in any specific case. Each orchardist must ascertain his own conditions and then confer with trustworthy manufacturers or their agents as to what will meet his requirements.*

\section{WATER MEASUREMENT}

The Miner's Inch.-Although the miner's inch, as commonly measured, is open to objection because of inaccuracy, from an engineer's point of view, it is so easily applied that it must remain a popular recourse. It consists in causing the water to flow through an opening,

*Full details of the cost and flow from pumps drawing from various depths and operated by various motors are given in the publications of the irrigation investigations to which reference has previously been made. Also, Circular 117, California Experiment Station: "The Selection and Cost of a Small Pumping Plant," by B. A. Etcheverry. 
the capacity of which is known, and which is readily capable of adjustment to the flow in any case. A simple form of this device and its use is shown in the illustration, which represents a board 1 inch thick, 12 inches wide, and about 8 feet long. The opening is 1 inch wide and 50 inches long, and the distance from the top of the board to the center of the opening is exactly 4 inches on the up-stream side. On the down-stream side the opening is beveled so that the hole presents sharp edges to the stream. A sliding board is hung upon the top of the first board, with a strip screwed along its upper edge, this sliding board being wide enough to cover the opening on the up-stream side. In the slot there is a closely-fitting block, made to slide on the beveled edges and fastened by a screw to the sliding board. It is obvious,

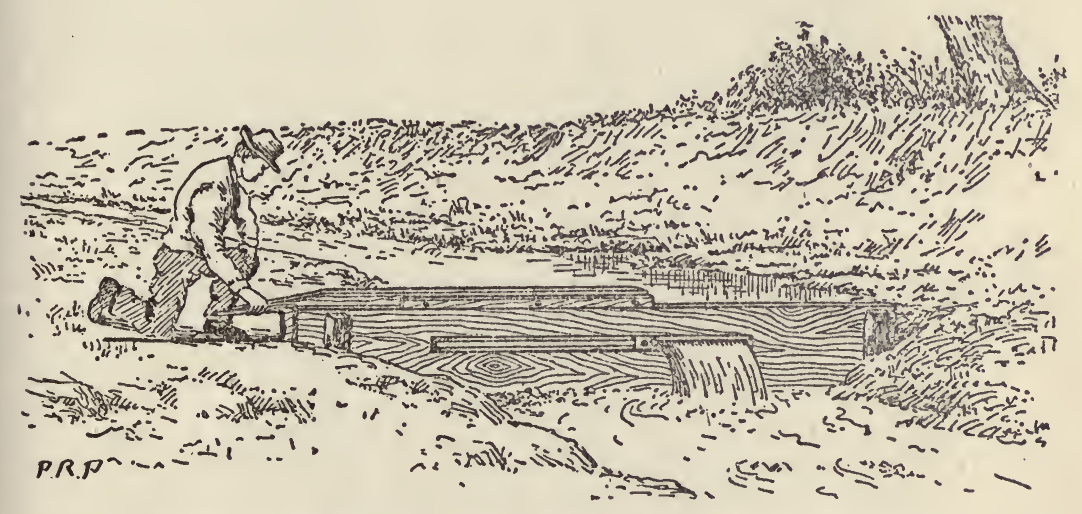

Measuring miner's inches in a small stream or ditch.

then, that when the sliding board is moved backward or forward, by means of its end, which is extended for a handle, the block moves in the slot and determines the length of the opening.

In operation the board is placed in the stream as shown in the figure, so as to dam the flow completely, and the sliding board is moved backward and forward until the water is all passing through the slot, the water being kept up to the top of the board, or 4 inches above the center of the opening. The length of the opening measures the number of miner's inches of water flowing through. If the flow is too great to pass through the opening 1 inch wide, the opening may be made wider, the water still to be kept 4 inches above the center of the opening. The laws of several States provide that in devices for measuring water for sale by the miner's inch the opening shall be 6 inches high and shall be provided with a slide as shown in the picture. The number of miner's inches then discharged is equal to the number of square inches in the opening. The assumption made that the discharge is proportional to the size of the opening is not true, but the 
error in measuring small quantities is not great enough to be taken into consideration. By converting the results of measurements in miner's inches to gallons, cubic feet, or some other familiar unit, it may be determined how long it will take the stream to fill a reservoir or cover a given field with the necessary depth of water. This unit is readily convertible into cubic feet or gallons or acre-inches of water, according to the time the water flows.

The following data will be helpful in computations: One miner's inch, as described above, equals 0.1496 gallons per second, 8.976 gallons per minute, 538.56 gallons per hour, $12,925.44$ gallons per day; 0.02 cubic foot per second, 1.2 cubic feet per minute, 72 cubic feet per hour. One acre-inch of water (that is, 1 inch in depth over an acre of surface) equals 27,152 gallons, or 3,630 cubic feet, and 1 miner's inch will supply this quantity in about 50.4 hours. Thus a simple calculation shows that a little stream of 5 miner's inches will supply enough water to cover an acre 2.3 inches deep in about 23 hours-a fair amount for one irrigation of soil of average character if it has not been allowed to become too dry before the application. In fact this is an average amount actually used for an irrigation of shallow-rooted plants like most field and garden crops.

Weir Measurement.-The term "weir" is not always understood by those who use it. The term can properly be used only for structures designed to allow the water to flow over the crest with a considerable fall on the down stream side. There are a large number of forms of weirs, taking their names from the shape of the weir notch, or the form of crest. The triangular weir has a V-shaped notch. The rectangular weir has a horizontal crest with vertical sides. Both of these forms of weir are good, when used by the expert irrigator or engineer who understands the principles and factors which enter into their calculations. In order to avoid the variable factors which enter into the calculations for the flow of water over weirs, Cipoletti invented the form of weir which has taken his name and which is in general use throughout the irrigated sections of the world.

The Cipoletti Weir.-The Cipoletti Weir has a thin horizontal crest, the sides of the weir notch sloping back from the vertical at an angle equal to one inch in horizontal for every four inches in vertical. This, for each additional inch in depth the weir notch widens on each side one-fourth of an inch, or a total addition of width of one-half inch. A weir having a length of crest of one foot, and designed to be eight inches in depth, will have a top width of notch of 16 inches.

When the weir box is placed, care should be taken to have the bottom of the notch or crest, level. An ordinary carpenter's spirit level may be used for this purpose. When the crest is horizontal, one end of the spirit level is placed on the center of the crest, and when level the other end will mark the point for the zero of the weir gauge. In rough work a nail may be driven part way into the side of the box, the top of the nail being level with the crest of the weir. A thin plate of brass is to be preferred to a nail. In other cases gauges are inserted on the sides of the flumes and properly marked in tenths 


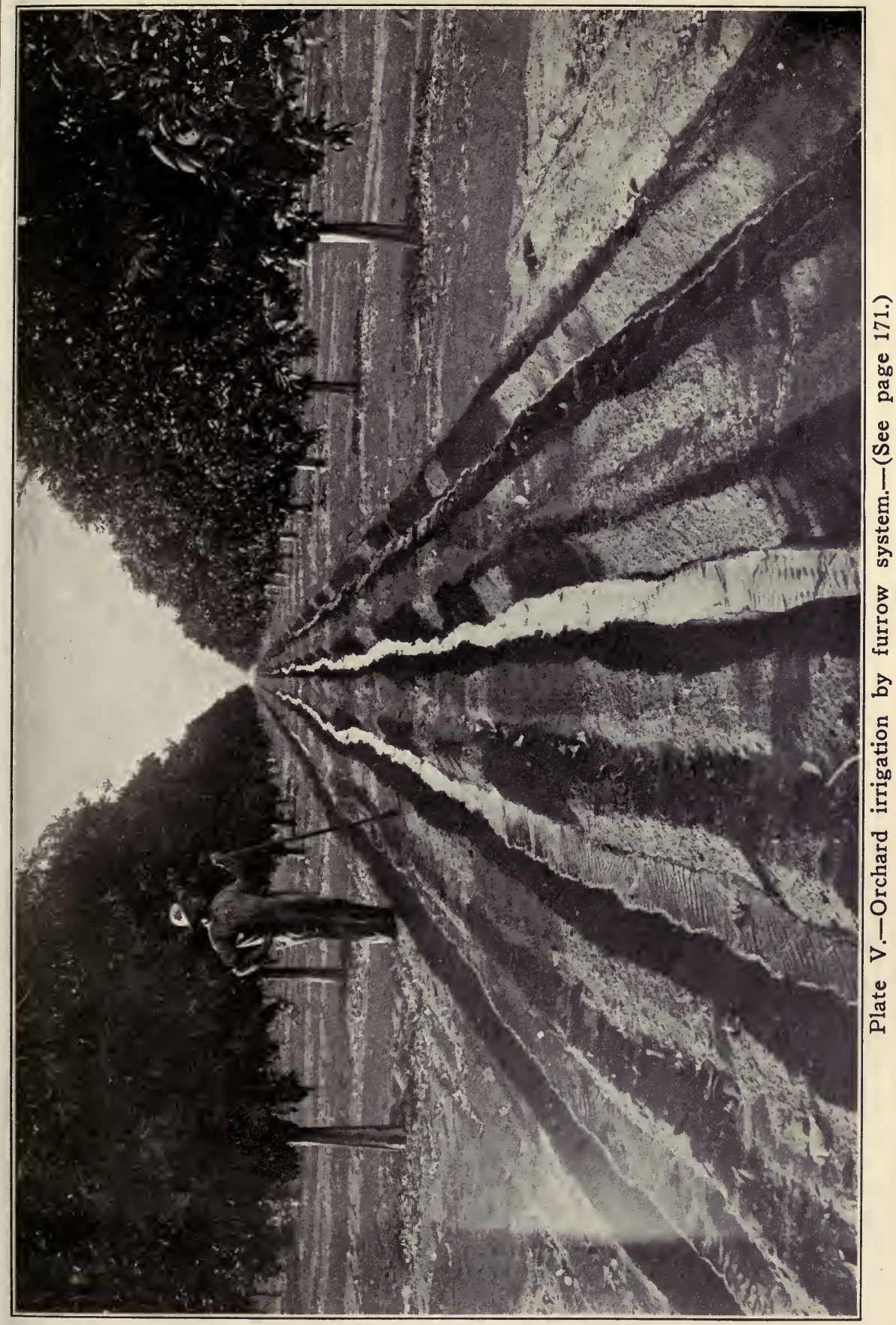



of feet or inches. At other times a post from 1 to 2 inches square is placed in the center of the box and several feet above the weir board. The top of this post is on a level with the crest. This is the method shown in the accompanying sketch.

How to Measure Water Over Weirs.-The method to follow can best be shown by examples. Let us suppose that a farmer has made and placed a box similar to the one shown in the drawing, after turning in the water and allowing it some time to attain a uniform flow he proceeds to the weir box and with an ordinary rule measures the depth of water flowing through the weir notch. Bear in mind that

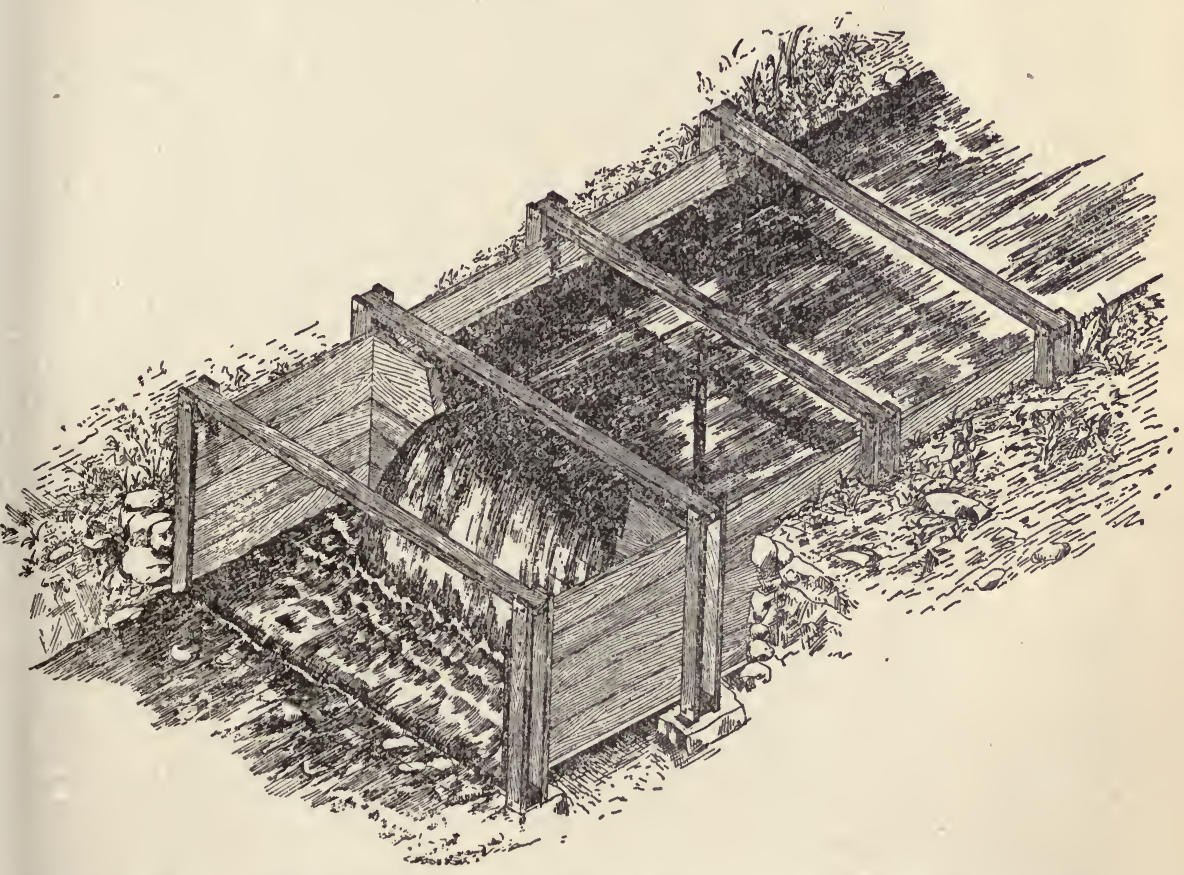

Weir box in operation showing post from which to measure depth of stream.

this measurement is not made at the weir board, but at the regular gauge, whether it be a nail, brass plate or post, as already described. We will assume that the depth as found by the rule is $31 / 2$ inches. Now by referring to the table below he follows down the first column until $3 \mathrm{r} / 2$ is reached. The weir used is one foot, and under the column marked " 1 -foot weir" and opposite the figure $3 \mathrm{r} / 2$ already found, he finds the cubic feet per minute or the gallons per minute flowing over a 1 -foot weir when the depth of water is $31 / 2$ inches. The equivalent flow in gallons per minute for any given length of weir and depth of water over the crest was obtained from the accompanying table: 
Discharge over Cipoletti Weirs

\begin{tabular}{|c|c|c|c|c|c|c|c|}
\hline \multicolumn{8}{|l|}{$\begin{array}{l}\text { Depth of water } \\
\text { on crest. }\end{array}$} \\
\hline 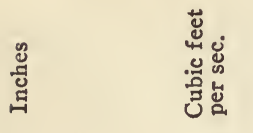 & 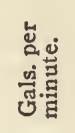 & 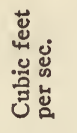 & 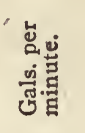 & 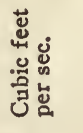 & 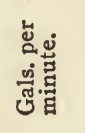 & 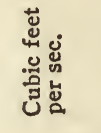 & 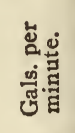 \\
\hline 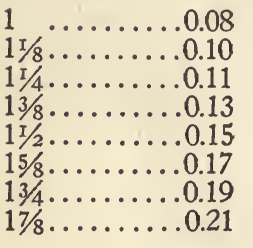 & $\begin{array}{l}36 \\
44 \\
51 \\
59 \\
67 \\
75 \\
84 \\
93\end{array}$ & $\begin{array}{l}0.12 \\
0.15 \\
0.17 \\
0.20 \\
0.22 \\
0.25 \\
0.28 \\
0.31\end{array}$ & $\begin{array}{r}55 \\
65 \\
76 \\
88 \\
100 \\
113 \\
126 \\
140\end{array}$ & $\begin{array}{l}0.16 \\
0.19 \\
0.23 \\
0.26 \\
0.30 \\
0.34 \\
0.38 \\
0.42\end{array}$ & $\begin{array}{r}73 \\
87 \\
101 \\
117 \\
139 \\
150 \\
168 \\
187\end{array}$ & $\begin{array}{l}0.24 \\
0.29 \\
0.34 \\
0.39 \\
0.45 \\
0.50 \\
0.56 \\
0.62\end{array}$ & $\begin{array}{l}109 \\
130 \\
152 \\
175 \\
200 \\
226 \\
252 \\
280\end{array}$ \\
\hline 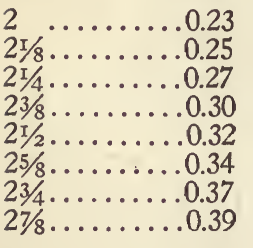 & $\begin{array}{l}103 \\
113 \\
123 \\
133 \\
144 \\
154 \\
166 \\
177\end{array}$ & $\begin{array}{l}0.34 \\
0.38 \\
0.41 \\
0.44 \\
0.48 \\
0.52 \\
0.55 \\
0.59\end{array}$ & $\begin{array}{l}154 \\
169 \\
184 \\
199 \\
215 \\
231 \\
248 \\
262\end{array}$ & $\begin{array}{l}0.46 \\
0.50 \\
0.55 \\
0.59 \\
0.64 \\
0.69 \\
0.74 \\
0.80\end{array}$ & $\begin{array}{l}206 \\
225 \\
245 \\
266 \\
287 \\
309 \\
332 \\
355\end{array}$ & $\begin{array}{l}0.68 \\
0.75 \\
0.82 \\
0.89 \\
0.96 \\
1.03 \\
1.11 \\
1.18\end{array}$ & $\begin{array}{l}308 \\
338 \\
368 \\
399 \\
431 \\
464 \\
497 \\
531\end{array}$ \\
\hline 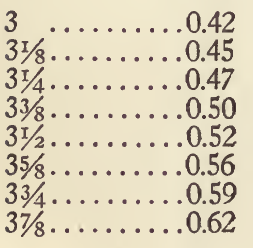 & $\begin{array}{l}189 \\
201 \\
213 \\
225 \\
238 \\
251 \\
264 \\
277\end{array}$ & $\begin{array}{l}0.63 \\
0.67 \\
0.71 \\
0.75 \\
0.80 \\
0.84 \\
0.88 \\
0.93\end{array}$ & $\begin{array}{l}283 \\
301 \\
319 \\
338 \\
357 \\
376 \\
396 \\
416\end{array}$ & $\begin{array}{l}0.84 \\
0.90 \\
0.95 \\
1.00 \\
1.06 \\
1.12 \\
1.18 \\
1.24\end{array}$ & $\begin{array}{l}378 \\
402 \\
426 \\
451 \\
476 \\
502 \\
528 \\
554\end{array}$ & $\begin{array}{l}1.26 \\
1.34 \\
1.42 \\
1.51 \\
1.59 \\
1.68 \\
1.76 \\
1.85\end{array}$ & $\begin{array}{l}566 \\
602 \\
639 \\
676 \\
714 \\
753 \\
792 \\
832\end{array}$ \\
\hline 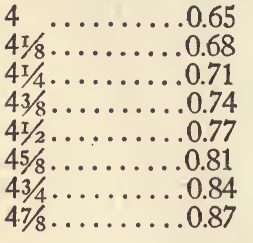 & $\begin{array}{l}291 \\
304 \\
319 \\
333 \\
347 \\
362 \\
376 \\
391\end{array}$ & $\begin{array}{l}0.97 \\
1.02 \\
1.07 \\
1.11 \\
1.16 \\
1.20 \\
1.26 \\
1.31\end{array}$ & $\begin{array}{l}436 \\
456 \\
478 \\
499 \\
521 \\
543 \\
564 \\
587\end{array}$ & $\begin{array}{l}1.30 \\
1.36 \\
1.42 \\
1.48 \\
1.55 \\
1.61 \\
1.68 \\
1.74\end{array}$ & $\begin{array}{l}582 \\
609 \\
637 \\
665 \\
694 \\
723 \\
753 \\
782\end{array}$ & $\begin{array}{l}1.94 \\
2.04 \\
2.13 \\
2.22 \\
2.32 \\
2.42 \\
2.52 \\
2.62\end{array}$ & $\begin{array}{r}872 \\
913 \\
956 \\
998 \\
1,041 \\
1,084 \\
1,129 \\
1,174\end{array}$ \\
\hline 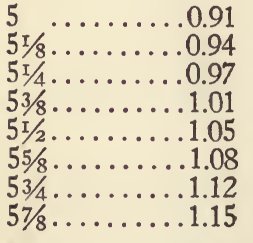 & $\begin{array}{l}406 \\
422 \\
437 \\
453 \\
469 \\
485 \\
501 \\
517\end{array}$ & $\begin{array}{l}1.36 \\
1.41 \\
1.46 \\
1.51 \\
1.57 \\
1.62 \\
1.68 \\
1.73\end{array}$ & $\begin{array}{l}609 \\
633 \\
656 \\
679 \\
703 \\
727 \\
752 \\
776\end{array}$ & $\begin{array}{l}1.81 \\
1.88 \\
1.95 \\
2.02 \\
2.09 \\
2.16 \\
2.23 \\
2.31\end{array}$ & $\begin{array}{r}813 \\
843 \\
874 \\
906 \\
938 \\
970 \\
1,002 \\
1,034\end{array}$ & $\begin{array}{l}2.72 \\
2.82 \\
2.92 \\
3.03 \\
3.13 \\
3.24 \\
3.35 \\
3.46\end{array}$ & $\begin{array}{l}1,219 \\
1,266 \\
1,312 \\
1,359 \\
1,407 \\
1,455 \\
1,503 \\
1,553\end{array}$ \\
\hline 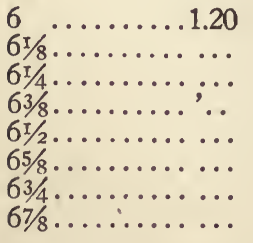 & $\begin{array}{l}534 \\
\cdots \\
\cdots \\
\cdots \\
\cdots \\
\cdots \\
\cdots\end{array}$ & $\begin{array}{l}1.79 \\
\ldots \\
\ldots \\
\ldots \\
\ldots \\
\ldots \\
\ldots\end{array}$ & $\begin{array}{l}801 \\
\cdots \\
\cdots \\
\cdots \\
\cdots \\
\cdots \\
\cdots\end{array}$ & $\begin{array}{l}2.38 \\
2.46 \\
2.53 \\
2.61 \\
2.68 \\
2.76 \\
2.84 \\
2.92\end{array}$ & $\begin{array}{l}1,069 \\
1,102 \\
1,136 \\
1,170 \\
1,205 \\
1,240 \\
1,275 \\
1,310\end{array}$ & $\begin{array}{l}3.57 \\
3.68 \\
3.80 \\
3.91 \\
4.03 \\
4.14 \\
4.26 \\
4.38\end{array}$ & $\begin{array}{l}1,603 \\
1,653 \\
1,704 \\
1,755 \\
1,807 \\
1,859 \\
1,912 \\
1,966\end{array}$ \\
\hline $7 \quad \ldots \ldots \ldots \ldots$ & $\ldots$ & $\ldots$ & $\ldots$ & 3.00 & 1,346 & 4.50 & 2,020 \\
\hline
\end{tabular}




\section{RANDOM SUGGESTIONS}

Without attempting an impossible thing, to-wit, to furnish explicit directions for the practice of irrigation, for much of it every man must learn for himself by experience, a few suggestions may be noted, even though more important ones do not come to mind.

Usually water should be prevented from actual contact with the trunk of the tree. Citrus trees are especially sensitive to such contact, and resent it by "gum disease," which was formerly far more prevalent in the State than now. Care must, therefore, be taken not to set trees which are to be irrigated, too low. It is better to raise them up a little and draw the earth up around them to prevent approach of the water, but this must not be overdone.

If possible, the ditch should be run on the shady side of the tree, because reflected sunshine from the water surface may burn the bark.

In examining soil to ascertain dryness, one must dig deeply, for often an upper layer will be fairly moist, if well cultivated, while lower layers, where the feeding rootlets are, will be arid. Therefore, when trees, or vines are suffering, dig far down in examining the soil.

In irrigating, thorough, deep soaking is necessary, and examination must be made to see if an artificial hardpan which prevents the descent of the water has been formed.

Be careful not to continue irrigation too late in the season. It will prevent the proper dormancy of deciduous trees, and if more fall irrigation is given citrus trees than they need for perfecting the fruit, the trees will continue growing tender shoots until they are injured by severe frosts. On the other hand, it is often desirable to give deciduous trees a draft of water after the fruit has been gathered, if the soil is so dry that the tree is likely to drop its leaves too soon, and wake from its dormancy with the first rains. Many times the fall blooming of deciduous trees, which is very undesirable, may be prevented by keeping them growing later in the summer by moderate irrigation.

If trees or vines, in regions usually irrigated, are to be grown without irrigation, it is important that the grower be more than usually thorough and constant with his summer cultivation. In trying the non-irrigation experiment, one should, of course, begin with young trees which have not been irrigated, and not usually expect success by withdrawing the water from trees which have been accustomed to it, and have developed a root system accordingly.

While waiting for an "irrigating system," young trees can be kept going with a water wagon. With a galvanized tank on a wagon three men went over a 60-acre orchard three times, taking four days for each irrigation, each day's expense being less than $\$ 10$. This made twelve days, or $\$ 120$, or $\$ 2$ per acre. One man went ahead to scoop out around each tree, and after the water was put on he covered it up, to keep the soil loose and prevent loss by evaporation. He could keep ahead because of the loss of time of the team going after water and returning. From the tank ran two large hose, the water being siphoned off at the basin near each tree till about four or five gallons of water were put on. Then the hose was bent back so the water could not flow out, and they advanced to the next tree. 


\section{SUB-IRRIGATION IN CALIFORNIA}

The word "sub-irrigated" is freely used in California to describe land which is moistened below by underflow or seepage from streams or springs, or from open irrigation ditches, traversing higher levels. This land is sub-irrigated, it is true, but there is no system about it, except the natural distribution of water, which is to seek its level. Some of our most productive lands are of this character, and where the soil and subsoil are fitted to the movement of this living water, and not apt to retain it up to the point of saturation, most satisfactory growth of deep-rooting field crops and of trees and vines are secured. But this is not sub-irrigation in the ordinary signification of the term.

Several systems of sub-irrigation by subterranean pipes have been devised by California inventors, but none have passed beyond the experimental stage, and no acreage has been continually operated.

\section{DRAINAGE IN CALIFORNIA}

There was for a long time a very erroneous popular generalization that California soils do not need drainage; that in a dry state the aim should be to retain the moisture, not to part with it. It is, of course, true that we have vast areas of naturally well-drained soil, upon which any money spent for drainage would be in a great part thrown away, but we have, also, both in the valley and on the hillsides, localities where, by peculiar character and conformation of the subsoil, water is held in the soil until evaporated from the surface, and the result is a boggy, miry condition, which prevents proper winter cultivation, and at the same time injures the roots of the trees or vines. This defective cultivation, added to the puddling effect of standing water, makes the soil dry out completely under the fervid sun of summer, and the result is that the wettest soil of the winter is the driest in the summer, and plants which are injured by soaking in winter suffer again from lack of moisture and sustenance in summer. Thus it is a fact, clearly proven by observation and experience, that thorough under-drainage removes surplus water in winter, and ministers to the retention of moisture in summer. More than this, a soil puddled by standing water can not present its contents in available form for plant nutrition, and besides, it loses the fertilizing effects of atmospheric currents, which pass through an open, well-dried soil. Wet land is cold and late in spring, and hot as a baked brick under the summer sun; it is no fiction of the imagination to say that well drained land is warm in winter and cool in summer - that is, cool to a degree which favors quick and free root growth, and cool enough to escape the parching effect of deeply baked soil.

These, and a host of similar considerations, which have made under-drainage popular in older countries, are of weight in California. Possibly, as a rule, because of our vast area of deep, kind loams, the proportion of land needing drainage in this State is less than elsewhere, and yet there is a vast extent of country to be improved by tilling. There have been large losses of trees from planting upon soils 
defective in this respect. The evil has resulted from excessive rainfall and excessive irrigation, either direct or by underflow from adjacent irrigations. In some places this latter movement of water has brought alkali to assist in the ruin of the trees and vines. The cure is drainage to sufficient depth and with good outlet for the drainage water.

Information on the construction of under-drains is too available through other sources to call for its presentation in this connection.

Drainage and Irrigation.-A special importance attaches to complete and systematic drainage in connection with irrigation. There is pressing need of such provision where the soil has become overloaded by seepage water from irrigation ditches, and it is well that people in such situations are waking up to the need of coupling drainage outlets with their irrigation inlets. Another matter closely allied to this is the action of alkali on soils thus artificially watersoaked. This has been made the subject of a special publication, to which allusion has already been made in Chapter III. Drainage is plainly essential, both in individual farms and in districts where the water level is rising too high, and the striking statements given below by Professor Hilgard should incite all to give immediate attention to the needs of vines and trees in this regard.

The following summary of drainage needs, and the advantage of providing drainage when needed, is made by Professor Hilgard:

In the valleys and plains of the arid irrigation countries the soils are predominantly of a light, sandy or silty nature, easily penetrated to great depths by water and air. With these the roots of plants also reach to such depths, drawing therefrom not only moisture, but also plant food, which in these soils is, as a rule, very abundant. The plants of the arid region thus are enabled to utilize nearly as many feet of soil mass as in the regions of summer rains inches would be drawn upon; and it is evident that this advantage, which postpones for a long time the need of fertilization, should not be lightly thrown away. Each farm in the arid region has several similar ones underground, which with proper management can be fully utilized.

But this presupposes that the water, air and roots can all penetrate under irrigated culture as they do in the natural condition. It means that the ground water level shall not be allowed to rise to such an extent as to prevent the penetration and healthy life of the roots in the depths of the soil mass. If by intentional or careless over-irrigation, or by leakage from the ditches, the water level is allowed to rise within a few feet of the surface, the wonderfully productive lands of the arid valleys are reduced to the same condition as are those of the humid countries; a shallow layer of surface soil, within which alone the roots can exercise their functions of plant nutrition. The natural result is that this layer soon becomes exhausted, and copious artificial fertilization is required to maintain profitable production.

And even this is the most favorable case. When, in addition, the upward movement of the soil water carries with it the entire mass of salts of various kinds which exist in all arid soils, and brings them within reach of surface evaporation, these "alkali" salts impregnate the soil to such an extent as to render the cultivation of many crops unprofitable, or sometimes altogether impossible.

Summarizing the advantages of systematic land draining it may be said that:

1. It prevents the drowning out of the deeper roots of plants by the rise or fluctuations of the ground water, by which the vineyards and orchards are so frequently rendered unprofitable.

2. It prevents, or at least limits definitely, the shallowing of the soil caused by high-lying ground water, resulting in the need of early and copious fertiliza- 
tion, which would otherwise not have been called for in many years. The annual cost of such fertilization would soon exceed the first cost of drainage.

3. Drainage does away definitely with the alkali evil. When drainage is established the land can easily be so handled as either to remove all the alkali, or to leave in the soil so much of it as riay be rationally considered beneficial, on account of its usual content of valuable and highly available plant food. To prevent the waste of much of this soluble plant food, the use of gypsum is also valuable; but subsequent swamping of the land would cause a return of the black alkali unless drainage were provided for.

In view of the facts that water-logged lands are still being sold to the unwary for fruit planting; that sometimes lands are offered with the attractive promise of an irrigation supply when they actually need a drainage system; that on such lands every year of large rainfall brings areas of trees into distress and inflicts considerable losses, these declarations of Professor Hilgard should be most carefully kept in mind. 


\section{PART THREE: ORCHARD FRUITS}

\section{CHAPTER XVI}

\section{COMMERCIAL FRUIT VARIETIES}

What fruit to plant, or what kind of a bearing orchard to buy as an investment, are questions which can not be answered, in this treatise. The planters on new land and the investors in improved land must answer them for themselves-forming their judgments after securing facts which seem to them a proper basis for such a business decision. It is the conviction of the writer that all fruits which have demonstrated commercial suitability in California, when properly placed under the soil, temperature and moisture conditions which favor their best growth and productiveness, may be counted as yielding nearly equal net returns, considering the investment in land, water, waiting for bearing and handling of the product. So far as the writer has observed, all our commercial fruits have reached maximum and minimum returns during the last quarter of a century which are practically identical. Therefore to plant good fruit in the best place for it, to handle the trees and products most intelligently, both in production and marketing, holds out substantially equal promise of profit. If it could be demonstrated that any particular fruit had the especial advantage over others in net returns, this advantage would immediately disappear because planters would rush to it and take away this advantage by undue increase of its acreage. Therefore the choice of fruits must remain an open question for each one to determine by his own experience and observation, at least to the extent of determining his own line of production.

It is one of the purposes of this treatise, as they will be disclosed in succeeding chapters, to impress upon the local planters the conviction that their clearest path toward satisfactory income lies in choosing varieties which have demonstrated two fundamental characters, viz.: adaptation to the locality and to the uses of the fruit trade-rather than in choosing novelties, no matter how alluring they may be.

It may surprise the casual reader to find that our production proceeds so largely upon old standard varieties. Anyone, however, who is acquainted with commercial fruit growing knows that it is neither wise nor easy to revolutionize an established and profitable industry by the substitution of new varieties for the old standards. It takes several years to determine whether a new variety is really trustworthy and suitable, and it takes much longer to get a large acreage in bearing either by grafting or new planting because people are slow and conservative in making changes. As the period of trial of each novelty passes, however, new varieties are accepted, if for any good reason found suitable, and become prominent as their merits justify.

Another reason why new varieties do not figure more largely in California fruit growing is the smallness of the amateur interest. 
There is, in fact, almost an absence of pure amateurs-enthusiastic critical, discriminating, athirst for novelties. Even suburban planters follow the lead of commercial orchardists and plant chiefly that which has shown adaptations to local growing conditions, and few are averse to making what they can by sale of small surpluses. The result is that California fruit growing is almost wholly commercial in spirit, policy and point of.view, which is perhaps only natural in a state where the fruit products reach an annual aggregate value of something like one hundred millions of dollars. The effect is to concentrate attention upon varieties which have achieved fame for profit, and to repress amateur devotion and indulgencies.

At the same time there is, and has always been, quite a disposition toward trial of novelties among commercial growers, especially manifested in search of specific characters which are seen to be desirable rather than desire for newness for its own sake, which is often a point of pride among amateurs. To this enterprising and discriminating search is due the prominence of some of the leading varieties, which are chance seedlings recognized as meeting special requirements and having grown great because they really did so. The California grower is, therefore, quite certain that he needs not varieties new throughout and of startling characters, but improved varieties which hold the good points of the old and add other points. For instance, he calls for trees resistant to disease, for improvement of the fruit in beauty, flavor and keeping qualities; for varieties, similar in kind, which fill gaps in the ripening season so that he can employ help continuously, and shippers and canners agree with him so that they can keep the cars moving and the cannery plants at work. The grower says he must be careful not to plant something different from what is already growing and selling well in his region, (nd this is also the advice of the trade to him. He can not risk much on varieties of entirely different types, although most growers are always doing a little experimenting. Nor should he undertake too many varieties, because a profitable orchard is not a pomological museum. There must be a large quantity of uniform fruit to make any district commercially prominent.

For these reasons the number of varieties now planted is but a fraction of what it was a third of a century ago, and, stopping at this point, one might get the idea of the California grower was a monument of conservatism and lacking in enterprise and adventure. Subsequent chapters will, however, show that he has very definite ideas of the suitability and desirability of the varieties which he chooses for planting.

It should therefore be noted by the reader that the preference for certain varieties, which is embodied in this statement, does not involve pomological standards as a leading factor. The claim is distinctly not made that these varieties are chosen exclusively on the basis of quality, beauty, hardiness or health. In the case of nearly all the fruits, there are other varieties which might equal or even surpass them in one or more of these respects. The choice is made because they are most profitable to grow; not alone because they are good, but because they 
are good for something. This particular suitability or serviceability may involve pomological considerations and commercial and manufacturing considerations as well. The planter must use these lists in connection with what he may find about the varieties in subsequent chapters, without neglecting to confer also with older growers in the district in which he may plan to plant.

Perhaps an intelligent use of the statement can be concretely suggested by briefly discussing the first group of varieties mentioned-the apples most approved in California. First comes the yellow Newton Pippin, and that means that most apples commercially grown are winter apples and this variety is, on the whole, the most profitable of them. But a planter in a hot interior valley should usually reject them, for all winter apples are apt to be unsatisfactory, and, if he plants apples at all, should choose early varieties, because they ripen early, thus escaping the highest heat and at the same time being ready for the early market. Similar comments might be made upon the varieties of other fruits.

During the year 1914 the writer had the advantage of revising all his earlier data on varieties through association with Professor B. B. Pratt, J. E. Coit, F. T. Bioletti and R. E. Smith as a committee of the California Experiment Station to assist the Bureau of Plant Industry in a revision of the variety list of the American Pomological Society, and desires to gratefully acknowledge benefits from such association.

The varieties grouped below are arranged not according to ripening season but in the order in which the greatest number of growers consider them worth planting:

Apples.-Newtown Pippin, Bellflower, E. Spitzenberg, W. W. Pearmain, Gravenstein, Red Astracan, W. Astracan, R. I. Greening, Rome Beauty, Jonathan, Winesap, White Astracan, Delicious, Arkansas Black, Baldwin, Tompkins Co. King.

Apricots.-Royal, Blenheim, Moorpark, Tilton, Hemskirk, Peach, Newcastle.

Cherries.--Royal Ann, Black Tartarian, Bing, Black Republican, Lambert, Chapman, Purple Guigne, May Duke, Centennial, Black Bigarreau.

Peaches.-Muir, Elberta, Phillips, Lovell, Salway, Early Crawford, Tuskena, Foster, Late Crawford, Orange Cling, Susquehanna, Sellers, Lemon, St. Johns, Henrietta, Hales, Alexander, Heath. Triumph.

Pears.-Bartlett, Winter Nelis, Easter, Du Comice, Glout Morceau, Hardy, Barry, Comet, Seckel, Winter Bartlett, Wilder.

Plums.-Wickson, Burbank, Climax, Hungarian, Tragedy, Kelsey, Yellow Egg, Red June, Giant, Washington, Satsuma, Jefferson, Grand Duke, Santa Rosa, Clyman, Formosa.

Prunes.-French, Imperial, Sugar, Robe de Sergeant, Italian, German, Standard, Silver, Splendor.

Raisin and Shipping Grapes.-Muscat, Tokay, Thompson, Emperor, Malaga, Cornichon, Black Prince, Sultana, Black Morocco, Sweet Water, Verdal, Gros Colman, Pierce. 
Figs.-White Adriatic, Lob Injir, (Smyrna), Mission, Brown Turkey, Purple Smyrna, Kassaba.

Almonds.-Nonpareil, Drake, IXL. Ne Plus Ultra, Texas Prolific, Languedoc, Peerless.

Walnut.-Franquette, Mayette, Concord, Eureka, Placentia, E1 Monte, Santa Barbara Softshell.

Orange.-Washington Naval, Valencia, Mediterranean Sweet, Paper Rind St. Michael, Ruby Blood.

Lemon.-Eureka, Lisbon, Villa franca.

Pomelo.-Marsh.

Olives.-Mission, Manzanillo, Sevillano, Ascolano, Nevadillo.

Blackberries.-Mammoth, Lawton, Himalaya, Crandalls.

Raspberries.-Cuthbert.

Strawberries.-Dollar, Brandywine, Jessie, Arizona, Marshall, Melinda, Banner.

Let it be understood that this compilation indicates the popularity of varieties in the State as a whole. It should not be taken as a guide to planting in any particular district, except for experimental purposes. Lists for localities should be made after careful studies of the chapters treating of the particular fruits chosen for planting. 


\section{CHAPTER XVII}

\section{THE APPLE}

During the last decade notable progress has been made in apple growing in California. The old idea that our conditions did not favor excellence in the apple has given away to full assurance that in wisely selected elevations and exposures the very highest points of size, beauty, flavor, keeping and shipping qualities are secured. Even before the wonderfully satisfactory test of both northern and southern California apples at the New Orleans World's Fair, it was clear that the right variety grown in the right place yields an apple in California than which a better can not be grown anywhere, and during the last five years California early apples have been in sharp request for shipment to all regions of the Northwest and British Columbia, and California winter apples have been sold at the highest prices east of the Rocky Mountains and in Europe. Shipments beyond State lines of above four thousand carloads in 1910 testify to these facts.

Localities for Apples.-Speaking generally, it may be laid down that the great valleys of the interior are not well suited to the apple; also, there are some situations which are much better than others. In the early regions of the Sacramento Valley and foothills, however, excellent early apples are profitably produced. In the great valley and lower foothill region of the State, the late apple usually lacks character and keeping quality. On the great plains the tree is liable to sunburn, or sun blight, as it is called. Some varieties, because of the character of their foliage, are less liable to this injury than others, and it is possible that this evil may be finally overcome by the selection of varieties with blight-proof foliage, as will be mentioned later. In the great valley, however, on the rich river-bottom land of the Sacramento and the San Joaquin and its tributaries, the apple roots deeply, attains good size, bears good fruit, with fair keeping quality, while but a few miles away on the plains it is inferior.

In the interior, adaptation to the apple lies at an elevation on the foothills on both the east and west rims of the great valley. Its limits are not well defined, but there are flourishing orchards at an elevation of about four thousand five hundred feet on the slopes of the Sierra Nevada Mountains, and from two thousand to three thousand five hundred feet is commonly regarded the best apple region of the mountains. The trees attain large size and bear heavily, and the fruit, of well-adapted varieties, is large, crisp, juicy and has exceptional keeping qualities. On the Tule River in Tulare County, at an elevation of 4100 feet, eight-year-old Winesaps have borne 300 pounds, Yellow. Newtown Pippins 250 pounds, and White Winter Pearmains 300 pounds per tree. At such elevations, however, there is constant danger of spring frost injury.

Along the coast the apple succeeds well from end to end of the State, and very close to the ocean excellent fruit is produced on good 
soil. There is a certain advantage in elevation in the coast region as well as in the interior, but the advantage is not so marked nor is the required elevation so great. Coast valleys in the central and upper portion of the State, where the soil is suitable, produce most excellent apples, but even here the lower hillsides, with deep, well-drained soils, are, perhaps, preferable to the floors of the valley. Departing from immediate coast influences and approaching the interior, with its greater heat and aridity, the greater elevation becomes desirable. The apple, excepting the very early varieties, does not relish the forcing heat which brings such perfection to the peach, but to insure late ripening and long keeping, with accompanying crispness, juiciness, and flavor, it must have atmospheric surroundings which favor slower development.

Localities for apple growing in southern California are to be choser with much the same rules as in the upper parts of the State. As has already been said, valleys in which coast conditions largely predominate produce good apples, on suitable soils, but away from the coast, proper elevations must be sought, and they should be above the so-called thermal or frostless belts. Good apples are grown on low lands near the coast in Los Angeles and Orange Counties. Sixty miles inland, in Sar Bernardino County, winter apples fail in the valleys, but are mosi excellent at a sufficient elevation upon the slopes of the surrounding mountains or in elevated valleys like the Yucaipe Valley above Red. lands, where a Rome Beauty of excellent quality was grown in 1903 to a weight of twenty-seven ounces and a circumference of fifteer inches. In the elevated interior of San Diego County, as in the Juliar and Smith Mountain districts, excellent apples are produced in large quantities and profitably carried long distances.

Second and Third-Crop Apples.-There is a peculiar behavios of the apple tree, most noticeable when winter temperature is mildest and that is blooming and fruiting out of season. In the case of early apples the second bloom may appear about the time the first frui ripens and the third bloom when the second crop is half grown. Ever such behavior may be followed by regular blooming the following spring. Second crops of apples are not of amount nor regularity enough to be of much economic importance, as the second crop o pears and grapes sometimes are. The third crop occasionally ripens An instance is on record at Chino, San Bernardino County, where it 1903 a tree ripened its first crop in June, and its last fruit was pickec on Christmas day following. Such behavior, of course, indicates con ditions ill suited to the apple.

Exposures for the Apple.-The choice of exposure for an appl orchard may almost be inferred from what has been said about locali ties. In regions with high summer temperature the apple will do bes on cool, northerly slopes, and this exposure becomes doubly desirabl when the location has high temperature with only moderate annua rainfall, or where the soil is not well adapted to the retention of mois ture. With such prevailing conditions, the apple will be grateful fo the cooler air and the greater moisture of the northerly slope. Wher the temperature is moderately cool, and the rainfall adequate, the mat 
ter of exposure is of less account, and the grower can make the existence of the best soil the test of location of his orchard. At elevations on the sides of high ranges where late cold storms are liable to rush down from higher snow fields, protection from the usual course of such storms, or from the course of cold winds generally, must be sought; and directly up the coast, especially in the northern part of the State, in certain places where the peach does not usually succeed even the apple needs protection, and the benefit of all heat available, and then a southerly or southeasterly exposure becomes desirable. The choice of exposure is thus seen to be largely a local question and to be determined by a knowledge of local conditions. A newcomer in a region can best learn these conditions by conference with older residents, or by personal observation of older orchards.

Soils for the Apple.-Experience with the apple in California confirms what has long been set forth as its choice of soils in older regions. If one avoids an extremely light, sandy soil on the one hand, and a very stiff clay or adobe on the other, he may plant apples on almost any soil which allows extension of the roots to a considerable depth without reaching standing water. The apple thrives in a moist soil, but it must be well drained, naturally or otherwise. A soil which may be called best for the apple is a deep, rich, moist, calcareous loam, but the tree will thrive on coarser materials. The subsoil, whatever its nature, must be sound and open to the passage of moisture. The most unfavorable condition for the tree is a subsoil of clay which holds water. There is some difference in varieties as to choice of soil. The Yellow Bellflower, for instance, will do well on a lighter soil than the Yellow Newtown Pippin.

\section{PROPAGATION OF THE APPLE}

The apple is chiefly propagated by root-grafting upon apple seedling roots either whole roots or root pieces. Budding is also practiced to a certain extent. For dwarf trees the Paradise stock is used. Repeated trials with working the apple on the pear, chiefly by top grafting, have secured growth of limited life but without fruiting.

The resistance of certain roots to the woolly aphis has been fully demonstrated by local experience in the use of the Northern Spy and Winter Majetin, chiefly the former. Seedlings of Northern Spy can not be relied upon as resistant to the woolly aphis. It is necessary to get a root actually grown from the Northern Spy wood. The best way to get a start is to buy some Northern Spy trees from some reputable nurseryman, specifying that they shall be Northern Spy root and top. With these resistant roots and wood growth for scions or cuttings can be grown. Resistant trees are made by root grafting the scion of the variety which it is desired to propagate upon a piece of Northern Spy root and then being careful that the scion does not send out roots of its own, but is wholly dependent upon the Northern Spy root. It is customary with nurserymen selling resistant trees to save the root pieces which are removed in digging and packing for subsequent propagation. It is also possible to get a resistant tree by start- 
ing from the cutting of a Northern Spy. To facilitate the rooting of these cuttings a small piece of any kind of apple root is put in by side graft near the bottom of the cutting. This acts as a starter, but the cutting will also make roots of itself. At the end of the first year then the cuttings are taken up, the piece of root used as a starter is cleanly cut away and the rooted cutting replanted; henceforth it is dependent upon its own roots and is resistant. The variety desired is then grafted in a little way above the ground surface so that there will be no danger of its making its own roots. By either of these processes it is more troublesome and takes more time to produce a tree with a resistant root than in the ordinary way, and for that reason trees on resistant roots are sold at a higher price. This may be the reason why resistant trees are not yet largely used in this State.

Other suggestions applicable to the growth of young apple trees are given in Chapters VIII and IX.

\section{DISEASES AND PESTS OF THE APPLE}

The apple is subject to various diseases and insect enemies which must be resolutely fought or they will render the trees unprofitable. Chief of these diseases are the "pear blight" and the apple scab, and the apple mildew. Of the insect enemies the codlin moth, the appleleaf aphis, the various leaf-cutting caterpillars and several scale insects must be kept in check and the latest approved means of reducing these troubles will be described in detail in later chapters.

\section{PLANTING AND CARE OF THE APPLE ORCHARD}

The chapters on planting, and pruning contain suggestions to which the reader is referred. Care should be taken to obtain trees with clean, healthy roots, not knotted and scarred by woolly aphis.

Distance in Planting.- The distance between the trees is of the highest importance. All the old apple orchards are overcrowded. More recently trees have been set at greater distances, and such planting is now generally advised. There is some difference of opinion as to proper distance, but certainly twenty-five to thirty feet is near enough, and some of the best new orchards have been planted at forty feet, the ground being used for a time with other crops or planted with early bearing trees, for which the soil is suited, between them.

Pruning the Apple.-The manner of shaping fruit trees described in the chapter on pruning succeeds admirably with the apple. Yearling trees are usually planted, and they are regularly pruned until proper form is secured.

Mr. C. H. Rodgers, during his life-time a leading apple grower of the Watsonville district, near the coast in central California, gave the following excellent outline of a simple and economical, yet successful, method for apple tree building under ordinary conditions: 
First Year: On planting cut the stem from 30 to 36 inches in height, with the terminal bud toward the southwest. In the spring, when growth begins, strip off all shoots from the ground up to about 20 inches. Above this point let all growth remain during the summer. If for any cause during early summer a bud does not start where wanted, a short transverse cut through the bark just above the bud will cause it to develop into limb.

Beginning of Second Year: Cut off all limbs except those selected to remain permanently. Two, three, four, and not more than five limbs should be allowed to remain, the number depending on their position. It should be the aim to distribute them evenly on all sides, and to give all possible space between limbs up and down the trunk. This latter precaution is to give room for expansion of limbs in after years. Cut back the limbs that are to remain, taking off from one-third to one-half of the previous season's growth. If the tree is of a spreading habit, and it is desired to have it grow erect, cut to inner buds. If desired to spread the top cut to outer buds.

Beginning of Third Year: Allow two or three lateral limbs to remain on each of the main branches. Top the tree again, taking off from one-third to one-half the previous year's growth. Continue this method during the first four years, at which time the tree should begin to bear, and if surrounding conditions are favorable, it will prove strong, vigorous and capable of sustaining a heavy load of apples. The after treatment will consist mainly in keeping the top properly thinned.

After coming into bearing there must be intelligent pruning according to the growth-habit of the variety. Some varieties, like the Yellow Bellflower, resent heavy pruning after coming into bearing, and slow growers like the Yellow Newtown Pippin, do not need it. On the other hand, varieties like the Winesap and Smith's Cider are apt to make long slim branches and bear at the ends. This can be corrected by cutting back to secure more short shoots which will bear better fruit. Some varieties, like the Jonathan, will make plenty of short spurs under this treatment, while others, like Rome Beauty and Rhode Island Greening, are persistent tip-bearers, but can be gradually drawn in without reducing the crop too much. The grower must study his varieties not only with reference to this but in forming the tree, cutting to an inside bud all varieties which naturally take a horizontal direction, and cutting to an outside bud varieties which have a tendency to send up tall, straight shoots. By thus throwing the new growth upward in the first case, and outward in the second, one can shape each kind to greater symmetry and strength for fruit carrying, and bring up all spreading varieties to a form which admits near approach of the plow and cultivator. This manner of shaping the tree must continue as long as seems necessary to secure a tree which will come to bearing age shapely and strong, and within reach.

Bearing trees should not be allowed to carry too many branches, and pruning will largely consist of thinning out surplus shoots and removing interference between branches. It is not desirable to shorten in the apple as is done with the apricot and peach. 
Summer pruning to reduce wood growth and promote bearing is practiced to a limited extent in some districts upon varieties inclined to shy bearing. In regions of the most intense summer heat, less pruning is admissible than in the coast and elevated regions. It is necessary that the foliage be dense to protect the tree and the fruit from sunburn. Nor does the tree seem to relish cutting back. Slight thinning out if the tree becomes too brushy, seems to be the best treatment in some of the hot valleys.

Thinning the Fruit.-One of the most important items in the handling of an apple orchard is the faithful thinning out of the fruit of all varieties which are prone to over-bear, and this work is now regularly provided for by the leading commercial growers. Only one apple should grow at a place and spacing of four to six inches is commended. Although this work is tedious and expensive, it is profitable, because of the improved price which can be had for the larger fruit which will be secured, and it is desirable in the effects of thinning on the tree. It will be relieved from the exhaustion of overbearing, induced to yield annual crops, and often saved from breaking down with a too heavy burden.

Cultivation and Irrigation.-All that has been urged in measures to secure adequate moisture supply has full force with the apple. Excepting the early varieties, it is a fruit with a long growing season and therefore requires continuous moisture to secure size and quality. Most California apples are grown on deep, retentive soils in regions of large rainfall and if this is conserved by thorough cultivation, good fruit can be secured. It is doubtless true, however, that apples in California would sometimes be improved by irrigation just as they are in interior and mountain districts.

Fertilizers have been thus far but little used in California apple orchards but they are manifestly needed.

There has arisen recently evidence of the unfinished character of the fruit in some districts because it has shown blemishes after picking and during shipment which can not be attributed to any parasitic encroachment. This is probably due to some unfavorable condition in the local climate or to some other stress upon the tree which prevents it from doing perfect work.

\section{GATHERING AND STORING APPLES}

The disposition in this State, as elsewhere, is to allow the fruit to hang too long upon the tree before gathering. It was long ago demonstrated that an apple for long-keeping must be picked a little in advance of full maturity. As late fall weather in California is so delightful, there is more temptation to delay the picking than where the approach of winter admonishes the grower to get his fruit under cover. Picking apples for shipment should be done just when the seeds begin to blacken and when the fruit yields to pressure. If left on until fully ripe, and the seeds all black, the fruit is apt not to keep well. This rule applies to fall apples for shipment to distant markets, or for apples to be stored at home. 


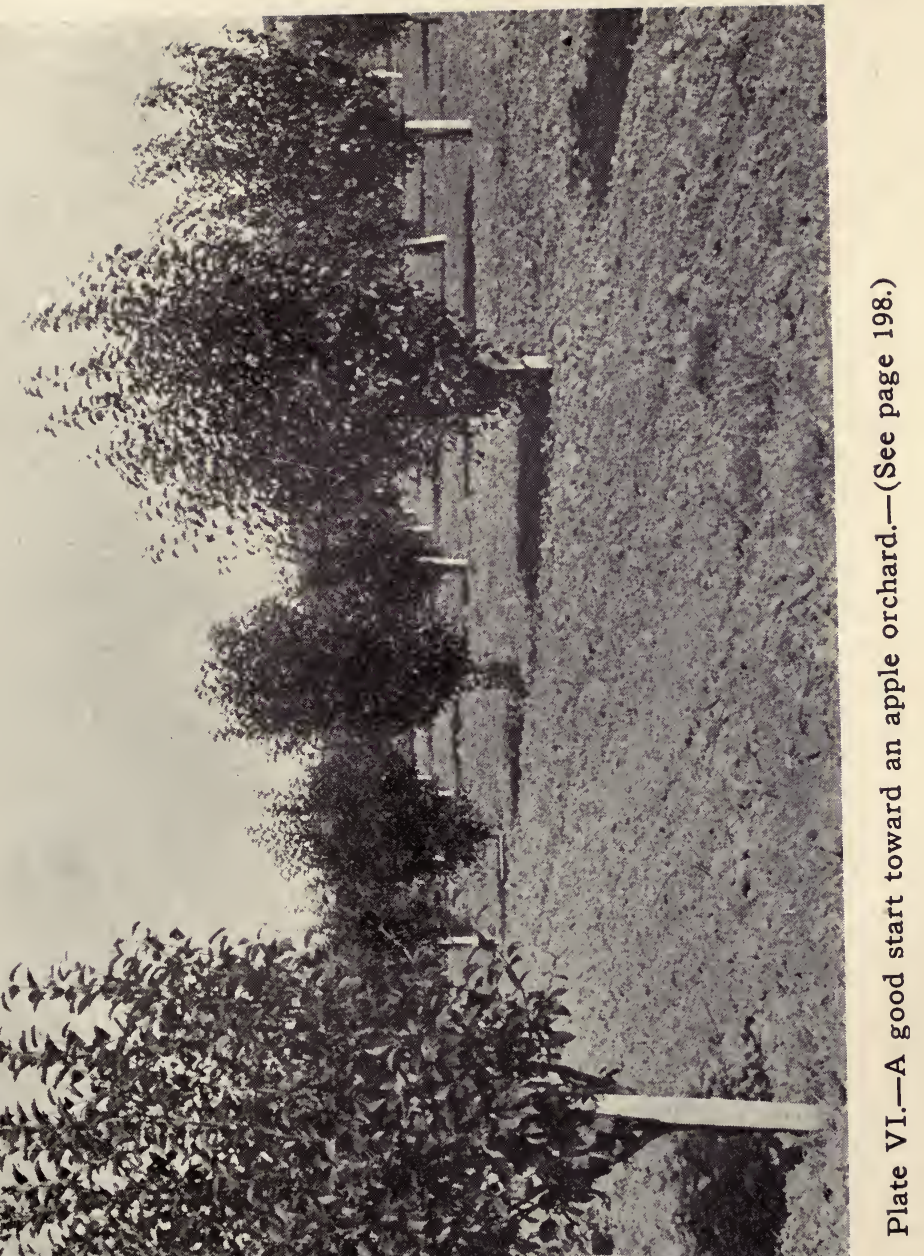



Mr. H. G. Keesling of Edenvale, Santa Clara County, gives a sketch of his way of handling apples on a small scale: In picking apples we insist on just as careful handling as in picking other fruits, and we find that the picking pail made of tin or light galvanized iron, holding about twelve quarts, or nearly twenty pounds of apples, is the best vessel to pick in, and we use them right through the season for cherries, apricots, peaches and even prunes. A pail of this size is not too heavy to handle even on high ladders, and it carries the fruit without bruising. Our plan is to pick and sort into boxes in the orchard. If a number of pickers are at work, then one or more men will do the sorting. As each picker fills his pail, he carries it a short distance to the sorting station, taking an empty one and returning to his work. The apples are sorted out of the pails and very carefully examined. The perfect apples go into one box, second into another and culls into another. They are then loaded onto a truck or wagon with springs and hauled to the house. A good sorter will keep pails empty for several pickers, all of course depending on the crop, size of apples, etc. I put my winter apples in redwood boxes, which, when piled one on top of another, five or six high and close together and covered with canvas or muslin, are in a condition to keep their flavor and juiciness a long time. Storing apples in boxes saves a lot of work in handling if they are to be examined or sorted during the winter.

Nearly all the ways of keeping winter apples have been tried in California. The main difficulty in keeping apples in good condition during the dry months of the autumn is the loss of moisture from the fruit by evaporation. This causes shriveling and operates against long keeping. It has been found by experience that apples keep perfectly until late in the spring by piling under the trees and covering with leaves, etc., allowing the rains to fall upon them. When dry north winds blow, the fruit should be sprinkled occasionally. They come out from the cover fresh, smooth, and plump, and for family use such rough storage will often answer a good purpose. For commercial storage, even on a small scale, however, good fruithouses are used. The requisites of such houses are an evenly cool temperature, moist air, and good ventilation, the fruit being open to free access of the air. In the mountains where stone is abundant excellent apple houses are made of it, which resist temperature changes notably.

Mr. Edward Berwick, of Monterey, apple grower of experience in the coast region, handles his fruit in this way:

The apples are carefully handpicked into baskets and at once transferred to ordinary apple boxes-just put in loose, not packed tight as for shipping. These boxes are hauled to the fruit houses with as little jar as possible.

This fruit house is built of rough boards (fastened on a heavy frame) with inch-thick battens covering the cracks, and rustic-nailed outside the battens, thus leaving an inch air-space between the boards and the rustic. It is of two storiesthe upper devoted to tools and stores, the lower used for fruit, and arranged with shelves accordingly. This lower story has only an earthen floor. One object of this is to give no lodgment for rats or mice, the other is to serve as a means of maintaining a cool, damp atmosphere. To this end it is kept well watered in apple-keeping season; and, to avoid mildew or mold, it is also liberally sprinkled with ground sulphur. By day, doors and windows are mostly kept shut, by night open; this, of course, is to exclude the heat and allow free circulation of the cool night air. 
A rather more open house is used in the coast region of southern California, by Mr. T. W. Ward, of Carpinteria :

It is a slat house made of strips $1 \times 2 \frac{1}{2}$ inches, put on one inch apart. The roof is similarly constructed. There are two passages, on either side of which are two shelves, one above the other, $i . e$., eight in all. The shelves are made of slats placed one-half inch apart, with sides a foot high. The apples are spread on these shelves a foot or more deep. The floor is made of slats, and there are bins on this also. The first must receive a thorough sprinkling weekly, unless sufficient rain falls. The slats are close enough to prevent birds doing damage, and the whole building is raised six inches from the ground.

In the monutain regions arrangements must be made for frost exclusion-a consideration which does not apply to the valley and coast.

\section{PICKING AND PACKING APPLES ON A LARGE SCALE}

Mr. C. H. Rodgers, whose pruning prescription has just been cited, gives the best methods of handling apples for market as follows:

In the matter of picking, experience has evolved a number of rules which should be strictly adhered to:

(1) Do not pull the apple off the tree. By so doing, the stem may be detached from the apple, thus making a second grade of what otherwise would be choice.

The proper method of plucking the apple is to grasp it with the full hand, not with the fingers only, and by a gentle twist and lateral movement detach it with the stem attached. Especially must finger pressure be avoided in the picking, as bruises thereby produced injure the value.

(2) The apple must never be dropped into a receptacle or from box to box, but should be transferred as carefully as so many eggs.

(3) Under all circumstances use vehicles having springs in moving the fruit.

Once within the packing-house the more perishable varieties should be handled immediately and forwarded to market, while the long-keeping varieties, especially those intended for export, should be held at least a month before sorting and packing. This latter precaution enables the packer to discover and eliminate all diseased and defective fruit-a thing that would be impossible if the fruit were packed at an early date after picking.

Three grades or qualities are recognized in the "trade"-first, second and third. First grade includes only perfect fruit. Second grade includes the fruit having a trivial surface blemish or stem absent. The third or cull class includes all wormy, badly bruised or skin-broken apples.

Though grading for size varies somewhat in different localities, in the Watsonville district, the leading apple producing center of the West, there are but three sizes recognized. These are $3 \frac{1}{2}, 4$, and $4 \frac{1}{2} 2$ tier. The unit of size is the 4-tier, which comprises all apples running from $25 / 8$ to $3^{\mathrm{T}} / 4$ inches in diameter, and derives the name from the fact that when packed in the box there are four rows of four apples each, both vertically and horizontally across the end of the box. Apples in excess of $3 \frac{1}{4}$ inches are classed as $3 \pi / 2$-tier size. The third size, or $4 \mathrm{~T} / 2$-tier, includes those apples ranging between $2 \mathrm{~T} / 4$ and $25 / 8$ inches in diameter. Both the $3 \mathrm{~T} / 2$-tire and $4 \mathrm{~T} / 2$-tier are packed in the manner known as "diamond" pack or "pear" pack. Apples smaller than $4 \mathrm{~T} / 2$-tier are thrown into the cull pile. The sorter ascertains the size by passing the apples through circular holes in a board.

In this state the standard box is made of pine. Its measurements are $93 / 4$ by 11 by 22 inches, and it holds about 50 pounds of fruit. A modified box of extra thick material, reinforced by iron straps, is frequently used for export trade. Redwood boxes are used only for cheap grades of apples packed for the local market.

After being sorted, the apples are passed to the packer, who, before placing them in the box, wraps each apple in a piece of paper prepared for the purpose. 
The apples must be so packed in the box as to permit the nailing firmly of the lid at each end, and at the same time allow a gradual swell of about threefourths of an inch at the middle of both top and bottom. On account of the resultant shape of the boxes, they can be stacked up with safety only on their sides.

The packed boxes after being neatly labeled, are next transferred to the cars and stacked four or five tiers high. An air space of three or four feet is left between the top tier and the roof of the car, also the entire space between the doors is left vacant for the better circulation of air. The boxes, after being systematically placed in the car are so braced with timbers as to prevent any movement. The usual carload consists of about 650 boxes. Refrigerator fruit cars are employed mainly for apple shipment, but no ice is used.

Summer and Fall Apples.-In some regions noted for early maturing of fruit, it is profitable to grow early apples, providing there are facilities for reaching profitable avenues of trade. Except to minister to some special local or distant trade which can be thus foreseen, it must be said that very early apples are hardly worth the attention of the commercial planter. These sorts are apt to come into direct contest with the magnificent peaches, grapes and other summer and autumn fruits, and suffer thereby.

On the other hand the fall apples, chiefly the Yellow Bellflower and Gravenstein, are so good and profitable in regions where they bear well that they are among the varieties which constitute our chief commercial reliance. Bellflowers are also encroaching on the field of winter apples because they come out so well from cold storage.

Winter Apples.-For large ventures in apple growing, in localities carefully chosen for especial adaptations, a few of the finest varieties of winter apples should generally be selected. It is the judgment of the most experienced apple growers, many of whom have old orchards including many varieties, that new plantations of winter apples should contain only about six sorts. Of these, in all parts of the State, two would be the Yellow Newtown Pippin and White Winter Pearmain; the other four would vary in different parts of the State, as can be learned from the table which will follow.

Apples for Long Shipment.-There has been for years quite an important trade in shipment of California apples to various ports in the South Pacific Ocean, and recently there has been a sharp demand for California apples for shipment to the eastern states and England. The characteristic size, quality and keeping of the fruit, together with the size and style of package, have strongly commended the fruit. The center of this trade is Watsonville, in a coast valley, in the central part of the State. The two apples which are most popular are the Yellow Bellflower and the Yellow Newtown Pippin. It is an interesting fact that these varieties by virtue of quality have overcome the popular fervor for a red apple, though at Watsonville some Red Pearmains are grown to fill orders which insist on having some color.

For the Interior Valleys.-In choosing varieties for the hot valleys of the State those making a heavy leaf growth are to be preferred. The Spitzenberg, for example, is a failure in the valleys of the interior, though satisfactory at points on the valley borders. From experience already had it seems likely that some of the Russian varieties, with 
thick, large leaves, will prove best for such situations. The behavior of the Astracans, the Duchess of Oldenburg, and others of Russian origin, are illustrations of this fact. Other varieties have been on trial for several years, but no great distribution of them has yet been attained.

\section{SELECTION OF VARIETIES FOR CROSS-POLLINATION}

Association for cross-pollination can be arranged with a number of our most popular varieties by consulting the following dates of blooming as prepared by Mr. Frederick Maskew based upon observations in the coast region of Los Angeles County.

Blooming Season of a Group of Popular Varieties.

\begin{tabular}{|c|c|c|}
\hline First bloom. & Full bloom. & $\begin{array}{c}\text { General } \\
\text { fall of bloom }\end{array}$ \\
\hline White Winter Pearmain & April 27 & May \\
\hline Red Astracan & April 30 & May \\
\hline Bellflower ... & April 30 & May \\
\hline Fall Pippin & May & May \\
\hline Rhode Island Greening & May & May \\
\hline Kentucky Red Streak... & May 10 & May \\
\hline Early Harvest...$\ldots \ldots$ & May & May \\
\hline Shockly ................. & May 15 & May \\
\hline Fameuse $\ldots \ldots \ldots \ldots \ldots \ldots \ldots$ April 27 & May 15 & May \\
\hline Ben Davis .......................... April 29 & May 15 & May \\
\hline$\ldots \ldots \ldots \ldots \ldots \ldots$ May 5 & May 17 & June \\
\hline Yellow Transparent $\ldots \ldots \ldots \ldots \ldots . . .$. May & May 16 & June \\
\hline None-such $\ldots \ldots \ldots \ldots \ldots$ May & May 16 & June \\
\hline Missouri Pippin ................. May 10 & May 20 & June \\
\hline Alexander ........................ 15 & May 25 & June \\
\hline Smiths Cider .................. May 15 & May 25 & June \\
\hline Transcendent Crab ................ Mar. 30 & April 7 & April 2 \\
\hline Hyslop Crab ..................... April 11 & April 22 & April \\
\hline Montreal Crab ............ & April 24 & May \\
\hline
\end{tabular}

This is a later range of bloom than will be found in many parts of the State, but the same relation may be expected everywhere.

\section{VARIETIES OF APPLES CHIIEFLY GROWN IN CALIFORNIA}

Of the hundreds of varieties tested in California comparatively few are now grown, as has already been suggested. Those named below have been reported by growers as succeeding in localities named with the description, or indicated in the table which will follow. The arrangement is, approximately, in the order of ripening.

Carolina Red June (Southern).-Medium size oval, irregular, inclined to conic; deep red covered with light bloom; slack in small cavity; calyx closed; flesh white, tender, juicy, subacid; core rather large.

Early Harvest (American).-Medium size, roundish; straw color with few faint white dots; stalk half to three-fourths inch, slender, set in moderate cavity; calyx in shallow basin; flesh very white, tender, crisp, pleasant. 
Early Strawberry (New York).-Medium size, roundish, narrowing toward the eye; skin smooth, deep red on yellow ground; stalk one and a half inches, rather slender and uneven, in deep cavity; calyx small, in shallow basin; flesh white, tinged with red next to the skin, tender, subacid, sprightly.

Red Astracan (Russian).-Large roundish; skin deep red, save greenish yellow in the shade; pale white bloom; stalk short, and deeply inserted; calyx partially closed and set in slight basin; flesh white, juicy and crisp, pleasant acid; tree hardy and vigorous, and an early bearer. The main reliance in California for an early apple.

White Astracan (Russian).-Large, roundish; skin smooth and nearly white, with faint streaks of red, and covered with white bloom; flesh white. Considerably grown in the Sacramento Valley and foothills for early shipment. Sometimes attains a weight of 29 ounces. Excellent in the Modesto district of the San Joaquin Valley.

Duchess of Oldenburg (Russian).--Large, roundish, oblate; yellow, streaked with red; calyx large, nearly closed, set in wide, even hollow; flesh juicy, subacid.

Gravenstein (German).-Large, rather flattened; a little one-sided or angular; broadest at base; stalk short, strong, deeply set; calyx large, closed, in a large basin; skin yellow, freely marked with light and deep red and orange; flesh tender, crisp, highly flavored, aromatic; a strong-growing and heavily-bearing tree; a standard fall apple in this State.

Red Bietigheimer (German).-Large to very large, oblate, slightly conical, regular, smooth, whitish or yellowish white, shaded with light and dark red, and purplish crimson in the sun; stalk short, rather stout, calyx closed in large, deep, slightly corrugated basin; flesh white, firm, juicy, brisk subacid. Declining in favor.

Maiden's blush (New Jersey).--Rather large, smooth, regular; yellow, with evenly shaded red cheek; stalk short, in rather wide, deep hollow; calyx closed in moderate depression; flesh white, tender, sprightly.

Fall Pippin.-Very large, roundish, a little flattened; stalk three-fourths inch, projecting considerably beyond the fruit (which distinguishes it from the Holland Pippin); calyx open, not very large, rather deeply sunk in round, narrow basin; skin smooth, yellowish green, becoming pure yellow; brownish blush and few scattered dots; flesh white, tender, mellow, rich, aromatic.

Alexañder (Russian).-Very large, showy, conical, greenish yellow, streaked with red in shade, bright red in sun; calyx large, in deep basin; stalk slender, long, in deep cavity; flesh yellowish white, crisp, tender, and juicy. Three vigorous, but not always a good bearer.

Gloria Mundi.-Very large, roundish, oblate; ribbed; greenish yellow. A popular show apple on account of great size attained in this State. (See table.)

Fameuse; syn. Snow Apple (Canada).-Medium size, roundish, somewhat flattened; deep crimson, nearly concealing pale yellowish ground; flesh snowy white, tender, juicy, slight perfume; stalk slender, one-half inch, in narrow funnel-shaped cavity; calyx small, in shallow, rather narrow basin; "tree vigorous, with dark wood; one of the finest desert fruits; succeeds particularly well in the foothills."-John Bidrvell.

King of Tompkins County.-Large, globular, angular, inclining to conic; yellowish, mostly shaded with red, striped and splashed with crimson; stalk short and stout, in large, somewhat irregular cavity; calyx small, closed; flesh yellowish, rich, juicy, vinous, aromatic; chiefly grown in mountain regions.

Rambo (Pennsylvania).-Medium to large, flat; yellowish white with pale yellow and red in the sun, with large, rough dots; stalk long, rather slender, curved, deeply set; calyx closed; in broad basin; flesh greenish white. Reported a failure in some counties.

Ben Davis.-Large, roundish, sides often unequal; light red and deep red on yellowish ground; stalk medium, rather slender, in deep, narrow cavity; calyx 
partially open. Commended as a market apple by the Southern California Nurserymen's Association. Grown in the upper Sacramento Valley; sometimes keeps until July 1.

Baldwin (Massachusetts).-Large, roundish, narrowing a little toward the eye; deep bright red over a yellow ground; a few russet dots; calyx closed and set in narrow basin; stalk one-half to three-fourths inch, rather slender, set in deep, even cavity; flesh yellowish-white, crisp, juicy, subacid. Best in northern and elevated regions; coloring varies greatly according to locality.

Hoover (South Carolina).-Large, roundish, slightly oblique; yellowish, mostly overspread with red, with conspicuous light dots; stalk rather long, in large cavity; calyx open in furrowed basin; flesh yellowish, juicy, crisp, acid.

Rhode Island Greening.-Large, roundish, a little flattened, pretty regular; dark green, becoming yellowish green; calyx small, woolly, closed, in shallow basin; stalk three-fourths inch, curved, thickest at the bottom; flesh yellow, fine grained; tender, crisp, juicy, aromatic, slightly acid; tree healthy and the variety widely popular.

Vandevere; syn. Newton Spitzenburg.-Medium size, oblate, slightly conic; fine yellow, washed with light red, striped and splashed with dark red and shaded with carmine in the sun; light bloom and peculiar gray specks; stalk short, in wide cavity; calyx small; flesh yellow, rich, sprightly, vinous.

Jonathan (New York).-Medium to large, roundish, conical or tapering to the eye; light yellow, nearly covered with red stripes and deep red in the sun; stalk three-fourths of an inch, rather slender, in deep, regular cavity; calyx in deep, broad basin; tender, juicy, rich, vinous; a great favorite in California; specially commended as a market apple by Southern California Nurserymen's Association; keeps till midwinter.

Winesap.-Medium size, roundish oblong; dark red with traces of yellow in the shade; stalk nearly an inch, slender, set in an irregular cavity; calyx small, in regular basin; flesh yellow, crisp, high, rich flavor; largely grown; tree a good bearer.

Stayman Winesap.-An old improvement on the Winesap now becoming more prominent. Some growers reporting favorablly on Winesap have this variety, which is larger and better, and the tree a stronger grower and more productive. Approved by Mr. Frank Femmons, of Madera county.

Ortley; syn. White Bellflower, etc. (New Jersey).-Large, oblong, greenish yellow, becoming fine yellow with slight blush; stalk medium, slender, set in deep, acute cavity; calyx closed, set in abrupt corrugated basin; flesh white, finegrained, juicy, subacid.

Swaar (New York).-Large, roundish; golden yellow with numerous brown specks; stalk slender, three-fourths inch, in very round cavity; calyx small, greenish, set in shallow basin; flesh yellowish, fine-grained; very rich, aromatic flavor and spicy smell.

Lawerer.-Large, roundish, oblate, dark red, covered with small dots; stalk medium, cavity deep, regular; calyx small, closed in medium furrowed basin; flesh white, sprightly, aromatic; a promising, late keeping variety.

Yellow Bellflower (New Jersey).--Very large, oblong, irregular, tapering toward the eye; smooth; lemon color, with blush; stalk long and slender, in deep cavity; calyx closed, in rather narrow basin; flesh tender, juicy, crisp, with sprightly subacid flavor; keeps well into the winter; tree a strong grower and healthy; one of the universal favorites of California.

Romanite.-Small to medium, roundish, conical, truncated; yellow, mostly covered with clear, handsome red; indistinct light dots; stalk slender; calyx in an abrupt basin: flesh yellowish, fine-grained, juicy, pleasant, subacid.

Esopus Spitzenburg (New York).-Large, oblong, tapering roundly to the eye; smooth, nearly covered with rich, lively red, dotted with distinct yellowish russet dots; on shaded side, yellowish ground with streaks and broken stripes 
of red; stalk rather long, three-fourths inch, slender, projecting beyond the base and inserted in wide cavity; calyx small and closed, in shallow basin; flesh yellow, rather firm, crisp, juicy, with a delicious rich, brisk flavor. A largely grown variety; tree a good, upright grower and healthy; fruit keeps fairly.

Hyde King.-Very large, glistening golden yellow with blush, very handsome. Ripens October 15 th in Humboldt county. Approved by A. F. Etter.

Smith's Cider (Pennsylvania).-Large, roundish, oblate conic; yellow, shaded and striped with red, sparsely covered with gray dots; stalk slender, in deep, rather narrow cavity; calyx closed, in broad, shallow basin; flesh whitish, juicy, crisp, acid; tree a strong grower, and fruit keeps till midwinter.

Rome Beauty (Ohio).-Large, roundish, approaching conic; yellow, shaded and striped with bright red, sprinkled with light dots; stalk one inch, in large, deep cavity; calyx partially closed, in deep, narrow basin; flesh yellowish, juicy, sprightly; fruit keeps late. Particularly fine in the mountain valleys of Southern California.

Missouri Pippin (Missouri).-Large, roundish oblate, slightly oblique, somewhat flattened at the ends; shaded, striped and splashed with light and dark red, often quite dark in the sun; many large and small gray dots; stalk short, small; cavity large, deep; calyx closed or half open, basin rather abrupt deep, slightly corrugated; flesh whitish, rather coarse, moderately juicy, subacid. Quite largely planted, but losing favor for lack of keeping quality in coast valleys.

Nickajack (North Carolina).-Large, roundish to roundish oblate, slightly conic, sometimes oblique; yellowish, freely striped and splashed with red, many large dots; stalk short, in large cavity; calyx partly open; flesh yellowish, fair quality; reported a shy bearer in high altitudes.

Northern Spy (New York).-Large, roundish, oblate, conical; pale yellow, purplish red stripes in the sun; stalk three-fourths inch, slender, in wide, deep cavity; calyx small, closed; flesh white, mild, pleasant; highly esteemed in a few localities, but abandoned in others for shy bearing.

White Winter Pearmain.-Large, roundish, oblong conic, somewhat oblique; pale yellow with slight blush, many minute brown dots; stalk short in deep cavity; calyx nearly closed; flesh yellowish, tender, crisp, juicy, very pleasant subacid, extra high flavor; grown everywhere and fruit keeps late; tree a strong grower and healthy. Greatly advantaged by cross-pollination.

Lady (French).-Small, regularly formed, flat; smooth and glossy, with brilliant red cheek contrasting with lemon yellow ground; flesh white, crisp, juicy and pleasant; chiefly used for ornamental purposes.

Black Ben Davis.-Resembles Ben Davis in tree and fruit, except that the latter is deeper colored, more symmetrical and of better quality. Highly praised by Mr. Frank Femmons, Madera county.

Delicious.-Resembling Bellflower; yellow; almost covered with dark red; very mild acid, quality good; a late keeper. Strongly approved in elevated districts.

Arkansas Beauty (Arkansas).-Medium to large, oblong conical, yellow and red stripes; fine, juicy, very good, subacid. Approved in Southern California.

Rawles Janet (Virginia).-Medium to large, oblate conic, yellowish, shaded with red and striped with crimson; stalk short and thick, in broad, open cavity; calyx partially open, in shallow basin; flesh yellow, tender, juicy, pleasant vinous flavor; tree healthy and prolific.

Stark.-Large, roundish, inclined to conic; sometimes elongated, sometimes oblique; greenish yellow, nearly covered with dark red or sprinkled with light and brown dots; stalk short, rather stout; calyx closed; flesh yellowish.

Yellow Newtown Pippin.-Large, roundish, oblate and oblaque, more or less flattened yellow with brownish red cheek; stalk very short; flesh firm, crisp, juicy, and with very rich, high flavor. Generally considered the best winter apple in California. 


\section{CRAB APPLES}

Hyslop.-Fruit large, growing in clusters; roundish ovate; dark rich red. covered with thick blue bloom; stalk long, slender; calyx closed; flesh yellowish.

Large Red Siberian.-Roundish ovate with large and prominent calyx; pale red and yellow skin.

Large Yellow Siberian.-Fruit similar in size to foregoing, roundish oval, flattened at base and crown; light clear yellow, inclining to amber, with warm cheek.

Transcendant.-Medium to large, roundish oval, flattened at the ends, slightly but regularly ribbed; golden yellow, with rich, crimson cheek, or nearly covered with red; delicate white bloom; stalk long and slender, in open, deep cavity; calyx closed; flesh creamy yellow.

Montreal Beauty.-Large, roundish, oblate; bright yellow, mostly covered and shaded with red; one of the most beautiful of crab apples.

Whitney's Crab.-Large, handsome, greenish yellow, striped with crimson.

\section{CALIFORNIA SEEDLING APPLES}

Skinner's Seedling (name approved by California State Horticultural Society, November, 1887); syn. Skinner's Pippin, Santa Clara King.-Originated with Judge H. C. Skinner, of San Jose. Fruit large to very large; form oblate, conic, slightly mixed; color rich lemon yellow, faintly striped with bright red; flesh yellowish white, very tender, juicy, sprightly, mild subacid; quality best. Season, September and October.

Marshall's Red.-Originated with J. L. Marshall, Brown's Valley, near Napa. Fruited first about 1877; introduced by Leonard Coates in 1884; no longer propagated.

Magoon.-Large, roundish oblong, deep red shading to light red on yellow ground, resembling Esopus Spitzenburg in form, flesh and flavor. Found in 1893 on place formerly occupied by S. E. Magoon, near Ahwahnee. Named by Frank Femmons.

Cook's Seedling; syn. Sonoma Seedling.-Brought to notice by O. B. Shaw, of Sonoma, as a seedling raised by David Cook from the seed of the Juneating. Above medium size, pale yellow striped with red; sharp acid flavor. Chiefly grown in Sonoma and Napa counties.

Lawton.-Seedling on place of Mrs. F. H. Lawton, one-half mile north of Sebastopol, Sonoma county. More symmetrical than Belmont or Waxen. Very promising show variety.

Tabular Showing of Adaptations.-An attempt is made to district the State for the apple, and for the other fruits which follow, in accordance with the scheme of climatic conditions described in Chapter I. This groups regions of nearest resemblance, and is more rational than any prescription according to county lines can be, for though some counties lie wholly in one climatic division, many more counties extend through two, and some even through three, such divisions. It is, therefore, a more promising proposition to encourage planters in any locality to study their climatic adaptations, not with regard to county lines but rather as related to the conditions of elevation, ex. posure to ocean influences and other factors which characterize natural belts, or areas, of similar horticultural fitness. 
Apple Varieties Approved by California Growers.

\begin{tabular}{|c|c|c|c|c|c|}
\hline VARIETIES. & $\begin{array}{l}\text { Northern } \\
\text { Coast } \\
\text { Region. }\end{array}$ & $\begin{array}{l}\text { Central } \\
\text { Coast } \\
\text { Region. }\end{array}$ & $\begin{array}{c}\text { Interior } \\
\text { valleys } \\
\text { and } \\
\text { foothills. }\end{array}$ & $\begin{array}{c}\text { Mountain } \\
\text { valleys } \\
\text { and } \\
\text { plateau. }\end{array}$ & $\begin{array}{c}\text { Southern } \\
\text { California } \\
\text { Coast. }\end{array}$ \\
\hline Alexander & . $\quad \ldots$ & $*$ & $*$ & ... & $\ldots$ \\
\hline Arkansas Beauty ......... & . $\ldots$ & ... & $\cdots$ & ... & $* *$ \\
\hline Arkansas Black ......... & . $\quad *$ & $*$ & $* *$ & $\ldots$ & $*$ \\
\hline 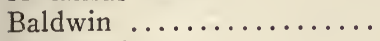 & $* *$ & $*$ & $*$ & * & * \\
\hline Ben Davis .............. & $*$ & * & $*$ & $* *$ & $*$ \\
\hline Bietigheimer.$\ldots \ldots \ldots \ldots$ & $*$ & $\cdots$ & $\cdots$ & $\cdots$ & $\cdots$ \\
\hline Black Ben Davis......... & - $\quad \cdots$ & $\cdots$. & $*$ & $*$ & $\cdots$ \\
\hline Cook's Seedling ........... & . $\quad \cdots$ & $*$ & $\cdots$ & $\cdots \dot{*}$ & $\cdots$ \\
\hline Delicious........... & . $\quad \cdots$ & $*$ & $*$ & $*$ & $\cdots$ \\
\hline Early Harvest .......... & - $\quad *$ & $*$ & $*$ & $\cdots$ & $*$ \\
\hline Early Strawberry ........ & . $\quad \ldots$ & $\cdots$ & * & $\cdots$ & * \\
\hline Esopus Spitzenburg ...... & $* *$ & $* *$ & $*$ & $* *$ & $*$ \\
\hline 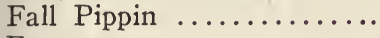 & . $\ldots$ & $*$ & $*$ & $\cdots$ & ** \\
\hline Fameuse...$\ldots \ldots \ldots \ldots$ & ** & * & * & ..* & $*$ \\
\hline 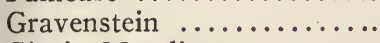 & $* *$ & $* *$ & $* *$ & $*$ & $* *$ \\
\hline Gloria Mundi ........... & . $\ldots$ & $\cdots$ & $*$ & $\cdots$ & $\cdots$ \\
\hline Hoover ................ & . $\quad \ldots$ & $* *$ & $*$ & ... & ... \\
\hline Hyde King ............. & * & -.. & $\cdots$ & .. & $\ldots$ \\
\hline Jonathan..$\ldots \ldots \ldots \ldots$ & $*$ & ** & $* *$ & $* *$ & ** \\
\hline 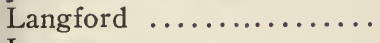 & - $\quad \cdots$ & $*$ & $*$ & $\cdots$ & $\cdots$ \\
\hline Lawver ................ & $*$ & * & $\cdots$ & $\ldots$ & ... \\
\hline Maiden's Blush ......... & . $\quad \ldots$ & $*$ & $*$ & .. & $*$ \\
\hline Marshall's Red .......... & .. $\quad \cdots$ & $*$ & ... & $\cdots$ & * \\
\hline Missouri Pippin ......... & . $\quad *$ & $*$ & .. & .. & $*$ \\
\hline Nickajack .............. & - $\quad \cdots$ & $\cdots$ & $\cdots$ & $\cdots$ & * \\
\hline Northern Spy $\ldots \ldots \ldots \ldots$ & - $\quad \cdots$ & $\cdots$ & $\cdots$ & $*$ & $\cdots$ \\
\hline Oldenburg Duchess ...... & . $\quad *$ & $\cdots$ & $*$ & $*$ & * \\
\hline Ortley $\ldots \ldots \ldots \ldots \ldots \ldots$ & . $\cdots$ & $*$ & * & $\begin{array}{l}* \\
*\end{array}$ & * \\
\hline Rawles's Janet........... & - $\cdots$ & $\cdots$ & **ं & $*$ & ** \\
\hline Red Astracan $\ldots \ldots \ldots \ldots$. & * & * & $* *$ & $*$ & $\begin{array}{l}* * \\
*\end{array}$ \\
\hline $\begin{array}{l}\text { Red June } \ldots \ldots \ldots \ldots \ldots \ldots \\
\text { Red Pearmain } \ldots \ldots \ldots \ldots\end{array}$ & & * & * & ... & \\
\hline $\begin{array}{l}\text { Red Pearmain } \ldots \ldots \ldots \ldots \\
\text { Rhode Island Greening.... }\end{array}$ & $* *$ & * & * & $\ddot{* *}$ & $\ddot{* *}$ \\
\hline $\begin{array}{l}\text { Rhode Island Greening.... } \\
\text { Romanite } \ldots \ldots \ldots \ldots \ldots \ldots\end{array}$ & . $\ldots$ & & $\cdots$ & $*$ & $*$ \\
\hline $\begin{array}{l}\text { Romanite } \ldots \ldots \ldots \ldots \ldots \ldots \\
\text { Rome Beauty } \ldots \ldots \ldots \ldots \ldots\end{array}$ & . $*$ & $\ddot{* *}$ & $* *$ & ** & $* *$ \\
\hline & * & * & * & * & $\ldots$ \\
\hline $\begin{array}{l}\text { Smith's Cider } \ldots . . . \ldots \ldots \ldots \\
\text { Skinner's Seedling . . . . . }\end{array}$ & . & * & ... & $\ldots$ & 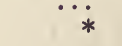 \\
\hline $\begin{array}{l}\text { Skinner's Seedling } . \ldots \ldots \ldots \\
\text { Stark } \ldots \ldots \ldots \ldots \ldots \ldots\end{array}$ & . & $\ldots$ & $\because *$ & $\cdots$ & * \\
\hline 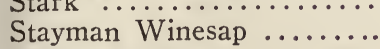 & $\cdots$ & .. & * & $*$ & * \\
\hline Tompkins King .......... & $* *$ & * & $*$ & ** & \\
\hline Vandevere $\ldots . \ldots \ldots \ldots$. & $\ldots$ & ... & $*$ & ‥ & * \\
\hline Wagener ............... & ** & ** & .. & $\ldots$ & * \\
\hline Wealthy $\quad . . \ldots \ldots \ldots \ldots$ & * & $\cdots$ & $*$ & $*$ & ... \\
\hline White Astracan ........... & $\because$ & * & ** & $\cdots$ & $\cdots$ \\
\hline White Winter Pearmain... & * & $* *$ & ** & $*$ & ** \\
\hline Winesap $\ldots \ldots \ldots \ldots \ldots$ & * & $* *$ & $* *$ & & ** \\
\hline Winter Banana ............ & $\cdots$ & $*$ & ** & $*$ & * \\
\hline Yellow Bellflower ......... & $* *$ & $\begin{array}{r}* \\
* *\end{array}$ & $* *$ & ** & $\begin{array}{l}* * \\
* *\end{array}$ \\
\hline Yellow Newton Pippin..... & $\begin{aligned} * * \\
*\end{aligned}$ & $* *$ & ** & ** & $\begin{array}{r}* * \\
*\end{array}$ \\
\hline Yellow Transparent ....... & $*$ & * & $*$ & * & * \\
\hline York Imperial ........... & $*$ & $\cdots$ & $\cdots$ & * & $\cdots$ \\
\hline
\end{tabular}

${ }^{*}$ Indicates that the variety is approved in the region resignated. ** Most highly commended. 


\section{CHAPTER XVIII}

\section{THE APRICOT}

California has nearly three million apricot trees which stand in the open air without protection of any kind and bear large, luscious fruit. That apricot trees can do this constitutes one of the unique features of California fruit growing and proclaims it different from fruit growing in other States, for, excepting a few localities in other parts of the Pacific slope, California has a monopoly of commercial apricot growing, and nowhere else in the world does the fruit attain such commercial importance. Although the apricot has been grown here from the earliest days of the American occupation, and though since the opening of the export trade in canned and dried fruits, the apricot has gained in popularity, the planting of apricot orchards has not proceeded recently with great rapidity, although indications are that our distant patrons are only just beginning to recognize the desirability of the fruit, and their demands will make it well-nigh impossible for us to extend our production beyond profitable limits. The reason by the apricot has not kept pace with the advance of some other fruits in California is to be found in certain limitations of suitable area which will be mentioned presently.

Though the apricot has some pests and diseases to contend with, they have thus far proved slight evils, and the tree is generally regarded as one of the healthiest and most vigorous, as it certainly is one of our most beautiful orchard trees. It is long-lived and attains great size There are here and there groups of trees nearly half a century old which have a height of fifty feet; the main trunks like forest oaks, and the first branches or limbs twelve and fifteen inches through. The smaller limbs and foliage are at least fifty feet across; a half dozen of them shade an acre of ground and they sometimes yield per tree a ton of fruit. But such trees do not meet orchard requirements and are only mentioned to show what the tree may do when it has its own way.

The apricot is a rapid grower and an early and heavy bearer in California. In the interior and in the southern coast valleys it yields a paying crop during its third summer in the orchard, and from eight to fourteen tons to the acre was reached for several years in succession, in Judge Blackwood's old orchard of Royal apricots, in Alameda County. The trees, even of some varieties which are uncertain bearers, are large and vigorous growers, and have warranted the suggestion that there is a use for the apricot tree for a windbreak for the protection of other trees. The trees may be planted near together in strong land and make a windbreak that will pay its way without regard to such fruit as it may incidentally produce.

Apricots are chiefly marketed as a dried fruit and the operation of drying will be described in the chapter devoted to such processes. The amount used in canning is, in a year of full production, about onequarter of that for drying, while the weight of fruit sold fresh to consumers, near and far, is about one-quarter of that used by the canners. 


\section{LOCALITIES FOR THE APRICOT}

In discussing localities for the apricot, reference is, of course only made to its growth as a standard orchard tree without protection of any kind. It shows even in California that it does not forget the conditions which destroy its thrift elsewhere, for late frosts in our upper coast counties render it, as a rule, unprofitable. Speaking broadly, the quarter of the State lying northward of the Bay of San Francisco and westward of the high ridge of the Coast Range is not suited for commercial apricot growing; though here and there are places where bearing may be regular and abundant enough to make trees satisfactory for home gardens. The mountain regions everywhere in the State above an elevation of about 1200 feet are also to be excluded. The lowest lands of the great interior valleys, except here and there, where frosts are prevented by proximity of broad streams of by favoring air currents, are unsuited for apricots, and the bottoms of small valleys whence cold air can not find drainage outlet, are also treacherous. It is evident then, that even in regions of general adaptation to the fruit local discrimination must be exercised in selecting lands for apricots, and the occurrence of spring frosts, which are usually governed by topography, must be guarded against. This is not the same problem which arises in the selection of land for citrus fruits, because apricots are not open to injury during December, January and February, and consequently they may be successfully grown in places where winter temperatures might injure the evergreen trees of the citrus family. Still, next to the almond, the apricot is most liable to frost injury of all our deciduous tree fruits, and commercial success depends largely upon the selection of a proper place for them. The occurrence of even light frosts during the blooming and setting, or soon after, may strip the tree of its burden of fruit without injury to even the softest of twig and leaf; consequently, regular bearing of the apricot can not be expected where the temperature is apt to fall four or five degrees below freezing point during the months of March and April, even though the duration of such temperature may be very brief. For this reason the area of California which is well suited to apricot growing is limited when compared with the great area of the State, though when counted by acres it is ample enough to supply all the fresh canned and dried apricots which the markets of the world can be expected to take at profitable figures.

It is often claimed that situations directly subject to ocean influences are best for the apricot. It is noted by many observers that the apricot "points its best branches to the ocean, in the very teeth of the constant breeze, and the landward limbs and twigs bend up and endeavor to reach the same direction. This is patent in every tree, and in the long orchard rows is very striking." This is taken to signify the special liking of the tree for the vicinity of the coast. It is well enough to interpret it that way, providing one does not lose sight of the perfect success of the apricot in the interior as well. It is true that the fruit near the coast attains higher color, and the less rapid growth of the tree makes it somewhat easier to handle, but the earlier ripening in the interior, coupled with freedom from fog and constant sun- 
shine for drying, are points of the highest industrial importance. The fact is that the apricot has a very wide range in California, and though the trees have been cut out at some points it has been chiefly because too frosty locations have been chosen or because some other fruit has seemed to be locally more desirable, for one reason or another.

In some valleys in the upper part of the State opening directly to the ocean, there is sometimes complaint of the cracking of the fruit on the sunny side. The alteration of sunshine and fog seems to have something to do with this, for in favorable years, when fogs are few, the fruit is sound.

Locations for early ripening of the apricot are to be chosen with reference to the influence of topography, as laid down in Chapter I. In a general way, it may be said, in regions directly subject to coast influences, both in northern and southern California, the apricot is late. On the west side of the Sacramento Valley, on slightly elevated places, in small, hill-locked valleys, the earliest apricots have been grown for years. Protected situations in the foothills of the Sierra Nevada, on the eastern rim of both the Sacramento and San Joaquin Valleys, share in the production of the earliest ripening fruit. There is probably about a month's difference in the ripening of the same variety in the earliest situations and in the coast valleys of both northern and southern California.

In the interior of southern California, in irrigated situations, on the west side of the Colorado River and in adjacent parts of Arizona, apricots rival in earliness the product of the famous valleys of interior northern California.

Recently a measure of success with the apricot has been attained in irrigated sections of eastern Washington, Idaho and Utah. If winter temperatures are low enough to keep the tree dormant and yet not injure the fruit buds and frosts are absent after growth begins, success ought to be attainable.

\section{STOCKS AND SOILS FOR THE APRICOT}

Because of the success with which the apricot can be budded on various stocks, it has a wide range in adaptation to different soils. Budded on the peach root it may be grown successfully on the light, warm, well-drained loams in which the peach delights. The peach root is, in fact, largely used for the apricot. It gives the tree quick growth and early fruiting, and the fact that the gopher does not like the peach root is a consideration with some planters. In growing stocks, pits of a strong-growing yellow peach are believed to yield more uniform and thrifty seedlings.

For deep, rich, well-drained, loamy soils, the apricot on its own root makes a magnificent tree. Apricot roots for budding are easily secured. The pits sprout as readily as corn. Sometimes, where cutting and drying are done in the orchard, the ground the next spring will be almost covered with a volunteer crop of seedling apricots. These little plants, taken up and set out in nursery rows in March, are ready for budding in June or July. Large numbers of trees are sometimes 
secured in this way. In the upper San Joaquin Valley there are situations in which the apricot seems more productive on its own roots than on the peach, and in the moister parts of the San Fernando and tributary valleys in southern California the apricot root has recently advanced in popularity. It is, however, rather more sensitive to soildrouth than the peach root.

When it is desired to grow the apricot in moister and heavier soils than have been described, or where a light soil is underlaid by a heavy, retentive subsoil, recourse should be had to the plum root. Only a non-suckering plum stock should be used. For this purpose the Myrobalan has been considerably used. Some growers complain that the root has a dwarfing effect on the tree, and object to its use. The manner of securing Myrobalan stocks has been described in the chapter on propagation.

Apricot and Almond.-The almond should as a rule be rejected as a stock for the apricot. Hundreds have tried it, and found that the scion never made a good union with the wood of the stock but was knit to it only by the bark, and is, therefore, easily broken off by the wind. It may grow well and sometimes gets to be two or three inches in diameter before it breaks off, thus wasting much time for the orchardist. Whole orchards worked in this way have been a loss and disappointment.

A few growers, however, approve the almond and use it with the idea that it gives larger fruit. It has been claimed that the Royal apricot will take well on the almond seedling by root grafting instead of budding, using the side graft. Cut off the top of the stock about four to six inches above ground, scrape away the dirt, bend the stock, and, with a sharp, thin knife, cut into the root to the center, making the cut perpendicular, so that the graft will be that way when inserted. The scion should be made wedge-shaped. After insertion, draw the loose earth around it, and the work is done until the graft has made a growth of eighteen to twenty-four inches. This is given as a record of experience, but still caution is urged against the use of the almond as a stock for the apricot.

The apricot may also be made to hold on the almond by double working, growing on the almond seedling first a shoot from a peach or plum bud and then working an apricot bud higher up in the new shoot.

In addition to the specifications of certain stocks for different soils, it may be remarked, in a general way, that the apricot seems to thrive better on a tolerably heavy soil, with enough sand to make it work easily, than on a very light soil. It does well on soil rather too heavy for the peach. It also enjoys moisture better and gives signs of distress unless its roots are fairly supplied all during the season, but it dislikes standing water and should not be planted on undrained situations.

\section{PLANTING THE APRICOT}

The apricot becomes a large tree in California, as has already been remarked, and it should be given plenty of room. Twenty-four feet each way is certainly the minimum distance for so large and longlived tree, and some orchards have been planted at thirty feet. If 
nearer planting is done it should be with reference to subsequent removal of part of the trees, which, however, is very seldom done. Twenty feet apart, with later removal of half the trees to double the distance was proposed by H. D. Briggs, of Azusa, in this way:

In setting out an orchard it seems advisable to double set the ground, as an apricot twelve to fifteen years old should have not less than 800 to 900 square feet of ground. This can easily be obtained by setting $20 \times 20$ feet; then when nine or ten years old remove every other tree, making them forty feet in the row, with rows twenty feet apart, of course, taking them out diagonally. The trees will very quickly tell the orchardist when they are too thick. When the outside rows have twice the fruit of those inside, it is quite evident that the time spent in pruning, etc., on half of the trees is worse than wasted. I have cut roots forty feet from a nine-year-old tree.

The apricot makes such rapid growth and so much depends upon giving it proper form, as will be seen presently, that one year's growth is all that should be allowed in the nursery. Some growers would rather have a dormant bud than a two-year-old tree, and cases have been reported from dormant buds outgrowing yearling trees planted at the same time in the same orchard. But in growing from a dormant bud in the orchard care should be taken to develop a short trunk, with properly-spaced branches, by pinching the side shoots near the ground. Trees started from dormant bud and allowed to branch from the ground, have developed very unsatisfactory form, and have, in some situations, lost their lower branches by the wind. The tree should have a low head, but a short trunk seems to give a better tree, and more elasticity to the branches.

\section{PRUNING THE APRICOT}

Of all the California orchard trees, the apricot seems most in need of the constant attention of the orchardist to give it proper shape and strength. It is a rampant grower, and in its zealous haste for size and fruitage it over-reaches itself and becomes the prey of specific gravity and wind force. Thousands of trees have been ruined by literally breaking to pieces with the weight of their fruit, and being torn by winds of only ordinary velocity. Thousands more have been rescued from such a fate by bolting the branches to each other. This excessive growth and consequent weakness of the apricot is greater in some parts of the State than in others, because of the difference in degree of forcing conditions, but everywhere the apricot needs watchfulness and timely aid in building up its strength. The general principles to be observed in securing branches strongly attached to a short trunk have already been discussed at length in the chapter on pruning.

There has been a very marked change during the last few years of the pruning of the apricot. Summer pruning, immediately after the fruit is picked, has become much more general, and winter pruning has proportionally decreased. The new practice is certainly more rational than the old. Young trees are winter pruned to promote low branching and short, stout limbs; bearing trees are summer pruned to promote fruit bearing and check wood growth-the excess of bearing shoots being removed by thinning during the winter. 
The apricot tree bears upon old spurs, like the plum; also upon the new wood, like the peach. This fact has to be borne in mind when winter thinning of the new growth is undertaken.

A very clear record of procedure is given by J. B. Neff, of Anaheim, Orange County, who built up one of the best apricot orchards in the State as he describes. This orchard was displaced to make way for English walnut trees which are more profitable in that district, but that was no fault of the pruning:

Pruning the apricot requires some skill and considerable judgment, which can only be formed by experience and observation of the habits of the tree. Trees of four to five feet in height are preferable for planting, and when planted should be trimmed to a single stem and cut off at eighteen inches from the ground. These will throw out shoots vigorously, and frequently two or three shoots from one bud. These shoots should be thinned out, leaving not more than four or five, no two of which should come from one bud, nor be directly opposite. The firsi. shoot should start twelve inches from the ground, the others in such a manner as to divide the space and make the branches balance, leaving the top shoot to form the central part of the tree.

It will be necessary to go over the trees several times the first year to remove shoots that may start where not wanted, but no general heading back should be done, as it tends to dwarf the tree; though if some of the limbs are making an overgrowth they should be pinched back to keep the head balanced.

In the pruning of the second year, the first year's growth should be cut back to within five or ten inches of the body of the tree, and all forks should be cut out, even if it necessitates forming a new head, as it is much better to lose some growth on a young tree than to take the risk of splitting down when the tree begins to bear fruit.

When the shoots start for the second year's growth, take off all that come on the under side of the limbs and thin to one, two, or three, as may be needed to balance the tree.

The second year will require much more attention than the first year, in order to keep off suckers and all lateral growth that may start on the under side of the new limbs, the object being to make the limbs grow as nearly upright as possible. The remark on heading back holds good for the second year also.

In pruning for the third year the second year's growth should now be cut back to within fifteen to twenty inches of the old wood, except the central stem, which may be left twenty-four to thirty inches long, depending on the number of laterals it may have thrown out. When the new shoots start they should again be thinned down to two or three on each limbs, and all taken off that tend to turn down or out at right angles, but do not take off the fruit spurs.

During the third season's growth, go over the trees about three times before July to remove suckers and lateral growth that may start on the lower side of the limbs, as the tendency in the third year is to make an immense growth of downward laterals, and these must be taken off so as to develop wood that is to be left for fruit. If the orchard is on good land and has been properly irrigated and cultivated there will be a few specimens of the fruit the third year, and as soon as these are gathered the trees should be summer pruned for the first time, care being taken that the land shall have been allowed to become moderately dry so that the trees may be partially dormant. If the downward growth of the laterals has been kept cut off, all that remains to be done is to cut off about one-half of all this season's growth all over the tree, using the same judgment as before with reference to prevailing winds and symmetry of tree. If this is properly done and water at once turned on the orchard, a new growth will be made and the fruit buds for next year fully developed.

The only pruning necessary in the following winter will be to take out any cross limbs and sprouts that may have been overlooked in the summer.

After the trees begin to produce regular crops they will not grow so vigorously, and the numerous prunings of the first three years will not be necessary, as almost all can be done by summer pruning until the trees get so old that they need the old wood taken out. This can be more readily done without damage 
to the trees where from 24 to 30 limbs are grown, than in the old method of leaving only eight or ten large limbs. A few limbs can be taken out each year until a full crop of new wood is again made.

The foregoing describes the development of the common vase form. A method of forming the apricot with continuous leaders is described in Chapter XII.

Winter Pruning of Bearing Trees.-The evident defect of many old apricot orchards is the failure of the low-bearing wood and the thicket of brush near the ends of long bare limbs. Such trees need renewal of the top by vigorous winter pruning, which should preferably be done toward the close of the dormant season rather than early in the winter as formerly. Old and unprofitable trees have been reclaimed in this way.

Winter pruning is still the regular method in some parts of the State where the conditions do not favor excessive growth of the tree and where summer pruning does not seem to be called for. The practice is to remove half or two-thirds of the new growth and thin out, by removing entirely enough new and old wood to prevent the tree from becoming thick and brushy. In shortening the bearing shoots it should be remembered that the larger fruits usually grow nearer to the tip than to the base of the shoot.

\section{THINNING THE APRICOT}

All free-fruiting varieties of the apricot must be thinned to secure size acceptable to purchasers. It is the experience of the oldest growers that though thinning is an expensive operation, it is very profitable. When half the fruit is taken off in thinning, the remainder reaches as large aggregate weight as though the whole were allowed to mature and thinned fruit is worth about twice as much per pound. Even if less weight is secured, and in most cases the purpose should be to get less weight, the tree is spared the exhaustion of over-bearing and the owner escapes a year of little or no fruit. A discussion of this subject is given in a previous chapter.

Where conditions are favorable, the tree will set more fruit than it can bring to full size, and for this reason thinning or spacing the fruit on the twigs by hand-picking, while the fruit is about the size of a pigeon's egg, is almost a universal practice among the best commercial growers. This is necessary to bring the individual fruits to the diameters required by canners or overland shippers and which they scale in price according to size: Extras, 21/4 inches; No. 1, 2 inches; No. 2, $1 \frac{1}{2}$ inches. Fruit of less size is hard of sale unless the crop happens to be very small. It has also been found that thinning to regulate size is quite as important when the fruit is to be dried by the grower as when sold as fresh fruit.

\section{IRRIGATION OF THE APRICOT}

Whether the apricot shall be irrigated or not is answered in the chapter on irrigation. In many locations, with proper pruning, thinning and cultivation, perfectly satisfactory fruit can be grown with the 


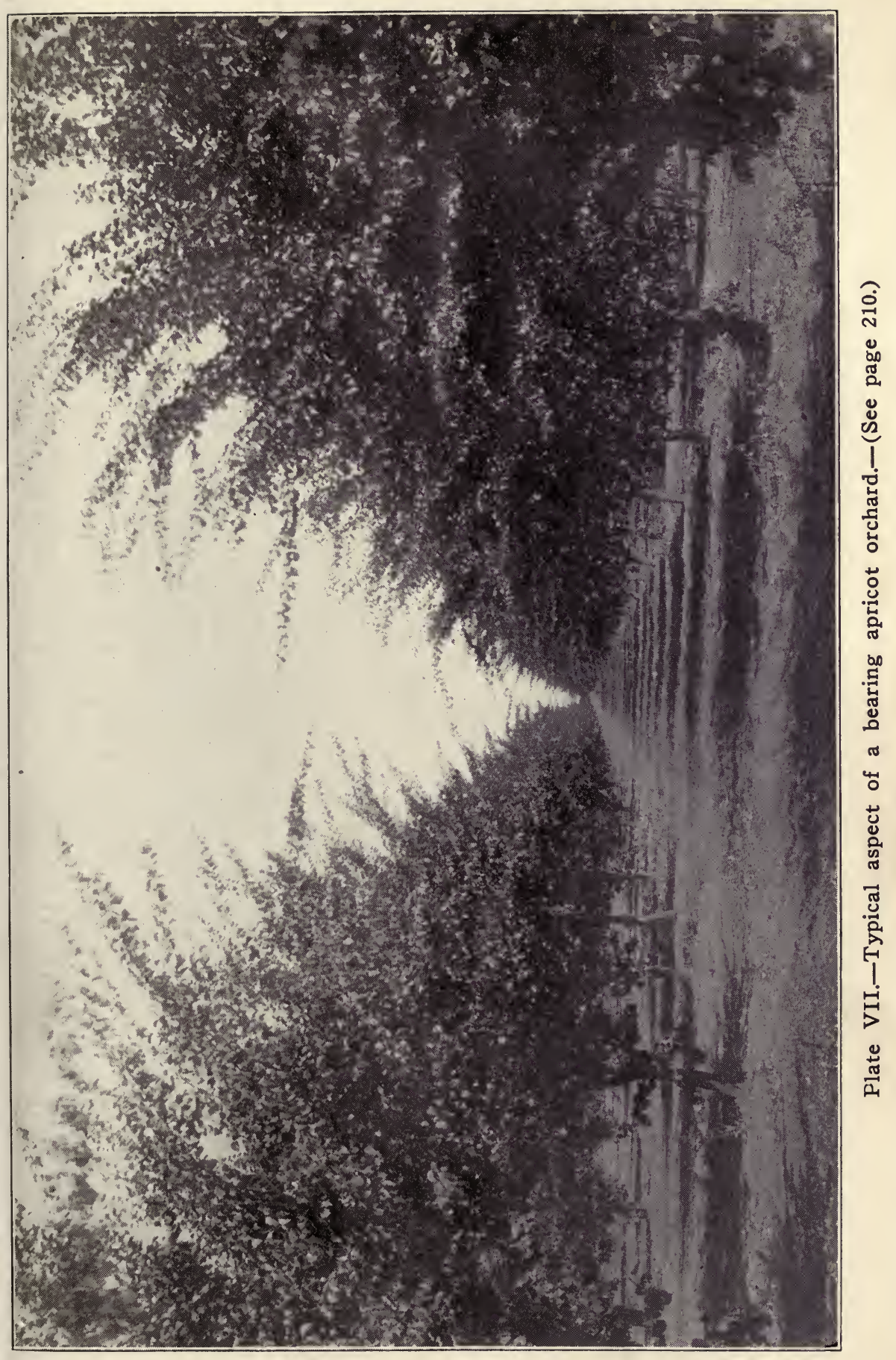



usual rainfall. In others a single winter irrigation will satisfy all the needs of the tree; in others a single irrigation just after fruit picking and summer pruning will carry : the tree through. It is a fact, however, that as the trees advance in age some supplement to the average rainfall is often desirable and in dry years irrigation is the saving of two crops. Some idea of the amount of water used can be had from the chapter on irrigation. The following account by Mr. Neff applies to this practice in Orange County, which is an average situation as to rainfall and atmospheric humidity, and is as good a general statement as could be made:

If rains are copious, winter irrigation may be dispensed with during the first two or three years after planting the orchard, but when the trees reach the age for bearing fruit the rain water should be supplanted by irrigation water until the soil is thoroughly wet 5 feet deep, and in order to have this, at least 20 inches of water, including rainfall, must be put on the land. Three irrigations should be given the trees during the first summer, but it is not necessary to wet more than a strip 5 or 6 feet wide along the tree rows. The orchard should have three irrigations during the second summer and a strip 12 feet wide should be watered, as the roots are reaching farther and the trees require a greater amount of water. The irrigation for the first two years should always be done before the trees show any want of water, so as to keep them growing vigorously.

All the space between the trees should be watered the third year and afterward; but two irrigations will be sufficient for the summer. The best time for the summer irrigation of bearing apricot trees is when the fruit is about half grown, which is usually about the second or third week in May.

If well watered at this time the fruit grows to its largest, and has time to ripen slowly as the ground gradually dries, until it has all the sugar which will go into the fruit. An orchard in full bearing that has been well watered in the winter should now have as much as full 100 inches of water for two hours on each acre (equal to four acre-inches).

The second irrigation should be given as soon as possible after the summer pruning is done, in order to start the trees growing and develop the fruit buds for the next year. This will not require so much water as the irrigation in May, but ought to be as much as 100 inches of water for one hour on each acre.

Winter irrigation of apricots on deep soils, as supplementary to rainfall and largely reducing summer irrigation, is a very satisfactory recourse in some districts.

\section{DISEASES OF THE APRICOT}

Though the apricot tree, as has been said, is regarded as one of the healthiest fruit trees, it is subject to some maladies. Trees perish from being set in unsuitable situations, and in these cases, if the evil be stagnant water in the soil, or penetration to alkaline subsoil, the root shows it. Sometimes, however, a branch or a whole tree withers and dies without apparent cause early in the summer, and while the root is still sound. The disease is evidently acute, but its cause is not known, nor a remedy proposed. It is an old trouble of the apricot, and not peculiar to California.

The so-called "gum disease" sometimes causes injury to trees. The best treatment is to cut away the diseased part down to healthy wood and cover the wound with common lead and oil paint, putting on sparingly so as not to flow over healthy bark.

Some years certain varieties in particular are blackened at the pit and rendered unsalable, but the trouble has not thus far proved serious 
generally, except with certain varieties which have generally gone out of use for that reason.

The worst injury to tree and fruit is done by what is called the "shot-hole fungus" (coryneum), from its perforations of the leaves as though by a charge from a shotgun. It makes ugly scars on the fruit, which renders it unsalable. The same disease also affects the leaves of cherries and plums. Thorough use of the Bordeaux mixture described in a later chapter will prevent this trouble.

A disease which is prevalent in some districts of southern California is called "black heart"; a pith disease which sometimes does great injury. No treatment except that of pruning back to healthy wood has thus far been proposed. Root knot is also a serious trouble of the apricot as of several other trees. It will be considered in the chapter devoted to disease of fruit trees.

Until recently the apricot has been generally free from scale insects, and it is not affected by those species which are worst on some other fruit trees, but recently it has been seriously infested in some places with black and brown scales, which will be considered in a later chapter.

The ripe apricot is sometimes seriously assailed by the diabrotica, a small green beetle, with twelve black spots upon its wing covers. Driving the insects away with smoke smudges has been used to some extent. Fortunately, the insect only occasionally occurs in large numbers.

Varieties Approved by California Growers.

\begin{tabular}{|c|c|c|c|}
\hline VARIETIES. & $\begin{array}{c}\text { Central } \\
\text { Coast } \\
\text { valleys }\end{array}$ & $\begin{array}{l}\text { Interior } \\
\text { valley and } \\
\text { foothills }\end{array}$ & $\begin{array}{l}\text { Southern } \\
\text { California }\end{array}$ \\
\hline Barry & * & & \\
\hline$\ldots \ldots \ldots \ldots \ldots \ldots \ldots \ldots \ldots \ldots \ldots \ldots$ & ** & $* *$ & $* *$ \\
\hline $\ldots \ldots \ldots \ldots \ldots \ldots \ldots \ldots \ldots \ldots \ldots \ldots \ldots \ldots \ldots \ldots$ & 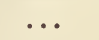 & * & ** \\
\hline 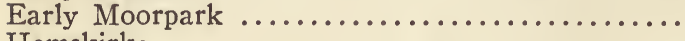 & & & ** \\
\hline 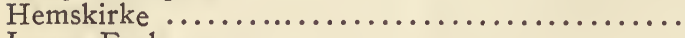 & ** & * & \\
\hline Large Early $\ldots \ldots \ldots \ldots \ldots \ldots \ldots \ldots \ldots \ldots \ldots \ldots$ & $\cdots$ & * & ** \\
\hline 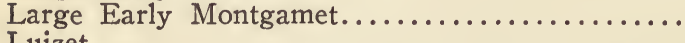 & * & * & .. \\
\hline 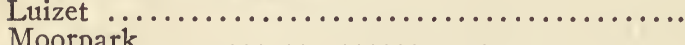 & *** & * & * \\
\hline 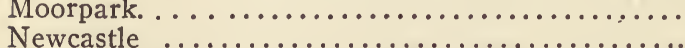 & & ** & \\
\hline $\begin{array}{l}\text { Newcastle } \\
\text { Oullin's Early } \ldots \ldots \ldots \ldots\end{array}$ & * & * & $\cdots$ \\
\hline 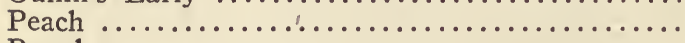 & & ** & * \\
\hline Royal,$\ldots \ldots \ldots \ldots \ldots \ldots \ldots \ldots \ldots \ldots \ldots \ldots \ldots \ldots \ldots \ldots \ldots \ldots$ & ** & ** & *** \\
\hline Routier's Peach $\ldots \ldots \ldots \ldots \ldots \ldots \ldots \ldots \ldots \ldots \ldots \ldots \ldots \ldots \ldots \ldots$ & ... & ** & $\cdots$ \\
\hline Spark's Mammoth $\ldots \ldots \ldots \ldots \ldots \ldots \ldots \ldots \ldots \ldots \ldots \ldots \ldots \ldots \ldots \ldots$ & $\cdots$ & $\cdots$ & * \\
\hline St. Ambroise $\ldots \ldots \ldots \ldots \ldots \ldots \ldots \ldots \ldots \ldots \ldots \ldots \ldots \ldots \ldots$ & * & ** & * \\
\hline $\begin{array}{l}\text { Tilton } \\
\text { Wiggin's Seedling }\end{array}$ & & * & 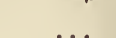 \\
\hline gin's seedling...$\ldots \ldots \ldots \ldots \ldots \ldots \ldots \ldots \ldots$ & $\cdots$ & & $\cdots$ \\
\hline
\end{tabular}

\section{VARIETIES OF THE APRICOT}

Though nearly all standard varieties of the apricot have been introduced and planted in this State, comparatively few are found on the list of the orchard planters. Many local seedlings have been brought to notice and propagated to some extent but are less used now than 


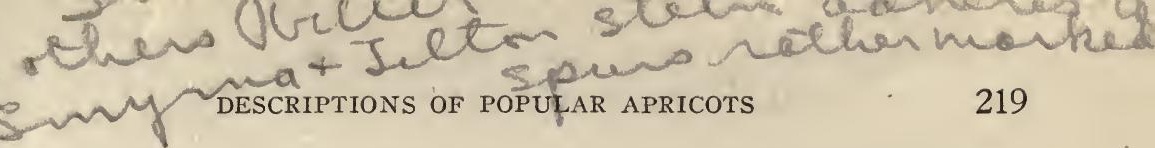

twelve years ago, and the disposition is to restrict planting to a few old varieties. There is, however, still a need of new varieties combining size, quality and regular bearing.

\section{VARIETIES OF FOREIGN ORIGIN}

Large Early.-A French variety; fruit of medium size, rather oblong and compressed; suture deep, skin slightly downy; pale orange in the shade, fine bright orange with a few ruddy spots in the sun; flesh separating readily from the stone, orange colored, rich and juicy; kernel bitter. This variety is especially popular in the southern coast counties, but in most situations has proved an uncertain bearer. Ripens before Royal.

Wiggin's Seedling.-Favored in Winter's district as best of early apricots; good size, fine color, solid red cheek, good bearer; ten days earlier than Royal.

Early Golden.-Origin unknown; small, roundish oval, with suture well marked and extending half way round; skin smooth, pale orange; flesh yellow, moderately juicy and sweet, with very good flavor; separates from the stone. This variety is reported favorably from some counties, but generally otherwise, and is not largely grown. Ripens before Royal.

Royal.-A French variety, and at the present time the leading California apricot. Of large size (when well thinined out), free stone, fine color and flavor, good bearer, and fruit ripens evenly, when well grown; a favorite with the canners, and an excellent variety for drying. Fruit roundish, large, oval, slightly compressed; skin dull yellow with orange cheek, very faintly tinged with red, and a shallow suture; flesh pale orange, firm and juicy, with a rich vinous flavor.

The Derby Royal is grown in the Winter's district; like the standard Royal but two weeks earlier.

There is a variety somewhat grown in Sacramento and Solano counties, sometimes called "White Royal," which is not liked by canners, because of its lack of color and flavor.

Large Early Montgamet.-Large, orange yellow, reddish on sunny side, firm.

Oullin's Early.-Early form of Peach apricot, large size, delicious flavor. Ripens in Amador county four weeks earlier than Peach.

Luizet.-Large, oval, distinct suture, one side higher than the other; orange with crimson cheek; flesh deep yellow, firm, rich. Especially approved in the upper San Joaquin.

- Blenheim or Shipley.-This is a valuable variety in this State, standing next to Royal in popularity; was described by John Rock as follows: "A very good variety, above medium, oval; orange, with a deep yellow, juicy and tolerably rich flesh; vigorous grower and regular, prolific bearer." This behavior of the variety was demonstrated fully in the University orchard at Berkeley, where it was for years the best of twenty varieties. It is now popular in all parts of the State. Fruit runs a little larger than the Roval, and is usually better distributed on the tree, but it must be well thinned. This variety has been approved by canners. Ripens a little later than the Royal. district.

Alberge de Montgamet.-Large and handsome, late, grown in Vacaville

Early Moorpark.-Very popular in Southern California, where its identity has been long in dispute, and is not yet fully determined. The Early Moorpark of Thomas Hogg is as follows: Roundish, inclining to oval, with very deep suture on one side, extending from base to apex. Skin yellow, mottled, and dotted with crimson on the exposed side. Flesh in all respects resembling Moorpark. Stone oblong, with a covered channel along the back, which is pervious. Kernel bitter. Ripens three weeks earlier than Moorpark.

St. Ambroise.-Large, early, compressed, deep yellow, reddish on sunny side. Juicy, rich, and sugary, with firm flesh when grown in the interior; apt to be coarse and to lack flavor near the coast. Condemned by canners for not processing well, and by dryers for loss of weight and for white color around the pit. It has served well as a shipping variety. 
Bergetti.-An undetermined variety introduced by Mr. Bergetti and widely distributed under his name in the San Joaquin.

Hemskirke.-A fine English variety quite widely grown in California; ripens later than Royal; described by Downing as follows: "Fruit large, roundish, but considerably compressed or flattened on its sides; skin orange, with red cheek; flesh bright orange, tender, rather more juicy and sprightly than the Moorpark, with rich, luscious, plum-like flavor; stone not perforate, rather small, and kernel bitter." Esteemed in California because the tree is more hardy and a more regular bearer than the Moorpark, and the fruit ripens evenly on both sides. Sometimes drops worse than other varieties.

Peach.-A variety from Piedmont of the largest size, about two inches in diameter, roundish, rather flattened, and somewhat compressed on its sides, with a well-marked suture; skin yellow in the shade, but deep orange mottled with brown on the sunny side; flesh of a fine yellow, saffron color, juicy, rich, and high flavored; stone can be penetrated like Moorpark, and has bitter kernel. This has been a very successful sort in the warmer parts of the State especially, and a favorite in the Sacramento Valley, but is now in less favor because of rapid ripening and inferior appearance in canning and drying. It ripens just ahead of the Moorpark.

Moorpark.-A standard of excellence and an old variety which originated in England. Fruit large, roundish, about two inches and a quarter in diameter each way; rather larger on one side of the suture than on the other; skin orange in the shade, but deep orange or brownish red in the sun, marked with numerous dark specks and dots; flesh quite firm, bright orange, parting free from the stone, quite juicy, with a rich and luscious flavor; stone peculiarly perforated along the back, where a pin may be pushed through; kernel bitter. In California the Moorpark reaches grand size, but has the fault of ripening unevenly in most localities. The tree is tender and bears irregularly, which leads to its rejection by most planters, though some growers cling to it because of its size and quality and occasional grand crops. The San Jose districts lead in the production of this variety, and in some parts of the Santa Clara Valley the Moorpark seems to ripen uniformly. The same behavior is reported from localities in the upper San Joaquin Valley, where it also seems to be a more regular bearer. The variety is almost wholly rejected in Southern California.

\section{VARIETIES OF CALIFORNIA ORIGIN}

Nerucastle.-Originated with C. M. Silva \& Son, of Newcastle, Placer county, in 1881; size medium, round, with spherical pit; freestone; not quite as large as the Royal, nor quite as rich in flavor, but more highly colored; rather darker on the side to the sun. Early, regular and good bearer, a medium grower, being more upright than the Royal. Its time of ripening has been reported as seventeen days earlier than the Early Golden, and twenty-five days earlier than the Royal.

Routier's Peach.-Originated with Joseph Routier, near Sacramento. Large, yellow in shade; deep orange, mottled or splashed with red in the sun; flesh juicy and rich, high flavor and a good market variety. Blooms a week later than Peach. Very popular in Sacramento and San Joaquin Valleys.

Spark's Mammoth.-Popular in Ventura county. Largest size, even larger than Moorpark; pale yellow, very tender, juicy and sweet; ábandoned for shy bearing.

Tilton.-Chance seedling first noticed about 1885 on place of J. E. Tilton, near Hanford, Kings county, and distinguished by regular bearing. Propagated and introduced by J. W. Bairstow, of Hanford. Fruit large; freestone; symmetrical, ripening evenly and one week to ten days later than Royal. Tree vigorous and prolific. Widely planted recently and very promising, though condemned for shy bearing in some places. Colors in advance of ripening and is often picked too green for best quality in drying.

Late Englehardt.-Chance seedling originating at La Crescenta. Propagated and introduced by W. B. Thorne of Tropico. Large as Moorpark, ripening evenly and twenty-eight days later than Royal. Claimed to be a very late bloomer, and thus escaping frosts which caught all other varieties at similar elevations. Planted chiefly in Los Angeles county. 


\section{CHAPTER XIX}

\section{THE CHERRY}

Although the amount of cherries grown in this State is small as compared with the aggregate weights of some other fruits, the cherry, from the growth of the tree and the size and quality of the product, is entitled to rank as one of the grand fruits of California. The size of the California-grown cherry is a matter of pride with the residents, and a marvel to visitors. It is related that, many years ago, one of the most distinguished Eastern pomologists, who was taken to an Alameda County cherry orchard during picking time, could not recognize the varieties, though he had himself propagated and shipped to California the very trees which were bearing the fruit, the size of which so far surpassed all his mental standards. And quality is commensurate with size. Whatever disagreement there may be concerning the flavors of our other fruits as compared with Eastern, the richness and excellence of the California cherry have never been impeached. Recently the shipment of cherries to eastern markets, the extension of the canning interest, and the uprising of a demand for maraschino-preserving have considerably enlarged the opportunity for profitable growth of the fruit.

Famous Old Trees.-The longevity and productiveness of the cherry tree in this State is naturally of interest. Cherries that were planted in some of the earliest settled parts of the State are still in full vigor. One of the famous trees is a Black Tartarian, which was brought from France by Dr. L. E. Miller, and planted by him in 1854, on land afterwards owned by Robert Hector in Placer county, just below Rattlesnake Bar, on the American River, about eight hundred feet above sea-level. It is described as above seventy feet in height, the branches covering a space between seventy feet and seventy-five feet in diameter. The trunk branches about six feet above the ground, and at that point has a girth of over ten feet. A close record of its crop, kept for a number of years when the tree was over thirty years of age, showed that it yielded from a ton to a ton and a half a year. Such trees are too large to be profitable, for the fruit has to be picked with the aid of extension ladders securely guyed, by men slung in swings from such ladders or the forks of the trees. At last reports there were about fifty of these large trees. Other large trees were to be seen near Woodside, San Mateo. County, and near Oroville and Chico in Butte County, some of which have borne a ton of fruit in favorable seasons.

\section{LOCALITIES FOR THE CHERRY}

In California there are many districts in which the cherry does not do well, and situations for the fruit must therefore be selected with discrimination. The chief product is made in the coast valleys adjacent to the Bay of San Francisco, including its extension east of the Coast 
Range, known as Suisun bay, for in all these regions there is a modification of climate due to the influence of ocean temperature and moisture. Away from these influences the cherry also thrives on the alluvial bottoms of large rivers and their tributary creeks, both on the low lands of the valleys and the foothills, while on broad valley plains and foothill slopes it is not usually satisfactory. In the mountain valleys cherries also thrive in suitable soils. In southern California at elevations where moisture is adequate and temperatures favor suitable winter dormancy of the trees, good cherries are profitably grown, while on the mesas and valleys below, where citrus fruits flourish, the cherry is an aggravation.

How far atmospheric conditions which are beyond control influence the growth and fruitage of the cherry, can not yet be fully determined, but ample trial seems to demonstrate the unsatisfactory character of the tree, manifested in small fruit and sunburned foliage, on the plains of interior valleys, although the soil is kept moist enough. There is, however, still the chance of securing varieties of the fruit which have been developed under conditions similar to those prevailing in the interior of California. The Russian cherries, which are largely grown in a region subject to high summer heat and dry air, will succeed in parts of California where the varieties originating in west Europe fail. Though this was suggested long ago, the effort has not yet been made.

\section{SOILS FOR THE CHERRY}

The cherry thrives in free deep soil, in which water does not stand too near the surface. It delights in deep deposits from old water courses, and does not dislike a moderate amount of sand. A loam underlaid by a sandy subsoil is acceptable if it is not allowed to dry out in the late summer, but a loam underlaid by clay has shown its unfitness by the early failure of the trees, because of standing water in the rainy season, while those on deep loam near by have remained vigorous and profitable. On the foothills it thrives in the light, mellow soil and fails in the tight clay either in soil or subsoil, as it does in the adobe of lower lands; and yet a clay loam of no great depth upon a clay subsoil may grow good trees if the clay be so disposed that surplus water from winter rains can escape and water is at hand to guard against summer drouth. Commercial orchards should have a good depth of sufficiently retentive soil. The great cherry trees which we have mentioned, are growing right on the bank of the American River, where the soil is a pure, sandy loam, in some places over sixty feet deep, as proved by an old shaft once dug near the center of the orchard.

But though the cherry dislikes a wet soil, it is particular about its water supply and insists upon enough, its requirements being greater than some other trees. During the dry year 1898-99, also in 1912-13, trees came into distress where they had never suffered before, and many large valuable trees died. The only new condition they encountered was lack of soil moisture. It thus appears that while the cherry is undoubtedly injured by excess of water in the soil 
it is still very exacting in its requirement of an adequate supply. If this can not be retained in the soil by cultivation, irrigation must be resorted to. Thus the cherry growers in the famous Willows district of San Jose, usually find it an advantage to give their trees an irrigation between the spring rains and the ripening of the fruit, and another irrigation after the fruit is gathered.

Irregularity in the moisture supply also causes the cherry to bloom and fruit unseasonably. There has been bloom in October and ripe fruit in January, due to the fact that trees become dormant in late summer from soil-drouth. January cherries may be evidences of salubrity but they betoken poor horticulture.

These facts show that the cherry must have water enough or it will not succeed. Sometimes young trees which have made a good summer growth die outright on leachy soils which dry out before the fall rains begin. On the other hand, there must not be. excessive moisture in the soil either from irrigation or by moisture. Cherry trees in southern California, planted with orange trees and given similar irrigation, have failed utterly. Planting on naturally moist land in low places has also failed, and observed facts some time ago led to the conclusion that at the south the cherry should be planted on well-drained land, which could be irrigated (as the behavior of the tree indicated its need of water), rather than on naturally moist land, because of the likelihood of excessive moisture in such situations. More recent experience has declared mellow, well-drained soils, of the higher lands well adapted to the cherry and on such soils, when well cultivated, cherries have done well without irrigation at Pasadena, Pomona and elsewhere. The commercial cherry product of southern California comes, however, from mountain valleys and high plateaux - the chief regions being the Yucaipe Valley above Redlands and the Mesa Grande region in the interior of San Diego County.

In California, as elsewhere, the Dukes and Morellos may succeed where the Hearts and Bigarreaux fail. The May Duke seems especially hardy, and bears well in Nevada, where other sorts fail utterly.

Delayed Fruiting of Cherry Trees.-Though the cherry in fav. orable situations bears early, the grower, especially on strong, rich lands, will often have many years of disappointment from falling blossoms and fruit. During this time the trees will be making marvelous wood growth, and this apparently suppresses the fruiting function. Usually these trees will ultimately bear when their exuberant growth declines. They can be thrown into fruit sooner by allowing the trees to go uncultivated, or by root pruning, through digging a trench around about eight feet from the tree, and severing the roots thus encountered, or by summer pruning. Because of this over-growth, growers give such strong soil to the apple or the pear rather than the cherry. Sometimes the non-bearing of the cherry is inexplicable. Though everything seems to be right, and the blossoms are profuse, the fruit will not stick. 
Lack of bearing is sometimes due to the need of cross fertilization. There is warrant for the claim that keeping bees in the vicinity of cherry orchards has increased the bearing. But varieties must be provided which will act as cross-pollinizers. The Royal Ann needs this assistance and will bear better when associated with Black Tartarian, Black Bigarreau, Bing, Lambert and probably others.

\section{EXPOSURES FOR THE CHERRY}

Exposures for the cherry are chosen both with reference to protection from frost injury and to early ripening of the fruit. The cherry blooms early; though hardly as venturesome as the apricot and almond. In protected situations, guarded from cold northerly winds, and open to sunshine on the south and southeast, the fruit advances to maturity very rapidly. In Vaca Valley about a month of good weather after the blossoming will ripen an early cherry and ripe cherries have been shipped as early as March 31. The pioneer cherry growers of Vaca Valley went there from their old homes in Napa Valley, because they could gather and market cherries in their new locations before the same varieties were ripe in Napa They chose places protected on the north and west by steep hills. The two things to secure are, apparently protection from the sweep of cold winds and elevation above the deposits of cold air, which occur in depressed places.

In localities where fruit ripens late, as near the coast, there is no need to seek forcing conditions, for the extra early varieties should not be planted except for family use. Early varieties are comparatively poor in quality, and will not sell profitably, as they will reach the market alongside the better later sorts from earlier districts. The place for the cherry in the later districts is on the most proper soil, according to the requirements which have been laid down, avoiding however, so far as possible, wind-swept spots and seeking amelioration of direct ocean influences by elevation or intervention of hills and windbreaks.

\section{PROPAGATION AND PLANTING THE CHERRY}

In the chapter on propagation is given a successful method of growing cherry seedlings. California cherry trees are almost exclusively propagated by budding on seedlings of the Black Mazzard. The Mahaleb root is more hardy than the Mazzard and is less subject to injury by soil saturation during the winter season. It is also more hardy against injury by summer drouth on shallow soils, which is one of the causes of die-back of the cherry tree in some parts of the State. While there may be particular places in which the Mahaleb is the better root, the conclusions of fifty years' experience in California cherry growing, which approve the Mazzard, are on the whole trustworthy. The Mazzard is a better grower and, where moisture conditions are fairly good, leaves little to be desired. The 


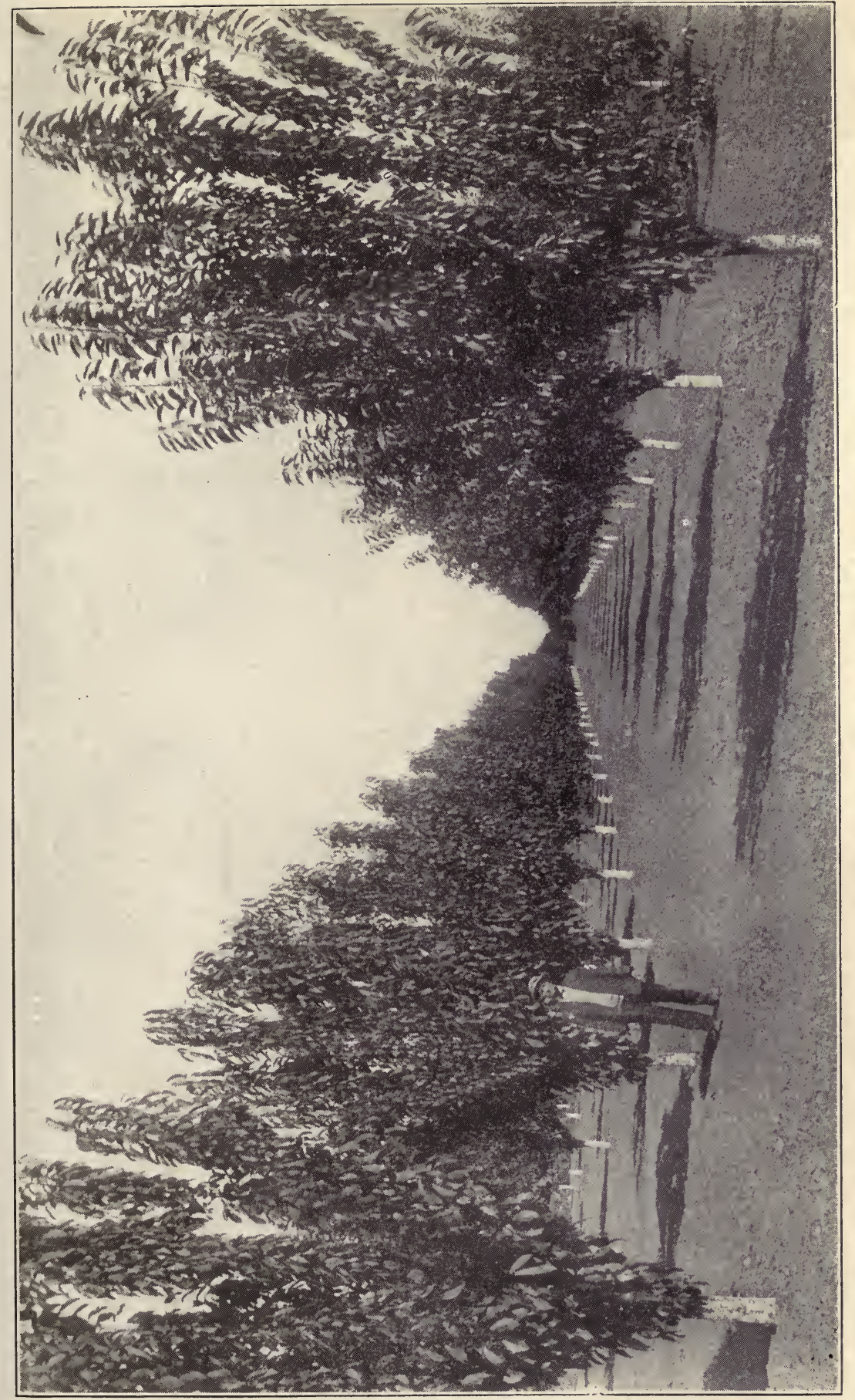

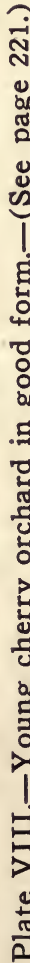



Mazzard, however, though credited with a dwarfing influence, does make a good sized tree under our conditions. Recently the Morello or sour cherry seedling, has found favor with some growers on the Sacramento River, although it is inhospitable to the buds of some varieties, like the Early Purple Guigne and Royal Ann, and double working has to be resorted to which is expensive, both in outlay and time. The Black Tartarian takes well on the Morello and other varieties can be budded upon the Tartarian top growth.

The planting of the cherry is covered by the general considerations already given for the planting of orchards. The distance which cherries should be set apart is a disputed point among planters. When planted twenty feet apart the trees have interlaced their branches when sixteen years old, and the spaces between the rows have been covered in like colonnades. In the Hayward region the branches of twelve-year-old trees set twenty-eight feet apart have nearly reached the other, though continually cut back. Much depends in the matter of distance upon the manner of handling the trees. The trees can be grown much nearer together by continuous pruning than where the usual way of cutting back for the first few years and letting the tree take it natural growth after that, is followed. James E. Gedney, of Mesa Grande, San Diego County, practices close planting and cutting back which may work better on his upland than on deeper, moister soils. He says:

I plant my trees twenty feet apart each way. My method is to plant thus closely and then keep my trees low, by cutting back every year; this facilitates gathering the fruit very much. I prefer this way to setting the trees farther apart and allowing them to attain too great a height. By the former method I secure fully as good, if not better, results per acre, to say nothing of the difference in gathering the fruit. Another advantage in keeping the trees headed low is that the wind does not affect them nearly as much as it does tall trees.

The best distances are 24 or 28 feet on such deep soils as have been described as best befitting the tree and though one may fix his distance in planting according to the method of pruning he proposes to follow, he should remember that the cherry is naturally a large tree, and most old orchards are now over-crowded.

As with other trees, orchard planters prefer trees with one year's growth on the bud in the nursery, because they usually get, then, a straight switch with well developed buds all the way down, and the head can be formed as desired. For garden planting, older trees, properly pruned in the nursery, can be used to advantage.

\section{PRUNING THE CHERRY}

All our best growers agree in the advantage of a low head for the cherry, and all aim to have trunks of young trees from the ground up to the limbs literally covered all around with leaves, which completely shelter the bark from the rays of the sun. In planting, therefore, the side buds are carefully preserved-not to be grown into branches, but to be cut or pinched back when they have come out a few inches, leaving just growth enough to clothe the tree with a 
covering of its own foliage. These spurs not only furnish leaves to shade the trunk, but soon become fruit spurs and bear well.

Low Heading with a Central Stem.--Some of the trees in the older orchards have been shaped by carrying up a leader with a regular system of side branches. Head back at planting to two feet, pinching off the shoots below the head as stated, and allowing the shoots which form the head to grow larger, but they too are all to be pinched except the leader, which is allowed to grow as long as it pleases during the summer. During fall or winter pruning cut back the leader to about twelve or sixteen inches from its starting point and cut back the side branches to about six or eight inches. This is done year after year, cutting back and thinning out the side shoots, pinching the laterals, and allowing the leader to grow, never interfering with it until the winter pruning and always letting it predominate over the side shoots. By cutting short, wood is increased, but at the end of six years the tree goes into fruit very rapidly. As the tree increases in fruit it decreases in wood, and by the time it is ten or twelve years old there will be but little cutting to do, except to shorten in and thin out, and this requires some judgment and experience, to know where to cut, how to cut, and when to cut. To shorten in, never cut down to an old fruit spur. It is very difficult to get healthy wood out of such; but whenever you can find last year's wood, there you can cut with safety anything that is less than one inch in diameter.

This system of pruning must be accompanied by constant pinching during the summer time. It should commence when the lower shoots are about six inches long, and be followed up closely all through the growing season. Those on the trunk should never get longer than eight or ten inches, under any circumstances. After these are pinched, let the trees rest ten or fifteen days, or until the branches in the top get a good start. Then pinch everything clean but the leader, in every main branch in the tree. The leader takes its own way all through the growing seasun, to prevent the effects of overpinching or checking the growth. If only the side shoots are kept back, the leader or head of the branches receives the current or flow of sap and maintains and carries on life and vitality in the tree. One object in pinching or spur pruning is to keep back surplus wood and create fruit spurs, throwing all the little twigs and branches into fruit, thereby utilizing all the wood the tree can produce, not allowing it to grow at the tree's expense, and then have to cut it off. And another object in side-shoot pruning is to make the tree produce fine large cherries, all closely nestling around the big wood, and no long, slim branches hanging down like weeping willow. All such branches are always more or less sunburnt on the top and full of worms, one of the evils tending to the destruction of the tree.

This method is commended to those who like a tree with a central leader, and are willing to give their orchards such constant attention. Unless pinching and consequent multiplication of shoots and foliage is faithfully followed such, a tree is apt to become tall and rangy and to expose the bark all the way up to sunburn and borers. 


\section{THE USUAL METHOD OF PRUNING THE CHERRY}

As we have said, all cherry growers agree on low heading and on the advantage of pinching the lowest shoots as soon as they make a bunch of leaves. In forming the head, and in after treatment, the usual method is quite different from that we have described. It follows the vase or goblet form, which has been discussed at length in the chapter on pruning. Of the application of this method to the cherry, W. W. Smith, in an address before the State Horticultural Society, said:

The cherry may be pruned the same as any other deciduous fruit tree until it is about five years old; after that the less pruning the better, except when necessary to cut out a dead or crossing branch. Pruning the cherry is more or less likely to produce gum (and this, decay), and should be avoided as much as possible. Cherry trees, however, should trained with low heads not to exceed eighteen inches from the ground to the first branches; fifteen inches is better. From three to five branches are enough to form the head of the tree; all others should be removed early. Three are better than five; two make a forked tree, which is likely to split in after years.

At the end of the first season we have a neat little tree with three to five branches. During the following winter these branches should be cut back to six to eight inches. The next season these should be allowed to produce two branches each (no more); then, at the end of the second season from planting out, we have a tree with from six to ten branches. The following winter the new growth should be cut back again to from twelve to eighteen inchesaccording to the amount of growth the tree makes-the less the growth the more you cut. The same process should be repeated the following winter, treating each branch as an individuual tree, until the tree is about five years old; it takes at least five years to get the head of a cherry well established. After this, as some varieties will persist in throwing out branches near the ground, they should be removed during the summer. At this age the tree, if well grown, will have top enough to shade its body from the sun, and there is no further need of branches on the main trunk.

If necessary to remove large branches it should then be done in midsummer, as that is the only season when the gum is not more or less exuded. We made it a rule to go over and dress up and prune our cherry orchard immediately after the crop is gathered-which in our part of the State is the last of May. All wounds made then by the removal of branches or otherwise will heal over the same season. All large wounds made at any time, however, should be coated over with paint.

The method thus described by Mr. Smith is that by which probably nine-tenths of the cherry trees of this State are shaped.

In the cherry there should be the same observation as to cutting inside and outside buds as with other trees; in fact, the outside bud is the rule, because so many varieties make a directly upward growth. In removing limbs, cutting to the collar or swelling at the base of the limb is especially important, also the covering of the wound to 0 prevent checking of the wood.

\section{GRAFTING OVER THE CHERRY}

Since canning of cherries began on a large scale, there has been a vastly increased demand for white cherries. The Royal Ann (a local name for Napoleon Bigarreau) has been the favorite. Other white sorts are also used for canning. This rise in favor of the white 
cherries has vastly increased their proportionate production as compared with the choice black and red varieties, which are still popular as a table fruit.

It is the experience of growers that the cherry is grafted over as easily as the pear or apple, if the tree is healthy. In large trees as many as fifty or one hundred grafts may be set, choosing the smaller limbs, even if you have to go pretty high in the tree. J. W. Cassidy, of Petaluma, used to advise grafting before the sap begins to flow in the winter, or if not done then, wait until the buds are well advanced or the tree in bloom. He has trees which were over thirty years old before they were re-headed, and they made fine tops of new and healthy wood, and produced abundantly. The cherry is in fact a very easy tree to graft by the usual top-grafting methods.

\section{PESTS AND DISEASES OF THE CHERRY}

The disease of the cherry which is most heard of is the "gum," or overflow and condensation of sap, which, if left to itself, often induces decay of adjacent bark and wood. Without attempting to explain the cause or causes of the unhealthy exudation, it may be said that prompt treatment of certain manifestations is desirable, and in others the tree should be cleansed from the flow. Where the gum exudes on the side of trunk or limb, the thin outer bark should be pared away with a sharp knife, the accumulation of gum and sap removed, and the wound painted with lead and oil paint, or covered with grafting wax.

Gum in the crotch should be cleanly brushed out when softened by the winter rains. If allowed to remain, it becomes sour and offensive and may injure the tree. In places where two or three limbs come out close together a kind of cup is formed, which will hold the gum from one year's end to another, and in its soft state, leaves, sticks, cherry pits, dust, and dirt will stick and hang and sometimes the mass becomes very foul. By this collection also, a nest is made for all manner of insects, bugs and worms. Another evil in letting the gum stay on is, if the rain does not wash it off clean, it runs down the trunk of the tree and makes the bark look bad, and if it is very thick on the bark when it dries, it will contract and crack the bark crosswise, and is very injurious to the tree.

Gumming in the crotch can be largely avoided by starting the young cherry as advocated in the chapter on pruning. Branches which emerge from the trunk at separate points and at wide angles seldom gum; those which are crowded together or emerge at acute angles gum badly. In shaping young trees a gumming joint sometimes may be clearly cut out and those branches selected to remain which start out at a wider angle; in older trees there is nothing to do but keep the fork clean, as already described.

There are cases reported in which gumming of old trees has been stopped by allowing the ground to lie uncultivated, weeds being cut 
down with the hoe. As a rule, however, the cherry thrives with good cultivation.

Die-back of the Cherry.-The dying back of cherry branches is more or less common in all regions, and the immediate cause thereof is not known. It is apparently sometimes a root trouble, as is the dying back of other fruit trees. This might have resulted from standing water in the winter in the soil, although the same condition may result from lack of sufficient moisture during the late summer and autumn. Anything which causes destruction of the root hairs is apt to cause die-back and other forms of unthrift in the top. Early vegetative activity in the branch, followed by frost, seems also to occasion die-back in some cases. Fortunately, this can occur without injury to the rest of the tree, though it is sometimes and in some places destructive to the tree in the end. The only treatment is removal of the affected wood, and if this can be done during the growing season, as soon as signs of injury appear, it is all the better.

The Gopher.-One of the most dangerous foes of the cherry is the gopher, for he seldom takes less than the whole tree, young or old. Traces of his presence should be constantly watched for, and killing methods described in a later chapter adopted. If a tree is seen to wilt suddenly, the probability is that a gopher has girdled it. Covering the wound sometimes saves the tree, but not usually.

Insects injuring the cherry will be mentioned in a subsequent chapter.

\section{VARIETIES OF THE CHERRY}

Many varieties of the cherry have been tested in this State, and many have been abandoned from one cause or another. Those most frequently starred in our table are the survivors in public esteem. As our reports have come from those who grow for market, possibly some sorts are too tender for shipment, but excellent for family use, are omitted, but will be included in the descriptions which follow the table. The claims to value upon which a variety is judged are several: Extra earliness, an important consideration in early districts for shipment, and elsewhere for local sale or family use; firmness to withstand mechanical injury by jarring in transit and durability to escape decay during the long journey to distant markets; firmness and fixed color to stand processing in the cannery, and to prevent coloring the juice; lateness to extend the cherry season.

In classification of cherries it was originally considered that there were four classes of cherries. The Hearts were the tender and half-tender sweet cherries, while the Bigarreaux were the firm-fleshed ones; but these have been so intermingled and blended together by hybridization that no distinct line can now be drawn separating them. There is really but one class of these, whose main characteristic is the large, vigorous growth of the tree. The Duke and Morello cherries, also wanting a natural division, really constitute but one class. 
Cherry Varieties Approved by California Growers.

\begin{tabular}{|c|c|c|c|c|c|}
\hline VARIETY. & $\begin{array}{l}\text { Upper } \\
\text { Coast } \\
\text { Region. }\end{array}$ & $\begin{array}{l}\text { Central } \\
\text { Coast } \\
\text { Region. }\end{array}$ & $\begin{array}{l}\text { Interior } \\
\text { valleys } \\
\text { and } \\
\text { foothills. }\end{array}$ & $\begin{array}{l}\text { Mountain } \\
\text { valleys } \\
\text { and } \\
\text { plateaux. }\end{array}$ & $\begin{array}{l}\text { Southern } \\
\text { California }\end{array}$ \\
\hline Advance & $*$ & * & * & $*$ & \\
\hline Bing .... & $* *$ & ** & $* *$ & * & $\begin{array}{l}\cdots \\
\ldots\end{array}$ \\
\hline Bl'k Heart (B. Bigarreau) & ... & * & * & ... & $\cdots$ \\
\hline Burbank $\ldots \ldots \ldots \ldots \ldots$ & $\ldots$ & $\ldots$ & ** & $\ldots$ & * \\
\hline Burr's Seedling............ & . & $* *$ & & $\ldots$ & * \\
\hline Centennial $\ldots . . \ldots \ldots \ldots$ & - $*$ & $*$ & $*$ & * & ... \\
\hline Chapman .............. & .. & $* *$ & ** & $\cdots$ & ... \\
\hline Cleveland. . . .......... & $\cdots$ & ‥ & $*$ & $\cdots$ & $\cdots$ \\
\hline Early Purple Guigne...... & * & * & $*$ & ** & * \\
\hline Elton $\ldots \ldots \ldots \ldots \ldots \ldots \ldots$ & $\cdots$ & * & $\cdots$ & $*$ & $\cdots$ \\
\hline $\begin{array}{l}\text { Knight }(\text { Early }) \ldots \ldots \ldots \ldots \\
\text { Lambert } \ldots \ldots \ldots \ldots \ldots \ldots\end{array}$ & $\cdots$ & **ं & $\therefore *$ & * & $\cdots$ \\
\hline Lewelling (B. Republican) & $*$ & ** & ** & * & ... \\
\hline Mezel $\ldots \ldots \ldots \ldots \ldots \ldots \ldots$ & * & * & ** & & \\
\hline Napoleon (Royal Ann).... & ** & ** & ** & ** & * \\
\hline Nonpareil. . . . . . . . . . . . & ... & & ** & $\cdots$ & .. \\
\hline Rockport $\ldots \ldots \ldots \ldots \ldots$. & * & * & * & $\cdots$ & .. \\
\hline Schmidt $\ldots \ldots \ldots \ldots \ldots \ldots$ & & * & * & $\ldots$ & $\ldots$ \\
\hline Spanish (Yellow)......... & * & & & & \\
\hline Tartarian (Black) ........ & ** & ** & ** & ** & ** \\
\hline Wood (Governor) ........ & * & * & $*$ & * & * \\
\hline Buttner's Yellow ........ & $\cdots$ & * & & $\cdots$ & $\cdots$ \\
\hline Eugenie $\ldots \ldots \ldots \ldots \ldots \ldots$ & . $\ldots$ & * & ** & ... & ... \\
\hline May Duke . & & * & * & * & ** \\
\hline Morello .... & * & * & * & * & * \\
\hline Richmond $\ldots \ldots \ldots \ldots \ldots$ & * & * & ** & * & ** \\
\hline
\end{tabular}

*Indicates that the variety is approved in the region designated.

** Most highly commended.

\section{BIGARREAU AND HEARTS}

Early Lamaurie.-Fruit large, dark purple; flesh rich, juicy, excellent. Downing says a week earlier than Early Purple Guigne. Has proved the earliest cherry in the University collection at Berkeley, and in Vacaville district. Not fully tested as to regular bearing.

Guigne Marbree.-"Fruit medium large, round, skin dark red; flesh purplish red, tender, juicy, delicate flavor."-Gillet. "A better bearer than Early Purple Guigne."-W. W. Smith.

Baumanns May (Early Black Guigne).-Rather small, deep rich red, becoming rather dark when fully ripe; tender, juicy, tolerably sweet and good.

Early Purple Guigne.-Small to medium size; purple; tender, juicy, and sweet. This variety is considered the earliest good cherry. It is reported a shy bearer in some localities.

Belle d'Orleans.-Above medium size, roundish, heart-shaped; whitish yellow, half covered with pale red, very juicy, sweet and excellent.

Early White Heart.-Below medium size, rather heart-shaped, skin dull whitish yellow, tinged and speckled with pale red in the sun; flesh melting, sweet, and pleasant when fully ripe.

White Tartarian.-Fruit of medium size, obtuse heart-shaped; skin pale yellow; stalk slender; flesh whitish yellow; half tender and very sweet.

American Heart.-Fruit pretty large, heart-shaped, often nearly four-sided and irregular in outline borne in clusters; flesh half tender; skin strong and adhering to flesh.

Werder's Early Black.-An early variety, moderately productive; tree vigorous, spreading; fruit large, black, tender, sweet and excellent. 
Knight's Early Black.- "Large, black, tender, juicy, rich, and excellent; high flavor; a shy bearer until the trees attain age."

Rockport Bigarreau.-Large; pale amber in the shade, light red in the sun; half tender, sweet and good; a very excellent and handsome cherry; good bearer; highly esteemed for canning and shipping.

Coe's Transparent.-Medium size, pale amber, red and mottled next the sun; tender, sweet and fine.

Cleveland Bigarreau.-A thrifty, strong, spreading grower, and productive large; clear red and yellow; juicy, sweet, and rich.

Black Tartarian.-Fruit of the largest size, bright purplish black. Flesh purplish, thick, juicy, very rich and delicious. Tree a remarkably vigorous, erect. and beautiful grower, and an immense bearer; the best of the black cherries.

Governor Wood.-Large; light yellow shaded with bright red; flesh nearly tender, juicy, sweet, rich and delicious; a vigorous grower and very productive.

Elton.-Large, pointed; pale yellow, nearly covered with light red; juicy, with a rich and luscious flavor; one of the best.

Black Eagle.-A very excellent English variety, ripening in June; large size, deep purple, or nearly black; flesh deep purple, tender, with a rich, high-flavored juice.

American Amber.-Fruit medium sized, roundish, heart-shaped; skin thin, smooth, light amber; delicately mottled and overspread with bright red; flesh tender and juicy, but not high flavored.

Yellow Spanish (Bigarreau Graffion).-Large; pale yellow, with red cheek in the sun; flesh firm, juicy, and delicious; one of the best, most beautiful and popular of all light-colored cherries.

Mezel, Monstreuses de (Great Bigarreau).-A foreign variety of the largest size; dark red or quite black; firm and juicy; late.

Pontiac.-Large; dark purplish red; half tender, juicy, and agreeable.

Burr's Seedling.-Large; yellow, shaded with red; sweet and rich; vigorous and great bearer; apparently does better near the coast than in the interior.

Oxheart.-Fruit large, obtuse, heart-shaped; skin dark red; flesh red, half tender, with a pleasant juice of second quality.

Napoleon Bigarreau (Royal Ann).-A magnificent cherry of the largest size; pale yellow, becoming amber in shade, richly dotted and spotted with deep red, and with a bright red cheek; flesh very firm, juicy and sweet. Tree a free grower and an enormous bearer. when properly pollinated. The great commercial cherry of California.

Tradescant's Blackheart (Elkhorn, Black Bigarreau).-Large, heart-shaped) ; deep, glossy black; very solid and firm; dark purple, moderately juicy.

Schmidt's Bigarreau.-The largest of all the Black Bigarreau cherries. Skin of a deep black color; flesh dark and very juicy, with a fine flavor.

\section{DUKES AND MORELLOS}

Early Richmond (Kentish).-An early, red, acid cherry; valuable for cooking early in the season.

Eugenie.-Medium large; heart-shaped; amber red; good quality; early.

May Duke.-An old, well-known, excellent variety; large, dark red, juicy, subacid, rich.

Arch Duke.-Fruit large, obtuse, heart-shaped; bright red becoming dark; flesh light red, melting, juicy, rich, subacid flavor, very good; tree more upright and vigorous than May Duke.

Late Duke.-Fruit large, flattened or obtuse, heart-shaped; white, mottled with red, becoming rich dark red when ripe; flesh yellowish, tender, juicy; hangs long on the tree.

Reine Hortense.- "It is one of the very largest of cherries; a beautiful, glossy red, or deep pink, when fully ripe; heart-shaped; a universal bearer, and when 
hanging on the tree no fruit is more beautiful; excellent for canning, but too soft and juicy for shipment."-W. W. Smith.

English Morello.-Large, dark red, nearly black; tender, juicy, rich, acid, productive and late.

Guigne Noir Luisante (Black Spanish).-Fruit medium size, round, heartshaped, glossy, blackish red; flesh reddish purple, tender, juicy, rich, acid.

Belle Magnifique.-Fruit large, roundish, inclined to heart-shape; skin a fine bright red; flesh juicy, tender, with sprightly subacid flavor; one of the best of its class; a fine table fruit when fully ripe.

Buttner's Yellow.-Medium, roundish; clear yellow; firm, late.

\section{PACIFIC COAST SEEDLINGS}

Lewelling-Black Republican (Black Oregon).-_"Seedling by Seth Lewelling, Milwaukee, Oregon, from seed planted in 1860; first fruited in orchard in 1864. Widely distributed in California. Large, black, sweet, with purple flesh; ripens ten days after Black Tartarian."-James Shinn. "Large, late black cherry, good flavor, long keeper; dries and ships well. Seems to succeed better on foothills than in the valley."-Robert Williamson. "Supposed to be a cross between Napoleon Bigarreau and Black Tartarian, having the solid flesh of the former and the color of the latter; very late."-John Rock. "I am of the opinion that the Black Republican and Lincoln came down from the seed of the Black Eagle, but I have little idea of what variety they were crossed with."-Seth Lewelling.

Bing.-Originated by Seth Lewelling, from seed of Black Republican. "Fruit large, dark brown or black, very fine, late; a good shipping variety."-Seth Lewelling. Tree vigorous, and foliage heavy. Fruit ripens so that trees can be cleaned at one picking.

Centennial.-A seedling of Napoleon Bigarreau, raised by Mr. Henry Chapman in Napa Valley, and fruited by him for the first time in 1876. Propagated and introduced by Leonard Coates, then of Napa, in 1885. It is larger than its parent, more oblate in form, and beautifully marbled and splashed with crimson on a pale yellow ground; exceptionally sweet and of remarkable keeping quality. Described by Committee of American Pomological Society (1885) as follows: "Size large, slightly oblate; amber, with dark crimson marbling; flesh, firm, sweet, and rich; quality best; condition excellent (after crossing continent by mail), showing its good shipping qualities." The Centennial has been little planted recently, because of superiority of Royal Ann.

The Oregon.-Seedling of Napoleon Bigarreau, by H. W. Prettyman, of East Portland, and named by. Oregon State Horticultural Society in 1888; described as larger than Napoleon; firm; dark red; "fit to eat earlier than Napoleon, but coming to full maturity somewhat later." Introduced in 1888 by W. S. Failing, Portland.

Lambert.-Seedling of J. H. Lambert, Milwaukee, Oregon, 1887 ; presented to Oregon State Horticultural Society; right to propagate sold to Oregon Nursery Co., 1896, and introduced by this company; very large, roundish, heart-shaped; stem long, slender, suture medium depth, acid; smooth, glossy, dark purplish; red flesh dark purplish red, firm, flavor rich, quality good. Ripens ten days to two weeks after Black Tartarian.

Andrews.-Fruited French seedling about 1896 by C. N. Andrews, Redlands. Grown in mountain valley near Redlands. Apparently a fine shipping variety locally named after the grower.

Paul.-Found by E. V. D. Paul, of Ukiah, on place purchased by him and previous owner could not account for its presence. Very large, black, mottled with dark red; late; remarkable shipping endurance demonstrated; diploma at Oregon Cherry Fair, 1907. Propagated and introduced by Leonard Coates Co., Morgan Hill, California, 1908.

Nonpareil.-Originated at Vaca Orchard; a fine, black, shipping cherry, owned by Earl Fruit Company at Vacaville and not distributed.

Early Burbank.-Originated by Luther Burbank; a seedling of Early Purple Guigne and sold in 1903 to a group of Vacaville growers. Very early, earlier than its parent variety. Large, rich deep crimson, resembling Black Tartarian in quality. Tree medium upright grower, large leaves, prolific. 


\section{CHAPTER XX}

\section{THE PEACH}

Until the great prune planting passion of the decade ago the peach was the greatest deciduous fruit of California judged by the total number of trees in service. When the bloom impression went forth that cured prunes could be put up in sacks more cheaply than wheat, people took to planting prune orchards by the section all through the wheat districts of the great valley, and bloom planters even carried the trees where no one would think of planting wheatcutting up shallow-clay upland sheep pastures and even yucca sand wastes into prune-growing colonies. Under such planting propositions it is little wonder our nurseries sold prune trees for twice the normal prices and still could not fill the demand. Figures of prune trees in orchards rushed far beyond the peach figures. This over-planting of prunes naturally brought loss and disappointment, and interest turned again to peach planting, so that now the peach has secured notable advance beyond the prune, as shown by statistics in Chapter VI. During the last few years the peach has had the call, the nurseries have had difficulty in keeping up with the planting demand for certain varieties, which will be discussed later, and the peach has demonstrated its right to attain again its old position by possession of a greater acreage than is given to any other deciduous fruit.

The peach was the first fruit to ripen on the improved trees brought here by the early American settlers, and the magnificence of the peach was consequently the key-note of the refrain which greeted the ears of the world in which the California gold cry was ringing early in the fifties. In fact, the gold from the mine and the gold from the tree were very nearly related. In old Coloma, where gold was discovered, there was a peach tree which bore four hundred and fifty peaches in 1854 , which sold for $\$ 3.00$ each, or $\$ 1,350$ for the crop of the one tree, and in 1855, six trees bore one thousand one hundred peaches, which sold for $\$ 1.00$ each. Some of these pioneer trees are said to be still living and bearing fruit.

\section{LONGEVITY OF THE PEACH IN CALIFORNIA}

There are many other facts to establish the claim that the peach tree, if planted in a suitable soil and situation and cared for with any devotion and skill, is not a short-lived tree in California. California is too young to mark limits of duration, but there are instances in the earliest-settled places in the State, where peach trees above fifty years old are still vigorous and productive. Some trees have, in fact, gone along in thrift until they have a bark below which looks like that of a forest tree, and framework of main branches sound and stalwart throughout because they have never been allowed to 
sunburn until protected by their own roughness, and have never been pruned with an axe, and never lost a limb no had a wound in to which decay could penetrate and descend to the root. When the peach has a fair chance in its aerial parts and is in a soil which favors health of the roots, it shows itself to be very long-lived in California. When trees break to pieces and show decay wounds, they are in bad places, and have suffered through natural stress or have been weakened by cultural errors.

In favorable soils the peach is stronger and longer lived in the root than in the top, and sometimes triumphs over neglect by discarding old, wind-broken, sun-burned and bark-bound branches, and forms a new head of its own. Such renewal is sometimes very rapid. In the interior valley new shoots on a cut-back Muir tree have grown twelve feet in one season, with a thickness of one and one-half inches at the base. Such shoots will bear the following summer and proper selection should be made from them to shape the new tree-all others being removed. Cutting back for a new head kills some trees, probably those which through hardship are weak in the root, but most peach trees take to it kindly.

It is through this disposition to renewal of good wood that the intelligent system of pruning which is now prevalent, ministers to the longevity as well as the profitability of the tree, aiding it to constantly renew its youth by restraining its exuberance, and at the same time furnishing it sound new wood on which to grow its fruit foliage. But while these are facts, there is some difference in opinion as to the point at which an old tree becomes less valuable than a young one. Along the Sacramento River some count about a dozen good crops as the limit, and thus replace the trees when about fifteen years of age. This is a point which may vary greatly, according to local conditions.

Early Productiveness.-Quite as important as the longevity of the peach tree are the facts of its rapid growth and early productiveness. It is the first of our fruit trees to attain size and yield a ") rofitable crop. In localities best suited to its growth it will mature some fruit the second summer in the orchard if the small shoots are not pruned away from the main branches, and during the third summer averages of forty to fifty pounds per tree have been secured from considerable acreages. These facts are stated to show what the peach of good variety may do in a good situation and soil and with the best of care. Of course they are not to be taken as average results, although greater than those given are sometimes attained. For example, on the rich, alluvial land near Visalia, an Admiral Dewey yearling tree planted in March, 1904, had in October, 1905, attained these dimensions: Near the ground the trunk was eleven and three-quarter inches in circumference, branching two feet from the ground it had four main branches, each seven inches in circumference; height of tree, twelve feet; spread of branches, ten feet. It grew near a crack in a cement ditch and so had all the moisture it could use, and being in a free, open soil was not impaired by standing water. 
As for possible productiveness of the peach, one Susquehanna tree in Kern county yielded twenty-seven forty-five pound pickingboxes-twelve hundred and fifty pounds in one crop-about four times as much as good trees may average.

\section{LOCALITIES FOR THE PEACH}

The peach has a wide range in California, and finds many districts suited to it in the several ways in which the trade delights it it. As compared with the apricot, the peach thrives in the sheltered valleys of the district north of the bay and west of the Coast Range, in which the apricot is of little commercial moment; it yields those peerlessly beautiful "mountain peaches" from one to two thousand feet higher in the Sierra foothills than the apricot can be trusted; it goes everywhere in the lower foothills and over the great valleys that the apricot will go, and beyond it also, because it is less restless in the spring and escapes some frosts which injure apricots. Counted from trees in orchard the peach is more than three times as great as the apricot.

Nearly every county in California reports the possession of peach trees. Above an elevation of four thousand feet on the sides of the Sierra Nevada, they may be subject to winter killing, and lower still the careful choice of situation has to be made to avoid frost at blooming time-the peach in such places being subjected to some dangers which beset it in the eastern States. Below these points, however, lies the great fruit belt of the foothills of the Sierra, where the peach is the chief fruit grown and its excellence is proverbial. Size, beauty, richness, delicacy of flavor and firmness, which endures carriage to the most distant markets, are all characteristics of the foothill peaches of California.

In the great interior valleys of the State wherever proper condition of soil and water supply can be found, the peach also thrives, the tree making a wonderfully quick and large growth, and the fruit attaining great size. The San Joaquin Valley is the greatest peach district in the State.

In the small valleys on the west of the great valley and on the eastern slopes of the Coast Range, there are also extensive areas suited to the peach, and sheltered places on the eastern and western edges of the Sacramento Valley have produced the earliest fruit for a long series of years. Recently the contest for the earliest fruit of these districts, with the foothill district on the east side of the Sacramento Valley and special locations in the upper San Joaquin Valley, has been quite close.

In the coast valleys, opening upon San Francisco Bay and the Pacific Ocean, the peach is also a leading fruit. Its success is greatest, however, where good shelter is had from direct coast influences. Even where open to these influences, good peaches can be grown by choosing the smaller range of varieties, which do well by protecting the trees from harsh winds, and by seeking elevation above depressed valleys, whose frosts are frequent. The occurrence of curl-leaf is a factor 
of much importance, which will be considered presently. In the coast counties north of the Russian River Valley the danger to the peach from unfavorable atmospheric conditions increases as one goes northward, and situations must be chosen with greater care. And yet by such exercise of care, peaches for home use and local markets can be successfully grown.

South of San Francisco Bay the coast influences soften as you proceed southward, and the peach draws nearer to the ocean, choosing, however, elevations and avoiding broad, wind-swept areas and narrow defiles where drafts and fogs are frequent. At considerable elevations, as on the Santa Cruz Mountains, some varieties of peaches are notably excellent. The general rule holds with the peach, as with other fruits, that coast influences retard ripening and the season of the fruit is late.

In some valleys and at elevations in southern California the peach is largely grown and high excellence attained while on the mesas and plains there is often too high a temperature which starts growth out of season and follows with a dormacy and die-back when the tree ought to be most active. It has recently been demonstrated that varieties like Lukens' Honey, descended from the Peen-to or flat peach of China, resists such irregularities better than the common sorts which are largely of Persian origin.

\section{SOILS AND EXPOSURES FOR THE PEACH}

Through the suitability of soils for the peach can.be somewhat extended by the choice of stock for budding upon; as will be considered presently, its range of soils is narrower than that of the apricot. The best peach soils are light, deep, sandy loams, rather dry than moist, but under all circumstances well drained. It will thrive on land with a considerable mixture of coarse sand or gravel, providing it contains also needed elements of fertility; for the rapid growth and heavy fruitage of the peach requires abundant nutrition. Though it accepts coarse materials both in soil and subsoil, it relishes fine sediment and perhaps finds no more congenial location than in the deep, sandy loam, or sedimentary deposit bordering the creek beds of our warm valleys, and will send its roots deep to secure long life and abundant fruitage. Such soils, whether along existing streams or deposited by prehistoric water courses, which have left their mark by the elevated ridges of rich sediment above the prevailing valley soils, are warm, deep, and thoroughly drained, and delight the peach. Peaches are grown very successfully on what is called hardpan in some parts of Fresno County, providing the hardpan is blasted as described in Chapter XI. In these situations the hardpan is near the surface and has a deep free soil below it into which the roots can extend.

At elevations on the hillsides there are free loams which result from decomposition of the underlying rocks, and on them the peach thrives, both where the soils themselves are deep and where the 
underlying rock is loose and open, permeable by roots and affording escape for water. Success has been reported even when holes are partly excavated in these rotten rocks as in the soft sand rock on the hills east of Vaca Valley, or in the broken chalk rock in what is called Blackburn Gulch, near Santa Cruz. The superior warmth of such soils is supposed to minister to earlier ripening of the fruit, though the escape from cold air by elevation is no doubt a greater factor to the end.

The influences of comparatively slight difference in elevation is very marked. E. R. Thurber, of Pleasant Valley, Solano County, had for many years a plat of peach trees on a natural terrace about seventy-five feet higher than the general level of his orchard. On the terrace peaches ripen and are disposed of before the same varieties ripen in the orchard below.

As in the valley a short distance to water is to be avoided, so on the hills too great percolation from higher levels is undesirable. Of course, natural defects of this kind can be corrected by adequate under-drainage.

Still, though such be the general soil conditions best suited to the peach, the tree can be well grown for home use or local markets on somewhat heavier soil, providing there is a good drainage, but drainage must be insisted upon, for thousands of trees have perished because planted in retentive soils without drainage. Alkaline soils which are usually rather heavy should, however, be avoided as the peach, when grown on its own roots, seems to be of all fruits most sensitive to alkali.

As to exposures for the peach the same rules hold as for other fruits which are liable to injury when in bloom or young foliage. Thus low places where cold air settles should be avoided, also low gulches through which cold drafts prevail. In frosty situations an incline away from the morning sun will often allow the trees to escape serious injury.

\section{PROPAGATION AND PLANTING}

The chapter on propagation gives the general method of growing and budding peach seedlings. In selecting pits, preference is usually given to those from strong-grownig, yellow peaches, at least for working on the same colored fruits, while others use pits of the Morris White, others the Strawberry, and others still will use only pits from vigorous seedling trees. In this State the peach is usually so healthy and vigorous, and the "yellows" not known, and less care may be needed in selecting pits; still there is certainly nothing lost by making every effort for a good stock.

The hard-shell sweet almond has long been used as a stock for the peach. It is held that it gives a hardier, stronger root, in dry soils especially.

When it is desired to grow the peach on moister soil than suits its own roots, the St. Julian plum may be used. The Myrobalan 
has been used to some extent, but experience generally does not favor any plum stock for the peach and our largest propagators have abandoned its use.

The so-called "peach-almond" has often been urged as a stock for the peach but has been little used, probably because the straight peach and straight almond are so satisfactory and available. It is a fruit having the pit of a peach but the pericarp of an almond, that is tough and tasteless and disposed to split like an almond hull. Early in the fifties a chance hybrid of this sort appeared in the nursery. of W. B. West, of Stockton, and its pits were used for nursery seedlings which, when budded to the peach, produced good trees. Trees bearing the peach-almond are found here and there over the State. Mr. Burbank has produced a hybrid of the Wager peach and the Languedoc almond.

Distance in Orchard.-Distance observed in planting peach orchards differs greatly, according to the views of different growers. Regarding the peach as a catch crop to plant between apricot, pear, cherry, walnut, fig or other slower-growing, larger trees, the trees may be set comparatively close; that is, with the latter trees at thirty to forty feet, and alternate rows of peach planted quincunx, and to be removed at the end of ten to fifteen years. If the peach is to have the ground to itself, some planters plant at eighteen feet in equilateral triangles, or twenty to twenty-four feet on the squares, the present tendency of the peach, as with other trees, being to give more room than was the custom a few years ago.

Age of Trees.-In planting peach orchards yearling trees are generally preferred, though June buds are freely used and more are planted in dormant bud than of any other kind of fruit trees. The reason for this is easily found in the disposition of the peach to make a tree the first year from the bud. It springs almost at once into a full outfit of laterals. Some growers employ this disposition to form a head the first year in the nursery. When the bud has grown out eighteen inches, pinch it off at the top and force out laterals, which make long growth the same season. When planted out in orchard the following winter, cut back to ten or twelve inches. In this any one can get a yearling with the equivalent of a two-year-old head on it. The common practice is, however, to let the growth from the bud proceed as it chooses, and when the yearling is set in orchard, cut back to a single bud the laterals which are desired to form the head and removing others. If there is a dormant bud on the stem where a branch is desired and it is obstinate in not starting, a cross-cut through the bark just above it may concentrate pressure and force it out. Proper starting of the young tree is promoted by cutting away cleanly all laterals which have grown from stem-buds. In such cutting back the dormant buds at the base of such a lateral should be preserved. The development of form from a yearling branched in the nursery is illustrated in chapter on pruning.

Planting Dormant Buds.-The chapter on planting describes the planting of yearling trees. The lifting of dormant buds from the home nursery and planting in orchard is described by P. W. Butler, of Placer County, as follows: 
Have the ground prepared and stakes placed in position in the orchard in early February, if possible, and begin the planting at once, while the trees are in dormant bud. Take no more trees from the nursery than can be planted in half a day. Plow a furrow on each side of the row, six inches from the trees, turning the soil from them, then two men with heavy spades or shovels, one on each side of the tree, can readily take it up without breaking many of the roots; and what are so broken should be smoothly trimmed with a sharp knife. Place the trees in a tub of water, near where they are to be planted, and take them from it only a few at a time. Put them in a basket or box and cover with wet sack, that they may be kept moist until placed in the ground.

On planting, place the bud one inch below the level of the ground, but do not cover it until after it has grown to the height of a few inches. The stock should be cut off at the bud with a thin, sharp knife (and not with shears, as is often done, as the latter method will sometimes split the tree), when it will take in moisture and not heal readily.

Some growers do not cut back the young seedling tree until growth has started out well on the dormant bud, girdling the bark above the bud to force its growth. The tender shoot is protected somewhat by the old stem to which it can be tied. The removal of the old stem should not be too long delayed, for the wound by its amputation should heal over the first summer.

Rather more care is needed in handling dormant buds both in planting and in their young life in the orchard. Lookout must be kept for suckers and against injury in cultivation. Success with dormant buds is notable. In good hands they commonly outgrow yearlings planted at the same time, and the percentage of loss from failure of the bud to start is very small. Of course, every bud should be examined before planting, to see that it has a healthy color.

In the selection of peach trees for planting, a clean, healthy root only should be taken. During recent years there have been a good many young roots affected with knots or swellings from some obscure cause. Such trees should be burned. If planted, the knot sometimes grows to an enormous size and little or no top growth is made.

\section{PRUNING THE PEACH}

As has already been stated, the peach will carry a top of great fruiting longevity if the grower will do justice to the tree by regular shortening of the growth and forcing out new wood, upon which alone fruit is found. Not only does regular pruning do this, but it promotes longevity and vigor in the framework of the tree upon which these bearing shoots come. Left unpruned, the peach soon becomes barkbound, and the bark itself becomes hardened and brittle. Lower shoots are apt to dwindle, and the tree becomes an umbrella of foliage and fruit held aloft by bare branches bark-burned by the sun, invaded by borers, exuding gum, covered with moss and lichens-a picture of distress and unprofitability because its owner does not give the tree a chance to re-invigorate itself with large fresh leaves from the new wood which alone can carry them.

Vase-Form with Continuous Leaders.-The common vase form of the peach tree is amply described in Chapter XII, where it is used 
to illustrate the development of the prevailing California method of shaping fruit trees. Recently there has appeared a different form which, though not new, is being given local names. It consists in building the tree with continuous ribs or leaders, a variation in the vase-form which has been used in Europe for centuries, perhaps. The following very clear account of it is prepared by Mr. C. F. Collins, horticultural commissioner of Tulare County:

Another method of vase form, known locally as the Sims method, has been practiced for many years by William Sims, of Farmerville, and is now practiced by the California Fruit Canners' Association in its large orchard near there, by Hunt Bros. in their orchards near Exeter, and by many individual growers in that vicinity. The trees are cut back to 18 inches at planting, and at the first winter's pruning four or five of the most upright-growing branches are left to form the head. These are cut to a uniform height and as great a length as the season's growth will allow. On a vigorous tree this will be about 6 feet. All laterals are removed from these.

The second winter retain one strong upright branch emerging from near the end of each branch of previous season's growth, and remove all laterals from the tree larger than a lead pencil. Toy these main branches at a uniform height of 10 or 12 feet from the ground, according to the growth made. Sometimes a lateral is allowed to grow from one of these main limbs to fill in an open space in the outline of the tree. At the end of the second winter's pruning, we have a low-headed tree with four or five main branches 10 or 12 feet long, and so upright that the tree is only 6 or 7 feet across the top. Enough small laterals are left for abundant shade. After this each season remove all large laterals, leaving only the small fruiting wood, and cut this back to the required amount.

Some of the claims made for this system are that no propping is required to support the limbs, and as these are so nearly perpendicular in growth the orchard is more easily worked close to the trees and the trees are more easily kept down to a proper height than when pruned by the ordinary method. This system certainly works admirably in the sections where it is used, and in my opinion, on true peach soil, where the trees are vigorous and grow large, strong limbs, it is far superior to the old vase form, but it is a question if it would prove as satisfactory in many of our orchards where the trees make a more feeble growth, and especially with a tree of as slender growth as the Muir. Of course, with a less vigorous tree it might require three or even four years to get the tree to a proper height by this method.

Special Points on Peach Pruning.-Whatever plan for low, vase-form be adopted, there are points which should always be borne in mind as deductions from the experience of successful peach growers in all parts of the State. A few instances will be given:

"The peach, fruiting only on wood of the previous year's growth, bears fruit farther away from the body of the tree each year, and the small shoots of from one-eighth to three sixteenths in diameter begin to decline when the fruit is removed. To have healthy growth, all of these small branches must be removed the first winter following their fruiting, when there is a greater tendency to form small new growths, which may fruit the following season. In the peach, it will seldom be found necessary to remove any interior branches, except suckers, until they have produced a crop, when they will begin to decline and should be removed.

"I would certainly not cut peach trees back less than one-half of the new growth in the winter pruning, and our trees are getting too large for their age even with that amount of pruning. This has suggested, in other localities, summer pruning or shortening in, with success in some places. So far my own experience is favorable. It will be noticed on trees kept growing rapidly that the fruit buds are near the ends of the shoots, and it seems to take away too many 
of these buds to cut back one-half in the winter pruning, but by cutting back about one-half the new growth in August, fruit buds are developed lower down, and where they would not be developed without the summer pruning."H. Culbertson, El Cajon, San Diego County.

"Prune the peach every year, cutting back and thinning out the center, using great care not to cut out too many of the little fruit shoots of new wood growing on the main branches, but removing the slender branches of the old wood, leaving as many branches of the new growth as the tree will support. In this case judgment must be used as to what the tree will support. The soil may be wet or dry, rich or poor, the grower must be the judge. To grow small fruit, prune lightly; to grow large fruit, prune with care and judgment. To get this judgment you must have some practical experience. I prefer doing the work when the sap begins moving in the spring of the year. All cuts heal over better then and the pruner can see how the buds are setting and use his own judgment as to how much wood he wants cut out."-R. C. Kells, Yuba City, Sutter County.

"Cutting back the peach must be more severe, as the growth of the new wood diminishes. Not more than five or six fruit buds should be left on a shoot, and if the fruit all sets, it must be also thinned. The trees should be trained low and their vigor encouraged by permitting a reasonable amount of young shoots to grow around the lower part of the main limbs. When this method is continued systematically every season, the trees will bear large crops of fruit, of good quality, for many years. When they are allowed to overbear for one or two seasons' the fruit will decrease in size, and soon become almost worthless; the trees will be enfeebled, and in consequence very liable to be attacked by disease. The only thing to be done in this case is to cut off the whole top of the tree, allowing it to form a new head. I have seen old peach orchards thus renovated, and the results are often very flattering, but it is far better not to allow them to get into a condition where this desperate remedy is necessary." -Leonard Coates, Morganhill.

Cutting Back the Peach Is not Shearing.--Some undertake the annual pruning of the peach by a shearing process, treating a fruit tree as one would a hedge-cutting everything to a line. There has been a good deal of this done in California, but it is wrong nevertheless. Shortening in the new growth of the peach each year is a proper practice. It is the first step toward preventing over-bearing of small, unmarketable fruit and saving the tree from profitless and injurious effort. Thinning the shoots by removing all but one when two or three start from the same point is also working toward large fruit and regular bearing in the tree. This shortening and thinning of the new wood must also be followed by thinning of the young fruit just after the natural drop and it is seen that the tree carries too many. Proper pruning can not be done by shearing because it is apt to shorten the strong shoots too much and the weak shoots too little. Each shoot must be cut by itself according to its growth and its ability to carry more or less fruit. Shearing, too, does not thin out the shoots but continually multiplies them until the tree is full of brush as a hedge.

\section{THINNING PEACHES}

Thinning out fruit on the peach tree is not only the secret of obtaining good, marketable fruit, but joins hands with pruning in preserving the health and future production of the tree. The importance of thinning has been urged in a previous chapter, but the following is a very strong statement, by Mr. Culbertson : 
In my own experience there is no single operation in connection with fruit growing of more importance than thinning. The past season, in order to test the difference in expense of preparing large and small peaches for drying, I timed the cutting, and found it took double the time; hence, double the expense, which meant a difference of about $\$ 15$ per ton of dried fruit. Add to this a difference of two cents per pound in price makes $\$ 55$ per ton. Suppose an orchard under good treatment produces a ton of peaches to the acre, then $\$ 55$ would represent the difference in profits. Unthinned or small fruit is certainly undesirable.

As to how much thinning should be done there are diverse opinions. Some take off one-half, others three-fourths. Some growers thin to meet a certain ideal, but find it difficult to explain in words. The common rule of leaving a specimen of fruit every four or six inches is a safe rule; that means many must come off. Different conditions of soils, climates, and irrigation vary the amount to thin out, more or less. More may be left where the tree is on land giving a strong, vigorous growth.

In thinning peaches $I$ have been practicing a method that gives good results and is easily learned. The peach bears on three sizes of branches, that are oneeighth, three-sixteenths, and one-fourth of an inch in diameter. The first has two peaches, the second three, and the third four; this, of course, after there has been a judicious course of pruning and the trees under irrigation; trees on dry land should have only one-half as many left. To reach this result often a dozen may have to come off, allowing only two to remain. The more there are the greater the necessity for thinning.

The time for thinning peaches is as soon as one can be sure which are likely to remain on the tree and which will drop off of their own accord.

\section{IRRIGATION OF THE PEACH}

As the peach is the greatest deciduous fruit of the interior valleys and foothills, it is also the deciduous fruit which is chiefly grown with irrigation. Most of the specific conclusions set forth in Chapter XV are based upon experience with the peach and the reader is advised to consider them from that point of view. One of the most important points of success in irrigating the peach is to use enough water earlier in its growth so that application need not be made within about three weeks of ripening. Enough water before that will usually insure size on properly thinned trees and the withholding of water near ripening will secure good quality. After the crop is gathered, irrigation can be resumed to continue the late summer growth for next year's fruit buds and to save the tree from injury during the long autumn drouth. Very great disappointment in thrift and bearing of peaches has resulted from lack of irrigation in the early autumn in the interior valley. Even in places where the trees make a good crop by rainfall or underflow, there may be great injury to the tree by inadequate soil moisture during the remainder of the growing season.

\section{WORKING OVER PEACH TREES}

The fashion in peaches changes from time to time according to the demands of the canners or the market for dried fruit. The grower often finds varieties which he first selected, less healthy, less pro- 
ductive, or, for other reason, less desirable than others. There is, therefore, often occasion for working over trees. Budding if often resorted to, buds being successfully set in quite old wood, providing buds from well-matured wood are taken. Wood buds from young trees unaccompanied by fruit are best, but because of greater certainty of securing the variety desired, it is common to take wood and fruit buds together from bearing trees. A larger cut of bud and adjacent bark is taken when working in old bark than for use on seedlings. When a branch is budded, it is sometimes broken at a distance beyond the bud and allowed to hang, the idea being to furnish the bud some but not too much sap. Some growers thus bud and break part of the branches, allowing others to remain unworked, to maintain the growing processes of the tree. These branches and those in which buds have not taken, are cut off and grafter the following spring. The almond is successfully grafted over with the peach, and this course has been followed with thousands of unproductive almonds.

Grafting the Peach.-Grafting the peach by the ordinary topgrafting with a cleft graft seldom succeeds. A side graft with saw and knife is better. It is described by J. W. Mills, formerly of the University Branch Experiment Stations, in southern California, as follows:

Saw grafting is rapidly taking the place of cleft grafting, for it does away with all difficulties arising from splitting, and there is no cavity left in the heart of the limb or tree. The process is to saw off the limb at the desired place as in cleft grafting, then saw across the corner and down the side at an angle of about 45 degrees and trim out with a sharp knife. Place the knife blade a little to one side of the saw cut, a little farther from the edge at the top than at the bottom, and by pressing on the knife the whole sides of the crevice will be trimmed smoothly at one stroke; this operation repeated on the other side of the saw cut will make a neat notch in the end of a solid limb. By cutting a little deeper from the saw cut at the top than at the bottom, and if the amateur does not trim his scion at the right angle, he can insert it gently in the crevice or notch and see just where to trim. If he is so slow that the fresh cut shows signs of discoloration, he can make a fresh surface by placing his knife parallel to the edges and shaving off a thin slice. He still retains the same angle, but the scion will set a little deeper, which is no objection. By cutting a thin layer off the top of the stump next to the notch will show exactly where the inside layer of bark is. The inside of the scion must be even with the inside layer of the bark of the stump or limb that is being grafted. If the scion is inclined slightly out or in at the top, it will make a correct union at some point and be sure to grow. If the inclination is very slight the union will extend over considerable length, and will make a much better start than if the union is at only one point, owing to the enlarged surface through which the sap is transmitted. One of the most important points in grafting is to have good wax and go over the grafts a few days after they are put in and rewax them.

\section{DISEASES OF THE PEACH}

Curl-Leaf.-The most prevalent trouble with the peach tree in California is the curl-leaf. It was noticed from the first planting of peach trees by Americans, nearly sixty years ago, and free conjecture as to its cause was indulged in until it was shown to be a specific fungus, 
and its prevention by washes of fungicidal character demonstrated. The treatment will be described in the chapter on tree diseases. The facts of its occurrence may be stated as follows:

Curl-leaf is much more prevalent in some sections than others, and in one place than another in the same section, and some sections are practically free from it. Some varieties are much more subject to curl-leaf than others; generally speaking, some curl nearly everywhere, others curl in one place and not in another, others are practically free from curl in all situations.

Curl-leaf occurs in various degrees. Mild cases do not seem to injure either tree or fruit; severe cases destroy the fruit and sometimes the tree itself. The disease is almost always at its height when the young fruit is about the size of small peas. If the curl is "bad," the fruit will fall to the ground, there not being healthy leaves enough to afford the required support. If, however, the curl is moderate and partial, only a part and sometimes none of the fruit will be lost. The disease, as is well known, is of brief duration, say twelve to twenty days, after which the trees resume a healthy appearance in every respect, and if the fruit has been able to survice the ordeal, it also appears to grow and become as perfect as if no check had been given to its growth. But it is better to save the tree the burden of a new foliage growth.

Mildew.-This disease, which occurs in the form of whitish felted patches on leaf and twig early in the spring, and finally affects the fruit, has long been troublesome in this State, and occurs on certain susceptible varieties in many localities from the coast to the Sierra foothills. Observation in this State has fully affirmed the statement of Downing, that the serrate, glandless-leaved varieties are liable, and those with good glands on the leaf stems are free.

The conclusion would be that where mildew prevails, varieties with serrate, glandless leaves should be avoided. But it has been found that some glandless-leaved varieties, although subject to mildew, resist curl-leaf. Therefore it may be worth while to combat the mildew. This has been done effectually by treatment which will be described in a later chapter. others.

As with curl-leaf, mildew is prevalent some years and slight in

The most serious disease which has thus far stricken the peach in California is locally known as the "peach blight," the work of a shothole fungus (coryneum). This also has been satisfactorily checked by spraying, as will be described in the Chapter on Diseases of Trees and Vines.

A common trouble of the peach known as "split-pit," has recently been studied by the California Experiment Station and the tentative conclusion has been reached that split-pits are physiological phenomena and not caused by disease germs. Whether this abnormal growth is due to weakness of variety or to cultural conditions is not determined. It is true that varieties differ in amount of splitting, and selection is being made to some extent on that basis. 
Peach Varieties Approved by California Growers.

\begin{tabular}{|c|c|c|c|c|c|}
\hline VARIETIES. & $\begin{array}{c}\text { Upper } \\
\text { coast } \\
\text { valleys. }\end{array}$ & $\begin{array}{c}\text { Central } \\
\text { coast } \\
\text { valleys. }\end{array}$ & $\begin{array}{l}\text { Interior } \\
\text { valley and } \\
\text { foothill. }\end{array}$ & $\begin{array}{l}\text { Mountain } \\
\text { valley } \\
\text { region. }\end{array}$ & $\begin{array}{c}\text { California } \\
\text { Southern } \\
\text { Coast. }\end{array}$ \\
\hline Alexander & * & * & ** & * & $*$ \\
\hline 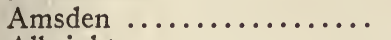 & ... & $\cdots$ & * & $\ldots$ & * \\
\hline Albright $\quad \ldots \ldots \ldots \ldots \ldots$ & ... & ... & * & $\cdots$ & $\cdots$ \\
\hline Bergen...$\ldots \ldots \ldots \ldots$ & . & ... & & .. & $*$ \\
\hline 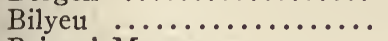 & & $\cdots$ & * & $\cdots$ & \\
\hline 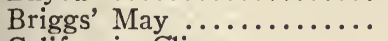 & * & $*$ & * & $*$ & * \\
\hline California Cling .......... & $\cdots$ & * & $\begin{aligned} * * \\
*\end{aligned}$ & $\cdots$ & $\cdots$ \\
\hline Decker $\ldots \ldots \ldots \ldots \ldots \ldots$ & $\ldots$ & 米* & $\begin{array}{r}* \\
* *\end{array}$ & $\dddot{* *}$ & $\ddot{* *}-$ \\
\hline Early Crawford $\ldots \ldots \ldots \ldots$ & ** & $\begin{aligned} * * \\
*\end{aligned}$ & $\begin{aligned} * * \\
*\end{aligned}$ & & $*$ \\
\hline $\begin{array}{l}\text { Early York } \ldots \ldots \ldots \ldots \ldots \\
\text { Elberta }\end{array}$ & *** & ** & ** & * & ** - \\
\hline Foster $\ldots \ldots \ldots \ldots \ldots \ldots \ldots \ldots$ & $\ldots$ & ** & ** & * & $* *$ \\
\hline George Fourth .......... & $\ldots$ & & & & $*$ \\
\hline George's Late Cling ...... & ... & * & * & $\ldots$ & * \\
\hline Gen. Bidwell ............ & ... & $\cdots$ & * & ... & $*$ \\
\hline Globe $\ldots \ldots \ldots \ldots \ldots \ldots$ & * & ... & & * & * \\
\hline Golden Cling (Sellers).... & & * & * & & * \\
\hline Hale's Early ............. & * & ** & ** & $*$ & * \\
\hline Heath Cling ............ & ... & $*$ & ** & ... & * \\
\hline Henrietta (Levy's) ....... & ... & * & ** & ... & * \\
\hline 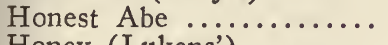 & $\cdots$ & $*$ & $\ddot{*}$ & $\cdots$ & $\because$ \\
\hline Honey (Lukens') ........ & $\cdots$ & -.. & * & .. & * \\
\hline Imperial $\ldots \ldots \ldots \ldots \ldots$ & * & .. & * & $\ldots$ & $*$ \\
\hline Indian Blood $\ldots \ldots \ldots \ldots$ & $\cdots$ & $\cdots$ & * & $\cdots$ & * \\
\hline Jones's Large Early ...... & $\cdots$ & $\cdots$ & * & $\cdots$ & $\cdots$ \\
\hline La Grange $\ldots \ldots \ldots \ldots \ldots$. & $\cdots$ & ** & $* *$ & $\ddots_{*}$ & $\stackrel{*}{*}$ \\
\hline $\begin{array}{l}\text { Late Crawford } \ldots \ldots \ldots \ldots \ldots \\
\text { Lemon Cling }\end{array}$ & $*$ & * & * & & $* *$ \\
\hline & & ** & ** & $\ddot{*}$ & ** - \\
\hline May Flower $\ldots \ldots \ldots \ldots$ & $\ddot{*}$ & * & * & $\ldots$ & \\
\hline McDevitt Cling ......... & * & & * & $\cdots$ & * \\
\hline McClish Cling ........... & * & $*$ & & $\cdots$ & ... \\
\hline McKevitt Cling $\ldots \ldots \ldots \ldots$ & $\ldots$ & $\cdots$ & * & ... & ... \\
\hline Mary's Choice .......... & ... & $\cdots$ & * & ... & ... \\
\hline Morris's White .......... & $\cdots$ & $*$ & $*$ & ... & * \\
\hline Muir $\ldots \ldots \ldots \ldots \ldots \ldots \ldots$ & $*$ & ** & ** & * & ** - \\
\hline Newhall $\ldots \ldots \ldots \ldots \ldots \ldots$ & $\cdots$ & * & * & ... & * \\
\hline Nicholl's Cling .......... & * & $*$ & * & $\cdots$ & * \\
\hline $\begin{array}{l}\text { Oldmixon Free } \ldots \ldots \ldots \ldots \\
\text { Orange Cling }\end{array}$ & $\cdots$ & $\begin{aligned} * \\
* *\end{aligned}$ & $\ddot{* *}$ & $\cdots$ & $* *$ \\
\hline $\begin{array}{l}\text { Orange } \text { Cling } \ldots \ldots \ldots \ldots \\
\text { Peen-to } \quad \ldots \ldots \ldots \ldots \ldots .\end{array}$ & $\ldots$ & $\begin{array}{l}* * \\
\ldots\end{array}$ & & & $\begin{array}{l}* * \\
* *\end{array}$ \\
\hline $\begin{array}{l}\text { Peen-to } \\
\text { Persian Cling } \ldots \ldots \ldots \ldots \ldots\end{array}$ & $\cdots$ & $\cdots$ & $\cdots$ & $\cdots$ & \\
\hline Phillips's Cling $\ldots \ldots \ldots \ldots$ & * & $\ddot{* *}$ & ** & $\cdots$ & $\ddot{* *}$ - \\
\hline Piquet's Late ............ & & * & * & & * \\
\hline Runyon's Orange Cling.... & $*$ & * & * & $\ddot{*}$ & * \\
\hline Salway $\ldots \ldots \ldots \ldots \ldots \ldots$ & * & ** & ** & ... & ** - \\
\hline 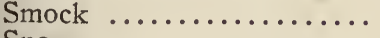 & ... & & ... & $\ldots$ & * \\
\hline Snow $. . . \ldots \ldots \ldots \ldots . . . . .$. & ... & * & & ... & * \\
\hline St. John .............. & ... & * & $* *$ & $\ldots$ & * \\
\hline 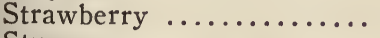 & - & * & * & $\ldots$ & * \\
\hline Stump...$\ldots \ldots \ldots \ldots$ & ... & * & * & $\ldots$ & ** \\
\hline Susquehanna ........... & ... & * & ** & ... & * \\
\hline Triumph $\ldots \ldots \ldots \ldots \ldots$ & $\cdots \ddot{*}$ & $*$ & ** & $\cdots$ & Wis \\
\hline $\begin{array}{l}\text { Tuskena (Tuscan) ........ } \\
\text { Wheatland }\end{array}$ & & ** & ** & & $* *-$ \\
\hline Wylie Cling $\ldots \ldots \ldots \ldots \ldots \ldots$ & $\cdots$ & $\cdots$ & $*$ & $\cdots$ & $*$ \\
\hline Yellow Tuscany .......... & $\cdots$ & * & $\cdots$ & $\begin{array}{l}\cdots \\
\ldots\end{array}$ & $\ddot{*} \dot{*}$ \\
\hline
\end{tabular}

**Indicates that the variety is approved in the region designated.

** Most highly commended. 


\section{VARIETIES OF THE PEACH}

Nearly all varieties of the peach have been tried in California, and, as with other fruits, it has been found that varieties must be chosen with reference to their success in special locations. Choice also has to be made according to the purpose of the grower, whether for early marketing, for sale to canners, for drying, or distant shipment or for late marketing. As with apples, there is little use of planting early varieties (unless it be for home or local use) except in very early regions. An early peach from a late region is killed by competition with better middle season sorts from the earlier regions.

Dates of Ripening of Leading Varieties.-The relative ripening of a large number of peach varieties, as noted at the University Experiment Station, at Pomona, will be useful to planters in determining proper succession of varieties, although of course the exact dates will not widely apply.

\begin{tabular}{|c|c|c|c|}
\hline VARIETY. & First Flower. & Full Flower. & Ripe. \\
\hline Briggs' Red May & March 4 & March 24 & June 16 \\
\hline Amsden's June .................. & March 8 & March 25 & June 17 \\
\hline Alexander $\ldots \ldots \ldots \ldots \ldots \ldots \ldots \ldots \ldots \ldots$ & March 17 & March 31 & June 17 \\
\hline Large Early York. & March 4 & March 18 & July 15 \\
\hline Yellow St. John... & March 4 & March 12 & July 17 \\
\hline Crawford's Early ............... & March 2 & March 15 & July 21 \\
\hline Foster $\ldots \ldots \ldots$. & March 4 & March 15 & July \\
\hline Oldmixon Free .. & March 7 & March 18 & July 25 \\
\hline Morris White ... & March 4 & March 15 & Aug. \\
\hline ......... & March 8 & March 23 & Aug. \\
\hline Susquehanna & March 4 & March 14 & Aug. \\
\hline Crawford's Late & March 2 & March 14 & Aug. \\
\hline ............... & March 4 & March 14 & Aug. \\
\hline Runyon's Orange Cling...$\ldots \ldots \ldots$ & March 2 & March 14 & Aug. \\
\hline California Cling ............... & March 2 & March 16 & Aug. 12 \\
\hline Stump the World & March 2 & March 16 & Aug. 12 \\
\hline Lovell & Feb. $\quad 28$ & March 9 & Aug. 13 \\
\hline Nichols' Orange Cling & March 4 & March 14 & Aug. 14 \\
\hline Seller's Cling ........... & March 2 & March 14 & Aug. 14 \\
\hline McDevitt's Cling $\ldots \ldots \ldots \ldots \ldots \ldots \ldots$ & March 2 & March 18 & Aug. 16 \\
\hline McKevitt's Cling .. & March 2 & March 19 & Aug. 19 \\
\hline Wilkin's Cling .......... & March 3 & March i4 & Aug. 20 \\
\hline Indian Blood. & March 5 & March 25 & Aug. 20 \\
\hline Yellow Tuscany Cling & March 5 & March 19 & Aug. 21 \\
\hline Lemon Cling & March 14 & April & Aug. 21 \\
\hline Smock's Late Free. & March 4 & March 18 & Aug. 28 \\
\hline Picquet's Late & March 5 & March 18 & Sept. \\
\hline Staley's California & March 2 & March 15 & Sept. \\
\hline Henrietta (Levy's) & March 6 & March 15 & Sept. 15 \\
\hline Heath's Cling & March 7 & March 14 & Sept. 15 \\
\hline Salway $\ldots$... & March 7 & March 9 & Sept. 18 \\
\hline
\end{tabular}

In an early region one can plant early, middle, and late varieties to advantage, and thus secure a very long-fruiting season. The peach season in interior districts begins at the first of June with the Alexander, and continues to the end of Nevember with local seedlings-giving six months of peaches. Of course, the very early and very late sorts are only of use for marketing as table fruit. The most important series 
is a fine succession of mid-season peaches suitable either for canning, drying, or distant shipment. Such a selection can be made from the tables and descriptions which will be given later.

Color is a most important item in the peach. While canners and Eastern shippers use the beautiful white peaches to advantage, the fashion for canning and drying is now strong in support of the yellowfleshed clingstone varieties. The yellow freestone peaches are also in greatest demand. The color about the pit is also an important point. Canners demand a peach, whether white or yellow, which is almost free from color at the pit, because the extraction of the red color dyes the juice; in drying, the demand just now is for a yellow peach with a red center, because the colors give the dried fruit a more attractive appearance. Of course there is a market for dried white peaches but the preference is for the yellow.

\section{PEACHES APPROVED IN CALIFORNIA}

The following are the peaches chiefly grown in California, arranged approximately in the order of ripening:

Briggs' Red May (California).-Originated as a chance seedling in nursery row, on the farm of John G. Briggs, on the Feather River, about one mile from Yuba City, about 1870. It was found to be about ten days earlier than the Early Tillotson, which was then the stand-by for an early peach. Fruit medium to large, round; white skin, with rich, red cheek; partially free, a standard early variety; subject to mildew.

Jones' Large Early (New York).-Large, roundish, flattened, white with deep crimson.

Alexander (Illinois).-Most widely grown as best early variety. Fruit medium to large; greenish white, nearly covered with deep red; flesh firm, juicy, and sweet; bears transportation well; pit is partly free.

Triumph (Georgia).-Medium sized, early, yellow, partial cling; very good.

Amsden (Missouri) - - Resembles preceding, but averages smaller; claimed by some to be slightly earlier; rather less liable to curl-leaf.

Honey (Lukens).--Medium, oblong pointed, white mottled carmine; very sweet; related to Peen-to.

Peen-to.-Flat peach or saucer peach of China; good in Southern California.

Early Imperial (California).-Originated by W. W. Smith, Vacaville, and planted to secure a yellow freestone earlier or larger than St. John. Most growers find it no improvement on St. John.

Yellow St. John (New Orleans).-Earliest yellow peach; averages smaller than Yellow Crawford, but classed as large; roundish, orange yellow with deep red eheek; juicy, sweet and high flavored; freestone.

Hale's Early (Ohio)--Medium to large, nearly round; skin greenish, mostly covered and mottled with red when ripe; flesh white, melting, juicy, rich and sweet; fair for local market and shipping; widely grown; freestone.

Strawberry (New Jersey).-Medium size, oval; stem cavity deeply sunk; suture extending half way round; skin almost wholly marbled with deep red; flesh whitish, juicy, rich and delicate; tree healthy.

Bergen's Yellow (New York).-Large, roundish; suture deep; orange yellow, red cheek; approved in Sonthern California.

Foster (Massachusetts).-Uniformly large, slightly flattened; slight suture; stem moderately depressed; flesh yellow, very rich and juicy; color deep orange, 
dark red in the sun; freestone; tree hardy and productive; very widely grown in California and popular. Ripens before Early Crawford, which it sometimes resembles, but is of better quality.

Craroford's Early (New Jersey).-Very large, oblong, swollen, point at the top prominent, suture shallow; skin yellow, with red cheek; flesh yellow, rich, and excellent; freestone; tree very healthy and productive; probably the most largely planted variety in California.

George the Fourth (New York).-Large, round, deeply divided by broad suture; sides unequal; skin pale yellowish white, dotted with red, and red cheek; flesh pale, red at pit, from which it parts freely; quality good. Somewhat troubled with curl-leaf.

Snow (American).-Large, globular; skin clear, beautiful, almost wholly white; flesh white to the free stone, juicy, rich, and sprightly.

Mary's Choice (New Jersey).-Large, yellow, resembling Early Crawford, but ripening later.

Red Cheek Melocoton (American).-Large, roundish oval, swollen point at top; yellow, with deep red cheek; flesh yellow, red at stone, which is free, juicy, good flavor. Approved in Humboldt and San Benito Counties.

Tuskena (Alabama or Mississippi).-Wrongly called "Tuscan" and "Tustin" cling in this State; largely planted in interior valleys and foothills; very large yellow cling; the earliest fine cling variety; very valuable for early shipping. Ripens with Crawford's Early.

Oldmixom Free (American).-Large, roundish or slightly oval; greenish or yellowish white, marbled with red; flesh white, tender, and excellent, juicy and rich; high flavor.

Honest Abe (California).--"Originated at Healdsburg, Sonoma County. Large, yellow, with red cheek; best quality; ripens between Crawford's Early and Late. Does not curl."-James Shinn.

Morris White.-Large, oval; skin white with creamy tint when fully ripe; flesh white to the stone, which is free; melting, juicy, sweet, and rich; especially good for home use and canning; somewhat subject to curl-leaf.

Wager (New York).-Lemon yellow tinged with red; flesh yellow, rich, juicy, sweet, excellent, having much the appearance and flavor of apricots; stone small and free from the flesh; quality best.

Muir (California).-Originated as chance seedling on the place of John Muir, near Silveyville, named and first propagated by G. W. Thissell, of Winters. Fruit large to very large; perfect freestone; flesh clear yellow, very dense, rich and sweet; pit small; tree a good bearer and strong grower, if on rich soil, to which it is best adapted; free from curl in Vacaville district; fruit a good shipper and canner, and peculiarly adapted to drying because of exceptional sweetness and density of flesh; yield, one pound dry from less than five pounds fresh. One of the best California seedlings. Claimed by some to be identical with Wager.

Muir variations with large flowers, with fruit like Muir, but not splitting at pit, reported by W. A. Rosander, Kingsburg; A. S. Coon, Fresno, and H. R. Shaw, Selma.

Muir Cling by W. R. Fletcher, Green Valley, Sonoma County. Commended by Green Valley Cannery; propagated by A. F. Scheidecker, Santa Rosa, 1907.

Wheatland (New York).-Large, roundish; skin golden yellow, shaded with crimson; flesh yellow, rather firm, juicy, sweet, and of fine quality.

Elberta (Georgia).-Very large; round-oval with deep suture; golden-yellow, faint red stripes; flesh yellow, fine, juicy, rich and sweet; tree prolific; perfect freestone.

Newhall (California).-_"Originated with Sylvester Newhall, of San Jose. Very large, skin yellow, with a dark red cheek; flesh deep yellow, juicy, and a rich, vinous flavor; ripens about one week before Crawford's Late; tree very hardy, healthy, vigorous, and not affected by curl; freestone."-John Rock. 
Stump the World (New Jersey).-Large, strong; skin creamy white, with bright red cheek; flesh white, juicy, and highly flavored. Commended for family use by the Southern California Nurserymen's Association. Curls somewhat in some localities; freestone.

Crawford's Late (New Jersey).-Very large, roundish, yellow with dark red cheek; flesh deep yellow, juicy, and melting; flavor rich and excellent; a popular and widely-grown variety, but very subject to curl-leaf in some localities; freestone.

Lemon Clingstone (South Carolina).-Large, lemon-shaped or oblong, having large, projecting, swollen point like a lemon; skin fine yellow; flesh firm, yellow with sprightly vinous subacid; slightly red at the pit, which adheres firmly.

Orange Clingstone.-Large, round; suture distinctly marked and extending nearly around the fruit; no swelling at apex, like Lemon Clingstone; deep orange color, with red cheek; flesh yellow, firm, juicy, with rich flavor; somewhat subject to mildew. Though largely grown, this variety has been largely supplanted by the following sub-varieties, which are seedlings from it.

Sellers' Golden Cling (California).-Originated on the farm of S. A. Sellers, Contra Costa County, and introduced by James Shinn. Very large, rich golden color; tree healthy; one of the very best of clings; ripens with Late Crawford.

Runyon's Orange Cling (California).- "Originated with Mr. Sol Runyon on the Sacramento River. Superior to the common Orange Cling. Runyon's Orange Cling has globose glands, and is not subject to mildew like the common sort. Fruit very large, yellow, with a dark crimson cheek; rich, sugary, and vinous flavor. Highly esteemed and extensively planted in the Sacramento region and elsewhere."-John Rock.

Nichols' Orange Cling (California).-Originated by Joseph Nichols, of Niles, introduced by James Shinn. Large, yellow, with purple cheek; flesh yellow and good. Tree healthy and a heavy bearer.

Peck's Orange Cling (California).-_Originated at Healdsburg, Sonoma County. Improved seedling of Orange Cling, of Downing. Large, handsome, yellow-fleshed, free from curl, hardy, vigorous, productive, superior for market or drying; planted more extensively in Santa Rosa Valley than in any other."Luther Burbank.

Stilson (California)._-“Originated at Marysville (?). Perfect shape; very large; red cheek with crimson stripes; yellow-fleshed, more highly colored than Susquehanna; table and market quality excellent; ripens after Crawford's Late; freestone."-P.W. Butler.

Susquehanna (Pennsylvania).-Large, nearly globular, suture half-round; skin rich yellow, nearly covered with red; flesh yellow, sweet, juicy, with rich, vinous flavor; freestone; tree healthy. Very widely distributed and popular.

McCowan's Cling (California).-Originated with Dr. McCowan, of Ukiah. Yellow cling; round, smooth outline; no suture; no red at pit, which is small; flesh firm, fine-grained, and sweet; not much subject to curl; fruit apt to run small unless carefully thinned; reported an irregular bearer in Alameda County; liked by canners; approved by Placer County.

Lovell (California).-Originated as chance seedling with G. W. Thissell, and named by him in 1882 ; propagated by Leonard Coates, of Napa. Yellow, freestone; size uniformly large, almost perfectly round; flesh fine, texture firm, solid, clear yellow to the pit; tree a good grower and bearer; superior for canning and shipping, and dries well. Said to curl in some places. "The richest peach I ever saw on a tray."-E. A. Bonine, Los Angeles County. The most popular yellow freestone for canning and drying. Sometimes reported as "heavier" than the Muir.

McKevitt's Cling (California).-Originated as a chance seedling in apricot orchard planted by M. R. Miller, on place owned later by A. McKevitt, Vaca Valley; named in 1882 by nurserymen who propagated it. A white clingstone; flesh very firm, fine-grained, sugary, and rich, high flavor, white to the pit; skin strong, and fruit excellent for shipping or canning; tree remarkably strong in growth and free from disease. Widely distributed. 


\section{McClish Cling.-Yellow cling, grown in Sonoma County.}

Wylie Cling.-An old seedling by John Wylie, Green Valley, Sonoma County, increasing in popularity in northern Sonoma County as superior to Orange Cling in not splitting at the pit, and not dropping from the tree. A fine peach for canning and drying.

General Bidwell (California).-Originated from a short shoot from a peach root upon which an apricot had grown and died, on Ranch Chico. Named by State Horticultural Society, September 4, 1886, and commended for :cultivation. Ripens one week later than Late Crawford and ahead of Salway and Picquet's Late. About the shape of the Orange Cling, but larger; very yellow with reddish cheek; flesh very solid, juicy, and rich; freestone and a small pit.

California; syn. Edreard's Cling (California).- "Originated in Sacramento. Very large, round, regular; orange, nearly covered with dark, rich red; flesh deep yellow; flavor delicate, rich vinous." $-C . W$. Reed.

Picquet's Late (Georgia).-Large to very large; round, sometimes a little flattened; yellow, with red cheek; flesh yellow, melting, sweet, rich and fragrant; freestone; not subject to curl-leaf.

Smock Freestone (New Jersey)._"Large yellow, mottled with red; moderately rich and juicy. A better drying peach than Salway."-E. A. Bonine.

La Grange (New Jersey) - Large, oblong; greenish white, some red on sunny side; not desirable in coast regions; freestone.

Salway (English).-Large, roundish oblate; suture broad, deep, extending beyond the apex; skin downy, creamy yellow, rich, clear, crimson cheek; flesh deep yellow, red at the pit; juicy, rich, sweet, vinous; freestone; a standard late peach in California; tree very healthy.

Phillips' Cling (California).-Originated with Joseph Phillips, of Sutter County; propagated by J. T. Bogue of Marysville. Fine large yellow cling, no color at pit, which is very small; exceedingly rich and high colored; described by Mr. Skinner, superintendent Marysville Cannery, as the best peach he ever used. The most popular yellow clingstone. Ripens progressively so that picking can cover two weeks without falling from tree. Requires good land and ample moisture.

Persian's Cling (California).-."Originated in Visalia, probably from seed of Heath Cling, and a few days earlier than its parent. Large; clear white skin and flesh, the latter very sweet; commended for canning."-J. H. Thomas; Tulare County.

Heath (Maryland).-Described by Downing as the most delicious of all clingstones. Very large; skin downy, creamy white, with faint blush of red; flesh greenish white, very tender and juicy, with most luscious flavor, best adapted to interior regions, or places free from curl.

Steadly (Missouri).- "Large to very large; white skin; flesh white at the pit, firm, rich, and good flavor; freestone. Produces very heavy yield of dried fruit." - I. H. Thomas, Tulare County.

George's Late Cling (California).- -Originated in Sacramento. Large; white flesh, colored around the pit; beautiful yellow color, striped and splashed with bright red; a very heavy and uniform bearer; a good shipper, and at its season of ripening there is no peach grown in Placer County that yields the grower so much profit."-P. W. Butler. Subject to mildew in some localities.

Yellow Tuscany (Dura cini, Tuscany).-A very large yellow cling; propagated by G. Tosetti, formerly of San Leandro; tree a strong grower and free from curl-leaf; very productive. On the basis of its behavior at the University Experiment Station at Pomona, this variety has recently been largely planted in Southern California. It is counted the best yellow cling for canning in that section. Ripens with Lemon Cling.

Albright's Cling (California).- "Originated with Mr. Albright, near Placerville. Very large; yellow, with bright cheek; rarely equaled in quality and flavor. Described as larger, more highly colored, of better flavor, better shape, and the tree a more prolific bearer than the Orange Cling." $-P . W$. Butler. Endures long shipment even after being well colored. 
McDevitt Cling.-"Originated with Neal McDevitt, of Placer County. Uniformly large, rich, golden yellow, becoming red when ripe; flesh very firm and solid; superior in flavor; excellent shipper; tree good and regular bearer.

Staley (California).-Very large; eleven and one-half inches in circumference; somewhat elongated and flattened laterally; rich, creamy white with very faint touches of light red; suture shallow, but almost continuous around the peach; stone small and perfectly free, cavity considerably longer than stone; flesh white to the pit, very juicy, fine, tender; flavor delicious. Originated as sucker from peach root from which prune had been broken off in Selma, Fresno County. Ripens twenty days after Salway, or four weeks after Susquehanna. A high-class white freestone. Introduced by F. M. Nevins, Selma.

Lewy's Late; syn. Henrietta (District of Columbia).-Above average size, yellow flesh, red cheek; late; clingstone. Very popular in San Joaquin Valley.

Bilyeu's Late October.- "Large greenish white with red cheek; flesh whitish, freestone; tree a rapid grower and attains great size; prolific bearer; fruit ships well, and where it will mature no peach can take its place; does particularly well in the foothills." $-P . W$. Butler.

Decker (California).-Grown for eastern shipment, in Vaca Valley, and in Sutter and Butte counties. 


\section{CHAPTER XXI}

\section{THE NECTARINE}

The nectarine reaches perfection under California conditions, as does its close relative, the peach. The fruit is, in fact, as Downing says, only a variety of the peach with a smooth skin; only a distinct, accidental variety of the peach; and this is rendered quite certain, since there are several well-known examples on record of both peaches and nectarines having been produced on the same branch. Nectarine pits usually produce nectarines again, but they occasionally produce peaches. Peach seeds occasionally produce nectarines; the Boston variety originated from a peach stone.* All these facts which are recorded of relation between the peach and nectarine have been verified by California observation.

The practice of growing nectarines is also exactly like that employed with the peach. It is propagated and pruned in the same ways, except that, as pointed out by Mr. Culbertson, the nectarine has more of a tendency to form short interior growths, and fruit buds are formed on the larger new growths, thus enabling the pruner to cut them back more closely, and yet have an abundance of fruit buds remain. The peach and nectarines are the same in natural adaptations and requirements, and in diseases, so that what has been given concerning the growth of the peach in this State has an apt application in the case of the nectarine.

The success of the nectarine worked on almond stock, as has been demonstrated by the experience of many, has led to the grafting over a good many unprofitable almond trees to nectarine, though this has not been done to the extent to which the French prune and some other plums have been worked on old almond stocks.

Comparative Production of Nectarine and Peach.-It may be wondered, considering the similarity of the peach and the nectarine, why the former comes so near being our leading deciduous fruit and the latter is the least grown, but one, of all temperate zone fruits, only the lowly quince being less in importance. The explanation is that the fruit buyer, both in California and at the East, prefers the peach, whether it be fresh, or canned, or dried, and some of those who have tried even a few acres of nectarines have found many occasions to wish the ground had been given to peaches. How much of this preference is due to lack of knowledge of the nectarine, and how much to its somewhåt different flavor, it would be difficult to actually determine.

That the nectarine would advance in popular favor has been prophesied for some years, because of the wonderful excellence of the nectarine as grown in our interior valleys, and the passing beauty of the amber translucency of the dried nectarine, both when sun-dried and when produced by machine evaporators. The excellence of the canned

\footnotetext{
*"Downing's Fruit and Fruit Trees," p. 565.
} 
nectarine has also figured in the anticipation. It is, however, questionable how far this anticipation has been realized, for it is estimated that the amount of dried nectarines is less than two per cent and of canned nectarines less than one-half of one per cent of the respective forms of peaches. Nor does the demand call for change in this proportion, for there is a slight advantage in the market value of the peach even in its great preponderance of supply. During the last decade many nectarines have been rooted out to be replaced by peach trees, or have been grafted over into peaches. There are, however, some growers who are confident that the nectarine will in the future rank much higher in the California fruit product. It would please growers and fruit driers and canners to popularize the nectarine, for its smooth skin makes it as easy to handle as an apricot, and the beauty of the product, which certainly exceeds that of the peach and is rather more easily attained, is very gratifying to the producer.

\section{VARIETIES OF THE NECTARINE}

Varieties of the nectarine, as of the peach, show different local adaptations, and are valued by growers accordingly. The varieties grown, are, however, comparatively few. The following have been found most satisfactory in California; the descriptions are somewhat condensed from Downing's treatise, modified to suit local growth, and arranged approximately in the order of the ripening of the varieties.

Advance.-Large, round, green, marked with red and brown; flesh greenish white; rich and well flavored. The earliest to ripen.

Lord Napier (English).-Large, pale, cream color with dark red cheek; flesh white, melting, tender and juicy, separating freely from stone; leaf glands reniform and flowers large. Especially commended as a heavy and regular bearer; pronounced best in flavor at Pomona Experiment Station.

Dowenton (English).-Leaves with reniform glands; flowers small; fruit large, roundish oval; skin pale green, with deep violet red cheek; flesh pale green, slightly red at the stone, which is free, melting rich, and very good.

Early Newington (English).-Leaves serrated without glands, flowers large; fruit large, roundish ovate, a little enlarged on one side, and terminating with an acute, swollen point; skin pale green, but nearly covered with bright red and coated with thin bloom; flesh greenish white, but deep red at stone, which adheres closely, juicy, sugary, rich, and excellent.

Hardwicke (English.) - Leaves with globose glands; fruit very large, roundish, inclining to oval; skin pale green, with deep violet red cheek; flesh pale green, slightly marked with red at the stone, melting, rich, and highly-flavored; freestone. This variety is a favorite in Southern California; described by the Southern California Nurserymen's Association as being the only satisfactory bearer.

Boston.-Raised from a peach stone by $\mathrm{T}$. Lewis, of Boston; tree hardy and productive; leaves with globose glands; flowers small; fruit large and handsome, roundish oval, bright yellow, with deep red cheek; flesh yellow to the stone (which is small and pointed), sweet, though not rich, with pleasant and peculiar flavor; freestone; a general favorite in California.

New White; syn. Large White-Leaves with reniform glands; flowers large; fruit rather large, nearly round; skin white with occasionally slight tinge of red; flesh white, tender, very iuicy, with rich, vinous flavor; stone small and free commanded wherever nectarines are grown in California, and more freely planted than all other nectarines combined. 
Stanwick.-Originated in England from seed brought from Syria. Large, roundish oval, slightly heart-shaped at base; skin pale, greenish white, shaded into deep, rich violet in the sun; flesh white, tender, juicy, rich, sugary, and delicious.

Humboldt.-Very large, bright orange yellow vigorously marked with crimson, flesh orange, tender; juicy, and highly fiavored. Described as one of the best of the newer varieties. Ripens late.

As the future for the nectarine seems to rest upon drying and canning of the fruit, the light-skinned, white or yellow-fleshed varieties without color at the stone, are most desirable. For drying there has been thus far a decided preference for freestone varieties, though possibly the present popularity for cling peaches for drying may extend to the clingstone nectarines. Much color, however, either in skin or flesh, will prevent the production of the beautiful translucent, amber hue of the dried nectarine, which is attractive to consumers. Color in the flesh is, of course, undesirable in canning, because of the discoloration of the syrup. These facts have had much to do in fixing the popularity of the varieties named in the foregoing list.

At present the largest orchards of nectarines are in the interior valley locations, which are also fine for the peach and are perfectly adapted both to the growing of the fruit and to the open-air, sun-drying of it. 


\section{CHAPTER XXII}

\section{THE PEAR}

The oldest deciduous fruit trees in California are pear trees, as has already been stated in the account of fruits at the old missions, and some of the trees are still bearing, though it is a century and a third since their planting. Trees planted by pioneers in the old mining districts have actually assumed semblance to adjacent oaks. Notable instances are found in the Stillwater district of Shasta County and elsewhere. Near San Jose there is a tree over half a century old, with a trunk seven and a half feet around and yielding annually about fifteen hundred pounds of fruit, some of which was exhibited at the Columbian Exposition.

The pear withstands neglect and thrives in soils and situations which other fruit trees would rebel against. It defies drouth and excessive moisture, and patiently proceeds with its fruitage even when the soil is trampled almost to rocky hardness by cattle, carrying its fruit and foliage aloft above their reach. And yet the pear repays care and good treatment, and receives them from California growers, for the pear has been one of our most profitable fruits. It is in demand for canning, for drying, and for distant shipment, and its long season and the slow ripening after picking allow deliberation in marketing, and admit of enjoying low rates for shipment by slow trains. One of the most striking demonstrations of the commercial suitability of the California pear is found in successful marketing in London. Solomons, who is called "London's greatest fruiter," said in 1903 that California Bartletts from Block of Santa Clara are the "best in the world." Even after crossing the continent they seemed to endure shipment across the Atlantic better than eastern pears.

The most obvious marks of the California pear are size and beauty. The most conspicuous example is the Bartlett, which is the pear of California, judged by its popularity, fresh, canned and dried. When well grown, its size is grand, and its delicate color, aroma and richness unsurpassed. What extreme in point of size has been reached is not known to the writer, but he saw at the San Jose Horticultural Fair, of 1886, thirteen Bartlett pears grown by A. Block, of Santa Clara, which weighed fourteen pounds, the heaviest of the group weighing twentytwo and one-half ounces. Other pears have made standard sizes in California far in advance of their records elsewhere. There was in 1870 a Pound pear sent from Sacramento to the late Marshall P. Wilder, president of the American Pomological Society, which weighed four pounds and nine ounces, and was reported by Colonel Wilder to be larger than anything previously reported in pear annals.* But California has recently done even better, for a pear from near Marysville in 1904 was reported as nine inches high, sixteen inches around the base

*“Tilton's Journal of Horticulture, March, 1871, p. 87. An, engraving of this fruit, natural size, was given in Pacific Rural Press, November 8, 1873." 
and five pounds in weight. Notes kept by the writer include five Vicar of Winkfields weighing four pounds eight ounces; nine Easter Beurre weighing twenty-four and one-half pounds, the heaviest single specimen weighing two and three-fourths pounds; thirty-five Beurre Clairgeau weighing thirty-seven pounds, the heaviest one, nineteen ounces; Seckel pears, nine and three-fourths inches in circumference-Downing's figures make the Seckel five and seven-eighths inches around.

\section{LOCALITIES FOR THE PEAR}

The pear has a wider range than the apple in local adaptations. It does as well as the apple in the coast regions, if suitable varieties are grown; it thrives far better than the apple in the interior valleys; it rivals the apple in the ascent of the slope of the Sierra Nevada, and gains from the altitude, color and late keeping, as does the apple. By rejecting a few naturally tender varieties, or by proper protection against the scab fungus, in regions where its attacks are severe, one can grow pears almost everywhere in California-providing pear blight can be held in check, as will be discussed later.

The choice of location is governed more by commercial considerations than by natural phenomena. The same facts which make the Bartlett the favorite variety with planters, also should regulate the choice of locality for growing it. These facts were expressed by the late C. W. Reed, of Sacramento, who was in his time one of the leading pear growers and shippers of the State, as follows:

In the Sacramento Valley proper there is but one variety of pear that will justify extensive cultivation, viz., the Bartlett. While nearly all varieties may be grown successfully, and many varieties may be desirable for home purposes, yet for profitable orchards we have to confine ourselves to this one variety, except in high altitudes, or localities where the fruit only matures very late. The reason for this will be better understood by the inexperienced if explained. The Bartlett pear having qualities that make it a universal favorite for shipping, canning, and for domestic market, no other variety is wanted while it is obtainable. With the difference in the time of its ripening in different localities that are adjacent, our markets are supplied with this variety about four months each season, viz., July, August, September and October. While this pear is in the markets, any other variety to compete with it must sell at very low prices.

Of course experienced pear growers, whose taste would soon cloy with a continuous diet of Bartletts, and who know fully the superior quality of other varieties which ripen soon after it, would dispute the position taken by Mr. Reed, but for present California taste and trade he is undoubtedly correct. As the canners and shippers and local consumers all call for Bartletts, and as they usually sell at the East for nearly twice the price of other varieties, the choice of location to secure a Bartlett, either very early or very late, is the part of wisdom, for either end of the season usually yields better prices than the middle. Some growers are even opening the Bartlett season by growing Clapp's Favorite, which sells well because it is taken for a Bartlett, and closing the season with the Winter Bartlett, a local variety recently introduced. The earliest Bartletts come from the interior valley sometimes as early as the last week in June; the next, from the valleys adjacent to the 


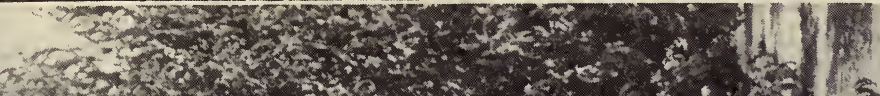

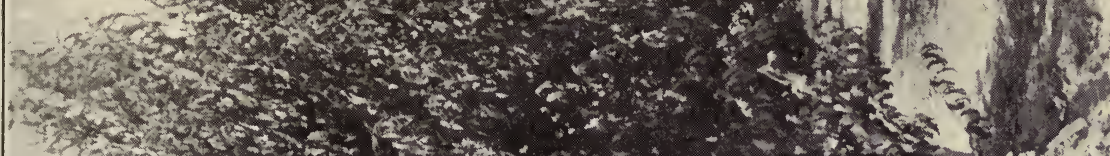

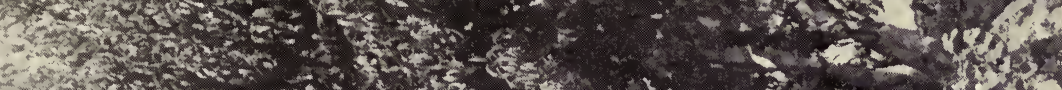

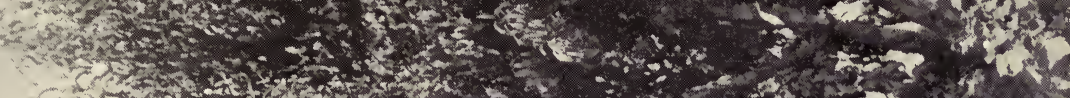

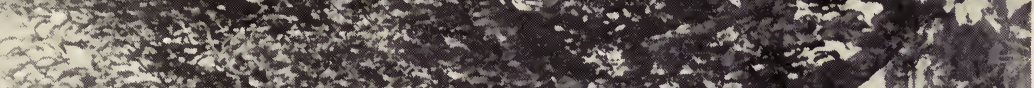

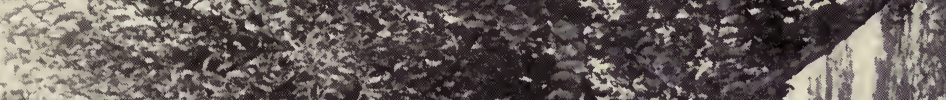

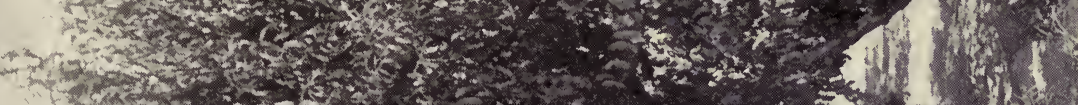

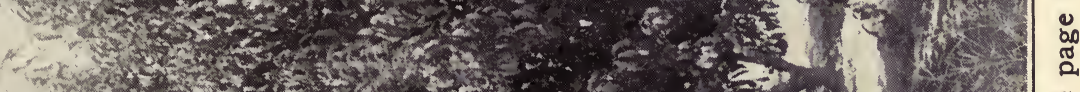

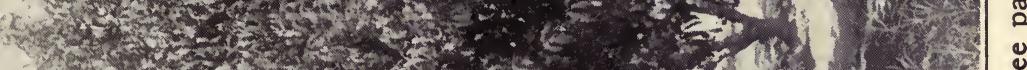

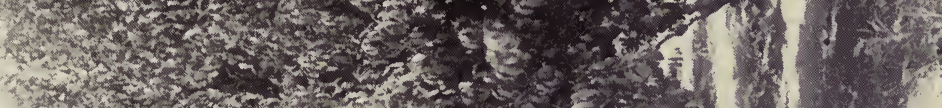

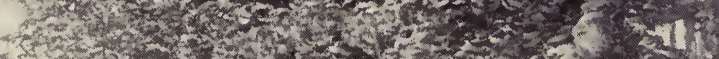

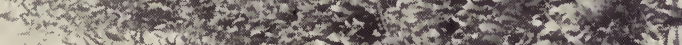

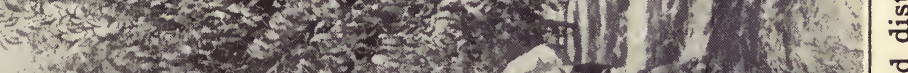

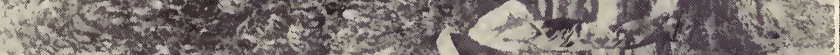

W

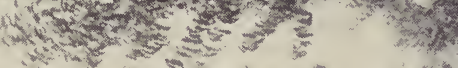

$$
\frac{2 \pi}{2 x}+2
$$

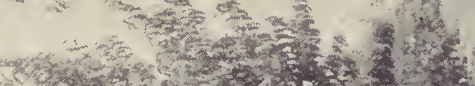

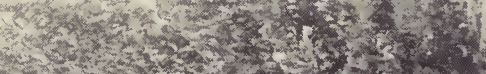

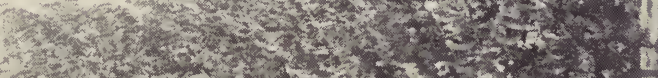

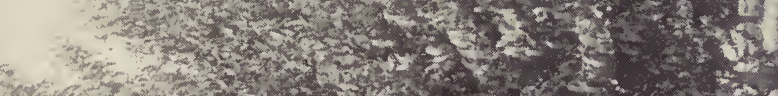

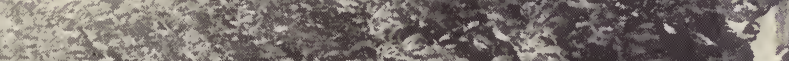

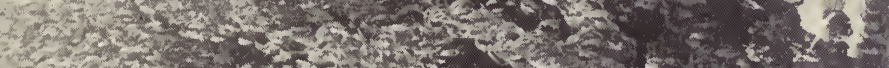

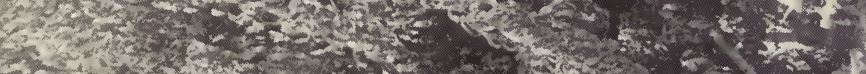

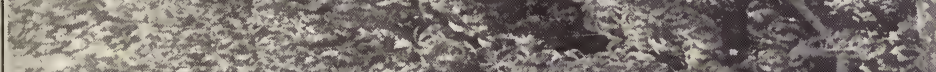

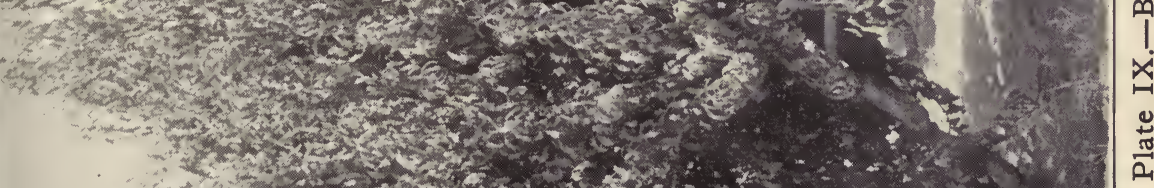

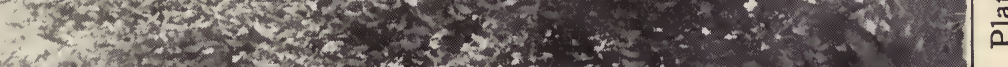

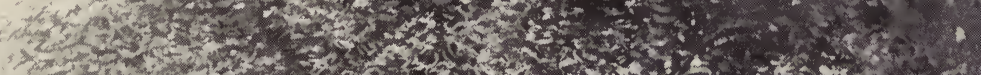

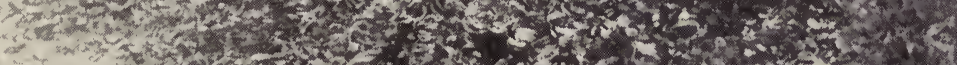

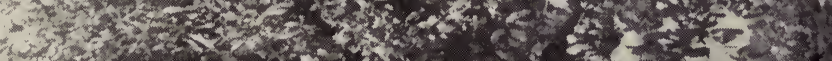

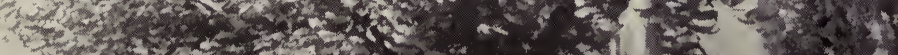

3 (n)

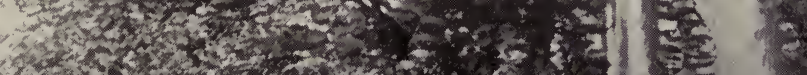

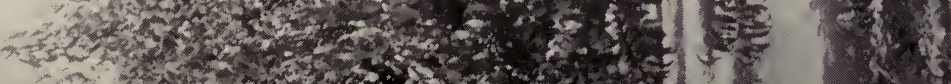

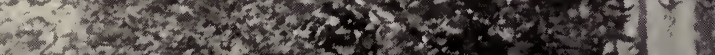

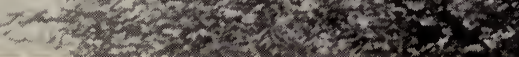



Bay of San Francisco; the next, from the higher foothills of the Sierra Nevada; and the last, so far as present experience goes, although some coast and mountain situations are quite late, reach the market from the Vacaville district. It is an interesting fact that this district, which has long been famous for marketing the first early fruits, should also market very late ones. It is true, however, that early fruits hasten to maturity and late fruits are retarded. Late fruits push along until about midsummer, then stop growing for a month or two during the hottest weather, and afterwards proceed on their course and finish up well. W. W. Smith, of Vaca Valley, has picked Bartletts as late as November 19, but that is unusually late. In years with heavy late spring rains the Bartlett ripens earlier in the Vaca Valley than in ordinary seasons, and when the fruit sells well in the East, the Bartletts are gathered green and shipped all through the season, as their first growth usually makes them large enough for this purpose.

There is produced in some situations a "second crop" of Bartletts and of other varieties, which is of account when pears are scarce and is sometimes dried with profit. For such fruit the bloom appears upon the tips of the shoots of the current season's growth. The fruit is sometimes coreless and has led to claims of "seedless pears." Bartlett pears have actually been picked in the foothills above Peatz in Butte County on February 25, 1905, and described as "fine, delicious and ripe." This fact must be regarded as a token of local climatic salubrity and not of economic or pomological account.

Bartletts can also be successfully held in storage for a time if fitted for it. The experiments of the United States Department of Agriculture, conducted in Southern Oregon, show that the Bartlett season can be extended from six to seven weeks by leaving the fruit on the trees two weeks longer than is at present the practice and by storing for four or five weeks at a temperature of $32^{\circ}$ or $34^{\circ} \mathrm{F}$. after the fruit has been precooled.

\section{SOILS FOR THE PEAR}

The pear, if it is not allowed to dry out entirely, will generally do well on shallow soil and over a tight ${ }_{2}$ clay hard-pan, where most other fruits would be unsatisfactory or fail utterly. The trees will thrive in clay loams, and even in adobe, if properly cultivated. In laying out fruit farms, which often include a variety of soils, even in comparatively small area, the pears and plums (if on the right stock, as will be seen), should be set in the lower, moister, stiffer soil, and other fruits on the lighter, warmer, and better drained portions. The pear, however, enjoys the better situation, though it will thrive on the poorer. The tree seems to attain its greater growth and heaviest bearing on the alluvial soils of the valleys and near the banks of rivers and streams. All pears will be later in maturing and have better keeping qualities if grown on a clay subsoil. Thus it appears that the pear will flourish whether the water is near or far from the surface. On wet land the apple is apt to die in a few years, or become worthless. On dry land the apple lives longer, but the fruit is small and tasteless. But the pear tree may bear good fruit, under the same conditions. 
It has been learned by experience that the pear will flourish on soil somewhat alkaline. At the University Agricultural Experiment Station at Tulare, this subject was demonstrated in detail. It was shown that though the pear endures a certain amount of alkali its limit of endurance may be often exceeded and there is little warrant to select alkali soil for pears, unless it be to fill a space that would otherwise be vacant in the orchard. If it is not too alkaline the pears will thrive. If gypsum be used in planting, somewhat stronger alkali will be endured than otherwise.

\section{PROPAGATION AND PLANTING}

The use of dwarfing stock for the pear has been nearly abandoned in this State, though in early years the quince was largely used. The most prominent orchard on quince stock is that of A. Block, of Santa Clara, where may be seen dwarf trees originally planted eight feet apart in squares, but now wider spaced by removing part of the trees; the remainder doing exceedingly well under liberal manuring and irrigation. It would, however, require special investigation to determine whether these trees are still dependent upon the quince or whether they have developed roots from the pear wood above.

It is quite possible that, at least for gardens, there may be in the future more use made of dwarf trees, but for commercial orchards there appears no need of dwarfing. The common conclusion is that it is better to have fewer trees and larger ones, but since the pear blight became an issue in this State the Angiers quince has been advocated as a means of maintaining a sound root and keeping the warfare above ground.

The following varieties are commended for cultivation on quince stock as dwarfs, experience proving them vigorous growers and abundant bearers in suitable localities: Bartlett, Beurre Hardy, Doyenne du Comice, Duchess d'Angouleme, Glout Morceau, Pound, Beurre Diel, White Doyenne, Easter Beurre, Winter Nelis, P. Barry, Winter Bartlett.

But the pear is usually grown in California on its own roots; that is upon imported French pear seedlings. It comes into bearing early enough, and is a long-lived tree unless badly attacked by blight. Trees are grown by either budding or grafting, as described in the chapter on that subject. Only good seedling roots should be used, and not suckers from old trees. The Japanese stock, so called, being seedlings from the Sand pear, of Asia, is now being widely used because of confidence in it as measurably blight resistant, while it is also less subject to attack by woolly aphis, has been used to some extent, but no definite results reported. Propagation upon rooted cuttings of the Le Conte, which has a measurable resistance to the pear blight, has been advised for the purpose of securing less susceptible roots, but Japanese seedlings are easier to secure and to work with than rooted cuttings. Mr. M. B. Waite, orchard pathologist of the United States Department of Agriculture, has in progress a test of many cross-bred seedlings in the hope of reaching a hardier seedling stock for pears than is now known. 
He also proposes top grafting the Bartlett upon Winter Nelis, Seckel, Angouleme, Lawson and other partially immune varieties to increase the chances of securing a somewhat resistant tree-body and sound roots. Fortunately the pear is readily grafted-over by the common. methods, and some growers are planting Winter Nelis on Japanese roots, expecting to top-graft them with Bartlett in the orchard.

Distance in Planting.-If the pears are to have the whole ground, it is usual to plant from twenty to twenty-four feet apart on the square. As the tree is slower to attain size and full bearing than the stone fruits, and as it is a long-lived tree, the pears are sometimes set twenty-four feet with plums in quincunx. Peaches and apricots are also set between pears sometimes, when the soil chosen for pears suits them also.

\section{PRUNING}

Usually the pear is grown in the vase form, as described in the general chapter on pruning. With regular, upright growers, heading low and cutting to outside buds results in a handsome, gently-spreading top, and effectually curbs the disposition which some varieties, notably the Bartlett, have to run straight up with main branches crowded together.

The development of the vase-form with a few continuous leaders, in a general way as prescribed for the peach in Chapter XX, is practicable. Such leaders are to be covered with short, fruit-bearing laterals. Thinning and shortening of laterals can be done by summer pruning.

As with other fruit trees, the pear must be studied and pruning must be done with an understanding of the habit of the variety under treatment. Irregular and wayward growers, which, in windy places, also have their rambling disposition promoted by prevailing winds, often give the grower much perplexity. The general rules of cutting to an outside bud to spread the tree, to an inside bud to raise and concentrate it, and to an outside bud one pear and an inside bud the next, if a limb is desired to continue in a certain course, are all helpful to the pruner. But with some pears, of which the Winter Nelis is a conspicuous example, it is exceedingly hard to shape the tree by these general rules, and some growers abandon all rules, merely shortening in where too great extension is seen, or to facilitate cultivation, and trust to shaping the tree when it shall have finished its rampant growing period. It will be interesting to cite a few methods of California pear growers:

"The Winter Nelis pear is an uncouth grower. Let the trees alone until they have borne a good, heavy crop, and the limbs come down and spread out nicely; this will occur in five or six years after setting. This will give you an idea what you want to do with the balance of the top that is not borne down with the fruit. My plan is to cut straggling branches, thin out so that the branches will not wind around each other, but don't cut the top, for you will find that the more you cut the more wood you get, and after the tree comes into full bearing is plenty of time to head back."-A. Cadreell, Petaluma.

"Our orchard in Napa county was not in a very windy place, but still windy enough to throw our Nelis trees nut of form. To get any regularity of shape, we cut off every year all the shoots growing low down on the leeward side, 
shortening in what are left as occasion may require, to an inside bud. On the windward side we rarely cut any branch out, but shorten in a little to an outside bud, frequently being obliged to cut back a strong shoot to a lateral which is growing outward."-Leonard Coates, Morganhill.

"It is hard to get a misshapen Winter Nelis tree into shape. Let the grower take his shears and go around the tree and examine the difficulty until he is conversant with it, and then commence to prune, not to heavily, though. Cut the limbs that lean too far leewards back a little with an inside bud, and train all future limbs toward the weather side of the tree; cut limbs this year so that the coming buds will form limbs growing in the direction of the weather side of the tree. But use moderation and take your time for it, and don't cut too many big limbs off three-year-old trees-none, in fact, if it can be helped. In bringing limbs to proper place, I have found a piece of cornstock the required length for the intended place, inserted endwise between the limb and the body of the tree to be spread, to be a very good brace, easily made, and not likely to injure the tree." -T. E. Oren, Santa Cruz.

These methods will suggest others by which one can bring the most irregular grower into shape. If the tree is cut at planting so as to form the head low, it may be safely left until bearing age for shaping. The tree naturally makes a viny growth of young wood, and the object of leaving it alone is that one limb holds the others more upright until the main limbs become large, or stiff enough to keep the shape; so they may be left, after being thinned out to form three to five limbs, as judgment may direct. Some trees will be best with three or four, others five.

The experience of pear pruning just cited has been secured in regions more or less subject to coast influences. In the hot interior valleys, with the pear as with the apple, care must be taken to prune so as not to open the tree too much to the sun, but to shorten in and thin out only so far as is consistent with maintaining a good covering of foliage.

The pruning of bearing pear trees is much like that of the apple, to be determined largely by the habit of the tree, and to secure a fair amount of fruit on branches with strength and stiffness enough to sustain it.

Summer pruning will promote fruiting either in a young or an old tree and some practice it to secure early bearing of young trees, but the common practice is winter pruning to secure strong wood and prevent overbearing.

\section{THINNING PEARS}

It is quite important to attend to thinning the fruit on overloaded trees. Even the popular Bartlett will often give fruit too small for profitable sale unless thinned. With pears, as other fruits, thinning should not be done until it is seen that the fruit is well set. Dropping off from natural causes sometimes thins the crop quite enough.

\section{IRRIGATION OF THE PEAR}

In some situations the pear needs irrigation, though it will endure drouth which would destroy most other fruit trees. There is no profit in small, tough fruit. As stated in the chapter on irrigation the wood 
growth and fruit show whether proper moisture needs are met or not. Early pears are advanced in development by irrigation in some parts of the State, and this is an important factor in their value.

\section{BLIGHT OF THE PEAR}

The pear blight appeared in the San Joaquin Valley about 1900. In 1904, after having nearly wiped out bearing trees in the southern counties of the San Joaquin Valley the disease began to devastate the orchards along the Sacramento River through the vast area of rich valley land which it traverses and on which is situated our most extensive pear acreage. In 1905 resolute warfare was made upon the blight, with a large appropriation of State funds, by the plant disease experts of the United States Department of Agriculture and of the California Agricultural Experiment Station, with the assistance of the local horticultural authorities. It was probably the greatest campaign ever made against a single tree disease although some insect warfares have been greater. The outlines of the plans followed and the results attained are to be found in the publications of the institutions engaged.*

In a later chapter on Diseases of Trees and Vines, an outline of procedure against pear blight will be given. It is apprehended that neither paying crops nor living trees can be counted upon in the future unless the disease is successfully kept under control by successful fighting or by recourse to some form of natural immunity which can be discovered or developed by plant breeding. It is probable that pears can not be grown in the future as cheaply and profitably as in the past and there is a certain amount of bravery or daring in pear investments at the present time. It is encouraging, however, to note that in California the disease shows signs of relaxing the virulence which characterized its first attacks and it is reasonable to believe that here as elsewhere it may be possible to have the blight and pears also. $\dagger$ The reader must keep himself continually of the various phases of the problem as they will arise, by careful study of California horticultural journals and of later publications from the official sources which have been indicated.

The very exuberance of the pear in California seems to increase the virulence of the blight. The long growing season with its continual production of new soft tissue, the unseasonable bloom which attracts bees to bring new supplies of blight germs, the break of new shoots from root, trunk and main branches-all these make the tree subject to repeated renewals of the disease in all its most vulnerable parts. How far growth can be repressed by scant cultivation or by summer pruning; how far suppression of later shoots and blooms is practicable and whether the tree can be depleted so that it can only make fair sized fruit and no surplus soft tissue for blight invasion-all these are cultural problems which make pear growing very interesting to the enquir-

\footnotetext{
* Reports of the California Commissioners of Horticulture, 1901 to 1906, including Reports on California Fruit Growers' Conventions for 1905-6-7, Horticultural Commissioner, Sacramento. Report of Plant Pathologist, University Experiment Station, Berkeley, 1906 and 1908. tThe character of such a fight and what it costs is graphically portrayed by E. A. Gammon in the Report of California Fruit Growers' Convention of 1909, and in Pacific Rural Press, June 22, 1910.
} 
ing mind. A Sonoma grower suggests that pruning may be used to control pear blight in the following manner: It is the natural tendency of the pear and apple while young to form fruiting spurs upon the body and larger branches of the tree. These fruiting spurs produce blossoms from year to year, which are in turn as liable to be visited by bees or other insects carrying the destructive spores of the disease as are the blossoms at the ends of the branches. It is evident, therefore, that a blossom situated upon the body or larger branches of a tree, becoming infected, would communicate the disease directly to the framework of the tree, with the result that it would be fatally injured; but if these fruiting spurs are all removed from the body and larger branches by pruning, the possibilities of infection in this way are overcome. The available means of gaining entrance to the tree by this parasite is confined to the smaller branches, which if affected can be cut away without severely injuring or disfiguring the tree. All suckers at the base of the tree should be removed at the point where they emerge from the trunk or the roots as they favor the entrance of blight to the root.

The scab fungus which seriously affects some varieties, and notably the Winter Nelis, in the Coast region, is identical with the scab of the apple and will be mentioned in the chapter on tree diseases. Because of the liability of the Winter Nelis, to this disease, and because of its irregular bearing in the Coast region, there have been many trees grafted over into varieties better suited to Coast conditions. The Beurre Clairgeau, because of its health, prolific bearing, and acceptability to shippers, was largely introduced in this way, but it has not sold as well as expected. Ordinary top grafting succeeds admirably with the pear. Clapp's Favorite and other varieties have also been worked upon Winter Nelis but they are apt to be more susceptible to blight than Winter Nelis so this old practice is now. of less value than formerly.

\section{GATHERING AND RIPENING OF PEARS}

Many pear growers make the common mistake of allowing the fruit to hang too long on the tree, instead of gathering and ripening in a cool, dark place. Pears should be picked at the first indication of ripeness, the first sign being a tendency of the stem to part from the spur when the pear is gently raised up. This test applies especially to the Bartlett. Picking at this stage and laying away in the dark ripens up the Bartlett well. When picked at this stage and sent overland by slow freight, they ripen en route and the boxes open well on the Eastern markets. There are a few varieties which shrivel if ripened under cover, but the rule is a good one, and the grower will soon note the exceptions. Many desirable varieties have, no doubt, been pronounced poor and insipid because allowed to ripen on the tree.

To ripen well, pears should be packed in tight boxes or inclosed in drawers. They do not do as well as apples on shelves open to circulation of air. As already stated, the oily-skinned apple endures exposure and maintains a smooth, ruddy cheek and sound heart in spite of wind, rain and rough weather. The pear under similar conditions decays rapidly. 


\section{POLLINATION OF PEARS}

As very few varieties of pears are largely grown in California and as the Bartlett generally bears well when grown in large acreages by itself, the Eastern claim that the Bartlett is self-sterile does not seem to be justified in California experience. Recent observations indicate that even at the east the Bartlett is self-fertile when conditions are favorable to setting of the fruit and self-sterile when they are otherwise. As conditions are usually favorable in California this may he the reason why its self-fertility is more conspicuous here than at the east. It has been individually noted, however, that the White Doyenne is a good fertilizer for the Bartlett. The Winter Nelis is one of the pears commercially worth growing which is most apt to be fruitless, but thus far association with other varieties has not been demonstrated to be a full remedy. For the assistance of those who desire to test pear pollination the following statement is made of the relative dates of bloom of a large number of varieies, based upon records kept in Tulare County:

\begin{tabular}{|c|c|c|}
\hline VARIE'TY. & First Bloom. & Full Bloom. \\
\hline Barry & March 10 & March 20 \\
\hline Bartlett & March 14 & March 24 \\
\hline Belle Lucrative & March 14 & March 26 \\
\hline Beurré Clairgeau ................. & March 11 & March 22 \\
\hline 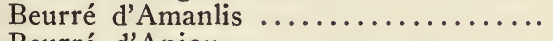 & March 10 & March 20 \\
\hline 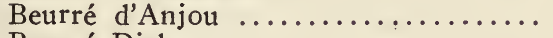 & March 16 & March 24 \\
\hline Beurré Diel $\ldots \ldots \ldots \ldots \ldots \ldots \ldots \ldots \ldots$ & March 20 & March 30 \\
\hline 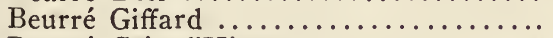 & March 16 & March 26 \\
\hline Beurré Gris d'Hiver............... & March 14 & March 24 \\
\hline 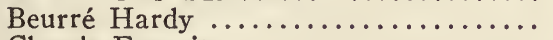 & March 18 & ch 28 \\
\hline 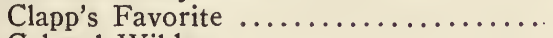 & March 14 & rch 24 \\
\hline$\ldots \ldots \ldots \ldots \ldots$ & March 14 & March 24 \\
\hline Counseiller de la Cour............... & March 12 & $\operatorname{ch} 22$ \\
\hline 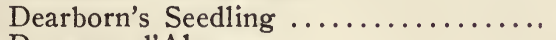 & March 16 & March 28 \\
\hline Doyenne d'Alencon...$\ldots \ldots \ldots \ldots \ldots$ & March 18 & March 28 \\
\hline Doyenne d'Eté $\ldots \ldots \ldots \ldots \ldots \ldots \ldots \ldots$ & March 18 & March 28 \\
\hline Doyenne du Comice................. & March 16 & March 26 \\
\hline Duchesse d'Angoulene $\ldots \ldots \ldots \ldots \ldots \ldots$ & March 12 & March 20 \\
\hline$\ldots \ldots \ldots \ldots \ldots \ldots \ldots$ & March 14 & March 20 \\
\hline 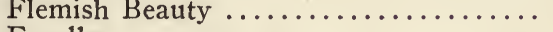 & March 20 & rch 30 \\
\hline Forelle $\ldots \ldots \ldots \ldots \ldots \ldots \ldots \ldots \ldots \ldots$ & March 2 & March 10 \\
\hline lerick Clapp $\ldots \ldots \ldots \ldots \ldots \ldots \ldots$ & March 16 & March 26 \\
\hline Howell $\ldots \ldots \ldots \ldots \ldots \ldots \ldots \ldots \ldots \ldots \ldots \ldots$ & March 14 & March 24 \\
\hline Jaminette $\dddot{M} \ldots \ldots \ldots \ldots \ldots \ldots \ldots \ldots$ & March 12 & $\operatorname{ch} 22$ \\
\hline $\begin{array}{l}\text { Joseph de Malines..................... } \\
\text { Keifer's Hybrid . . }\end{array}$ & $\mathrm{Ma}$ & March 22 \\
\hline $\begin{array}{l}\text { Keifer's Hybrid } \ldots \ldots \ldots \ldots \ldots \ldots \ldots \ldots \ldots \\
\text { Kennedy } \ldots \ldots \ldots \ldots \ldots \ldots \ldots \ldots \ldots \ldots\end{array}$ & March 2 & $\operatorname{ch} 10$ \\
\hline$\cdots \cdots \cdots$ & March 14 & March 24 \\
\hline$\ldots \ldots \ldots \ldots$ & March 12 & March 22 \\
\hline n.m.n. & March 16 & March 26 \\
\hline ........... & Feb. 28 & March \\
\hline Louise Bonne de Jersey............... & March 10 & March 20 \\
\hline$\ldots \ldots \ldots \ldots \ldots \ldots \ldots \ldots \ldots \ldots \ldots \ldots \ldots$, & March 14 & March 24 \\
\hline lon & March 18 & March \\
\hline Souvenir du Congrés... & March 20 & March 29 \\
\hline Swan's Orange ........ & March 14 & March 24 \\
\hline Ver & March & March \\
\hline White Doyenne $\ldots \ldots \ldots \ldots \ldots \ldots \ldots$ & March 14 & March 24 \\
\hline$\cdots \cdots$ & March 12 & March 20 \\
\hline
\end{tabular}




\section{VARIETIES OF THE PEAR}

Though large collections of famous Eastern and European pears have been brought to California, the peculiarity of the local market and demand for canning and shipping has led to concentration upon very few sorts. The pears chiefly grown in California are the following, arranged approximately in the order of their ripening:

Harvest; syn. Sugar Pear (American).-Small, roundish, pale yellow, brownish in sun, brown and green dots; flesh whitish, rather dry but sweet; tree upright, young wood olive yellow brown.

Madeleine (French).-Medium, obovate pyriform, stalk long and slender, set on the side of a small swelling: pale yellowish green, rarely brownish blush; calyx small, in shallow, furrowed basin; flesh white, juicy, delicate.

Wilder Early (American).-Small to medium, yellow with red cheek; sweet, and good. Recently introduced and profitable for local sale in San Diego county. Should not be confused with Col. Wilder, a California seedling which has gone out of use.

Bloodgood (New York).-Tree short, jointed, deep reddish brown wood; fruit medium turbinate, inclining to obovate, thickening abruptly into stalk; yellow, sprinkled with russet dots; calyx strong, open, almost without depression; stalk obliquely inserted, without depression, short, fleshy at its base; flesh yellowish white, melting, sugary, aromatic; core small.

Clapp's Favorite (Massachusetts).-Tree a strong grower; young shoots dark reddish brown; fruit large, slightly obtuse pyriform; pale lemon yellow with brown dots; flesh fine, melting, juicy, with rich, sweet delicate, vinous flavor; resembles Bartlett, but lacks musky flavor.

Dearborn's Seedling (Massachusetts).-Young shoots long, reddish brown; under medium size; roundish pyriform; smooth, clear, light yellow, with few minute dots; stalk slender set with very little depression; calyx spreading in shallow basin; flesh white, very juicy, melting, sprightly.

Laze'son; syn. Comet (New York).-Medium to large, bright crimson on yellow ground; flesh fine, rich and sweet.

Souvenir du Congrés (French).--Large to very large (exceeding Bartlett and Clapp's Favorite, to both of which it bears a strong resemblance); skin smooth, bright yellow when fully ripe, brilliant carmine in the sun; flesh resembling Bartlett, but has not the musky flavor; firm to the core; tree a good grower, but somewhat subject to smut.

Bartlett (English).-Tree a strong grower, early bearer, and healthy; fruit large, smooth, clear yellow, sometimes with delicate blush; stalk moderately long, stout and inserted in shallow cavity; calyx open; flesh white, fine grained, juicy, buttery, highly perfumed (musky), vinous flavor.

Beurré Hardy.-Large, long obovate, sometimes obscurely pyriform; skin greenish with thin, brown russet; stalk an inch long; cavity small, uneven, oblique, basin shallow; buttery, somewhat melting, rich, slightly subacid; tree a strong grower.

Flemish Beauty (Belgian).-Large, obovate, often obscurely tapering to the crown, very obtuse, surface slightly rough, with some reddish brown russet on pale yellow ground; flesh juicy, melting, and good if picked early and ripened in the house.

Seckel (Pennsylvania).-Rather small, regularly formed, obovate; brownish green, becoming dull yellowish brown, with russet red cheek; stalk slightly 


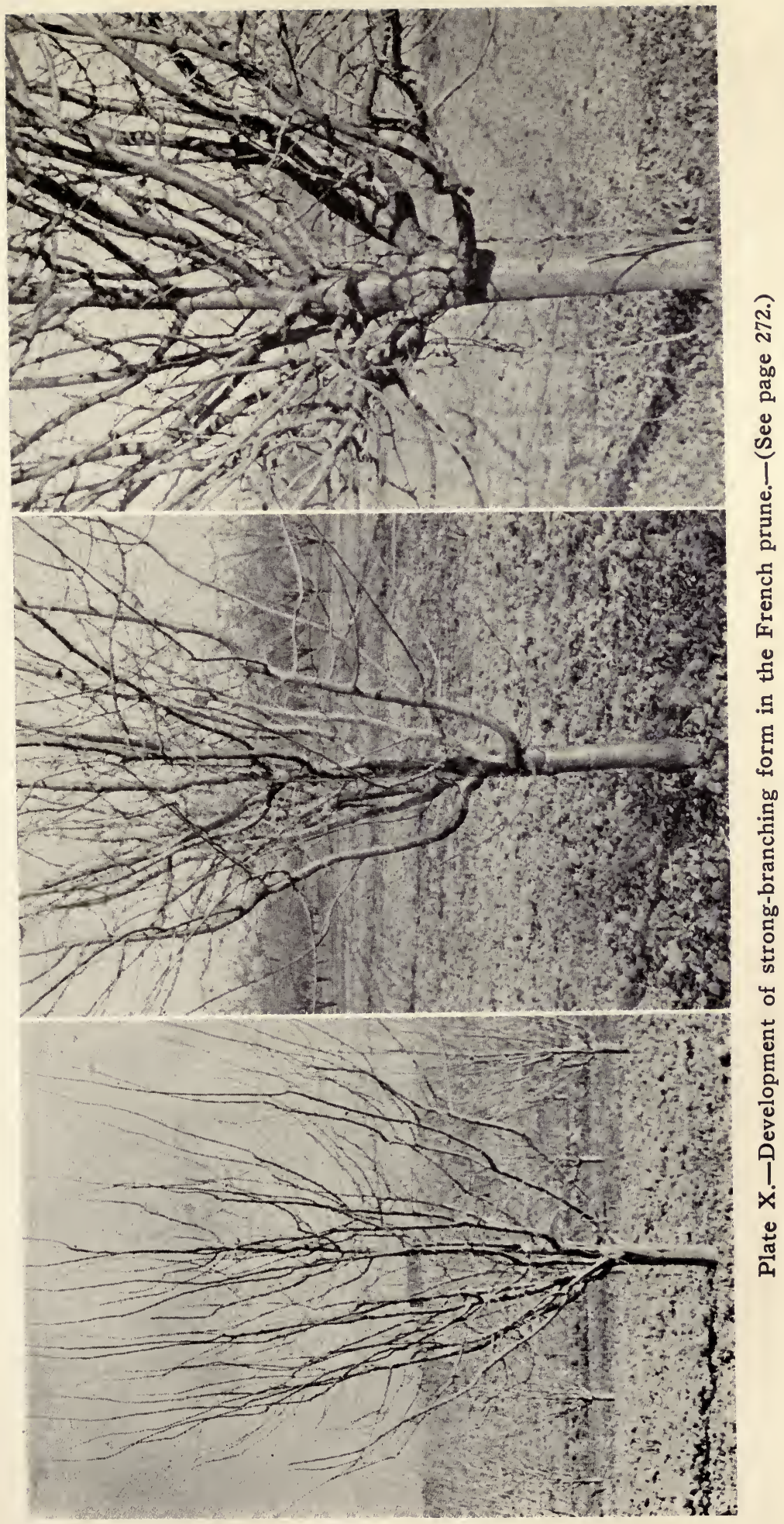



curved, and set in strifling depression; calyx small and set in a very slight depression; flesh whitish, buttery, very juicy and melting, with peculiarly rich, spicy flavor and aroma.

Howell (Connecticut).--Rather large, roundish pyriform, light waxen yellow, often with finely-shaded cheek thickly sprinkled with minute russet dots and some russet patches; stalk medium, without cavity and sometimes lipped; sometimes in small cavity; calyx open in large, uneven basin; flesh whitish, juicy, brisk, vinous.

Duchess d'Angouleme (France).-Very large, oblong obovate; somewhat uneven, knobby surface; dull greenish yellow, streaked and spotted with russet; stalk long, stout, bent, deeply set in irregular cavity; calyx set in somewhat knobby basin; flesh white, butterv. and juicy, with rich flavor.

Louise Bonne of Jersey (France.) -Large oblong pyriform, a little one-sided; glassy, pale green in shade, brownish red in the sun, numerous gray dots; stalk curved, rather obliquely inserted, without depression, or with a fleshy, enlarged base; calyx open in a shallow uneven basin; flesh very juicy, and melting, rich, and excellent ; very prolific.

Beurré Diel (Belgium).-Large, varying from obovate to obtuse pyriform; skin rather thick, lemon yellow, becoming orange yellow, marked with large brown dots and marblings of russet; stalk stout, curved in rather uneven cavity; calyx nearly closed in slightly furrowed basin; flesh yellowish white, a little coarse-grained near the core; rich, sugary, buttery, delicious.

White Doyenne; syn. Virgalieu (French.)-Medium to large, regular, obovate; smooth, clear pale yellow sprinkled with small dots, sometimes red cheeked; stalk brown, little curved, in small round cavity; calyx small, closed in shallow basin; flesh white, fine-grained, buttery, rich, and high flavored.

Beurré Bosc (Belgium.)-Large pyriform, a little uneven, often tapering long and gradually into the stalk; skin pretty smooth, dark yellow, dots and streaks of cinnamon russet, slightly red on one side; stalk long, rather slender, curved; calyx short, in shallow basin; flesh white, melting, buttery, rich, with slightly perfumed flavor.

Onondaga; syn. Swan's Orange (Connecticut).-Large, obtuse, oval pyriform, neck very short and obtuse, body large and tapering to obtuse apex; flesh melting, sprightly, vinous. A vigorous, upright grower, healthy; yellow shoots; sells well in distant markets.

Beurré Clairgeau (France.)-Large, pyriform, but with unequal sides; yellow, shaded with orange and crimson, thickly covered with russet dots, sometimes sprinkled with russet; stalk short, stout and fleshy, inserted by a lip at an inclination almost without depression; when lip is absent, the cavity is uneven; calyx open; flesh yellowish, buttery, juicy, granular, sugary, perfumed, vinous; apt to ripen early for a winter pear.

Beurré d'Anjou (France).-Large, obtuse pyriform: stem, short, thick, and fleshy, in a cavity, surrounded by russet; calyx small, open in small capity, russetted; skin greenish, sprinkled with russet, sometimes shaded with dull crimson, brown and crimson dots; flesh whitish, not very fine, melting, juicy, brisk, vinous flavor, perfumed; tree a fair grower, but somewhat affected by fungus.

Dana's Hovey; syn. Winter Seckel (Massachusetts).-Small, obovate, obtuse pyriform; greenish yellow or pale yellow, with much russet and brown dots; stalk rather short; a little curved, set in slight cavity, sometimes lipped; calyx open and basin small; flesh, yellowish, juicy, melting, sweet, aromatic.

Vicar of Winkfield (France.)-Large and long pvriform; pale yellow, fair and smooth, sometimes with brownish cheek and marked with small brown dots; stalk slender, obliquely inserted without depression; calyx large, open, set in a basin very slightly sunk; flesh greenish yellow, juicy, with good sprightly flavor. 
Doyenne du Comics (France.) - Large, varying, roundish pyriform, or broad, obtuse pyriform; greenish vellow becoming nine yellow, shaded with crimson, slightly marked with russet spots, and thickly sprinkled with russet dots; stalks short, stout, inclined and set in shallow cavity, often russetted; calyx small, open; basin large, deep and uneven; flesh white, fine, melting, aromatic. Very profitable during last few years in eastern shipments.

Glout Morceau (Flemish).--Rather large, varying in form, but usually short pyriform, approaching obtuse oval; neck very short and obtuse; body large and tapering towards crown; often considerably ribbed; green, becoming pale greenish yellow; stalk stout, moderately sunk; calyx large, basin distinct, rather irregular; flesh white, fine-grained, buttery, melting, rich, sweet, and fine flavor.

Block's Acme (California seedling, br A. Block, of Santa Clara).-Large and very handsome, surpassing Beurré Clairgeau in size and color; regularly formed, pyriform, skin pale yellow, covered with russet all over, which becomes a fine glowing red on the side exposed to the sun; flesh white, crisp, and melting, juicy, sweet, and slightly musky; a pear that will rank foremost with our best shipping pears; reserved by originator for his own export trade.

Winter Nelis (Belgium).-Medium, roundish, obovate, narrowed in near the stalk; yellowish green, dotted with gray russet and a good deal covered with russet; stalk rather long, bent, and set in narrow cavity; calyx open in shallow basin; flesh yellowish, white, fine grained, buttery, very melting, and full of rich, sweet, aromatic juice.

P. Barry (California seedling, by B. S. Fox).-Fruit large, elongated pyriform, a little obtuse; skin deep yellow, nearly covered with a rich golden russet; stalk of medium length and thickness, set rather obliquely on a medium cavity, sometimes by a lip; flesh whitish, fine, juicy, melting, sweet, slightly vinous and rich. An early and prolific bearer. December to January. P. Barry is recommended for planting by the Southern California Nurserymen's Association. It is, to some extent, displacing the Winter Nelis as a more healthy tree and a more certain bearer. Has sold well in eastern shipments.

Easter Beurré (France).-Large, roundish, obovate obtuse, often rather square in figure; yellowish green, sprinkled with many russet dots and some russet patches; stalk rather short, stout, set in an abruptly sunken, obtuse cavity; calyx small, closed,. but little sunk among plaited folds of angular basin; flesh white, fine grained, very buttery, meltıng, and juicy, sweet, rich flavor; was successfully shipped from California to England as early as 1872. Bears more regularly but usually sells for less than Winter Nelis.

Pound.-Large, pyriform, yellowish-green with red cheek, esteemed for cooking; reaches enormous size in this State, as already noted.

Kieffer and Le Conte.-These pears, recently introduced as especially hardy varieties, are grown to a limited extent in all parts of the State, but are usually condemned as inferior to the European varieties. The Kieffer is best in interior regions.

Crocker's Bartlett (California).-Chance seedling on place of L. L. Crocker, Loomis, Placer county. Introduced by Mr. Crocker in 1902. Described in year book, 1905, of U. S. Department of Ágriculture; medium to large, oblong, obovate, pyriform; rich golden yellow, somewhat russetty; quality very good; keeps until March. Claimed to be blight-resistant and regularly productive.

Winter-Bartlett (Oregon).-Chance seedling in a dooryard in Eugene, Oregon. Introduced in California by Geo. C. Roeding, of Fresno. Closely resembles Bartlett in shape and appearance and flavor but a little coarser; ripens four months later than Bartlett in interior situations in California and promising as a winter pear. Abandoned by some growers for coarseness and disliked by canners for developing a pink color. 
Pear Varieties Approved by California Growers.

\begin{tabular}{|c|c|c|c|c|c|}
\hline VARIETIES. & $\begin{array}{l}\text { Upper } \\
\text { coast } \\
\text { valleys. }\end{array}$ & $\begin{array}{c}\text { Central } \\
\text { coast } \\
\text { valleys. }\end{array}$ & $\begin{array}{l}\text { Interior } \\
\text { valley and } \\
\text { foothill. }\end{array}$ & $\begin{array}{l}\text { Mountain } \\
\text { valley and } \\
\text { plateaux. }\end{array}$ & $\begin{array}{c}\text { California } \\
\text { Southern } \\
\text { Coast. }\end{array}$ \\
\hline Angouleme (Duchess) .... & * & * & * & * & * \\
\hline 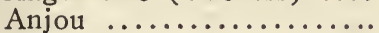 & * & * & * & * & \\
\hline 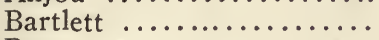 & ** & ** & ** & ** & $* *$ \\
\hline Bosc $\ldots \ldots \ldots \ldots \ldots \ldots$ & * & $*$ & * & & \\
\hline Clairgeau .............. & * & * & * & $*$ & $\dot{*}$ \\
\hline Clapp's Favorite ......... & $\cdots$ & $*$ & * & ... & $\ldots$ \\
\hline Comice $\ldots \ldots \ldots \ldots \ldots \ldots$ & * & ** & * & & \\
\hline Easter Beurré $\ldots \ldots \ldots \ldots \ldots$ & * & $* *$ & ** & & $*$ \\
\hline Flemish Beauty ......... & * & * & * & $*$ & * \\
\hline Glout Morceau ........... & * & ** & * & & * \\
\hline Hardy, Beurré .......... & & * & * & & * \\
\hline Howell $\ldots \ldots \ldots \ldots \ldots$ & $\dot{*}$ & * & * & $\cdots$ & * \\
\hline Kieffer ................ & .. & ... & * & $*$ & * \\
\hline 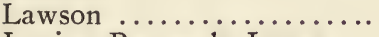 & & $\cdots$ & ** & $*$ & $*$ \\
\hline Louise, Bonne de Jersey.... & * & $*$ & & & \\
\hline Madeleine .............. & $\cdots$ & * & * & * & * \\
\hline P. Barry $\ldots \ldots \ldots \ldots \ldots$ & ** & ** & * & * & * \\
\hline Seckel $\ldots \ldots \ldots \ldots \ldots \ldots$ & * & * & * & ** & $* *$ \\
\hline Souvenir de Congrés....... & * & * & * & ** & $\ldots$ \\
\hline Vicar of Winkfield......... & * & * & & $\cdots$ & $\ldots$ \\
\hline Wilder, Early ........... & $\cdots$ & * & * & $\ldots$ & * \\
\hline White Doyenne .......... & * & * & * & & \\
\hline Winter Bartlett .......... & * & * & * & $\ldots$ & * \\
\hline Winter Nelis . ........... & * & ** & ** & * & ** \\
\hline
\end{tabular}

*Indicates that the variety is approved in the region designated.

** Most highly commended. 


\section{CHAPTER XXIII}

\section{PLUMS AND PRUNES*}

The plums of California are exceptionally fine in appearance and of high quality. Both tree and fruit have thus far escaped the parasites which have wrought greatest injury on the eastern side of the continent. The curculio has never been found here, and the "black knot," though detected in some of the indigenous species of the genus prunust has never been observed in our orchards. The tree suffers, it is true, as do most other fruit trees, from various pests and diseases but their work is a light affliction compared with the ravages of the curculio and black knot which Eastern plum growers have to contend against. Because of reduced planting during the last few years, the plum stands second in point of number among the fruit trees of California, for, as noted in Chapter XX, the peach now holds first place. Of the plums, at least four-fifths of the acreage are those varieties designated as prunes. This is, of course, owing to the profitable shipping demand for our prune product, while ordinary dried, pitted plums are expensive in production and do not always command good prices. There is, however, a large trade at the East in our fine plums in a fresh state. Some varieties stand shipment well, and are large, handsome and in some cases possessed of unique characters, resulting from Mr. Burbank's work with the Japanese species as will be noted later. Considerable shipments of fresh plums have been made from California to England.

By choosing varieties ripening in succession, the plum season extends from May to December, thus enabling the California plum grower to strike the Eastern markets both early and late. It is on record also, that second crop plums have ripened. In 1904 Judge Leib, of San Jose, sent to Luther Burbank, on December 1, ripe fruit from a tree which ripened its first crop on July 4, of the same year.

There is also considerable demand for plums by the canners who do not use, however, the varieties in chief demand for shipping.

\section{LOCALITIES FOR THE PLUM}

The plum has an exceedingly wide range in California. The trees are thrifty and profitable even from the immediate vicinity of the coast and in coast valleys, where the sea winds and fogs intrude eastward across the great interior valleys, and upwards upon the sides of the Sierra Nevada. In the upper half of the State, at least, wherever there

*All prunes are plums, but all plums are not prunes. A prune is a plum which can be dried without the removal of the pit without fermenting: The result being a fleshy pulp with a high degree of sweetness. All plums which will not do this are not prunes, even though the word may appear in their California common names.

$\nmid$ Found on prunus demissa, in Yosemite Valley and in Coast Range in San Mateo County, by Dr. H. W. Harkness. Report State Board of Horticulture, 1883, pp. 54, 55. 
is sufficient moisture in the soil, good plums can be grown. The tree is quite hardy, but in situations open to the sweep of the winds there has been found to be decided advantage in belts of sheltering trees for protection. At some points subject to direct coast influences, there is sometimes loss by cracking of the fruit. It is seldom encountered in the interior valley, except near the rivers or in draws where the damp coast air makes its way through. It seems to be worst where there are marked differences in atmospheric humidity within short periods of time. Where the percentage is quite uniformly high or low there seems to be less trouble. Some years conditions usually restricted to more exposed coast situations prevail in the interior valley, and the result is unusual prevalence of mildew and other moist fungi and cracking of fruit also, though they have no relation to each other except that the same conditions favor both. Only certain varieties are thus affected, and they can be avoided where the trouble is found to exist.

It was for a long time held that southern California was not adapted to the growth of the plum, but the experience of the last few years has shown that the conclusion was too broad. The "French prune" demonstrated its success adjacent to the Coast in Santa Barbara County, and elsewhere, in the low, rich lands of the Santa Ana Valley, of Orange County, in the interior at various points on the rim of the San Gabriel Valley, in Los Angeles County, notably at Pomona, and still farther inland in the San Bernardino Valley, but the Southern California prune product is small because the land and water can be more profitably used for other fruits. There is, however; difficulty in some dry uplands where the tree is shy in fruiting and subject to serious gumming; but this is encountered locally in all parts of the State. Irrigation does not always overcome these troubles, and yet, no doubt, the arrangement of proper moisture conditions is important. The tree should be helped to make one good growth and to ripen its wood in the fall. To have growth checked by drouth and a second start made later in the season is not desirable.

Still it must be admitted that prune planting in the interior, proceeding with such rapidity, has encountered some soils and situations in which bearing has not been altogether satisfactory. New planters should confer with older residents before making investments in prune planting in interior valleys and foothills.

All the foregoing observations are based upon the behavior of plums of European origin; descendants of the prunus domestica. One of the grandest contributions to the extension of the range of the plum in California was the introduction of the Asiatic species, prunus triflora and simoni. Varieties of these species directly introduced or locally developed by Burbank and others, have proved productive in places where the domestica varieties were abandoned as shy or sterile. To estimate the value of these varieties one has only to visit the home fruit gardens of southern California or inspect the fruit stands of Los Angeles which are continuous exhibits of fine specimens of these varieties in their seasons. Even in places where the domestica varieties are largely grown the Asiatic varieties are also prominent as is shown 
by the fact that the Wickson, a Burbank triflora-simoni hybrid, is the leading shipping plum of California, and shipping plums are chiefly grown in the central and northern regions of the State. Other notable Burbank plums of recent introduction will be included in the descriptions of varieties at close of this chapter.

\section{SOILS AND STOCKS FOR THE PLUM}

With the plum, as with the apricot, the subject of soils and stocks are intimately related, but the whole matter has been wonderfully simplified by the experience of the last few years. This relief has come through the adoption of the myrobalan, or cherry plum (prunus myrobalaria) has a general all-around stock for plums and prunes. Before this practice was taken up the effort to grow the plum on its own roots generally resulted in getting an orchard full of suckers, and to avoid this, plums were worked on peach roots wherever this root would succeed in the soil to be planted. But some varieties of plums do not take kindly to the peach, and then "double working" (putting first on the peach a plum which is known to take well and then on that plum wood the variety desired) was followed. The use of the myrobalan does away with the suckering nuisance, and the need of double working.

There was considerable discussion a few years ago as to what is the true myrobalan, and it must be acknowledged that some of the refined distinctions formerly claimed have been abandoned. Seedlings grown from the seed of the myrobalan vary as do other fruit seedlings, both in fruit and in foliage and habit of trees, and perhaps this fact has given rise to the distinction between "true" and "false" myrobalan, so-called. Practice has proceeded without much reference to the discussion, and our nurserymen now have large, thrifty myrobalan trees from which they secure their seed supply. Growing stock from myrobalan cuttings is but little practiced. The myrobalan is now the accepted plum stock for California, except in light, alluvial, well drained soils, where, for the French prune, peach or almond may be preferred. Though described by some authorities as a dwarfing stock, it is found to be sufficiently free growing in California to suit all purposes, and to form a good foundation for full standard trees, though the peach and almond roots in proper soils give a quicker and greater growth. Experience has shown that the myrobalan root thrives in this State both in low, moist, valley lands; in comparatively dry lands; if not too light and leachy; and in stiff upland soils.

In some soils especially adapted to the peach, peach roots are preferred as stock for the French prune, but, as already said, all plums can not be worked directly on the peach root, the Robe de Sergeant, Columbia, Yellow Egg, Washington, and Sugar Prune, for example. Sometimes the bud or scion may make a large growth, but the two woods do not unite, and the trees break off sooner or later.

Some work the plum on the apricot root, and report success when the soil suits the apricot root, and the gophers do not get at it. But it 
sometimes happens that the French prune parts from the apricot root even after growing some time upon it. There are, however, instances of the French prune thriving, and, apparently making good union with the apricot root and some of the softer wood varieties, like the Sugar Prune, take kindly to it.

Some plums do well on the almond root and some do not. The French prune succeeds admirably both when worked on young almond stocks and top grafted in old almond trees. Success is also reported with the Fellenberg on the almond. But the almond root is suited especially for warm, dry soils. Excellent results from the use of almond stock are reported from the interior valley and the Sierra foothills.

Propagating by Sprouts.-The French practice of growing certain varieties of the plum by means of sprouts from the base of old trees was successfully followed in this State by Felix Gillet, of Nevada City, and was strongly commended by him as securing a tree which will not gum, which is one of the reasons why the same practice prevails in France. Sprouts growing at the foot of old and large trees, and but few are found to each tree, are taken off and planted close together in a bed to make them root well, and the ensuing spring planted in nursery rows, where they are trained like any other trees, and transplanted where to remain, when branched. For this method it is necessary that the parent tree should be upon its own roots, else one is apt to get suckers from a wild stock. Sprout-grown trees can not, however, be defended unless some special point like that claimed by Mr. Gillet can be attained by them.

\section{PLANTING AND PRUNING}

As with other trees, there is difference of opinion as to the best distance apart for plum trees. The present tendency is toward wider planting; not nearer than twenty feet is the usual advice, and on rich. land, twenty-two or twenty-four feet is better.

The plum, in California, is a most rapid grower ; six to ten feet from the bud or graft in a season, and about as much after the first winter's cutting back, is not at all unusual. At this rate of progress then, the tree soon runs up and away, in a spindling, sprawling fashion, unless severely cut back for the first few years. Neglected trees of some varicties show long, streaming branches, arching outward, and exposing the bark to sunburn (to which it is very sensitive), breaking the tree to pieces as the fruit gets weight, and, even if supported by props, breaking off at the bearing of the prop. This condition of the tree can only be obviated by low heading and moderate cutting back each year, with due regard to limiting the amount of bearing wood to get large fruit. For such plum varieties the suggestions on forming the tree and subsequent treatment in Chapter XII will be found helpful. This reference to repressive treatment for brittle-wood plums is emphasized by experience with the Sugar Prune and Golden Prune, of which Mr. Leonard Coates of Morgan Hill says: 
"The sugar prune is a great bearer, but the tree must receive very different pruning from that of the French prune. The annual growth must be shortened in every winter from one-half to two-thirds, and all laterals and fruit spurs must be cut back at every pruning. Of course, judicious thinning out of wood is also needed. This will result in a very large, showy, product for long-distance shipments, as fresh fruits, which have brought satisfactory returns."

Pruning the French Prune.-Growers of the French prune, and other varieties of similar growth of strong and pliable wood, have reached substantial agreement as to the best practice. The old method of cutting back bearing trees has been abandoned. Cutting back the young tree to secure sufficient low branching is followed by thinning

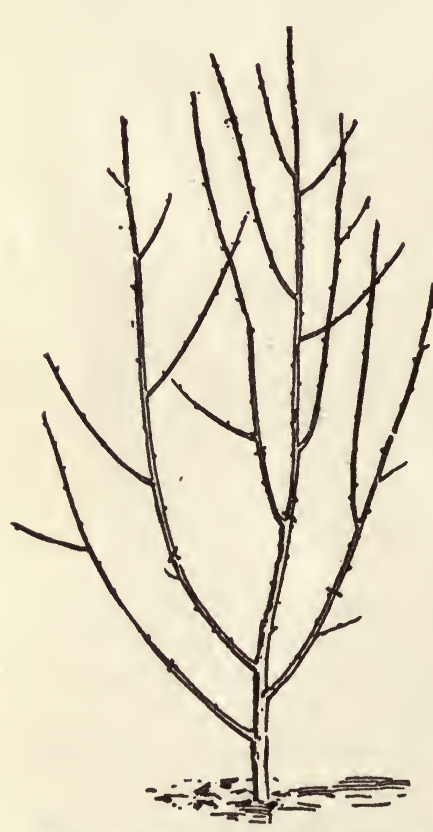

Pruning after first summer's growth in orchard.

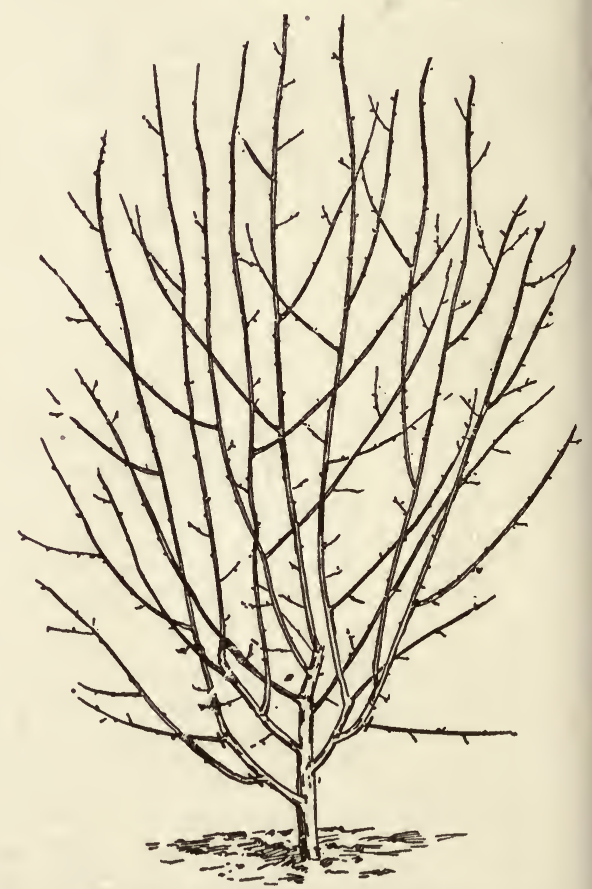

Growth during second summer in orchard.

of shoots from this low head so that the tree shall not become too dense or carry too much bearing wood. The strength in the head depends upon proper spacing and arrangement of the branches as insisted upon in the chapter on pruning; and large, well-ripened fruit, which is essential to successful and profitable drying, is conditioned upon avoiding excess of branches and admission of sufficient light to the tree.

A rather longer central stem is retained than in the old style, and a central stem throughout is admissible if one prefers it and does not desire to dispense with it as the first step toward securing a more open tree. Some retain the longer stem at planting, others cut back to 
eighteen inches, develop three side branches upon that and train the branch from the top bud for a lengthening of the stem, and bring out more branches upon that the second year, and then dispense with its farther extension. The engravings on page 272 show this method of developing the head of a young French prune. The tree was cut back at planting in orchard to a straight switch about eighteen inches high. At the end of the first summer this showed the form in the first picture, which is marked for the first winter pruning. The second engraving shows the branching developed from this during the second summer's growth, also marked to prune away some undesirable branches. Upon a tree of this form further cutting back is not desirable as it has enough well-placed branches to form the tree.

How long cutting back shall continue depends partly upon the locality and partly upon the notion of the owner. In interior localities the tree grows with great rapidity and branches more freely. During the third summer it will bear some fruit if not cut back the previous winter, and, where growth is so rapid, there is little danger of injuring the tree by early bearing. In the coast valleys cutting back may continue another year, and fruiting be thus postponed a year to get another summer's freer wood growth.

Though cutting back may properly cease early with the French prune, it is a great mistake to allow the trees to go unpruned. Removal of defective wood, prevention of branch crowding and overbearing are of the highest importance, as insisted upon in the chapter on pruning. Removing surplus laterals at their starting points, and cutting back leaders to laterals already grown and not to encourage new branching will result in a more open tree, which is generally very desirable.

Special Studies of Varieties in Pruning.-The points just advanced apply especially to the management of the French prune. In addition to what has been already said about the Sugar prune, the grower must be exhorted to study the habit of the variety he has to deal with. The general rules for handling trees with different habits of growth are applicable to a certain extent to the plum. When to apply a rule or make an exception must be learned by observation and experience. Some plums, like the Silver prune, have something of the growth habit of the peach, and this is also very true of some of the Japanese varieties. Cutting back in winter and pinching in summer are both useful facts in securing lower branching and low-growing fruit spurs.

The formation of the vase-form with continuous laterals, as discussed in Chapters XII and XX is being successfully practiced by some growers with plum varieties which need short pruning.

Grafting the Plum.-The plum has been grafted and regrafted in the constant effort to secure varieties promising superiority in various directions. Within the scope of their affinities plums graft easily by common top-grafting methods, and if the roots are strong the new growth is so rapid as to need special attention. Mr. Luther Bowers gives these hints about pruning such growths: "From practical experience I have found out that the Sugar prune wood should only be 
summer pruned and only cut while the shoots are tender, or so soft that the top can be pinched out; this will cause the top to be well branched and this should be done at least twice during the first year of the graft. This system will avoid long, slender limbs. After a graft is two years old I would never cut the top off of a limb. If a tree gets too thick a top, I would cut out some of the main branches."

\section{THE PLUMCOTS}

One of the most striking achievements of Mr. Burbank from the fruit grower's point of view is the cross of the plum and the apricot, which he has very fitly named the "plumcot." He has combined in a single fruit enough of the diverse characters of two fruits so that the ordinary observer can recognize the combination clearly and distinguish the gift of each to it. Mr. Burbank has secured several such crosses, the first of which to be made public is the "Rutland," introduced by Mr. George C. Roeding in 1907. The fruit is about the size of an ordinary apricot with a deep purple velvety skin. One of its striking features is the brilliant red flesh possessed of a strong sub-acid flavor rendering it suitable for cooking, jellies and jams, and it is in good demand for such uses. When fully ripe, it is an excellent dessert fruit possessing an apricot-plum flavor. The amalgamation of the apricot and the plum has produced a fruit unique in character, and its economic value is still to be determined by the exploitation of its uses.

\section{HOW A PRUNE QUEST DISTURBED THE PLUM FAMILY IN CALIFORNIA}

Referring to the distinction between plums and prunes cited at the opening of this chapter, and to the extent and methods of the great prune industry of the State which will appear in Chapter XXXVIII, it may be stated here that the California prune product was obviously undertaken in emulation of the globe-trotting French prune, which had attained position as the leading commercial dried fruit of the world long before California arose on the horticultural horizon. Naturally, French settlers in California bethought themselves of transplanting this great industry to their new home, and Mr. Louis Pellier introduced scions from the district of Agen to his place near San Jose in 1856. the product was good, and planting for a large output was entered upon, though slowly at first. There was disappointment over the fact that, while all fruits came surprisingly large in California, the dried prunes were smaller than the great French prunes in cartons and canisters which sold for great prices. Had we secured the true French prune; did they not have larger ones which they were holding back from us? This was the great question of five decades ago. Some nurserymen of that day had spirits of enterprise larger than their consciences. If the people demand larger prunes they must have them, 
surely. Because of the small average size of the prunes of Pellier's introduction, they christened that variety "petite prune d'Agen," which was subsequently corrupted into "petty prune"-a free translation and a mispronunciation at the same time, for a prune which seemed to be too small and inferior. The people must have something large, and propagators offered trees of the "gros prune d'Agen," or the "Hungarian prune." It was a double misnomer, because Europe does not have any "gros prune d'Agen" and the variety did not come from either France or Hungary, but was the old large light red, English plum, properly called Pond's Seedling, re-christened in California to meet a long-felt want. But it did not meet such a want; it would not dry sweet nor fleshy, but became merely a skin and pit, with a sour streak between. Still the question persisted: Have we the true French prune? It was definitely settled by the late W. B. West of Stockton, who visited France in 1878, and after close examination of the trees, announced that the variety grown in California was really the prune d'Agen, and that we had made no mistake so far as getting the main standard variety of French prune was concerned.

But still we needed a variety which would run more to large sizes, and how to get it, with sweetness and flesh, characters which would resemble the best French product, was, and even now is, still a question. One of the early introductions to meet this end is now generally known as Robe de Sergeant. Here again confusion attends the name. Robe de Sergeant is one of the synonyms of prune d'Agen, and yet the fruit we secured was different. Much discussion was given to the elucidation of this problem, and the conclusion seemed to be that the variety is grown in France, but in another district, and is generally considered inferior to the prune d'Agen. Still it runs larger, and has sold well, even though of distinctly different quality, and would probably have cut a much larger figure in California prune production if it had shown itself to be more free and regular in bearing. Next came the "prune d'ente, or Imperial epineuse," introduced at about the same time by John Rock and Felix Gillet, which has been quite widely planted, but because of shy bearing, especially when attacked by the thrips, and because of the difficulty in drying such a large prune, this variety, of which so much was expected, has fallen into disfavor for the low lands of the Santa Clara Valley, though on the mountains west of this valley and in the prune valleys north of the Bay of San Francisco it has proved very popular and profitable when planted on uplands.

Other introductions made much earlier, like the German and Italian, also fell out of the race very early, for shy bearing and for different flesh characters. Although the latter leads in Oregon and other States north of us, it is out of California calculations. The conclusion of the whole matter now is that we have never secured from abroad a better than the one which came fifty years ago-the true prune d'Agen. We have learned to grow it better, to seek places where it grows larger and in full quality; to use irrigation when it is needed by the tree to do its best; to guard against overbearing by reducing the amount of bearing wood and excessive branching; to strengthen the soil by fertilization, and to grade the fruit into sizes which commend themselves to 
different demands. Here we are again, doing our main business at the old stand, but knowing how to do it better. Have we anything more to expect? Probably nothing from old varieties, for we have prospected them all from a prune-making point of view, taking Coe's Golden Drop plum, or its seedling, for the Silver Prune, and canceling all others as possibly good plums for various uses, but not for prunes.

Probably our only expectation lies along the line of plant breeding, although nothing to supplant the prune d'Agen has yet been attained. The Giant prune is a large red plum; several Oregon prunes are simply large red plums. The standard of sugar in the prune d'Agen as grown in California is from 15 to 23 per cent of sugar in the fresh juice, according to degree of ripeness and localities in which the fruit is grown. The sugar in Pond's Seedling and in the large red plums just named is less than 10 per cent-sometimes very much less. But percentage of sugar in the juice is not the whole story; there are tissue or flesh characters which are essential also. Mr. Burbank's Sugar prune answers the sugar requirement; it is a free bearer and early ripening variety, and it dries easily though large, and the small dried product thus far made has sold well, but it has not the fine grain nor distinctive flavor of the prune d'Agen, and the pit is large and rough. It becomes a good plum for shipping and possibly for other plum purposes. But Mr. Burbank has held the plum family in training, and his latest introduction, the Standard prune, seems to be making good, and in 1914 is being widely planted.

Others are also working at the problem, and the next generation of California prune growers may attain what the last and present have striven for. The most promising line at the present time is the search for better types of the prune d'Agen which are found here and there, arising from natural variation. Mr. Leonard Coates of Morgan Hill is giving particular attention to this subject, and has demonstrated the existence of very superior variations.

\section{POLLINATION OF PLUMS}

The shy bearing of certain plums is probably due to lack of pollination, either through the self-sterility of the variety or lack of acceptable pollinating agencies. Bearing can be induced in many cases, no doubt, by either planting or grafting-in of effective pollinating varieties. But this is not always profitable. For instance, there are many instances proving that the Tragedy can be brought to greater bearing by the presence of Clyman, but an early variety like the Clyman is not worth growing for sale in a late district, though in an early district both are valuable as shipping plums and should be planted together. The Hungarian is well pollinated by the Peach and the Grand Duke plums. The bearing of Wickson is greatly increased by association with Climax The pollination of plums not not yet, however, been given as much attention as of other fruits. 
Plums and Prunes Approved by California Growers.

\begin{tabular}{|c|c|c|c|c|c|}
\hline VARIETIES. & $\begin{array}{l}\text { Upper } \\
\text { coast } \\
\text { valleys. }\end{array}$ & $\begin{array}{c}\text { Central } \\
\text { coast } \\
\text { valleys. }\end{array}$ & $\begin{array}{l}\text { Interior } \\
\text { valley and } \\
\text { foothill. }\end{array}$ & $\begin{array}{l}\text { Mountain } \\
\text { valleys and } \\
\text { plateaux }\end{array}$ & $\begin{array}{c}\text { California } \\
\text { Southern } \\
\text { Coast. }\end{array}$ \\
\hline Abundance & & & * & & ** \\
\hline Agen, Prune d'........... & ** & $* *$ & ** & $* *$ & ** \\
\hline 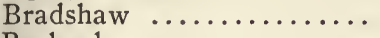 & $*$ & & $*$ & .. & \\
\hline 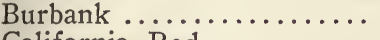 & * & ** & ** & $\cdots$ & ** \\
\hline California Red $\ldots \ldots \ldots \ldots$ & $\cdots$ & $\cdots$ & * & $\cdots$ & $\cdots$ \\
\hline Chalco $\ldots \ldots \ldots \ldots \ldots \ldots$ & $\cdots$ & $\cdots$ & $\begin{array}{c}* \\
* *\end{array}$ & $\cdots$ & $\cdots$ \\
\hline Climax..$\ldots \ldots \ldots \ldots \ldots$ & $\cdots$ & * & $\begin{array}{l}* * \\
* *\end{array}$ & $\cdots$ & \\
\hline Clyman $\ldots \ldots \ldots \ldots \ldots \ldots$ & $\cdots$ & & $*$ & $\cdots$ & $\cdots$ \\
\hline Columbia ................. & $\cdots$ & $\ddot{*}$ & * & $\cdots$ & $\cdots$ \\
\hline Damson........... & * & * & * & $*$ & *i* \\
\hline Diamond...$\ldots \ldots \ldots \ldots$ & $\cdots$ & * & ** & $\cdots$ & $\cdots$ \\
\hline Formosa $\ldots \ldots \ldots \ldots \ldots$ & ... & * & *** & & $\cdots$ \\
\hline German Prune...$\ldots \ldots$. & * & * & * & * & * \\
\hline Giant $\ldots \ldots \ldots \ldots \ldots \ldots$ & $\cdots$ & * & ** & & $\cdots$ \\
\hline Golden Drop, Coe's....... & & * & * & * & $\cdots$ \\
\hline $\begin{array}{l}\text { Golden Prune } \ldots . \ldots \ldots \ldots . . . \\
\text { Grand Duke }\end{array}$ & $\cdots$ & $*$ & ** & $*$ & $\cdots$ \\
\hline & $\cdots$ & $*$ & & $\ddot{*}$ & $\cdots$ \\
\hline Hale $\ldots \ldots \ldots \ldots \ldots \ldots \ldots \ldots$ & $\cdots$ & & $*$ & $\cdots$ & * \\
\hline Imperial Epineuse ....... & * & *** & * & $\ldots$ & $\ldots$ \\
\hline Imperial Gage $\ldots \ldots \ldots \ldots$. & & & * & & $\ldots$ \\
\hline Italian Prune $\ldots \ldots \ldots \ldots \ldots$ & * & * & * & $*$ & * \\
\hline Jefferson.$\ldots \ldots \ldots \ldots \ldots \ldots$ & * & ** & * & ... & \\
\hline Kelsey $\quad . . \ldots \ldots \ldots \ldots \ldots$ & $*$ & $*$ & ** & & ** \\
\hline Peach $\ldots \ldots \ldots \ldots \ldots \ldots$ & $*$ & $*$ & $*$ & * & $\cdots$ \\
\hline $\begin{array}{l}\text { Pond (Hungarian) } \ldots . . . \\
\text { Red June }\end{array}$ & $\begin{array}{l}* \\
*\end{array}$ & $*$ & $\begin{aligned} * * \\
*\end{aligned}$ & * & $\begin{array}{l}* \\
*\end{array}$ \\
\hline $\begin{array}{l}\text { Red June } \ldots . . . \ldots \ldots \ldots \ldots \\
\text { Robe de Sergeant.......... }\end{array}$ & . & $* *$ & ** & & 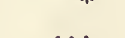 \\
\hline 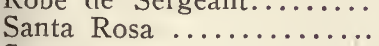 & $\ldots$ & * & * & $\because$ & $\ddot{*}$ \\
\hline 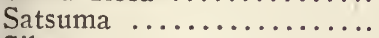 & * & * & * & $\ldots$ & ** \\
\hline$\ldots \ldots, \ldots, \cdots, \cdots$ & * & * & * & $\ldots$ & $\ldots$ \\
\hline 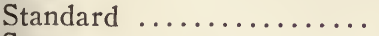 & & * & * & $\ldots$ & $\ldots$ \\
\hline$\ldots \ldots \ldots \ldots \ldots \ldots$, & * & ** & ** & * & * \\
\hline Tragedy $\ldots \ldots \ldots \ldots \ldots \ldots$ & & ** & ** & & * \\
\hline Washington...$\ldots \ldots \ldots$ & * & * & * & * & * \\
\hline Wickson $\ldots \ldots \ldots \ldots \ldots \ldots$ & * & ** & ** & * & \\
\hline Yellow Egg $\ldots . . \ldots \ldots \ldots \ldots$ & * & ** & ** & ** & * \\
\hline
\end{tabular}

*Indicates that the variety is approved in the region designated.

**Most highly commended.

\section{VARIETIES OF PLUMS AND PRUNES}

As with other fruits, comparatively few varieties of the plum are largely grown in California, and the list is continually changing by rejection of old varieties and introduction of new which are largely of Burbank origin:

Simon (Prunus Simoni).-Medium to large, roundish, flattened, with cavities at base and apex; brick red, small yellow spots; stalk stout and short; flesh yellow, adhering to flattened pit; largely grown for shipment in early interior regions where it has good quality; lacks flavor and cracks badly near the coast. Being displaced by Burbank varieties.

Climax (Burbank).-Large; very early; heart-shaped; deep red; flesh yellow. Popular for shipping in places where it does not crack badly. Growing in favor. 
Clyman (California seedling introduced by Leonard Coates).-Large, roundish oblong, flattened suture indistinct; mottled reddish purple, beautiful blue bloom; freestone; flesh firm, dry and sweet; prolific; the leading early plum for shipment.

Red June (Japanese).-Medium to large, deep red flesh light yellow, firm, good quality. The best of the early Japanese plums.

California Blue.-Seedling of Peach plum by W. W. Smith, Vacaville; large, good flavor; early; "first really good blue plum."

Tragedy (California seedling).--Medium to large, suture shallow, wide and extending beyond apex; dark purple; flesh yellowish green, sweet and well flavored; freestone. Very valuable for shipping from early regions in all parts of the State. Largest in amount of shipments in 1913.

Abundance (Japanese).--syns. Yellow-fleshed Botan, Mikado of Hinclay.Large, globular with point at apex; cherry color covered with white bloom; flesh yellow, juicy anl rich. Popular for shipment from eary regions.

California Red (California seedling).--Introduced by J. T. Bogue, of Marysville. Large, light red, firm flesh and small pit. A good shipping plum.

Peach (French, prune peche).--Very large, roundish oblate, regular, flattened at ends; suture distinct, shhallow; color varying from salmon to light brownish red; stalk very short, cavity narrow, shallow, flesh rather coarse, juicy sprightly, free from the nearly round, very flat, much furrowed stone; shoots smooth. Becoming less prominent variety for early eastern shipment.

Femmonzi (probably domestica seedling).-Found in pioneer orchard of $\mathrm{Mr}$. Crooks, Madera county, foothills. Grown and made public by Frank Femmons, for whom it was named by Leonard Coates. A very large, handsome, dark plum, sweet and rich and good in shipment..

Royale Hative (French).-Medium roundish, slightly wider at base; light purple stalk half an inch long, stout, scarcely sunk; flesh amber yellow, with rich, high flavor, nearly free from the small, flattened, ovate stone; shoots very downy. Grown as an early market plum for eastern shipment.

Bradshaw.-Large, obovate, with obtuse suture on one side, sometimes with very slight neck; dark purple, with light blue bloom; stalk three-fourths inch long; cavity narrow; flesh a little coarse, becoming light brownish purple, at first adhering, but becoming nearly free when fully ripe; juicy, good, slightly acid; tree vigorous; shoots purple, smooth. Reported from Sacramento county as blooming late and seldom injured by frost. Not in high favor.

Green Gage (French).-Rather small, round; suture faint green, becoming yellowish green, usually with reddish brown dots and network at base; stalk half to three-fourths inch, scarcely sunk; flesh pale green, melting, juicy, exceedingly rich, and flavor excellent; shoots smooth.

Burbank.-Tree imported from Japan by Luther Burbank. Named "Burbank" by Professor Van Deman. Tree usually vigorous, with strong, upright shoots, and large, rather broad leaves; comes into bearing very early. Almost globular, being five and a half inches around horizontally, and five and five-eighths inches around vertically; rich cherry red, slightly mottled with yellow, and freely dotted with same tint; flesh deep yellow, juicy, very sweet, and of fine, somewhat peculiar, but very agreeable flavor; pit is very small, three-fourths by a trifle over half an inch in diameter. Next to Tragedy in amount of shipments to the east in 1913.

Duane's Purple (New York).-Very large, oblong oval, longer on one side; slightly narrowed towards the stalk; reddish purple, bloom lilac; stalk threefourths inch; slender; cavity narrow, flesh juicy, moderately sweet, and moderate flavor, mostly adhering to stone; shoots very downy and leaves large and downy underneath.

Washington (New York).-Very large, roundish oval, suture obscure, distinct at base; yellowish green, faintly marbled, often with pale red blush; stalk half to three-fourths inch; slightly downy; cavity wide, shallow; flesh rather firm, sweet, mild, very rich and luscious, free from the pointed stone; shoots downy; very vigorous.

Grand Duke.-Large oval, necked; deep purple with blue bloom; flesh greenish yellow, fair quality. A good shipper, following Hungarian in ripening. 
Wickson.-A crossbred by Luther Burbank; form suggests the Kelsey, but more symmetrical; in ripening the color develops from a deep cherry red down to a rich claret as full ripeness is attained. The color is solid and uniform. The flesh is of amber tint, very juicy and translucent; the pit is small and shapely, the flavor is striking and agreeable, but likely to be deficient near the coast. The most popular shipping plum in the State, judged by number of growers reporting favorably, but not largest in eastern shipments in 1913.

Yellow Egg; syns. White Egg, White Magnum Bonum (English).-Very large, oval, narrow at ends, necked at base, suture disttinct; stalk one inch, not sunk, surrounded by fleshy ring at insertion; light yellow; bloom thin, white, flesh firm, rather acid until fully ripe, and then sweet, adheres to the pointed stone.

Jefferson (New York).-Large, oval, base slightly narrowed, suture slight; greenish yellow, becoming golden, with reddish cheek; bloom thin, white; stalk one inch, but little sunk or not at all; flesh rich yellow, very rich, juicy, high flavored and luscious, adheres partly to its long, pointed stone; shoots smooth; tree a slow grower, but productive.

Columbia (New York).--Very large, nearly globular, one side slightly larger; brownish purple, reddish brown where much shaded, with many fawn-colored dots; bloom blue, copious ' stalk one inch, rather stout; cavity small; flesh orange, very rich and sweet, free from the stone, which is very small and compressed. Shoots downy, stout, blunt, spreading; leaves nearly round.

Satsuma; syn. Blood Plum of Satsuma.-Introduced and first fruited in this country by Luther Burbank, of Santa Rosa. Described by Prof. H. E. Van Deman, U. S. Pomologist, as follows: "Leaves more lanceolate than those of Kelsey; fruit averages about two. and a quarter inches in diameter, nearly round, and but slightly sutured on one side; surface dark red, under a thick bloom; dots rather conspicuous and numerous; flesh dark purplish red, which has caused the name of 'Blood Plum of Satsuma' to be given by some; stone very small and pointed." Chiefly grown in Southern California for local use and trade.

Red Magnum Bonum; syn. Red Egg.-Large, oval, tapering to the stalk; suture strong, one side swollen; deep red in the sun; slight bloom; stalk one inch, slender, cavity narrow; flesh greenish, coarse, subacid; shoots smooth.

Diamond.-Large, deep purple; handsome but not high quality; ripens after Japanese and sells well at the east as a cooking plum.

Imperial Gage (New York).-Medium size, oval, suture distinct; stalk threefourths inch, slightly hairy, evenly sunk; green, slightly tinged with yellow, with marbled green stripes; bloom copious and white; flesh greenish, juicy, melting, rich, and delicious, usually free from the oval, pointed stone; tree very vigorous and productive; shoots long upright, slightly downy; leaves with slight shade of blue. A popular canning variety.

Damson (English).-Small, roundish, oval; purple, with thick blue bloom; melting, juicy, subacid.

German Prune (Common Quetsche, Germany).--"This name has been applied in this State to numerous plums and prunes which are sold under it. The fruit of the true German prune is long oval, and swollen on one side; skin purple, with thick blue bloom; flesh firm, green, sweet, with a peculiar pleasant flavor; separates readily from the stone."-John Rock. Complaint is made in many localities of the tendency of the variety; to drop before ripening, almost the whole crop sometimes dropping.

Kelsey, Japan.-Trees brought from Japan by the late Mr. Hough, of Vacaville, in 1870, and purchased by the late John Kelsey, of Berkeley, who propagated and fruited them for several years. First wide distribution was made by W. P. Hammon \& Co., in 1874, who named the fruit after Mr. Kelsey. The following description is by H. E. Van Deman, U. S. Pomologist, from California and Florida specimens: "Tree upright in growth, leaves narrow, twigs brownish gray. Fruit from one and a half to two and a half inches diameter, heart-shaped, with a distinct suture on one side from stem to apex; stem is short, and set in a depression at the larger end; colors mixed yellow and purple, which vary in depth, but rarely make a brilliant appearance, covered with a bloom; flesh yellow, 
very firm, and clings to the stone, which is rather small, and nearly always partly surrounded by a cavity; when fully ripe the quality is very good." Very widely grown; is in less favor than formerly in interior valleys where color is not well developed. Where the fruit is of good color it is profitable for shipping and is highly regarded everywhere for domestic use.

Quackenbos (New York).-Large, oblong oval; deep purple; suture faint; stalk short, slightly sunk; slightly coarse, sprightly, sweet and subacid; partly freestone.

Victoria (English).-Large, obovate, suture distinct; color a fine light reddish purple; stem half inch, cavity rather deep and narrow; flesh yellow, pleasant; clingstone; next to Pond's Seedling in size, beauty and productiveness.

Hungarian Prune; English Pond's Seedling; Gros Prune d'Agen (English).This variety was brought to San Jose probably about 1856 , and in some unaccountable way was first contrasted with the French prune and called the "great prune of Agen ;" afterwards, also in a mysterious way, it took the name of "Hungarian prune." It is still marketed by these names both here and at the East. The true name is English Pond's Seedling. Fruit very large, ovate, slightly tapering to stalk; skin thick, reddish violet, with numerous brown dots, and covered with handsome bloom; rather coarse, juicy, sweet; a very showy fruit; tree a strong grower and nrolific bearer; fruit has a tendency to double; sells well in local and distant markets on its style and is largely grown. Third in amounts of eastern shipments in 1913.

Giant.-Burbank seedling; very large, dark crimson upon yellow ground; flesh yellow, flavor good; freestone. A shipping plum, disappointing as a drying plum.

Splendor.-Burbank seedling; medium size but larger than French prune; clear red, drying dark, does not shake from the tree; earlier than French prune.

Sugar.-Burbank seedling, introduced in 1898; large and sweet; sugar in fresh fruit 23.92 per cent; not of highest quality as a cured prune but sells well; also good for shipping; oval, slightly flattened; dark purple with thick white bloom; freestone; early. Needs pruning and thinning to secure size.

Standard.-Burbank cross of Sugar and Tragedy. Large, purple, blue bloom; flesh fine grained, amber juicy and sweet; freestone; earlier than French prune. Satisfactory shipping plum and considered of great promise for drying.

Imperial Epineuse syn. Clairac Mammoth.-Introduced in 1884 by Felix Gillet and in 1886 by John Rock. Described by Mr. Rock as follows: "Uniformly large size, reddish or light purple, thin skin, sweet and high flavor." Described by Mr. Gillet: "Uniformly large, more oval than the French prune; nearly of the same color but somewhat lighter or reddish nurple; earlier than the French and with thinner skin." Fruit grown by Mr. Rock analyzed at the State University in 1898, showed 20.4 per cent of sugar against 18.53 per cent average of three analyses of French prune. Blooms about ten days earlier and ripens about three weeks earlier than the French prune.

There has been quite widely planted another prune called Imperial which is very inferior in sugar content and likely to prove much less satisfactory.

Prune d'Agen; syn. Petite Prune d'Agen; French Prune, etc.-This is the drying prune at present most widely grown in this State. It is described by John Rock as follows: "Medium-sized, egg-shaped, violet purple, very sweet, rich, and sugary; very prolific bearer." The leading drying-prune of California, commonly called "California French prune." Its standing is sketched in detail on a preceding page.

Robe de Sergeant.-Though this term is given in Downing as a synonym of prune d'Agen, and seems to be in French a synonym for the d'Ente prunes; another prune grown in this State from an importation by John Rock, is quite distinct from the foregoing. Mr. Rock describes the variety as follows: "Fruit medium size, oval; skin deep purple, approaching black, and covered with a thick blue bloom; flesh greenish yellow, sweet, and well-flavored, sugary, rich and delicious, slightly adhering to the stone." This variety makes a larger, darkercolored dried prune than the prune d'Agen, and has sold in some cases at a higher price. It has recently been in disfavor in coast valleys for defective bearing, but is more satisfactory at some interior points. 


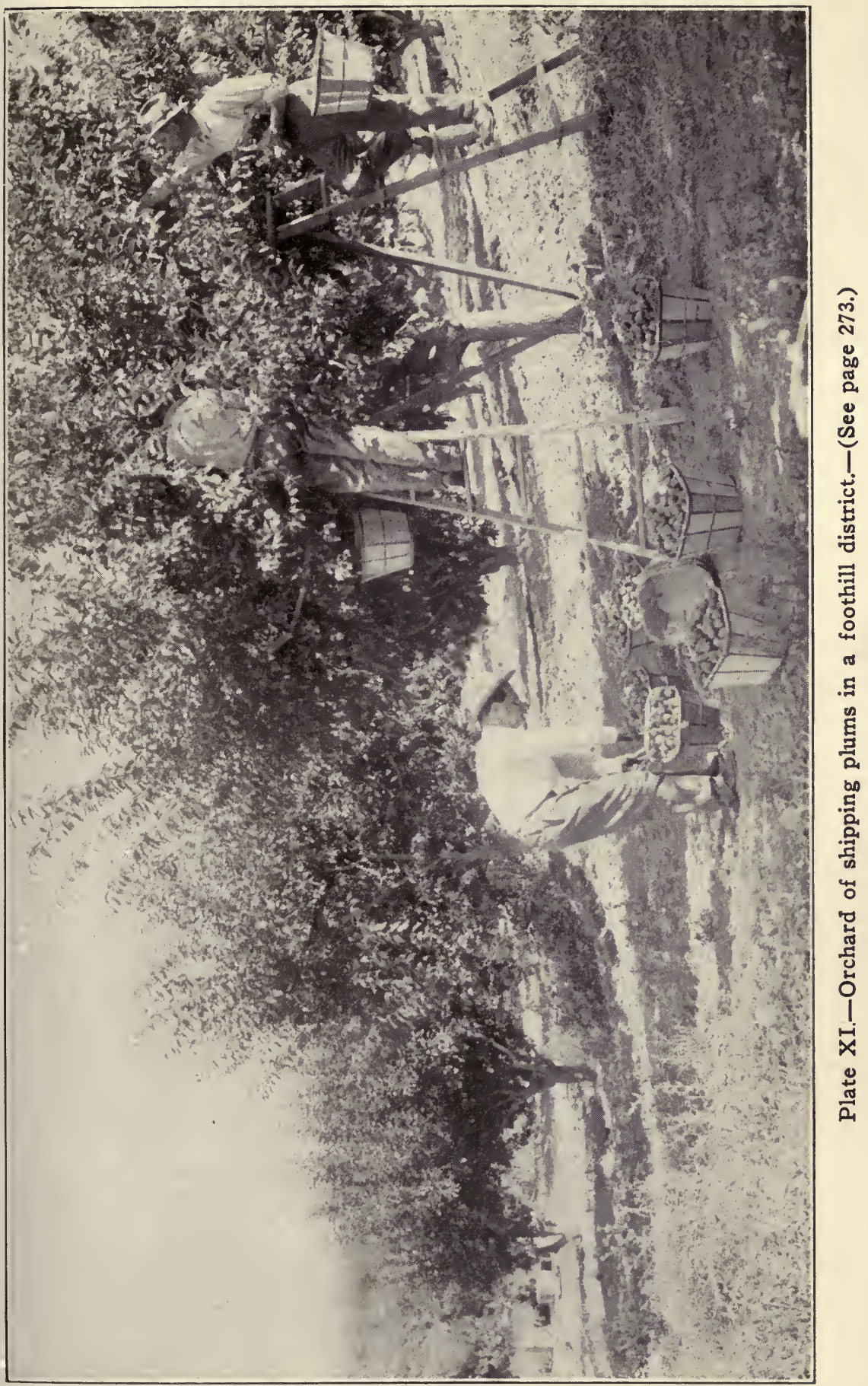



Bulgarian.- "An undetermined variety grown under this name, chiefly in the vicinity of Haywards, Alameda County; above medium size; almost round; dark purple; sweet and rich, with pleasant acid flavor; tree a vigorous grower, and an early, regular and profuse bearer."-John Rock.

Coe's Golden Drop (English).-Very large, oval, suture distinct, one side more enlarged, necked; light yellow, often dotted red to the sun; stalk threefourths inch, rather stiff; flesh yellowish, firm, juicy, and rich, closely adhering to the pointed stone; shoots smooth, rather glossy. A standard late variety for canning.

Silver Prune (Oregon).-Originated with W. H. Prettyman, who says: "It is a seedling from Coe's Golden Drop, which it much resembles, but it is much more productive." Profitable as a bleached prune, but defective in bearing in some California districts. A red variety by bud variation is reported by Mr. J. G. Grundel of Alma.

Golden Prune.-Originated from the seed of Italian prune by Seth Lewelling, of Milwaukee, Oregon, and described by him as larger than Italian; light golden color; exquisite flavor; dries beautifully. Chiefly grown in Contra Costa county. Good for drying and canning. Requires short pruning.

Santa Rosa.-By Luther Burbank, described by S. F. Leib as follows: "It is a fine grower, the wood is very tough and the limbs will not break. It is a sure, regular bearer and bears always most abundantly. It does not have any off years. The fruit runs remarkably fine, even in size, and astonishingly smooth and clear of any defects. It is beautiful, delicious, and a very fine carrier to Eastern markets. It will keep well in hot weather for a week after it is ripe, so there is no occasion to pick it half ripe in order to ship. I intend to plant it very largely myself, and to the exclusion of all other shipping plums." Introduced by George C. Roeding. Largely planted for shipping purposes.

Formosa.- "Unusually large, thick, healthy, light green foliage; strong, hard, wiry wood; blooms with the Burbank and Abundance, and always escapes late spring frosts, and alwavs bears profusely even when continuous rainy weather prevents full pollination in most other plums. No disease has ever found lodgment with Formosa. The fruit is of uniform size, averaging about six inches in circumference one way by eight the other. Fruit yellow with a pale bloom until nearly ripe, turning to a clear rich red. Flesh pale yellow, unusually firm, sweet, rich, delicious, with a delightful apricot flavor, nearly freestone. Formosa has been very thoroughly tested for its keeping qualities, which are unequaled except by Santa Rosa, Wickson, Burbank, and a few others."-Luther Burbank. This variety is notably making good in the Placer County foothills as a shipping plum.

Gaviota.-Burbank cross of Japanese and native American; very large, deep red; flesh yellow, firm and sweet; pit small; rather late bloomer. Favored in the Vacaville district for shipping.

Bavay's Green Gage; syn. Reine Claude de Bavay (French).-Large, round oval, greenish yellow, spotted with red, with small violet-colored longitudinal veins; flesh rather firm, juicy, sugary, rich, of fine quality, adhering slightly to the stone; shoots smooth, leaves roundish, shining; a free grower and very productive.

Ickworth Imperatrice (English).-Large to medium obovate, purple, with irregular streaks of fawn color; stalk medium; flesh greenish yellow, sweet, juicy. rich, mostly adhering to the rather small stone; shoots smooth; very late, hangs long on the tree, and keeps well; endures long shipment well.

Fellenberg, syns. Large German Prune, Swiss Prune, Italian Prune.-Medium size, oval, pointed and tapering at both ends; suture small, distinct; dark purple, with dark blue bloom; stalk one inch, scarcely sunk; flesh greenish yellow, juicy, sweet, delicious, parts from the strne; tree a free grower and very productive; late, excellent for drying. But littie grown in California, but largely in Oregon.

Coe's Late Red.; syn. Red St. Martin.-Size medium, roundish, suture distinct on one side; skin light purplish red, or dark red; bloom thin, blue; stalk threefourths inch, scarcely sunk; flesh rather firm, crisp, rich, vinous; very late, shoots downy. 


\section{CHAPTER XXIV}

\section{THE QUINCE}

The quince enjoys California conditions to the utmost, and rewards the grower with large crops of very large and beautiful fruit. A quince weighing a pound is no curiosity, and it is unlikely that any city of the world can show such fine quinces at such low prices as San Francisco. The lesson from this fact is that the fineness of the fruit, and the evident adaptation of the State to its growth, should not alone be considered by the planter. The local consumption of quinces is naturally small, and it is chiefly for home preserving jelly making. The commercial jelly makers use apple juice as the basis of nearly all their jellies, only using a little quince for flavoring, and some housewives follow the same course. The hope for profitable sale of the fruit in large quantities must therefore rest on distant markets, and though those well acquainted with the growth and sale of the fruit in the cities of the Mississippi Valley, have predicted a great demand for the California quince in that territory, experiences of shippers thus far have been varied, and not such as to induce the extension of our quince production, at present at least.

But though the quince in California has at present narrow commercial limitations, a few trees should find a place in every orchard, for family use or local sale.

\section{CULTURE OF THE QUINCE}

The quince is readily grown from cuttings. Take good-sized shoots of well-matured wood of the current year's growth, after the leaves drop in the fall, and set out at once in nursery row in moist alluvial soil, or in any loose soil which is well drained and can be kept moist enough by cultivation or irrigation.

Quinces are planted at all distances apart, and are grown either as bushes or trees. Undoubtedly the best way is to plant about fourteen or sixteen feet apart, and prune into low standard tree form. This can be done much as already advised for other fruit trees. An annual cutting back of about half of the new growth, while forming the tree, will strengthen the trunk and limbs and prevent the running out of long leaders, which droop to the ground on all sides when laden with fruit, and are often broken by the weight and the wind. Owing to the disposition of the quince to throw out several small shoots at a single point, it is advisable, when forming the tree, to remove all buds but one, just as the growth is starting. This will give one good, strong branch where it may be needed, instead of several weak ones. Pinching off shoots which start out too vigorously, or at undesirable points is, of course, advisable. 
Soils for the Quince.-As the quince grows naturally in moist, though not wet lands, many persons think it always does best in springy ground or along the banks of rivulets; but though moist soils are preferable to dry, such positions are not essential to obtaining large crops of fine fruit. In fact, the quince, like most fruit trees, prefers a well-drained location, and does best on a soil which can be freely worked. It thrives when fanned by the ocean breeze and does fairly well in the interior, providing it has moisture in the soil, and in some situations will doubtless require summer irrigation.

\section{VARIETIES OF THE QUINCE}

Though notably all varieties of the quince are introduced by our nurserymen and carried by them in small stock, most plantations are of the "apple" or "orange" variety. The following may be enumerated, however, as growing in this State:

Apple or Orange.-Large; bright yellow; the best. August and September.

Rea's Mammoth.-A very large and fine variety of the orange quince; a strong grower and very productive.

Smyrna.-Introduced from Smyrna in 1897 by George C. Roeding of Fresno; large, lemon yellow, handsome, tender and delicious after cooking; keeps well; tree a strong grower, with heavy foliage.

Pineapple.-Originated by Luther Burbank and distributed by him in 1899; the result of a long effort to secure a quince which would cook tender like an apple. The name comes from its flavor, which is suggestive of the pineapple. Resembles Orange quince, but is smoother and more globular.

Portugal.-Very large, and fine flavor, turns a fine purple or deep crimson when cooked.

The Chinese Quince.-A most extraordinary fruit, oblong, of immense size, often weighing from two to two and one-half pounds; growth rapid and distinct.

West's Mammoth-O Originated by W. B. West, of Stockton, from seed received from Boston in 1853: of the Orange quince family; round, clear yellow; very large; fine flavor and for the class a very good keeper.

Champion.-Fruit very large, fair and handsome; tree very productive, surpassing any other variety in this respect; bears abundantly when young; flesh cooks as tender as an apple, and without hard spots or cores; flavor delicate, imparting an exquisite quince taste and odor to any fruit with which it is cooked. 


\section{PART FOUR: THE GRAPE}

\section{CHAPTER XXV}

\section{THE GRAPE INDUSTRY IN CALIFORNIA}

The grape grows in all parts of California, from near sea level on the coast to an elevation of 5000 feet or more on the mountains. It is contented, too, with nearly all fertile soils, from the deep valley loams, where the great fat, firm-fleshed grapes are grown for raisin and table grape shipments, to the shallow soils of the high foothills and mountain slopes, where the grapes are less in quantity, but of superior aromatic qualities. This wide adaptation gives an immense area suited for grape culture, but the chief reason for the achievement and the promise of the grape in California is in the fact that the European species, Vitis vinifera, thrives, and thus the California grower has command of all that Europeans have accomplished in centuries by developing special varieties of the species for special purposes. The grapes of the States east of the Rocky Mountains are only grown in California in a small way because the European varieties are the only ones from which raisins can be made; they also furnish the world's wine and brandy, and they give size, beauty, and shipping quality beyond all comparison with American varieties. Wherever wealthy Eastern connoisseurs choose grapes for their glass houses, they select European varieties; the Californian grows his "hot house grapes" in the open air. He also grows most of them without the cost of trellising, because the European varieties generally will bear well in short-pruned, bush form. California has a large acreage of grape vines, and planting has been very active during the last few years, because good prices have prevailed, especially through the increased opportunity for fresh grape shipments. At the same time, new economic and commercial problems are continually arising and the industry has to readjust itself to new conditions. discussion of such problems does not come within the scope of a cultural treatise like this. It is the duty of the grower to keep himself up to date on such subjects by faithful reading of California periodicals and by participation in public assemblies in the grape interest. Concerning cultural difficulties, the protection of vine from its enemies and problems in vinification, special researches are constantly pursued by the University Experiment Station at Berkeley and publications are furnished on application.

The culture of the grape is one of the great branches of California horticulture. Its three chief divisions are: Grapes for the table, grapes for wine, and grapes for raisins. In all these branches the product has far exceeded local requirements and has become an important item in the export trade of the State. The attainments of the industry can be roughly measured by the statistics of the shipments of grapes, raisins, 
wine and brandy, which are given at the close of Chapter VI. The California Board of Viticultural Commissioners reports the quantities and products of California grape products in 1913, as follows:

Table grapes, 7,363 carloads at $\$ 950$ per car................ $6,994,850$

Wine and brandy, $42,134,988$ gallons, estimated selling for . . . . . . . $15,000,000$ Raisins, 65,000 tons (below normal), estimated selling for $\ldots \ldots \ldots \ldots 4,840,275$

Total.

$\$ 26,835,125$

\section{THE GRAPE AREA OF CALIFORNIA}

The grape has a very wide range in California. If the immediate seacoast and the higher altitudes on the mountains be excepted, the grape may be planted with a good chance of success anywhere if soil and local topography be suitable. As has been shown in Chapter I, the vine can approach quite close to the ocean if some shelter from prevailing cool winds be afforded, and quite high on the mountains if one keeps out of depressions where late frosts are frequent. In planting the grape in doubtful situations much depends upon choice of proper varieties. For example, in the cool air of the coast region and the short summer of the higher altitudes, early maturing varieties must be the main reliance, for late sorts will not receive heat enough to bring them to full maturity.

Away from immediate coast influences, and up to perhaps three thousand feet or more on the sides of the Sierra, the grape is successfully grown both upon the floors of the valleys and upon the hillsides. But there is still need of choice both of special locations and of varieties according to the purposes which the grower has in view. The coast valleys of the upper part of the State produce good table grapes, but they are unfavorable for the raisin industry because of the deficient sunshine and excessive atmospheric humidity of the autumn months. The best raisins are made in the dry, heated valleys of the interior, and the conditions which there develop the fullest quality of the raisin grape also develop the sugar in some kinds of wine grapes beyond a desirable percentage. Here again the choice of suitable varieties intrudes itself, for the varieties which yield light table wines in the coast valleys may yield heavy "heady" wines in the interior. Valleys, too, as a rule, although they yield larger crops of grapes and greater measure of wine than similar area on the hillsides, must yield the palm for quality to the warm soils of the slopes. And here enters the business proposition whether large amount and less quality is better than less amount and higher quality. To this there can be no general answer. It depends upon the disposition which is to be made of the crop, and the demand for it.

The coloring of certain varieties is a matter underlying their profitable production for fresh shipments and this is determined by local conditions concerning which the best information is actual observation of their effects. These few facts out of many which could be stated will serve to enforce the fact that wide as is the range of the grape, both localities and varieties for certain purposes must be intelligently chosen. 
Much has been learned during the last few years, but it will require the experience of another generation, perhaps, to make the matter clear.

Soils for the Grape.-The grape will thrive on a great variety of soils, in fact, on any of those enumerated as fruit soils in Chapter III. There are thrifty vineyards on the light, deep valley loams, on the heavy clayey loams, on adobe, and on the red soils of the foothills. Even on shallow soils the grape will do well if given sufficient moisture, and on rocky subsoils it thrives if there be crevices for the roots to penetrate, or if the rock be shattered to admit the roots to permeable substrata. Standing water during the active period of the vine is, however, unfavorable to growth, and alkali is adverse to satisfactory results in wine making. Almost any soil which does not hold excess of water or is not tainted with alkali will do for the vine, although the plant appreciates good, deep soil, and will grow and bear fruit in proportion to its supply of it. Of course the economic question of ease of cultivation enters into the choice of soil for the grape, as for other fruits, but its claims are obvious and need not be enlarged upon.

Length of Grape Season.-By choice of early and late varieties the grape season extends over half a year in California, without recourse to artificial means of preservation. Where the fall rains are not very protracted, the late varieties sometimes remain in good condition on the vines until the winter pruning. Good grapes have been picked from the vines as late as the middle of January. In the Lodi district of the San Joaquin Valley, a great center of the grape shipping industry, overland shipping begins about August 10, with Black Prince. Tokays from light soil soon follow, then from the heavy soil, and in about two weeks everything is going at full blast, keeping up strongly for nearly two months. Then it decreases greatly and continues into November with the later varieties, like Cornichon, Emperor and Ferrara. There are also a number of other shipping varieties grown than those named, but Tokays far outnumber all the rest put together. There is much latitude in grape gathering, for grapes can stay on the vine for some time without deterioration; besides there is the "second crop" from later blooming which is of advantage in shipping, but objectionable in handling for raisins, brings less price for wine making. Tokays may be picked during a period of two months in the same vicinity because of the "second crop" and because the ripening comes earlier or later according to soil, as noted above. Even the intrusion of a light fall rain, such as they have in the interior, may help size and color, but a heavy rain is destructive and constitutes a constant menace in the late ripening districts near the coast, while occasionally injurious to the interior also.

Shipping Grapes in Sawdust.-The table grape industry of California seems to be entering upon a notable extension of its marketing season and area through the demonstration by the United States Bureau of Plant Industry, that California grapes can be profitably packed in drums, holding about $35 \mathrm{lbs}$. of grapes, packed in redwood sawdust, properly prepared and held safely in cold storage for winter sale in the East-as Spanish grapes in cork dust are handled. Better results are 
attained with sawdust than with cork dust. Full details are given in Bulletin 35, U. S. Dept. Agr., December 31, 1913, which the interested reader should secure. The shipments of 1912, about thirty carloads, or 8,596 drums, sold in New York at an average of $\$ 2.90$ per drum, which, after deducting all costs of packing, transportation, storage and commission, would net the grower about $\$ 60$ per ton for the grapes. The grapes thus handled were Emperors. Thus far it seems to be demonstrated that Muscats can be sold this way until November 15th, Malagas until December 15th, Emperors until about January. It is expected that if California can commercially grow the Almeria, the grape used in Spanish shipping, it can be safely held in storage until April 1. 



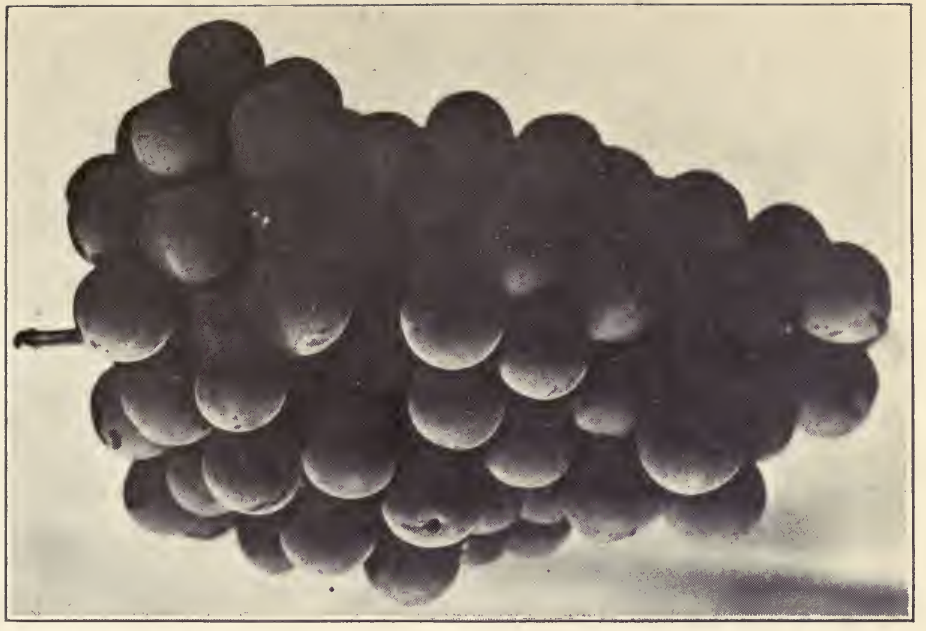

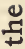

ฮี

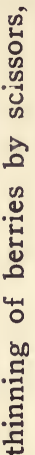

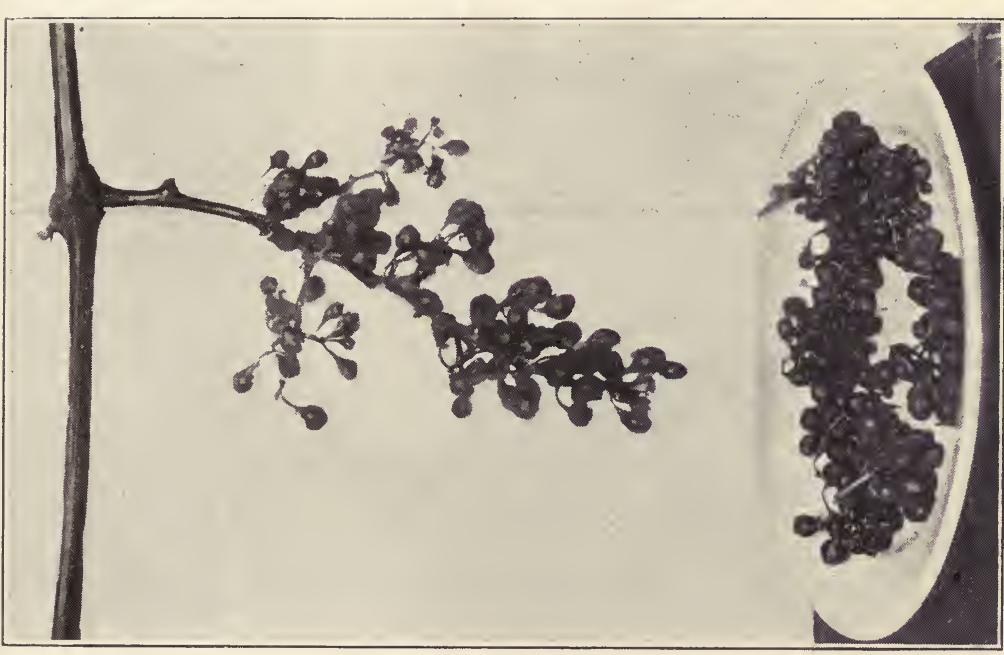

亲

की

至

\&

సี

声 。

苂 \&

च

岳!

1 क

of 2

है

웜엉

v

는 졈

嵌

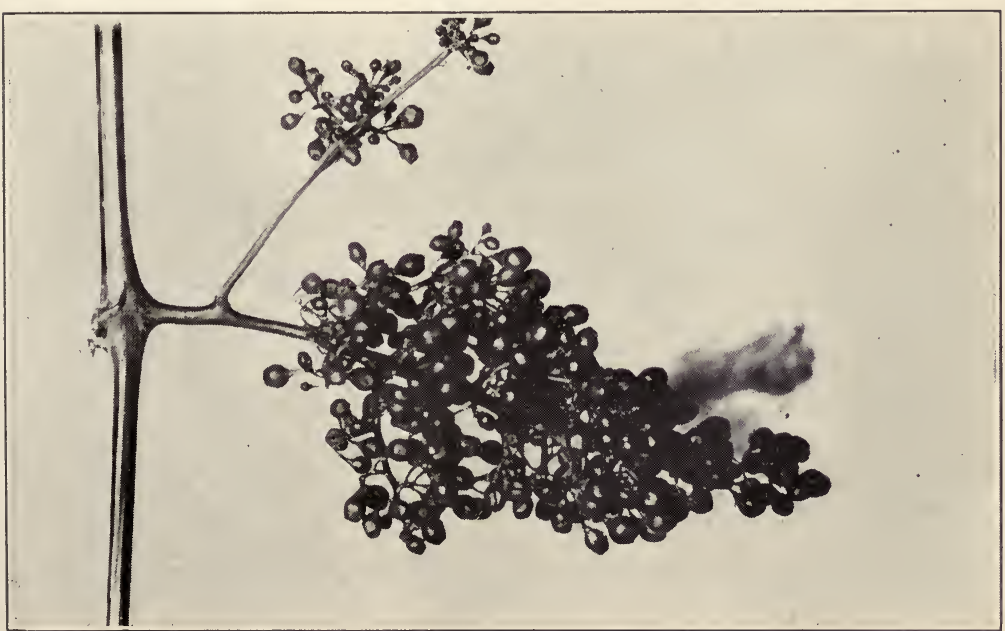

范 
The length will depend altogether on the soil and climate where they are to be planted. They should be of such a length that when planted the base of the cutting will be at the level where the conditions are most favorable to root formation. If the base is too deep, it will be too wet and too cold to develop roots. Roots will start higher up

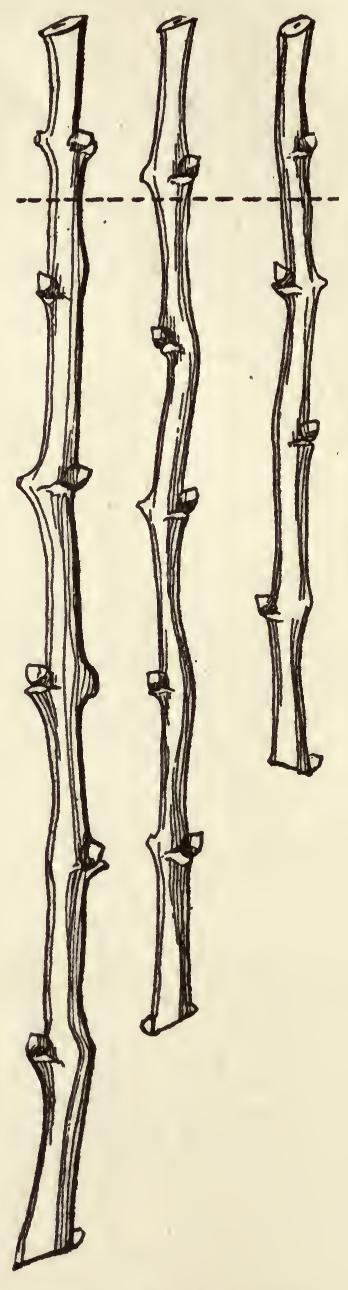

Properly made cuttings.

and the bottom part will be wasted, or worse still, may decay and injure the vine. If the base is too near the surface the whole cutting may dry out and die before its roots have developed sufficiently to supply it with water.

In the moister soils of the cooler districts a cutting 10 inches long is sufficient for direct planting in the vineyard. In the drier and warmer 
interior a 14-inch to 16 -inch cutting is better, while in the driest soils of the warmest districts it is often necessary to have a cutting 18 to 20 inches long. For planting in the nursery a 12 or 14 -inch cutting is about the most convenient. . If the soil of the nursery is wet and cold more of the cutting should be left above ground; if, on the contrary, the soil tends to be hot and dry the cutting must be planted deeper and even covered up completely.

It is not necessary, or possible, to make every cutting of exactly the same length, because they should all terminate at each end at a node. A vine cane consists of nodes where the buds are and internodes between them. The pith is interrupted at each node by a woody partition (called the "diaphragm") which extends through the cane at each bud. In making a cutting, therefore, we should cut exactly through a bud both at the top and at the bottom. This will leave the woody partitions, which will prevent decay at the bottom and drying out at the top. If removed, the pits in the upper internode will be exposed to alternate wetting and drying, and may decay, thus weakening or killing the bud below.

In planting, the cutting should be placed with just one bud above the surface of the ground, as indicated by the dotted line in the accompanying engraving. It is a great mistake to leave more than one bud out of the ground, as this increases the danger of drying out.

Making and Caring for Cuttings.-Cuttings can be taken from the vines at any time after the fall of the leaf and before the spring flow of sap begins. The earlier cuttings-those taken before January -are more likely to make a successful start and after-growth than those cut later in the season.

It is common, however, to defer preparation of cuttings till the pruning is done, be it early or late, and this will generally answer the purpose, if care be taken to secure the cuttings immediately at the pruning; but if the branches be allowed to lie upon the ground for days, exposed to sun, wind, or frost, before the cuttings are secured, their chances of growth are seriously lessened, and a good part of the failures in planting are due to such cuttings.

Cuttings should be taken from the short-jointed, well-ripened wood of the previous year's growth, cut squarely and smoothly as already described. Cuttings from the outer ends of long canes are not so likely to root, nor to grow so vigorously, as those from stronger wood, trom three-eighths to five-eighths of an inch in diameter generally.

Keep them dormant until the time comes to set them in the vineyard, else the tender shoots may get broken. To keep them back, place them, at the pruning in trenches, about as deep as the length of the cuttings, on the north side of a close board fence or a building, cover with loose earth, and over that throw some straw and boards. Take care that the trenches are in moist but not wet ground as too much moisture rots the cuttings. If the ground has not been moist enough, and the cuttings seem dry or withered, plunge them in water to within three or four inches of their top, for a few days before setting, and do not let them dry again before planting. 
Rooting Cuttings in Nursery.-What has been written is in reference to cuttings designed for placing in permanent position in the vineyards, but, for the most part, applies as well to the preparation of cuttings for the nursery. For nursery treatment, however, shorter cuttings can be used than for field planting, because of the better cultivation and more generous moisture conditions which are usually provided.

In preparation of ground for the rooting of vines and the planting of cuttings therein, the suggestions in Chapter VIII are directly applicable, as, to secure rooting of the cuttings, there is just as great need for deep and fine working of the soil, pressing of it around the cutting, and for careful culture during the growing season, as there is for such treatment of fruit-tree seedling or root graft. It is just as necessary, too, that the rooted cuttings should be carefully lifted and guarded from drying out while on the way from the nursery to permanent place. The reader is, therefore, referred to Chapter VIII for suggestions on preparation, laying out, and care of nursery ground intended for the rooting of grape cuttings.

To secure vines upon resistant roots recourse has recently been made by some growers to the cutting-graft which will be mentioned presently.

There is a growing tendency to use rooted vines instead of cutting in planting out vineyards, for, although the former costs several times as much as the latter, either in the time of the grower or in cash outlay, the balance is believed to be usually on the other side, when the uniform stand and more satisfactory growth secured by rooted vines are considered.

\section{BUDDING AND GRAFTING THE GRAPE VINE}

Working over the grape vine is largely practised in this State and is easily accomplished. The occasion is twofold; Replacing undesirable varieties with those of better quality, or in better market demand, and in bringing the vinifera varieties upon roots which resist the attacks of the phylloxera. The employment of resistant stocks has proved eminently satisfactory in this State, the resistant stock having been successfully installed even in the hole from which the dead vinifera root had been taken. For this reason resistant roots are largely relied upon in the planting of new vineyards in infested districts, and also used to some extent in regions where the insect is not now found, by those who fear and desire to provide against its coming. And yet in the large planting of vineyard in the interior valley which has recently been undertaken very little attention has been paid to resistant roots. It is so cheap to proceed with simple cuttings of the variety desired, and the vine comes into bearing so soon, that most planters are willing to take the risk of infection with phylloxera for it may be long delayed and several profitable crops may be realized before its arrival. This is a question which each planter must answer for himself.

Budding the Grape.-Buds can be readily made to grow in grape canes, though budding is not largely used. Success can be had 
with the same method of budding that is common with fruit trees as described in Chapter IX. Insert the bud (which is taken from a cane of the previous season's growth) in the spring as soon as the bark will slip well on the stock, and before the run of the sap is too strong. Keep the cuttings in a cool place so their growth will be retarded, and then seize upon just the right condition of the stock, insert the bud under the bark of a cane of the previous season's growth, tie it around with a string, and the bud starts readily without further treatment, when its growth shows its ability to take the sap, the top of the stock is removed.

Herbaceous budding is also practicable. It consists of taking buds from the current season's growth and working them upon canes also of current growth by the usual shield budding process. Mr. Thomas

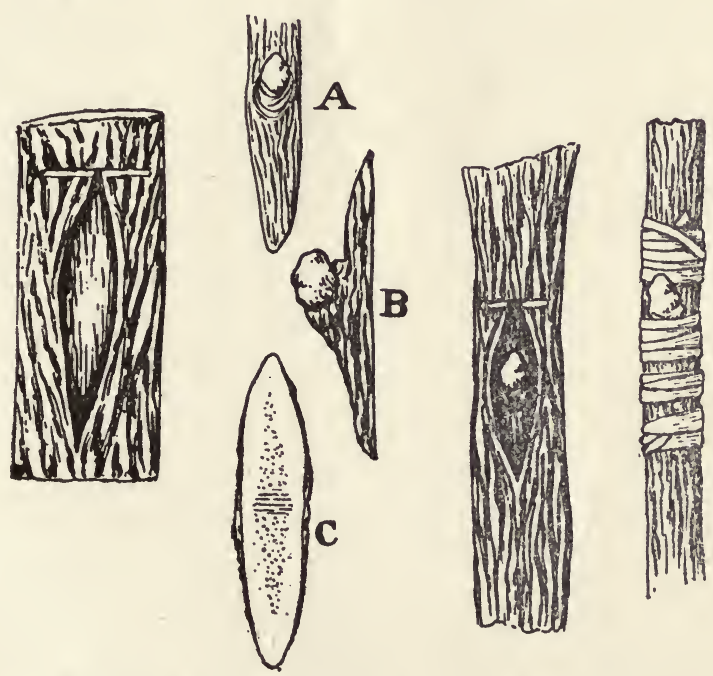

Budding from previous season's growth.

Casalegna of San Martin succeeds well with this under these conditions: All buds put in from July 15 to August 15 start the same year, but may be injured by fall frosts. Those put in from August 15 to September 15 remain dormant until the following year, unless the stock is exceptionally vigorous. Budding is most successful in the month of August. The buds should be taken from canes which have reached the stage of maturity indicated by the pith turning white and just before the bark turns yellow.

Grafting the Vine.-Grafting in old vine roots is a simple operation, and is performed in various ways. The principles involved in vine grafting are similar to those affecting tree grafting, as described in Chapter IX. The processes employed are also similar, but the graft requires less binding and waxing is dispensed with, because the graft is made beneath the surface of the ground, and is, therefore, less subject to accident, exposure, and drying out. 
Grafting in the Old Stump.-This is resorted to when the character of the vineyard is to be. changed. Out of the many ways for working into old stumps, one introduces the scion by a side cut into the stock without splitting across as shown at $C C$ in the adjacent engraving. The earth is removed from the old vine down to its first lateral roots, and the top is sawed off cleanly a few inches above the first laterals. A cut is then made into the side of the stump with a knife and mallet, as shown in the figure. The scion is then cut long enough so that one bud will remain above ground when the surface is leveled again, the bottom of the scion being given an oblique wedgeshape, so as to fit the crevice in the stock. Some care is needed in shaping the wedge of the scion, so that the surfaces are in contact will give good results. If the stock is well made and the end of the scion so adjusted that the stock will pinch it when it is pushed into place, nothing more will be needed except to smear over the cut surface of the stump and the joint of the scion and stock with clay or with a mixture of two parts clay and one part fresh cow manure. If the scion is held firmly and sealed in with this mixture, it usually needs no tying, and the hole can be carefully filled with loose earth, with a strong stake to mark the place of the graft, and to which the new growth can be securely tied afterwards.

Another common method of grafting beneath the ground is to split the stump across its center, as is done in top-grafting fruit trees as shown in Chapter IX, and one or two scions inserted. If two are used and both grow, the weaker one is afterward suppressed. In this cross cleft graft some grafters rely upon the stock to hold the scion without tying, and daub it over with the clay mixture, care being taken to fill and cover the split in the stock to exclude water. Others put a ligature around the split stump. Strips of cotton cloth answer well for this purpose. Tying offers better security from knocking out the graft with the cultivator.

In grafting into very tough old stumps, some growers leave a slim wedge of wood in the cleft with the scion to prevent the stock from closing too forcibly upon the scion.

Side Grafting.- Side grafting the vine is commended by some growers. It consists in inserting a graft by a cut into the side of the stock, the method being essentially the same as that employed with fruit trees, as described in Chapter IX, excepting that in side grafting the vine the top is not amputated, but is allowed to bear its crop and is then removed the following winter. The next summer the scion will bear a crop, and the vine is worked over without cessation in its bearing.

Herbaceous Grafting.-This term is applied to a graft in which the scion of the current season's growth is set by a cleft graft into canes also of the current season's growth, while both scion and cane are elastic, but not too soft. The method has not been usually successful in this State, apparently because of the dryness of the summer air. Still some satisfactory results are reported. Mr. Casalegna of Santa Clara county whose success with herbaceous buds has been noted, does well also with soft-wood grafting by the whip-graft method. He says 
it is most successful in June, provided the scions are hard enough. The pith must be white. In a strong-growing vineyard grafting may be done in July. The leaves are taken off the scions when they are cut. If they are to be used immediately they are placed in water; if to be carried some distance they are placed in a wet sack. Tie the grafts

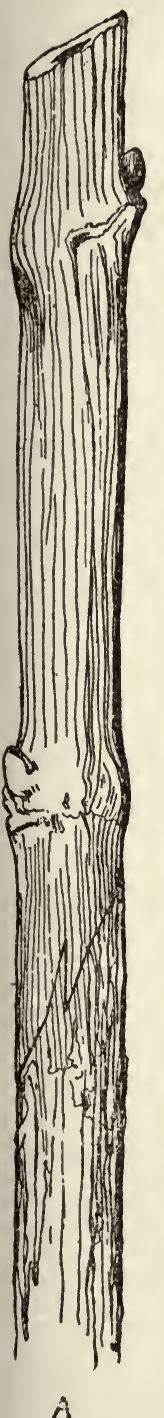

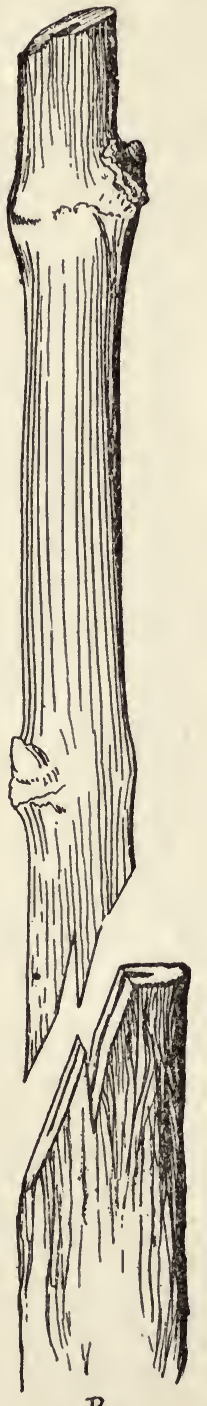

B

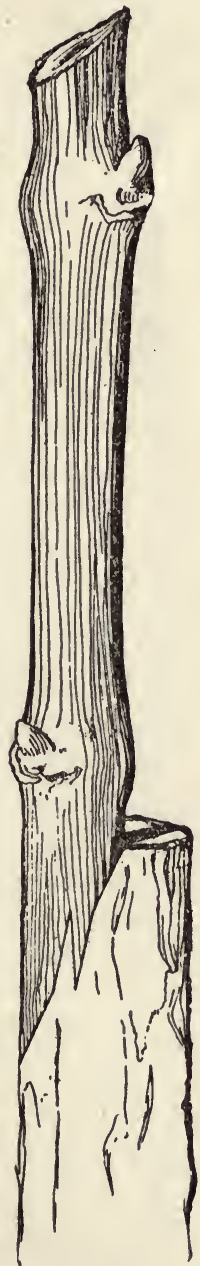

B B

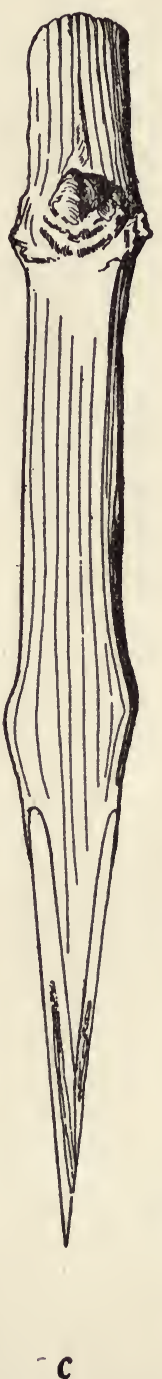

C

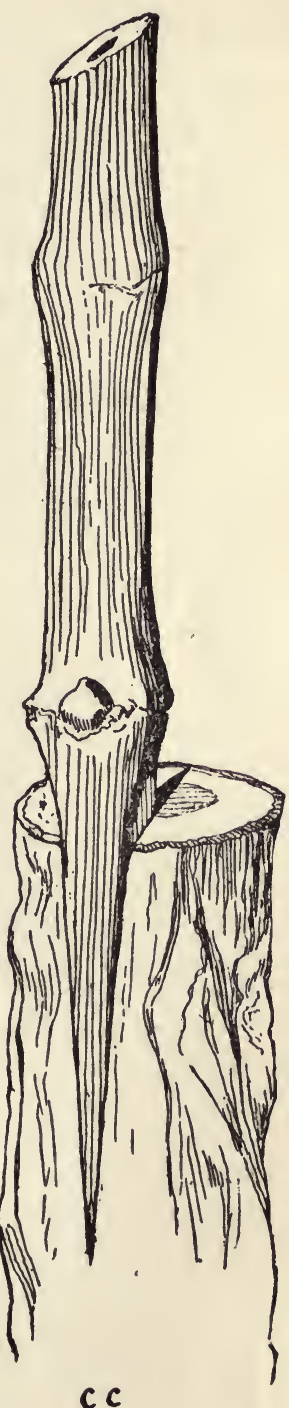

$c c$

Methods of field grafting grape vines.

A. Whip graft for stocks 1-2 to 2-3 of an inch in diameter.

$B, B B$. Whip graft for stocks $2-3$ to $3-4$ of an inch in diameter.

C, CC. Cleft graft for stocks 3-4 of a ninch in diameter and for old stumps. 
with German knitting yarn, not with raffia. The season for herbaceous grafting will, of course, vary according to the locality. Hot weather immediately following the work is fatal to most of the grafts If two or three cool days follows the insertion of the scions he obtains an almost perfect stand.

Care of Scions.-Scions should be kept cool and moist enough to prevent drying but not wet enough to cause decay, as has already been described in the keeping of cuttings.

Time of Grafting.-Grafting into old vine stumps is done in February, March and April in different parts of the State, March being the month usually chosen for the work. If a spring graft fails, the stump may be regrafted in August or in the following spring. In regrafting, the stump is cut off again below the previous cleft. The time for the work is when the sap has ceased flowing, usually from the first to the tenth of August.

Resistance to Phylloxera.-The recourse to resistant roots to escape the phylloxera has been attended with some disappointment because the wild roots at first widely used proved only partially resistant. Recently, in the main through employment of French selected varieties of the American wild species, stocks with satisfactory resistance, larger growth and vigor and adaptation to different California soils have been secured. Notable success has been attained in the habilitation of vineyards on the basis of resistant roots. As this undertaking involves considerable outlay and as success depends taking advantage of the latest demonstrations of the affinities of varieties, the adaptations of roots to soils and the suitability of varieties for special purposes, the latest information should be had from the University Experiment Station at Berkeley, before making investments.

The Cutting Graft.-Grafting the desired variety upon a resistant cutting, putting these cutting-grafts through a callusing bed and then planting the grafted cutting in nursery for rooting is an accepted French method which is being successfully employed in California. This has advantage in time gained and in securing a full stand of vines as compared with grafting upon cuttings already rooted in place in the vineyard though the latter has been successfully practiced.

If cutting-grafts are placed directly in the nursery many will fail. For this reason it is always best, except at the extreme end of the grafting season, to "stratify" the grafts in a "callusing" bed, where conditions of moisture, temperature, and aeration can be controlled. This callusing bed is usually a pile of clean sand placed in the south end of wall or building surrounded by a board partition where there is no possibility of its becoming too wet by the flow of water from a higher level or from an over-hanging roof. It should be protected, if necessary, by a surrounding ditch. It should be furnished, with a removable cover of canvas or boards to protect it from rain and to enable the temperature to be controlled by admission or exclusion of the sun's rays. A waterproof wagon-cover, black on one side and white on the other, is excellent for this purpose. 
The bottom of the callusing bed is first covered with 2 or 3 inches of sand. The bundles of grafts are then placed in a row along one end of the bed, and sand well filled in around them. The bundles should be placed in a slightly inclined position with the scions uppermost, and the sand should be dry enough so that it sifts in between the grafts in the bundle. The bundles of grafts are then covered up completely with sand, leaving it at least 2 inches deep above the top of the scion. There should be but little more moisture present for callusing than in the sand used for keeping the cuttings over winter. Too much moisture will stimulate the emission of roots and starting of buds without aiding the callus formation, which is a perfectly distinct process from the formation of roots.

Grafting of Resistant Stocks after Rooting.-Grafting on resistant roots differs from working in old stumps in the size of the wood to be operated on, and in the fact that the graft must be set higher up because it is not desirable to have the scion strike roots of its own, for the obvious reason that depending on such roots would make the vine no longer resistant. The advantage of covering the graft with earth is, however, still to be enjoyed, for the earth can be raised in a little mound around the graft, to be removed when the graft has taken well. For this reason grafting on resistant roots is usually done at or near the surface of the ground.

The common cleft graft is used when the stock is large enough to give a split strong enough to hold in the scion. In grafting smaller stocks the whip graft is used both in making cutting-grafts and in grafting cuttings already rooted. This graft is variously treated. It is covered with clay by some, by others with grafting wax; but the common experience is that grafting wax makes too tight a joint, and holds in surplus sap, which begets disease. The use of a wax band specially adapted to ruling conditions has proved very successful, but the easiest and usually most satisfactory way is to wind with soft twine or raffia which will decay and loosen as the graft enlarges, but care must be taken to cut the band if it has not decayed at the time of transplanting.

\section{LAYING OUT THE VINEYARD}

Vines are planted in rectangles, generally in squares, but sometimes at a less distance in the rows than the rows are from each other. The stakes which are to represent the future vines are in either case placed by the same methods of measupring or marking off. All the methods described for clearing and preparing lands, in Chapter VII, and for laying off ground in squares, described in Chapter $\mathrm{X}$, are applicable to vineyard ground. The measuring wire therein described is the means usually employed for laying off. A special contrivance which has been used to some extent on level ground is thus described:

The marker most in use is made in the form of a sled, sixteen, fourteen, or twelve feet long, with three runners so placed as to mark rows eight, seven, or six feet wide. These runners should be made about three feet long, of some hard wood (Oregon pine will do), two inches thick and firmly nailed to two planks placed upon them of the lengths first above named. Upon these should 
be bolted two strong pieces of joist in the form of wagon hounds projecting in front far enough to receive a stout pole like a wagon tongue, well braced and fastened with an iron rod. Care must be taken that the motion of the machine is steady and true in all its parts. With a well-made marker, a gentle team, and a careful driver, excellent work may be done.

Distance of Planting.-There is as much difference of opinion and practice in fixing the distance between vines as between orchard trees, but usually more room is given than formerly. Planted in squares, the distance varies from seven to ten feet, with eight feet as most prevalent, taking the State as a whole. Planting in rows is also adopted to some extent. Such plantations are made with the vines seven by ten or eight by ten feet, four and one-half by eleven feet, etc. There is a great variation in the distances. Some advantages of the row system are as follows: Greater space to spread trays for raisin curing; plowing can be done with double team and larger plows; the brush can be gathered and burned between the rows instead of carrying it to the avenues; sulphur and materials for spraying can be brought in by team to any part of the vineyard; empty boxes can be distributed and filled ones gathered up without carrying, etc. Planting in rows recommends itself not only for planting new vineyards, but also for changing old vineyards from seven by seven feet to three and one-half by fourteen feet, or from eight by eight feet to four by sixteen feet, giving opportunity to change from a vinifera-root vineyard to a resistant-root vineyard. This can be accomplished by planting resistant roots in the alternate rows to be preserved, right between the two old vines.

Number of Vines to the Acre.-However the vines be set, it is very easy to calculate the number of vines which an acre will accommodate. Multiply the distance in feet between the rows by the distance the plants are apart in the rows, and the product will be the number of square feet for each plant, which, divided into the number of feet in an acre (forty-tree thousand five hundred and sixty), will give the number of plants to the acre.

Aventues in the Vineyard.-For convenience of access with team and wagon there should always be avenues through the vineyard. They are usually arranged so as to cut up the vineyard into blocks about twice as long as broad, if the vineyard be on the level land. Of course, on hilly lands the avenues should be located for ease of hauling. The avenue is made by leaving out a row of vines, and, therefore, the exact size of the block will depend upon the distance between the rows. Some advise having not more than forty vines between the avenues. Planting in rows, with wide spaces between the rows, renders few avenues necessary.

\section{PLANTING CUTTINGS AND ROOTED VINES}

Various means are used for planting cuttings. An essential condition to successful growth is to have the lower part of the cutting well embedded in the soil, as it will not root unless in close contact with the earth. To lack of care in this regard most failures are due, and for lack of surety that such contact is made the various contrivances for 
speedy planting, such as the planting bar, are widely condemned; an excavation of the hole and refilling with fine surface earth, just as advised in Chapter XI, for planting orchard trees, is commended as the safest practice. Much, however, depends upon the soil. In loose, free soil such a use of a bar or "sheep's-foot" as will be presently described may be satisfactory, while it would be impracticable on firmer soils, both because of the difficulty of insertion and because the packed condi-
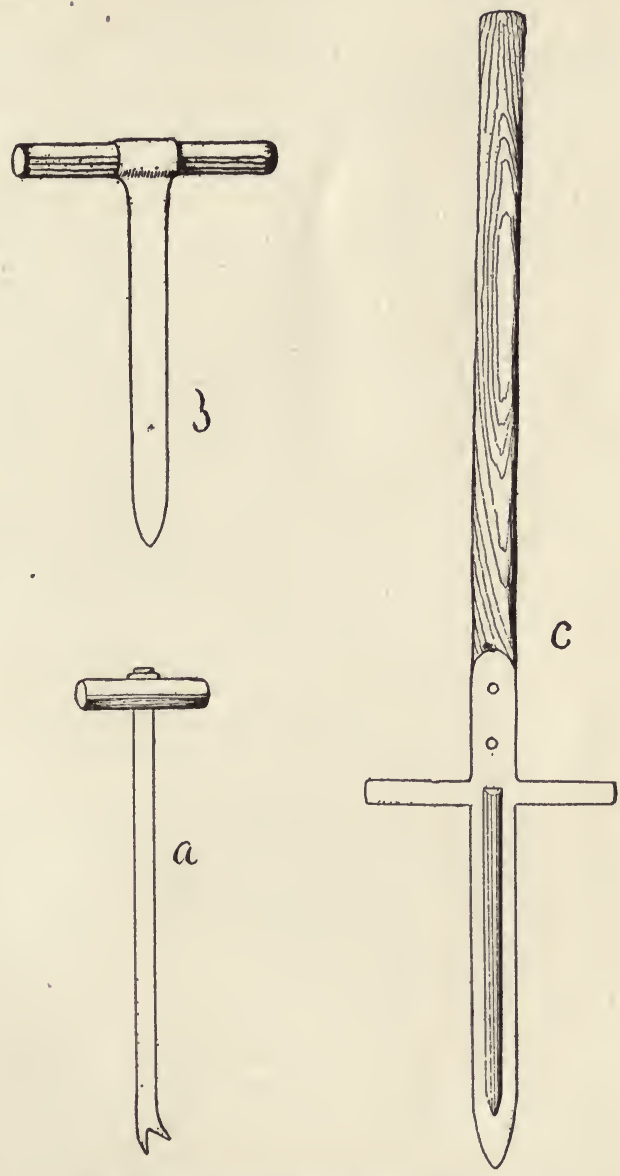

Dibbles for planting vine cuttings.

tion caused by the forcing in would not favor root extension, and not desirable on shallow soils because the contact of the better surface soil with the bottom of the cutting will stimulate the growth of the cutting, and is, therefore, very desirable.

The post-hole auger and a device for taking out soil as a "trier" takes out a sample of cheese or butter, have also been used to some extent, but not widely, in making holes for cuttings. 
Other forms of dibbles for planting vine cuttings relate particularly to the setting of cutting-grafts in a light deeply-worked nursery soil, but may be also used to advantage in placing cuttings in the vineyard if the soil favors such a method. They are described by Mr. Bioletti as follows:

Two of the best forms of nursery dibbles are shown in the engraving. The first, "a" consists of a piece of round one-half-inch iron, 18 inches long, furnished with a wooden handle at one end and a curved double point with a V-shaped cleft in the other. The bottom node of the stock is caught in the cleft and the graft forced down to the desired depth. Unless the ground is very light the other dibble, "b," is preferable. It consists of a sword-shaped piece of iron 18 to 20 inches long and 2 inches wide, furnished also with a handle. The usual way of using it is to press it into the ground to the desired depth, open the hole a little with a lateral thrust, withdraw it and insert the graft. The dibble is then pushed into the ground again at about an inch to one side of the graft and by another lateral thrust the earth is pressed tightly around the graft. This takes more time than is necessary with the other form of dibble, and usually done carefully there is danger of failing to make the soil close around the base of the stock, which is thus left surrounded by an air space. Grafts left in this way are apt to become moldy and fail to make good roots. The figure "c" is a planting dibble to be used with hand and foot like a spade. All such contrivances are only suited to light soils which crumble and settle easily. On heavier soils, digging holes and placing the soil around the roots or the base of the cutting by hand is indispensable.

Planting Bar and Sheep's-foot.-The following methods, described by Dr. Gustav Eisen as prevailing in the raisin districts of the San Joaquin Valley, on sandy, loamy soils, will well illustrate similar methods wherever followed:

The planting bar consists of a bar of hard iron, sharpened at the lower end and furnished with a cross handle at the other. The length of the bar is about three and a half feet, width about two and a half inches, and thickness a third to half an inch. If less than this the bar will bend. The planting is done by pushing the bar perpendicularly in the ground. After withdrawing it, insert the cutting and push it down to the bottom. Fill up the hole by again inserting the bar in the ground close by and pressing the flat side against the hole.

The sheep's-foot consists of a round rod with cross-handle at the upper end. The lower end of the rod is slightly flattened, bent and forked. The planting is done by fitting the forked end over the butt-end of the cutting, and immediately pushing cutting and rod together to the desired depth in the soil. A slight twist is now given to the sheep's-foot. This loosens it from the cutting and allows it to be withdrawn. A tamp with the foot fills the hole. Great care must be taken in withdrawing the sheep's-foot, lest in doing so the cutting should be lifted also, and this will leave a fatal air chamber at the lower end. The slight twist given the rod before withdrawing loosens it and leaves the cutting undisturbed.

For planting in dry situations some careful planters run water and fine earth into the hole made by the bar after inserting the cutting; others run in fine sand dry and then pour on water. In using water in this way one must take care that he uses sand or sandy earth, and not clay, for a succeeding dry spell may bake it, and the cutting will be worse off than if not puddled. 
Planting Rooted Vines.-Planting rooted vines is governed by the same rules commended for planting trees in Chapter XI, so far as preparation of holes, care in placing and firming the soil around the roots, etc., is concerned. In handling rooted vines there must be greater care in packing and transportation to prevent the roots from drying, and in carrying to the field it is generally advised that the plants be kept in a pail or other receptacle with water. The vine roots are very small and tender, and success will largely depend upon good care of them. At planting all dead or injured roots should be trimmed away, healthy roots shortened so that they can be placed well in the hole, and the top reduced to a single cane cut back to two eyes.

When to Plant.-The exact time to plant can not be stated, for the condition of the soil and the local season-points are the best guides. Planting can be done much later as a rule in the coast regions than in the interior, because the soil is usually later in getting into good condition of mellowness and warmth, and the late rains are usually heavier. It is certainly not advisable to place cuttings in cold, wet soil, and dry soil will quickly destroy their vitality. The suggestions given in Chapter XI should be carefully considered. The planter must use good judgment in choosing his time for planting, aided in forming it by the best local experience he can get.

Cultivation of Vineyard.-General suggestions concerning the cultivation of the vineyard have already been given in Chapter XIII. in which methods employed in California, and which are practically the same for all fruits, are described. 


\section{CHAPTER XXVII}

\section{PRUNING AND CARE OF THE VINE*}

Most of the varieties of vinifera grown in California at present thrive under the short pruning system. There are exceptions, however, which will be noted later. The prevalence of the short pruning system frees our growers from the expense and inconvenience of trellises. Though in the early years of the vine stakes are used, our older vines stand by themselves and are as independent of support as our fruit trees. The vines are, in fact, shaped upon something the same model as our fruit trees, the so-called "goblet form" of the French being our prototype.

\section{THE FIRST YEAR}

During the summer of the year the vines are planted, no pruning or training of any kind is needed in most cases. For this reason it is nearly always unnecessary to stake the vines when they are planted. The only exception to this is when strong-rooted vines are planted in a rich, moist soil in which they will make a very large growth the first year. In this case it is desirable, though not quite necessary, to stake the vines immediately after planting to adopt the method of summer treatment described below for the second year.

In most cases it is best to allow all shoots to remain to feed the vine and to insure a good root growth the first year.

Staking.-In the autumn or winter following planting, the vines should be staked, either before or after pruning, but in any case some time before the buds start in the spring.

The kind of stake used will depend on the variety of vine and on the method of pruning to be ultimately adopted. For ordinary shortpruning, the stake should be of such length that, after being driven into the ground, sufficient will be below the surface to keep it firm and prevent its being loosened by the force of the wind acting on the vine which is tied to it, and sufficient above the surface to extend one or two inches above the height at which it is intended to head the vines. It should be from one and one-quarter to one and one-half inches square, according to the length.

In firm ground, for small-growing vines such as Zinfandel, a stake $1 \frac{1}{4}$ by $1 \frac{1}{4}$ inches and 27 inches long will be sufficient. This will allow 15 inches to be driven into the ground and leave 12 inches above, which is enough for vines to be headed at 10 inches. If the ground is loose or sandy a 30 -inch stake driven 18 inches into the ground will be needed. For strong-growing varieties, such as Carignane or Tokay, especially when planted in rich soil, a stake $1 \frac{1}{2}$ by $1 \frac{1}{2}$ inches and 36 inches long will be necessary, and 15 or

*The detailed instructions and accompanying illustrations in this chapter are taken from the excellent writings of Mr. F. T. Bioletti for the California Experiment Station, and embody the teachings of long experience and wide observation. 
18 inches of this should be left above the ground. This will permit the heading of the vines at 15 inches.

If the vines are to be trellised with one wire, a 36-inch stake driven 18 inches into the ground is the proper length. If two wires are to be used, a 48 -inch stake will be needed, leaving 30 inches above the surface.

If the vines are to be pruned long and the canes tied to the stake, a 5 -foot stake will usually be needed, and this must be stronger, 2 by 2 inches square. This stake should be driven 2 feet into the ground.

These dimensions are all smaller than are usual in California, but are quite sufficient for all practical purposes. The stake should be placed 1 to 2 inches from the vine on the side opposite to the

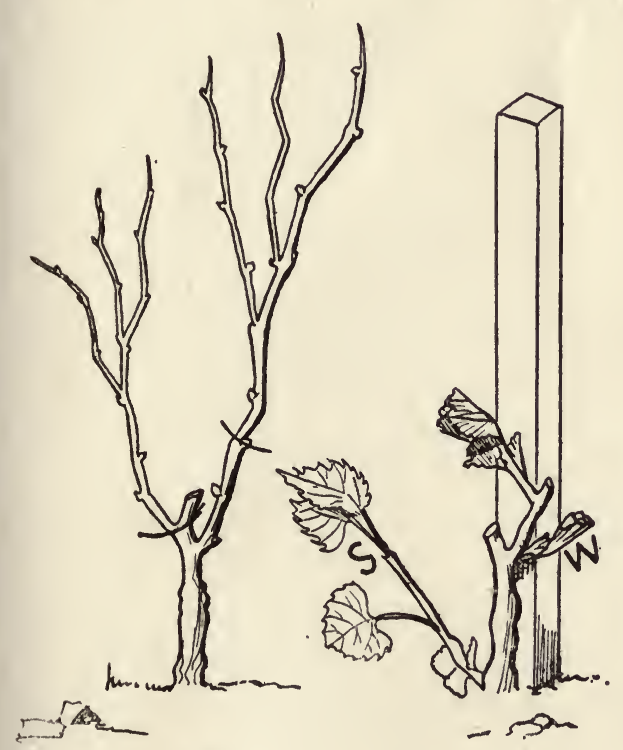

a

$\mathrm{b}$

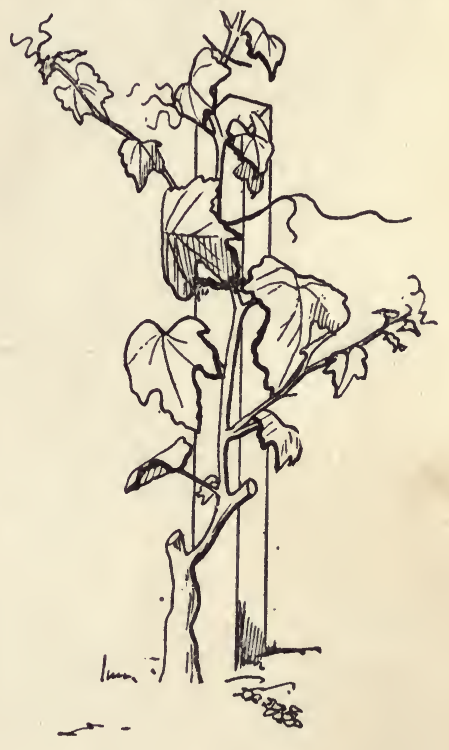

c

Fig. 1. Treatment of an average vine during second season.

a. Winter pruning.

b. Spring pruning-removal of suckers (S) and thinning of shoots (W).

c. Summer treatment-tying to stake and topping.

prevailing heavy winds. The force of the wind will thus keep the vine pressed against the stake and the tying material less liable to break.

First Winter Pruning.-In California, the young vines may be pruned at any time after the leaves have fallen, except in sections very subject to spring frosts, where it is sometimes advisable to defer the pruning until after the top buds of the canes start.

The way the vines are to be pruned will depend altogether on the growth they have made. If the growth has been small the tops are pruned exactly like rooted vines before planting. All the canes are removed entirely, except the strongest, and this is cut back to two buds. (see Fig. 1, a). 
Any vines which have made a strong growth and possess at least one cane of which sufficient length is well ripened may be pruned for tying up. All the canes are removed entirely, except the strongest, and this is cut back to 10,15 , or 18 inches, according to the height at which it is intended to head the vine (see Fig. 2, a). The top cut is made through a bud, just as in making cuttings. This will facilitate tying up and insure the healthy growth of the top bud.

Sometimes, even when the vine has made sufficient growth, the canes are prostrate or crooked and none can be tied up straight to the stake. In this case the vine must be pruned like a weak vine-that is, thinned to one cane and this câne cut back to two buds.

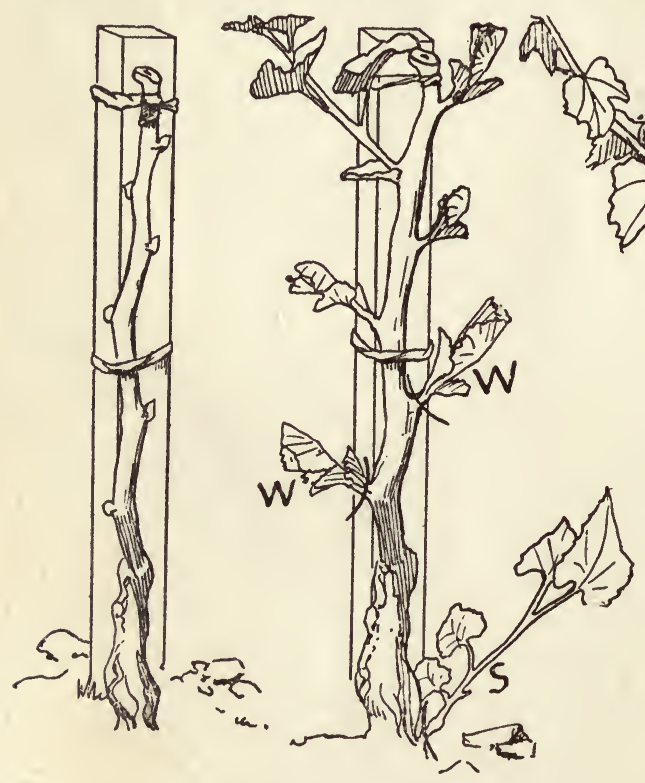

a

$\mathrm{b}$

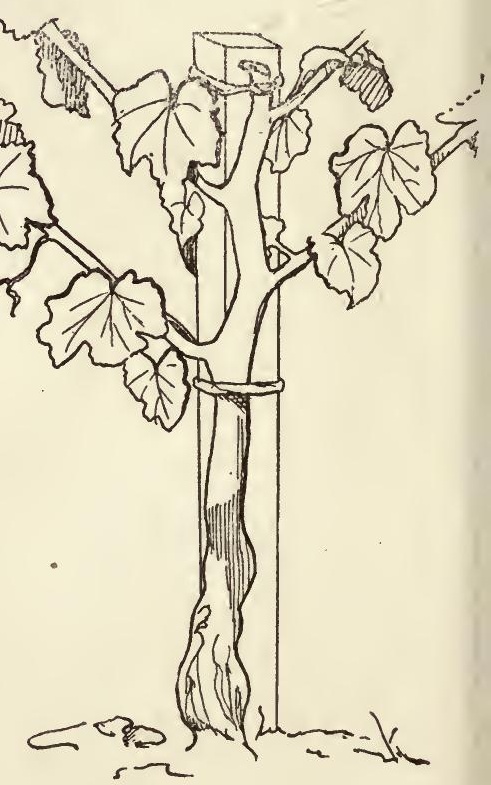

c

Fig. 2. Treatment of an average vine during the third season or of a vigorous vine during the second.

a. Vine pruned to one cane and tied to stake.

b. Removal of sucker (S) and lower shoots (W) in spring.

c. Vine in summer at time of pinching.

In no case should two canes of any length be left, and in all cases where it is impossible to obtain the full length of well-ripened wood for tying up, the cane should be cut back to two buds. It is very bad practice to leave some of the canes of intermediate length, as this causes the vines to head out at various heights and produces an irregularity of shape which can never be remedied and which interferes with regular pruning, cultivation and other vineyard work.

The idea to be kept in mind is to cut back each winter nearly to the ground-that is, to two buds-until a cane is produced with a length of well-ripened wood and good buds equal to the height at which the vine is to be headed. It is very important that this cane should be 
straight, healthy and well-ripened, as it is from it that the trunks of the mature vine develops. All the vines on which a cane has been left should be carefully tied up. Two ties will be needed in most cases. A half hitch should be made around the cane below the swelling left by the bud which has been removed, and the cane tied firmly to the top of the stake. Another tie is made about half-way down the stake. The lower tie need not be very tight, and in any case the tying material should not be passed completely around the cane, except above the top bud, or the vine will be strangled when it commences to grow (see Fig. $2 a$ ). Any kind of string or twine, sufficiently strong to withstand the pressure of the growing vine in a heavy wind, may be used. Binding twine, or a single strand of good bailing rope, is suitable. No. 16 or 17 galvanized wire is preferred by some and is better than string, if care is taken to remove the bottom ties the following year before they strangle the vine. Wire is a little more expensive and takes a little longer to put on than string, but holds the vines better and can be used for several years.

\section{SECOND YEAR}

Summer Pruning.-The treatment during the second and third spring and summer is of great importance to the future welfare of the vine. A little judicious care at this period will avert many troubles in later years. It will be necessary to go over the vineyard four or five times to do the suckering, topping, and tying which are necessary.

The shoots starting from the vines which have been cut back to two buds should be thinned to a single one. This thinning should be done as soon as possible in such a way that it is never necessary to remove a shoot more than 3 or 4 inches long (see Fig. 1, $b$ ). If the thinning is deferred until the shoots are a foot or more long the vine will be weakened by the removal of so much foliage. If the thinning can not be done early, it is better not to do it at all. The object of this thinning is to throw all the force and growth of the vine into the cane which is to form finally the trunk of the vine. If it is done too late not only does the growth not go into this cane, but the vine is weakened so much that this cane does not grow so well as it would have done without thinning.

The first thinning can be done with the first hoeing, and the second with the suckering. The suckering consists of the removal of all shoots which come from below the ground. These also should be removed as early as practicable, both to avoid weakening the vine by the removal of mature leaves and also because a young sucker is much more easily separated from the vine at this time. Every sucker must be cut or broken off at the point where it originates. If a little piece of the sucker is left, several new suckers will start at the same place. The more completely the suckering is done during the first two years, the less trouble in this respect there will be in later years. This is particularly true of grafted vines.

A few weeks after the first thinning, the single shoot which has been left will have grown 10 or 15 inches. At this length it should be 
tied up to the stake (see Fig. 1 c). If this tying is neglected or deferred too long, a heavy wind is very liable to break off the whole shoot. A piece of string tied rather loosely about the middle of the shoot is all that is needed. If the vines are to be headed high. (18 inches) another tie near the top of the stake may be necessary.

For vines which are making only moderate growth this is all the treatment needed during the summer. Strong-growing vines in rich soil, however, should be topped.

Topping.-The object of this is to force the shoot to send out laterals at the right height above the surface of the soil, to be used as spurs during the following year. This topping is an operation which requires a good deal of judgment. If the topping is done too soon, laterals will not start, but a new terminal shoot will be formed. This is not a serious defect, however, but simply necessitates a second topping two or three weeks later. Neither will the laterals start if the topping is done too late, or if they start they will not mature, and the vine is weakened by the removal of foliage without any compensating advantage.

Until experience has shown the proper time for the variety and locality, it is best to top when the shoot has grown to from 8 to 12 inches above the top of the stake, and if necessary top again later.

The shoot should be topped within 1 or 2 inches of the top of the stake, if the stakes have been chosen and driven as advised above (see Fig. 1,c). This will insure the growth of laterals just where they are needed for the next winter pruning.

The vines on which a cane has been left and tied up during the preceding winter must be treated a little differently. The removal of underground shoots or suckers is the same. Instead of thinning out the shoots to a single one, as for the vines just described, all the shoots should be left to grow, except those too near the ground (see Fig. 2, b).

As a rule, all shoots between the ground and the middle of the stakes should be taken off. It is even more important that this should be done early than in the case described above. If the lower shoots are allowed to become large and then removed, not only is the vine weakened by the removal of mature leaves, but the stem of the vine is suddenly exposed to the direct rays of the hot sun and is very liable to injury. This injury does not show by the peeling off of the bark as with fruit trees, but by a general weakening and dwarfing of the vine.

The shoots coming from the upper half of the cane are to form the spurs for the following winter pruning, and can often be left to grow without further treatment.

If the growth is very rapid and succulent, however, it is necessary to pinch them, or the first heavy wind may break them off (see Fig. 2, C.)

Pinching consists in the removal of 1 or 2 inches of growth at the extreme tip of the shoot. This delays the growth in length temporarily and gives the shoot time to strengthen its tissues before its length gives too much leverage to the wind. This pinching usually has to be repeated at least once. 
Pinching may be replaced by topping a few weeks later, but the latter is somewhat weakening to the vine.

In all summer pruning-that is, the removal of green shoots and leaves-of young vines, two things should be kept in mind: First that all summer pruning is weakening; second that the object of summer pruning of young vines is to direct the growth as much as possible into those parts which are to become permanent portions of the mature vine. The weakening effect is almost nil if the shoots or tips are removed when they are very small, but may be very serious if large shoots are removed or heavily topped. When a large shoot covered with leaves is removed it is a total loss to the vine. When a small shoot is removed the food materials which would have gone into that shoot are diverted to the shoots that remain, and the vigor and size of the latter are increased.

\section{THIRD YEAR}

Winter Pruning.-After the leaves have fallen at the end of the third summer every vine should have a well-formed, straight stem with two, three, or more canes growing from the upper part, and the formation of the "head" or crown should commence. Any vines which have not been brought to this condition must be pruned like two or one-yearold vines, as the case may be.

If the work up to this point has been well done, the formation of the head is a simple matter. It consists in leaving two, three, or four spurs, arranged as symmetrically as possible near the top of the vine. The stronger the vine, as evidenced by the number, length, and thickness of the canes, the larger the number of spurs and buds that should be left.

A spur consists of the basal portion of a cane, and normally of two full internodes. This leaves two buds besides the base bud. The number of buds to leave on a spur depends on the strength or thickness of the cane from which the spur is made. A thin, or weak, cane should be cut back to one bud or even to the base bud. A strong cane, on the other hand, should be left with three buds besides the base bud.

The pruning of each vine requires judgment, and it is impossible to give an inflexible rule to follow. The ideal of a perfect vine should be kept in mind and each vine pruned as nearly in accordance with this ideal as circumstances permit. Fig. 3 represents nearly perfect threeyear-old vines consisting of two or three symmetrically placed spurs of two buds each near the top of the stem.

Sometimes it is necessary to leave a spur lower down (see Fig. 3, $b$ ). This spur will be removed the following year after it has produced two or three bunches of grapes. Sometimes a vine may be very vigorous but have only two canes properly placed for making spurs. In this case the spurs should be left longer-three buds and even in extreme cases four buds long.

In stump pruning there is a difference of practice as to low heading according to locality. In the interior regions the vine is now headed almost at the surface of the ground; in the coast regions there is usu- 
ally a stump of one to two feet or more. As with trees so with vines, the practice is to prune to make lower heads than during the early years of California fruit growing.

Long Pruning.-Some varieties grown for market and for raisin making do not thrive if pruned by the short-spur system. Notable among these are the Sultana, Sultanina, (Thompson's Seedless), Emperor and Sabalskanski. There are also a number of wine varieties which must be pruned long. Whatever the variety of vine and whatever the system of pruning to be ultimately adopted, the treatment for the first two and even three years is practically identical and is that which has already been described in detail.

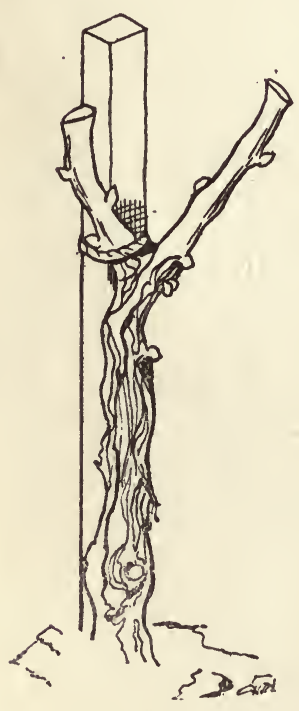

a

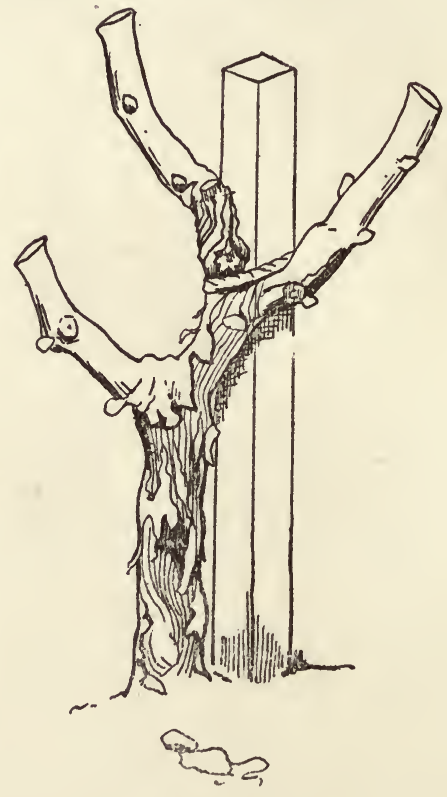

b

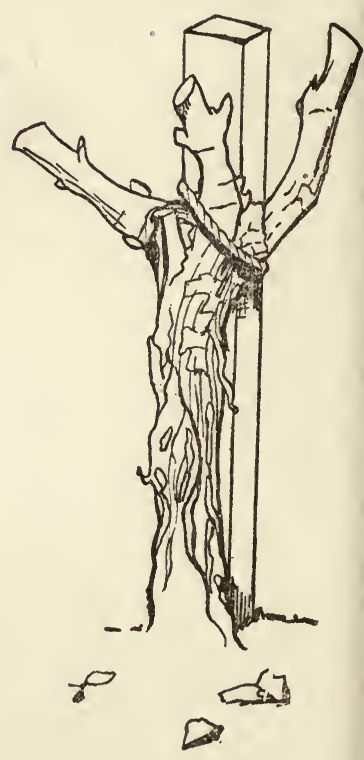

c

Fig. 3. Three-year-old vines after pruning.

a. Average vine with two spurs.

b. Vigorous vines with three spurs, the lowest of which is to be removed the following year.

c. Vigorous vine with three spurs.

Long pruning admits of degrees, but it usually signifies using a five or six instead of a four-foot stake and leaving the selected canes from eighteen inches to three feet longer instead of cutting back to two or three buds, as in short pruning. These long canes are securely tied to the long stakes.

With varieties needing long pruning the first two or three buds next the old wood do not bear fruit, hence the need of leaving buds farther removed from the old wood to secure it. This habit of the vine invites the practice of growing a long cane for fruit and at the same time providing for wood growth for the following year's fruiting by cutting 
another cane from the same spur down to two or three buds. By this practice the wood which has borne the fruit is cut back to a bud each winter and the cane which has grown only wood is pruned long for the fruit of the following summer. A modification of the practice is to prune the canes from some of the spurs long, and from other spurs short, thus making the spurs alternate from wood bearing to fruit bearing from year to year. Unless some method is adopted to promote the growth of strong canes from near the head of the vine, long pruning becomes unsatisfactory. According to the common way with those vines which are known to require longer canes for satisfactory bearing, such canes are selected when the vine is well established and two, three, four, or more canes four or five feet long are tied up vertically to a high stake. This process is repeated the next year and the next, and the result is, with the Sultanina at least, that after the second or third year all the bearing wood is at the top of the stake, and the vine must be pruned short again or suckers and watersprouts left as long canes. Neither way is satisfactory.

Two methods have been successfully used to insure the growth of new fruit wood every year in a position where it can be utilized. The first consists in bending the fruit canes into a circle. This diminishes the tendency of the sap of the vine to go to the end of the fruit canes. The consequence is that more shoots start in the lower parts of the fruit canes. All the shoots on these canes are made weaker and more fruitful by the bending, and at the same time the sap pressure is increased and causes strong shoots to start from the wood-spurs left near the bases of the fruit canes. These shoots are used for fruit canes at the following winter pruning, and new wood spurs are then left for the next year.

The tying and bending of the fruit canes require great care, and repeated suckering and removal of watersprouts are necessary to insure a strong growth of replacing canes on the wood spurs. This method can be used successfully only by skillful hands.

The other method requires some form of trellis. The most practicable trellis is a wire stretched along the rows about $11 / 2$ or 2 feet above the surface of the soil. For very vigorous vines in rich soil a second wire 12 inches above the first is advisable.

The pruning is the same as for the method just described. The fruit canes, however, instead of being bent in a circle and tied to the stake, are placed in a horizontal position and tied to the wire. The horizontal position has the same effect as curving in promoting the starting of more shoots on the fruit canes and the consequent production of more bunches of grapes. At the same time the buds on the wood spurs are forced to start, and not being shaded they tend to grow vigorously. It is best to tie the shoots from the wood spurs in a vertical position to the stake, and they should not be topped. This system of pruning is not only theoretically correct, but is easy to explain to pruners, and can be carried out much more perfectly than the first method with ordinary labor.

Whatever system of winter pruning is adopted with Sultanina, careful summer pruning, suckering, sprouting, and topping are necessary for the best results. This variety has a tendency to send out large 
numbers of suckers from below ground and watersprouts from the old wood. These shoots are usually sterile, grow vigorously, and unless removed in time divert the energies of the vine from the fruit and fruit shoots. Two or three times during the spring the vineyard should be gone over carefully and all sterile shoots which are not needed to balance the vine or to replace weak or missing arms should be removed. This removal of shoots should be done in such a way that no shoot longer than 12 inches is ever removed. If the watersprouts are allowed to grow large their removal weakens the vine. The shoots which are to give fruit canes for the following year should not be topped. The shoots from the horizontal fruit canes on the trellises, however, will set their fruit better and are less likely to be broken by the wind if they are pinched or topped early.

\section{SUMMER PRUNING AND SUCKERING}

Summer pruning or topping of bearing vines is usually practiced. Some follow the pinching process, by which the terminal of the growing cane is nipped off with the thumb and finger when it has grown out about two feet. Others wait longer and then slash off the ends of the canes with a sickle. The tendency is to leave summer pruning until too late and to slash off wood indiscriminately, to the injury of the vine. Summer pruning, if done early enough, and this would be while the growth is still soft at the point of removal, will induce the growth of laterals and will shade and improve the fruit, and at the same time thicken the growth of the main cane and strengthen its connection with the spur. Slashing of canes too late in the season deprives the fruit of the service of enough leaf surface for the elaboration of the sap, often seriously checks the growth of the vine, and in hot regions, induces sunburn. The first summer pruning should be done soon after the bloom, but not during blooming. The second could take place whenever the canes or laterals extend beyond the length necessary to shade the grapes.

Suckering is an important process and usually has to be attended to at least twice in the season. It consists in removing all shoots from old wood which are not provided for at the previous winter pruning. The growth of these suckers takes sap which should go to the other canes. All such shoots should be rubbed or pulled off while they are still soft; if a sucker puts out at a point where it would be desirable to have a spur to balance the head of the vine, it should of course be allowed to grow, to be cut back to two buds the following winter. By such selection of suckers new spurs are secured to replace old and failing ones.

\section{GENERAL NOTES ON PRUNING}

Longer or shorter pruning produces effects not only upon the amount of early ripening of the fruit of certain varieties, but upon quality, as shown in the wines. Such effects have to be discerned by local observation. 
It is a very difficult matter to lay down any rule for pruning a vineyard, so much depends on the age of the vines, the different varieties, and the quality of the soil. A basis on which to build a theory on the subject might be found in and through an understanding of the quantity of grapes that may be expected from a vine, as the secret of pruning is to keep a just medium between the production of grapes to the injury of the vine and its wood and an overproduction of wood to the detriment of the crop. In older vines a proportion should be maintained between the vigor of the vines and the crop desired; each bud may be considered good for two bunches of grapes the ordinary size, and upon this estimate may be obtained. It must be borne in mind that the result of overloading the vine is detrimental to its vigor and health, while the reverse will not injure it, but will lessen the profits for that season, often giving greatly increased returns in after years.

Close attention should be given to the growth of the wood and fruit of the preceding year. If the canes are very large and the bunches of grapes poor and there are many suckers, it indicates that more eyes are necessary. On the contrary, if the canes are small, and the bunches of grapes numerous and straggling, and the ripening not even, it indicates that the number of eyes left should be less.

Pruning may be regulated to produce a good second crop of grapes or to prevent the formation of a second crop. The second crop is often desirable in raisin and table varieties, but undesirable in wine varieties.

Attention should be paid to the tools used in pruning. Let the blades be kept sharp and thin; large shears are very apt to bruise the wood more than small ones.

Pruning is done after the fall of the leaves and before the swelling of the buds, usually in January and February. Early pruning has a tendency to make the vines start growth early, consequently in frosty situations pruning is often deferred till late in the winter-as late as the middle of March in some cases. But it is inconvenient to do a large amount of pruning so late. At any time in the winter the canes can be cut back to 15 or 18 inches and the vineyard cleaned up. When the outer buds on these long spurs start, cut back to the two buds next the old wood and they will start 15 or 20 days later than if the vines had been pruned short earlier in the season. This practice has been followed with marked advantage in some regions liable to late spring frosts. The bleeding which follows late pruning is no loss to the vine, according to observation of late-pruned vines.

The proper treatment of vines injured by spring frosts is clearly the immediate removal, by a sharp downward jerk, of the frosted shoots. Careful experiment has proved that the vines that were not stripped did not do as well as the others, as the dormant buds in many cases did not come out, and those that came out were not healthy and strong, and hardly had any grapes. The damaged shoots that were not removed died gradually, and at the junction with the cane new shoots came out without any grapes at all, while two-thirds of a crop came on the frostbitten vines, which were stripped of the damaged canes; hardly and first crop and only a second crop on the vines which were not attended to. 


\section{DISEASES OF THE VINE}

One of the most prevalent diseases of the vine in California is caused by a fungus which affects leaves, canes, and berries, and is locally known as "mildew." This disease is recognized by grayish white coloring of the affected leaves, which, as the disease progresses, shrivel and dry up; the young cane also blackens and dries, and the berries show whitish patches, which become darker colored and the berries crack open. The usual remedy for the trouble is finely-ground or sublimed sulphur applied several times during the season. Definite reference may be found in the chapter on diseases of trees and vines.

The Bordeaux Mixture and other copper preparations are sometimes useful upon grape-vines, as will be cited in the chapter on plant diseases.

Coulure.-A frequent misfortune of the vine, and for which no remedy is yet known, is coulure, a term signifying the failure of the fruit to set or to remain on the cluster. This occurs in varying degrees from the loss of a few berries to the almost complete clearing of fruit from the stem. It is worse with some varieties than others and in some localities than others. The trouble is believed to arise from various causes.

There is, also, occurring with more or less frequency, a reddening and death of the vine leaves, supposed to be identical with the trouble known to the French as "rougeole." The leaves show light-colored spots at first, which afterward turn red and finally involve the whole leaf or cane, and sometimes the whole vine. It usually occurs in midsummer, and is not necessarily fatal in its effects.

Root Knot.-An evil occurring on the main stem of the vine, generally near the surface of the ground, is an excrescence of woody character commonly called "black knot." There has been much discussion as to the cause of this abnormal growth, without full agreement among observers. Some attribute the knots to injuries to the stump in cultivation, others to outbursts of sap which the short pruning system does not give top growth enough to dispose of, and to various other causes, but the agency is now definitely known to be invasion by bacteria. This is analogous to the "crown knot" of fruit trees which will be mentioned in the chapter on plant diseases.

Anaheim Disease.-There has prevailed for several years in California a mysterious disease of the vine known as the "Anaheim disease," because its evil work first appeared in that vicinity. It destroyed many thousand acres of vines and led to the abandonment of grape growing in some regions in southern California. The fullest statements concerning its performance can be found in Bulletin No. 2, U. S. Department of Agriculture, Division of Vegetable Pathology, by Newton B. Pierce, 1892, and Farmers' Bulletin No. 30, 1895. Fortunately during recent years the trouble has been less aggressive but neither its nature nor satisfactory treatment has been fully demonstrated. The latest available information concerning it and other troubles of the vine can be had by application to the University Experiment Station at Berkeley. 


\section{CHAPTER XXVIII}

\section{GRAPE VARIETIES IN CALIFORNIA}

Large collections of grape varieties have been brought into California during the last forty-five years. They were sought in all grape countries, and from such wide experimental planting a few have survived in popular esteem and are now chiefly grown. Being derived from different countries, they came bearing many names. Some of these have been preserved, some wholly lost, and replaced with local appellations. The result is that our grape nomenclature is full of confusion. Some varieties have been identified by the means of the standard French grape literature; others are apparently unknown to the compilers of that literature. It is, therefore, impossible to-day to determine a number of our most popular table and shipping grapes, as well as some of the wine varieties. The relative importance of the most popular table and raisin grapes is shown in the accompanying tabulation of reports from growers who were asked to name the grapes which they considered best worth planting in 1914:

\section{TABLE AND RAISIN GRAPE VARIETIES APPROVED BY CALIFORNIA GROWERS}

\begin{tabular}{|c|c|c|c|c|}
\hline GRAPES. & $\begin{array}{l}\text { Central } \\
\text { Coast } \\
\text { valleys. }\end{array}$ & $\begin{array}{c}\text { Sacramento } \\
\text { valley and } \\
\text { foothills. }\end{array}$ & $\begin{array}{l}\text { San Joaquin } \\
\text { valley and } \\
\text { foothills }\end{array}$ & $\begin{array}{l}\text { Southern } \\
\text { C'alifornia. }\end{array}$ \\
\hline$\ldots \ldots \ldots \ldots \ldots \ldots \ldots$ & * & ** & ** & $*$ \\
\hline$\ldots \ldots \ldots \ldots \ldots \ldots \ldots \ldots$ & * & ** & * & ** \\
\hline$\ldots \ldots \ldots \ldots \ldots \ldots \ldots$ & * & ** & * & ** \\
\hline 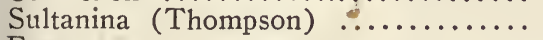 & $*$ & ** & ** & ** \\
\hline 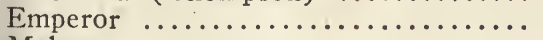 & & ** & ** & \\
\hline 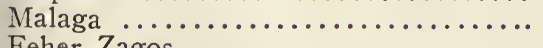 & $*$ & ** & ** & *** \\
\hline 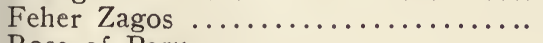 & - $\ldots$ & & * & \\
\hline 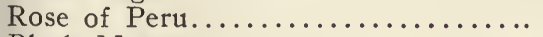 & & $* *$ & * & * \\
\hline Black Morocco .................. & $*$ & $*$ & * & * \\
\hline 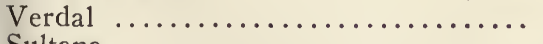 & . $\ldots$ & * & & * \\
\hline Sultana $\quad . \ldots \ldots \ldots \ldots \ldots \ldots \ldots \ldots \ldots \ldots \ldots$ & . ... & * & * & \\
\hline 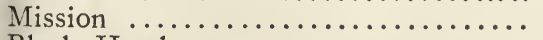 & * $*$ & * & * & * \\
\hline 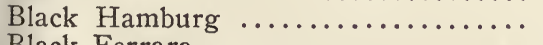 & * & * & * & * \\
\hline 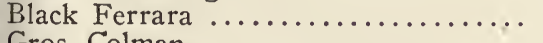 & ... & * & & $\cdots$ \\
\hline Gros Colman $\ldots \ldots \ldots \ldots \ldots \ldots \ldots \ldots$ & - $\quad \cdots$ & $\cdots$ & $*$ & $\cdots$ \\
\hline 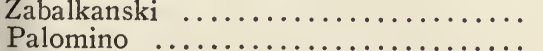 & $\cdots$ & $\ddot{*}$ & * & $\cdots$ \\
\hline Sweetwater $\quad \ldots \ldots \ldots \ldots \ldots \ldots \ldots \ldots \ldots \ldots$ & * & $* *$ & $\dot{*}$ & $\cdots$ \\
\hline 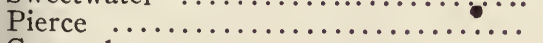 & * & $\ldots$ & $\ldots$ & * \\
\hline Concord $\ldots \ldots \ldots \ldots \ldots \ldots \ldots \ldots$ & * & ... & ... & ** \\
\hline 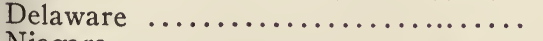 & * & .. & ... & * \\
\hline Niagara,$\ldots \ldots \ldots \ldots \ldots \ldots \ldots \ldots \ldots \ldots \ldots \ldots \ldots \ldots \ldots \ldots$ & * & $\ldots$ & -.. & \\
\hline 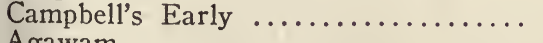 & $\cdots$ & $\cdots$ & ... & * \\
\hline Agawam $\ldots \ldots \ldots \ldots \ldots \ldots \ldots \ldots$ & $\cdots$ & $\cdots$ & $\cdots$ & * \\
\hline
\end{tabular}

* Indicates that the variety is approved in the region designated.

** Most highly commended. 
The last six named are grapes of popular eastern types. They are of exceedingly small commercial importance and are chiefly grown in southern California; not because eastern varieties are better suited to local conditions there than in other parts of the State but because there are more people who enjoy them.

In order to characterize our leading table grapes, descriptions will be quoted from the best available local authorities, as follows:

Early Black July; syns. Madeleine, Madeline Noir, etc.- "Leaves rather small, light green above and beneath; bunches small and compact; berries small, quite round; skin thick, black, covered with a blue bloom; flavor moderately sweet, but not rich nor perfumed. The earliest grape, and chiefly valued for dessert on that account."-Hyatt.

White July; syn. Luglienga.- "Vine strong-growing and sensitive to frost; leaves of medium size, deeply five-lobed, dark green, glabrous on both sides, sharply toothed, the terminal tooth of each lobe very long and acuminate; bunches of medium size, well filled; berries of medium size, oval, at first green, becoming yellow with overripeness, with thin skin, crisp, firm flesh, and agreeable flavor."Bioletti. The Luglienga, which means July grape, is one of the earliest grapes known.

Early Madeleine; syn. Madeleine Angevine.-"Moderate grower, with longjointed, brown wood; leaf medium, deeply lobed, dark green above, tomentose below; young points reddish, woolly, slender; bunch medium, compact, shoulddered; berry medium, oblong, yellowish green, transparent, rather thick skin, sweet and juicy. Vine a shy bearer when frost is prevalent."-Husmann.

Chasselas Dore; syn. Fontainebleau, Sweetwater.-"A rather vigorous grower, with medium or somewhat slender canes of a reddish-brown color; young shoots of garnet color, nearly or quite glabrous; leaves rather below average size, a little longer than wide, glabrous above and nearly so below, except for a few hairs on the main nerves, with well-marked sinuses, the petiolar one often closed, the petiole long, rather slender, and rose-colored; bunches of medium or overmedium size, conico-cylindrical shouldered, more or less compact; berries medium to large, with firm but tender skin, small seeds; of delicate flavor and texture, at first crisp but becoming soft with full maturity. The grapes are of a clear green color, tinged with a beautiful golden bronze where exposed to the sun."-Bioletti.

Chasselas Rose.-Fruit resembling foregoing, except that both bunch and berries are usually smaller, and flavor is more pronounced.

Chasselas Victoria.- "Vine vigorous, very short-jointed and brittle, and bears well with short pruning; wood grayish yellow, thick and strong, leaf light green, deeply lobed and shining; young shoots with numerous laterals; bunch very large and heavy, often weighing five pounds, shouldered, very compact; stem brown, very thick; berry medium, round, pale lilac, purple, with lilac bloom, juicy, vinous, refreshing."-Husmann.

Palomino; syn. Golden Chasselas. - "The vine quite largely grown as 'Golden Chasselas' is undoubtedly identical with the Listan, or Palomino."-Hilgard. "Vine a fair grower; wood close-jointed; leaf medium, oblong, deeply lobed, bright green above, grayish grain and tomentose below, stem short, young points with reddish tint and woolly; bunch large, conical, rather loose and shouldered; berry round, full medium, sometimes flat, pale green with yellowish tinge; thin skin, juicy and sweet, resembling Chasselas."-Husmann.

Black Malvoise.-"Vine a strong grower; wood long-jointed, rather slender, light brown; leaf medium size, oval, rather evenly and deeply five-lobed; basal sinus moderately open, with parallel sides, upper surface smooth, almost glabrous, lower surface lightly tomentose on the veins and veinlets; bunches large, rather loose, branching; berries large, oblong, reddish black with faint bloom; flesh juicy, flavor neutral."-Hilgard. Widely grown as an early table grape.

Mission.-"This variety, grown at the old missions, has never been determined, nor its exact source ascertained. It is regarded by some as a most delicious table grape. It can be found in small areas in every county of the State adapted to the grape. Vine a strong grower; wood short-jointed, dull dark to brown to 
grayish; leaf above medium size, slightly oblong, with large, deeply-cut, compound teeth basal, sinus widely opened, primary sinuses, shallow and narrow, secondary sinuses ill-defined, smooth on both sides, light green below with light, scattered tomentum."-Hilgard. "Bunches slightly shouldered, loose, divided into many small, distinct lateral clusters; berries medium size, round, purple black, heavy bloom; exceedingly sweet, juicy, and delicious ; seeds rather large ; skin thin." - Hyatt.

Muscatel; syn. White Frontignan.- "Vine of medium size, with strong, spreading canes; canes reddish-brown, with short internodes; leaves of medium size, thin, five-lobed; glabrous except for a few hairs on the lower side of the wellmarked ribs; bunches long, cylindrical, regular, compact; berries round, goldenyellow, becoming amber-colored, very sweet and of marked aroma. Ripens a little later than the Chasselas."-Bioletti.

White Muscat of Alexandria.*_"Vine a short, rather straggling and bushy grower, well adapted to short stool pruning, as it forms rather a bush than a vine; wood gray, with dark spots, short-jointed; leaf round, five-lobed, bright green above, lighter green below; young shoots a bright green. The laterals produce a second and even a third crop; bunch long and loose, shouldered; berry oblong, a light yellow when fully matured, transparent, covered with white bloom, fleshy, with thick skin, very sweet and decidedly musky."-Husmann. The leading table grape of California. Rejected for irregular bearing on some mesa lands in Southern California.

Muscatel Gordo Blanco.- "Muscatel Gordo Blanco has a closer bunch and rounder berry than the Muscat. The skin is softer and the pulp is not quite so hard. The berry inclines to be a little darker in color and not nearly so green when it is ripe, and I think not quite as long as the Muscat of Alexandria. If the Muscat would set as well as the Muscatel, the difficulty would be obviated. One very important difference is that when you come to dry them, the Muscat of Alexandria loses the bloom very rapidly. The bloom comes off when you come to dry and pack them. But the Muscatel does not lose its bloom. The Muscat of Alexandria has to be dried a little more than the Muscatel to bring it into a keeping condition under the same condition of ripeness." $-R$. B. Blowers.

"The growth of the Muscatel or Gordo Blanco vine is low and spreading, with no upright branches in the center; clusters heavy, and, when perfect, close and shouldered; berries round and large (the greatest circumference being at the center), a crease often being found at the apex of the berry; color green, or, when fully ripe, amber green or yellow. Distinguished from Muscat of Alexandria by low depressed growth of vine, closer cluster, rounder berries, and by thicker and finer bloom. The Muscatel is the choice raisin grape for the San Joaquin Valley, and for the interior generally."-Dr. Eisen.

Huasco Muscat.-A variety brought from Chile, but after wide trial in California, seems not superior to the other White Muscat varieties previously mentioned. Its dense cluster is not well adapted to raisin making. It is said, however, to be less subject to coulure.

Feher Szagos.-."Vine a strong grower and heavy bearer; branches erect but slender; leaves glossy, entire; bunches medium to small, pointed, and solid; berries greenish amber, medium oval, pointed, with thin skin and few small seeds; flesh not firm, but dries well and makes a good raisin."-Dr. Eisen.

Larga Bloom; syn. Uva Larga.-A variety of Muscat said to be named because of the length of its berries, but held by some growers to be indistinguishable from Muscatel Gordo Blanco. An excellent raisin grape, but now chiefly grown as a table fruit in the Santa Cruz Mountains.

White Malaga.- "Vine a strong grower; wood reddish brown, short-jointed; leaf medium, leathery smooth, deeply lobed, light shining green above; bunch very large, loose shouldered, long; stem long and flexible; berry very large, oval, yellowish green, covered with white bloom; thick skin, fleshy."-Husmann. Grown

*There is much doubt about the White Muscats as grown in California. Some claim inability to distinguish between certain grapes of the Muscat type which are being grown in this State under distinctive names; others pronounce them clearly different varieties. The matter can not be adjusted at present. 
in Southern California in situations where the Muscat does not do well; also elsewhere as a table grape, and to some extent in San Joaquin Valley for raisins.

Sultana; syn. Seedless Sultaona.- "Vine vigorous, upright; leaves large, fivelobed, with rather large sinuses, light colored, and coarsely toothed; bunches large, long-cylindrical, with heavy shoulders or wings, well filled when not cultured, but not compacted; berries small, round, firm and crisp, golden-yellow, and without seeds."-Bioletti. In California the variety is apt to have some seeds It has more acid, and therefore greater piquancy of flavor, that Thompson's Seedless, but the latter has recently far outstripped it in popularity among growers.

Thompson's Seedless; syn. Sultanina.-Named by Sutter County Horticultural Society, after W. Thompson, Sr., of Yuba City, who procured the cutting in 1878 from Ellwanger \& Barry, of Rochester, New York. It was by them described as "a grape from Constantinople, named Lady Decoverly." When it fruited in Sutter county, it was seen to be superior to the Sultana, and has been propagated largely. It was first widely distributed by J. P. Onstott, of Yuba City, and others, and is now to be found in all parts of the State. The variety is described by Dr. Eisen as follows. "Oval; greenish-yellow ; as large as a Sultana; seedless, with a thin skin; good, but not strong flavor, and without that acid which characterizes the Sultana grape and raisins; bunches large or very large; vine an enormous bearer." Mr. Bioletti considers the variety identical with the Sultanina of Asia Minor, and gives this description: "Vine very vigorous and with large trunk and verv long canes; leaves glabrous on both sides, dark yellow-greer above and light below, generally three-lobed, with shallow sinuses, teeth short and obtuse, bunch large conico-cylindrical, well filled, on herbaceous peduncles; berries under medium; ellipsoidol, crisp, of neutral flavor, with moderately thick skin of a fine golden-yellow color."

Flame Tokay; syn. Flame-colored Tokay, Flaming Tokay.-"Vine a strong grower, large in all its proportions, wood, joints, leaves; wood dark brown, straight, with long joints; leaves dark green, with a brownish tinge; lightly lobed; bunch very large, sometimes weighing eight to nine pounds, moderately compact, shouldered; berry very large, oblong, red, covered with fine lilac bloom; fleshy and crackling, firm; ripens late."-Husmann. The leading shipping grape of the State, quality low. Defective in color in some localities.

Black Hamburg.- "Bunches very large, from six to ten inches in length, very broad at the shoulders, tapering to a point gradually; berries very large, round, slightly inclining to oval; skin rather thick, deep purple, very black at maturity; very sugary, juicy, and rich."-Hyatt. A very popular market grape.

Rose of Peru; syn. Black Prince (?).- "Vine a strong grower, with dark brown, short-jointed wood; leaf deep green above, lighter green and tomentose below; bunch very large, shouldered, rather loose; berry round, large, black, with firm and crackling flesh, ripens rather late; a very handsome and productive variety, of good quality, but not adapted for long shipment."-Husmann.

Moscatello Fino; syn. Moscatello Nero, Black Muscat.-"Leaves of medium size, with deep upper and shallow lower sinuses, glabrous above, slightly downy below, and very hairy on the veins, teeth long and sharp; bunches large to very large, long, loose, conico-cylindrical, and winged; berries very large, on long, thin pedicels; skin well colored, thin but tough; flesh soft and juicy, with delicate Muscat aroma. An excellent table grape. It is a heavy bearer, and produces very fine-looking bunches of dark colored grapes. Rather late."-Bioletti.

Purple Damascus; syn. Black Damascus._-"Vine a medium grower; wood light brown striped with darker brown, short-jointed; leaf round, five-lobed, smooth, light green above, tomentose beneath; stem reddish, large, long and woody; bunch large, loose, shouldered; berry very large, oblong, dark blue, covered with lighter bloom, meaty, skin thick, ripens late."-Husmann.

Purple Cornichon; syn. Black Cornichon.- "Vine a heavy grower, with thick, light brown, short-jointed wood; leaves large, longer than wide, deeply fivelobed, dark green above, and lighter and very hairy below, coarsely toothed, and with short, thick petiole; bunches very large, loose, on long pedunckles; berries large, long, more or less curved, darkly colored and spotted, thick-skinned, and 
on long pedicles. Desirable on account of its attractive appearance, curious shape, excellent shipping qualities, and late ripening."-Bioletti.

White Cornichon.-Resembles Purple Cornichon in shape and flavor, but has very thin and tender skin, which makes it better for the table, but poorer for shipping. Leaves not deeply cut; smooth on both sides.

Emperor.- "Vine a strong, vigorous grower; leaves very large, with five shallow lobes, short, obtuse teeth glabrous above, woolly beneath, light green in color; bunches very large, long conical, loose, with large, dull purple, oval, firm berries."-Bioletti. An excellent shipping grape, largely grown by R. B. Blowers, of Woodland, Yolo county, by whom its merits were first announced. Pronounced unsatisfactory because of irregular setting and non-ripening in localities near the coast in Northern California, and generally condemned in Southern California. Seems best adapted to interior situations and is chiefly grown for shipping in the San Joaquin Valley.

Black Ferrara.-A large black grape; large bunches; berries cling well to the stem, thick-skinned, flavor superior. An excellent local market variety and longdistance shipper.

Gros Colman; syn. Dodrelabi.-"Vine strong-growing, with dark brownish wood; leaves very large, round, thick, very slightly lobed, shortly and bluntly toothed, glabrous above, close-woolly below; bunches large, short, well filled, but not compact; berries very large, round, dark blue, with thick but tender skin. Remarkable as having the largest berries of any round berry variety known, and is probably the handsomest black table grape grown. The grapes have good keeping qualities, except that they are liable to crack."-Bioletti.

Black Morocco.- "Vine a strong grower, with thin, spreading canes, leaves, under medium size, very deeply five-lobed, even when very young, the younger leaves truncate at base, giving them a semi-circular outline, with long, sharp teeth alternating with very small ones, glabrous on both sides, bunches very large, short, shouldered, and compact; berries very large, round, often angular from compression, fleshy, of neutral flavor, dull purple color or colorless in the center of the bunch. Remarkable for the number of second-crop bunches which it produces on the laterals. Late in ripening and of very fine appearance; a fairly good shipping grape, but difficult to pack on account of the size and rigidity of the bunches. The grapes are of an agreeable crispness, but lacking in flavor." -Bioletti.. Vine quite subject to root knot.

Verdal; Aspiran Blanc.- "Vine of medium vigor and rather hardy; canes somewhat slender and half erect; leaves of average size, glabrous on both surfaces, except below near the axis of the main nerves, sinuses well marked and generally closed, giving the leaf the appearance of having five holes; teeth long, unequal, and somewhat acuminate; bunches large to very large, irregular long-conical, without any or with small shoulders, well-filled to compact; berries yellowish-green, large to very large, crisp, with thick but tender skin, agreeable, but without marked flavor."-Bioletti. Largely grown as a late table grape; in good condition; in some regions as late as November.

Almeria.-"Vine vigorous; leaves of medium size, round and slightly or not at all lobed, quite glabrous on both sides, teeth obtuse and alternately large and small; bunches large, loose or compact, irregular conical; berries from small to large, cylindrical, fattened on the ends, very hard and tasteless."-Bioletti. The grape cultivated at the University Experiment Stations under this name is one of the several varieties which are shipped in such large quantities from Malaga and Almeria packed in cork-dust. The grapes ripen late and attain about 20 per cent of sugar. They have remarkable keeping aualities. Vine needs long pruning, and is only adapted to hot, interior situations.

There are many other vinifera varieties which are grown to a limited extent either for raisins or for table use. Among these are the Canon Hall Muscat, the White Tokay, White Champion, Cinsaut, Zabalskanski, etc., for table use; the White and Black Corinth, for drying, of which the white is much more productive than the black, but the product sells for less than our other seedless raisins and therefore is 
not largely undertaken. With grapes, as with other market fruits, the planter usually confines his attention to a very few popular kinds. The relative standing of grape varieties in the raisin industry is shown by the California Board of Viticultural Commissioners' statement of the raisin product of 1913 :

Pounds of raisins.

Muscats ............................... 96,588,798

Thompson (Sultanina) . ................... 18,290,922

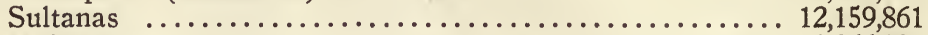

Malagas ................................ 1,266,983

Faber Szagos ................................. 529,707

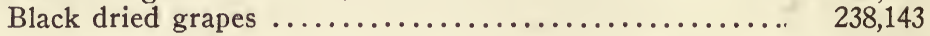

Total...............................129,074,414

\section{EASTERN GRAPES}

Though many of the improved varieties of the grape species indigenous east of the Rocky Mountains, and of the hybrids between these species and the vinifera, have been introduced in California, their growth for table fruit is almost of insignificant proportions, and does not constitute even a respectable fraction of one per cent of our grape area. The popular taste decidedly prefers the vinifera varieties. There is, however, a variety believed to be of local origin, which is worthy of mention as follows:

Isabella Regia.-"A remarkable, giant-leaved, and very prolific sport of the Isabella, originating by bud-variation with Mr. J. P. Pierce, of Santa Clara. The berries, like the leaves, are of extraordinary size, and when ripe the fruit is exceedingly sweet and strongly aromatic. It is, therefore, acceptable as a showy, perfumed table grape, much liked by some, but readily surfeiting those who are accustomed to the vinifera grapes.. The berries are too soft for shipment to any distance, but all things considered, keep fairly."-Hilgard. Grown as the "California Concord" in the Los Angeles region.

\section{WINE GRAPES GROWN IN CALIFORNIA}

Progress is being continually made in the propagation of varieties yielding wine, and in the manufacture thereof. The hosts of considerations involved in this effort are beyond the scope of this work and largely beyond the writer's knowledge. The grapes which are chiefly commended by growers for the kinds of wine to which they are adapted are as follows: Zinfandel, Alicante, Bouschet, Petite Sirah, Colombar, Grenache, Valdepenas, Palomino, West's Prolific, Semillon, Carignane, Green Hungarian, Burger, Mondeuse, Riesling, Cabernet, Chardonay, Cabernet, Mataro, Sultanina, Blaue Elbe, etc. 


\section{PART FIVE: SEMI-TROPICAL FRUITS}

\section{CHAPTER XXIX}

\section{DATE CULTURE IN OUR DESERT AREAS}

Ever since the arid, semi-tropical regions of the United States became known through the narratives of explorers, the date palm (Phoenix dactylifera) has been projected as a plant likely to demonstrate commercial value in American like that which it has held for centuries in the arid regions of Asia and Africa. This idea was also cherished even at an earlier date by the Spanish missionaries who brought the palm to California, as will be described presently. During the last decade the problem of introducing and establishing a date-growing industry has been taken up more seriously and systematically than ever before and must now be looked upon as approaching successful solution. As, however, the undertaking is still in an experimental stage and appeals to relatively few people as a line of investment, no attempt will be made to discuss the date in detail. Instead, citation will be made of sources whence the reader who desires them can easily secure information of date-growing methods in the old countries, discussion of their suitability to our conditions and suggestions of ways to enter upon dategrowing in California.*

The date palm was brought to California by the padres, and the oldest date trees in the State are the survivors of their early plantings. Such trees are found at the San Diego Mission. They are conjectured to be more than a century old, and they have survived drought and neglect, making unsuccessful effort at fruiting, for, according to common report, the fruit does not ripen, but whether owing to the unfavorable conditions indicated, or to lack of fertilization of the bloom, is not known. There are trees at Ventura, on the site of the garden of the old mission at San Buena Ventura, about forty feet in height and ten feet in circumference at the base, with long, graceful fern-like leaves, which put forth about thirty feet from the ground.

The ill success of these old trees in the direction of fruit bearing probably long prevented further attention to the date as a profitable growth. Still there were date palms grown from seed of the commercial date planted here and there for ornament or out of curiosity, and in due course of time the fruit appeared. The first public exhibition of California dates known to the writer was made at the Mechanic's

*The Date Palm and its Utilization in the Southwestern States, by Walter T. Swingle, Bulletin 53, Bureau of Plant Industry, U. S. Dpt. of Agr., Washington, 1904.

Date Varieties and Date Culture in Tunis, by T. H. Kearney, Bulletin 92, Ibid, 1906.

Date Growing in Southern California, by S. C. Masin. Report of Riverside Fruit Growers' Convention, State Horticultural Commissioner, Sacramento, 1908.

Date Growing in the Old and New Worlds, by Paul B. Popenoe, Altadena, California, 1913. A fine, illustrated treatise.

Also publications of Arizona Experiment Station, Tucson, and California Experiment Station, Berkeley. 
Institute Fair, in San Francisco, in September, 1877. The fruit was grown on the south bank of Putah Creek, the northern boundary of Solano County, the situation being slightly above the level of the plain of the Sacramento Valley, which lies east of it. The plants were grown by the late J. R. Wolfskill, from seed of commercial dates purchased in San Francisco, and planted in 1858 or 1859 . The seed germinated readily, and the young plants were set out in a row about one hundred feet south of Putah Creek, on a rich, fine, sandy loam lying about twenty-five feet above the bed of the creek. The plants received good cultivation but no irrigation. This treatment was combined after the property passed into the hands of the late S. C. Wolfskill, the plants being allowed to remain in the row as originally planted, and they have attained great size, considering their crowded condition.

Another bearing date palm stands about a mile eastward of the situation just described, near the residence of the late J. R. Wolfskill. It was grown from seed of the date of commerce, which was planted in 1863, and the tree bore its first fruit in 1880. Near it stands a taller date palm, which bears staminate bloom. This latter tree was originally one of the row previously described, and was successfully moved to its present situation after attaining considerable size.

The fruit of the two bearing palms differs notably in appearance. That of the first mentioned tree is of bright yellow color and angular outline; that of the second tree is wine red, with smooth surface.

During recent years the date has fruited at many places in California and Arizona. There is little doubt that it will succeed in any of the interior regions which have a sufficiently high summer temperature, and even the so-called Colorado Desert may be dotted with groves of date palms as portions of it now are with groves of the majestic fan palm of California.

Soils and Waters for the Date--Until recently the date palm has only been planted on good orchard land, but, according to experience.in date-growing countries, the tree does not require rich soil, but on the contrary, will thrive in a soil poor in humus-too poor and too purely mineral for any other fruit tree; and it produces the finest and best flavored dates, nourished by water too alkaline for man and beast to drink. These observations warrant the trials of the tree which are now being made in situations not adapted to other fruits.

\section{PROPAGATION OF THE DATE}

The date palm grows readily from the seeds of the dried date of commerce, and, as has been intimated, the trees now fruiting in this State have been obtained in this way. By the use of seed one gets, however, only seedlings, and the chance of thus securing a really fine variety is probably not greater than with other fruit tree seedlings. In date-growing countries the best varieties are propagated by rooting the off-sets, sprouts, or suckers which appear at the base of the old palms, To secure the best foreign varieties such plants must be imported. The first successful enterprise of this kind was accomplished in the summer 


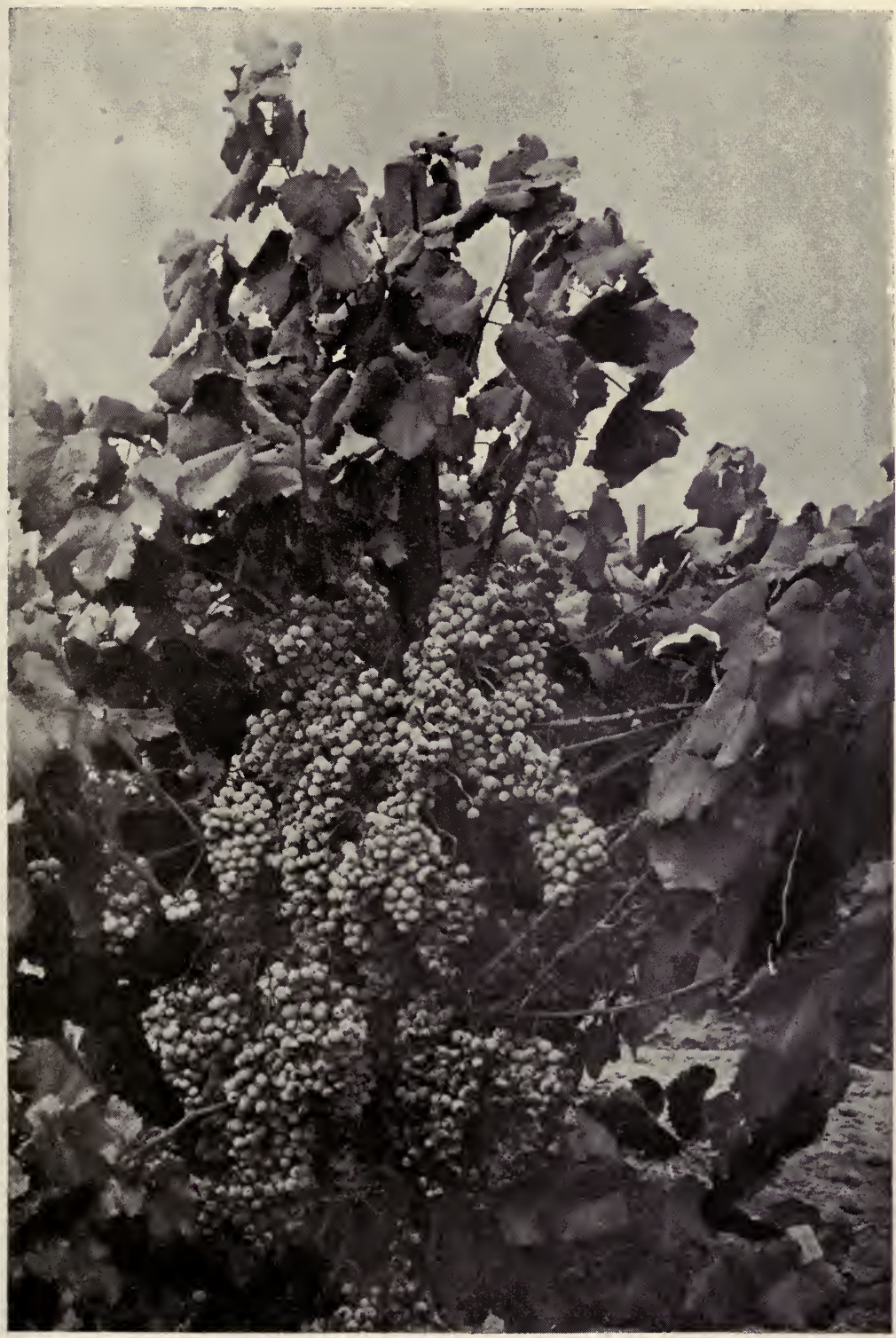

Plate XIII.-Profuse bearing of vine by long pruning. (See page 308.) 

of 1890 by the United States Department of Agriculture, under the direction of H. E. Van Deman, then chief of the Division of Pomology. The plants were divided between New Mexico, Arizona and California. The plants for California were sent to the Department of Agriculture of the University of California, and were planted at the experiment stations at Tulare and at Pomona, some of them being subsequently transplanted to the United States Date Garden at Mecca in the Colorado desert region. Upon fruiting a number of these plants, they seemed to be only seedlings and not the best foreign varieties, as represented.

A full account of this effort and its outcome is given in Bulletin 29, of the Arizona Experiment Station.

The United States Department of Agriculture undertook arrangements in 1899 for new importations, which were successfully made, but all that was anticipated in securing valuable varieties was not attained. Director R. H. Forbes, of the Arizona Experiment Station, writes in Timely Hints No. 72 (April, 1908):

"Several "of the Old World varieties which have thus far fruited have failed to make good for various reasons. Some have required a longer growing season to mature; others have soured in damp or cool weather, and still others do not appeal to the American consumer. A few varieties have proved acceptable in all respects, and to such as these we must look for future commercial developments."

Of the foreign varieties thus introduced the following have been produced in small commercial quantities: Rhars, Tedalla, Birket el Haggi and Deglet Noor.

During recent years large importations for commercial planting in southeastern California have been made by Mr. Popenoe, after searching foreign investigations.

Growing Palms from Seed.-Seeds taken from the dried dates of commerce germinate readily; in fact, seedlings frequently appear in the gutters of unpaved streets where the seeds have been thrown during the rainy season. Director Forbes, of the Arizona Station, says that seeds will come up more promptly if first stratified. This may be done by taking a gasoline can or deep box and placing three inches of sand in the bottom after making a number of holes in it for drainage. The seeds are placed upon this layer and the can or box filled with sand, the whole then being put in a sheltered place and kept moist from three to six weeks, when the seeds will be soft and ready for prompt growth when planted. The seedlings may be started in nursery rows for transplanting after one to three years, or if frequent irrigations may be relied on, in the field where the trees are to remain.

Dr. W. T. Swingle, whose work on date growing has already been cited, gives the following suggestions on the growing of seedlings and their subsequent handling to determine sex and to select bearing palms of desirable type:

The seed should be planted rather thickly in well-drained beds of fertile soil, free from alkali. These beds should be watered frequently, as the young date seedlings need an abundance of moisture. If properly cared for, the seedlings will reach a height of from 12 to 18 inches the first year and can be transplanted into the permanent orchard the second year. 
These seedlings should be set out in rows about 30 feet apart and placed 5 or 6 feet apart in the row. Ordinarily field crops can be grown between the rows until long after the palms come into bearing. After three or four years, when the young palms begin to flower, the male trees can be dug up and destroyed, thus thinning out about half of the trees Then when the female trees come into fruit, those which yield decidedly inferior fruit can also be removed, so that finally about one-fourth of the original number of seedlings will be left standing. The spaces between the seedlings will be irregular and offshoots can be taken from the best sorts and planted where the largest gaps occur. By preventing offshoots from growing on the poorer sorts they will yield more fruit and finally can be destroyed and replaced by offshoots from some of the better sorts. In this way, by degrees, the orchard can be improved without expense for offshoots aside from the labor of planting them.

Rooting Suckers.-Suckers taken off in warm weather and watered freely usually take root readily. Care should be taken not to let the plants dry. Director Forbes gives these points: Suckers should not be taken from the parent tree until they have attained a diameter of 5 to 6 inches and a weight of 15 to 20 pounds. Suckers should be removed by cutting in and down along the line of cleavage between them and the main trunk, with a strong chisel or a flat-pointed bar. If possible the cut should be carried down so as to bring away at least one or two sound roots. The leaves should be closely pruned and for shipment the cut bases had better be protected against drying out by layer of wet moss or similar material. In planting, the sucker should be set in previously irrigated and well settled soil to the depth of its greatest diameter, taking care that the center of the palm is not below the irrigating water level.

For convenience in irrigating, a shallow basin of earth should be made about the sucker, in which, to lessen evaporation and the rise of alkali, a mulch of fine barnyard litter three or four inches deep should be spread. The soil about the newly transplanted suckers should be kept constantly wet by frequent irrigations. Suckers should be cut and transplanted April to August, inclusive, but not during or approaching cool weather.

Bearing Age of the Date.-There is much difference in the ages at which the seedlings have come in fruit in the hands of different growers. Fruit has been reported on seedlings six years old and even on plants four years from the seed. Such early maturity must not, however, be generally expected.

Blooming of the Date.-The date palm is dioecious, and, its staminate (male) and pistillate (female) blooms appearing on different trees, it requires the association of the two for perfect fruiting. Growing plants from seed, as already stated, leaves the grower in doubt as to the sex of his plants until they bloom. Usually one obtains a large preponderance of male plants. In propagating from suckers the new tree is of the same sex as the parent. It is advised to have about one male to twenty female trees. The pollen can be transported long distances and maintains its vitality for a long time.

Artificial fertilization of the bloom of the bearing palm has been found of advantage in this State and was probably first practiced by J. R. Wolfskill. Though the staminate tree was but a few feet away from the pistillate, the male bloom was broken in pieces and hung to 
the leaves of the female tree near to the pistillate flowers. It was found that the parts of the date cluster which are nearest to the suspended male blooms have more perfect fruit than the more distant parts. Other California date growers have had similar experience.

In Winters the bearing date palms bloom in April and May, and the fruit ripens in November.

Ripening the Date--Dr. A. E. Vinson reports that the Arizona Experiment Station has demonstrated that by pasteurization of the ripe fruit, it is freed of the obnoxious insects that naturally infest the date. The heat drives the small beetles from their shelter beneath the skins and they drop to the bottom of the heater where they die. In pasteurizing, the temperature is raised sufficiently to destroy all insect eggs and at the same time to improve the keeping quality of the fresh fruit by checking fermentation. The date, heated after it has been ripened at natural temperatures, becomes more palatable than the raw material and does not cloy the appetite so quickly. These improvements alone have greatly extended the possibilities of marketing the fresh fruit and of its becoming a staple among the people of this country.

Beauty of the Date Palm.-The date palm in fruit is a beautiful sight. The glauceous green pinnate leaves arch outward. Between two of these emerge the bright orange-yellow polished fruit stalks, which divide into a spray of slender bright yellow stems a foot or so in length; and thickly set upon these in clusters are the various colored fruits covered with a rich bloom. It is a sight not easily forgotten by a lover of nature, and especially by one reared in a northern zone, the characteristic vegetation of which is so different. 


\section{CHAPTER XXX}

\section{THE FIG}

The fig is, perhaps, the grandest fruit tree of California. Its majestic size and its symmetry make it a crowning feature of the landscape, and its dense foliage renders the wide space embowered by it a harbor of refuge from mid-summer heat, both for idlers and for the industrious. On adjacent farms in Pleasant's Valley, Solano County, there are large fig groves; one serves as a shelter for the packers of fruit from the contiguous orchard, and the other incloses and shades a croquet ground. Measurements of large trees are abundant, for old trees are numerous in the interior of the State, both in the valley and on the slopes of the Sierra foothills. At Knight's Ferry, in Stanislaus County, there is a fig tree sixty feet in height, with branches of such length as to shade a circle seventy feet in diameter. The trunk at the base is eleven feet around, and nine feet at a distance of three feet from the ground. A little higher the trunk divides into seven or eight large branches, each of which is nearly five feet in circumference. At thirty feet from the ground the limbs are seven and eight inches through. The largest grove is in the neighborhood of Knight's Ferry, and consists of fifteen massive black fig trees, which, though set sixty feet apart, mingle their branches overhead and form a network through which, in the summer, hardly a beam of light can pass.

Such groves are frequently seen in the older settled parts of the State. Perhaps the most interesting single fig tree is that on Rancho Chico, quite near the residence of General Bidwell. It was planted in 1856 , and has attained a marvelous growth. One foot above the ground the trunk measures eleven feet in circumference; the wide-spreading branches have been trained toward the ground and, taking root there, banyan-like, they now form a wonderful enclosure over one hundred and fifty feet in diameter, the tree is loaded every year.

The crop on these large trees is proportionate to their size and, entering their area in the morning during the ripening season, one can scarcely step without crushing figs, though the fruit may be gathered up each day and placed in the sun for drying.

\section{REGIONS SUITED FOR THE FIG}

Though there are still many fine points to be determined as to what situations and conditions favor the production of the very finest figs, and there are indications that there is possibly much difference, it may be truly said that a very small part of the State is really unsuited to its growth. If one shuns the immediate coast of the upper part of the State, where the summer temperature is too low for successful ripening, and keeps below the altitude of the mountains where winter killing of the tree is possible, he can grow figs almost anywhere. 
Selections of varieties adapted to particular situations has much to do with the success of the fig, as with other fruits, and, therefore, a broad statement of adaptability must be received with such an understanding. The intrusion of the coast influences borne eastward by the winds of summer, as described in Chapter I, gives a night temperature too low for ripening of some varieties, which turn sour upon the trees. Present indications are that the finest dried figs, having the thinnest skin and the nearest approach generally to the fig of Smyrna, the commercial standard for dried figs, will be produced in the drier portions of the valleys and foothills. Even in Southern California fig-souring is quite prevalent, and selection of locations must be circumspectly made. More time is requisite for the final demonstration of these matters, although years have already been devoted to the problem.

\section{SOILS FOR THE FIG}

As it must be left with the future to determine the mooted point as to the influence of special situations upon the bearing of the fig, and the more minute characteristics of the fruit, so more experience is needed to deconstrate the comparative effects of different soils. It might seem, from the fact of the age of our trees in different parts of the State, that time enough had elapsed to determine these points, but it must be remembered that all our oldest trees are of the very hardy variety found at the missions, and conclusions drawn from them as to all varieties are unsafe.

The fig will thrive in any soil that one would think of selecting for any of our common orchard trees, and, in fact, the fig succeeds on a wider range of soils than any one of them. One is safe in planting figs for family use, or for marketing, wherever the summer temperature is high enough to ripen the fruit well, and the winter temperature high enough to preserve the life of the tree. This applies merely to the successful growth of the fig; to secure ripening at a time when the fruit can be profitably sold for table use, is another question.

The selection of soils especially suitable to the production of the best figs for drying involves more considerations than rule in the growth of table fruit. For drying, the fig should attain a good size, but should not contain excess of moisture. In some parts of the State the first crop of figs in the season has been found unfit for drying. The second, and, in some localities, the third crop, appearing later in the season, when the moisture supply of the soil is reduced, dry well. This condition of the first crop is, however, affected by local conditions, for there are places in the Sierra foothills where the soil moisture has to be replenished early in the season by irrigation to prevent even the first crop from falling prematurely, and subsequent irrigation brings to perfection the second and third crops. The fig tree needs plenty of moisture in the soil, but not too much. As with other fruits, if the soil does not retain the needed amount naturally, it must be supplied by irrigation wisely administered. 


\section{PROPAGATION OF THE FIG}

The fig grows very rapidly from cuttings, and this is the chief method of propagation. Cuttings should be made while the tree is fully dormant, in the winter, of well-matured wood of the previous season's growth, giving preference to the stocky, short-jointed shoots, and making the cuttings about six to eight inches in length. The cut at the lower end should be made at the joint, or where solid wood is found. The planting; and care of the cutting is essentially the same as of vine cuttings, already described. If well made and cared for, a very satisfactory growth is made the first season, and the trees are ready for planting out in permanent place the following season.

Single Bud Cuttings.-If one desires to multiply a new variety very rapidly single eye cuttings will make plants. This is, also, analogous to single-eye grape cuttings, as already described.

Budding the Fig.- The foregoing means enable one to propagate a fig so rapidly that recourse is not had to budding, as in propagating other trees; still, budding is feasible, either on small plants or on young shoots of old trees which it is desired to bud over.

The fig may be budded by the common shield method, as used for ordinary fruit trees, as described in Chapter IX, but owing to the tendency of the fig bark to shrink in drying, the bud should be closely bound in with a narrow waxed band, to exclude the air. As the bark is thick, it is often desirable to cut out a little of the edges closest to the bud when in place.

Another method of budding the fig is by annular or "ring budding," a method also relied upon with the walnut and chestnut. Annular budding is done in the fall. A circular ring of bark is taken off from the stock by the aid of a budding knife, by running two circular cuts around the stock, and a longitudinal one between the two circular cuts; the ring of bark taken off must be at least one inch wide, and from that up to two inches. A like ring of bark is taken off in the same manner from the scion of the variety to be budded in, and from a branch of the year, or preceding one, well in sap, and having about the same diameter as the stock. The ring should have on its one or two buds. It must fit exactly the space prepared on the stock, and more particularly at the lower circular cut, so that both barks will exactly unite at that point. When the ring is too long, a little bit of it might be cut off with a very sharp knife till it fits well; if the ring is too large for the stock, a longitudinal strip would be cut out, and if too narrow, such a strip, if with a bud on so much the better, will have to be used to fill up the empty space. One must be vary careful while drawing the knife around the stock not to go too deep into the wood to injure the cambium layer, or to weaken the stock. Tie a bandage pretty firmly over the whole. After two or three weeks the bandage has to be taken off, and, in the ensuing spring, the top of the stock or limb is cut down three inches above the budding.

Another way of working such trees is by "whistle budding," which is done in the spring, when the sap is well up. The stock and the scion must be both of the same size and well in sap. The top of the stock 
is cut down to several inches from the ground; a circular ring of bark is then taken off, and a corresponding ring from the scion, but without a longitudinal cut, is put in its place. In inserting it care should be taken that the top of the stock, which is to receive the ring from the scion, be very smooth, and the latter is then easily pushed down around it and bandaged. In the case of the fig, it is especially desirable to use the latter method when the sap is up, because if the top of the stock is not removed, the exudation from above sours around the bud and prevents the union of stock and bud.

To prepare an old tree for budding over, the limbs may be cut back in February to within two to six feet of the trunk, covering the ends with paint or grafting wax. Allow two shoots to start near the end of each of these amputated limbs, and rub off all other shoots. But the shoots when they attain the thickness of one's finger, taking green buds from the growth it is desired to introduce, or let them grow and bud in the fall, whichever is most convenient; or bud in the growing shoot, and rebud in the fall where buds have failed.

Grafting the Fig.-The fig can be grafted by the cleft-graft method, as described in Chapter IX, but the cleft should be made to one side of the stub and not through the central pith. Especial care must be taken in excluding the air. Fill the cleft between the scions with warm wax, which will run in and fill the cavity. Then bind the stock with wax bands, taking the greatest care to cover the exposed wood surface, the cut end of the bark (which in the fig is very prone to shrink and draw back), and as far down the stock as the bark has been split.

Cut the shield from a limb of about $1 / 2$ inch in diameter, length of shield about $11 / 2$ inches, its thickness from $1 / 8$ to $1 / 4$ inch, and its bud near the middle of the shield. Do not remove the wocd from behind the bud. Make a cut in the stock, through the bark and into the wood, its length and width a little greater than those of the shield. Insert the shield into the cut, so that the inner bark of the top of the shield and cut will coincide, so that one side of the shield and cut-and both sides, if practicable-will coincide. Place the flap of the cut over the shield (removing a part of the flap so the bud will not be covered), and fasten flap, shield and stock together very firmly with twine, and protect them with paper tied around them. They may be grafted in that mode, whenever dormant buds are found, for the shields. Twenty-four shields were inserted at several times, during one spring, and there was only one failure.

A method of bark grafting applied to the fig by George C. Roeding of Fresno and approved by him after several years of successful experience, affords an excellent way of grafting over large trees. It does away with splitting the stock and therefore hastens the barking-over of an amputation. The branches to be grafted are cut off within 18 to 24 inches from the point of divergence from the main body of the tree, allowing at least two branches to remain, one of which should be on the southwest, if possible, so that the grafts will be protected from the afternoon sun.

After having sawed off the branches, the stumps neatly smoothed over with a sharp knife, so as to leave a clean, smooth surface, particularly along the edge, two, four or six scions should be placed on tach stock, the number, of course, being regulated by the size of the stump. Cut out a V-shaped piece of bark. The distance from the top of the stock to the point of the V should be about $11 / 4$ inches. 
Another method is to make slightly outward and downward cuts into the stub with a sharp knife, so as not to cause a split, but rather deep, clean cuts, into which the wedge-shaped scions are firmly pushed and a cord wound around the stub to hold all strongly in place before waxing thoroughly. This form of grafting will be shown in the chapter on the walnut, for it is very successfully used on that tree also.

The form of side-graft with a saw cut as described in the chapter on the peach is also available. A form of bud-graft, that is, budding with a large shield into old bark, is also successful. Judge Rhodes of San Jose describes his method, both with the olive and the fig, in this way:

Select a scion of the proper size, never be smaller than an ordinary lead pencil. As a rule scions from two-year-old wood, with very little pith and with a diameter of about $1 / 2$ inch, will give the best results. The scions should have a sloping cut at the lower end, with the bevel all on one side and not like a wedge. The bevel should be as long or a little longer than the $\mathrm{V}$-shaped opening in the stock and should fit snugly into this opening, so that the bark on both edges of the scion touches the bark of the stock. After the scions are placed, wrap tightly with five or six-ply cotton twine, and cover the wounds as well as the stub with liquid grafting wax. Wax the top of the scion to prevent drying out. If waxed cloth is used it must be removed before the warm weather sets in or the bark will be smothered and will die. After the scions have become well united, which takes from two to three months, the strings should be cut. This method of grafting can not be made successfully until the sap begins to flow, say from the latter part of February to the first of April. The scions should never be more than four inches long.

Seedling Figs.-Figs are readily grown from the imported fig of commerce. Dr. Gustav Eisen of San Francisco, our leading writer on the fig, gives the following explicit directions for growing the fig from seed:

Cut open imported Smyrna figs; wash out the seeds in warm water, those that float are empty and worthless; those that sink are generally fertile. Sow these in shallow boxes of sand and loam mixed, and place in a frame under glass. In three weeks they will be up and must be very sparingly watered. Set out next season in nursery row. In three years from the seed such plants will be found to bear.

The tendency of the plants grown from Smyrna figs is to revert to the wild type, and there is a small chance of securing good varieties.

\section{PLANTING AND PRUNING THE FIG}

The chief point to observe in planting the fig trees is to get them far enough apart, because of the great spread of branches which they attain. Of course they may be planted twenty feet apart if the owner intends to remove alternate rows, but to plant at forty feet, or even farther apart, with other fruit trees or vines between, on the plan of alternate or double squares, described in Chapter $\mathrm{X}$, would be the best 


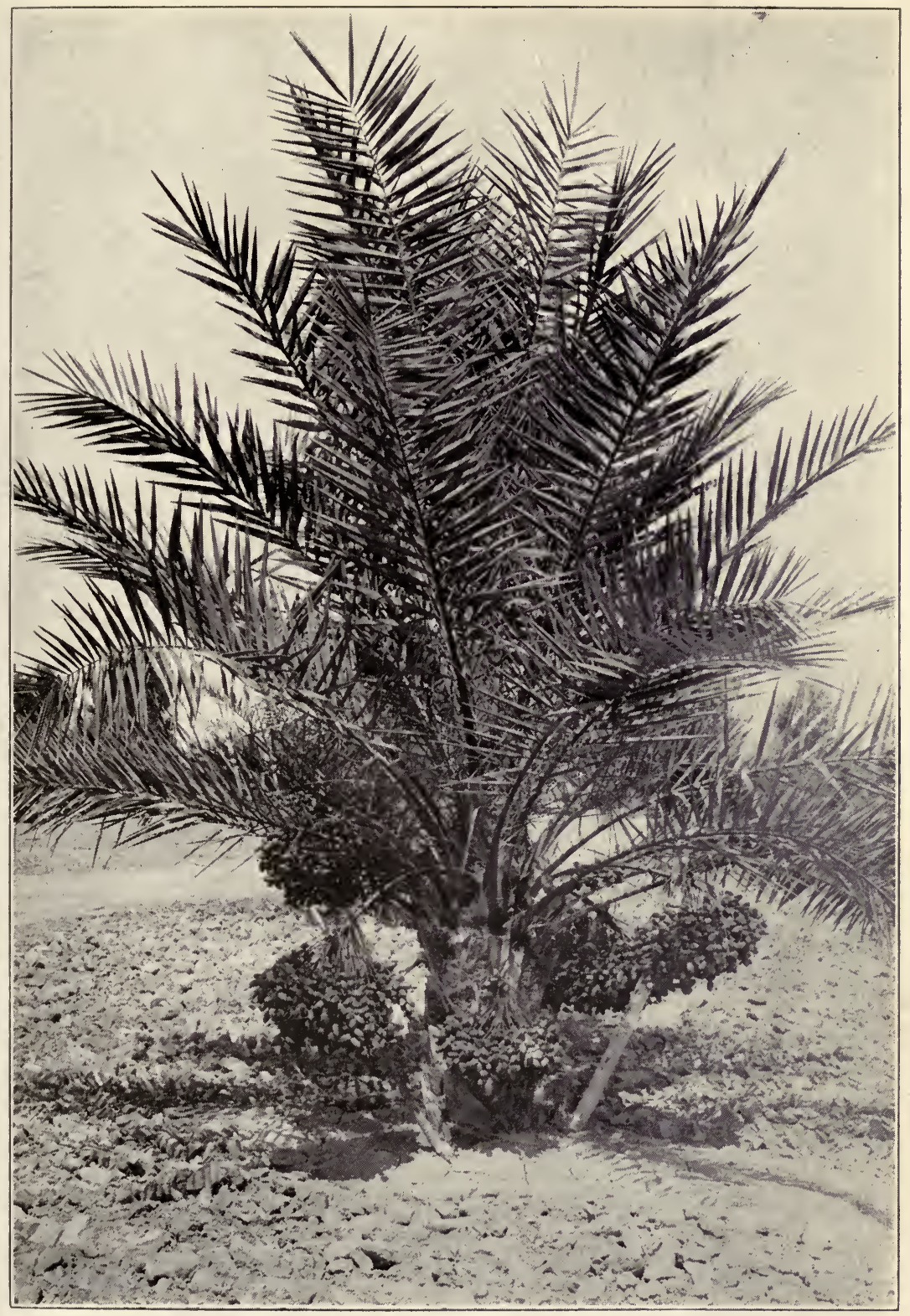

Plate XIV.-Bearing of the young date palm in California. (See page 319.) 

way to lay out a fig orchard-the intermediate growths to be removed as the figs require more room.

Very handsome effects are produced by planting the figs along avenues to inclose orchards of other fruits. Fig trees are grand for shade around buildings, and wild or Capri figs are desirable to plant in this way for a purpose which will be mentioned later.

In transplanting fig trees extra care must be taken to keep the roots from drying. After planting, the stem must be diligently guarded from sunburn, to which it is liable in the warmer parts of the State.

Pruning the Fig.-The fig requires very little pruning after its shape is outlined. There is difference of opinion and practice as to the height at which the head should be formed; some head nearly as low as already advised for common orchard trees; others, having in mind the immense thickness attained by the limbs, and their disposition to droop, head as high as four to six feet, which is the better way to proceed when the trees are wide-spaced and expected to attain large size.

In shaping the tree, branches should be brought out at a distance apart on the stem, so that there may be room for their expansion without crowding each other, and care should be taken not to leave too many main limbs. Three limbs, well placed around the stem, are enough. The branches putting out on the under side of these limbs should be suppressed, and those growing upright, or obliquely upright, retained. As the fig has pithy shoots it is very desirable to cover all cuts with paint or wax. After getting the general shape of the tree fixed, there is little need of pruning except to remove defective branches or those which cross and interfere with each other and to prevent the interior of the tree from becoming too dense. It is better to remove branches entirely than to shorten them; or, in shortening, always cut to a strong lateral: Stubs left at pruning are very undesirable in the fig.

Cultivation.-Young fig orchards are cultivated as are other fruit areas. Old trees which completely shade the ground are usually left to themselves, without cultivation, except cutting out weeds. Irrigation is governed by local conditions, as already stated. In starting the orchard it is exceedingly important that the young trees should not be allowed to suffer from drying out of the soil.

Bearing Age of the Fig.-The fig often, and, perhaps, usually, begins its bearing very early, in the most favorable situations in this State. Some fruit is often had the second year, and a crop worth handling the third year. Still, it is wiser not to calculate definitely upon such returns, for four or five years sometimes pass without a satisfactory crop. We have, also, instances of "barren fig trees," which persist in "dropping their untimely figs," year after year, during their youth. How much of this is due to variety, and how much to locality, is not definitely known, but successful fruiting has been secured by grafting over barren trees, using scions from bearing trees growing adjacent to them. This has no relation to the subject which will be next discussed. 


\section{CAPRIFICATION*}

Caprification consists in suspending the fruit of the wild or Capri fig in the branches of the tree of improved variety, that the pollen may be carried by an insect from the former to the later. Until the present decade in California has never been able to produce dried figs equal to the fig of commerce or the Smyrna fig. This was, at first, thought to be due to lack of the Smyrna variety. After painstaking effort this variety was introduced. Trees grew readily from the cuttings; fruit appeared upon them and dropped before maturity. Doubt then arose as to whether importers had not been deceived, and other efforts were made which resulted in other importations. These also cast to the ground immature figs. Discussion turned then upon the fact of caprification-the necessity of having the fruit of the Capri or wild fig adjacent to the fruit of the Smyrna fig so that insects from the Capri might visit the fruit of the improved variety and pollinate its inclosed flowers, which, appearing upon the inner wall of an almost closed cavity, could not be reached by ordinary visiting insects. The wild tree had already been introduced and were freely growing near the others, but this fact availed nothing-the figs fell just the same from the Smyrna trees. In $1890 \mathrm{Mr}$. George C. Roeding, of Fresno, essayed to demonstrate the fact that the lack of the pollination was the secret of failure, and he succeeded in introducing the Capri pollen into the eye of the Smyrna fig, and secured thereby the retention of such pollinated figs upon the trees, and when ripened and dried these had the Smyrna character. The demonstration was complete that California could not grow Smyrna figs without the pollinating agency found to be essential to success in Smyrna. This agent is a minute wasp called the blastophaga-an insect so minute that it can make its way through the mesh of ordinary cheese-cloth and can enter the almost closed eye of the young Fig-so minute that a magnifying glass is necessary to give one any clear idea of its outline. For years constant effort has been made by various parties to secure the introduction of this insect. Urgent appeals were made to the United States Department of Agriculture, after private undertakings failed, to secure the insect alive or otherwise in form for permanent residence. In April, 1899, the feat was accomplished, the blastophagas being received from Algiers as collected and forwarded by W. T. Swingle to Mr. Roeding. Their offspring appeared in large numbers during the summer and fall of the same year. On the basis of this achievement the commercial production of a true Smyrna fig in California began and has rapidly developed. Mr. Roeding gave his product the musical patronymic "Calimyrna," which now adheres also the the variety from which it is produced.

*In a general treatise like this only a passing reference can be made of this subject, which is perhaps the most interesting in the whole realm of entomo-horticulture. The fig grower should secure the following monographs: "Smyrna Fig Culture in the United States," by L. O. Howard. Year Book of U. S. Dept. of Agr. for 1900; "The Fig-its History Culture and Curing," by Gustav Eisen, Bulletin No. 9, Div. of Pomology, U. S. Dept. of Agr., 1901; "The Smyrna Fig at Home and Abroad," by George C. Roeding, Fresno, Cal.. 1903; "Some Points in the History of Caprification in the Life History of the Fig," by W. T. Swingle; Report of Riverside Fruit Growers' Convention, 1908; "The Latest development in Fig Culture," by G. P. Rixford, Pacific Rural Press, December 18 and 25, 1909; also December 17, 1910, and July 13,1912. The saine journal has an excellent discussion of caprification methods in its issue of January 10, 1913. 
It is an interesting fact that after this strenuous work was successfully accomplished it was ascertained that the fig wasp had really reached California without assistance before 1880 and has been established in San Joaquin county since that remote date.

To avail himself of the benefits of caprification, every grower of varieties which require it must also grow suitable Capri figs and establish the insect in them. California nurserymen supply these and the insects also when the trees are of suitable age to receive them.

\section{FOES OF THE FIG}

The fig is freer from insect pests than other fruit trees, and yet it is a mistake to consider it wholly free. The writer has seen the leaves well covered with a lecanium scale and has found a moth larva boring in the pith of the young shoots; still, practically, the fig tree in California has not yet suffered from insects.

The gophers have a pronounced appetite for fig roots, and their presence should be carefully watched for. Swine have a liking for fig bark. The trees of the grand grove planted at Hock Farm, on the Feather River, by General Sutter, were completely girdled from the ground as high as a pig could reach by standing on its hind legs. Figs make good food for hogs, and plantations have been made with this in view, but if the hogs are to be harvesters, it will be well to protect the stems of the trees from them.

\section{VARIETIES OF THE FIG}

The fig presents what may be termed an aggravated example of the confused nomenclature which pervades California fruits. Dr. Eisen has made a commendable effort to bring order out of chaos by a study of foreign records and locally-grown fruit, and has published a catalogue of varieties chiefly grown in California, with descriptions of each in Bulletin 5 of the Division of Pomology of the U. S. Department of Agriculture.* The following enumeration is largely restricted to varieties which have been commercially propagated:

Adriatic.-Size, medium, roundish; neck medium; stalk short; ribs obscure; eye open, with red iris; skin very thin, greenish in the shade, yellowish in the sun; pulp bright strawberry red or white, with violet streaks in the meat; varies in quality according to location. Very productive and often very profitable as dried product. Apt to sour if weather is dark or showery during ripening. This variety is not identical with that known in Italy as Adriatic.

Agen.-Medium size, roundish; skin bright green, cracking longitudinally when ripe, showing white bands; flesh deep red, very rich; a good bearer, but very late, requiring a long hot season.

Angelique; syn. Angelica.-Medium, pyriform; ribs, prominent; yellowish white; pulp white, with rose-colored center; leaves five-lobed. A very good variety in some of the coast valleys.

*A much fuller discussion of fig varieties is to be found in Dr. Eisen's Bulletin 9, already cited. Many notes are made of the fruiting in the late John Rock's collection on the grounds of the California Nursery Co., at Niles, Alameda County, of many introduced varieties which have not been commercially propagated in California. 
Bardajic.--"Very large, obovate, pyriform, long neck and stalk, skin very thin, grayish green; pulp rich crimson, fine table fig and largest of Smyrna class."-Roeding.

Belloma.-Large, pyriform, dark purple, red flesh, fine flavor when dried.

Black Bulletin Smyrna.-Large, obtuse, pyriform, short neck, long stalk, light purple, flesh pink, luscious.

Black Smyrna.-Small, globular, short stem, pulp dark amber, good for home use.

Bourjassotte, White; syn. Barnissotte, White.-Medium, round and somewhat flattened, eye large, sunk; skin waxy, green; pulp bright red. A very fine fig. Tree very large.

Brown Turkey.-Large, turbinate, pyriform, with hardly distinct neck; stalk short; apex flattened; ribs few; slightly elevated; eye medium, slightly open, scales large; skin smooth, greenish to violet-brown in sun, with darker ribs; pulp dark rosy red, quality good, and tree a good bearer. Brunswick is frequently confounded with this fig. A distinct variety is grown in Vacaville as Brown Turkey, which is named by Dr. Eisen "Warren."

Brunswick.-Very large, pyriform, with swollen cheeks, one of which is larger than the other; apex very obtuse; neck and stalk very short; ribs distinct; but not much elevated; eye medium, open; skin pale amber, with violet tint; pulp amber. An early, large fig, but lacking flavor. Very common; requires rich, moist soil.

Celeste, White.-Very small, amber; suitable for preserves.

Celeste, Blue; syn. Violette.-Small, ovate, turbinate; ribs few, but distinct, especially near apex; eye raised, rough; color dark violet amber, without reddish blush; bloom confined to the neck; skin thin; pulo deep rose; meat amber, sweet, but lacking in flavor.

Checker Injur._-"Roundish, oblate, short neck, flesh reddish, skin greenish yellow, very thin, dries well."-Roeding.

Col. de Signora Bianco.-Medium sized, pyriform; long ribbed neck; skin green, changing to yellow; flesh deep red, very rich and luscious a strong grower; late, suited for a warm region.

Dauphine.-Large, round turbinate. purple with blue bloom; flesh amber.

Doree.-Medium, oblong, bright yellow, flesh rose-color.

Dottato.-Medium ovate, pyriform; neck well set; stalk very short or none; ribs low; skin smooth; eve medium; skin thin, yellowish green, meat white; pulp yellowish amber, sometimes with violet flush. One of the best figs for drying; tree a strong grower, requiring moist, rich soil. Lately introduced into California.

Drap d'Or.-Large, pyriform, with very low neck and stalk; ribs elevated; apex obtuse and concave; color light violet-reddish amber, not dark; pulp rosy red. A fig of very fine quality; especially useful for confections and crystalizing; not identical with Brunswick.

Du Roi-Above medium; round, pyriform; stalk very short; eye large or variable, with scales standing out; skin smooth, pale bluish green; pulp amber, with rosy streaks and exceedingly minute seeds. Related to Marseillaise and Athens, and one of the very best figs in California for drying.

Early Violet.-Small to very small, round turbinate; neck distinct but short; stalk medium to long; ribs distinct, elevated; skin rough; violet-brown, with thin pearl-colored bloom; pulp red. This variety bears almost continuously and is preferable to the Ischias and Celeste.

Genoa, White.-Above medium, pyriform; neck small; stalk short; ribs indistinct; skin downy; eye very small; skin pale olive-green; pulp pale rose. One of the better figs, quite distinct from Marseillaise.

Gentile.-Very large; ovate pyriform; neck short but distinct; stalk very short; skin uneven, with ridges; eye very large, open, with projecting scales; color greenish yellow, spotted with white; pulp amber, streaked with rose; seeds 
few but very large. Only the first crop of this variety ripens. It is of the San Pedro tribe. One of the best early figs.

Grosse Grise Bifere.-Medium ovate pyriform; neck very short; stalk short; ribs distinct; eye small; skin downy, dark violet amber, pale olive in shade; the bloom is separate by a distinct line from the apex; pulp deep red. A tender, good fig.

Hirtu du Japan.-Medium size, roundish with long stalks; skin very dark; flesh opaline; quality best; very prolific.

Ischia, Black.-Small; neck short; stalk medium; skin smooth; color dark violet black, greenish around the apex; neck dark; eye medium, open; bloom thin, dark blue; pulp red. Of fair quality but small size.

Ischia, White.-Size below medium, round, with small neck; stalk very short; eye open; skin smooth, bluish green, with brown flush, pulp rosy red. Common in California.

Kassaba.- "Medium to large, globular, flattened, short neck and stalk, pale green, pulp reddish, very sweet, dries well. Tree handsomest of Smyrna varieties." -Roeding.

Ladaro.-Very large, oblong, pale yellow, brown cheek, flesh deep red, rich and sugary.

Magdalen (Madeleine).-Below medium, round; ribs distinct, rough, disappearing around the eye; stalk longer than the fig, eye open, large; skin greenish yellow; pulp amber white. A very delicious fig, superior to the Ischias and Celeste. Not synonymous with Angelique.

Marseillaise, Long.-Large, longer than wide; skin thick, with brownish shade; pulp dull red. Requires moist soils. A fair fig, which dries well. Not related to either Black or White Marseillaise.

Marseillaise, White.-Medium ovate, pyriform; neck short; stalk medium; ribs numerous and distinct; apex flattened; eye large, open; skin downy, pale yellowish green, mottled with white; pulp amber, with a few large seeds. One of the best figs for drying. Requires sandy, rich soil.

Mission, Black.-Medium to large, turbinate; neck long; stalk short; ribs distinct; eye prominent, open; skin rough, deep mahogany violet, with red flush; pulp not fine, red but not bright or brownish amber; sweet, but not highflavored; common in the Southern States, California and Mexico. The oldest fig in this country. Very regular and prolific in bearing, and free from souring while sun drying.

Monaco Bianco; syn. White Monaco.-Large, rounded, turbinate; flattened, neck small but very distinct; ribs numerous; eye very open; skin dark bluish green, with thin bloom; pulp dark-red rose. A most excellent fig for table, one of the best in California.

Mouissouna.-Globular, turbinate, flattened, dark violet, blue bloom, pulp red, soft and sweet.

Pacific White.-An unknown variety found growing on a farm in Placer county. Medium size, fine grained, very sweet, dries well, but the skin is thicker and more tough than the imported fig. That and its small size are the only objections to it. It is quite widely distributed in Southern California.

Pastiliere.-Large, 3 inches by $1 \frac{1}{2}$; elongated, pyriform, with long neck; stalk short; eye closed, surrounded by an elevated iris; skin rough, hairy, with blue bloom; pulp red. Fine for preserves.

Ronde Noire.-Large, round, but, irregular; neck distinct, short; eye small; skin smooth, waxy, dark violet brown; pulp amber. Greatly to be recommended as a table fig. Is not related to Black Ischia or Osborn Prolific.

Ronde Violette Hative-Large, globular, with no neck; glossy green, shaded violet brown; eye large; flesh amber, surrounding rose center.

Rose Blanche.-Large, roundish, flattened; long stalk; brown or white ground; flesh bright red. Suitable for table and drying.

Royal Vineyard.-Medium, pyriform, long, slender neck; reddish brown, blue bloom; eye large, open; flesh bright red. 
San Pedro, White; syn. Brebas.-Very large, round, flattened at apex; stalk and neck short; eye open; skin thick, tender, of a bright yellow color or greenish in the shade, without bloom; pulp amber. A remarkable and handsome fig. Only the first crop matures without caprification. Suited only for table use. Requires moist, rich soil.

San Pedro, Black.--Very large, elongated ovate, with no stalk, but with wellset neck; skin smooth, violet black with green neck, pulp red, coppery, tinted violet. For table use. The largest fig known.

Smyrna (Fig of Commerce, Drying Fig of Smyrna, Calimyrna).-Of several attempts to secure the true Smyrna fig, or the variety which produces the wellknown Smyrna fig of commerce, that made by the San Francisco Bulletin, and managed by G. P. Rixford, has achieved most prominence, and is now generally conceded to have proved successful. Fourteen thousand cuttings were obtained through United States Consul E. J. Smithers, in 1882, and a large part of these were distributed throughout the State. A later direct importation of fig cuttings from Smyrna was made by the Fancher Creek Nursery, of Fresno. These trees have already borne fruit, as has been described in a preceding paragraph on caprification. In the summer of 1890 cuttings imported from Smyrna by the United States Department of Agriculture were sent to several parties in this State. As the fig insect has fully established itself, as described on a previous page, this variety will establish itself as the leading drying fig, here as a Smyrna, and a very important industry will be established upon it. Other figs previously called Smyrna in this State are misnamed.

The Rixford Fig.- There are, however, new varieties of direct Smyrna parentage attracting attention in this State. The planting of Smyrna fig seed by Mr. E. W. Maslin, at Loomis, in 1886, has yielded several varieties which Mr. W. T. Swingle described in the Pacific Rural Press of February 27, 1909, as of decided promise. These new varieties, which might be called self-sealed figs, show a drop of pellucid gum completely filling the very narrow mouth of the fruit when it matures. As the figs dry and shrivel on the tree and fall to the ground, the drop of gum hardens and hermetically seals the mouth. Such figs do not sour, since the germs causing fermentation are unable to effect an entrance to the pulp within. The skin is pale in color, very thin and tender, often translucent and amber colored in the figs that have cured on the ground. The pulp is light amber colored, full of fertile seeds, sweet and of excellent flavor. The drop of hardened gum that closes the mouth it usually from one-sixteenth to one-eighth inch in diameter, sometimes concealed just within the mouth, but usually partly protruding outside.

Verdal, Round.-Below medium, round pyriform without stalk or neck; skin smooth, waxy, bluish green; eye closed; pulp dark, blood red. A small fig, but valuable for canning and preserves; better than the Ischias or Celeste. It does well in the Santa Clara Valley, but is inferior in the interior of the State.

White Endich.-A re-named variety. Medium, golden yellow, pulp white tinged with pink; tree prolific and long ripening season.

Verdal Longue.-Medium, oblong, turbinate; stalk and neck short; eye closed; yellow, ribs brownish; flesh red; sweet aromatic. bearer.

Zimitza.-Large, pyriform, greenish, yellow amber flesh; good grower and

There are many undetermined varieties of the fig grown here and there in the State. Some may be finally identified, others may be new. Some of them yield an excellent dried fruit and should be more carefully experimented with. The outlook for the fig seems to rest upon successful caprification, although recently there has been increased success in profitable drying of other varieties. 


\section{CHAPTER XXXI}

\section{THE OLIVE AND ITS GROWTH IN CALIFORNIA}

The olive is another of the old mission fruits and though the tree and its products have been constantly under discussion since the American occupation, and though experimentation has been constant, it was not until 1885 that the tide of popular favor turned strongly toward the olive. For twelve years thereafter planting proceeded with enthusiam amounting almost to infatuation, until the acreage in olives ten years ago reached such a figure that even the most enthusiastic ceased from further planting, because the future of the products of the olive was by no means clear. The competition of olive oil with cheaper salad oils worked greatly to the disadvantage of the higher-priced article, but as deception is now ruled out by recent purefood legislation, cheaper oils can no longer be sold under the name of the olive, and legitimate producers will henceforth be protected.

The difficulty of producing pickled ripe olives with good keeping qualities is also vastly greater than anticipated. In addition to these troubles the sterility of the trees in some situations, through frost or other agencies, discouraged many growers. It is probable that during the first decade of this century the uprooting of trees far exceeded the planting and the olive acreage decreased considerably. At the same time there was much progress attained in the building and equipment of oil mills and pickling establishments, and in mastery of processes which yield acceptable products-all of which have favorably influenced the demand and price of the fresh fruit. The fact is, the olive was boomed in California along spectacular and speculative lines, and the industrv had to outlive the mistakes which were made. California will produce profitably, good olives and olive products in suitable places and through the efforts of masterful men and women who can rise to the requirements of production. This view is justified by the experience of the last few years. During the year 1913, the price for good pickling olives ranged from $\$ 140$ to $\$ 160$ per ton, while fancy fruit reached $\$ 200$ and upwards. Such prices were never previously attained and it is believed that even one-half of these values would be profitable, if the trees were fairly productive. This is the ruling factor in the business for olives have proved very shy bearers in many places. Wise investments seems to be to enlarge profitable areas by planting under conditions demonstrated to be favorable.

The olive tree has survived a temperature of 14 degrees Fahrenheit in California, but the fruit is injured by a slight fall below the freezing point. This may render unprofitable the late varieties which carry their fruit-ripening into the winter months.

The olive tree will thrive throughout the larger part of California, and it has been shown that it will grow in a soil too dry even for the grape-vine, and too rocky for any other fruit tree, but the growth of the tree and the bearing of fruit will be proportional to the amounts 
of plant food and moisture, and it is idle to expect fruit without irrigation if the soil can not hold water enough for the tree. There must be moisure enough in the soil to hold the fruit plump and smooth. Olives shriveled by drouth will usually become smooth again after fall rains; but shriveling, either by drouth or frost, leaves the fruit not in the best condition for pickling.

On foot-hill slopes the trees bear fruit earlier than in the rich valleys, although in the latter the trees attain larger growth. Trees in the interior bear sooner than on the coast, and ripen their fruit earlier in the season.

The olive tree is now thriving in California in a great variety of soils. It is productive, if frosts are not too severe on moist valley lands, while on hillsides, even where excavations had to be made between boulders, or into disintegrating rock, the tree has exhibited thrift and content with the situation. But the conclusion should not be drawn that the olive relishes poor soil. It may thrive with loose rocks or boulders, but it finds among them the elements it needs including an adequate supply of moisture. It is not to be inferred that the olive will succeed on sterile soil.

The olive is chiefly grown with clean summer cultivation. Growing the tree in irrigated alfalfa, as discussed in Chapter XIV is objectionable because of the danger of delaying the ripening of the fruit and bringing it farther into the rainy season, which is a very great disadvantage.

Although the relations of soils to the qualities of oil have been investigated by the University of California Experiment Station and some interesting results published, we have not had experience enough in this State to demonstrate the influence of soils on the quality of the oil, but trade results have shown that good oil has been made from fruit grown on some of our best valley fruit soils, deep and naturally well drained, as well as from fruit grown upon drier uplands, and the production on deeper, richer lands is much larger.

\section{PROPAGATION OF THE OLIVE}

Olives are propagated from seed, and from cuttings of various kinds and sizes. The growth from seed is seldom practised in this State, because growth from cuttings is easy, and furnishes the variety desired without grafting.

Growing Olives from Seed.-The olives should not be planted with the pulp, but cleaned of this either by letting them rot in a pile or by putting them into an alkaline solution to cut the oil. A simple way to hasten germination is to break the pits, taking care not to hurt the germ. An instrument similar to the nut cracker has been invented in France which is said to work well. When the kernels are deprived of their shell, they may be kept moist in a compost, or mixture of cow-dung and sandy soil, and sow thickly in the month of April. If it is thought to be too much work to take the kernels out of the pits, they must be soaked for twenty-four hours in a solution 
of one-half pound of concentrated lye to the gallon of water. Most of the seeds sprout the first year. Planting the naked kernels gives the quickest result. Without using this artificial means some of the seeds may remain dormant at least for two years. Clipping the tip of the shell, so as to open a small aperture so that moisture can enter readily, will often cause nine-tenths of the seed to grow within three months.

G. P. Hall, of San Diego, gives the following hints for those who desire to experiment with seedling olives:

After the pulp has all been taken off with lye water (in order to remove all the oil and to roughen the shell), the seed is washed in clean water and then kept in moist sand till planting time, which is in February and March. They will not all germinate the first year, so it is best to preserve the seed-bed. Some assist the germination by cracking the pits in an iron vise; one turn of the screw generally splits the shell. Leave the pit in the cracked shell when you plant in the prepared seed-bed. Some use boxes perforated to insure drainage; the boxes are filled to within three inches of the top with good sandy loam, then the kernels, cracked or otherwise, are spread over the top and an inch or more of sand covers the whole, which must be kept constantly moist. The cracking of the kernels greatly accelerates the germination, and a person can prepare 600 or more seed in a day. Take seed from best trees and avoid dwarfs of any kind. Use any good kinds of either pickling or oil olives as you may desire.

Large Cuttings.-There are two chief methods of propagating the olive from cuttings now practised in California. One uses wellmatured wood, and the other young wood which has just passed out of the herbaceous state. Practice with hard wood proceeds by taking cuttings of sound wood about a foot long and one-half to one inch in diameter, and rooting them as already described for vine cuttings, in Chapter XXVI. A fresh cut should be made at the bottom of the cutting and if the bark has shriveled at all the cuttings should be put in water for a time before planting. These large cuttings sometimes remain dormant for a year or more, and recent propagation has been largely by the small-cutting method.

Small Cuttings.-Propagating by small cuttings serves an excellent purpose in rapid multiplication of varieties; it enables the grower to handle a large number of plants in a small space, and the plants from small cuttings have a symmetrical root system quite resembling that from a seed. These cuttings are made from very small shoots and both the tips and the lower cuts are used. In the engraving the figure on the left is a tip cutting; the next, a cutting lower down the shoot. These figures are about natural size, and show clearly how the cuttings are made. They are placed closely in boxes of sand about four inches deep, rooted under glass or in a lath house, and after a few months are potted in small pots, or may be reset farther apart in boxes of soil or in the open ground. In January or February, the wood seems to be in the best condition in Berkeley, but such condition may come at other times in other parts of the State. From such cuttings the trees will be of good size for planting in permanent place the next year. It is very important to take the small cuttings just, when the wood is in the right condition, not too soft nor too hard. 


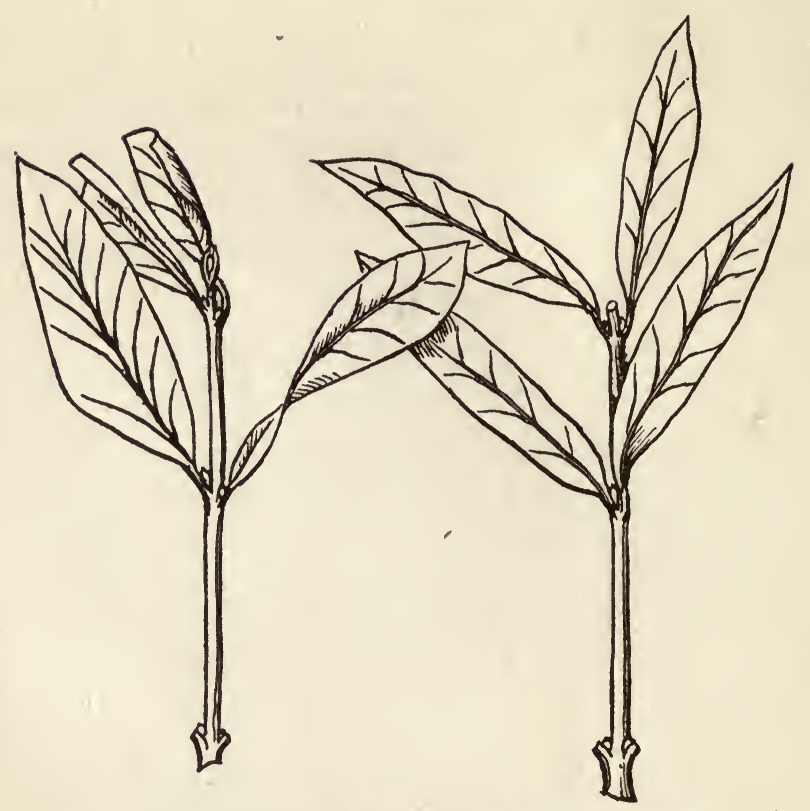

Propagating the Olive by small cuttings.

How to determine this point can not be described; it must be learned by experience.

Growing Trees from Truncheons.-New varieties secured from the south of Europe generally come in the shape of truncheons, which are long sticks of hard wood. They may be planted entire, or be sawn and split into large cuttings (for olive cuttings, even in firewood shape, will grow if properly treated), though better trees come from small cuttings. If the truncheons are bedded a few inches below the surface in moist, warm soil, shoots will appear which can be worked up into small cuttings when they reach the proper condition.

\section{BUDDING THE OLIVE}

Since the planting of a large area of Redding Picholines and the fruit found to be that of a wild or poor seedling olive and not a superior named variety, there has been a demand for working over the trees into better varieties. Besides, many of the imported varieties have proved disappointing and a change to a variety profitable in the region is imperative. The method of budding commonly employed with fruit trees does not usually yield a high percentage of success with the olive, and other ways have been adopted with much better results.

Budding may be performed at any time of the year when the sap flows freely. If done late in the summer, the buds lie dormant through the winter. Best results are obtained when the buds are inserted 
early in the spring, as the operation can be performed to a much better advantage, and the buds will grow to some height before winter. When inserted in large orchard trees, or in limbs of large trees, the shoots from the inserted buds are allowed to grow until they have attained such a size as will justify in the removal of the entire top.

Twig Budding.-Twig budding is very successful. The bud is cut deep into the wood, in order to give the bud as much bark as possible. The leaves are partly cut off ; then, with the sharp point of the budding knife, the greater part of the wood inside of the bud is removed, as shown in the picture. If part of the wood is not removed, then the bud can not take, as the wood in it prevents the two barks (the inner bark of the bud and the inner bark of the stock) from uniting. When the wood has been partly removed from the bud, the bud is inserted into the stock, as budding is done in the regular,

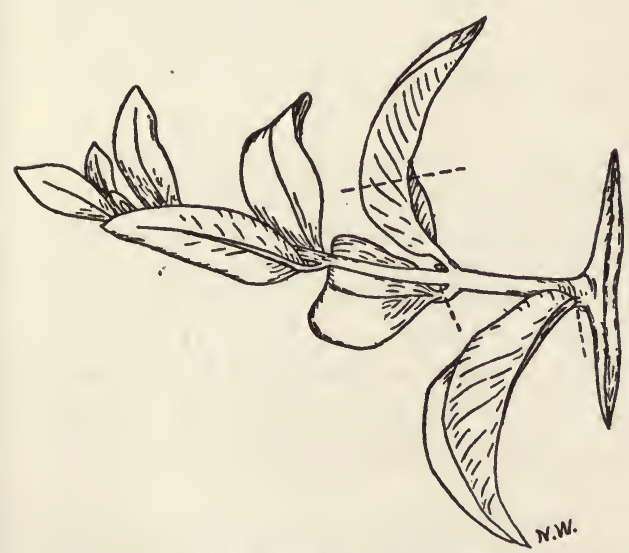

Olive: Twig-Bud as cut.

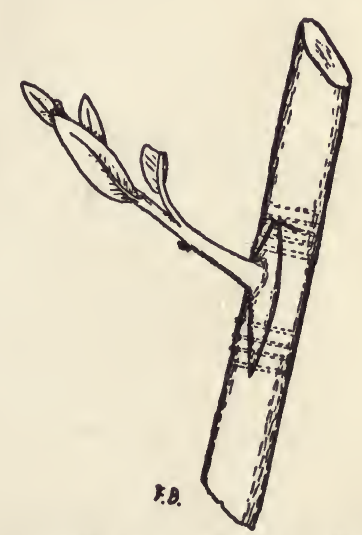

Olive: Twig-Bud Inserted.

ordinary way, and tied tight. A small tip-twig may be used or a longer twig, cut back to the lowest bud and part of the foliage cut away, as shown in the engraving. At the end of three or four weeks the string is removed, and part of the top of the stock is cut back to force the bud to start. As the bud grows, the foliage of the stock is gradually removed, until the bud is able to take up the entire flow of sap; it is then left to grow, and it may be protected by tying to a long stub of the branch which may be left for that purpose above the point of insertion. When the bud has grown out strongly, what remains of the stock above the bud is cut smooth, close to the bud, to allow it to heal over.

Flap-Budding the Olive.-Although the use of a twig with a pushing bud is to be commended strongly a dormant bud can be forced into activity quite successfully. Mr. C. A. Wetmore describes this method:

Late in the spring, when advancing warmth has set the sap to moving freely, observe a tree of the kind you desire to propagate. Notice parts of limbs with 
vigorous growth and clean bark free from little lateral twigs. Where a leaf adheres to such limbs or where one has been and fallen off, there is a small bud or eye. Such dormant buds or eyes are lifted from the wood together with a small piece of the bark about one-third or half an inch square. On the properly selected limbs of the trees you wish to bud into, make three cuts with a knife so as to include a space a little longer than the piece of bark to be inserted. Two parallel cuts are in line with the limb; one is across the top. Lifting the flap of bark thus made carefully with dull side of a blade, the cambium layer of the wood will be exposed. Slip in the piece of bark with the dormant bud and press it close with the flap made on the limb. Tie the flap firmly to cover the whole piece inserted with raffia or strips of cloth or soft twine. At the end of one week cut the ligatures and suffer the flap to curl up and dry. If the insertion has united to the wood, it will remain green and fresh-looking about the dormant eye. If this freshness continues after exposure, the bud has taken. Then cut with a knife or saw into the limb above the bud to induce the sap to force the growth. If any one fails, the limb need not be cut into, but may be budded in another place. The following winter, cut off the limb above the bud if it has sprouted well, and, as sufficient new wood is made, the old branches of the tree may be cut away.

\section{GRAFTING THE OLIVE}

Grafting is also used in working over both large and small olive trees. Good success can sometimes be had with the ordinary method of top grafting, as described in Chapter IX, using scions not larger than a lead pencil and inserting them in April. The olive can also be successfully grafted in the bark according to the method shown in Chapter IX. This graft is used for working in the top of the tree, but it may also be used at the surface of the ground, covering the cut surfaces with earth when the scions are in place. Judge A. L. Rhodes, of San Jose, gives the following explicit account of his success with a bark graft, as follows:

The stock, where cut off, may be from half to two and one-half inches in diameter; the scion about one-quarter inch in diameter, the lower end to be formed by an oblique cut of about one and one-half inches. Split the bark of the top of the stock about one inch, raise the bark at the sides of the split slightly, insert the point of the scion between the bark and wood of the stock, at the split, and press it down the length of its oblique cut. Fasten it by binding twine around both stock and scion, about ten times, very firmly. Apply grafting wax to top of stock and scion.

If the bark of the stock be three or more years old, make two slits in it, about one and one-half inches in length, the width between them equaling the width of the oblique cut of the scion, raise the bark between the slits, cut off about half of it, by a sloping cut, then insert the scion and press it down, and bind with twine and apply grafting wax, as above directed. Cotton wrapping twine is of sufficient strength.

Stocks the diameter of one inch or more should receive two or more scions. Scions gathered a short time before their insertion are the most successful. The twine around the stock and scion should not be loosened until it indents the bark of the stock. Protect the graft from sun and wind. Wrap paper around stock and scion, the paper to extend a few inches above the scion-or place the paper, in the form of a bag, over scion and stock-and secure the paper with twine, tied around the stock in a slip-knot.

Bark grafting may be performed at any time when the bark of the stock can be readily raised-whenever the bark will "slip." I grafted in that mode in each week of April and May and the first of June, and in September. Failures not 5 per cent. Twelve scions inserted about the middle of last September are 
all growing. Shield budding is the most successful in the spring. I prefer the bark grafting, as the shield buds may not start for months, or even for a year.

Side Graft on Small Wood.-A satisfactory graft can be made with an oblique cut, as shown in Chapter IX, which is superior to a split of the stock, because on a small stock the spilt is apt to continue farther than desirable when the scion is pushed in. With the slanting cut in the stock the scion can be firmly pushed into place without splitting. The union of inner barks of scion and stock must be made on one side when the stock is larger than the scion. This graft is tied in and waxed, or a waxed band may be used. In working small wood at the ground surface, the earth should be drawn up around the graft.

Grafting on Rooted Cuttings.-Good trees are made by grafting in the nursery or rooted Picholine cuttings. Such cuttings are made from wood, say, one-half inch in diameter. Using a young scion with a starting bud. Such a scion makes a salable tree with one year's growth.

\section{PLANTING THE OLIVE}

There is nothing gained by planting out the olive too early in the spring. Both cuttings and rooted plants will do better if planted after the soil becomes well warmed, and after the heavy rains of the winter are well over. Of course the time when this condition comes is different from year to year, and varies, also, according to locality and situation. During the first summer the young plants will need occasional watering in some situations; in others, merely mulching, or keeping the surface finely stirred, will suffice.

Olive trees are planted at different,distances, but the ruling intervals are twenty to twenty-five feet. This will allow the trees to bear a number of years before they crowd each other; and then removing alternative trees gives ample distance for future growth. But it is clearly the part of wisdom to hold the olive to a low growth in order that the fruit may be cheaply gathered, and this may be done by proper pruning.

Transplanting Old Olive Trees.-It is often desired to move olive trees of considerable size and the method outlined below is, applicable to olives and citrus fruits and, in a general way, to all evergreen trees:

After the heavy rains are over and the ground is getting warm-say in February-cut back not less than one-half of the top. Trench all around the tree to a depth of two or three feet, according to the size of the tree; the inside edge of the trench about two feet from the trunk of the tree. At the same time remove top earth down to the roots to reduce the weight. This trenching cuts off all side roots and gives room to get in to handle the ball of earth, which rope up with barley sacks under the ropes so as to hold the ball from breaking. When this is done well, dig in and under the tree from the bottom of the trench so as to cut off the bottom roots. Carefully pull and pry the ball to see that it will wobble a little in the hole to show that all the roots are cut. The get ropes and sacks under the 
ball, rig up a derrick and block and tackle and lift the balled tree out of the hole, land it on a low truck and haul it to a new hole previously made ready, and lower it into place by the same tackle which lifted it. Fill in with fine earth and water-settle it to fill all cavities-covering with loose earth several inches to keep from drying. Be sure the tree has plenty, but not too much, moisture during its first summer in its new place.

Large deciduous trees can be removed when dormant with much less labor, but it should be done in the fall-as soon as the soil is deeply wet by rains. It should be understood, however, that moving large fruit trees, either evergreen or deciduous, is seldom commercially desirable.

\section{PRUNING THE OLIVE}

Pruning policies as insisted upon in Chapter XII, have direct bearing upon the commercial growth of the olive. The development of the tree according to principles there laid down is practicable and desirable. After proper low form is secured, satisfactory bearing will depend upon regular pruning to secure new bearing shoots and thinning to prevent the tree from becoming too dense and bushy. The olive bears upon wood which grew the preceding year, and upon no other. It is just as important, then, to secure a good supply of such shoots as it is to secure new bearing wood for the peach, and the ways to do it, by cutting back and thinning out, are much the same. Keep the tree from running out of reach of a step-ladder; prevent it from becoming a brush-heap, for both these acts are essential to the growth of good bearing wood, low down. At the same time it must be remembered that too severe cutting-back forces the growth of branches which form only wood buds and fruiting is postponed. The secret is to prune enough to induce plenty of new growth but so that not much excessive, non-bearing, new growth results. This is secured by regualar and moderate pruning; some growers systematically cutting back the new growth one-half to two-thirds.

Trees which have been allowed to form umbrella-like tops may be brought down to business again by cutting back the main limbs and making selection from the many new shoots which appear, but by proper, regular pruning a tree can be so trained that the removal of large limbs is seldom necessary. The times to prune the olive are just after the gathering of the fruit or just before new growth starts in the Spring.

Developing the Vase Form.-Explicit suggestions as to the development of a low, vase-form tree may be helpful to inexperienced growers. The following is from a foreign writer, whose illustrations are presented herewith:

When the young tree has attained some height, it is the practice to cut off the top, so that the main stem shall be about four and a half feet in rich soil, or three feet in poor soil or in locations exposed to strong winds. Six or eight branches are left to form the head. The process of shaping the tree then proceeds, as shown in the engravings. Fig. 1 shows the young tree to be cut at the 


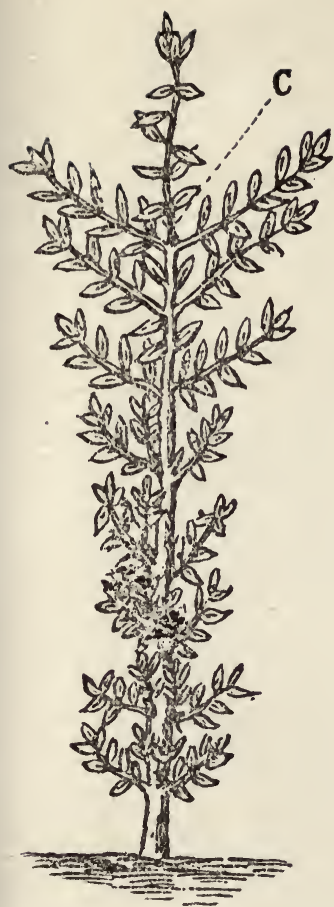

Fig. 1.

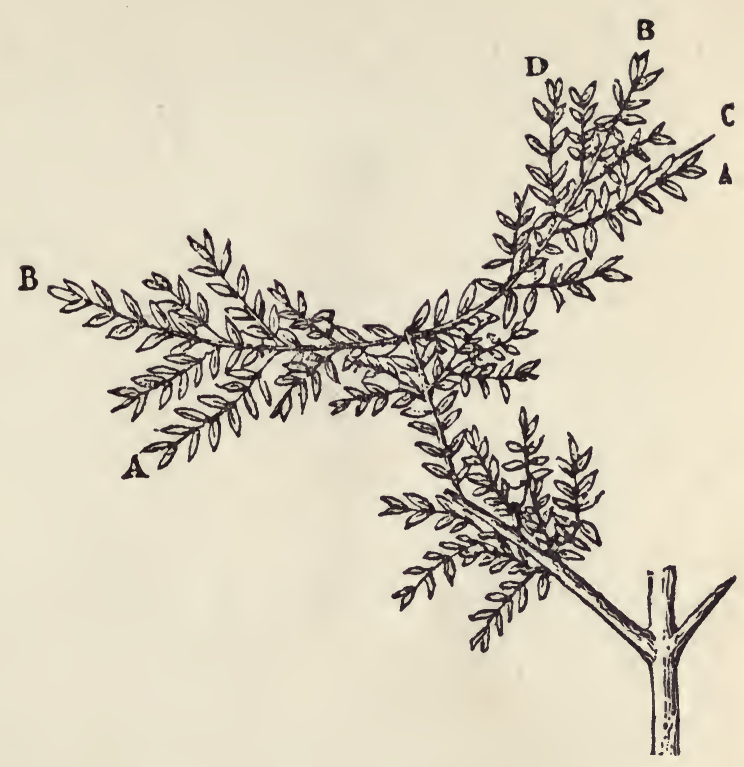

Fig. 4.

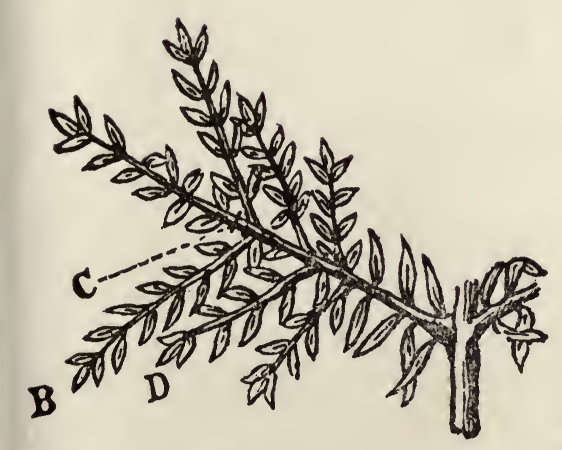

Fig. 2.

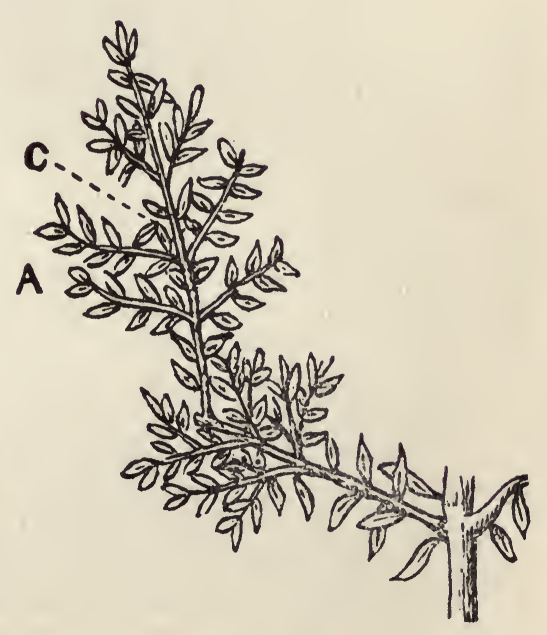

Fig. 3. 
point marked by the dotted line $C$. Six branches, three on each side, are left, and the lower twigs shortened. Each of the branches left develops, during the year, as the one shown in Fig. 2, which is then cut at C again, and the shoots B and D are shortened. This process starts out the upper shoots, and appears the following year as $A$ in Fig. 3 , and it is again cut at $C$. This causes the two upper shoots to develop, and at the end of the year they appear as shown at $B B$ in Fig. 4, Thus they stand at the fourth year's pruning, and each of them is cut at $C$, and $A$ is shortened and $D$ allowed to develop. By this time the tree has a spherical or vase form, and exposes much surface to the sun, which is desirable.

The young branches that spring in the form of a cross on the more vigorous branches, bear only wood buds; the others, which are weaker, bear fruit buds on their whole length and burst into blossom at the spring of the second year. The latter never blossom again in the same place, but the shoot extends itself and forces two lateral ones. These new shoots bear the following spring, and so on. It must therefore be always borne in mind that the olive bears only on the two-year-old wood. If the new shoots are formed every year, the olive will bear annually; but in years of good crops, the sap employed to nourish the fruit only produces a number of very diminutive shoots, and the next crop is a short one. The pruning ought to favor the growth of young lateral shoots, either by shortening the terminal ones, suppressing the "gormand," or fruitless shoots, or by reducing in a certain proportion, each year, the fruit-bearing shoots, if we wish for a crop every year. The shortening of a branch is made immediately above an outside bud in an oblique direction, the interior one being suppressed. The suckers at the root of the tree should be continually cut off.

Concerning the time for pruning, the best season is said to be when the winter frosts are well over and just before the sap starts in the spring. By early pruning the sap is made to act upon the buds unfavorably situated on the tree, brings them out, and also develops latent buds on the old wood. Thus one is enabled to prevent the tree from becoming covered with naked limbs.

\section{THE FRUIT AND ITS PRODUCTS}

The agricultural experiment station of the University of California was occupied for many years in the growth of olives and close examination of olive products both by laboratory and practical test. The publications of the station discuss the operations of oil making and pickling and the suitability of varieties and for the purpose of this treatise outlines will be drawn from these sources.

Gathering the Fruit.-Olives should be picked carefully and at the right time. For green pickles they should be picked very soon after they obtain full size, but before they have begun to color or soften. For ripe pickles and for oil making the fruit should be gathered when it contains the maximum amount of oil. This is soon after the olives are well colored, but before they have attained the deep black which signifies overripeness. If the olives are gathered too green the oil will be bitter; if too ripe, it will be rancid. When they can be easily shaken from the tree they are ripe enough. If they commence to fall without vigorous shaking they are overripe. For whatever purposes the olives are to be used they should be carefully gathered by hand, and imperfect, immature, or bruised fruit rejected. Sound fruit is required for high-grade oil or for handsome pickles with good keeping quality. 


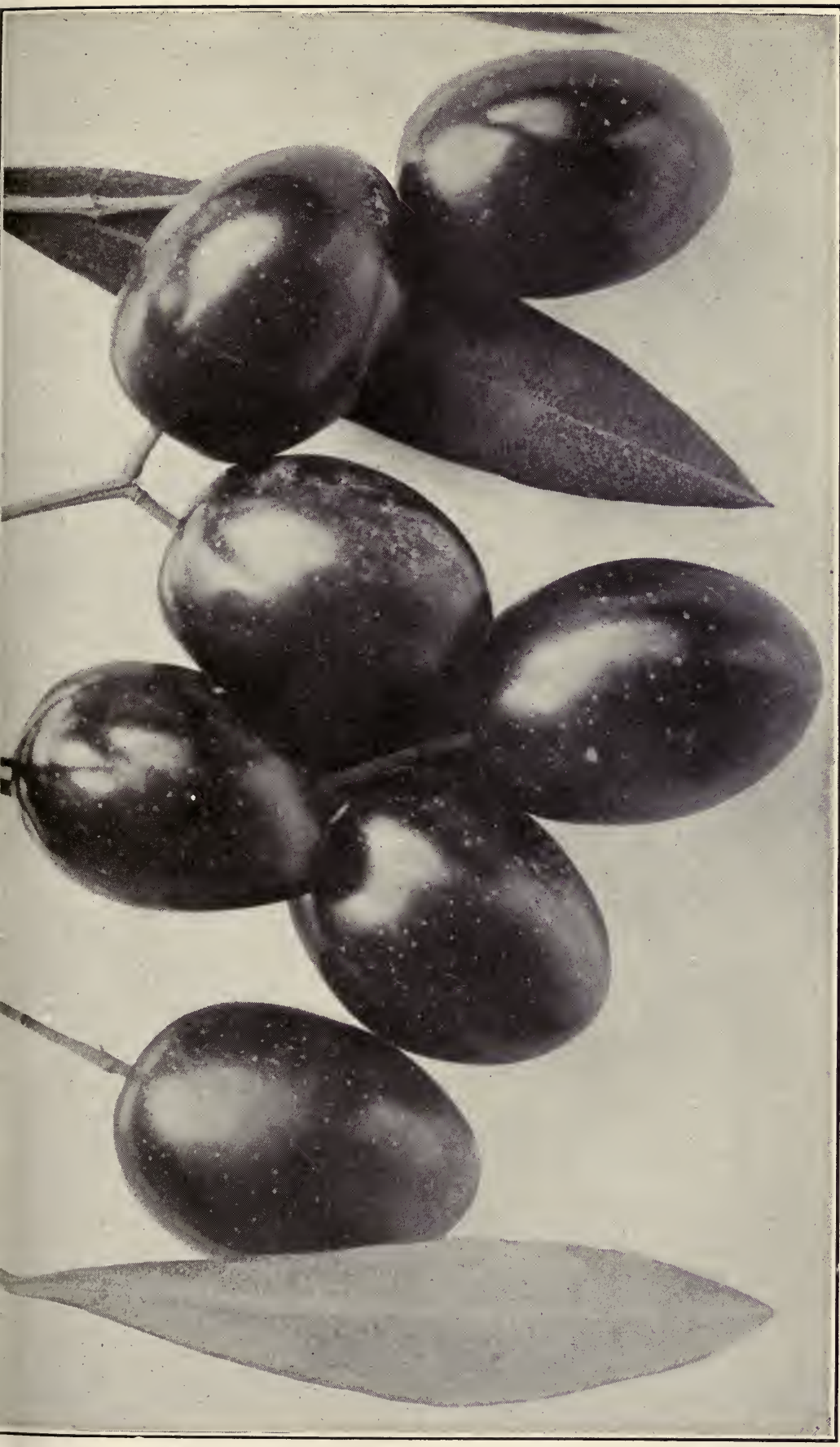

ริ

๑

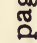

है

I

2

:

हึ

I

동

on

क

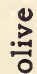

范

है

i

营 



\section{THE MANUFACTURE OF OLIVE OIL}

Olive oil is made in this State with apparatus of both Californian and Europeon design, and, as a rule, there is made only one, and at most but two, pressings of the pomace, which is then used for fattening swine. In the frequent working over of the pomace, and the close extraction of the oil, as practiced in Europe, we have done little as yet.

Olive oil is made on a small scale by a number of parties who use home-made contrivances, or small, portable cider machinery for the crushing and pressing. During the last few years quite a number of mills have been erected at several points in California and they have made a market for the olives produced by growers who do not care to undertake manufacture. A detailed account of oil making, including descriptions of buildings and machinery, published by the University Experiment Station has been out of print for several years, but can be consulted in libraries.* As this is available to those who desire such specific information, only an outline will be undertaken for the information of the general reader.

Drying.-Extraction of oil from fresh olives gives the best oil, but it is somewhat troublesome, and it is customary to partially dry them. This partial drying is also useful to keep the fruit for some time or for shipment before crushing. Place the olives in layers not more than three inches deep, on trays that are stacked in a dry, wellaired room, protected from the wind and the direct rays of the sun. Turn daily until the fruit becomes well wrinkled. This requires about eight or ten days, according to the degree of temperature. The partially dried fruit may be stored in a dark room where the temperature does not rise above sixty degrees Fahrenheit, for three or four weeks without any serious deterioration of oil. To hasten the drying process, artificial driers, constructed on the same principle as the fruit or hop driers, are sometimes used. The olives are placed in a single layer upon trays, and the drier is kept at a temperature of about one hundred and twenty degrees Fahrenheit; at over one hundred and thirty degrees Fahrenheit the quality of the oil may be impaired. The drying takes about forty-eight hours-more or less-according to the nature of the fruit.

Crushing.-The olives are usually crushed by heavy stone rollers revolving in a circular depression in a bed of masonry into which the fruit is placed. Chrushers with corrugated bronze or bronzed metal rollers are now made that perform their work in a very satisfactory manner, breaking up the flesh and pits very thoroughly. As they are all of metal they absorb no oil and are easily cleaned. It is very essential that the flesh should be crushed thoroughly in order to break up the cells and permit the oil to be pressed out.

Pressing.-When the revolving crusher has reduced the olives to a mass, the pomace is shoveled up from the bed of the mill and prepared for pressing. Instead of the fabric of woven esparto grass which is used abroad, coarse linen cloth is used. A certain amount of the

* "California Olive Oil: its Manufacture," by G. W. Shaw, Bulletin 158. University Experiment Station, Berkeley, Cal. 
pulp is put in each cloth, so that when the cloth is folded back it makes a cheese about three feet square and three inches thick. Ten or more of these cheeses are placed one above the other, with slats between, and the pressure applied gently at first. From the liquid which runs out first is made the very finest oil, known as "virgin oil." The pressure is then increased very gradually until the full power of the machine is reached. This presses out the second quality of oil, which is generally mixed with the first. After obtaining all the oil possible by the first pressure the "cheese" is taken out, thoroughly broken up in hot water, and again pressed. This yields the third quality, which is very much inferior to the first and second. Sometimes the "cheese" from the first pressing is thoroughly broken up with cold water and pressed again before being treated with hot water. In this way a little oil is obtained that differs a little from the second quality, and may be mixed with it. After this a certain amount of oil still remains in the "cheese," but it can be extracted only by very powerful hydraulic presses, or by chemical means, and then is of very inferior quality, and suitable only for burning or for soap making.

Settling and Clarifying.-The liquid from the press is dark colored, and it is conducted into a receptacle for settling. Much of the foreign matter quickly separates, the oil appearing on the top. The oil is removed to other receptacles in which it can stand from two to five months for perfect separation of undesirable sediment. These settling tanks may be of well-tinned metal, or of cement lined with glass or other impervious substance. The first settling is conveniently made by means of a funnel-shaped apparatus, which by its conical shape facilitates the rapid deposition of sediment. After standing for twenty-four hours in this apparatus the major part of the sediment is deposited and can be drawn off at the bottom. It is well, before running the oil into the settling tanks, to pass it through two or three inches of cotton wool. This is accomplished by means of a funnel with a perforated, horizontal cross partition, upon which the cotton is placed. It takes, generally, about one month for the oil to settle sufficiently in the first tank, after which it should be drawn off carefully into the second, and so on until it is sufficiently bright. Three rackings are usually sufficient.

Olives are sometimes ground and pressed in portable cider mills or ground in barley crushers for oil manufacture on a small scale. As the above description shows, oil making is a simple process, and may be carried on at home with rude devices. It is, however, a process requiring care and cleanliness, and intelligent personal attention.

\section{PICKLING THE OLIVE}

Olives are pickled in a green state, as is the case with the imported olives; or in a ripe state, as largely undertaken in California. No one had any conception at first of the difficulties attending the production of pickled ripe olives which would have the keeping quality demanded in an article of commerce. It is now clearly seen that treating olives to extract the bitterness and to secure firmness, good flavor and keeping 
quality is one of the most difficult propositions in our horticultural manufacturing, and we can but admire the wisdom of the Spaniard in teaching Anglo-Saxons to enjoy green olives. To succeed with the ripe olive requires the utmost patience, experience, and intelligence, and one who undertakes it must not get weary of the most exhaustive study of difficulties that may arise and how to meet them. When the most careful picklers with the best appliances sometimes lose hundreds of dollars worth in spite of all they know about it, the difficulty of the matter may be appreciated.

The following is an outline of the pickling of olives as drawn from the University publications by Professor Bioletti:

The pickling of olives involves three steps: I. Treatment with lye to neutralize the acidity and bitterness. 2. Repeated soaking in water to remove the excess of lye. 3. Salting by soaking in brines of gradually increasing strengths.

(1) Neutralization. Soak the olives for twelve hours in a solution made by dissolving $1 \frac{1 / 2}{2}$ pounds of caustic potash in 12 gallons of water. Very bitter olives may require a second treatment with fresh lye (Mission, Manzanillo, Sevillano); sweeter olives may require a weaker lye (Ascolano, Columbella). With soft olives, $1 \frac{1}{2}$ pounds of salt should be added to the lye. The olives should be kept submerged by means of a floating cover and the lye drawn from the bottom and poured over the top two or three times to insure equal treatment.

(2) Soaking. When, by examination of the color of the flesh of the olives, it is found that the lye has penetrated nearly to the pit, they should be removed to pure water. This water is renewed at least once a day until the lye is removed, which requires two or three days. With soft olives, a brine containing about two pounds of salt to twelve gallons of water should be used for removing the lye.

(3) Salting. When the lye and most of the bitterness have been removed the olives should be placed in stronger brine. If the olives are to be canned or bottled, a brine containing about five pounds of salt to twelve gallons of water is sufficiently strong. If they are to be kept in barrels ten or twelve pounds of salt will be needed. The olives must be prepared for the strong brine gradually by being placed in new brines every three to four days. Each brine should be about two per cent stronger than the last.

Precautions.-Strong lye tends to soften the fruit. A second treatment is weak lye is better for bitter olives than a strong lye. Salt has a tendency to harden the fruit and can be used to counteract the softening effect of the lye with very ripe olives. Strong brine will shrink and shrivel the olives, unless they are prepared for it by solutions of gradually increasing strengths.

Color.-With ripe pickles, the object is to have the color as uniform and dark as possible. Uniformity is obtained by pickling each variety by itself, by having the fruit as evenly ripe as possible and by exposing the olives to each solution in as uniform a manner as possible. Spotting comes usually from unevenness of the lye treatment, and especially from allowing some olives to float on the surface. Depth of color is obtained by having the olives thoroughly ripe. By exposing the olives to the air for several hours between the various solutions, both the depth and uniformity of the color are increased.

With green olives, the air should be excluded as much as possible, until the final brine is reached. A little vinegar improves both the color and flavor of green olives. Some picklers advise the addition also of a few bay leaves. 
Pure-Water Process. - The best pickled olives are made without the use of lye, but this process is only practicable with olives whose bitterness is easily extracted, and where the water is extremely pure and plentiful, and even then it is very slow and tedious. It omits the preliminary lye treatment. The olives are placed from the beginning in pure water, which is changed twice a day until the bitterness is sufficiently extracted. This requires from forty to sixty days or more. The extraction is sometimes hastened by making two or three shallow, longitudinal slits in each olive, but this modification, besides requiring a large amount of expensive handling, renders the fruit peculiarly susceptible to bacterial decay and softening. Altogether the pure-water process can not be recommended for California, as it is too expensive and uncertain.

Green Pickles.-Green pickled olives are made by essentially the same processes as are used for ripe olives. The extraction of the bitterness requires the same care. The olives are pickled soon after they have attained full size, and before they have shown any signs of coloring or softening. They contain at this time comparatively little oil, and are in every way much inferior to the ripe pickles in nutritive value. They are not a food but a relish. They are rather more easily made than the ripe pickles, as there is less danger of spoiling.

\section{CANNING THE RIPE OLIVE}

The use of heat and hermetical sealing after the pickling process is completed is a recourse to avoid the difficulties of ripe pickling and canned olives, put upon the market in the same form as other canned fruits, have recently become popular. There àre special canneries for their preparation at several points in the State and the general canneries are also handling olives in considerable quantities. The process is in the main like that of canning other fruits, but special points have to be learned through experience. The University investigation of the effect of heat on the olive shows that ripe pickled olives, heated to 175 degrees F., kept perfectly for thirty-two months. By heating them still higher in sealed cans on bottles they can be kept indefinitely with as great facility as any other food product. The heating does not injure the flavor and the texture, but, on the contrary, improves them. Olives, preserved by heating do not require such strong brine, and it is only necessary to add as much salt as the palate requires. The heating causes some of the coloring matter to diffuse into the brine, so that the olives are made a little lighter colored. With time, however, the colored matter diffuses out in the same way from unheated olives, so that at the end of a year the heated olives are actually darker in color than the unheated.

\section{VARIETIES OF THE OLIVE GROWN IN CALIFORNIA}

Many varieties of the olive have been brought to California from southern Europe during the last thirty years. Fifty-seven varieties have been analyzed and elaborately reported upon by the University 
experts, and of these about fifteen varieties at first promised to rise to commercial account but many of them have been dropped. It is an interesting fact, however, that in spite of all the efforts put forth to secure a better olive than the old Mission variety, this old sort still heads the list. Several varieties at first popular have been abandoned because of an interior decay of the pulp.

The following are the varieties now favored in California on a commercial scale, arranged approximately in the order of their present popularity: Mission; Manzanillo; Ascolano; Sevillano; Nevadillo.

Other varieties sometimes used for pickling are: Oblitza; Macrocarpa ; Picholine ; Columbella ; Regalia ; Goodal; Atro-rubens ; Verdale ; Santa Caterina.

These may be taken, then, as practically all the varieties to which planters are giving attention. Of course future experience may produce changes in this list, but there is little present indication that it will be much enlarged. Descriptions of many other varieties are retained, largely for old acquaintance sake :

The Mission Olive.-By this name is signified the variety found growing at the old missions in California. Samples of the fruit and leaves sent by F. Pohndorff to Don Jose de Hidalgo Toblada, a noted Spanish authority on the olive, led to the classing of our mission varieties with the Cornicabra-Cornizuelo varieties of Spain, and its value was confirmed. It has long been known that the s)-called Mission olive embraced several varieties, or sub-varieties at least.

Common or Broad-Leaved Mission Olive.-The variety of olive most generally known as the Mission; ovate, oblique-sometimes very much so-the pit straight or slightly curved, fruit very variable in size, growing singly or in clusters of two or three, or even five; time of ripening, late, in the coast region sometimes not before February, but generally in December; in warm localities in November; tree vigorous and a good bearer, preferred by picklers.

Redding Picholine.-Imported by the late B. B. Redding. A perfect oval in shape, ripens early, several weeks earlier than the common Mission; dark purple or black when ripe; in pickling the pulp loses the bitterness quickly, the fruit being very pleasant. This variety was propagated extensively, in the State and, until fruiting was supposed to be a large pickling variety, but it proved to be a small seedling - a shoot coming from the root below the graft, probably. It has produced oil of good quality. The smallness of the fruit is its irremediable defect. It roots very readily from cuttings and is used as a stock on which to graft improved varieties.

Picholine d'Aix.-Fruit medium, enlongated, tapering toward apex which is pointed; reddish black when ripe.

Picholine de Stt. Chamas.-Oblong, reddish black; highly esteemed in France for quality when picked.

Oblonga.-Imported by John Rock from France. An olive of a peculiar, club-like shape, being narrow at the stem end, broad at the point, rounded and strongly oblique; generally pointed at both ends. The pulp loses its bitterness comparatively quickly in picking. This olive ripens quite early-at least two or three weeks earlier than the Broad-leaved Mission; color, dark purple.

Pendoulier.-Large, oval, slightly curved at apex end; desirable for pickling early ripening in October in the interior valley and in November in coast valleys.

Manzanillo No. I.-Imported by F. Pohndorff from Spain, large regular rounded oval; pit straight, strongly pointed at the apex, nine-sixteenths of an inch long, five-sixteenths of an inch thick. Ripens early, several weeks! earlier than the Broad-leaved Mission. The fruit grows on long stems. The pulp parts readily with its bitterness, and is exceedingly rich when pickled. Excellent in the San Joaquin Valley both for oil and pickles. 
Manzanillo No. 2.-Imported by F. Pohndorff from Spain. As the name ("small apple") indicates, this variety is nearly round, with a pit of rounded oval shape, rather squarely cut off at the base. This variety ripens earlyseveral weeks earlier than the Broad-leaved Mission olive; the fruit grows generally singly on long stems; a trifle larger than Mission; quality of pickles excellent; not a good bearer in all places and ripe fruit subject to rot in hot regions.

Gordal.-A popular pickling olive in Spain, medium size, early.

Rubra.-Imported by John Rock from France; ovate, slightly oblique, looks a good deal like a small Mission olive; pit straight, pointed; ripens three to four weeks earlier than the common Mission variety; is of a jet black when ripe. This tree begins to fruit quite young, and is a prolific bearer. Very hardy and prolific even in dry situations.

Atroviolacea.-Medium size, black, chiefly valuable for oil.

Uvaria.-Imported by John Rock from France. Oval, regular, and rounded on both ends; pit straight, heavy, late; later than the common Mission olive; color dark purple or black when ripe. The name, "grape-like," is well chosen, the fruit growing in clusters, as many as seven together, and in shape themselves resembling the grape. Very prolific.

Pendulina.-Imported by John Rock from France. An even, oval shape, rounded at both ends, quite variable in size, many fruits remaining small and undeveloped; pit has small, sharp points often at both ends. Fruit grows in clusters of from two to five; the pulp parts very readily with its bitterness. Larger and more ovate than Pendoulier. Tree a strong grower; fruit desirable both for oil and pickles.

Columbella.-Imported by John Rock from France. General form, broadly oval; very even in size, remarkable for the peculiar pale yellow color which all the fruit assumes before turning fully ripe and becoming dark purple; pit small, straight and sharp pointed; the pulp contains little bitterness; flavor very rich; ripens late, later than the Broad-leaved Mission. Tree hardy in dry places and a prolific bearer. Also called "Columella."

Polymorpha.-Imported by John Rock from France. Very large, ovate, oblique, and pointed; light colored; pit square at the base, strongly pointed at the apex; flesh firm; ripens very early; fruit grows on strong stems in clusters of two or three. Tree not a strong grower, but productive.

Lucques.-A variety specially adapted for pickling, though producing oil of good quality; strong-growing tree and hardy; sometimes shy bearer when young; fruit shiny black, curved; product called "Crescent Olive."

Nevadillo Blanco.-Imported by F. Pohndorff from Spain. Oval, slightly oblique, pointed, resembling somewhat a Mission, but is generally more elongated in proportion to its diameter than the latter; pit small, curved, and generally pointed at both ends; the fruit is borne in clusters of three to five; ripening not much earlier than the Mission; a fine oil olive, largely planted, but disappointing in some regions as a shy bearer and subject to frost injury.

Oblitza.-Imported by the late G. N. Milco from Dalmatia; resembles the Pendoulier, and may be identical; fine in the San Joaquin Valley; very large oval, but broad and rounded at both ends; grows in clusters; tree a good grower, hardy and productive; fruit excellent for pickles; ripens in November in the interior-about the same as the Mission.

Sevillano.-Recently largely planted as the variety exported from Spain as the "Queen olive." Very large; only useful in pickling; when ripe, bluish black; clingstone. Tree a strong grower, leaves deep green, greenish white underneath. Described by Mr. Roeding as a regular bearer, but requires deep, rich, welldrained soil, and will not stand much cold. Sells at high prices because of great size; ripens early.

Ascolano.--"White olive of Ascoli." Very large, "large as a French prune and much like one in shape" (Bioletti). Excellent for pickles, but not desirable in color of either green or ripe pickles, and has to be carefully handled in pickling. Reported a good bearer and has recently been quite largely planted. 


\section{CHAPTER XXXII}

\section{THE ORANGE}

The orange leads all fruits of California. Near the close of Chapter VI will be found the numbers of trees and value of product, as reported by the United States census of 1910, and the railway shipments beyond State lines for a term of years, upon which the supremacy of the orange rests. In view of this leadership it seems fitting to take a little wider range in the discussion of the significance of the orange in the development of California than has been indulged in the consideration of other fruits, and this is justified by the fact that the orange is the chief exponent of the horticultural resources of the State and its attributes in this direction are shared in varying degrees by the other fruits. The greatness of orange growing in California becomes, then, in various ways the token of our advancement in one of the highest of the agricultural arts, and in the mastery of long-distance commercial distribution of fresh fruits. These achievements are also a demonstration of the quality of our agricultural citizenship.*

The estimate of total production in boxes, and of market value at selling points, of California citrus fruits for a decade is as follows, the citrus year being from November 1 to October 31 :

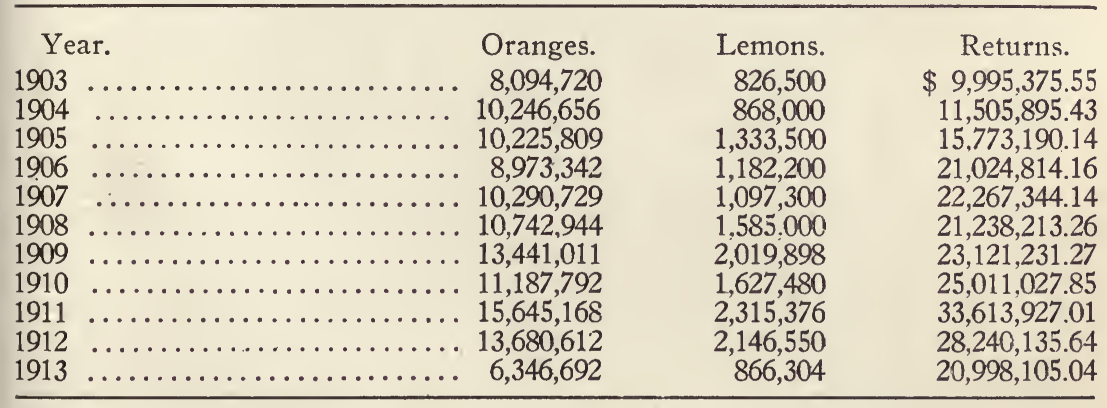

The descending scale since the maximum product of 1911 is due to frosts in 1912 and 1913, in which the decreased quantity was only partly covered by higher prices prevailing in those years. The 1914 product is expected to occupy its proper place in a regularly ascending series.

\section{THE ORANGE INDUSTRY OF THE WORLD}

From the beginning the orange has reigned as king in the international fruit trade of the world. The grape has always been and is still, greater in the value of its contribution to commerce and in the

*The Orange Industry "Encyclopedia Americana," Scientific American, New York, 1904. "The Orange in Northern and Central California," California State Board of Trade, San Francisco, 1903, etc., by the author. 
distance is safely traverses, but the grape rules not as fruit, but through its manufactured products, while the orange carries its natural beauty, fragrance and flavor unchanged around the world. From the earliest times the orange has not only been accepted in northern climes as a symbol of tropical and sub-tropical salubrity and sumptuousness, but by its own distinctive characteristics as a fruit it has won recognition as befitting the highest uses of mankind. By its nature too the orange ministers to its own commercial popularity. It endures long shipment; it ripens slowly and through a season of several months which constitute the winter in northern latitudes when local fruits are scant or absent and the refreshment in the citric juices most welcome. The production of such a commercial commodity has from the earliest times constituted an important industry.

It is a significant fact that though the orange thrives in the tropics it does not resent the slight touch of frost which characterizes semitropical situations. It is also significant that the fruit grown in semitropical countries, especially those which have a more or less distinctly marked two-season climate, differs in character from the strictly tropical orange and is firmer, heavier, more sprightly in flavor and with much better keeping and carrying qualities. The tropical orange has but small commercial importance; the semi-tropical orange rules in the markets of the world. That the semi-tropical orange should have this distinctive character is most fortunate, for it ministers directly to the will for industry which is superior in semi-tropical countries. By the seven degrees of frost which the orange tree will endure without injury, it has gained the seventy degrees of north latitude through which its fruit freely seeks a market. Because, though the tropical orange would reach most distant markets in small quantities, it could never attain the commercial supremacy which the fruit now enjoys.

The sweet orange is a native of eastern Asia and was carried thence to India and to Asia Minor. It possibly reached Portugal from India through the early Portuguese navigators. Thus the distribution of the fruit was westward. The history of modern commercial orange growing consists of a series of progressive movements always trending westward and gaining in volume-the newer centers of production outstripping the older and ultimately largely displacing their product from the greatest markets of the upper divisions of the temperate zone. When the Moors introduced orange growing into Algeria and Spain they displaced the traffic from Asia Minor and gave the Mediterranean region for several hundred years undisputed possession of the markets of the north of Europe and possession also of the American demand when that arose. When the Spaniards and Portuguese carried the orange to the West Indies and to Florida they laid the foundation for an industry which American enterprise developed in Florida until that district not only contended with the Mediterranean region for American markets, but was planning to invade northern Europe by direct shiploads when the demonstration came that the climate of northern Florida and of the Gulf coast westward was too treacherous for commercial ventures in orange growing - at least with the then popular varieties and methods of propagation. But as the Florida supply failed through 

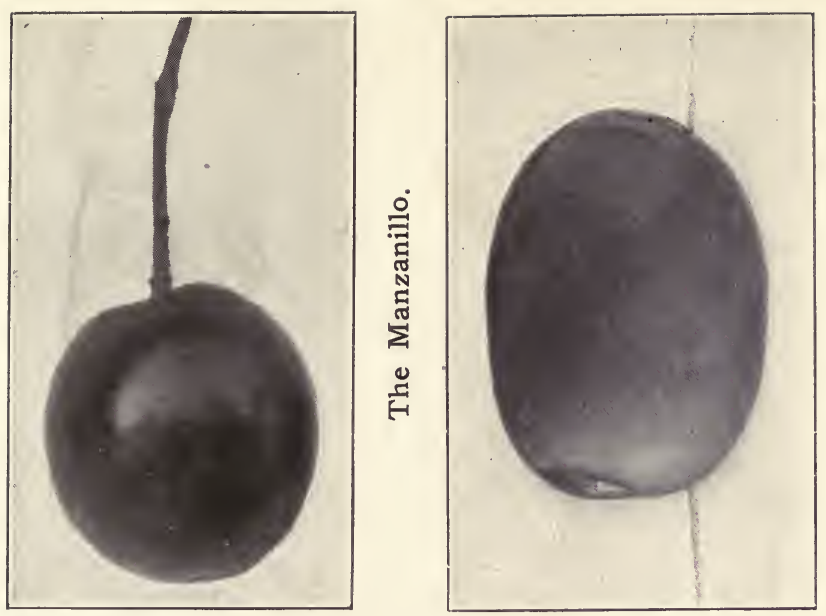

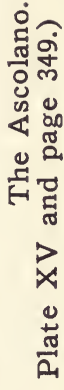

กั

ฮั

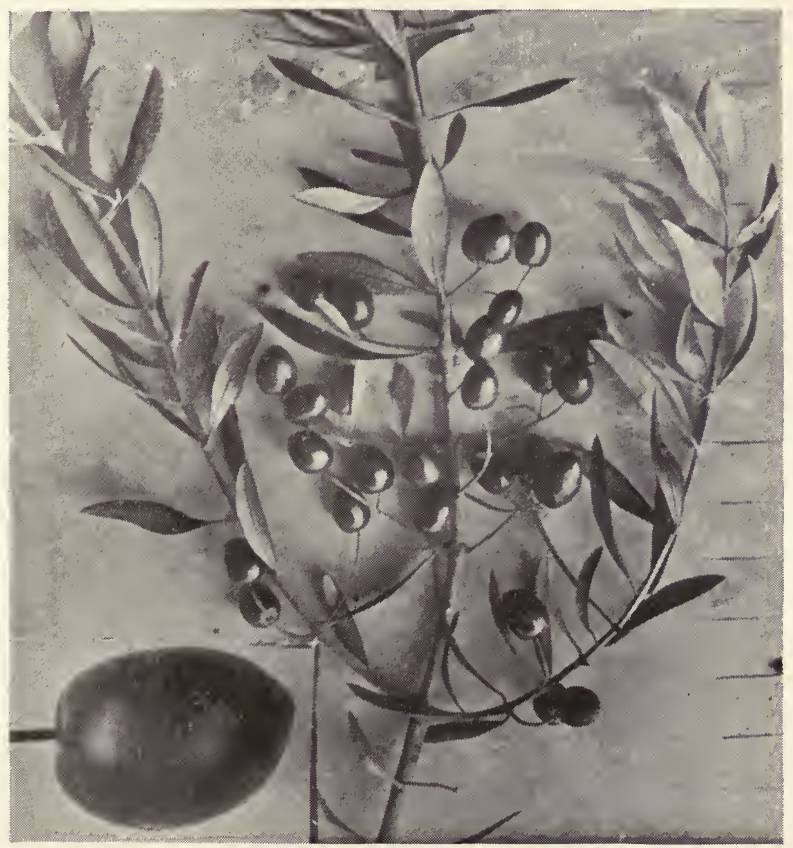

雚

.

홀

竞

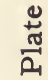



the severe freezing of 1895 , California came forward and, though Florida has re-established her citrus industry, California is now not only supplying the larger part of the oranges consumed in the United States, but is selling the highest priced oranges in the London market against a world of competitors.

In support of this contention let it be noted that the most important statistical statement of the world's citrus fruits in the English language was published April 15, 1914.* It shows that the annual citrus fruit crop of the world is equal to from ninety to one hundred million boxes of California size, which would fill two hundred and thirty to two hundred and fifty thousand carloads of California capacity. Of these the United States and Spain produce thirty per cent each; Italy twentyfive per cent; Japan and Palestine each less than five per cent; the balance, scattering. The acreage both in California and Florida is increasing. The normal product of the United States is approximately 78,000 carloads, and California, in view of recent plantings, 50,000 carloads. All the countries thus mentioned as contributing to the world's commercial product are endowed with semi-tropical and not with tropical climates.

\section{RELATION OF CALIFORNIA TO THE WORLD'S ORANGE PRODUCT}

Competition with the product of California is working hardship in the Mediterranean region because this region can more than supply Europe, and needs American markets as an outlet. Italy has exported six million dollars worth of oranges and lemons in a year, but recently prices have declined and the interest is depressed. Every effort is being made to secure relief from local taxation and from duties imposed by north European countries. The Spanish product of oranges and lemons which ranks with that of the United States, has to meet heavy tariffs in all countries except the United Kingdom and the belief at Valencia is (U. S. Commercial Relations, Vol. 2, 1902, page 686) that the limit of British consumption of Valencia oranges at paying rates has been reached; in fact, the British markets collapsed under the heavy shipments of 1901. When it is stated that the value of oranges imported into the United Kingdom in 1900 was $\$ 10,603,950$, and such a free buyer has more than enough, it can be realized how important it is to the Mediterranean producers that the populous countries of central Europe should hold less strictly to agrarian interests which aim to hamper the entrance of food supplies even if they can not themselves produce them. Manifestly the American product can only enter such markets with a fancy product which will win an extra price, except as a little difference in the ripening season may afford an opportunity.

The commercial position of the orange in the United States is also such as to awaken apprehension, because of the decreased import duties under the tariff of 1913. It will require time to demonstrate what the

*Bulletin 11, California Citrus Protective League, Los Angeles, "The World's Production and Commerce in Citrus Fruits and Their By-products," by F. O. Wallschlaeger, Secretary. 
full effect will be. The product of the West Indies is a direct menace to the Florida product, which meets it in point of market season, and the American product, which is undergoing expansion at the hands, of American capitalists, is constantly feared by the California growers because the Mexican railway will give it quick entrance to the great central states and constant advantage in distribution to the East and the Northwest. The orange from the West Indies and South Florida is different from the California orange in main ripening season and in character of the fruit, but the differences do not give full relief. With the late ripening varieties, the California grower extends his shipments into the autumn and thus laps upon the early fruit from Florida and Jamaica, while the parts of California which bring earliest maturity to the fruit are shipping before the southern fruit is cleared away. In fact, California can keep the markets supplied with oranges fresh from the trees and in prime condition the year round.

As to the difference in oranges grown under humid and arid conditions, the moisture being supplied by rainfall in one case and by irrigation in the other, there has been shown in the arid region orange a superior density, thinness and texture of rind, higher sugar and higher acid percentages and a more sprightly or vinous flavor. The popular conception of the superior sweetness of the orange grown in humid countries is due not to a greater amount of sugar in the juice, but to less amount of acid. The following are the determinations of sugar and acid of fully ripe Southern California and Florida navel oranges:

California Navel .......................

Florida Navel

Total sugar, per cent.

7.46
Citric acid, per cent.

1.45

0.95

Of course, the quality of an orange is largely inherent in the variety, but all varieties are similarly changed by growth under humid or arid conditions of climate and soil, and this modification becomes a factor of much industrial importance. This fact is strikingly illustrated by the standing of the navel orange in California. This variety has been grown for a century or more as the chief orange in Bahia, Brazil, whence it was taken to California. In Brazil it demonstrated no shipping qualities, and according to Burke (U. S. Special Consular Reports, Vol. 1, page 411) would need to be picked before maturity if to be shipped; while as grown in California and Arizona it is picked at full maturity and is successfully shipped all over the United States and to Europe. An examination of the Bahia district in 1913, by A. D. Shamel and associates, showed that the orange which is the foundation of the export industry of California is, in its home, only an article of local trade.

Orange growing in Florida is recovering from serious reverses. The product of 1894 was about $4,000,000$ boxes. Then came the disastrous freezing in December of 1894 and February, 1895, with a temperature of 14 degrees Fahrenheit at Jacksonville, and in the latter year only 75,000 boxes were shipped. In 1912-13 the product was about $8,000,000$ boxes, produced in the central and southern parts of the State. In Louisiana the freezing of 1895 nearly annihilated the 
citrus fruit interest and only recently has there been any disposition to resume production on a commercial scale. In Texas there is a rising orange industry on the basis of hardy Japanese varieties, largely. Its future is a matter of conjecture. In the southwestern corner of Arizona there is a small orange industry which is successfully shipping navel oranges to distant markets. Conditions favor early ripening and an advantage is secured by sale in advance of the main Californa product. From California the shipments of oranges beyond State lines in 1911 were about $16,000,000$ boxes. The orange industry of the United States is now largely supplying the home demand for the fruit. Imports of oranges reached their highest value in 1883 at $\$ 3,010,662$, and have since then declined. The value in 1913 was $\$ 227,827.50$ much less than one-tenth of the imports of thirty years ago.

The orange industry of the United States is unique in the high social and financial standing of those who have engaged in it, and in the striking features of its development. Both in Florida and in California large scale production was first undertaken by northern men who had gained wealth and had lost health in the pursuit of it. They brought capital and commercial ability to the ventures which they exploited. The professional classes of the north also participated largely in the work, bringing scholarship, insight and experience in organization. There were a few also who possessed horticultural experience, but the other classes largey predominated. The result has been the development of an industry characteristically American in spirit and new in methods. It has borrowed very little from the practices of old world orange growers. Free from tradition and prejudice it proceeded rapidly upon the results of original investigation and experiment, establishing a system of culture and of commercial handling of the product which are without precedent in the older orange regions of the world.

\section{THE ORANGE A STATE AFFAIR IN CALIFORNIA}

Thus far the discussion has been based upon the achievements of Southern California and the efforts made to assign them due credit for greatness and uniqueness. The relation of Southern California to other parts of the State in orange growing is not less important and significant.

Citrus fruit trees have been successfully grown in suitable situations in northern California for nearly half a century. There is a famous orange tree at Bidwells Bar, in Butte County, which was started from an Acapulco seed in Sacramento in 1855, and planted out in Butte County in 1859 , which has been generally made to stand sponsor for the demonstration of citrus conditions north of the Tehachapi Mountains, but it is not entitled to all the distinction which has been heaped upon it. In the fifties there were other orange and lemon trees growing in widely separated northern localities-in the valleys of the San Francisco Bay region, also near the rivers and among the low foothills on both sides of the Sacramento Valley. It was, even in early days, the proper thing to include citrus trees among ornamental dooryard plant- 
ings and one reason why the demonstration at Bidwells Bar was so widely accepted as complete, when it was brought forward as a guaranty for commercial planting in the later eighties, was because similar instances of successful old trees existed in many and widely separated places.

This question naturally suggests itself: Why, if such early demonstration was had, was large commercial planting of the orange delayed at the north until after southern California became famous for its orange product? Several good reasons can be adduced. In the first place a disposition toward wider planting did at one time arise and quickly subsided. In the later seventies when the general rush to fruit growing, which has resulted in the present vast extension of the interest, began, citrus fruits were not overlooked. There was a sharp demand for orange trees. Southern California nurseries had a large overstock of trees budded on China lemon roots which southern California planters had learned to despise as forcing excessive growth of tree, and large, coarse fruit. The natural tendency of such a root, exaggerated by excessive irrigation in the nursery, gave a stem as thick as a broom stick and higher than a man in a few months' time, and these soft monstrosities were sent north by carloads, by astute tree speculators, and sold to unwary planters, who thought they were getting a great deal for their money. Such trees were planted in all sorts of situations and their broad leaves made a fine display as soon as planted. There were fond anticipations of evergreen orchards everywhere from the swamps to the hillsides. Then came the cold winter of 1878-9. The temperature in places reasonably situated was not very low-not lower than is frequently encountered in southern California and not low enough to injure well placed old trees, though it did destroy some illplaced ones and helped to define suitable situations for citrus culture in the north as such temperatures have also defined them at the south. But the degree reached was fatal to those soft trees on a lemon foundation almost everywhere, and the disappointment of the new planters who based calculations upon them, discouraged them from further efforts toward citrus culture for some time. It was not a logical conclusion because a careful inquiry made after the frosts in 1879 elicited careful written statements from sixty-nine orange growers, living in thirty counties and fully justified this conclusion, which was at that time published: "this mass of testimony shows that orange growing is no longer an experiment in the north, and that, notwithstanding the severe frosts of such winters as this, orange and lemon trees can be profitably cultivated in nearly every county in the State, and by selecting favorable localities, no district, except it be situated in the high Sierra, need be without these most beautiful and useful fruits."

But there was another and more logical reason why the well suited lands in the central part of the State were not at that time given to citrus fruit culture. Citrus fruits require irrigation everywhere; deciduous fruits, including the grape, do not require irrigation except in places of shallow soil or light rainfall. Without waiting for irrigation facilities then, hundreds of thousands of acres of deep valley loams were immediately available for the planting of deciduous fruits. The 
growers understood these fruits, while the orange to an English-speaking people was an unsolved problem. The long list of deciduous fruits had varieties to suit the taste and ambitions of all planters and the opportunity for selling many different fruits and their different products seemed illimitable. "The world for a market" seemed a reasonable proposition, for deciduous fruits and their products had been shipped to eastern markets since the first overland railway was opened in 1868, and very large prices were attained, just often enough to be alluring. No citrus fruits had been shipped out of the State on a commercial scale, and no one knew that they could be, profitably. The central and northern districts threw their full strength into the deciduous fruit interest and the result has justified the effort, for, at the present time, the annual shipments of deciduous orchard fruits fresh dried and canned; the grape, both fresh, as raisins and as wine and brandy, has reached a total value of about sixty millions of dollars-almost all of it from the regions of California north of the Tehachapi Mountains. The engrossing requirements of this grandly successful undertaking gave northern growers, packers and capitalists no leisure to think seriously of citrus fruit planting - that was left for a decade and a half to the special attention of the southern California people, and they developed it splendidly for the settlement and upbuilding of their portion of the State-reaching a total value of product sold beyond State lines, of about eighteen millions of dollars. The chief reason, then, why, although citrus adaptations were demonstrated very early in the upper part of the State, the commercial planting was largely postponed to the present decade, was that the people were too busy developing a greater fruit industry to which their conditions were superlatively suited.

During the last few years new interest has arisen in citrus fruit growing in northern California and all the scattered experiences of the last half century are becoming of inestimable value in guiding this planting aright. There are several important reasons why the north has now turned to the orange.

First: Deciduous fruit production has reached large volumes, margins have become reduced to those which assert themselves in any well established and extensive industry, and some of the early glamour has gone out of it. It will henceforth proceed soberly, and consequently safely, to grand aggregates which no one can foresee, but it is readily demonstrable that with the present rush of population to the more wintry districts of the Pacific slope, the opening of Asiatic connections and the victories being attained each year in the distant East and in Europe, our production of deciduous fruits and their products will go steadily forward. Increased interest in citrus fruit planting in northern California is in no sense a menace to the deciduous fruit industry. It is merely a new graft upon a very vigorous industrial stock.

Second: Owing to natural conditions which will be briefly explained presently, orange growing can be pursued at the north without competition with the main crop in southern California. The northern California crop will be consumed before the bulk of the southern crop moves from the trees. 
Third: All California oranges have characteristics and qualities which are recognized as of distinctive excellence, and therefore have a commercial advantage which enables them to compensate the high grade American labor which is employed in their growth, packing and marketing, and leave a reasonable return to require the grower's effort and investment. This being so, the production, so long as protection is continued in adequate amount, justifies extension of the effort to produce an American orange for Americans.

Fourth: Semi-tropical fruits are nature's demonstration of the existence in a place of a climate which promotes health, comfort and a maximum of physical and intellectual attainment in mankind. Probably all that is urged against tropical climates as enervating and depressing of human standards is true, but not a word of it applies to an arid semi-tropical climate, in which the blessing of dry air and freedom from the debilitating effect of temperature extremes rejuvenate the old and weary and bring the young to stature and stalwartness which all newcomers notice in the rising generation of Californians. Of the existence of such conditions a well-grown orange of the California type is unimpeachable evidence. It has brought a hundred thousand people and a hundred millions of capital to southern California which would not have come otherwise. In the conscious strength with which northern California has recently awakened to make systematic effort for settlement and development, the orange is accepted as an exponent of the possession of those natural characters of sky and air and soil, constituting the most desirable environments of human life-the highest desirability in the location of a home.

Fifth: It is but a corollary of the foregoing that the successful and profitable production of citrus fruits is par excellence the motive force in promoting colony efforts and in drawing into horticulture the class of people which constitutes the most desirable element in the upbuilding of a great State-people who know what is noble and desirable in human life and desire it for their children; people who know how to secure what their aspirations and tastes approve; people who by intellectual force and training and by successful professional and industrial experience are prepared for attainment in the higher horticultural arts and in the new commercial efforts which make those arts profitable. The splendid development of southern California communities upon a horticultural bases points the way to achievements in other suitable parts of the State, and the citrus fruits become then the token, not alone of superior natural endowments, but of the type of manhood which can use them to the best advantage. None know this better than the southern California people themselves, and it is a demonstration of the desirability both of the natural resources of northern California in citrus lines and of citrus fruit culture itself, that in all the newer citrus regions at the north, there are to be found among the leading planters and promoters, southern Californians who have sold their early plantings at the south at high prices to newer comers and have started anew in the northern districts, where they find cheaper land, more abundant water supply and fruit which is marketed at an earlier date. 


\section{DISTRIBUTION OF CITRUS CONDITIONS IN CALIFORNIA}

The claim has been made above that citrus culture conditions exist in suitable situations in central and northern California from Shasta to San Diego county, and historical evidence has been cited to prove it. It is so surprising that practically the same climate should be found through a distance of between seven and eight degrees of latitude that many, even of those who have lived in California, do not appreciate the fact, nor know the explanation of it. An effort is made toward such explanation in Chapter I of this work. Even at the risk of repetition the subject will be reviewed with special reference to the occurrence of conditions affecting the growth of citrus fruits.

First: California is not only blessed with benign ocean influences, but northern California is additionally protected from low winter temperatures by the mountain barrier or the Sierra Nevada, extending southward from the multiplied masses of protecting elevations in the Shasta region, while Southern California enjoys the protection of the Sierra Madre and other uplifts on the north and east of her citrus region. Northern blizzards are, therefore, held back from entrance to California and are forced to confine themselves to southerly and easterly directions over the interior parts of the Pacific slope, while the great blizzards of the northwest traverse the Mississippi Valley and, if they have sufficient impetus, extend to the gulf and carry destruction to semi-tropical growths even in northern Florida. The ocean then bringing warmth and the high mountains defending against cold, combine their influences to give nearly the whole length of California semitropical winter temperatures.

Second: Although this striking similarity does exist, in citrus districts north and south, there is another even more startling proposition involved and that is the influence exerted by the presence of the coast range as the western boundary of the great interior valley of the State, and intervening between that great valley and the ocean. The several ridges of the coast range with their enclosed small valleys serve as a colossal wind-break against northwest winds, which might otherwise, now and again, bring a temperature too low for citrus fruits, where now they are safe from injury. The chief effect of these mountains is to protect the northern interior valleys and foothills from the raw winds of early springtime and to allow the sun as he crosses each day higher in his course, to expend the increasing heat directly in promoting vernal verdue. The result is a protected interior region in central and northern California, of quick growth in all lines-early pasturage, early grain harvest and early fruit ripening. The valleys of southern California, which have thus far been largely developed, have no high range between them and the ocean. They are open on the west because the coast range of mountains takes a sharp turn eastward in the southern part of the State and afterward curves southward, passing along the eastern side of the chief productive region. The influence of this opening of the valleys of southern California is not so unfavorable as such opening would be at the north, because ocean winds are 
gentler and warmer there, and there is winter service rendered by this eastward trend of the southern California mountains, as has been said, but the fact remains that the absence of high barriers against ocean influences retards the springtime and causes a slow development of summer conditions and late ripening of fruits, while the presence of high barriers at the north so hastens springtime and summer heat, that early summer fruits in California are shipped from the north to the south-a thing which does not occur anywhere else in the northern hemisphere. It is due to this same early start which the local topography gives to the orange, followed by the high summer heat which is essential to the development of a good orange, that the orange reaches an acceptable commercial condition at an earlier date in suitable interior places at the north and is at present being almost wholly shipped to eastern markets before free movement begins at the south. This early marketing also relieves the growers of much anxiety and costly frost fighting, because the fruit, which is always more susceptible to injury that the tree, is out of the way before the frost period, which usually begins about Christmas, is reached.

There is in southern California, east of the mountains, a district which has thus far been but scantily developed where protection from ocean influences tends to early ripening of fruits. The same is true of some parts of Arizona adjacent, and small quantities of early fruits move westward and northward from that region. That region is not in view in this discussion, for too little has been accomplished in citrus lines to warrant conclusions which the present confident planting in that part of the State will soon supply.

Third: Still another feature of local topography must be mentioned as influencing citrus conditions north and south and explaining why winter temperature has fallen no lower at the north that at the south. At the north the snow fields of the high mountains are farther from the valleys and mesas, where citrus fruits are grown than they are at the south. The benches and low foothills of the Sacramento Valley, for instance, are forty to fifty miles from the high range to the east of them and there intervene countless ridges of high foothills and small valleys, and before the citrus plantations can be reached by the descending air currents they are considerably warmed by rustling over so much land which has been warmed by the ampler winter sunshine. From many of the southern citrus regions one looks almost directly upward and outward upon the grand snow-clad mountains, whose crests are but fifteen to twenty-five miles away. It is a splendid scenic effect-ripening oranges and dazzling snow fields in the same glance of the eye, but it is sometimes not so grand as a pomological proposition.

Fourth: Another protective influence for citrus fruit trees during the frosty period of December and January, is the low canopy of land fog which covers the interior valley of central and northern California much of the time at that season of the year and checks the radiation of ground heat which is apt to take place rapidly under a clear sky. Though the nights are thus often protected from frosts, the day temperature is held low, which is also of account, because the citrus trees are held dormant, which is desirable, as there is no fruit to ripen. On 
the other hand, the higher day temperature in southern California is valuable in that district because the later fruit is still maturing. The winter aspects of the trees in northern and southern California are therefore quite different; in the north, the dark green of dormancy; in the south, the gold of the fruit and the oft-protruding light green of the winter wood growth. In both regions all growth conditions seem good; each after its own kind, and the two, in a sense, complimentary.

During the last few years there has been rapid extension of orange planting, particularly in the suitable lands on the eastern rim of the San Joaquin and on both sides of the Sacramento valley. The orange product of the northern district is normally about one-eighth of the State product, though in 1913 it rose to about one fourth of it because it was marketed before the frost period. It will increase rapidly, for of about two million trees now growing, only have half attained bearing age and planting is still being freely undertaken. Three-quarters of all the trees north of the Tehachapi mountains are in the citrus districts of eastern Tulare County.

\section{THE GENERAL OUTLOOK FOR THE ORANGE}

At the present time orange growing has a very promising outlook. The prospect for much larger consumption, at the east and abroad, is very encouraging. The orange is passing from its old status as a luxury to its proper recognition as a staple winter fruit for dwellers in cold climates. For such use the agreeable acid and sprightly flavor of the California fruit especially commends it. The consumption of the fruit per capita, away from California, is still small and will be greatly increased when people know better its desirability and the reasonable prices at which it can be secured. This wider distribution is to be confidently expected and the rapid increase in population through the great west and north is each year giving California growers nearer markets of growing consumptive capacity. It will be of great advantage to the whole country, as well as to California, to have production steadily increased.

Some considerations in this line are given to the closing pages of Chapter VI. To fully appreciate the advantage of California in supplying the whole northern half of the North American continent, especially with early fruits and semi-tropical fruits, one should calculate the exceedingly small area of suitable fruit land in California as compared with the area of the half-continent indicated, which is our logical consuming territory and in which development and population are now increasing so rapidly. Take a map of the continent, place your thumb over the area of California and gaze at the uncovered area, north of the fortieth parallel, if you please, and estimate the duty of California in coming decades. Millions will multiply on the continent, but the safe area for tender fruits will never increase an acre. From this point of view, it really seems impossible for California to create an oversupply of fruits which are distinctively her province to grow. There are likely to be temporary surpluses and problems in distribution, but a general oversupply seems unreasonable. 
The development of the orange industry in California to utilize the splendid natural adaptations which have been discussed, to make good the large investments which have been made, and to afford a field for the profitable employment of the high quality of American citizenship which has entered the list of producers, several things are essential. First, the advancement of horticulture art and science. Second, the maintenance for a time at least, of a sufficient protective tariff, as has already been suggested. Third, the extension of co-operative handling and distribution of the product as now embodied in the successful operation of the California Fruit Growers' Exchange and its auxiliaries in all the producing districts. Fourth, the pursuit of systematic and intelligent inquiry and experiments into the durability of the fruit in transit as affected by cultural and commercial practices, and the reformation of policies and methods in accordance with the results of such investigation as conducted by Mr. G. Harold Powell, formerly of the United States Department of Agriculture and his associates. Every grower of oranges should familiarize himself with this work.*

\section{SOILS AND SITUATIONS FOR THE ORANGE}

While citrus conditions do exist through large areas of California, there is still danger of loss and disappointment through unwary individual investments and unwise locations of citrus colony enterprises. The tracts of land for orange planting even in a favorable citrus climate are limited in area and every citrus climate has numerous places where local meteorological conditions will prove destructive to the profit of the enterprise, if not to the life of the trees. The orange is a hardy tree, judged within its temperature limits, but there is no money in a tree which is subjected to any kind of hardship. For this reason the selection of a good depth of strong, free loam should be made, for such is essential not only to good growth of the young tree, but to its support through the long productive life which the orange enjoys. Depth of good soil is not only a storehouse of plant food, which will postpone the use of purchased fertilizers, but it is a reservoir of water so that irrigation can be applied in larger amounts at longer intervals. While it is quite possible to grow an orange tree and to secure good fruit on shallower soils, if conditions are kept just right by frequent use of water and fertilizers in just the right amounts, such conditions impose heavy burdens in their constant requirements of extra care and expenditure, and these are handicaps of no small economic importance. The tree can not live upon climate as a man may, because a tree can not speculate; it must have a good foundation in the earth as well as a good outlook in the sky.

Growing orange trees on defective soils has brought disappointment and loss in all parts of California. Ample supplies of irrigation water available have encouraged over-irrigation where trees have been planted above hardpan, and drainage is absent. Dying-back and yellow leaf

*"The Decay of Oranges While in Transit from California," by G. Harold Powell, Pomologist in charge of Fruit Transportation and Storage. Bulletin 123, Bureau of Plant Industry, U. S. Dept .of Agr., Washington, D. C., 1908. 
have appeared in some groves and have been accounted for by digging to find the roots bedded in mud and slush. All plantings over clay subsoils should be guarded against this danger. Digging deep holes and filling them with good soil is setting a trap for the future failure of the tree unless the deep hole is properly drained by the nature of the subsoil or by artificial provision. On the other hand, planting over a gravelly sub-soil is often disappointing, because the water passes through the sub-soil as through a sieve and the tree shows distress although generous amounts are applied to the surface. Wide observation through the State teaches that such warnings are needed by the unwary. There has also been injury to the trees from planting over sub-soils carrying excess of lime.

Local temperature conditions even in sections generally suited to orange culture should be carefully ascertained. Frosty places must be avoided. A few feet difference in elevation may change profit to loss, but one must not therefore draw the hasty conclusion that all small elevations are favorable. The experience of the last few years shows that nothing is, on the whole, more dangerous than the warm bottom land in a small elevated valley which seems naturally protected on all sides. There are many such places which are far more treacherous than the uplands of the broad valleys, which may be considerably lower. The benches around the sides of the small valley may be safe and the bottom of the same valley dangerous because there is no adequate outflow for cold air to the large valley below. Look out for small valleys which have divides of crumpled hills where they debouch into the main valley. Cold air can be dammed and held back; consequently the low land of a small valley may be worse than lower land in the main valley, because in the latter there are air currents which prevent accumulation of cold air in particular places. These air movements make some plantings on the upper plains of the main valley safe, though the whole region may seem to the eye rather flat and low, but, of course, broad sinks of the main valley may also be dangerous. Too great elevations are to be guarded against. Where one approaches the reach-down of mountain temperatures and loses the warming influences of the valley mesas, the danger line is at hand.

An ample water supply is essential. Small waterings which may bring satisfactory growth to a young tree are no measure of the needs of a bearing tree. The orange is using water all the year, as discussed in Chapter XV. Its crop requires nearly a year to reach maturity. Both in leaf growth and fruit growth it nearly doubles the activity of the deciduous tree and all the time it is pumping water with its roots and pouring forth water into the air through its exposed surfaces. No investment in orange planting can be profitable without assurance of adequate water supply.

\section{PROPAGATION OF THE ORANGE}

The orange is grown from cuttings, layers and seeds. Growth from the seeds is the method almost exclusively followed, and by far the best, but the others will be mentioned briefly. 


\section{Growth from Cuttings.-The method of propagation is described in Chapter VIII.}

Growth from Layers.-The orange roots readily by layering, the drooping branches being partly cut through, buried in the soil with the terminal shoots above the ground. Layers must be kept moist. Layering may be employed to obtain a few plants easily, but, otherwise, it cuts no figure in propagation. Layers and cuttings, of course, reproduce the original variety without recourse to budding.

Growth from Seed.-The orange is grown upon seedlings of the bitter orange or orange of Seville, generally called "Florida sour stock"; of the common or sweet orange and of the pomelo or Grape Fruit. Good plump seed should be selected in growing orange seedlings either for their own fruitage or to use as stocks for budding.

When seedlings for fruiting are grown, select seed from a choice variety in a situation where other citrus species are not grown; but the orange can not be trusted to come true from seed, and, more than this, the seedling class for fruiting purposes has been practically abandoned as unprofitable to plant, though fruit from old seedling trees is occasionally sold at a profit.

In securing seed the fruit is allowed to rot and when thoroughly decayed, it is pulped by mashing in a barrel, and the mass is washed, a little at a time, on a coarse sieve, the pulp passing through, and the seed being caught on the wires, and pieces of skin thrown out. The plump seed will sink if thrown into water, and the imperfect can be skimmed from the top. The seed should never be allowed to dry, and unless it is to be sown at once, should be stored by mixing with moist sand, from which it can be afterwards removed by sifting; or it can be kept for a time in water, changing the water from time to time to prevent souring. The best time for sowing orange seed is after the ground has become warm in the spring.

Orange seedlings are grown either in boxes or in the open ground. In either case a rich sandy loam which will not bake should be secured or artificially made by mixing sand with rich garden loam. Boxes of about two square feet area and six inches deep are convenient to handle. The bottom should have holes, or sufficient crevices for good drainage. Fill the boxes about four inches with the prepared soil, place the seeds about an inch and a half apart each way, sift over them about an inch of the soil, or a little less of the soil, and a layer of clear sand if it is at hand. It is essential that the soil should be kept moist, and light sprinkling daily, or every other day, with water that has been warmed by standing in the sun, is desirable. Seed can be sown in boxes in the house at any time if plenty of light and heat are given. If the boxes are to be out-of-doors, it is best to sow in the spring, and to rig up a cover of cheap cotton cloth, suspended about a foot above the surface, to prevent effect of frost at night, and of burning by sun heat by day. The seedlings usually appear in about six weeks, and with good care in weeding and keeping sufficiently, but not excessively, moist, they will make a growth of about a foot the first season. Some growers collect the boxes in a sheltered place, and build over them a 
lath house, tacking on old sacks or other cloth, to shield them from the sun and frost. The lath house keeps animals from running over the boxes, etc.

Growing seedlings in an open bed involves about the same operations. To guard against intrusion, it is advisable to make board sides to the bed about a foot high, and to make lath frames which will reach across, resting on the edge boards. A cloth sun-and-frost shade is also desirable, to be laid over the lath frames when it seems needed. Beds should be made narrow enough so that one can easily reach half way across from each of the long sides for weeding, etc. In open seed beds it is usual to broadcast the seed evenly, using about one gallon to 200 square feet, which should give about 5000 seedlings.

The Orange Nursery.-Planting out in nursery is usually done after the ground is thoroughly warmed in the spring, and the seedlings are then about a year old. The preparation of nursery ground and the planting out of the seedlings can be done as described in Chapter VIII. Orange seedlings should, however, be given greater distance apart than is usual for deciduous trees, because the orange remains longer in the nursery, and because it is often desirable, when taking up, to sack the ball of earth embracing the roots. If the roots are not to be sacked, about nine inches will do between the plants; if to be sacked. the distance should be twelve or fifteen inches. The rows should not be too close in the orange nursery. If horse cultivation is to be used, at least four feet between the rows should be allowed, and even greater distance is desirable. In taking the seedlings from the seed beds, a few should be lifted at a time, and their roots kept shaded and moist until the ground closes on them in the nursery row. To get an even stand in the nursery, small and weak plants should be placed by themselves, or set in boxes to take another year before going into nursery.

Young trees in nursery are very liable to frost injury, and it is wise to protect them by some sort of a cover during the winter. A framework covered with cypress brush is often used, the whole being cleared away in the spring, to allow of summer cultivation. Cultivation of trees in nursery is about the same as with deciduous fruit trees. The horse should be used, and the surface kept perfectly pulverized. The cultivator should follow irrigation as soon as the soil will admit of it. Frequency of irrigation of nursery depends, of course, upon local conditions. Some give two or three irrigations, by running the water in a little trench alongside the rows, at intervals of two weeks, for a time. after planting, and then irrigate once a month during the summer. It is important that irrigation should not be continued too late into the fall, because the young tree should harden its wood before cold weather. Nor is it desirable that the growth be too rapid. A good growth of sound wood is better than extra size.

Length of Time in Nursery.--Seedlings are usually budded after being one or two years in the nursery, or at two to three years from the planting of the seed. At a convenient time in the winter the lower shoots and thorns are removed from the seedlings, so as to leave a clear stem of about six inches for the convenience of the budder. 
Intensive Work in the Citrus Nursery.-In addition to the foregoing general reflections the reader may be interested in a specific sketch of pushing a citrus nursery as described by Mr. R. E. Hodges:

Mr. Allen Dodson, of Los Angeles county, put 17 hay-rack loads of the rottenest manure he could find on a sandy space $290 \times 60$ feet. This had to be worked and reworked to leave no lumps of manure. The sand is necessary to prevent baking. Then he planted $81 / 2$ bushels of seed, watered carefully about every other day and kept the weeds down for six weeks before they came up. They grew about a foot before cold weather came. Around the seedling bed were set upright $2 \times 4$ s twenty feet apart and 6 feet out of the ground for head-room. From post to post were $1 \times 3$ boards on which lath were nailed upright about $11 / 2$ inches apart. Above these laths is a two-foot open space and then a roof similar to the sides but made in 20 -foot squares so it may be easily removed. This open space (to get head-room) should also be lathed, on the south and west at least, because the low winter sun shines directly onto the seedlings and may turn them yellow. The shadows under the lath are perpetually moving with the sun so that direct light never stays long at a time on a given tree. The movable roof makes it easy to set up the outfit on new ground. One year Mr. Dodson sold 185,000 seedlings from a certain plat and tried it again the next year on the same place, getting only 4000 salable ones. He had used only a third as much manure the second year, thinking to have some advantage from that applied before.

\section{BUDDING THE ORANGE}

The orange root is the best foundation for an orange tree, and the seedling sweet orange has been the main reliance. The seedling of the Florida sour orange is now being used almost to the exclusion of other stock, for its general hardiness and thrift and to escape gum disease. It has not been entirely free, though conceded to be less subject to the trouble. Oranges have also been worked upon pomelo seedlings, which force a strong growth, root deeply and are satisfactory. Of course, many lemon, and recently many pomelo trees, have been worked over the orange, but in these cases the orange root was below the other wood. All lemon roots are not suitable for the orange. The Japanese practice of dwarfing with the citrus trifoliata has never prevailed in this State. Recently the trifoliata stock has been used to a small extent to secure earlier ripening of fruit, and the tree claimed to be sufficiently free growing, but the experience favors the other roots for standard trees.

Budding is almost exclusively adopted for working in desirable varieties. The best time to bud is about the time the new growth starts on the seedling in the spring, though some practice budding in midsummer and fall. Good, well-matured buds only should be used; those from both base and tip of the shoots are frequently defective. Buds should be taken only from fruiting branches; not from sterile sucker growth, and from trees which are known to bear abundantly a good type of fruit. For spring budding, buds can be taken from fruiting trees and kept dormant in moist sand in a cool place until the seedlings show a sap-flow suitable for budding.

The method of budding described in Chapter IX is that usually employed in budding citrus trees, and the rules for loosening the ligature, etc., are similar. Midsummer buds are apt to have soft growth at the coming of cold weather; fall buds remain dormant until spring; 
spring buds start to grow almost immediately, and have the benefit of the whole summer season for growth and maturing of wood.

After the bud has started out well, the top of the stock should be removed at a short distance above the bud, and suckers on the old stock should be continually looked for and removed. The tender shoot of the bud may be protected by tying to the stub, and when the growth of the bud has become strong enough to support itself, the old stock is smoothly sawn away above the bud and the wound covered with liquid grafting wax or paint.

The care of budded trees in nursery is similar to that of the seedlings of the previous year. If too great a tendency to branch low down is observed, the tips of the lower shoots should be pinched, but it is not desirable to under-prune much; the retention of the lower branches thickens the stem. Sometimes a very rank growth on the bud will need a stake to strengthen it or to protect it from blowing out. The intrusion of gophers and other vermin should be resolutely and persistently guarded against.

Working Over Old Trees.-Old orange trees can be transformed into improved varieties either by budding or by grafting, as described at the close of Chapter IX, though re-working by grafting has been almost entirely superseded by budding. The common way to bud over an old tree is to cut back part of the branches and force out new shoots, the best of which are selected for budding and the others removed. Sometimes only a part of the tree is removed at first, and when the new buds have grown out on that, the other part is similarly treated. Others remove the whole top except a single low branch to maintain sap circulation until after the new buds start.

Recently the practice of budding into old bark has been widely adopted as the quickest way to secure a new tree. As with working into old bark in other trees, it is necessary to take an older bud and a larger, thicker shield of bark behind it, than when budding into younger stock. Some remove the wood from the back of the shield, but generally it is not done. The following is an outline of practice approved by the Redlands Horticultural Club:

Keep the buds carefully in a damp cloth. Slide the bud upward, above the cross section. Bind around the bark, steering clear of the buds proper, a wrapping of waxed cloth, already prepared, three-fourths of an inch wide. When enough of this has been wrapped about the tree trunk or branch to keep the bark and the bud in place, rub the end of the muslin strip with the handle of the budding knife, down upon the muslin already wound about the bark. This will hold the waxed wrapping firmlv in place. From ten days to two weeks after the buds have been inserted, cut off the entire top of the tree, above the buds, and cover the stump of trunk or large branch with grafting wax -applied hot-with a brush.

As soon as the wax is put on-and it must be put on as soon as the top is sawed off-whitewash the tree, over the waxed cloth, also over the bud, over every part of the tree that is left, except the stump ends, to which the hot wax has been applied. Immediately the buds will begin to grow. From ten days to two weeks after whitewashing take off the muslin wrap, and, if the work has been done carefully in accordance with the above directions, 90 per cent of the buds will develop-perhaps more. A prime necessity for this work is a razorsharp budding knife. 
There is a variation in practice in cutting back the stock above the bud. Instead of cutting back at once, heroically, as just described, some girdle the branch or cut back part of the top at a distance above the bud, cutting down to the bud after it shows good strong growth, tying the young growth to the stub at first to protect it. Others insert the bud in the fall, cutting back to start the bud after the fruit on the old top is gathered. It is very important to watch for suckers below the bud and remove, to pinch them back, to make a bunch of leaves. The growth from the bud itself often needs pinching to induce low branching. Twig-budding can also be used on the orange by the method already described for the olive.

Budding in old trees is best done in the spring, when the sap flow is strong, but, as stated, can be done in the fall and the bud allowed to lie dormant until spring.

\section{PLANTING ORANGES IN ORCHARD}

As already stated, orange trees are planted out a greater age than deciduous fruit trees. Budded trees are given one or two years' growth in nursery and one or two years' growth on the bud, which, added to the year in seed bed, makes them three to four years of age from the sowing of the seed. Seedlings, to be planted out as such, are allowed two years' growth in the nursery, which makes them three years old from the seed. For this length of time and the unusual care involved in their growth, taking up from nursery and the preparation for carriage, orange trees of planting age are of much greater cost than deciduous fruit trees.

Since the growing of seedlings for their fruit has practically ceased, the distance between the trees in orchard planting has ranged from twenty to twenty-four feet. All the varieties now propagated are quick to bear fruit, and if properly shaped will find ample space in these distances-the greater distance on the richer soil as a rule.

Preparation of land for orange planting by deep and thorough cultivation and laying off to secure straight rows by the square, quincunx, and hexagonal methods have been quite fully discussed in Chapter X, and Chapter XI has suggestions for planting, many of which are applicable to the setting of orange trees. There are, however, special methods employed in lifting the orange trees from nursery rows and in placing them in permanent position, which will be outlined.

The orange, in common with other evergreen trees, is exceedingly sensitive to exposure of its roots, and for this reason the handling of the young trees is very different from that of ordinary orchard trees. Three ways are employed for securing this constant moisture of the roots, as follows:

Packing in Wet Straw.-As fast as the trees are lifted from the nursery ground by digging carefully so as to loosen and secure all the roots possible, they are packed in damp and partially rotten straw, 
proper receptacles being at hand so that the roots are not exposed by carrying them any distance. In taking up, all roots bruised by the spade are cleanly cut with a sharp knife. The tap-root is cut away at a depth of a foot or so from the surface. This use of wet straw, if faithfully carried out, will answer well in taking trees short distances for planting, but the use of a puddle on the roots before packing in damp straw gives additional assurance of success. Large shipments of trees have been made by packing roots in damp moss in specially made chests holding 100 to 125 trees. These chests have top and one side on hinges so that the roots can be thoroughly bedded in the moss as filling proceeds. The hinged side and top are then brought together and closed with hasp and staple.

Puddling the Roots.-This method is also used for deciduous fruit trees, as mentioned in Chapter XI. It consists in having a thin puddling of loamy soil with preponderance of sand rather than of clay, into which the roots are dipped as soon as the tree is lifted from the nursery. This mixture, which should be about as thick as cream, may be made in a hole in the ground, or, better still, in a box or cask large enough to allow complete plunging of the roots. As soon as dipped, and with all the mud which will adhere, the roots are packed in wet straw. If the trees are to remain thus packed for any length of time the greatest care must be observed to keep the straw damp, and water must be applied gently to avoid washing the puddle from the roots.

Sacking the Roots with a Ball of Earth.-This is a very satisfactory way to move orange trees, and if it is well done, the tree does not wilt, and may be moved long distances and handled more freely than the puddled roots. To ball and sack trees, dig a trench along one side of the row about six inches away from the trees, and about a foot and a half deep. By careful digging under each tree from this trench the tap-root is reached, and severed by a cut with a sharp spade. The side roots are also cut by thrusting the spade down on the three sides not opened by the trench. The top earth is carefully removed nearly down to the highest lateral roots, and after being sure that the roots are severed all around, the tree is lifted out with the ball of earth which encloses the remaining roots. This ball is rounded off carefully and then placed on a half of a grain sack or other piece of burlap, the corners of which are drawn up and tied around the stem of the tree with baling rope. It is also an additional surety of safety to allow the baling rope to run under and around the ball to aid in holding it together. The balled trees must be carefully handled so as not to break the ball which would result in tearing to pieces, as well as exposing, the roots.

The manner of handling the trees depends somewhat upon the character of the nursery soil. Successful balling of course requires a certain amount of adhesiveness in the soil.

One can not be too careful in the handling of orange trees. Though they will stand harsh treatment when in permanent place, they must be most carefully transplanted. Lifting from the nursery when the soil 
is too dry, exposure of the roots, or careless planting, will consign the tree to a slow, sickly growth, and often kill it outright.

Cutting Back at Transplanting.-The rule of reducing the top to compensate for the loss of roots, is vital in moving orange trees, but sometimes cutting back is carried too far and subsequent growth is checked rather than promoted. Some growers cut back the young trees a little while before lifting them from the nursery. Some take off all leaves after planting out, and claim that growth starts sooner and more strongly, but it is doubtfull whether defoliation is advisable, except in case of wilting, when it is necessary.

Digging Holes and Setting Trees.-The same considerations which require extra care in lifting trees for nursery, rule in putting them in permanent place. All authorities on the subject specify exceptional care in preparing the tree holes as a profitable investment on the part of the planter. Large and deep holes are commended, provided the planting is done in a deep, free soil. Deep holes would be more injurious than beneficial in a tight sub-soil, unless drainage were furnished, but there are good orange trees now bearing in such places - good enough at least to be an ornament and acceptable fruit producers for family use.

On large-scale planting in deeply prepared soils, holes large enough to allow good spreading of the roots are sufficient. Handling the soil at planting has been fully described in Chapter XI, and the importance of bringing the soil into firm contact with the roots has been urged. The use of water in planting citrus fruit trees is especially desirable. Trans-planting should be done just as the growth is starting in the late spring or early summer, and this is the opening of the dry season and rains can not be expected. Therefore, when the roots are arranged and the top soil lightly trampled around them, water is run in the hole and the earth compacted around the roots by water settling. After the water has settled away, the hole is filled and the surface left loose to prevent evaporation.

These instructions apply to the planting out of trees which are taken up with long roots and puddled. In planting out balled trees, the sack is not removed, but after the tree is embedded in the earth, the tying rope is cut. The sack soon decays in the soil.

Orange trees can be successfully transplanted at different times of the year, but the best time, as just stated, is after the ground gets well warmed by the spring sunshine. The date at which this condition arrives depends upon locality. Experience seems to indicate that the young orange tree is in best condition to transplant just as the new growth is starting out, and preferably when it has not grown out more than two inches. In setting the tree, it should stand not lower than in the nursery. Root injury is sometimes due to deep planting.

Great care must be taken that transplanted orange trees do not become dry after planting. The methods of irrigation are described in Chapter XV. Good cultivation should also attend the orange from its first planting onward. This subject is fully discussed in Chapter XIII. 


\section{PRUNING THE ORANGE}

All the considerations urged in Chapter XII in favor of low-headed and strongly branched trees apply to the orange, though the habit of the tree in growth and bearing requires different pruning policies. The orange tree is more disposed naturally to assume a good form than most other trees, and for this reason most experienced growers declare their belief in pruning as little as possible. This is unquestionably good policy providing attention enough is given to securing a shapely and convenient tree, and to overcome the tendency in the young tree to run out very long shoots which result in unsymmetrical shoulders with hollows beneath them and obviate a weeping habit, which interferes with irrigation and cultivation and prevents the development of good bearing space above. It is so easy to bring the orange tree into good form by a little hard thinking about what shape is desirable and a little timely cutting and pinching to secure it.

It has long been held that the orange tree should assume a compact wall of foliage. The interior of the tree being considered merely the framework to support this and pump sap into it. Recently the importance of removing dead wood from the center of the tree is being urged, even though the cost is considerable, and a rational thinning of branches to admit light and air in sufficient amount to secure good fruit nearer the center of the tree is also being advocated and practiced to some extent. All these progressive tendencies carry the orange nearer to the application of the principles of pruning which are discussed in detail in Chapter XII, including the rational recourse to more severe pruning or cutting back to induce a growth of new and stronger wood for subsequent bearing. In other cases cutting back of varieties inclined to make a rank wood growth, like the Valencia Late to encourage a better supply of fruiting wood, is being advocated. It seems clear that there are important ends to be gained by more pruning of the orange tree, and by earnest thinking and close observation along this line.

It has been held that it was necessary to prune the young tree to quite a high head at first to allow for the natural droop of the branches and the result is seen in many young trees with slim stems and umbrella-like tops. It is better to develop a stout stem by allowing for a time a low growth upon it and then raise it later by removal of the lower growth which has done good service and outlived its usefulness. By wise under-pruning it is possible not only to secure a shapely and convenient tree but also to so train the lower growth that it shall present good, low bearing wood without groveling in the dust.

Unquestionably the drooping habit of budded orange trees is largely due to their treatment. A grower who does not believe in pruning allows the branches to extend too far horizontally, and the weight of the foliage and the early fruiting brings the branches to the ground, To relieve the lower branches of the young tree of a part of this weight will enable them to assume a better direction, and this slight relief at first will prevent much branch-sawing in later years. The young tree 
as it comes from the nursery usually starts upon an upright course. If stopped at about three feet it can be brought along to develop strong and well-arranged branches, much as has been described for deciduous fruit trees in Chapter XII. The adjacent engraving, Fig. 1, shows a young tree in planting condition, stopped at three feet and needing only a slight cutting back of the laterals to be ready to begin its orchard life. If young trees are transplanted short distances and at the right time they do not need so much cutting back as is commonly given them. If allowed to grow from the start shown in Fig. 1, pruning only to

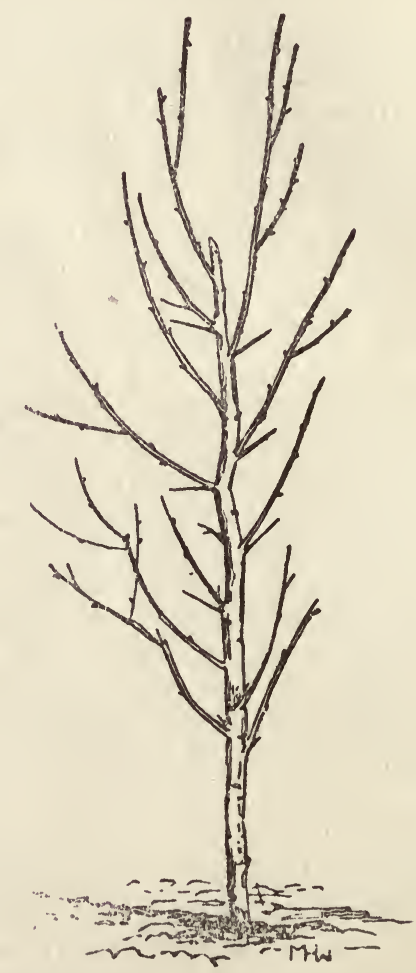

Fig. 1. Orange tree at planting in orchard. Three feet high

prevent long branches from running out at random, and removing branches which may start strongly from near the base, the tree will assume the branch-form shown in Fig. 2 and at from two to three years after planting in the orchard. At about this age the removal of lower branches begins, as they have served their purpose in shading the trunk and bearing the first fruit. These branches are removed one by one until, when the tree is five years in the orchard, it has lost all branches below the two-foot line except the one branch marked "a" of which the upright-growing part will be retained. The higher branches 


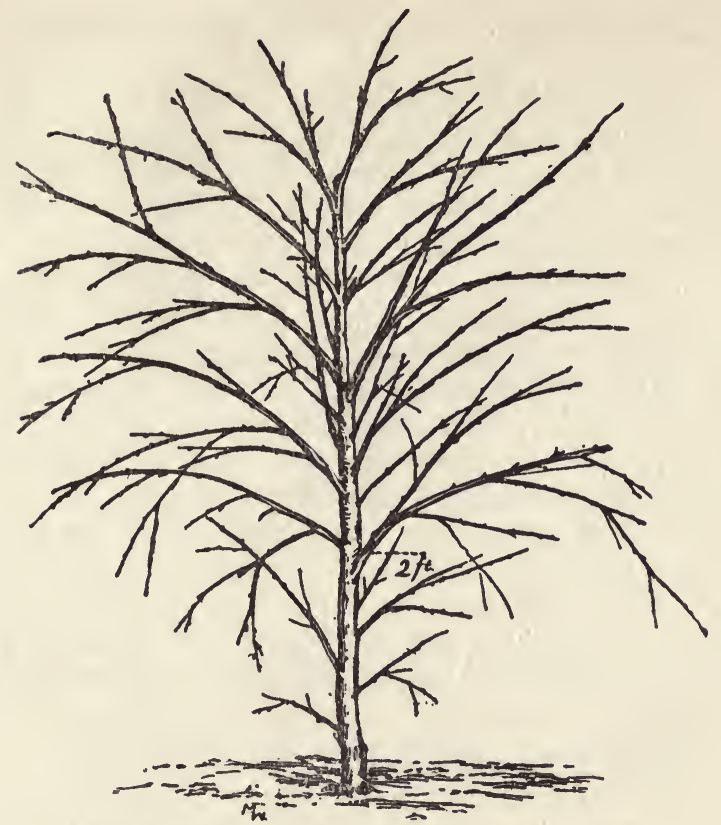

Fig. 2. Branch form of orange before removing lower branches.

assume the more horizontal habit, too great out-shooting is repressed and at about five years orchard-age the tree attains a height of about twelve feet and if of the general form shown in Fig. 3. The next few months will bring its foliage to the ground to remain there or to be under-trimmed, as the notion of the grower may be.

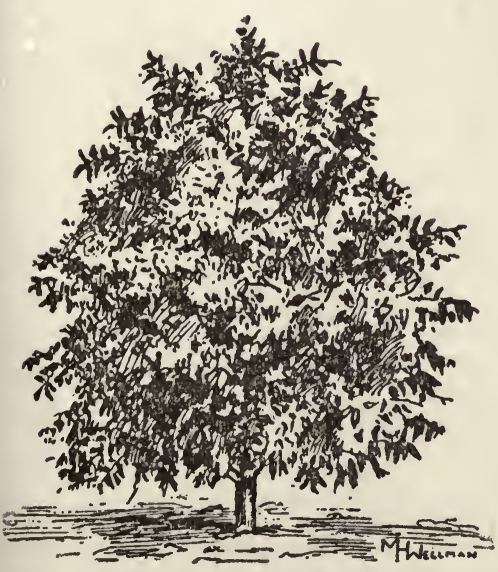

Fig. 3. Good form of five-year-old orange tree.

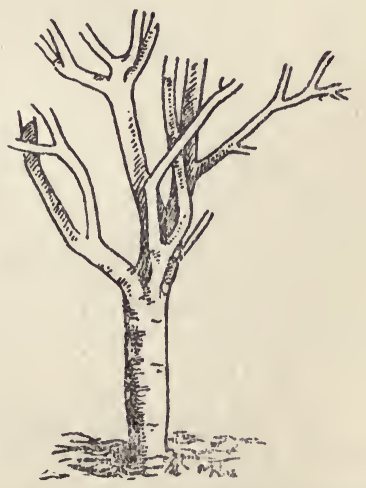

Good form secured by training 


\section{BUILDING DOWN AND NOT SAGGING DOWN}

It is perfectly feasible and rational to secure a good form of low tree without removal of large branches and without relying upon the sag of the branches from a high head. It requires rather more watchfulness and attention and study of the subject than some growers desire to give, but the results when attained are very satisfactory. The method is that of J. H. Reed, of Riverside, and has been followed by him for a number of years with his own orchard and others of which he has had charge. It will be found readily intelligible with the help of the sketches. Mr. Reed would begin with a young nursery tree like that already shown in Fig. 1. He does not believe in much cutting back before planting providing a fair amount of roots are left in the ball at transplanting. If the tree has been properly planted and cared for, it will soon begin to put out new growth, usually first along the stem, the strongest growth being lowest down. As many of these young branches along the stem as are not desired for permanent branches, are rubbed off, the earlier the better, at least before woody fiber is deposited in them. Mr. Reed rubs off promptly all below a point about two feet from the ground, if there is a prospect of getting sufficient good branches above that point. If not he saves them down to eight or even twelve inches lower if need be.

Mr. Reed's idea is to build the head along a considerable length of the trunk and not have the branches bunched at the top, and this is the same idea that is urged in the development of the trunk of the deciduous fruit tree in Chapter XII. He finds it impossible to do this in the nursery because if it is attempted to form a head 18 or 24 inches along the upper portion of the stem instead of one bunched near the top of it, the lower branches will appropriate most of the sap and the upper portion will not be well developed; while if this upper story is well established in the nursery the lower portion can be built on without detriment to the upper, if nutriment sufficient for both is furnished. Fig. 4 will show approximately the branch-form of the young tree at about six months from planting and the shoots with which the buildingdown is begun. The first step is to check the drooping habit. Upon this point Mr. Reed says :

The common notion that the branch of the Navel orange naturally tends down is a mistake which grows out of the fact that in its rapid growth the new part of the stems and large leaves are so loaded with sap that they pull the stems from their natural upright position, and, unless relieved, hold them there till the deposit of woody fiber fixes the branches in the drooping or unnatural position. If the tips of these rapidly growing branches, with their heavy leaves be clipped at the right time, the branches will spring back to the erect position, where they will remain to send out new branchlets. It is wonderful how the orange tree can be molded like a thing of wax by pinching and clipping here and there, if done at the right time.

Fig. 5 shows the result of this clipping of heavy shoots to allow them to assume a more upright growth and the encouragement of new shoots below the two-foot mark. Fig. 6 gives the branch form of a five-year-old tree, with its lower story of bearing wood well developed, 


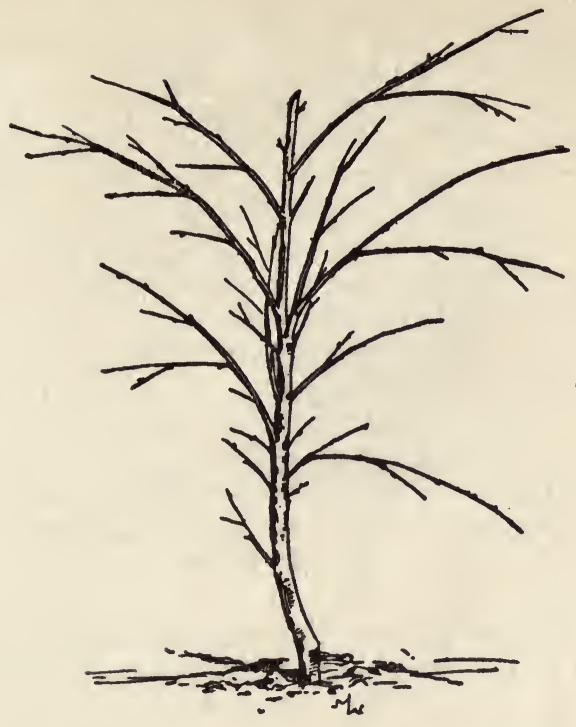

Fig. 4. Branch form of orange six months after planting.

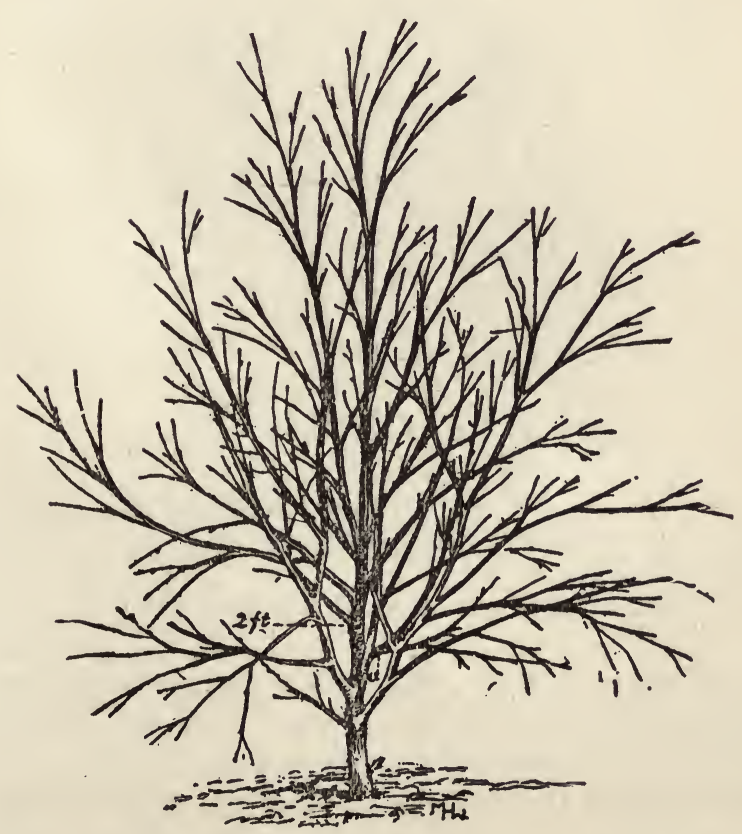

Fig. 5. Tendency of clipped branches to rise. 


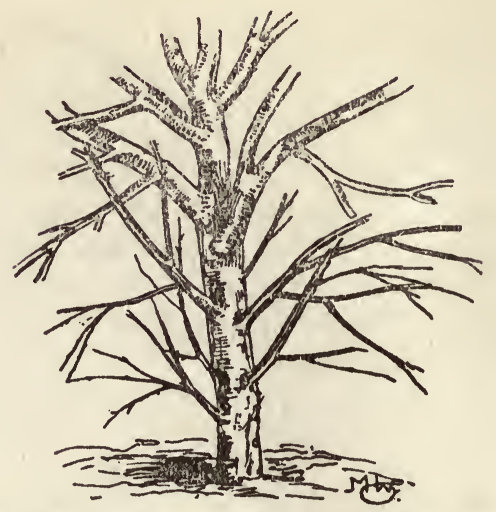

Fig. 6. Branch form of five-year-old tree built down.

and Fig. 7 is the foliage-form of the same tree, about fifteen feet high, with its leaves and fruit reaching to the ground. As to how low the branches should be allowed, Mr. Reed says that until recently he has thought it best to keep the lower branches clipped back so that the fruit would not touch the ground, but he is persuaded that it is better to let them come to the ground even if considerable fruit rests on it. He finds

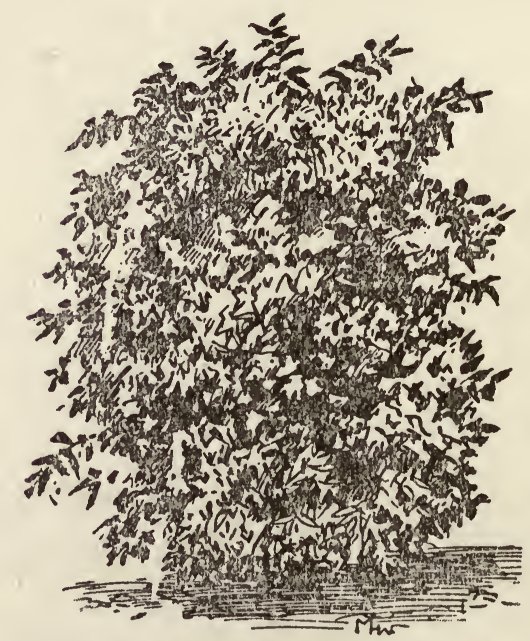

Fig. 7. Foliage form of five-year-old tree.

that many of the best orchardists do this, and claim that there are really less culls among the fruit on the ground than on the less-protected branches above.

Later Pruning of the Orange.-After the form of the orange is well established the aim should be to preserve a compact, symmetrical and convenient form. It is desirable that weak wood should be re- 
moved; dead interior branches, which have given up the struggle for the light, should also be removed. It is an appalling undertaking to get into the inside of an old orange tree and saw off and drag out the dead wood but, as already stated, the conviction is growing that this should be done. Of the growing shoots there is a reasonable amount of thinning to be done. The clipping back of ambitious shoots multiplies laterals. There should be a good cover of leaves, but the crowding of leaves on leaves excludes light and air and weakens the tree by lessening the vigor of leaf action. Dead twigs which appear among good bearing shoots should always be removed.

The gourmand shoots or suckers should be repressed, unless, by clipping, one can be turned into a branch where a branch is needed. The importance of resolutely removing sucker growths even from trees in which the wood may have become hardened is being recognized as a fixed policy in pruning. Good trees have been diverted from bearing fruit of good type to that which is in all respects inferior, by allowing supremacy to sucker-growths. Growth of new fruit wood from old is desirable, but suckers from stem or main branches, showing the marks of wild growth should be extirpated.*

\section{PRUNING FROZEN TREES}

The widespread freezing of citrus trees which occurred in January, 1913, demonstrated that the orange tree is hardier against a drop of the mercury to 12 or 15 degrees Fahr. than any Californian supposed. Very few trees were seriously frozen, though very many lost good fruiting wood, even with the temperature lower than has been experienced for sixty years, and the crop being gathered in 1914 demonstrates the quick recovery of the trees. Policies in the pruning of frozen trees and in treatment of rare injuries like bark-splitting, are being carefully studied through field studies and actual tests, by the citrus experts of the California Experiment Station, and popular instruction will appear in due time through that channel, even though the occasion for the use of it may not recur for half a century.

\section{DISEASES OF THE ORANGE}

The orange is thus far subject to few diseases in California. The most grievous is the so-called gum disease, which is analogous to the gumming of other trees and will be discussed in the chapter treating of tree diseases. Cracking of the fruit is a trouble which has never been fully explained.

There are several serious insect enemies of the orange, which will be discussed in the chapter on that subject. The "black smut," which makes leaves and fruit unsightly in some parts of the State, is a fungoid growth upon the exudations of insects, and can be prevented by removing its cause.

*Detailed discussions of the subject are given in the Pacific Rural Press, January 17 and March 14, 1914. 


\section{VARIETIES OF THE ORANGE}

Though many varieties of the orange have been introduced in California, but few are largely grown. During the last fifteen years there has been a pervading disposition to concentrate upon the Washington Navel, and, except to get other varieties either earlier or later to extend the season, there seems little reason to go beyond the Navel for commercial purposes. Not only have recent plantings been predominantly of this variety, but old trees of other kinds have been very largely budded over to it, and this work is still going on at a rapid rate.

As already claimed in the opening pages of this chapter, the Washington Navel is the greatest commercial orange in the world. As it goes from California into the world's commerce it is a combined product of grower's skill and climatic conditions operating upon its own natural qualities and characters. Neither of these factors alone could achieve its present position. The navel mark is neither peculiar to it nor determinative of it, for there are other navels which are inferior here and our navel is inferior elsewhere: and even in Bahia, whence it came, it has no such quality and standing, because in coming to California it passed from humid, tropical to arid, semi-tropical environment. As already suggested, the tropical orange is not in the same class with the semi-tropical from the point of view of commerce. Trade in tropical oranges is local or limited; trade in semi-tropical oranges is world-reaching. The orange produced in an arid, semi-tropical climate is dense and compact, firm and better in keeping and carrying characters. It is also of more sprightly flavor and richer composition, as shown earlier in this chapter. Such an orange, enclosed in a thin skin of silky texture and beautiful finish, comes very close to an ideal upon which to found an industry.

Although California has apparently no need for changes of type in oranges and has worked diligently and long for the attainment of the types which are at present supreme in her industry, there is still opportunity for improvement within the types. Such improvement is probably to be attained not by hybridizing, but by selection. The Washington Navel, like other members of the citrus family, is keenly disposed to variation and some of its variations have been named and propagated as the lists below will show, but variations appear as degradations as well as improvements. The pursuit of such and other improvements, and their opposites also, is now being systematically taken up at the Citrus Experiment Station at Riverside which is a branch of the University of California Experiment Station at Berkeley, and by the Bureau of Plant Industry of the United States Department of Agriculture. Preliminary publications by Mr. A. D. Shamel in the Reports of the California Fruit Growers' Convention for 1911 and 1912, are of deep interest and can be had from the California State Commissioner of Horticulture, Sacramento.

Relative Importance of Orange Varieties.-The relative importance of the orange varieties grown in California, and the shipping season of each, are indicated by the Citrus Protective League as follows: Washington Navels, 27,000 carloads in November and December from 
northern and central California and from December to June in southern California; Valencias, 8,500 carloads, from June to October inclusive; Seedlings, 1,000 carloads, from March to July inclusive; Mediterranean Sweets, 2,000 carloads, from March to July inclusive. Other varieties, including St. Michaels, Thompson Improved Navel, Ruby Blood, Malta Blood, Jaffa, Tangerines and Navelencias, comprise about 1,500 carloads.

\section{DESCRIPTIONS OF VARIETIES}

Washington Navel (Bahia, Riverside Navel).-Fruit large, solid, and heavy; skin smooth and of a very fine texture; very juicy; high flavored, with melting pulp; is practically seedless, only in exceptional cases are seed found; tree is a good and prolific bearer, medium thorny, a rapid grower, although it does not attain a very large size; bears when very young; commencing to bear as early as one year old from the bud; ripens early. This variety was imported from Bahia, Brazil, in 1870, by Mr. W. Sanders, of the Department of Agriculture at Washington, and in 1874 two trees were received from Washington by Mrs. Tibbetts, of Riverside, Cal. Trees were also received about the same time by Alexander Craw, but the Riverside trees were first in fruit, and the excellence of the variety being at once recognized, it was propagated rapidly and took the name Riverside Navel from the place where its characteristics were first made known. As it came to be grown largely in other districts as well, a broader name, Washington Navel, recognizing its receipt from the national capital, was adopted.

There is much tendency to variation in the Washington Navel, and subvarieties are to be found involving departures in the direction of thinness and silkiness of rind, etc., as well as interior characters. The first to become prominent of these is Thomson's Improved Navel with A. C. Thomson, of Duarte, Los Angeles county, claimed to have produced by a process of propagation, but which is believed to be a natural variation. It is a very refined fruit; generally held to be too fine for ordinary handling, and not otherwise superior to the Washington Navel.

Golden Buckeye Navel.-Introduced by Mr. R. M. Teague, San Dimas. Young wood, slender but strong; tree of striking appearance; fruit marked with bands of deeper color, skin very smooth; pulp aromatic with suggestion of pineapple flavor.

Golden Nugget Navel.-Also by R. M. Teague. Young wood willowy and slender, tree umbrageous; fruit smooth, solid, thin-skinned, rather oblong and good size, rich golden color; pulp free from rag, and delicious; said to average larger than common Washington and to be very promising.

Navelencia.-Another navel variation held to be a cross with Valencia by Thomson. Resembling Navel but more drawn out at the stem end. Large, handsome and later than Navel. Growing in favor.

Australian Navel.-A coarser type of the Navel introduced from Australia in 1874 by Lewis Wolfskill, of Los Angeles, and largely propagated formerly. It has now been practically abandoned for the Washington Navel. It seems to be of more account at some points in the San Joaquin Valley than elsewhere. Recently held to be undistinguishable from inferior types produced by sucker growths on Washington Navel trees.

Jaffa.-A variety introduced in early years but largely discarded. It has been recently propagated in the San Joaquin district, where it has shown desirable characters. Resembles Valencia. Late but is earlier in ripeningfollowing the Navel.

Valencia Late (Hart's Tardiff).-Medium size, oblong, pale yellow; flesh rich, deep yellow, sprightly and crisp; tree a strong grower, slightly thorny. Ripens late, and valuable for late shipping. 
Malta Blood.-Fruit small to medium, oval; flesh fine texture and flavor, streaked and mottled with red; few seeds. The tree is thornless and regular and heavy bearer.

Ruby.-Medium size, roundish; when ripe often reddened by deep red pulp within; juicy and snrightly, often rather acid; tree vigorous, thornless and a good bearer.

Mediterranean Sweet.-Fruit medium to large, pulp and skin of fine texture, very solid and few seeds; ripens late, often not until May or June. The tree is thornless and of dwarf habit of growth and is inclined to overbear. It was at one time the most widely-distributed variety in the State, next to the Washington Navel, but has recently been largely budded over. It was introduced and named by T. A. Garey, of Los Angeles.

Paper Rind St. Michael.-Fruit small, round, very firm and very juicy; pale, thin skin; very elegant in appearance. It ripens late and keeps well on the trees as late as August; tree is of dwarf habit, medium thorny, a good bearer, and very desirable.

Kumquat (Citrus Japonica).-Fruit very small, oblong or olive shaped, rind thick, yellow, smooth; sweet-scented; very little pulp; containing many seeds; tree dwarf (a bush), a prolific bearer. Chiefly used for conserves.

Dancy's Tangerine, or Kid Glove.-Leaves broad, fruit flat, small to medium, reddish; skin separating readily from the pulp; flesh juicy and aromatic.

Satsuma (Unshiu Oonshiu).-A considerable acreage of this variety, planted ten years ago on dwarfing stock, has availed very little commercially. At present there is disposition to grow the variety on sweet orange stock because of its earliness, but this practice has been recently condemned by Florida experience, where it is held that it must be grown on the trifolicata root. Tree quite hardy, fruit irregular in size, but usually medium size, flattened; rind easily detached; fine texture, sweet and nearly seedless.

Mandarin, Willow-Leaved.-Medium sized, flattened, deep yellow; flesh dark orange, skin loose; tree compact, ornamental.

King.-Of the Mandarin class; large, rough skinned, skin and segments loose, pulp high flavored, sweet, aromatic.

\section{STANDARD PACK FOR CITRUS FRUITS}

A standard orange box in California is $12 \times 12 \times 26$ inches outside measurements with a fruit space $11 \mathrm{I} / 2 \times 11 \mathrm{I} / 2 \times 24$ inches, and its weight is usually estimated at 72 pounds. A standard California lemon box is $10 \mathrm{~T} / 2 \times 14 \times 27$ inches outside measurements, divided also into two compartments. Pomelos are packed in the orange box, while the Tangerines, Mandarins and other kid glove varieties are packed in half boxes of the orange size, two of which are cleated together for shipment. In rare instances they are packed in quarter boxes, four being cleated together.

A carload of packed oranges varies some in number of boxes in keeping with the size of the car. A 36-foot car will take 336 boxes, the 40 -foot car 384, and the 42-foot 408. In lemons the 36-foot car will take 288 boxes, the 40 -foot car 312 , and the 42 -foot car 336 . When packed the boxes are placed on end two tiers high and six rows wide, with a space for ventilation between each and every box, made feasible by nailing small strips of lumber of sufficient strength crosswise of the car.

In oranges the regular sizes are $126,150,176,200,216$; small "off-sizes" 250, 324, 360; large "off-sizes" $64,80,96,112$. The relative 
value of the different sizes in the regular trade is given by Mr. Powell, whose excellent publication on the marketing of oranges is cited in the chapter on that fruit, in this way: "A car of Washington Navel oranges may contain boxes of all sizes. A car is called a "standard car" when it contains not more than ten per cent of each of the following sizes: $96,112,250$, and not over twenty per cent of the 126 size. The remainder of the car may be divided among the 150, 176, 200 and 216 sizes. If a car is sold to a dealer at a fixed price per box it is usual to allow a discount of 25 to 50 cents per box on the excess in the 96 , 112,126 and 250 sizes, and a discount of 50 cents per box on the 48 , $64,80,288,300,324,360$ and 420 sizes. The rule concerning the sizes in a standard car may vary with the season, with the section and with the general size of the fruit taken as a whole. When oranges of either the large or small sizes are scarce, they are at a premium, and the proportion in a car may be raised without discount.

\section{A CITRUS PACKING HOUSE}

To convey an idea of the systematic handling of citrus fruits in the California packing houses, with the advantage of the best architectural construction and arrangements and using up-to-date machines and processes, is beyond the scope of this treatise. At some future day, no doubt, some one will undertake an adequate publication on that subject. To give the reader a glimpse of this branch of the citrus industry, the following description by Mr. J. F. Adamson of the new plant of the Pomona Fruit Growers' Exchange, the second of two large houses owned by that association. There being nearly 300 active members, it becomes necessary that the fruit be handled with expedition, and at the same time with extreme care, the first so that there be no congestion of of teams, and the second to prevent injury to the fruit:

The fruit in the field, or picking boxes, is hauled in on spring wagons covered from the sun by canvas wagon sheets, and is delivered directly to a gravity conveyor, on which the boxes roll without jar to the point where they are piled. It is manifestly impossible to regulate the delivery to the rate of packing, so the fruit has to be piled as it comes in and taken to! the washer as needed. Passing through the washer, the fruit is rinsed off under a spray of clean water, and goes directly to the drier, in which it remains for fifteen minutes, being moved along under a gentle blast of dry air. At the end of this time it is delivered to the sorting table, dry, and without having been handled or bumped, except the handling of the boxes from wagon to conveyor, and from pile to washer.

The sorting of fruit must of necessity be a hand job, as after all, there is a certain amount of expert human judgment used in the separation of grades in fruit or other produce which can not be done by machinery. In the sorting table of this house, there is, however, an element of novelty. The fruit comes before the sorters on the usual belt, but the similarity ends there. In the ordinary sorting system the fruit is carried along in front of the sorters, and each man picks off the grades of fruit as the belt moves along. The man at the farther end of the belt picks over the fruit left on the belt by the first, second, and third or fourth man, making an extremely limited system. In the new table, however, the first man has a small table in front, of him to which he switches as much fruit as he can handle, and from that table he sorts into grades, and the fruit that passes him to the other man has not been picked 
over, and there is no limit to the men that can be worked without the drawback of repetition.

From the sorters the fruit goes to the usual sizers, over a complete set of automatic weighers. All grades and culls are weighed and recorded on the grower's load check, and the fruit has lost its individuality, being mixed by grades, and handled and sold only under those grades and the brands correspond to them.

In all this travel the fruit has been guarded from the least bump. No long steep inclines are used which would set the fruit in motion that would require stopping, but is carried through almost every stage of the proceeding on belts.

Under the sizers are located the empty packing boxes, fed in from the basement by conveyors and always handy to the packers. The full boxes are carried from the packers by power-conveyor to the nailing presses, where the covers are put on the box strapped with steel ready for shipment.

Fruit that is to be pre-cooled then goes by gravity conveyor to the refrigerator-rooms in the basement, where they are kept for three or four days in a cold blast until the temperature of the fruit is lowered to 35 degrees Fahrenheit, when it is loaded into the cars by means of a power conveyor.

This packing house is actuated by one of the latest models of crudeoil engines, of 90 horsepower, and costing for fuel about ten cents per hour. This power is used to operate a 25-ton refrigerating machine, two large fans for air circulation, all the conveyors, and an ice hoistan estimated load of 85 horsepower. The establishment cost over $\$ 65,000$, and is evidence that the citrus-fruit industry has passed from temporary expedient to permanence, just as the handling of fruit has passed from slipshod methods to a system characterized by the utmost care and forethought. 


\section{CHAPTER XXXIII}

\section{THE POMELO OR GRAPE FRUIT IN CALIFORNIA}

This citrus fruit achieved a very sudden interest in California because of the prices commanded by Florida pomeloes about twenty years ago. When this supply of eastern cities was cut off by the serious frost injuries in Florida there arose a passion for planting the trees in California, and a considerable acreage was planted, and as the tree is a very rapid grower and precocious in fruit-bearing, large shipments were made in 1898, but the results were not satisfactory, and since that time, although pomeloes sometimes sell to good advantage, the demand is on the whole light and irregular. The local consumption of pomeloes in California is increasing but does not warrant much acreage. For these reasons early plantings have been largely grafted over to the orange.

The fruit is a Shaddock (Citrus decumana). Varieties have been secured of smaller size and of blander flavor than the coarse, sour and acrid fruit which is suggested by its name. An effort has been made to secure the favorite varieties, and a large list has been planted in this State, but the fruit does not command the place at the East which was awarded to the Florida product. As a tree the pomelo most nearly resembles the orange and its culture is the same. As for varieties, California experience has been too brief and fitful to demonstrate particular value or adaptation in them. Among growers there is no wide agreement as to superiority from a commercial point of view. Amateurs can find many desirable sorts in our citrus nurseries.

Mr. A. P. Griffith of Azusa is perhaps the most enthusiastic and confident of California growers that the pomelo grown in this State will attain wide popularity in the United States. His grove is now seven acres in extent, nearly all of the Nectar variety which is of the type which he believes should be grown to win public favor. He concludes that the market wants a fruit not smaller than 80 's and prefers them to stop at 64's. A fruit flat-round that will cut up in two halves each of which will stand upright on a plate, cut side up, seems especially desirable. A fruit that is spherical or pear-shaped is not desired. There are several varieties whose similarity in type would seem to warrant including all under one name. These, with the addition of the seedless, comprise all there is of intrinsic value among California pomeloes. If all other varieties not of this type were budded over, and thus taken out of the market, Mr. Griffith believes the demand would increase.

Marketing Grape Fruit.-The California problem seems to be not only to grow grape fruit acceptable to consumers in distant markets but to place the fruit upon those markets when they are not otherwise well supplied. Recently better results have been attained by shipping during the Valencia season than earlier. When California growers 
give as much attention to meeting the demand as they have to the orange and the lemon, it is to be expected that the grape fruit will become commercially more satisfactory.

Varieties.-The following comprise about the only varieties grown in this State. Most growers prefer the Marsh Seedless:

Nectar.-A Florida seedling, grown at Duarte; flat-round, heavy; peel smooth and bright, pale-lemon color; oil cells small and numerous; flesh dense, firm texture, abundant'juice, vinous, excellent. bitter-sweet element distinct; few seeds and little rag; sizes from 42 to 80 to the box.

Imperial.-Medium to large, peel very smooth, medium thin and of fine texture; little rag, juice abundant, fine aromatic flavor, good keeper and shipper. Tree upright and heavy bearer.

Marsh Seedless.-Medium size, 52 to 64 to the box; thin rind; almost entirely seedless; flesh dark and rich, a late keeper and early and abundant bearer.

Triumph.-Medium size, peel smooth, clear, thin and fine grained; very juicy, heavy and good flavored; juice free from bitterness; very little rag; an early and prolific bearer. 


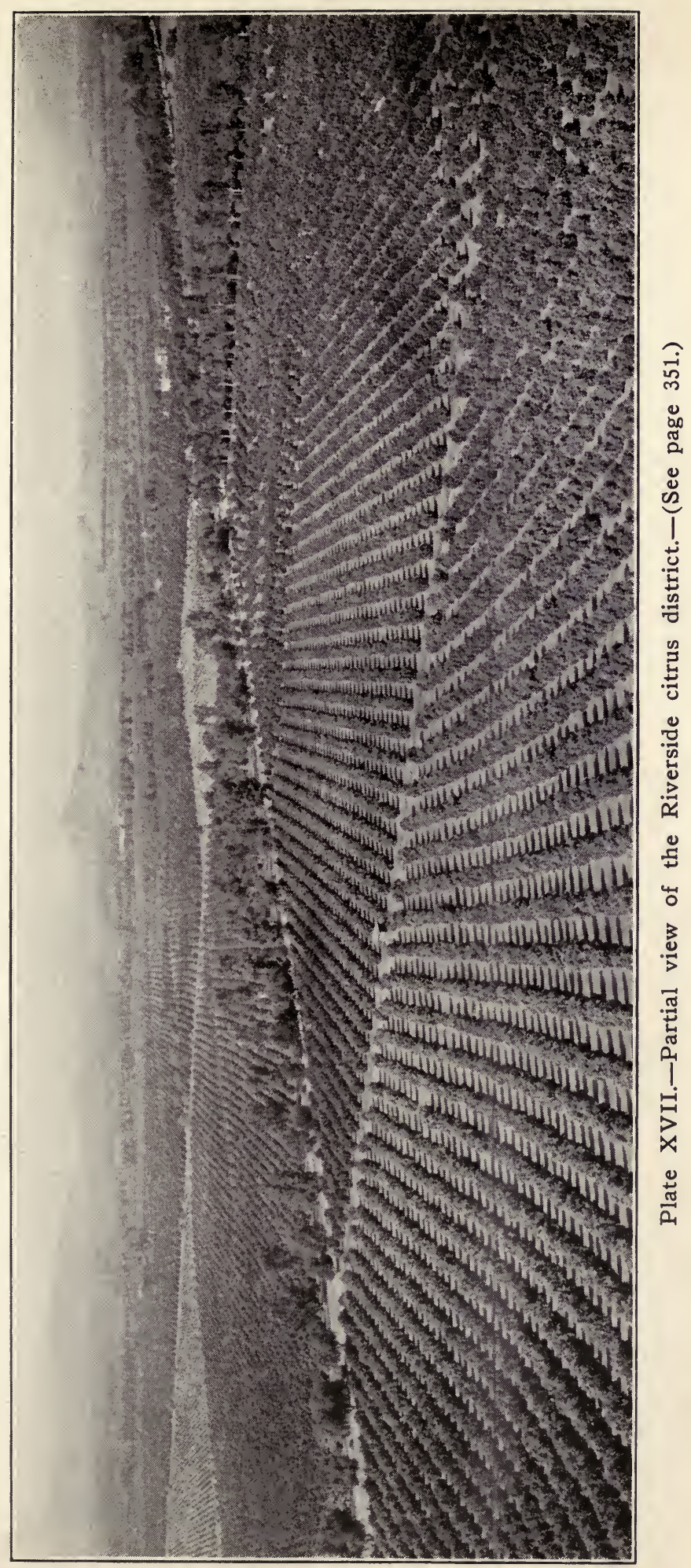





\section{CHAPTER XXXIV}

\section{THE LEMON AND MINOR CITRUS FRUITS}

Lemon growing is a very unique and distinctive branch of California horticulture, which in the present advancement of culture and preparation for the market, well illustrates the originality and invention which the California fruit grower has displayed in his undertakings. Lemon growing in California is old because it rose at the old missions in the second century back of us, but successful lemon growing as a great industry is new and constantly assuming new phases. For the old seedling lemons were bad, and though enterprising growers soon learned that fact and set about getting better ones, it took years to secure them and to learn how to grow and handle them so that the Californian could compete with the Sicilian fruit in the markets of the United States. Nor was time the only thing sacrificed-hundreds of thousands of dollars were lost before the California grower could put upon the market a good lemon, fit to stay good for a sufficient length of time. Unprofitable plantings; expensive curing houses, which did not cure well; countless experiments which yielded only loss and disappointment-all these are wrecks upon the rock of American lemon growing.

Naturally Californians sought first to know how lemons were grown and handled abroad. At cost of great effort and outlay they learned practically nothing that they could do and a great deal that it was not necessary to do. Then they assumed a more rational mood-a disposition to discern what principles are involved in the problem, and to apply them in their own way according to conditions locally prevailing. Along this line grand success has been attained by a few masterful men conducting large lemon enterprises or smaller undertakings of their own, while the mass of lemon planters, for one reason or another, have never reaped the reward they expected. On the whole, it may be said that lemon growing is a much harder and more exacting enterprise than orange growing, and for this reason many have new-topped their trees to oranges and thus escape difficulties which they could not overcome.

With the aid of the protective tariff the most resolite and capable have attained success, and the California lemon became known and highly esteemed upon its merits everywhere. The tariff has somewhat reduced the effect of cheap labor in Italy and cheap water transportation from the Mediterranean region, and our lemons could sometimes compete with the foreign product not only in the west but even in the cities of the Atlantic seaboard. All this has been accomplished within two decades and it is a notable result. One measure of this fact may be found in the shipment of over six thousand seven hundred carloads during the season of 1910-11. The California lemon has, however, not yet attained such mastery of American markets as the orange has, for there is a value of about six and one-half millions of dollars in imported 
lemons, while imported oranges are only worth one-twenty-fifth as much. The California contention that the lemon should be encouraged with increased protection to enable producers to push the issue of an American lemon for Americans to a successful termination, was rejected by the tariff of 1913 . In 1914 the future of the California lemon is not clear. The product of 1913 was reduced by frost to the figures of a decade ago, though in 1911 it was four times as great. The exact figures are given at the opening of Chapter XXXII. Whether the lemon can weather the storms of all kinds which have arisen remains to be demonstrated.

The best pack of California lemons has a uniformity of size, a finish of skin, a juiciness and keen acidity which is unrivaled in the world. Numerous careful tests have been made of the California lemon in Atlantic cities in comparison with the best south European product, and the superiority of the American fruit has thus been demonstrated.

\section{SITUATIONS AND SOILS FOR THE LEMON}

The lemon does best in a practically frostless situation. Such places are found in largest area in the southern half of the coast regions of California, but also exist at favoring elevations in the interior. The moderating influence of proximity to salt water, and the effect of local topography and environment, which give frost-free nooks or belts, are elements favoring the lemon grower. In such situations the lemon blooms and fruits continuously throughout the year.

While the lemon requires a less extreme of low temperature than the orange, it also thrives with a less extreme of high temperature and less duration of it. It apparently does not require as much heat to develop acid, which is the charm of the lemon, as it does sugar, which is essential to an acceptable orange; therefore, a coast situation which may not yield a sweet orange may produce a good lemon, although it is a fact that in the southern coast region, where the largest commercial production of lemons is now achieved, the orange also does well. Another advantage of the lower summer temperature is that the continuous ripening is not interfered with, as it is by high summer heat, which hastens maturity and brings the mass of the fruit to marketable condition in the winter-a season when the demand for the lemon is very small. This objection is, however, being measurably overcome by the proper storage and treatment of the fruit for sale, at a considerable interval after picking, as will be mentioned presently. But both the curing and storage of lemons are more easily secured in the more equable temperature and moister air of the coast region.

The lemon delights in a sandy loam, and probably our best orchards are on such soil, but the trees thrive in other soils. There is a difference of opinion among growers as to what soil is to be especially sought for. There are profitable lemon orchards in southern California located upon deep clay loams, and even upon strong red clay soils. As with some other fruits, the choice of soil is, to a certain extent, governed by the stock on which the lemon is worked. 


\section{PROPAGATING AND PLANTING}

The prevailing stock for the lemon is the orange seedling, either the sweet or sour stock, under the same conditions that each is preferred for the orange, the sour stock resisting measurably the effects of illdrainage of heavy land or flat, low places. The orange root thrives on a greater variety of soils than the lemon, and produces a healthy lemon tree where the lemon on its own root would fail. The growth of orange seedlings for budding has been described in the last chapter. If lemon seedlings are desired they may be grown in the same way. Plants either for permanent growth or for stocks for budding can be grown from cuttings, as explained in Chapter VIII. The budding of the lemon is practically the same as of the orange, which has been described. An old tree can be changed from one variety to another by the methods described for the orange, and oranges can be worked into old lemon trees and good fruit secured if the lemon itself be growing upon an orange root, which is likely to be the case with trees planted during the last decade.

Planting of the lemon is the same as that of the orange. The distance in planting varies from twenty to twenty-five feet. Irrigation of lemon and orange trees is also similar.

\section{PRUNING THE LEMON}

The pruning of the lemon is essentially different from that of the orange, because the habit of the tree is different. The lemon requires constant attention to bring it into good bearing form and keep it there; the orange, after it is well shaped, simply needs attention to encourage it to retain the bearing form to which it seems naturally disposed. The orange provides itself with satisfactory bearing wood, as a rule; the lemon devotes itself, even when it is old enough to know better, to a rangy rambling wood growth with bearing wood upon the ends of willowy rods where it is swept about in the wind and burned in the sun, instead of nestling it neatly among the leaves as the orange does.

The rational proceeding with the lemon is, then, to develop it at first into a low, stocky and strong form, such as is described in Chapter XII for deciduous tree. This may be secured by pinching so as not to allow running out of long branches at first, or it may be secured by severe cutting back of the long growths of the young tree. In either case low branches will be secured. Make good selection from these branches to form a symmetrical tree and cut back the growth which comes upon them to cause it to branch in its turn. In this way plenty of good, strong wood is secured low down, and with short distances between the laterals. Strong, upright shoots (wrongly called "suckers") which break out at points where branches are not desired, should be rubbed off or cleanly cut away. Having secured about the right branching in about the right places no strong sprouts should be allowed, and the tree should be encouraged to make smaller laterals, which will be the bearing wood. It must be admitted, however, that this rational plan of restricting wood growth and directing the energies of the tree to fruit 
has in some cases been pursued too far and the tree has resented repression by diminished thrift. The pruner must allow freer growth of shoot to secure better foliage. The pruning of the lemon as of other trees must always be pursued with judgment rather than by recipe.

When the adequate growth of bearing wood within reach is borne in mind it appears that the pruning of the lemon involves many of the considerations urged in Chapter XII for deciduous fruits; the method of making a strong, short trunk, the arrangement of branches, the prevention of long growths, the encouragement of low, bearing twigs, the thinning of twigs to prevent the tree from becoming too dense, the points to be observed in cutting back, not by shearing but by treating each branch according to its position and vigor-all these must be borne in mind by the lemon pruner. It must also be remembered that the work must be resolutely continued and the tree always prevented from wild growth and kept down to bearing on the smaller twigs, which are promoted and retained for that purpose. The building-down process described for the young orange is easily applicable to the lemon.

Old lemon trees which have been allowed to grow away into a long, rangy form and to bear fruit too high for profit, can be brought down to good form by severe cutting back and after-treatment of the new shoots, keeping the smaller horizontal growths and cutting out cleanly the strong upright shoots, or cutting them back if more branches are needed. The time for pruning the lemon depends upon the end in view; if a young tree, to promote wood growth, prune at the opening of the growing season in the spring; in older trees, to repress growth and advance fruiting, prune in midsummer.

When it is remembered that harvesting lemons is a continuous operation as will be stated presently, keeping the bearing wood of the tree within easy reach is more imperative from an economic point of view than with trees from which fewer pickings gather the crop. Adjacent engravings give suggestive views of rationally controlled lemon trees. Pruning is also related to preventing infection of the fruit with lemon rot fungus spores from the ground. Fruits which touch the ground or upon which dirt is splashed by rains is first infested. The whole question is discussed in Bulletin 190 of the California Experiment Station.

\section{PREPARATION OF LEMONS FOR MARKETING}

The lemon as taken from the tree is not in condition for marketing except to packers who wish to undertake the curing. To secure best results in quality and in keeping properties, the lemon should be carefully cut from the tree as soon as proper size is reached. To allow the fruit to hang upon the tree until lemon color is assumed, gives a lemon which is deficient in juice, oversized, apt to develop bitterness, and prone to decay. Two and five-sixteenths rings are used for winter pickings and $2 \mathrm{I} / 2$ for spring and summer, never more than six weeks being allowed to elapse between pickings, and the fruit is usually picked once a month. By careful attention to this, desirable sizes and good-keeping stock are obtained. Neglect of this is the weak point of 
many of the lemon growers of California. Mr. C. C. Teague, manager of the Limoneira Company of Santa Paula, Ventura County, the largest lemon growing concern in California, has made close examination of practice among lemon growers, and concludes that the carelessness with which picking is done is almost criminal. In grove after grove which he visited at least 50 per cent. of the values had been lost by allowing the fruit to hang on the tree too long. Not only on account of large sizes would it have to be discounted 50 cents per box, but the keeping quality of the lemon which is allowed to mature on the tree is never good. Good results can not be obtained, even by the best methods of keeping lemons, unless the fruit is picked at the proper time and properly handled. Mr. Teague says a lemon should be handled as carefully as an egg.

If gathered before the color begins to turn properly cured lemons may be kept for months, and they will improve in market qualities, by a thinning and toughening of the skin, and by increase of juice contents. This curing of the fruit, as it is called, is accomplished in many simple ways. If the fruit is gathered and placed in piles under the trees, where, with low-headed trees, it is completely shaded by the foliage, it processes well and comes out beautiful in color and excellent in quality, providing it is a good variety. Some have trusted wholly to this openair curing under the trees, merely protecting the fruit by a thin covering of straw, or other light, dry materials. Others let the fruit lie a few days under the trees, carefully shaded from the sun, and place it in boxes or upon trays, and keep it months in a darkened fruit-house, providing ventilation but guarding the fruit against draughts of air. Gathering the fruit while still green and packing with alternate layers of dry sand, has given excellent marketable fruit, but of course the handling of so much sand is too expensive nor is it at all necessary.

Much attention has been given to lemon storage in southern California, and many curing and storage houses have been constructed. Naturally there is great variation in design and method of operation. The essential conditions to be secured are exclusion of light; regulation of temperature; ample ventilation, under control, however, so as to prevent entrance of air which is too dry or too hot; convenience and cheapness of handling, for the lemon is expensive in handling at best during the months of storage which is often desirable. Some of these conditions are relatively of much more importance in the interior than in the coast region, because heat and dry air reach occasionally extremes which are not experienced near the ocean which is a great regulator of temperature and atmospheric moisture. For these reasons a much simpler system of storage is now in large use in the coast district, while in the interior suitable special buildings or basements are apparently necessary. Anyone entering upon lemon handling should certainly visit establishments now in satisfactory use and learn by careful observation of their suitability to his purposes.

Near the coast, and so far toward the interior as ocean influences extend in adequate degree, the building of special curing houses has been abandoned and some quite expensive structures have been turned to other uses. An objection to house-storage lies in the fact that the 
fruit it is apt to be massed in the house and that which is just picked given the same ventilation as that which has been in the house several months, when, as a matter of fact, lemons in different stages of curing require radically different treatment as regards ventilation. As a result of this treatment some of the fruit is usually wilted from receiving too much air, while the greater portion of it is badly decayed from receiving too little.

Proper ventilation is the keynote of success in keeping lemons, and after extensive and expensive experience along the old lines, Mr. Teague of the Limoneira Company, already cited, concluded that lemon handlers had been on the wrong track in believing a low temperature first in importance. If the ventilation is right the temperature will take care of itself. Mr. Teague decided that proper conditions for keeping lemons lie just between the points where they wilt and where they sweat, inducing neither if possible, for too much moisture induces decay and too little causes shriveling. The fragment of the stem left on the fruit by the cutter may be used as a test; if it adheres, the conditions are right for slow curing; if it detaches easily, the best keeping quality is not being secured.

The Limoneira Company was first to equip a house on the open air plan. The house is $300 \times 100$ feet. The flooring is 2 -inch planking and the roof covered with gravel-paper roofing. The building has no sides whatever, allowing free circulation of air. The fruit for storage is put into regular shipping boxes, piled in blocks of 560 boxes. There is a double row of these blocks on either side of a 20 -foot space which extends to the entire length of the building, and which answers the double purpose of a work room and an air space. The boxes are so piled as to permit of the circulation of air around each box. Each block of fruit is covered by a canvas $10 \times 10 \times 20$, made box shape by a canvas cover and four canvas curtains on rollers, the openings at the corners being closed by lacings as desirable. The ventilation is controlled by raising or lowering the canvas, and each block of fruit can be given exactly the ventilation that it requires, irrespective of the other fruit in the house. By this method 50 or 100 cars of fruit can be handled and kept in as good condition as if there was only one. Each block being numbered, a complete record of the lemons from each of the six sections of the ranch is kept from the time it is picked until the fruit is shipped. The fruit is all washed in a lemon washing machine, and is piled up in the house wet, just as it comes from the machine. The canvas covers are not dropped over it, however, until it is thoroughly dry. An idea of these curing tents can be had from an adjacent engraving which shows them on both sides of a central space which is used for packing the fruit in the shipping boxes.

With proper curing facilities lemons picked in November and December may be kept until the following July. Later pickings may not keep so well and may be marketed first. Of the finer points in lemon handling, however, there is much which must be learned by experience.

Forced curing of lemons, by which green fruit may be colored in about two weeks, is done by burning oil stoves in a closed room. The change is effected by the products of combustion and not by the heat 
alone. The process is described in detail in Bulletin No. 232 of the Bureau of Plant Industry of the United States Department or Agriculture, February 13, 1912.

\section{VARIETIES OF LEMONS FOR CALIFORNIA}

During the earlier years of California lemon growing there were continuous efforts put forth to secure better lemon varieties. During the last decade three varieties have been accepted as satisfactory and nearly all others have been dropped. The three are Eureka, Lisbon, and Villa Franca, arranged according to present degree of popularity in Southern California, where nearly the whole commercial product is now made, although some plantings have been undertaken farther north, chiefly in the citrus belt on the east side of the San Joaquin Valley.

Eureka.-A native of California, originated by C. R. Workman, at Los Angeles, from seed imported from Hamburg in 1872, only one seed growing, from which buds were put by him on orange stock. Distributed by $T$. A. Garey, of Los Angeles. Tree very free from thorns. Fruit medium size, sweet rind, a good keeper, few seeds; very popular, especially in coast regions. Less popular in the interior because of scant foliage.

Lisbon.-Imported from Portugal; first grown by D. M. Burnham, of Riverside. Fruit uniformly medium size, rather oblong, fine grain, thin sweet rind, strong acid; few seeds; a good keeper; tree is a strong grower, with compact foliage, prolific bearer, but starts bearing late; quite thorny, but thorns decrease in size as the tree grows older: popular at interior points especially, and Eureka trees in the Tulare region are being grafted over to Lisbon.

Villa Franca.-Imported from Europe. Medium size, oblong, slightly pointed at the blossom end, rind thin, without bitterness, acid, strong, juicy, nearly seedless. Tree thornless, branches spreading and somewhat drooping, foliag* abundant; withstands lower temperature than other imported varieties.

\section{THE LIME}

The lime (Citrus medica acida) has proved much less hardy than the lemon. It has been killed in situations where the orange and lemon have not been injured. Unless adequate protection is thought worth the effort, there is little use in planting the lime, except in a frostless situation. Such localities are found near the ocean in southern California, and here and there at proper elevation around the Bay of San Francisco and in the interior both north and south; still the growth of the lime must be counted very hazardous. There is less inducement to experiment with the fruit from the fact that the Pacific Coast markets are well supplied with Mexican limes, usually at prices which leave no opportunity for competitors.

Limes are grown from seed, the variety usually coming true from seed. The trees are small and are frequently grown in hedge form. The common variety is the Mexican. The Imperial, a large, rather hardy variety, is favorably reported by several growers. Bearss Seedless is being successfully and profitably grown by $\mathrm{Mr}$. R. Gallegos at the Mission San Jose in Alameda County. 


\section{THE CITRON}

This fruit (Citrus medica cedra) is little grown in California, although it is quite hardy and could be produced over a large area. The only use for the fruit, which resembles a monstrous lemon, is in its candied rind, and no one has deemed it worth while to push competition with the imported candied citron, though very fine experimental lots have been produced, and the interest of the fruit-preserving establishments in the product recurs periodically. There have been collections of citron trees imported from the Mediterranean region by the United States Department of Agriculture planted at several points in southern California. As yet no considerable product has been reached. There is, however, no cessation of interest, and experimental planting continues, with a prospect of satisfactory attainment ere long. Samples of the candied article have been approved by experts as very satisfactory.

An outline for the preparation of candied citron is as follows: The fruit, before assuming a yellow color, and also when bright yellow, is picked and placed in barrels filled with brine, and left for at least a month. The brine is renewed several times, and the fruit allowed to remain in it until required for use, often for a period of four or five months. When the citrons are to be candied they are taken from the barrels and boiled in fresh water, to soften them. They are then cut into halves, the seed and pulp are removed, and the fruit is again immersed in cold water, soon becoming of a greenish color. After this it is placed in large earthen jars, covered with hot syrup, and allowed to stand about three weeks. During this time the strength of the syrup is gradually increased. The fruit is then put into boilers with crystallized sugar dissolved in a small quantity of water, and cooked; then allowed to cool, and boiled again until it will take up no more sugar. It is then dried and packed in wooden boxes.

\section{ORNAMENTAL CITRUS SPECIES}

There are grown in this State for curiosity or ornament various minor citrus species, including the Bergamot and the dwarf ornamental sorts from Asia. There are, of course, the ornamental species grown by florists for their fragrant bloom. 


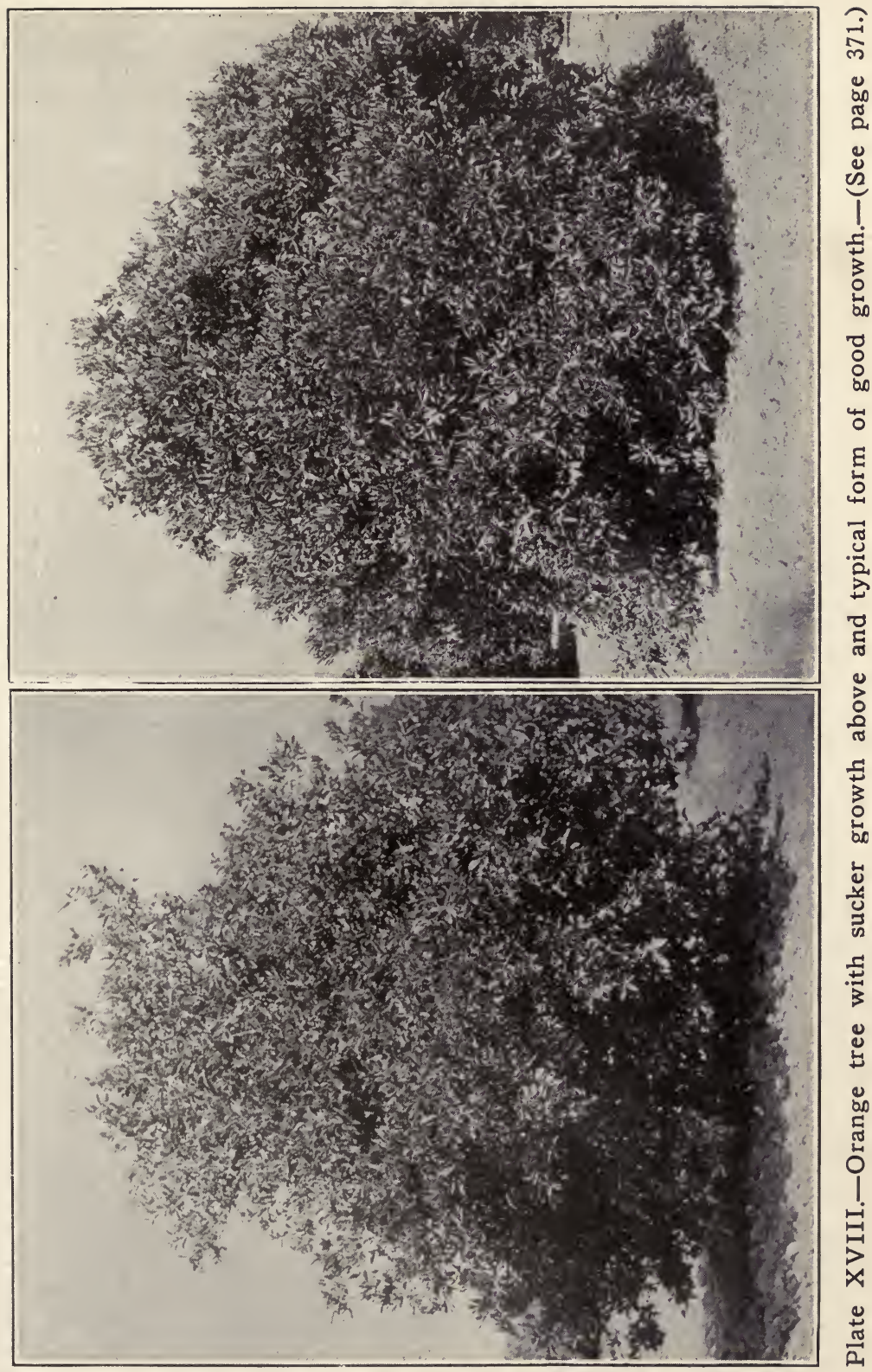





\section{CHAPTER XXXV}

\section{MINOR SEMI-TROPICAL FRUITS IN CALIFORNIA}

A number of interesting fruits are now grown in this State which, for one reason or another, have not yet attained any great commercial importance, although some of them are rapidly advancing in popular esteem and likely to gain much higher place in the markets. Others will probably never be grown except for home use and garden ornament.

\section{THE BANANA}

The banana has been a favorite plant for experimental culture for many years, and though good fruit has been grown at various points in the State, the culture is too hazardous to warrant large investment, and if this danger was not present, the abundant supplies available from the islands of the Pacific would probably reduce the profits to a narrow margin. The banana can be trusted only in protected situations and in small numbers which can be given special attention. With these conditions the banana may yield very acceptable fruit for home use and be an ornament to the garden. Its beauty is, however, seriously impaired by winds, which whip its tender leaves into shreds and give the plant an unkempt appearance.

The largest number of bananas are seen in Los Angeles and Santa Barbara, and one grower at an elevation near the latter place reports his table supplied daily throughout the year with the fruit of the Cavendish species, which is the most commonly grown sort. The Yellow Martinique or Yellow Costa Rica, the Orinoco, the Hawaiian Lele, Hart's Choice, and a large-fruited variety known in Los Angeles County as the Baldwin, are also approved by growers. How to grow bananas in the garden, according to the experience of the late S. H. Gerrish, of Sacramento, is as follows :

By experiment I have found that the banana will live-if in a proper soilwithout injury to the roots, at a temperature as low as sixteen degrees Fahr.; the stalk will stand a temperature of twenty-five degrees without injury, and the leaves are not wilted until the air is chilled to thirty degrees. My method has been to supply the richest food for this gigantic plant and force it to its extreme growth. Every one has old chip dirt, ashes, boots, shoes, clothes, and manure, which are often a nuisance. Dig a big hole, bury this up, in the center of the mass place a pailful of sand, and plant the fresh bulb. This is to preserve the dormant plant from the wire-worms and insects, which will not attack the growing plant. As the plant grows, give it an abundance of water and all the slops of the house. Any kind of manure, fresh or old, ashes, leaves, and vegetables will soon disappear and be absorbed by this gigantic king of plants. As the rainy season approaches, pile all the leaves and twigs of trees around the plants. It protects the bulbs and makes the soil rich for next season. 


\section{THE CHERIMOYER OR PERUVIAN CUSTARD APPLE}

The oldest cherimoyer (Anona cherimolia) is growing in Santa Barbara. The fruit was introduced about fifty years ago, and the parent tree has for many years produced abundant fruit in such perfection that the seeds have readily germinated, and the trees thus propagated have been in successful bearing in several Santa Barbara gardens. The leaves are oval and pointed at both ends; flowers solitary, very fragrant, and having a greenish color. Good specimens of the fruit are three or four inches in diameter, often heart-shaped, grayish brown or nearly black when fully ripe. The flesh, in which thirty or forty brown seeds are found, is soft, sweet, and pleasant to the taste, being most palatable when near decay. Mr. I. H. Cammack, of Whittier, describes the pulp as of the consistency of ice cream or a custard flavored with a blending of pineapples and bananas. If it has a fault it is too rich. Apparently it has no particular season for ripening, yet the best specimens seem to be found in Santa Barbara in April and May. The cherimoyer is also found in gardens in San Diego and Los Angeles counties. It needs a well-protected situation. The fruit has been marketed on a limited scale in Los Angeles, and larger plantations have been made, especially in the Cahuenga Valley, near Los Angeles. The plant comes true from seed and the tree bears in its fourth year, and should have as much room as an orange tree. Mr. C. P. Taft, of Orange, points out the fact that much can be gained by selection and propagation from the most satisfactory trees, as follows:

Cherimoyers found in the gardens of southern California are almost always seedlings, and generally shy bearers. There is but one named variety, so far as I am aware, the Golden Russet. This is very prolific and frequently attains large size. Specimens above one pound in weight are not uncommon. The qualitr is as good as any, but is variable owin to the season and time of ripening. much cold having a marked deteriorating effect. The normal shape is like that of the strawberry, and the variations from the normal are equally abundant; in fact, in this respect the cherimolia is quite extraordinary, as the same tree will have on it fully matured fruit from less than an inch in diameter up to six or eight inches. In size the tree averages about the same as the peach. The market is a good one, large fruit commanding $\$ 3$ per dozen or more, while the smaller ones sell by the pound at a relatively lower price.

\section{THE CHOCHO OR CHAYOTA*}

The chocho plant is fruiting in Santa Barbara County, for Mr. Kinton Stevens, of Montecito, who obtained the seed from Samoa. Sechium edule is the botanical name of this plant, but it is perhaps better known as "choco," "chocho," "chayota," and "Portuguese squash." It belongs to the order cucurbitacae, and is a perennial vine, resembling in growth and fruit our summer squash or vegetable marrow. It is a very prolific bearer. Both the fruit and the great yamlike tuber are used as food by man and beast in the West Indies, where it is considered a wholesome article of diet. The roots often weigh as much as twenty pounds. They have a flavor similar to the yam, and

*An interesting illustrated account of this plant by K. A. Ryerson may be found in the University of California Journal of Agriculture, April, 1914. 
are considered a greater delicacy than the fruit, which in a raw state resembles the chestnut in flavor, and under favorable conditions weighs over three pounds. The proper way to grow them is to plant the whole fruit, as they have but one seed, and they produce fruit in three months, under favorable conditions. The vine is exceedingly rapid in growth and may cover a thousand square feet in one summer.

\section{THE GUAVA}

Two species of guava have been quite widely tried in this Statethe strawberry guava (Psidium cattleyanum) and the lemon guava (Psidium guayava). The former is the hardier, and, in fact, seems to be about as hardy as the orange, and it has fruited in widely-separated parts of the State; the latter is quite tender, and is at present only grown in favorable places along our southern coast, and even there it is found inferior in quality and usefulness to the strawberry guava.

Mr. C. P. Taft, of Orange, has confidence in the lemon guava through the selection of better varieties. It is far larger than the Strawberry, and of quite attractive appearance. Sometimes the color is almost white, sometimes quite green, and frequently of a bright yellow, often with a red check. These variations are only what is naturally to be expected from seedlings, and almost no others have yet been planted. Mr. Taft has fruited quite a number, perhaps a hundred, and finds it to possess qualities which if properly selected and developed will cause it to equal the Strawberry guava in hardiness and flavor and early ripening.

Mr. D. W. Coolidge, of Pasadena, president of the California Association of Nurserymen, at the annual meeting of that body in Fresno, November, 1913, gave his judgment of the standing of the guava in (alifornia as follows:

The guava is a plant of great value as an ornamental. Its glossy green foliage is scarcely less attractive than its large snowy white, jasmine scented flowers. While many varieties of the guava are found growing in our section, few of them to my mind have any real value. The strawberry guava type is the hardiest and by all odds the best, and all that have had opportunity to test it agree with me in saying Guava lucidum is the best. This is a yellow strawberry guava of a distinctive flavor, and were it not for the large seeds possessed by all guavas would be considered an ideal desert fruit. This particular variety, too, is most prodigious in its bearing qualities. I have known a plant three years from seed to produce more than a quart of fruit, and we have had in our nursery plants eighteen months from the time the seed was placed in the ground with a number of fruits on them. The fruits will average an inch or more in diameter, always round, and are of a bright lemon color. The ordinary strawberrr or red guava is worth while growing for jellies. Another desirable strawberry guava is the Gurava araca. This in appearance is similar to lucidum, but is much later. While lucidum ripens from September to November, I have never known araca to ripen before the middle or last of December. This one is the more susceptible to frost.

The guava grows quite readily from the seed, and grows from cuttings under glass. In regions of generous rainfall and on retentive soil it does not require irrigation, but it must have sufficient moisture at command. A light loam seems best adapted to the shrub. 


\section{THE FEIJOA}

Along with the guava should be mentioned the Feijoa Sellowiana, a member also of the myrtle family. In habits of growth it is much the same as the guava and while the foliage is not so handsome, being of a generally silver gray effect, the flower is very showy. In May it sends forth a great profusion of blossoms, which may be called red, white and blue, unless one desires to be perfectly accurate, in which case the blue would have to be changed to purple. The petals are unusually thick and fleshy and are very sweet to the taste. The highly perfumed fruit, about one and one-half to two inches or more in length, comes in November. The flavor is delicious, like the strawberry, but lacking the acid. The seeds are very small, almost unnoticeable; quite a contrast in this respect to the guava. F. W. Popenoe, Altadena, Los Angeles County, has published an excellent monograph on this fruit.

Mr. Coolidge'gives this appreciative sketch of the plant and fruit:

For eight years I have been watching a fruit which was introduced supposedly as a tropical or semitropical fruit, but we find it does not belong to this class at all. Feiioa, cominr from Uruguay and Southern Brazil, is a fruit of extreme hardiness. I have in mind one plant that has stood a temperature of four degrees above zero without any injury whatever. I know of other plants growing on the desert that have endured a week's temperature of 115 degrees without any injury, so we can safely class it as a hardy fruit. So far nothing but seedlings have been grown, and these vary greatly as to bearing qualities and size of fruits, but there are individual plants growing in our section that fruit regularly and produce fruits of a good size. Within a few years we will have plants of the feijoa producing fruits on the average as large as an ordinary hen's egg. The fruit ripening in December gives it a status that no other fruit of its character has. I have kept the fruits in good condition for four weeks after picking. It is one of our finest ornaments with its grayish green persistent foliage and charming white and red flowers.

\section{THE GRANADILLA}

The granadilla is the term applied to the edible fruit of a species of passion vine (Passiflora edulis) which is quite hardy, and is growing in different parts of the State. The fruit is about the size of a small hen's egg, purple exterior when ripe, the thin, brittle shell inclosing a mass of small seeds covered with a brilliant yellow pulp, mildly acid, and of very agreeable flavor. Very good jelly has been made of the fruit. Another passion vine with large pink flowers is very widely distributed in California, and bears a large, yellowish-brown fruit with edible pulp.

\section{THE JUJUBE}

The jujube (zyziphus jujube), from the fruit of which the delicate paste of the confectioner is, or should be, made, was introduced by Mr. G. P. Rixford in 1876, and is fruiting regularly and freely in several parts of the State. The plant is easily grown from seed or cuttings. The orange-red berries are produced three years from planting, and ripen in November and December. They are edible fresh or dried. As yet the fruit has not been turned to commercial account. 


\section{THE LOQUAT}

The loquat (Eriobotrya Japonica) is widely grown in California as an ornamental plant, and a small amount of fruit is profitably marketed each year. During the last twenty years a very marked improvement in loquats has been achieved by painstaking effort by Mr. C. P. Taft, of Orange, whose experience is freely drawn upon in this chapter. Mr. Taft's work has demonstrated that this fruit is susceptible of improvement in size, flavor, appearance, in bearing habit of the tree, and in direction of early and late varieties, and in all these directions not only in the line of better fruit, but fruit which commands in the market several times the value of the common types. Upon the basis of the new varieties the season for the loquat is from February to June, the bulk of the crop coming in April and the first half of May. The Advance Loquat was the first of the new varieties to attract attention. It is very prolific. The fruit is often as much as three inches in length, and from one inch to one and one-half inches in diameter; it being of a peculiar pear shape. The clusters frequently contain twenty specimens. Its color is a bright orange yellow when fully ripe, and it should never be picked until it is so. The flavor is distinct and very sweet. Many compare it to the cherry. If not bruised when handled it will keep easily two weeks, growing sweeter by the process, and will eventually shrivel up without decay, thus proving itself capable of being shipped long distances. Mr. Taft has named the following varieties:

Blush.-Very large, much like Advance resistant to diseases.

Advance.-Yellow, pear-shaped, from two to three inches in length, clusters very large, very sweet when fully ripe.

Premier.-Salmon-colored, oval, large, but not as large as the Advance, sweet, but peculiar flavor .

Victor.-Largest, color pink to red, probably the best for canning.

Pineapple.-Very large, round, immense clusters; flesh white, skin yellow.

Commercial.-Very large, pear-shaped, yellow with white flesh.

\section{THE PERSIMMON}

The persimmon of the southern states (Diospyros Virginiana) was introduced into California in early days some time ago, as there are trees thirty to forty feet high growing on Rancho Chico. The widelydistributed species, however, is the Japanese (Diospyros Kaki), of which many varieties are now fruiting in different parts of the State. The tree is quite hardy, and fruits freely both along the coast region and in the interior. It easily takes the form of a low standard, and with its large, glossy leaves during the summer, and its immense, highcolored fruit clinging to the twigs after the leaves have fallen, it is a striking object in the orchard or in the house garden.

Persimmons grow readily from seed, but in most cases the improved varieties must be reproduced by grafting on seedlings either of the Japanese or American species. The tree seems to thrive in any fair fruit soil, taking very kindly to close soils if well cultivated. The amounts of fruit now reaching our markets are increasing and a demand is found for certain amounts at fair prices, but there is no object 
now apparent for large increase in production. This fruit, so highly esteemed in the Orient and so highly praised by travelers, has not become as popular as expected on this coast, nor have the great markets at the East required more than a carload or two a year so far. Such limited shipments have, however, sold well in the large Atlantic coast cities, where a considerable number of Orientals have congregated. Americans who wish persimmon at all seem to prefer the smaller but more piquant Virginia species.

Recently, however, the local demand has increased because of the large numbers of Japanese who are now upon the Pacific Coast and a shipping demand for the fruit from Seattle to the Hawaiian Islands and other Pacific ports, has arisen. Local sale in San Francisco and Los Angeles is profitable in a small way. The removal of astringency while the fruit remains firm has been successfully accomplished by Mr. George C. Roeding of Fresno, following a Japanese method. It is simply to place the fruit in tubs, from which saki, or Japanese "rice beer," has been lately removed. The tubs are hermetically sealed, and the fruit left in them from eight to ten days. When it is then removed, it is found to have altogether lost the puckering power. Mr. Roeding says that he used eight large saki tubs, each of which would hold twenty-five gallons, and in those treated one thousand pounds of persimmons.

Perhaps the largest single persimmon-producing proposition in California is that of Ira Avery, in Placer County, which is thus described by Mr. R. E. Hodges:

A mile down grade into the American River canyon, protected on all sides by magnificent hills, Ira Avery's father-in-law planted Japanese persimmon trees in 1876, and three of them are still thrifty and bearing. It was in 1887 that Mr. Avery bought the ranch and planted 50 more. In the years since then, many persimmons have been planted in whatever nooks of the ranch were available until he now has 1500 trees ranging from one to 38 years old. Four hundred were planted last snring, and one of these bore fruit the same season. the first four or five years, however, all fruit should be picked off. It is during this time, too, that all the pruning is done, just to shape the tree. Picking begins the middle of September and lasts till December. The fruit is then unripe, hard as a green tomato, colored, but not the same as they become later. They are wrapped in papers like peaches, and packed in peach boxes, holding about $30 \mathrm{lbs}$; t the largest size being highest priced. The Tane Nashi variety pays best on account of its size and earliness. Seven main varieties are grown and shipped, as follows: Tane Nashi, Hachiyu, Hyakume (the most important), Maru Kaki, Mikado, and Edoishi.

Some trees yield over 30 boxes, many are not yet in bearing; the crop in 1912 was 2200 boxes, in 1913 1500, because the trees bear lighter in alternate years.

New York is the best market, Pittsburg, Philadelphia and Boston being good, while the demand is light in the Middle West. Many are sold in San Francisco, where the average net in 1913 was $\$ 1.081 / 2$ per box, while the net on eastern shipments was $\$ 1.35$. The fruit is too hard to eat even after shipment to New York, where it must be stored some time until well ripened.

\section{THE PINEAPPLE}

Casual experiments with the pineapple in the open air in this State have been made for a number of years, the fruit being occasionally produced. Most has been accomplished by Mr. J. B. Rapp, of Holly- 
wood, Los Angeles County. Mr. Rapp's place is in the Cahuenga Valley, and in that part of the valley which is famed as frostless, where even beans and tomatoes survive winter temperatures. Mr. Rapp set out his first twenty-five plants in 1891, and a number of his neighbors also set out groups of plants, but two years finished up all but his. In 1893 the first of his plants fruited, but they did not seem to take kindly to the situation at first. They grew very slowly and the first fruit only weighed half a pound. After setting out his own acclimated plants, they have done better each year, and the fruit which Mr. Rapp has sold recently has weighed from two to four pounds each. If the strongest offsets or suckers are planted they bear inside of a year, and Mr. Rapp is endeavoring to have his fruit set from May to November, as the fruit setting at other times in the year is usually undersized on account of the slow growth during the winter and early spring. It seems probable that the pineapple resents the dry air of our summer as well as the lack of winter heat, and a lath covering and a summer spraying may be desirable. It is very doubtful whether the fruit can be profitably grown in this State on a commercial scale.

The pineapple thrives best on a fine sandy loam, but will grow well on many soils if well drained and cultivated. The plants can be set three by three or four by five feet, so as to allow cultivation both ways while the plants are young. Plants are secured from "suckers," which come from the root, from "slips," which grow on the stem just below the "apple," and from "crowns," or the tufts of leaves at the top of the fruit. Suckers are said to bear in one year, and slips and crowns in two years. Strong suckers are best for planting, and they should be set out early in the spring as soon as the danger of cold weather is over.

\section{THE POMEGRANATE}

This fruit (Punica granatum), famed in literature and art, is grown in various parts of the State, and certain amounts are profitably sold. The shrub or low tree, in good soil, will reach the height of twenty feet. It is a hardy plant, easy of propagation from seed or cutting. The beauty of the tree, not taking the fruit into account, has caused it to be planted in many gardens. Exposed to the raw sea winds it does not bloom well not set with fruit, and is best adapted to the warmer regions of the interior, where it is an early and abundant bearer. The variety chiefly cultivated is a bright orange color, but there is found a large variety of them, varying from almost pure white with a faint blush, to dark red. The fruit ripens in the warmer parts of the State, north and south, in October.

Pomegranates for eastern shipment are proving profitable in the Porterville district, whence nine carloads were shipped in 1913, and the packers reported the supply scant of the demand. In that year the greater part of the fruit shipped netted about $\$ 2.00$ for four-basket boxes, or at the rate of approximately $\$ 75$ per ton net to the growers. An especial feature was made of fancy packages, which proved acceptable to the trade. 


\section{THE STRAWBERRY TREE}

The Spanish madrono (Arbutus unedo) is now quite widely grown, chiefly as an ornamental shrub or tree. The growth is exceedingly beautiful if kept free from scale insects, the fruit ranging as it ripens through shades of yellow, orange, and deep red, and contrasting beautifully with the glossy evergreen foliage. The fruit is of pleasant flavor.

\section{MELON SHRUB}

This plant (Solanum Guatemalense) is a small, half-herbaceous shrub from the table-land of Guatemala. The fruit is yellow, splashed with violet, somewhat of the shape of the egg-plant, but is usually seedless, and is readily propagated from cuttings. There are thriving plants in many protected places in the State, and some fruit reaches the market, but few seem to like the flavor, which is something like a tomato and melon mixed. Its greatest use will probably be for salads.

\section{THE MELON TREE}

The melon pawpaw (Carica papaya) has been widely introduced experimentally in this State, and many situations are found unfitted for its growth, but satisfactory fruiting has been secured at several places in southern California, especially if protected the first year it will stand light frosts afterwards. With Mr. Cammack, at Whittier, Los Angeles County, it ripens fruit the third year from the seed-the fruit being pleasant to eat as one would a mushroom. The large figlike leaves and the peculiar markings of the trunk make the tree a very striking object.

\section{THE PRICKLY PEAR}

The tuna, or fruit of the cactus (Opuntia vulgaris), is produced in nearly all parts of the State except on the mountains. It was one of the old mission fruits, and was enjoyed by the early mining population until better fruits were available. It is about as large as a mediumsized pear, and has a pleasant acid flavor is one succeeds in escaping the prickels in getting at the interior of the fruit. The tuna is still a commercial article in a small way.* Plants are grown readily from cuttings of the fleshy leaves.

Quite a distinction must now be made between the foregoing and the smooth or spineless fruits which are superior in quality as well as unarmed with prickles, and therefore readily handled and eaten. Varieties more or less innocent in this respect were introduced from the Mediterranean region many years ago, and propagated to a limited extent. Recently Mr. Luther Burbank of Santa Rosa has undertaken special work with the cactus, both for fruiting and forage purposes, and has attained remarkable results which are attracting wide attention, and upon which producing enterprises are being undertaken.

*The tuna as food for man, by David Griffiths, Bulletin 116, Bureau of Plant Industry. 


\section{THE ALLIGATOR PEAR}

The avocado, or Aguacate of the Mexicans (Persea gratissima) has proved hardy in several districts in the State, north and south. It is not likely that it will be satisfactory without high summer heat and freedom from heavy frosts. It is, however, one of the most promising of its class of fruits, as it is known to epicures, and its marketing at a high price is reasonably assured. Mr. J. C. Harvey, of Los Angeles, gives this interesting account of it:

It is a handsome evergreen tree, and, in the typical form, bears elliptical leaves from two and one-half to three inches in width, narrow toward the base, and about six inches long. In some varieties the new growth is of a reddish brown, ultimately becoming deep green. The fruits are pear-shaped, about the size of a Bartlett pear, and contain a single, rather large seed. When ripe, the skin, which is much thinner than that of an orange, parts easily from the pulp, which is of a moderately firm though buttery consistency, and forms, with lime juice or pepper and salt, one of the most delicious salads known to epicures. Indeed, the fruit is a perfect mayonnaise in itself. Few persons fail to like it, even at first, and in countries where it is common, it is esteemed above all other vegetable productions, both by natives and foreigners alike. The pulp is quite rich in a bland and most agreeable oil, said to be very nutritious. The tree attains a height of from twenty-five to thirty-five feet, and forms a handsome object when liberally cultivated. The tree is a gross feeder. Goodsized trees carry a large crop, which, after attaining a certain size, can be picked at intervals of a week or two, extending over a period of two or three months, the fruits in each instance ripening in a week or ten days after gathering; and a very remarkable fact is that the quality or flavor of the last picking seems just the same as the first.

The alligator pear must be considered as one of the most promising fruits included in this chapter; it is now being planted largely and it may prove the most profitable of the grouip. Efforts are in progress for improved varieties by selection from fruiting seedlings and propagation by budding.

The seeds may be started in small pots, and shifted before they become potbound into small redwood boxes. As the plants attain the size of a lead pencil and larger, bud them at any time during the reason when the bark will slip and when budwood is obtainable, using care not to cut or injure the cambium layer. The bud is tied with wax cloth, and trained up as in any other budded tree. As the bud unites, say in from two to four weeks (as the case may be) the seedling is partly cut back to force out the bud. The strings are left on until the bud starts. Care must be taken not to use too much water in growing the young trees.

The literature of the alligator pear is increasing and should be consulted.*

Two general types of avocado are, in 1914, being rather sharply contrasted; the thick-skinned, being advocated as more likely to be profitable in California because its covering favors long shipment and because it is better known at the east, as the Florida product is of that

*The Avocado, by G. N. Collins. Bulletin 77, Bureau of Plant Industry, U. S. Dept. of Agr. 1907. Consult also Yearbooks of the U. S. Dept. of Agr. for 1905 and 1906. The Avocado in Southern California and the Development of the Avocado Industry by $F$. W. Popenoe, Altadena, Los Angeles county. New varieties of the Avocado for California, by K. A. Ryerson, University of California Journal of Agriculture, November, 1913. Mr. Ryerson gives original descriptions of fourteen varieties which he considers superior for commercial planting. 
type; the thin-skinned, because of its greater frost resistance. Californians have still much to learn of this fruit both in its cultural and commercial aspects. At the California Nurserymen's convention of 1913 Mr. J. S. Armstrong of Ontario gave the following review of varieties now being propagated:

The thin skinned sorts are much the hardier, some excellent types of which are now fruiting with us. The best of these I may mention: The Harman, the Ganter, the Northup, the Fowler and the Chappelow. Last winter (1913) young trees of these hardy types withstood from five to fifteen degrees of frost without serious injury where the trees had been allowed to harden up. One twelveyear-old seedling tree in the colder part of the valley withstood a temperature of 12 degrees above zero. The ends of the branches were nipped, but the main branches were not injured.

The thick skinned varieties are not as resistant to extremes of heat or cold, however, as the former mentioned types, hence are recommended only for planting in more favored localities. Among the best known thick-skinned varieties are the Taft, Lyon, Wagner, Meserve, and Walker. Of these the Taft is undoubtedly the best. It is pear shaped, weighing a pound or over. On Mr. C. P. Taft's grounds near Orange the original is a large spreading tree which usually bears several hundred fruits annually.

As there is an uncommonly great variation in the time of blooming and also in the period required for the fruit of different types of trees to come to maturity, an orchard may be obtained by selection which will bear continuously. This is of course very desirable to the consumer and immaterial to the market grower, as there is plenty of demand at all times.

\section{THE WHITE SAPOTA}

There are two old trees in Santa Barbara, one believed to have survived from the mission planting in the early part of the last century, the other half as old, of the white sapota (Casimiroa edulis). Dr. Franceschi commends the tree for every garden. Mr. Harvey of Los Angeles describes the sapota as growing with him from seed from Vera Cruz as follows:

This tree endures slight frosts unharmed. It is indigenous in northwest Mexico and is remarkable among the Aurantiacea, producing green colored flowers, and superficially bears little resemblance to an otherwise well-marked order of plants. The fruits are the size of apples, and are esteemed in that portion of Mexico where it is common; according to some botanical authorities it is not considered altogether wholesome, possessing narcotic properties. The pulp is described as possessing a delicious, melting, peach-like taste.

Mr. Taft reports the sapota as bearing well with him. The tree is an exceedingly rapid grower, much after the habit of the walnut, but evergreen. In August and September the peach-like fruit, greenish yellow, with large seeds shaped life those of an orange, matures well and is excellent, this being the normal season for ripening. At other times fruit is often found, but is apt to be worthless and even dangerous. As it will not ripen well off the trees and must be quite soft when eaten, it will never be of much market value. 


\section{THE TREE TOMATO}

This plant (Cyphomandra betacea) is a native of Central America and is of shrubbery habit, growing five or six feet high, with large, shining leaves, often a foot long. The flowers are fragrant, of a pale flesh color, with yellow stamens, and are followed by fruit the shape and size of a duck's egg, at first of a purple tint, but gradually assuming a warm, reddish color as it ripens. When ripe the fruit may be used raw as a tomato is. If the skin is removed and the fruit stewed with sugar, it has a slight sub-acid flavor which is very refreshing. It makes a fine jelly. The plants bear the second year from the seed and the fruit ripens continuously for several months. The seeds should be started just as are those of the common tomato, and the plants set out eight or ten feet apart.

\section{THE KAI APPLE}

This name is applied to the fruit of Aberia Caffra, a native of Natal and Kaffaria, a tall shrub, yielding an edible fruit of a golden yellow color, about an inch in diameter. It is commended as a hedge plant, as it is densely clothed with strong dry spines. The leaves are small and of a rich green hue. The fruit, which is produced freely in the warmer parts of the State, is chiefly used for making preserves.

\section{OTHER FRUITS}

The foregoing enumeration does not include all the exotic fruits which have found a place in California soil. There are many more, some of which are demonstrating their fitness to add to the graces or the gains of our horticultural life. The caricas, carissas, eugenias, hovenia, etc., are all gaining places in California gardens. Even the more strictly tropical mango, the monstera, sapodilla and the like are claiming the attention of amateurs. Of the mango in southern Cali-. fornia the best account is by F. W. Popenoe, Altadena. 


\section{PART SIX: SMALL FRUITS}

\section{CHAPTER XXXVI}

\section{BERRIES AND CURRANTS IN CALIFORNIA}

In suitable soils and situations, and with proper care and cultivation, the small fruits sustain the general reputation of California by the size and quality of the product, and by the long-continued and abundant fruiting of the plants. Probably nowhere else in the world do small fruits better repay generous treatment than in this State, and probably nowhere do they suffer more from neglect. There are parts of the State, of course, where some small fruits, left to their own resources, thrive and bear abundantly, but, speaking of the State as a whole, the price of success is intelligent devotion on the part of the grower.

There are localities in California which favor almost continuous growth and fruiting of some of the small fruits, and it is no fiction to say that in such a place one may have raspberries and strawberries upon his table every month of the year. Such situations are the thermal belts, which are practically frostless, and, by securing favoring moisture conditions in the soil and proper varieties of the fruits, the existing temperature conditions will produce the results indicated. Though this be the case, the profitable growth of small fruits is not, of course, restricted to such situations, but the largest commercial enterprises are carried on in places where the summer-crop rule prevails, but the bearing season is much larger than in the eastern States.

Small fruits for family use may be grown on all fertile soils, and therefore they should be produced on every farm. Growing for market on a large scale involves considerations of suitability of soil and climate, ease of cultivation, water supply, and facilities for transportation, which will probably occur to any one who gives the matter the thought and personal observation of existing small fruit farms, which such an important commercial venture should command.

It is often claimed that soil for small fruits should be deep and rich of the types generally called garden soils. There is an advantage in this because of amount of plant food and retention of moisture when well cultivated, but at the same time shallow soils even when overlying hardpan, which may not suit deep rooting trees or garden roots, can be profitably used for small fruits if water and fertilizers are intelligently used. This will be stated more fully in the discussion of the strawberry, but the general fact is pertinent to the growth of other small fruits also.

Preparation of soil for small fruits should be most thorough and careful. Even more generous work than that commanded in Chapter $\mathrm{X}$ for trees and vines should be done. It is the more necessary to work deeply because subsequent culture of small fruits must be shallow. 


\section{THE BLACKBERRY}

The blackberry is a great favorite in California markets. It thrives in all parts of the State, and the plant is best suited of all small fruits to yield generously without irrigation, though it relishes sufficient moisture and repays it with fruit. There is great difference in practice as to supplying water artificially. The growth of cane, and the size and appearance of the fruit, will show the observing grower what should be the practice in his situation, and the general suggestions as to irrigation in Chapter XV are applicable. There are regions in which blackberries are irrigated weekly throughout the summer, and others in which the berries are gathered from June to November without irrigation. Of course, with such wide local variations there can be no general rule for practice. Let the grower simply bear in mind that if he does not get good, plump, and glistening fruit and good strong growth of new canes at the same time, he should give irrigation. The requirements of the plant during the fruiting season are great, and they must be met. Many failures are due to lack of irrigation when needed.

Propagation.-Blackberry plants are secured by digging up the shoots which come late in the summer from old stools; securing therewith a bunch of fibrous roots with a portion of the main root three or four inches long. To propagate on a large scale dig up the roots entirely, and, cutting them up with pruning shears into pieces about two inches long, plant them in a well-prepared bed in the garden or nursery. Place the root cuttings about two inches apart and cover about three inches deep with well-ptlverized soil, the depth being regulated, of course, according to the nature of the soil, deeper in light than heavy soils. A light mulch will assist in retaining moisture. The time for this work is at the dormant period of the plant. One summer's growth gives good plants for setting out.

The Mammoth and Crandall are propagated by rooting tips of canes.

Planting out Blackberries.-Blackberries should be planted in rows far enough apart to admit of the use of the horse and cultivator. As the constant tendency of the plant is to extend itself in the growth of new canes, the rows should not be less than six to eight feet part, and the plants about three feet apart in the row. The plants soon occupy the full space in the row, and cultivation is only possible between the rows. Some growers plant blackberries as they do grapevines, seven or eight feet apart both ways, and then cultivate with the horse both ways. Planting in rows is usually thought better. The number of plants to fill an acre at different distances can be calculated as described in Chapter XXVI for grapevines.

D. Edson Smith, of Orange County, who had much experience with small fruits, described his method of laying out and planting on a large scale, with a view to irrigation, as follows:

Plow deeply and harrow thoroughly several times before setting out. Lastly, open a trench with your plow where the row is to be, twenty inches deep; go along with a basket of plants, a four-foot lath and a shovel, and set a plant in this trench every four feet and fill the dirt around it with the shovel. If this trench is too deep in places for the length of the plant root, fill in with a little 
dirt; if not quite deep enough in places, scoop out a shovelful. Aim, in preparing the ground with plow and smoother, to leave it dishing each way toward the row of young plants, so that irrigating water turned in at the upper end will run along the row of plants as in a trough. Aim to have the ground around the set plants a few inches below the general level of the land. After the plants are all set in a row, go along with a rake if there are but a few plants, or with a horse-hoe is there are many, and fill in the trench between the plants. It is a pleasure to set out plants in this way, and such deep, rich, well-stirred soil delights the plant roots, so that they grow rapidly in every direction, and the plants throw up their heads in a manner entirely satisfactory to all concerned. If the ground is dry, or there is no rain soon after setting out the plants, irrigating water should be turned down the row or at least a quart or two of water poured around each plant; then, before the soil hardens, stir it well with cultivator and hoe. All future care resolves itself into frequent waterings and frequent stirrings of the soil. Allow no weeds to appear, and keep three inches of surface soil well loosened with the horse and hoes. These small fruits require frequent waterings, especially when forming fruit and during the fruiting season.

Cultivation.-Thorough cultivation of the surface soil is essential for retention of moisture. After the plants attain size, cultivation should be secured with as shallow-cutting tools as possible so as to prevent injury to the roots, which not only weakens the plant, but increases the growth of suckers between the rows. A horse-hoe with a long knife running horizontally, or with duck-foot teeth, well sharpened, answers well in keeping the ground clear of weeds and suckers, and the surface loose. Due regard must, however, be paid to securing sufficient depth in this surface layer to prevent the soil beneath baking hard and drying out, as discussed in the chapter on cultivation.

Frequency of cultivation depends upon irrigation, for the cultivator must always follow the application of water. The spaces in the row which can not be reached with the cultivator must be kept clean from weeds, and free from baking, by the use of the hoe. It is advisable that the cultivation be the cleanest possible, for moisture exhaustion by weeds can not be afforded.

Pruning and Training.-There is a little difference in the way of training blackberries practiced in this State. Of course this does not include the "let alone" system, which is not followed by any good grower. The difference lies mainly in the use or disuse of artificial supports for the canes. In either case the pruning of the canes is similar in kind but different in degree, for if no supports are used, the canes are headed lower.

At planting out, cut back the cane to near the surface of the ground and mark the plant with a small stake. At first the top growth should not be checked, but when new canes grow out strongly they should be pinched at the tip to force out lateral branches for fruiting the next year. Those who intend to tie canes to a stake or trellis let them attain a height of five or six feet before pinching off the terminal bud; those who intend to teach the cane to stand alone pinch when it is about four feet high. All agree to pinch off the ends of the lateral branches at about eight to twelve inches from the main stem. This pinching of blackberry canes may be done by the watchful grower of a few plants, with the thumb and finger, but thrifty blackberry plants are such rapid cane growers that in large plantations cutting back is often done with a sickle or corn hook or sharp butcher-knife, several times in the course 
of the summer. It is also advisable to thin out the suckers with the hoe while cutting out weeds, leaving only about as many as it is desired to have for fruit the next season. This method gives stout canes, with plenty of short side branches, well supplied with buds, which will send out fruiting shoots the following spring. If supports are used, the four to six canes which are left to each stool are gathered within a looselydrawn bale rope and tied to the stake; or if a trellis is used, the branches are brought up to the wire or slat so that the distance is about evenly divided between the shoots.

Mr. Claud D. Tribble of Elk Grove gives the following advice for handling the Lawton, which is the chief upright variety grown in California :

Give the plants some support the first season by a stake, and the second season the permanent stakes are put in, one on each side of the plant. In driving them, allow the tops to be wider than the bottom so the berries can be picked easily. Large wires or wooden cleats are used to support the vines or canes. The posts are about six feet long and driven in the ground until solid, making the top about five feet high. During the first season's growth there is very little pruning except thinning to the desired number of canes. The second year the canes are allowed to grow above the stakes and then tipped to cause laterals to form, which are cut back to less than 12 inches to produce berries the following season. The old wood is cut out each season and the new shoots trained as before. If the old canes are cut out as soon as the crop is gathered, it is done more economically and the young plants grow better.

Though these systematic methods of summer pruning are practiced and advocated by the most careful growers, it should be stated that there are large plantations which are conducted upon a more simple system. The pruning consists in cutting out old canes in the winter, and the only summer pruning is slashing off these canes which interfere with cultivation. The canes are sometimes held up by tying bunches of them together with ropes. Of course this system costs less than the more careful one which has been described, and yields profit enough to induce adherence to it. No doubt quite as great weight of berries could be had from a smaller area by a better system of growing.

After the leaves fall, the canes which have borne fruit during the summer are all cut off even with the surface of the ground with longhandled pruning shears or with a short hooked knife with a long handle, and all debris removed from the rows.

Application of Manure.-The blackberry loves very rich ground, and plenty of well-rotted stable manure or compost, as described in Chapter XIV, should be applied. It is a good plan to apply in a thick covering all over the ground and between the canes as soon as the patch is cleaned up in the fall. The early rains carry down the soluble parts of the manure, and later in the season the whole is plowed in between the rows, leaving a foot or more next the plants to be carefully forked in, as the digging fork does not cut the roots like the spade.

Mulching.- The mulch, to keep the ground moist and to obviate summer cultivation, is very satisfactory where it is thoroughly done. Apply coarse manure or partially-rotted straw and the like, after the last spring cultivation, and use the hoe to keep down weeds and suckers which come up between the rows. Some growers use mulch close to the canes, cultivating the remainder of the ground between the rows. 
Bearing Age and Longevity.-If blackberry plants are well treated the first year after planting out, there will be considerable fruit the following summer. How long the plants will bear satisfactorily depends, also, on situation and treatment.. Sometimes the plants fail early; even with good, generous treatment in good soil, the old stool becomes weak, the shoots are thin, and the fruit small. Some count about eight years as the profitable age of the plant, and then cut out plants and give the land a change. Of course berry growers prepare for this by frequently making new plantations.

Irrigating Blackberries.-Though a good part of the blackberry crop is grown in regions of heavy rainfall without irrigation, the use of water is sometimes very profitable. Mr. T. B. Cannon, near Los Angeles, works in this way:

Mr. Cannon aims to get Crandall blackberries from June 15 to November, which is possible by his system of irrigation and pruning. So the water is applied in winter and about every ten days until the berries are as big as peas, cultivating between times. The rows are eight feet apart and the plants four, so that one-horse cultivation is possible until the fruit gets so heavy as to bend over into the onen space. When ripening time begins in June, a ditch is dug close to the vines on each side of the row, for irrigation. This gets water pretty well where it is needed, the ditches are out of the way of the cultivator after the first crop is off, and they are shaded so as to avoid evaporation in the hot summer. Water is applied every four or five days during the heavy picking, then every ten days till the main summer crop comes on, when it is applied twice as often again. Irrigation when the berries are turning black makes them larger and of better color. While the juice might thus be considered diluted, this is the sort of berry people buy, and the proportion of juice to seeds is what makes a desirable market berry, when markets are near by. Such practice might make undesirable fruit for canning or shipping.

Varieties of the Blackberry.-Comparatively few kinds are largely grown. The Wilson Junior, Lawton, and Kittatinny were formerly the prevailing kinds, ripening in the order named. The Erie is favored by some as a middle season variety. The Early Harvest has been favorably reported by a number of growers. These have, however, been largely superseded by a renamed variety, Crandall's Early, which is the earliest of the improved varieties, and has a very long fruiting season. The fruit was named after Dr. J. R. Crandall, of Auburn, who first fruited the variety from plants given him by a stranger hailing. from Texas, and the proper name of the variety is probably Texas Early. It is a strong, vigorous, hardy plant, very productive, of firm, handsome berries; resembles Lawton in canes, leaves, and flavor of fruit; not given to sprouting from running roots.

Another variety which has advanced in favor is the Oregon Evergreen, introduced from Oregon but not native nor originated in that State. The late John Rock described it as follows: "Origin unknown; beautiful; cut-leaved foliage, which it retains during the winter; berries large, black, sweet, rich, and delicious. It continues to ripen from July to November, which makes it one of the best berries for family use." It loses size and quality notably on scant moisture.

Some effort has been made to secure improved varieties of our native blackberry, and a most striking result has been secured by Judge J. H. Logan, of Santa Cruz, by crossing the wild berry with Crandall's 


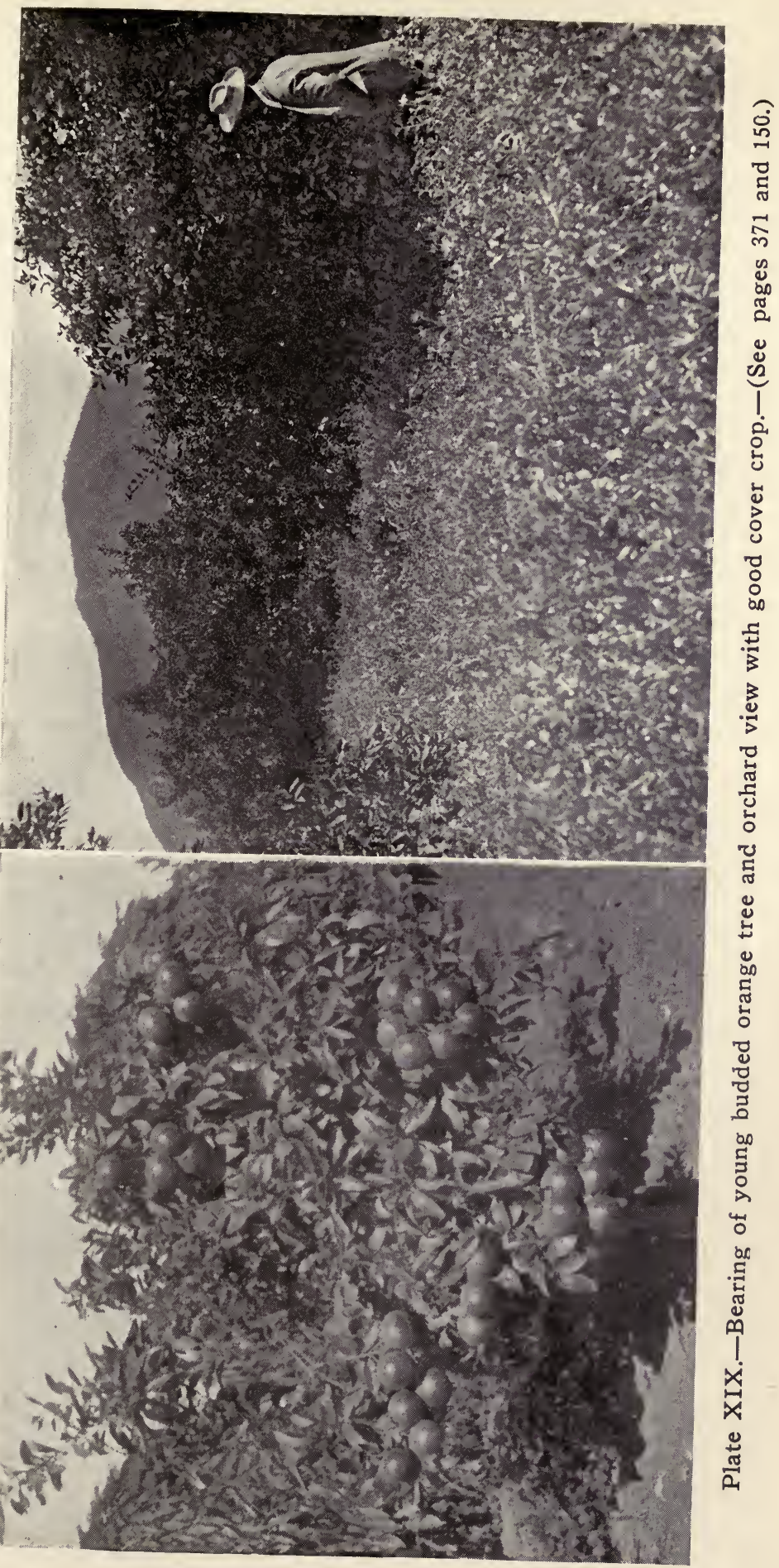



Early, producing a fruit so large that it has been named "Mammoth" by its originator. The canes of the Mammoth are very peculiar, being very large and thickly covered with small, short spines. The canes start early in March, grow thick and stout until about five feet high. They then take on a running habit and grow from twenty-five to thirty feet in a season. Late in the fall the tips or stolons seek the ground and take root. The Mammoth is not an evergreen like its Texas parent, although it does not entirely lose its leaves in winter. It begins to grow and flower very early in spring and ripens its fruit the last of May, some weeks earlier than the Lawton. The fruit is more acid than the Lawton, but, when perfectly ripe, is sweet and of superior flavor. When cooked or canned the flavor is identical with the wild berry of California. This variety is often wrongly called "Black Loganberry."

The Himalaya is a blackberry of wonderful growth and prolificness, highly praised by amateurs, but not yet fully made out from a commercial point of view. It is magnificent on a garden fence or trellis.

The Dewberry.-The improved varieties of the dewberry, or trailing blackberry, are now quite widely known and highly praised, but are chiefly grown for home use. Some growers use trellises; others train the vines along rows on the ground surface. The following is the method of Mr. A. M. Munger, of Fresno, and includes irrigation arrangements :

For planting the Lucretia dewberry, prepare the ground by plowing deen and cultivating until the dirt is thoroughly pulverized. Set the plants about three inches deep and four feet apart, in rows, leaving a space of six feet between the rows. Plant between February 15th and March 15th. Irrigate as often as once a month, always thoroughly cultivating after each irrigation. By so doing a sufficient growth is secured to produce a good crop the second year. Immediately after the first rainfall, generally in October, the vines should be pruned by cutting back within about sixteen inches of the base of the vine.

In February of the second year, plow between the rows with a small onehorse plow, turning the furrows toward the vines, but using a shield so as not to cover them. Follow immediately with a hoe, drawing the dirt up under the vines and forming a ridge. This ridge should be high enough to keep the vines up out of the water when irrigating. After this ridge is formed, water should be run quite often, as the dewberry requires a great deal of water to mature properly. The vines should be irrigated as often as three times at least during the spring. The fruit begins to ripen in Fresno about May 25th, and continues about one month. The dewberry roots readily from the tips without covering if the soil is loose and moist. If many plants are desired it is advisable to cover slightly, and the tips will root as soon as the soil is moistened by the fall rains.

A trellising method for dewberries consists in driving posts to stand about three feet high for the dewberry can not be trained up as high as other berries. Make a half hoop of coarse wire extending from the root of the plant over the arms at top of the post and training the plants on this. The rows are usually six feet apart and the plants six feet apart in the rows. The vines should be well thinned to produce large berries.

The dewberry which has recently been most largely planted in central and southern California is known as the Gardena because of the 
prolificness and profitability of a patch grown by W. M. Gray, of Gardena, Los Angeles county. Its qualities are vigorous growth, hardiness, regular and profuse yield of large, early berries.

Pruning and Training Trailing Berries.-With reference to the handling of trailing blackberries and the blackberry-raspberry hybrids which are continually becoming more popular, it may be said that there is no one best way. There are several good ways, according to the desires and convenience of the growers, and this is the reason why there is an apparent conflict in which all contestants may be right, each from his own point of view. The varieties are sufficiently alike to be discussed together. What seem to us the essentials in pruning them are these:

First, they all bear on canes which grow the previous year, and the fruit comes on laterals which break from them. In this mild climate there is continuous break of laterals which may cover quite a long period and the same wood may seem to be bearing twice. This second bearing is of so little account that the general rule to remove old wood after its main fruiting is a good one.

Second, the wood which grows this year will therefore bear next year, and will send out bearing laterals sufficiently with a number of treatments. The new cane may be pinched at any time during growth and it will then send wood laterals (not fruit laterals) at once and each of these laterals will have the same character that the main shoot would have had if it had not been pinched; that is, it will send out fruiting laterals at the same date the following season.

Third, it does not matter whether you make the new growth bunchy by laterals following pinching or whether you let it run out and cut off part of it at the end of the growing season or whether you shorten it in and at the same time cut away closely all the laterals which it may have made on its own account when it was running out. In all cases there will be dormant buds enough to give fruiting shoots on whatever part of the cane you reserve.

Fourth, the way you prune, then, depends upon serving your own convenience in the training of these shoots up to a post, along on a wire or along on a ridge on the ground-whatever suits you best to keep the fruit out of the dirt, and to promote such cultivation as is desirable, etc., will be accepted by the plant as not interfering with its starting fruiting shoots from whatever dormant buds you have allowed it to retain on the wood which it matured the previous season.

Fifth, there is in addition the application of the principle that good large fruit is the product of a plant which is not carrying too much bearing wood; that is, is not endeavoring to perfect too much fruit at the same time. For this reason, as well as for convenience, it is désirable not to allow a plant to retain all the cane it grows, but to shorten it or to remove the laterals or part of them or to shorten the laterals or in any other way to require the plant to direct its energy to the better development of fewer fruits.

Sixth, growers are, of course, influenced by different considerations. Amateur growers delighting in running vines on fences or trellises 
would not prune as would a commercial grower, who can not have canes running all over his fields. The amateur can pinch a main shoot and send the laterals up the arms of a fan-shaped trellis if he likes and make an object of rare garden beauty, and he can reduce the excess of bearing wood by cutting away the parts of the laterals which run beyond his arms or extra ones beyond those he can carry on his trellis. From the point of view of the plant, he does the same thing that the commercial grower does when he comes along with his scythe or sickle and cuts away indiscriminately all the growth which goes beyond the space where it is convenient for him to have the fruit.

Seventh, do not be too particular about exact methods to imitate; try rather to discern principles which may be served by many different methods.

Six Months Fruiting of Crandalls.-One way in which the principles just outlined may be applied to Crandall's Early which is our leading blackberry, considering the State as a whole, is described by Mr. R. E. Hodges as the practice of Mr. T. B. Cannon, of Los Angeles:

Briars eighteen to twenty-four inches tall, having good root systems, are set with a spade in finely pulverized soil as soon after they are dug as possible to avoid wilting, to the same depth as they were before, four feet apart in rows eight feet apart. The first year they require no pruning. The space between rows is occupied by cabbage, beans, corn, potatoes, etc.

In the second year three or four new canes come up. When these are four feet high, cut off six inches, and laterals start from the leaf axis. When these are thirty inches long their end buds are cut off and they send out blossoms which bear fruit that fall. These same laterals bear the main crop in their second year, besides sending out new laterals near the base of the old ones about the time the berries are well set. The new laterals bear the second crop, which comes on about the time the first crop is gone. The third crop, borne on the new canes, which have been cut back meanwhile to three and one-half feet, blossom from August till freezing, and the berries ripen from September on.

Mr. Cannon has followed this plan with satisfaction on two or three patches, the largest of which is a half-acre set seven years ago in the young family orchard. The first crop from this half-acre in 1913 was 180 crates. The second and third crops usually together equal two-fifths of the first. The second is about half of the third. Two years ago the third alone almost equaled the first. And the berries sometimes sell at $\$ 2.80$ per crate after October.

Growing Crandalls in Hedge Rows.-A method which aims at economy in getting a main crop, without providing for succession, is that of Mr. J. B. Wagner, of Pasadena. He uses no trellises, but leaves the old canes in the hedge-row to support the new growth, in this way:

On the day before berry-picking, all protruding new growth is cut back as close as possible to the blanket of bearing canes which covers the mat of dead ones. This removes all hindrance to picking. Do not leave stubs of new wood above the bearing vines, because it raises the general level of bearing wood in a year or two, so high that the pickers can scarcely reach over and up to the center of the row. When picking is over, both sides of the hedge-row are trimmed off clean till it is left only two feet wide. New growth soon leans out over this and covers it, ready for a crop next year with the berries well outside and handy for the pickers. Every six years he trims the whole hedge back to a height of two feet and clears out all the cane over a year old, and this renews the plantation. 
Training the Mammoth.-A good way with the Mammoth, grown in a commercial way, is described by Mr. Tribble as follows:

The Mammoth is one of the earliest of blackberries, very productive and is one of the most delicious. The plants are propagated from rooted tips which appear on the ends of the vines during the winter months. Mammoths are usually trained on a wire trellis made by driving posts at each hill and nailing on cross-arms to support the wire, which is stapled to each end of the crossarms. The laterals are trained along the wire about half way between the hills, and then cut off to cause them to throw out fruit spurs. As soon as the crop is taken from the old canes they should be taken out and the new canes trained on the trellis as before.

Training the Himalaya.-Success with the Himalaya blackberry is wholly dependent upon proper pruning, and whether it be grown upon an arbor or a fence or upon a low trellis, the same principles must be applied. This variety bears on the same laterals each season and reaches its maximum production the fourth or fifth year. This variety is the latest to ripen with us, coming in after the Mammoth blackberries have all gone. Methods of high and low trellising are thus described :

The high trellis is made on posts 7 feet high and 30 feet apart. Two wires are stapled on the posts, one $3 \mathrm{r} / 2$ feet from the ground and the other at the top. Rows should be planted 10 to 12 feet apart and about the same distance in the rows. The long runners should be trained and woven in these wires to hold up the load of fruit. The second year the runners will throw out laterals thickly along the main canes; these fruit spurs are from 2 to $21 / 2$ feet in length, projecting outside the main canes where the berries are all exposed to the picker's reach. Not over four canes should be allowed to grow in each hill. After reaching the desired length they should be tipped.

The low trellis method is as follows: New vines grow from the roots some 30 or 35 feet in a single season. These are trained on the ground by stakes under the wires which support the bearing vines, so they do not interfere with cultivation and picking. Not too many are allowed to grow. They will throw out a fruit bud about every eight inches, a large number of which will develop next year into spurs bearing 30 to 60 berries. It will also throw out numerous laterals which are cut off before laying the vine on the wires in winter. If they were left, and wound around the wires, they would tend to choke out the fruit spurs, besides being dangerous to pickers. Suckers are kept down, and there is a clear path for the pickers, lined shoulder high with luscious blackberries.

\section{THE CRANBERRY}

Though attention has been given to experiments with the growth of the cranberry in California for many years, it has not been demonstrated that the culture is successful or profitable. Cranberries have been produced, and the fruit shown at fairs, but beyond this nothing has yet been accomplished. It would seem to be a fair conclusion that even in the most moist regions our summer air is too dry to suit the plant.

\section{THE CURRANT}

The currant reaches perfection in size and quality in parts of California adapted to its growth, but its area is comparatively small. The plant does not thrive in the dry, heated air of the interior either at the north or south. It does well near the coast, especially in the upper half 
of the State, and is grown for market chiefly, on lands adjacent to the Bay of San Francisco. The comparatively cool and moist air of the ocean favors it, but even here the sunburn, which is the bane of its existence in the interior valleys, occasionally injures the fruit. Away from the coast, currants are grown to a limited extent along the Sacramento and San Joaquin rivers, near their confluence, but not in the hot valleys whence they flow. On the foothills, too, where the plant has a northerly slope, or other cooling influence, and sufficiently moist soil, it will do moderately well. It is quite possible that the currant may be satisfactorily grown for home use, or for local market in parts of the State where at present one does not find it, providing the moderating effect of elevation and northerly exposure, coupled with the shade of trees; be secured, but even then the hot north wind of the early summer may often injure the fruit. So far as the metropolitan market is concerned, it does not matter that the currant area is limited, for existing plantations produce all, and sometimes more, than can be profitably disposed of at present. It is possible, however, that the future may show a larger demand, for the pure food laws are likely to prevent the further selling of apple jelly with a currant color and flavor under the name of currant jelly.

Propagation.-The currant is readily grown from cuttings. As soon as the bush drops its leaves, and the ground is in condition, as to moisture, secure the cuttings about a foot in length from straight wood of the last growth, and place them in nursery or in permanent place, in good sandy or garden loam, deeply spaded and well broken up. Set the cutting firmly in the earth, six or seven inches deep. If they are to be trained as small trees, every bud below where the lowest limb is to start should be cut out-even to the end of the cutting undergroundotherwise they will be continually throwing up suckers. If they are to grow as bushes, the natural and more productive form of the currant, set them as they are taken from the parent bush.

Planting and Care.-Currants are usually grown in rows about five or six feet apart, the plants standing two and a half or three feet apart in the rows. Most of the currant plantations are between orchard rows, the partial shade of the trees being considered desirable. It is claimed that currants do best when interplanted with cherry, apricot, apple, and pear, not so well when associated with plum and peach, and the almond is least desirable-possibly because the almond is often given less cultivation than the pulpy fruit or is grown on lighter, drier soils. The cultivation is such as is usually given to the orchard, except that in heavy soil the plow is not allowed to come near the cuttings the first season for fear of tearing them from their rooting. After the first year the plow is used in the winter and the cultivator in summer.

Currants will repay generous applications of well-rotted manure, and relish sufficient moisture in the soil. Where this can not be had from rainfall, and retained by cultivation and mulching, irrigation must be resorted to.

Pruning.-If the currant is to be grown in tree form, the branches from the upper buds of the cutting should be shortened in at 
the end of the first summer, and branches growing horizontally should be removed. The weaker shoots in the head are thinned out, but not so much as to leave the top too open. If the plant is to grow as a bush, the only winter pruning will consist in removing dead wood, and thinning the new shoots as may seem desirable, and cutting back about half of the new growth. Summer pinching of the new growth is desirable, as it causes the fruit to set closely and tends to a thick growth of foliage also, and this is necessary, for the bark is liable to sunburn, and the best fruit is that which is well sheltered by the leaves. Another advantage of the bush form is the less likelihood of killing by borers, which is imminent when the growth depends upon a single stem. Well cared for bushes will bear profitably until about ten years old. Replanting should be made on new ground.

Bearing.-The currant bears a quantity of excellent fruit the second year from the cutting, and reaches its fullest product about the fifth to the eighth year, when the yield in the Hayward region is said to range from one and a half to three tons to the acre.

Varieties.-The Cherry currant is the prevailing variety, although the old sorts, the Red and White Dutch, the Red and White Grape, etc., are grown in some localities, and Fay's Prolific is approved by some growers. Pomona is one of the best of the newer red varieties and the old Fertile de Palluau is reported as doing better than others in hot, interior situations. Black currants are but little grown, the market demand for them being very light.

\section{THE GOOSEBERRY}

The Gooseberry is another fruit with somewhat circumscribed area in this State. In localities which favor it, the fruit is often found very profitable, but the demand does not warrant any great increase of product. Though the gooseberry thrives in some situations which do not suit the currant, they may both be described as averse to the hot and dry parts of the State. Still, for home use or local sale one can grow certain varieties of gooseberries successfully, by protecting them from too great exposure to the sun, and by keeping the soil sufficiently rich and moist. The choice of varieties is of the greatest importance, as will be mentioned presently. At present the chief supplies of the gooseberry, as of the currant, are produced in the country adjacent to San Francisco Bay, though thriving and profitable plantations are found elsewhere near the coast, here and there in the interior, and at considerable elevations on the slope of the Sierra Nevada.

Propagation, Pruning, etc.-The gooseberry is grown from cuttings, very much as already described for the currant. The common and the best method is to start the cuttings early in the winter, though some have succeeded with cuttings taken in the spring just as the new growth is starting out. Disbudding the lower part of the cutting if it is desired to train in tree form is also practiced with the gooseberry, but a smaller percentage of cuttings is found to grow after disbudding. 
Gooseberries are planted out and cultivated as already described for currants, and the requirements of the plant in soil, moisture, and manuring, are much the same.

If the gooseberry is to be grown in tree form, constant attention to removal of suckers is necessary; if in bush form, it will only be necessary to remove too old wood and thin out the new shoots. Suckers should be removed clean from the stem, so as to eradicate the latent buds, and pulling off with a gloved hand, when the suckers become woody enough to withstand breaking, is advised. As with the currant, the borer is a constant menace to the life of a gooseberry plant confined to a single stem.

Diseases and Pests.-The gooseberry is subject to insect depredation both in wood and fruit and leaf. The prevailing trouble, however, and that which causes the failure of so many foreign kinds, is trie mildew. To escape this nothing is usually done except to select varieties not subject to the disease, but susceptible varieties can be protected by spraying just as the leaves are opening and once a month afterwards with potassium sulphide half an ounce to the gallon of water. This does not stain nor poison the fruit. The cooler and moister the air the less the mildew.

Varieties of the Gooseberry.-The American varieties, Downing and Houghton's Seedling, chiefly the latter, constituted for a long time the main varieties marketed in San Francisco. Early experiments with collections of English varieties showed that most of them were failures because of mildew; still a few of the green and white sorts, notably the Whitesmith, have succeeded. The proportion of large berries now being marketed is much greater than formerly, and the superior price warrants especial effort to produce them.

A large English variety, which was brought to California many years ago by the late John W. Dwinelle, is now the most widely distributed large kind. Its true name was lost and it has been propagated under various names, viz., Dwinelle, Kelsey, New French; but the name Berkeley, adopted by W. P. Hammon, in his wide distribution of it in 1884, now prevails. It is large and handsome, very prolific, ripens early, and is usually free from mildew.

The Champion, an Oregon seedling grown by Seth Lewelling, is medium sized, very smooth, and thick fleshed, the seeds being few and small. They are entirely free from mildew, and are clean, bright and beautiful. The Columbus, a New York variety, is large and of good quality and resists mildew well.

\section{THE MULBERRY}

Nearly all varieties of the mulberry have been introduced in California and grown rapidly and thriftily. Most attention has been paid to those varieties most suitable for feeding silk-worms, but the fruiting varieties are also grown here, though the fruit has assumed no commercial importance. The mulberry is grown readily from cuttings. The fruiting varieties thus far chiefly distributed are the Downing, Everbearing, the Persian, the New American, the Russian, and the 
Black Mulberry of Spain. All these bear large and desirable fruit. The last named, introduced by Felix Gillett, of Nevada City, is grown quite widely. The mulberry has a long season; the Persian ripens in Tulare the last of May and continuously thereafter until October.

\section{THE RASPBERRY}

The raspberry is another of the great small fruits of California. It thrives over a great area of the state; in fact, there are few situations in which it can not be grown with at least a measure of success if proper attention is given to retention of moisture in the surface soil, and to giving the plants partial shade in the heated valleys, and the cooler exposures in the foothills. The raspberry, skillfully pruned and generously fed and cared for, is almost a constant bearer, as has already been intimated. It is a continual delight in the home garden, and always brings a high average rate in local and metropolitan markets.

The culture of the raspberry is in the main like that of the blackberry, as already described. The red varieties, which are the kinds almost exclusively grown in this State, are propagated by suckers and root cuttings like the blackberry, but the "black caps" are propagated by layering the cane tips during the growing season, and this method is also necessary in the Loganberry and other blackberry-raspberry hybrids. Bending down a cane with its branches and covering lightly with soil and with a light mulch to retain moisture, will result in free rooting of the buried parts, and one can sometimes secure a dozen plants by the layering of a single cane with its laterals.

The pruning of the raspberry is also by the renewal system, as advised for the blackberry. The topping off of new canes, when they reach about three feet in height, the subsequent pinching of the laterals which are thus forced out, the resolute thinning out of sprouts so that but three or four strong canes are allowed from one root, the faithful repression of all weeds, the maintenance of a loose surface layer of the soil by very shallow cultivation, the free application of manure and of water unless a continually moist condition near the surface can be secured by cultivation and mulching-all these are among the essentials of cultivation which will secure abundant fruit and a long bearing season. However, as has already been stated with regard to blackberries, there are large plantations which pursue a less careful system of cultivation especially in the moderate heat and drouth of the coast district.

Continuous bearing of the raspberry may be secured in those varieties which endure the treatment, by cutting out a cane as soon as its fruit is gathered, the force of the plant being then devoted to the fruiting of a second cane, which has previously been pinched, and a third shoot is pinched and allowed to mature its wood to carry over and bear the first crop of the following year. A succession of sprouts is gained by pinching off the tips of some as soon as they have grown up a few inches, which results in the growth of later shoots lower on the stems. In this way a succession of fruit is obtained.

The Cuthbert and other strong-growing varieties, after the pinching at about three feet from the ground, will send out laterals which will 


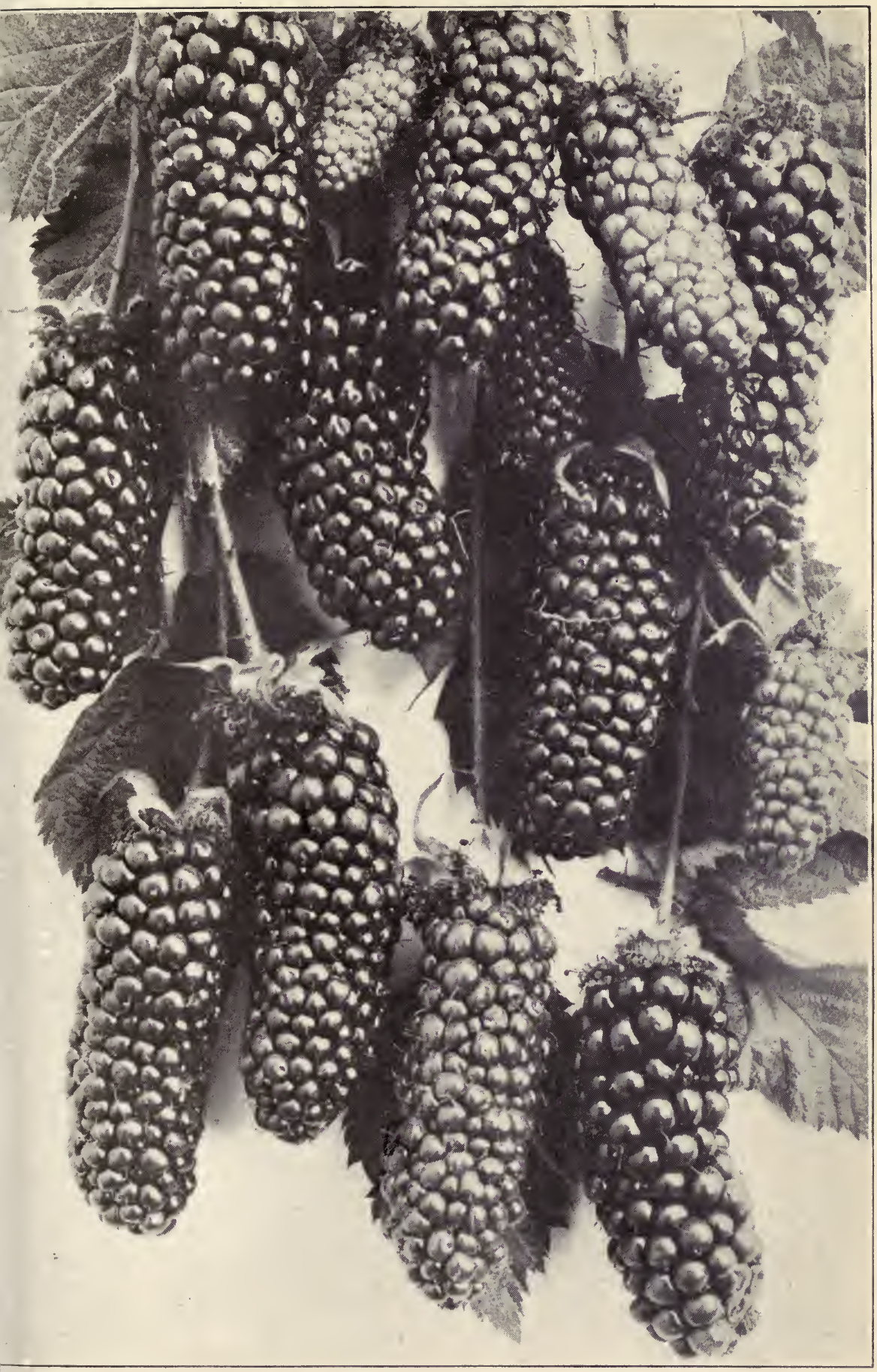

Plate XX.-The Mammoth Blackberry.-(See page 408.) 

bear late in the fall, and the same cane will bear a crop early in the following spring, when its career is ended and it should be removed.

Raspberries are planted about three feet apart in rows, and the rows about six feet apart. They can be well grown nearer together than is required for blackberries.

A Raspberry Trellis.-Mr. Louis F. Scribner, a well-known berry grower of Pasadena, commends a double-wire trellis made in this way:

At each end of a row a post should be deeply set and firmly anchored by brace or anchor wire, and a $2 \times 3$ redwood or cedar post should be set about every 20 feet in the row and a wire stretched on each side about three feet from the ground. These wires when stretched will be the width of the post in distance apart, and serve to keep the canes erect and in the row where they belong, so that cultivation and picking will not destroy the tender growth. The field should be gone over at intervals and canes drawn up between the wires when long enough.

Behavior of varieties under pruning varies greatly. Surprise can be pruned to advantage when dormant, but if cut back when sap is flowing and weather is hot they often die. This variety will bear two crops if handled right and none at all if abused. A variety like the Idaho needs very little if any cutting back, and the Superlative likes to swing gracefully over the trellis. The good old Cuthbert should be cut back with care, leaving as much cane as can be supported by the wires. The Red Antwerp is much like the Cuthbert. The Ruby, Miller, London, Herbert and St. Regis are all good varieties and require about the same care. The latter variety gives out an abundant sucker growth, which is not desirable when not confined.

Varieties of the Raspberry.-The old varieties have been largely replaced by the Cuthbert, which is the universally popular and most largely-planted sort, having been found trustworthy as a grower and as a free and constant bearer. The good points of the Cuthbert, as representing the experience of many California growers, include the following: A profuse grower, with healthy and rich foliage, which protects fruit from sunburn; an excellent bearer with the fruit well distributed through the bush; the fruit comes off easily, and does not crumble, is of fine flavor, and ships well. The Hansel and Red Antwerp and several newer varieties are grown to a limited extent; and the Barter, a renamed variety, the identity of which is unknown, has always retained a degree of popularity in the foothill region of Placer County, where it first appeared.

The Black Cap varieties thrive fairly in most parts of the State but do not sell well in the markets, and are only grown for home use. The golden or yellow raspberries are also out of favor because they are shy bearers and cut no figure in the California product.

Blackberry-Raspberry Hybrids.-Two crosses of California origin have been widely distributed and have demonstrated great value.

The Loganberry was originated by Judge J. H. Logan of Santa Cruz, and is a cross between the California wild blackberry and a red raspberry, thought to be the Red Antwerp. It was a chance hybrid developed by growing plants from the seed of the wild blackberry in 1881 . The plant was multiplied by its originator and fruited for more than ten years, plants being meantime given to Mr. James Waters, of Watsonville, who grew it on a commercial scale and was gratified at the results of his marketing of the fruit. The variety was first given to the 
public through the University of California in 1893 and has since then been propagated by nurserymen and sold in large quantities. It has proved a most valuable fruit in all parts of California, and has commanded the attention of pomologists and growers all over the world. The Loganberry is an exceedingly robust grower, and has unique foliage and cane growth as well as fruit. The fruit is strikingly large and handsome; sometimes an inch and a quarter long, with the shape of a blackberry, and sometimes the hue of a dark red raspberry. Its flavor is unique and peculiar, and gives to many tastes suggestions of the combination of blackberry and raspberry flavors. The culture of the Loganberry is like that of the dewberry-both in growth and propagation, rooting readily from cane tips without covering, unless many plants are desired and then a covered cane will root at each joint.

The Phenomenal is a hybrid which has recently been largely grown for a trade which prefers a less sharp acid than that of the Loganberry. It is one of the notable achievements of Mr. Luther Burbank, of Santa Rosa, and is a cross between the California dewberry and a red raspberry. It is exceedingly large, bright crimson, very productive and of delicious flavor. The fruit comes in large clusters and single berries have weighed four to the ounce. In shipping it holds shape and color well. The Phenomenal is counted about one week later than the Loganberry.

Trellising for Hybrid Berries.-The discussion and methods for training the trailing blackberries, already given at much length in this chapter, apply also to the Loganberry and Phenomenal. A special arrangement commended for the Loganberry is the following:

Plant the rows eight feet apart and vines four feet apart in the row. Use common fence posts for trellising, setting them sixteen or twenty feet apart, four feet high after they are set; then measure from top of post one foot down and nail a two-foot crosspiece; use three wires, stapling one to the top of the posts and one on each side to the end of the crosspieces. This gives a nice oval surface with three times the room as having the wires one above the other on the post. In the spring raise the vines and distribute them evenly and tie them firmly to the wires.

\section{THE STRAWBERRY}

"Strawberries all the year round" is the trite expression by which the charms of the California climate are characterized. It is no fiction, for in the wonderfully-even climate of regions adjacent to the coast and in thermal belts in the interior, the strawberry plant blooms and bears almost continuously, providing proper moisture conditions are maintained in the soil. There are, however, more or less well-defined crops, and "strawberries all the year" does not mean a uniform supply; nor does it mean that everywhere in California can one expect such constant fruiting. In the very hot interior situations the plant rebels against the atmospheric conditions of midsummer, even though the ground be moist; and in frosty places the plant becomes dormant during the wintry portion of the year. The conditions of constant growth and bearing are moderation of temperature and of atmospheric and soil moisture throughout the year. 
Situations and Soils for the Strawberry.-Bearing in mind the conditions described, the strawberry can be grown anywhere in California. The native species, as mentioned in Chapter V, flourish from the sand of the ocean beach to the rich valleys of the Sierra, just below the line of perpetual snow, and the deduction is that wherever fertile soil and sweet water can be brought together in California, the strawberry will reward the grower.

Strawberries do well on a variety of soils, but as a rule a deep, moist, loamy soil will yield best results. Boggy or swampy spots should be avoided unless drainage is provided, and in this way most excellent strawberry ground may sometimes be secured. Land which will produce good potatoes or corn will generally yield good results with strawberries, provided irrigation is furnished. In many regions the plants will hardly survive the summer without irrigation and everywhere a succession of crops during the season depends upon irrigation. It is the common experience that light, warm soils yield the earliest and highestflavored berries, and heavy soils the later and larger ones; but the size of the berry depends more upon the supply of available moisture, and immense fruit can be produced on loose, open soils by free irrigation. And yet the heavier soil, both because of its usually superior fertility and retention of moisture, is preferred for the strawberry. The largest producing regions for the San Francisco market in the Santa Clara and Pajaro Valleys are comprised mainly of low-lying, heavy valley soils, naturally moist and rich, and furnished with abundant water supply for irrigation. And yet in southern California the chief market crops are produced upon light sandy loams with water equal to the needs of the plants upon such a footing. It must be remembered that the strawberry is a shallow-rooting plant and must have moisture retained near the surface. Some loose soils, especially on uplands, are almost out of the question for strawberry growing. They are so leachy that they will not hold moisture near the surface though one should stand with a hose and almost continually pour it on. The plants would also dry up though the water were running near by in a ditch. To grow strawberries it is often an advantage to have a shallow loam over a clay or hardpan, for then the tight layer below will prevent the escape of the water below the reach of the roots. If this can not be had, the best way to grow strawberries on leachy soils for home use is to mulch and sprinkle.

Propagation of the Strawberry.-Seedlings undertaken in the hope of originating valuable new varieties are easily grown by taking off the outside layer of the choicest berries, which carries with it the small, yellow seeds. Wash these from the skin and cover them slightly in a sandy soil partially shaded and kept moist by sprinkling, or a light mulch, and the plants are readily grown. As with seedlings of other fruits, few, if any, will be found superior to the parent variety.

Plants for setting out are secured by taking off the small growths rooted from runners. The strongest plants are those nearest to the parent plant. When these are allowed to root in small pots plunged into the soil, they are called "pot-grown," and are superior for planting out, but they are not largely used in this State. When plants of 
any variety are desired for new beds or fields, a row or more are allowed to send out runners, during the summer, and these are fit for taking up and replanting the following winter or spring.

Laying off Ground for Strawberries.-The essentials are deep and thorough pulverization of the soil and grading of the surface so that water will flow slowly in the ditches. Suggestions as to location of grade lines may be found in Chapter XV. The inclination which answers for water distribution may be very slight; about two inches to the hundred feet answers on the level lands of the Pajaro Valley, while in the foothills much greater fall is made use of, and on hillsides rows are located on contour lines and not in straight lines. A grade of three and three-quarters inches to the hundred feet is sometimes used. The triangle described in Chapter XV can be used to fix the grades.

Of course, in grading the field it is often necessary to give adjacent blocks opposite inclinations to provide for the return of the water. On hillsides, where the water is carried down a ridge to a flume, it is usual to keep the water always running away from the flume, and only enough is taken out to reach the ends of the small ditches. A grade of six inches to the rod is practicable for hillside irrigation, but of course only a small flow of water is employed.

There are various ways of laying out strawberry beds and plantations. Some give flat cultivation and lay out in single rows two and a half to three and a half feet apart, and in some districts flat culture is unquestionably the best. Others lay out in double rows a foot and onehalf to two feet apart, and between each pair of rows the soil from the center is drawn up to each side, making a low ridge or level a little higher than the surface on which the plants are set. This levee serves as a walk between the beds and holds back the water upon the bed when irrigated by flooding. Another, and generally adopted plan, is to have the plants in double rows on a slight ridge, while between the beds is a furrow which serves as a walk and for irrigation. This is accomplished by throwing up the soil with the plow into ridges about two feet wide, with a double furrow between. On the sides of these ridges the plants are set, and often on the top of the ridge between the rows of strawberries a single row of onions or lettuce, or some other vegetable, is grown the first year. In irrigation the water is drawn up from the trenches by the roots and by capillary attraction, and the upper surface does not bake as it would by flooding if the soil be heavy. In hoeing out weeds and in fruit gathering, the workman walks in the ditch and does not pack the soil around the plant by tramping. This is the best method for laying out for large plantations. The rows are a uniform distance apart across the field, whether the space between be a ridge or a ditch. The method of making the beds a little lower than the general surface of the ground, answers best on free, open soils with perfect drainage. Cultivation can be reduced by covering the depressed surface of the bed with mulch of fine, clean litter, such as chaff, cut straw, etc. This retains moisture and gives the berry a clean surface to rest on. Such a bed is an excellent arrangement for the home garden.

In all arrangements the plants are set at less distances in the rows than the rows are from each other. Probably the prevailing distance is 
one foot between the plants; the range is from eight to eighteen inches in the practice of different growers, and determined, of course, largely by the habit of the variety. A vine like the Sharpless, with a spreading growth and long fruit stem needs, perhaps, sixteen inches which some growers give it, while the smaller, more compact, Longworth Prolific, may do well with half that distance.

Planting Strawberries.-Strawberry plants are set out either in spring or fall, or at any time in the winter when the ground is warm or in good condition. Fall planting usually gives a fuller spring crop though planting as late as February has brought two crops the following spring and summer, and planting in April has secured fruit the same year, but it is better to prevent it and induce more growth. Spring planting is in April and May. In the drier parts of the State, early fall or winter planting is more essential than elsewhere. If the ground is dry, water should always be used in planting. This may be given by thorough irrigation of the ground before planting, or a little water may be used in setting each plant. At planting it is usually best to remove all leaves from the plant, shorten the roots to three inches or less, and be sure the plants do not dry while planting progresses. As with handling rooted grape-vines, it is advisable to carry around the plants in a vessel which has water in it. If the plants have been received by mail they are invigorated by soaking in water a few hours before planting.

In setting the plants, scoop out a little excavation with the hand or a trowel, spread the roots well, cover with fine soil, being sure that the crown of the plant shall not be below the surface when the soil is leveled. Too many strawberry plants are buried, not planted. Some plant very rapidly by using a dibble to make a hole, into which the roots are dropped and soil pressed around them by using the dibble alongside; others set the plants on the side of the furrow, trusting to the next furrow to complete the covering. Nearly all ways succeed if the plant is not set too deeply and the ground is moist at planting and not allowed to dry out afterwards-providing good, strong plants are used. In buying plants it is often poor economy to buy the cheapest.

Staminate and Pistillate.-In associating varieties be sure the pistillate varieties are not set by themselves. Some sorts have perfect flowers and are self-fertilizing; others have only the pistillate element in the bloom and must have the staminate adjacent in another variety. Several varieties largely grown in California have perfect flowers, though some pistillate sorts have been locally approved. This matter should be looked into before planting a variety. Pistillate varieties should have staminate kinds in every fourth row.

Care of the Strawberry Plantation.-Herein lies the secret of success with the strawberry: Neglect has led to disappointment and condemnation of the strawberry, where intelligent care would have rendered it a constant delight. The cheap elements of proper care may be thus enumerated:

Retention of moisture very near the surface by careful, shallow cultivation or by mulching, persistent destruction of weeds, and compensation for summer evaporation by frequent irrigation. The plants 
during the bearing season should never be allowed to show any leafshriveling from drouth. Frequency of irrigation depends upon local conditions. Irrigation at intervals of four to ten days, according to the soil, are the outlines of prevailing practice.

Constant removal of runners from all plants except those it is desired to multiply to furnish new plants or to fill the rows. Pinching of runners should always accompany picking or hoeing of weeds, and on the garden bed there can be no excuse for neglect in this respect. The young plants should be faithfully freed from runners to strengthen them up for bearing.

Though, as already stated, strawberries may in some locations be had all winter, it is better practice, as a general rule, to lay the plants away for a rest. The market season in the regions supplying the San Francisco market extends from April to December, and fruit is continuously shipped during that period. At the approach of winter in the last-named month, it is usual to go over the beds with a sickle, cutting off the old crops of leaves close to the root crown, carefully cleaning up the plantation for the heavy rains. In most cases it will be a great advantage then to cover over all with a light coat of good manure, which the winter rains will leach down into the soils. The result of the fall clipping and enriching will be an early and strong start of the plant in the spring, and a most abundant fruitage.

Duration of the Plantation.-Strawberry plants well cared for and not visited by insect pests, have a long, productive, and profitable life in California. Twelve-year-old plants are sometimes reported as still producing abundantly. It is customary to count from five to eight years as the profitable life of a plant, though some growers replant after two bearing years. The effective duration depends directly upon preventing growth of plants and too close matting of the rows.

Varieties of the Strawberry.-Though all new varieties are tried by California growers, and quite a number may be considered successful either for market or for home use, only a very few may be said to be widely grown. In the Watsonville district, which largely supplies San Francisco, the Melinda, which some growers hold to be indistinguishable from the Dollar, is chiefly grown, with Brandywine as a distant second. In the southern Alameda County district the Banner is grown exclusively by some large growers. In the Florin district, near Sacramento, growing berries to ship all through the northern States of the coast and eastward to Colorado, the Dollar is grown almost exclusively, with a few Jessies for extra early. Other varieties which do well in the interior valley are: Longworth, Lady Thompson, and Tennessee Prolific. In the Fresno district, Marshall and Jessie are chiefly grown.

In the districts near Los Angeles, the Brandywine prevails. It is best for shipping and is held to be sweeter than at the east; and next to it, in the commercial fields at the south, are Excelsior and Klondike. The Arizona Everbearing is declining in popularity although still favored by some growers in Southern California.

Three old kinds which still hold favor for home use and local sale are the Longworth Prolific, the Sharpless, and the Monarch of the 
West. The Sharpless is the most widely grown; the Monarch shows better size and color in southern California and on the Sierra foothills than in the regions adjacent to San Francisco, although it is still grown therein to some extent. The Longworth is an old favorite, early, productive and hardy, and its style has become very popular in the markets. Wilson's Albany also holds favor.

It has been demonstrated that varieties show marked difference in behavior in different soils and situations. In planting for market or home use the planter will be safer in making his largest plantations of the varieties commended by leading growers and well informed nurserymen, and at the same time he should put out experimental plants of other varieties.

The most notable work for new varieties in California is being pursued by Albert F. Etter, of Briceland, Humboldt County. $\mathrm{He}$ is crossing cultivated varieties with local wild species and is thus introducing factors not employed hitherto with results which promise to be notable. His first named variety is Rose Ettersburg, which is a cross of a third generation Sharpless $x$ Parry with a novel type of Fragaria Chiloensis. It has remarkable drouth resistance and thrift on poor soils. The blossoms are often as large as a silver dollar, the berries large, often $11 / 2$ inches in diameter, and bluish pink in color. Single stocks from sets 18 months out measured 22 inches high and over 10 feet in circumference. Mr. Etter's work is described in detail in the Pacific Rural Press for August 22 and 29 and September 5 and 19, 1908; May 18, October 5 and November 2, 1912 , and is very interesting. The variety, Ettersburg No. 80, was distributed by this journal in February, 1913, and has already disclosed very important characteristics. 


\section{PART SEVEN : NUTS}

\section{CHAPTER XXXVII}

\section{NUT GROWING IN CALIFORNIA}

Two nuts have risen to large commercial importance in California: The English walnut and the almond. Other nuts than these, except peanuts, have never attained great acreage, although several have succeeded and promise to become popular.

The commercial production of almonds and walnuts in California during the last nineteen years has been estimated by the trade as follows, in tons of $2,000 \mathrm{lbs}$.

\begin{tabular}{|c|c|c|}
\hline Year & Almonds & Walnuts \\
\hline 1895. & 825 & 2,310 \\
\hline 396. & .. 1,605 & 4,115 \\
\hline 397. & . 2,375 & 85 \\
\hline 98. & .. 450 & 5,660 \\
\hline 999. & .. 2,320 & 5,530 \\
\hline 00. & . . 2,740 & 5,430 \\
\hline 1 & .. 1,5 & 10 \\
\hline 1902. & . . 3,270 & 8,520 \\
\hline 1903. & . . 3,200 & 5,500 \\
\hline 1904 & 800 & 7,590 \\
\hline & .. 2,125 & 5,750 \\
\hline 1906 & 900 & 6,125 \\
\hline 1907. & 750 & 6,500 \\
\hline & . . 2,900 & 8,500 \\
\hline & .. 1,500 & 8,000 \\
\hline & . . 3,300 & 9,600 \\
\hline & $\ldots 1,700$ & 12,500 \\
\hline & .. 3,000 & 11,250 \\
\hline & 1.100 & 11,750 \\
\hline
\end{tabular}

The walnuts are chiefly grown in Santa Barbara, Ventura, Los Angeles and Orange counties in southern California. The almonds are from interior counties in the central regions of the State. Reasons for the extreme fluctuations in production of the almond will be suggested in the discussion of that nut which will follow.

\section{THE ALMOND}

The almond has an interesting history in California, but it can be outlined in a few sentences. The importation of the best European varieties began very early, and a number of them had been planted in 1853. They proved irregular bearers, though the trees grew thriftily and in some cases showed fruit very soon after planting. 
The barren almond trees were largely grafted into prunes or made into firewood and the conclusion was reached that to secure regularity and abundance in fruiting, locations for almond orchards must be sought with the utmost care, and that the secret of success lay in the location. After that local seedlings seemed to demonstrate their value in regular crops, and in characteristics and qualities superior to foreign kinds. Large planting was then undertaken on the ground that the choice of soil and situation, and the selection of trustworthy varieties, are both factors of success, but that possibly more lay in the choice of variety than of location. This belief led to the wide planting in locations now seen to be unfitted by reason of frosts and losses were again encountered. Now it seems to be fully demonstrated that no matter what variety is planted locations for the almond must be selected with great care. It has also been demonstrated that association of varieties promotes pollination and satisfactory bearing.

Situations and Soils for the Almond.-Almonds are now doing best on the higher lands in coast valleys, free from fogs and protected from direct winds, but subject to tempered breezes; also at various points in the interior valleys and foothills. The general proposition that low lands in small valleys should be avoided, and bench or hillside situations preferred, seems to be a safe one. Lands directly upon the coast have not proved satisfactory. In the large interior valleys of the Sacramento and San Joaquin, almonds are successfully grown on flat valley lands but little lifted above sea level and from such plains the chief product comes. Why almonds are safer on the low lands of a great valley than of a small valley is explained by the discussion on page 16 .

The almond prefers a loose, light, warm soil, and heavy, poorlydrained soils should be avoided. Though they need moisture enough to make good, thrifty growth they will produce good crops on soils that are too light or dry to grow satisfactory peaches, apricots, nectarines, cherries, or similar pulpy fruits. The almond is, however, a very deep-rooting tree, and may succeed by reaching deeply for moisture rather than by denying itself, as some think. The tree certainly suffers and is barren from drouth in some cases, and a certain amount of irrigation in midsummer is very desirable. Almonds are doing well in alfalfa with irrigation-the alfalfa making a good growth and still leaving soil-moisture enough to cause the nuts to open their hulls better than on drier ground with clean cultivation.

Propagating, Planting, and Pruning.-The almond is propagated from seedlings grown as described in Chapter VIII, and budded as described in Chapter IX. The almond root is preferred, though the peach answers well. The apricot root should be avoided.

For planting out, trees in dormant bud are very successful if given proper care. Yearling trees are, on the whole, best and usually those which have made a moderate instead of a very large growth are to be preferred. The almond makes a comparatively large tree and should 
have plenty of room-not less than twenty-four feet apart (though some plantations are made at twenty feet), and thirty feet is better.

Old almond trees are readily worked over to other varieties by grafting and by budding into new shoots forced out by cutting off large branches. Methods with the peach described in page 242 are applicable to the almond.

The pruning of the almond is very simple. The tree should be headed low and pruned during the first three years, as described in Chapter XII, to secure a shapely, strong tree. After the third year little pruning is required except to thin out objectionable branches by winter pruning. Suckers, or rank new growth, in the center of the tree should be removed, unless more branches are needed; such growth bears little and takes away strength from bearing wood. There is danger of allowing the trees to become too dense. Shortening in, as practiced with the peach, is sometimes proposed for the almond, for the thrift of the tree and the size of the nut, but growers have not had courage enough to assume the increased cost of production which would be involved.

The cultivation of the almond orchard is the same as commended for other fruit trees, and as the trees are often planted in naturally dry soils, the greater care in cultivation is needed to retain sufficient moisture to give good size to the nuts. In certain locations, of course, irrigation will be necessary, but usually a light rainfall will answer if good cultivation is given.

Gathering, Hulling, and Bleaching.-Almonds are gathered by spreading canvas under the tree and jarring the branches separately with a side stroke from a soft wood pole; the few nuts remaining can be displaced by striking with a light stick. The gathering should be done after the hulls have burst open, but should not be delayed until the nuts are badly discolored. Discoloration of the nut depends upon local atmospheric conditions and is worst in regions subject to moist winds or fogs from the ocean, and they often extend considerable distances into the interior valleys. On the dry plateaux adjacent to the Mojave Desert perfectly bright almonds are produced naturally, but at these elevations frost injuries are frequent and notable.

For the greater part of the almond product, bleaching is apparently demanded by market requirements, but it must be carefully done as described on the following page. Various home-made contrivances are used for bleaching, such as piling up several of the slat-bottom trays one upon another, placing around them sides made of boards so as to hook together at the corners, cover the top with a damp canvas, and burn the sulphur in a hole in the ground below the bottom tray.

Webster Treat, formerly a large grower of almonds, describes his sulphuring-house for almonds :

My bleaching house is about twenty-five feet by eight feet, and I generally put in about four thousand pounds of almonds and expose them to sulphur fumes for three or four hours. The house is boarded with tongue and groove flooring, inside and out, and roofed with well-laid shingles, and has a flue about two feet high on the apex, to help draft the sulphur smoke up. The floor is of one-bythree-inch stuff, set up edgewise, three-eighths of an inch apart, or just wide enough to admit the fumes from the sulphur burning below, and narrow enough 
to prevent the nuts from falling through. The floor is about two and one-half feet above the ground, and the lower space is boarded up with tongue and groove also and fitted with small doors every five feet, so that the sulphur pans can be placed underneath the floor.

Sulphur fumes are applied until the nuts are of a light yellowish color; the proper shade is to be learned by securing approved samples from some trustworthy dealer.

The following explicit account of handling almonds on a large scale is by Mr. J. P. Dargitz, of Acampo, San Joaquin county:

"When the hulls on the nuts are loose from the shell, as will be indicated by their bursting open, it is time to begin gathering if you wish to hull them. If they get too dry you will have to wet them before hulling or you will break the shells. If you wish to shell them then, the drier they get the better. It will not pay to begin until the nuts about the crotches of the trees are ready and they will be the last to ripen. When they are all ready you can get all at one gathering. Have some sheets made of heavy unbleached sheeting or light duck or sail cloth. Mine for large trees are $15 \times 30$ feet, handled by two men to a sheet and two sheets to a tree. Spread the sheets under the tree, one on each side, lapping the edges where they join. Then the men take willow or bamboo poles and by jarring the limbs cause the nuts to fall on the sheets. Always strike the limbs sideways, for if you strike a glancing blow down the limb, you will reduce next year's crop. The object is to get the nuts and disturb the foliage as little as possible. When the nuts are all off the tree, the men toss their poles to the next tree and then gather up the sheets, one man at each end of each sheet and, lifting them, carry them to the next tree where the process is repeated. When enough nuts are in sheets to fill several lug boxes, the boxes are placed on the ground side by side, and the sheets are emptied into them. These boxes are then stacked up so as to be easily seen, and the teamster hauls them to the sheds where machines for hulling are located. In the very small orchards the hulling is usually done by hand at an expense of about 2 cents per pound. For orchards slightly larger there are small hullers in the market at from $\$ 100$ to $\$ 300$, which can be operated by hand or by a small gasoline engine or electric motor. For larger orchards, machines costing up to $\$ 800$, which can hull up to two or three tons per day, are in the market.

After the hulling more or less hand sorting is required, as the small machines do no sorting, while the larger machines do more or less perfect sorting of the hulls from the nuts.

Bleaching.-After this hand sorting, the nuts are spread out in the sun to be thoroughly cured before bleaching. After they are cured so that the kernel will break without bending, they are ready for bleaching. The bleaching requires the dampening of the shells. This is accomplished by immersing quickly in water or by a fine spray from a hose nozzle, or by putting on trays and running in the sulphur house and then introducing low-pressure steam-not more than 30 pounds-into the sulphur house for 15 to 30 minutes. The moisture from this low-pressure steam heats or dampens the outer portion of the shell, and then while they are both hot and damp-the steam being 
shut off-the sulphur fumes from burning sulphur is introduced in the sulphur house for perhaps 15 to 30 minutes and gives the required brightening to the shell. If the almonds have been immersed before bleaching it will be necessary after bleaching to spread them out in the sun for several hours and stir them occasionally to get rid of the excess moisture. If they have been dampened by a fine spray hose they may or may not need this drying, but they must be perfectly dry before being sacked. However, if the steam process is used, the nuts can be taken immediately from the sulphur house and sacked, and the heat that is in the shell will evaporate the small amount of moisture in the process of sacking.

The steam process costs only about one-half as much as the other process of bleaching. If the shell is wet through, or if the kernels are not well cured, or if the shells are open and the nuts immersed and there is more or less water inside the shells, the sulphur will materially injure the kernels and they will not keep well, but will soon become rancid.

Almonds that are to be shelled for commercial purposes should not be bleached under any circumstances, as the only object to be dccomplished in bleaching is the brightening of the shell, and for shelling purposes the shells are not marketed.

Pollination and Late Blooming.-The advantages of cross pollination between different varieties and the surer bearing of late blooming varieties are related subjects which are worthy of close attention. Mr. J. P. Dargitz, whose harvesting method has just been given, has pointed out that, according to his observation as a rule all the papershells are deficient in pollen, and will not bear well if planted alone and he concludes that the only two which warrant planting in his section are the Nonpareil and the Ne Plus Ultra. The latter is not so good, a nut, and needs much more moisture than the other. The Nonpareil will give good results if planted with some other variety as pollenizer. It is one of the very best of nuts and is well worth planting. Mr. Dargitz regards the Texas Prolific the very best pollenizer, and when planted in alternate double rows with the Nonpareil will cause it to set fruit freely in spite of the fact that the Nonpareil begins blooming two weeks earlier than the Texas but still has bloom to catch the Texas pollen when it is ready.

This late blooming of the Texas places it in the same class for surety with Drake's Seedling. Both nuts are medium soft shell and small but are being largely chosen because of sure bearing and large product. As to the relative return per sack, Mr. Dargitz says :

The Nonpareil at 14 cents per pound, the Drake Seedling at 11 cents per pound, and the Texas Prolific at 10 cents per pound, will each bring about $\$ 10.50$ per standard sack, the IXL at 13 cents per pound about $\$ 9$ per sack, and the Ne Plus Ultra at 12 cents per pound about $\$ 8.50$ per sack.

The relative values of varieties is also shown by the prices fixed by the California Almond Growers' Exchange at Sacramento for the opening sales of the 1913 crop as follows: * Nonpareil, 19c; IXL, $18 \mathrm{r} / 2 \mathrm{c}$; Ne Plus Ultra, $17 \mathrm{r} / 2 \mathrm{c}$; Drakes, $14 \mathrm{r} / 2 \mathrm{c}$; all f. o. b. warehouses. 
Varieties of the Almond.-Almonds should bear well every year, hull easily, have clean, thin, soft shells, and a smooth, bright, and plump kernel. Almonds with long, single kernels are preferred in general to those which have double ones. These are the characters which ruled in the selection of new varieties by the pioneer propagator of new almonds, A. T. Hatch, formerly of Suisun. In $1878 \mathrm{Mr}$. Hatch planted out about two thousand five hundred seedling almond trees grown from the bitter almond seed. He afterward budded all the seedlings but about three hundred which were left to bearing age unbudded. The fruit of these seedlings was of all degrees of excellence. A few of the best of them were selected for propagation and naming, and they constitute the chief part of the acreage which is now bearing almonds, but other late bloomers and sure bearers are being preferred in current planting.

Excellent seedling almonds have also been produced by other growers. The following list includes the sorts most widely grownthe following being chiefly approved by growers, in the order given: Nonpareil, Drake's, IXL, Ne Plus Ultra, Texas Prolific, Languedoc, Peerless :

$I X L .-$ Tree a sturdy, rather upright grower, with large leaves; nuts large with, as a rule, single kernels; hulls easily, no machine being needed, nor is any bleaching necessary; shell soft, but perfect; largely discarded for shy bearing, but desirable in some places.

$\mathrm{Ne}$ Plus Ultra.-Large and very long in shape; heavy and regular bearer; soft shell; hull free.

Nonpareil.-First called Extra. Of a weeping style of growth; smaller foliage than the IXL, but still forms a beautiful tree. An extraordinarily heavy and regular bearer, with very thin shell, of the Paper Shell type. Ripens early and can be gathered before other varieties are ready.

Lewelling's Prolific.-Originated with the late Mr. John Lewelling; "tree a great bearer; of drooping habit; nut large and good; soft shell; hull free."Leonard Coates.

Harriott's Seedling (or Commercial).-From Visalia, where it is a surer cropper than elsewhere; shell softer than the Languedoc; nut long, of peculiar shape, quite large; kernel sweet.

King's Soft Shell.-Originated in San Jose; shell very thin and soft; regular and abundant bearer.

Princess.-The finest of the Paper Shell class; long, oval, kernel large, white and sweet.

Languedoc.-Nut large; shell thin; kernel sweet; condemned for irregular bearing.

Paper Shell.-Medium size; shell very tender, easily broken between the finger and thumb; kernel large, white and sweet.

Drake's Seedling.-Originated with Mr. Drake, of Suisun, of the Languedoc class; very prolific, and a regular, abundant bearer. A late blooming variety.

Golden State.-Originated by Webster Treat. A large soft shell, somewhat longer than the Languedoc, with a full, smooth-skinned meat; parts from the hull readily. An early variety, but in less favor than formerly.

Peerless.-Resembling IXL: Popular in Yolo County for regular and heavy bearing.

Texas Prolific.-Brought from Texas by Robert Williamson, of W. R. Strong \& Co., of Sacramento, about 1891, as a seedling originated at Dallas, and the only almond which would bear there. Planted at Acampo by Robert Adams, who 
had charge of the company's nurseries at that place; fully discussed by J. P. Dargitz, of Acampo, and described by the introducer as resembling Languedoc in tree and nut, but with softer shell; which is filled with very sweet kernel. Blooms two weeks later than Drake. Mr. Dargitz reports fifteen years without failure to bear and usually prolific.

Jordan Almond.-This long, hard-shelled almond, notable because of its long, slim kernel for "salted almonds" and imported at high cost for that purpose, has been introduced both through government distribution and private enterprise, notably that of the late John Rock, of the California Nursery Co., of Niles. Too little has yet been seen to determine the value of the variety in local production. It is especially favored to the Coachella Valley.

\section{THE CHESTNUT}

The chestnut is not yet produced in large amount in California, and certain quantities of the nuts are annually imported, the American, Italian, or Spanish and Japanese all being found in the San Francisco markets. Of chestnuts grown in California, the Italian predominates, and the Japanese is more common than the American, which is slow of growth and late in fruiting, as compared with the other kinds. Judging the success of the Italian, it may be said that a large area of California is well suited for the growth of the chestnut, as there are bearing trees in nearly all parts of the State. The chestnut succeeds on heavy, clayey soil, even if it be rocky.

Chestnut trees are readily grown from the seed, and thus grown come into bearing from six to eight years, though the Japanese sometimes bear sooner. The growth of chestnuts from the seed is described in Chapter VIII. In growing from seed of the improved varieties, there is a tendency toward reversion, and budding and grafting may be resorted to; and can be done by the methods described in the chapter on the fig. The chestnut can also be grafted with the ordinary cleft graft. Buds or scions should be taken from trees which are fruiting satisfactorily, and in this way seedlings which have a tendency to bear empty burs can be turned to good account. Chestnuts can be grown in the nursery until several years old, providing they are lifted at the end of the first year, the tap-root cut off, and the trees reset, giving them rather more room than during their first year's growth. In permanent plantings the trees should have plenty of room, as they ultimately attain great size. Trees at Grass Valley, Nevada county, when about twenty years old, fifteen inches in diameter of trunk, and forty feet high, and reported to bear a barrel of nuts to the tree regularly. Felix Gillet of Nevada City, has for many years made a specialty of propagating a large collection of the improved French varieties of the chestnut, known as Marrons, which were distributed to some extent. The chestnut chiefly grown is the Italian but it has not attained any considerable product as yet. The chestnut, aside from its desirability as an orchard tree, can be commended as a tree for hillsides or a shade tree for waysides of pastures, and should be more widely planted in California. The chief product is in the foothill district east of the San Joaquin valley. 


\section{THE FILBERT}

The best English cob-nuts have been quite widely tried in California without successful results. Improved Spanish and French varieties of the filbert were early introduced by Felix Gillet, of Nevada City, and have been favorably reported by him as to growth and bearing. A few other growers in foothill situations have reported success, but as a rule disappointment has attended ventures with the filbert. The most favorable regions for farther experiment are apparently the north slopes of the Coast Range, from Santa Cruz northward, and other cooler and moister situations, as well as at an elevation on the Sierra foothills where Mr. Gillet found them satisfactory.

\section{THE PEANUT}

During the last few years the peanut product of California has notably increased, and the crop is a popular one in some parts of the San Joaquin Valley and southern California. The nuts are considerably grown between the rows in young orchards and vineyards, as well as upon ground wholly given to them. The following explicit directions are given by R. M. Hargrave, a grower in Orange county. Some slight modifications in practice may be needed, according to locality, as, for example, in time of planting, which is usually a little earlier than the date given:

Planting.-The best time to plant peanuts is about the middle of May, say, 10 th to 15 th, in rows about three to four feet apart and sixteen to twenty inches the other way, and not cover too deep-three to four inches. Peanuts planted the middle of May ripen evenly and are of uniform size. Very early peanuts ripen unevenly, and the first nuts that set on get so ripe they turn to a pink color, and if the land is a little sandy the stems get soft, lose their strength, and will not lift the nuts from the ground.

It takes about thirty pounds of the California or White Virginia, and fifty pounds of the Tennessee Reds to plant an acre. Tennessee peanuts can be planted much closer in the rows. The California peanut is the best to plant, as it yields three or four times as much as the Tennessee Reds do, and has more ready sale.

The Quality of Land.-Peanuts require a rich sandy soil loam that is known as upland. Damp land gives the nuts a straw color, and they are not as good probably as those raised on higher land. They require no irrigation, except on very sandy land, where some have found it profitable; but, as a usual thing, when irrigated the ground is liable to get hard, making the nuts crooked, ill-shaped, and many times coloring them.

Cultivation.-Peanuts should be cutivated about the same as corn, not allowing any weeds to grow in them, keeping the ground loose and mellow, and when the spikes begin to form, they should not be disturbed. If they are, it causes the nuts to blight or not fill out. The blooms do not require to be covered.

Harvesting.-Peanuts should be harvested when ripe, and not allowed to stand too long, in hopes that the last ones set out will fill out and ripen, as you lose more than you gain. The little ones spoil the sale of the crop, and many are left in the ground that get over-ripe. Peanuts should be cut or plowed out and thrown into windrows, nuts down, and let lie a week or ten days, and then sacked, as the best nuts are cured in that way, and they do not mold so badly, and cure a better color. They must not be allowed to get wet. The tops are good feed if stored away in a shed for winter usè. All kinds of stock like them, and small nuts can be left on the vines. They make the best chicken feed. An average yield is about twenty-five to thirty sacks to the acre, forty pounds to a sack, but many have raised fifty sacks, with extra care and good land well adapted to. 
peanuts. The price for picking is from 30 to $35 \mathrm{c}$ per grain sack holding from 40 to 45 pounds. The nuts are cleaned in revolving drums, and followed with a grain fanning mill which blows out the light ones.

Concerning the peanut situation in California in 1914, Mr. C. E. Utt of Santa Ana, who has been the largest grower in the State for many years, gives the following specific statement.*

My experience in growing peanuts in this State covers a period of many years. The last two years I was in the business I planted 400 acres each year. They netted me a tidy little loss, wiping out all the profits I had ever made growing peanuts, for occasionally the crop has proven profitable.

A few peanuts have been grown in California in a great many localities for perhaps fifty years, and most of the attempts to produce them have proven unprofitable and been abandoned. At one time Orange County produced something like twenty carloads annually, now not more than one carload a year, and it is perfectly safe to say that peanuts will never be grown in the Pacific States to any appreciable extent, because the cost of production is so much higher here than in Japan. At one time the Pacific Coast market was supplied by peanuts from Virginia. About twenty years since the Japanese began importing and now sell over 95 per cent of the unshelled peanuts used west of the Rocky Mountains. Virginia still supplying the bulk of the shelled Spanish nuts. The price at which Japanese nuts are laid down on the wharf at San Francisco, tariff paid, ranges from three cents to four and one-half cents per pound. The cost of producing peanuts at any of the Pacific States will range from three cents up, probably averaging now about six cents. The price of labor in Asia will probably always enable them to undersell us in the market.

Mr. Utt gives the following cultural hints: Peanuts require sandy sediment to give the best results. It is better to shell the seed and plant one kernel in a hill, 18 inches apart in the rows which are three feet apart. Plant the seed two or three inches deep, in good moist earth so as to insure germination. Plant as soon as frosts are over, in April or May, as they need about six months to complete their growth. There is very little land that will produce crops without irrigation. If irrigation is necessary, it must be by the furrow method, and no flooding must be permitted.

\section{THE PECAN}

The pecan, by rapid growth, early fruiting, and general thrift, seems to be the member of the hickory family best fitted for California conditions. A tree grown from a nut planted by J. R. Wolfskill, on Putah Creek, in 1878, was, when twenty-five years old, over fifty feet high, with a trunk twelve inches in diameter, growing luxuriantly and bearing freely. Still older trees, also very satisfactory in growth and bearing, are to be seen at Chico and Visalia. The pecan, though grown for thirty years by different parties around the Bay of San Francisco, either does not bear or keeps the nuts hanging on until sometimes they sprout on the tree. The wider extremes in temperature or in humidity in the interior seem to teach the tree better habits of growth and rest, and moist lowlands in the great valleys seem best for pecan planting. As yet, California has no marketable product of pecans but the total number of trees in the State is insignificant.

Pecan trees grow readily from the nuts if these are fresh. Planters should secure nuts of selected varieties (for there is a great differ-

\footnotetext{
*Rural Californian, March, 1914.
} 
ence in size and quality) direct from growers in the Southern States, and plant as soon as received, in the early winter, or if conditions are not favorable for planting, the nuts should be stored as described in Chapter VIII. Nuts planted in good nursery ground in rows as there suggested, and covered about two inches or a little deeper in dry, loose soil, and then mulched to retain moisture, will germinate freely. The trees should be transplanted to permanent place at the end of the first year and then usually the tap-root can be retained, as some growers deem very desirable; if the trees are to be put in permanent place later they should be transplanted in the nursery and the tap-root cut off. The nuts can, of course, be planted at once in permanent place if one will take the extra trouble necessary to properly care for them.

Pecans are polished by putting them in a revolving barrel only partly filled, so that as the barrel turns they fall upon and rub each other producing a beautifully smooth surface. When it is desired to give an artificial color, a little "Spanish brown" dry paint is put into the barrel. If the natural coloring is desirable, a little sharp sand put into the barrel assists in the scouring and produces the desirable uniformity of color. This barrel is rigged up like a revolving churn, with a side door in the staves and a gudgeon on each head which is fitted into proper bearing on two posts; proper arrangement being made for a pulley or crank by which the apparatus is turned either by hand or motor power.

\section{THE PISTACHIO}

The pistachio nut (Pistachia vera) was introduced a number of years ago but no results have been reported. The species upon its own root makes a low shrub and is slow of growth. We have also imported the Pistachia terebinthus, from which is derived the "chio turpentine," the stock the true pistachio is grafted upon in Europe and which is growing thriftily at several points in the State. The pistachio needs more time to declare its California career.

\section{THE ENGLISH.OR PERSIAN WALNUT}

The nut which is signified in California when the term walnut is used, is the English walnut or Madeira nut (Juglans regia) and its many varieties. This tree makes a grand growth in California. Specimens are seen here and there, which, at about twenty years of age, are from fifty to sixty feet in height, with a spread of branches of forty to sixty feet, and in some cases bearing four to eight hundred pounds of nuts. Much larger and older trees can be found in the coast and interior valleys of central California where the nut was first planted, although, as has already been stated, the nut has thus far been produced in large quantities only in regions adjacent to the coast in southern California. The equable temperature and moisture of the southern coast seems to specially favor the nut, but it must not be inferred that success can only be attained in such situations. A number of French varieties, which have been widely enough distributed to 
test their growth, have been found to thrive in many situations where the old Los Angeles variety is a failure, and there is at present quite a disposition to larger plantings of the walnut in all California valleys, either as a sole occupant of the land or as border trees around fruit orchards. At the South the walnut area has largely increased in those situations where the tree shows most satisfactory bearing qualities, and newer varieties of California origin, like the Improved Soft Shell, constitute most of the present area. In all untried places, or in all places where the old Los Angeles Walnut has failed, trial should be made of the hardy French varieties, which will be described farther on. Recently considerable planting has been done in the coast and interior valleys and foothills of central California upon the quite fully demonstrated success of these varieties. It is, however, very desirable to secure satisfactory depth and retentiveness, without excess of water. in the soil. The walnut, on its own seedling root, abhors drouth as well as standing water.

Soils for the Walnut.-The walnut makes most rapid growth upon a deep, rich, moist, loamy soil, and shows its appreciation of good things of the earth as do other fruit trees, and yet it attains satisfactory size and bearing in less favorable situations. Thriving trees can be found in the clays and decomposed granite soils of the foothills, as well as in the valley silts and loams. Adequate moisture must, however, be had, and the walnut cannot be commended for dry, neglected places nor for soils which overlie leachy subsoils described on page 33.

Propagation.-The walnut tree grows readily from nuts treated as described in Chapter VIII. In the main the use of seedlings has hitherto prevailed, and the nut has been looked upon as coming sufficiently true from seed. Recently, however, this has changed rapidly, and budding or grafting to secure a high, uniform grade and to secure fruitfulness in spite of the blight is commanding wide attention. In growing young trees it is being considered desirable to bud or graft rather high so as to get a trunk of the hardier variety which is used as a stock.

Excellent results have been obtained by using the California black as a stock for the English walnut, and in that case budding or grafting must be resorted to. Many instances of the success of the English walnut on our native stock might be cited, but the most notable tree known to the writer is to be seen on the grounds of John R. Wolfskill, on Putah Creek, in Solano county. He put in a bud in 1875 and the tree has reached immense size and large product. Since then many large native black walnuts have been top-grafted with the English walnut with notable success, not only in orchards, but along highways where the native black walnut has been planted for shade and ornament.

Mr. F. S. Leib, of San Jose, who has given much attention to stocks for the English walnut, believes that the cross of the California black and the Eastern black walnuts, and the California black walnut straight, afford the best seedlings for roots for the English walnut, but advises close selection to secure the best growth. This is his prescription: 
"The Royal hybrid (every cross between the native Eastern black walnut and the native California black walnut is called a Royal hybrid), is, in my opinion, the most magnificent growing tree in the walnut line, and I believe that in the future some Royal hybrids can, by persistent selection, be sufficiently fixed to furnish the strongest possible roots of substantial uniformity on which to graft. At present I know of only one tree, picked out from many hundreds, which is sufficiently fixed to grow a fair percentage of seedlings equal to itself. Only from one to two per cent of the seedlings of most Royal hybrids is equal to the parent, and the percentage of even the best straight California is only from fifteen to twenty per cent, and the poorest give less than one per cent.

"At present, we, by a system of sprouting, are able to eliminate four-fifths of the weakest growing nuts, and we plant the remaining fifth in the nursery. Twenty-five per cent of such remaining nuts from our selected trees of Royal hybrid blood, and fifteen to twenty per cent from our selected California trees, grow three to four feet in the first year. The three to four foot seedlings in the nursery are grafted when one year old, and the remaining smaller trees are left for another year; and for this reason a small per cent make a disproportionately large growth of root compared to its moderate top the first year, and make an immense growth of top the second year.

"All Paradox hybrids (English walnuts crossed with California black), make a large root and but a moderate top the first year, as do many seedlings from the Royal hybrid, and as do very, very few from the straight California. These few trees, which make good the second year, three or four per cent, possibly, of the trees left to grow another year in the nursery, are grafted at the end of the second year, and the balance thrown away as unworthy to be grafted and given a chance in orchard form; for a tree which is to have in the orchard from sixteen hundred to thirty-six hundred square feet can not be too good, either as a root or top."

Budding the Walnut.-In working on the native California seedling stocks, Mr. Clowes, of Stockton, buds by the common method, removing the wood from the inside of the plate of bark, as advised for the orange. Twig buds as used with the olive are also successful, and ring budding works well on shoots of a year's growth, which have at least attained the thickness of the middle finger. Mr. Gillet advises that the buds should be set at the base of these shoots where the wood is perfectly round. The bandage should pass above and below the bud so that the bark under it may be pressed down close upon the stock, and this is more surely gained by shaving off the base of the leaf stem, below the bud, about to the point where it would separate when the leaf naturally falls off.

Mr. A. W. Keith, of Selma, has hit upon a very interesting method of preparing walnut buds. In taking a fresh bud from the new growth of the walnut he found the large leaf stem a serious impediment in firmly placing a shield bud upon the cambium of the stock and binding it there. Shaving it away with a knife left too much exposed tissue. If the leaf stem would drop off as it does when mature and leave a 
healed-over scar, the result would be a flat surface with only the bud protruding, and this could be easily bound in place so as to exclude the air. He tried cutting off the compound leaf, leaving a stub of an inch or so, and was delighted to find that a stub thus left became dry and parted from the stem just as a mature leaf does in the autumn. By cutting off the leaves in this way about August 15, the stubs part readily before September 1, and then he takes off clean, flat buds and uses them just as he does in budding the peach, except that he leaves no wood behind the bud. He takes rather a large shield and puts in under the bark of the stock through a "T" cut, then wraps well with a strip of cheese cloth and waxes over the cloth with ordinary grafting wax. The wrapping is applied so as to cover most of the bark slit, everything beyond being waxed over. The bud is then allowed to remain dormant until the following spring.

Mr. Pennington of Vacaville has been very successful in budding by cutting buds in the regular shield form, about $11 / 4$ inches long, cut from branches $1 / 2$ to $3 / 4$ inch in diameter, so as to get wide buds with but little wood left in them. He also prefers to have stocks large, and considers a stock $1 \frac{1}{2}$ inches in diameter not too large. He uses ordinary budding twine for tying, but does not use wax to cover the incisions. He says it is best to put off budding as late as it is possible to get a good flow of sap, as then the weather is likely to be cooler, which is an important factor, and the growing season is about finished, which will allow the twine to be left in place all winter, which he considers advisable.

Buds are more apt to succeed when pushed upward from the crosscut than downward from it. The cuts would then be an inverted "T." No preparation of the budding wood is made, except that mature buds are taken. About 90 per cent of the buds placed are said to grow.

Spring budding can be done much more successfully if the bud sticks are cut while dormant, in February, and kept in a cool place until the trees begin to grow. The bud sticks will have also shown life, and the buds can be taken from them in the usual manner and inserted in the growing stocks. These buds will begin to grow at once and frequently make a growth of four to six feet during the season. As soon as the buds show they have united with the stock, the stock should be cut above the bud and waxed.

Grafting the Walnut.-Grafting into black walnut seedling root can also be well done by a triangular cut into the edge of the root stump, as described for grafting into grape-vine stumps in Chapter $\mathrm{XXVI}$. In the case of the walnut, close binding with a wax band is desirable.

Large walnut trees can be worked over either by budding or grafting. If by budding, the large limbs are cut back in the winter, and in autumn following, buds are put in, as just described, on as many of the new shoots as may be desired.

In grafting, the common cleft graft is used with a degree of success, particularly when the split is not made through the pith, but at one side; the scion should be whittled so as to show as little pith as possible. This is done by cutting down to a point at one side and not 
in the center of the scion. Care should be taken to cover all exposed surfaces.

Grafting over is desirable either for substituting a better variety of English walnut, or for working over a California black walnut into an English variety, and as much attention is now being paid to blight resistant, interest is sharpened in grafting methods. Mr. J. B. Neff, of Anaheim, who has looked into the subject deeply and worked over many old trees, gives the following practical suggestions on this work:

If the trees are from three to five inches in diameter they may be cut off at about four feet above the ground and below the branches, then four or five scions may be placed in one stock, or three or four of the branches may be cut back to within 10 to 24 inches of the trunk and two or three scions placed in each. All the other branches should be removed from the trunk.

Old trees of from 12 to 20 years should have the branches cut at places where they are from three to six inches in diameter, and from five to eight stubs left, which will be from three to six feet in length and should have as many as six scions in the large stubs, the other branches being removed before the scions are put in place.

In sawing large branches it is necessary to make two cuts, the first being some distance above or outside the final cut, to prevent splitting the stub, or the trunk, when the severed part falls.

The scion should always be of solid, mature wood, that is, with as small pith as can be had readily, and must have good living buds. Each scion should be about one-quarter inch in diameter and have at least two buds. The growth having buds close together is best, as shorter scions can be used.

To receive the scions use a heavy butcher knife and mallet to split the stubs, placing the knife across the stub as if a chip one-half to five-eighths-inch thick was to be taken off. Then depress the handle of the knife to an angle of 30 to 45 degrees and split the edge down to $2 \frac{1}{2} 2$ to 3 inches, allowing the knife to reach the farther side of the stub, but not making the split entirely across the stub. Open the cleft with an iron wedge $1 / 2$ to $5 / 8$-inch wide and thickest on one edge, placing the thickest edge toward the outside.

Trim the cleft in the stub with a sharp knife so it will be smooth. Then cut the scion so as to fit perfectly and place it so the inner bark (the cambium layer) of both will be on the same line, or at least will cross twice, then remove the wedge and put hot wax over all the cuts on both stock and scion at once.

The scions should be examined frequently and any excessive flow of sap wiped off, and the stub re-covered with wax as soon as dry. Excessive flow of sap for several weeks will cause the loss of the scions, as the callus can not form in water. This may be controlled by boring one-fourth inch holes in the body of the tree near the ground. Three or four holes four inches deep will be sufficient to control the flow of the largest trees. No damage is done to the body of the tree, as the holes soon grow over.

The last two wax formulas on page 78 are largely used in walnut grafting. For grafting in the stems of seedlings or in the smaller branches of young trees a side graft as described on page 80 is successfully used as are also several styles of cleft grafting. One which is used by Mr. R. Wiltz and others at San Jose consist in splitting a short stub of a small branch which has been cut about four inches from its attachment to a larger branch of stem. In this case the split can only extend to the closely knit wood in the crotch and the scion is pushed down strongly to the bottom of such a split and it is held tightly. The two pieces of the deep split are not cut away but are allowed to protect the short scion which is between them and if buds start on these pieces they are allowed to grow a little to keep the stock from dying back. When the scion starts well they are removed. 
A method of side grafting (so called because the stock is not cut across but a cleft made in the side of it) has been very successfully practiced by Mr. Weinshank, of Whittier, both with nursery seedlings and on branches of large trees. His work has shown eighty to ninety per cent successful in the nursery and even more on branches of large trees. The following is a condensed description of the method:

The scion is prepared as for a whip graft (page 81), cutting a cross obliquely and making another cut right straight down with the grain in the scion. Then, instead of cutting the stock completely across like the scion, just simply make a little cut on the side. Do not cut into the pith of the wood at all. Then, place the two together by pushing the tongue of the scion (made by the cut on its shorter side) into the lip cut in the stock; tie with a string and wax over. The same method on larger trees is performed exactly in the same way, except that the lip of course on the larger limbs, which are three or four inches through, would be considerably larger, and the scion instead of being placed directly in the middle of the lip, or in the center, would be placed on the side so as to have the cambium of the scion connecting with the cambium on the stock at least on one side. Three or four, or sometimes five scions are put on a large limb, and in most cases three or four scions would grow. After the scion has reached a certain stage of growth, this string will commence to bind, and must be cut down right over where the scion is. The wax on either side will tend to hold the string, but as the wood naturally grows, the strings will draw apart, and it will not bind as it would if the string was not cut.

Side grafting with a saw-cut, as described for the peach on page 243, also works well with the walnut.

Treatment After Grafting.-A fuller account of the protection of scions after setting and during their early growth is given by Mr. Edwin Gower of Fowler, Fresno County, thus explicitly:

Take two folds of burlap, punch holes for the scions to pass through, making a hood inclosing and covering the entire stump, letting it extend down the sides a short distance; tie a string around the stump to hold it in place; by using this method the wax will remain in perfect condition. In valleys, where the wax melts, a portion of the stump is exposed and runs down the crevices, destroying the tender tissues of the scion.

After the scion is growing rapidly, cut the string; do not remove it. This allows the scion to grow without the string cutting it. If small trees, drive a stake down to tie the new growth. On old trees nail four sticks equal distances apart around the stump, letting the sticks extend four or five feet beyond the stump, wrap some string around these sticks; this acts as a net enclosing the growth of the scion, which grows phenomenally on black walnut. I have seen instances where they grow fifteen feet in one year. This net acts as a support and keeps the scion from either breaking or splitting off by the winds. It is sometimes advisable to nip off the end of the branches to check the growth. Cut them back vigorously for two or three years, until such time as they are well attached to the stump.

I have used the bark graft, also the cleft graft, but I prefer the former, as the scion seems to grow more rapidly and reach maturity sooner.

Planting Walnut Orchards.-There is much difference in practice in planting out walnut trees in permanent place. Some advocate the use of trees two or three years from the seed, getting as much of the tap-root as possible; others allow the tree to remain in nursery 
until it throws out laterals, which is usually done the fourth or fifth year. Two-year-old trees are generally preferred, but walnut trees of many times that age can be successfully transplanted if the work is carefully done. Walnut trees are usually set forty feet in squares, though some give the large-growing varieties fifty feet. Planting in hexagonals at forty-five feet distance gives very satisfactory results. Some growers plant in squares at thirty feet distance, intending to remove alternate trees as they crowd each other, first cutting back, for a time, the trees which are finally to be removed.

On the Bishop Ranch in the La Patera section of Santa Barbara County several years ago, every other walnut tree was cut out of a twenty-five-year-old grove, the trees originally being set twenty-five feet apart. The advantages were at once apparent, the grove yielding ten per cent more the following season than they did under the old system and the crops have been getting better ever since. Sixty feet is about close enough for mature trees in a walnut grove and when the ground is so shaded that the sun and air cannot penetrate the trees will not thrive.

Intercultures with the Walnuts.-In the southern walnut regions it is common to grow beans, squashes, etc., between the rows of trees until the latter reach bearing age; root crops which attract gophers should be avoided. Inter-planting of smaller, early-fruiting trees is also practiced to a considerable extent.

Walnuts in Alfalfa.-Dr. W. W. Fitzgerald, of Stockton, an experienced propagator and planter of walnuts, says:

To my mind, walnuts and alfalfa, on proper soil, are a very desirable combination. While your walnut trees are growing you get good and quick returns from your alfalfa. The roots of the alfalfa loosen up the soil, carrying nitrogen with them to improve its condition. But on light soil, which is best for alfalfa, the walnuts do not do so well, and on heavier ground you have to be careful to have good drainage. One should by all means use the Royal Hybrid root when growing walnuts with alfalfa.

On heavy land it is best to plant the trees and let them grow one year before planting alfalfa, thus avoiding the danger of waterlogging the roots on recently Dlanted trees in irrigating alfalfa. A strip should be cultivated on each side of the walnut rows, or a space dug round the trees to prevent the alfalfa from growing close to the tree, which would be a detriment.

Before planting the walnuts, the land should be leveled so as to irrigate nicely, and more especially if it is to be interplanted with alfalfa or berries. With alfalfa the checks should be spaced at the distance you wish to plant the trees.

Pruning the Walnut.-The walnut is usually headed higher than ordinary orchard trees, but preference is now given to starting the first branch at about four feet from the ground instead of six feet as formerly. All the pruning needed is in shaping the tree as described for the fig. Upward trend of the branches should be secured, sometimes by cutting out the shoots which grow downward, sometimes by tying them up for a time to the central stem until they are stiff enough to retain this position. Placing branches on the stem according to the principles advanced in Chapter XII, should be borne in mind. The stem should be protected from sunburn until the foliage accomplishes this. Whenever shoots are killed back by sunburn or frost, they should be cut off cleanly below the black mark which shows how far the injury 
has extended. If this is done, the die-back down the branch is usually prevented. As the trees become older, removal of some of the interior limbs may be desirable to admit more light to encourage inside bearing.

Cutting Back for a Strong Start.-Dr. Fitzgerald gives specific suggestions for starting the young tree by cutting back at planting, rather than trusting to the growth in nursery, as follows:

Previous to planting, the tops of the trees should be cut off from 12 to 15 inches above the ground, according to the number of buds on the lowest part of the trunk of the tree, and the cut waxed. After these buds make a growth of four to six inches, select the most thrifty one to make the butt for the future tree and pinch off the tops of each of the other limbs. This will stop their growth and leave them with a few leaves to shade the butt of the tree.

When cutting trees back this way, it is absolutely necessary, especially on the strong-growing hybrid root, to sucker your trees often until the top gets a good start. You should dig down four to six inches around the tree to cut off each sucker close with a sharp knife, for if you break them off, adventitious buds will start out again and by cutting them off with a sharp knife you will remove all these buds. This should be done the first time just as soon as the buds begin to swell on any of the trees, and every tree should be suckered, for if you allow these sucker buds to start, being lower than those of the graft, they get the first sap and oftentimes prevent the buds above from starting. This suckering should be done about once every ten days, or just as sure as you let the suckers grow, they will keep back the growth above the graft or will prevent it starting altogether.

The tree will probably make a growth of four to twelve feet the first season. The next year it will not make growth in height, but will throw its side branches. Some think it necessary to cut off the top of a tree to make it branch out, but my experience has been that if you will leave the tree alone two or three years, it will throw out its side limbs and make a perfect tree. The only pruning that is necessary is to cut off the limbs that are too low and any limbs that cross and would chafe against each other.

After the tree gets older and is bearing, the limbs of the tree should be pruned to open up in the center, allowing the sun and air to go through the tree, the same as with other fruit trees. With some trees that are inclined to go to wood and do not throw out enough fruit buds, you may cut back one-third of the limbs, which causes them to throw out new limbs on which the fruit buds form.

Bloom and Bearing of the Walnut.-The walnut has its staminate and pistillate blooms separate, but both occur on the same tree. Successful fruiting depends upon the appearance of these two forms of bloom, without too great interval of time, and although there seems to be a quite a retention of vitality, the lack of bearing of some varieties has been found to be due to the fact that the catkins disappear too long before the pistillate bloom was sufficiently developed to receive the pollen.

The bearing age of the walnut depends upon the variety. Some of the French varieties are very precocious and have borne fruit in nursery row at two and three years old, but the pistillate blooms were then fertilized from catkins growing on older trees. The practical bearing age of the seedling English walnut in this State may be rated at six to eight years, according to the variety. Trees grafted with scions from bearing trees fruit much sooner.

Harvesting Walnuts.-Gathering walnuts is done in different ways; some gather them from the ground at intervals during the months of September and October; others use poles and clean the trees at one operation; some go over the ground three times; first, picking up what 
have fallen; second, picking up what have fallen and striking the limbs lightly to dislodge others which are ripest; third, picking up again and then knocking off all that remain on the trees. In this way gathering lasts a month or six weeks. Walnuts, after gathering, are treated by some growers as described by F. E. Kellogg, of Santa Barbara County:

As fast as gathered the nuts are placed in slat-bottomed trays, $6 \times 3$ feet, by six inches deep, about fifty pounds in a tray, being thoroughly shaken up once or twice a day. If the weather is very hot, they should be dried in the shade. When the nuts are dry they are passed through an inclined revolving grader, making about twelve revolutions per minute, having a one-inch mesh wire screen, and all that fall through this are called "seconds." The lower end of the grader dips into vat of water, thoroughly wetting the nuts and washing them to a certain extent-entirely sufficient for paper shells and soft shells,- and usually enough for hard shells. A system of buckets attached to the drum of the grader then elevates the nuts to a chute, which discharges them into a large box $4 \times 4 \times 8$ feet high, with an inclined slat bottom two and one-half feet above the ground. While in this box they are subjected to the fumes of sulphur for twenty to thirty minutes for the purpose of improving the color. The second grade walnuts are also put through the washing and sulphuring process. The nuts are next drawn off from the bleachers into the drying trays, piled one on top of the other, to prevent the sun from shining directly on the nuts, and remain there for ten or twelve hours, until the nuts are thoroughly dried off. The trays are then emptied into a hopper, from which the nuts are drawn off into bags containing something over one hundred pounds each; the bags are securely sewed up and stamped with the producer's brand, and the nuts are ready for shipment.

\begin{abstract}
Col. A. S. Heath, of Carpinteria, uses a moist bleach and a drier afterward, as follows:

When the nuts leave the rotary washer they are entirely free of hulls and clean. They are taken to the bleaching boxes, and here subjected to the very minimum amount of sulphur necessary, and cold steam, being in the bleachers about one hour. From the bleachers they are taken to the steam drier. In the drier are 100 drawers, each capable of holding 40 to 50 pounds of walnuts. These drawers have wire bottoms to allow the heat to pass through them. In the basement beneath the drier Mr. Heath has some modern furnaces constructed, and during the course of the drying about 130 degrees of heat is maintained. The nuts are kept under this heat for eight hours. During the drying it is possible to watch the progress by pulling out drawers for inspection.
\end{abstract}

\title{
follows :
}

J. B. Neff, of Anaheim, describes large handling of walnuts

The nuts are allowed to ripen and the hulls open on the trees. If the trees have had sufficient irrigation and the weather is normal, a large part of the nuts will fall clean from the hulls and will have to be picked off the ground once a week in dry weather, but should be gathered oftener in damp weather. The gathering can be hastened by gently jarring the branches with a pole which has a light flat steel hook on the end. The hook is passed over the branch and pulled quickly so as to jar the branch without swinging it. Any hulls that cling to the nuts may be taken off by hand. The nuts are placed in trays made of sides 1 by 6 in. by $6 \mathrm{ft}$. and ends 1 by 6 in. by $2 \mathrm{ft} .10$ in., with laths $1 / 2$ inch apart for bottoms. The sides are allowed to project for handles, and the tray measures 3 to 5 feet. The imperfect nuts are picked out and the good nuts dried in the shade when the weather is hot. Damp localities require some artificial heat, but care must be taken to have only a mild heat, as the nuts will be damaged if high temperatures are maintained.

Grading and bleaching is usually done in the Association warehouses, as considerable outlay for apparatus is required and some experience is necessary to get the best results. When the nuts are thoroughly dried they are passed through a revolving screen having meshes $3 / 4$ inch square at the upper end and 1 inch square at the lower end. The small meshes take out the dirt, and the 
1-inch meshes take out the small nuts, which are called No. 2 nuts, while those going over the screen are known as No. 1 nuts. The bleaching is done either by dipping or spraying, using a solution made of five pounds of salt in 100 pounds of water and passing the solution through an electric cell with a current of 95 to 110 volts and at a temperature of 90 to $95^{\circ} \mathrm{F}$., the variation depending on the purity of the salt.

Varieties of the Walnut.*-Of walnuts of California origin there are two classes, which are called "hard" and "soft" shell, but the accepted commercial product is largely composed of the soft-shell class. Several varieties of French walnuts are now being widely distributed. An attempt will be made to give some of the distinctive points of each variety mentioned:

Common English Walnut; Los Angeles Nut, etc.-This is the ordinary English walnut of commerce. It was planted at an early day in Los Angeles County, but is now largely replaced by the Improved Soft Shell.

Santa Barbara Soft Shell; Sexton's Soft Shell.-Originated by Joseph Sexton, who gives this account of its origin and characteristics: "The winter of 1867 I bought in San Francisco a large sack of English walnuts. I raised about one thousand trees that season, and planted two hundred of them the following spring, in orchard form, at Goleta. Sixty of them proved to be the soft-shell variety. The soft shell is a little later in starting in the spring than the common nut, and blooms about ten days later. It commences to fruit at six years old from the seed, and some have been known to fruit as young as the fourth year. The hard shell commences to fruit about the ninth year, and bears full crops alternate years. The soft shell is not as strong a grower as the other walnut; it being so prolific, retards its growth. It is a superior nut; the kernel is white. The shell is thin, rendering them easily broken by the hand, at the same time strong enough to bear transportation to any part of the United States."

Ford's Improved Soft Shell.-G. W. Ford, of Santa Ana, propagated an "Improved Soft Shell," gained by selection from the variety of Joseph Sexton, which has been largely planted.

Santa Rosa.-A seedling by Luther Burbank, which has been distributed quite widely; but by variation in its seedling, its character is somewhat ill-defined and the name is applied to several types, some of which are less desirable than the one receiving the name.

Proeparturiens.-Introduced in California in 1871, by the late Felix Gillet, of Nevada City, and afterward by other parties, and widely distributed. Its chief characteristic, as its name indicates, is early bearing. It blooms from two to four weeks later than the common Los Angeles seedling; it shows both kinds of bloom simultaneously, and has the characteristics of ripening its wood well, and is a good nut, but it has been little planted recently because of its disposition to run to small sizes.

Mayette.-This variety chiefly constitutes the imported Grenoble walnuts. It is large, roundish, with a broad base, on which the nut will sit up; shell thin and white; kernel full and rich; a good bearer and late bloomer; local Mayette seedlings are being named; one is the "San Jose," by R. Wiltz, of San Jose-a fine nut, believed to be blight resistant.

Concord.-Seedling of Cluster: of the Mayette type, grown by Messrs. Westgate and Hutchinson of Concord from seedling tree by Felix Gillet. Introduced by Mr. Leonard Coates in 1908. Claimed to be blight resistant.

Franquette.-This French variety has risen to great favor and has been largely planted upon the successful experience of Mrs. Emily M. Vrooman, of Santa Rosa, and the extensive effort at its distribution by the Oregon Nursery

*The fullest account of English walnut varieties grown in California, historical and descriptive, is given in Bulletin 231, University Experiment Station, Berkeley, by R. E. Smith. Unfortunately the publication is out of print and must be consulted in libraries. 
Co., of Salem, Ore. It is a large, elongate-oval nut with shell rather thick and kernel of high quality. It is a late bloomer, and escapes blight to a certain extent.

Bijou Seedlings.-Several of these are being grown. Willson's Wonder, introduced by F. C. Willson, of Santa Clara, is a large, smooth, desirable nut, and the tree is reported an early and prolific bearer.

Other French Varieties.-Other French varieties introduced by $\mathrm{Mr}$. Gillet and others include the following: The Cluster, which fruits, as its name indicates, in long bunches, sometimes as many as fifteen in a bunch; otherwise the tree resembles the common English walnut. The Parisienne is a beautiful variety, the nut large, broad, and shapely; the tree blooms very late. All the foregoing varieties and the Franquette, Serotina, Barthere, Mesange, Gant and Chaberte, were introduced by Mr. Gillet in 1871 .

Kaghazi.-A variety called Kaghazi was grown and propagated for several years by the late James Shinn, of Niles, who described it as follows: "Very much larger than the ordinary kinds, and thinner shelled. The tree is late in putting out leaves and blossoms, and is, therefore, especially good for places that are in danger of late frosts."

Japanese Walnut; Juglans Sieboldiana.-This species, native of the north of Japan, was introduced to California about 1860, and a tree grown from seed planted about that time is growing at the Tower House, in Shasta County. Recently the good points of the tree have been more widely recognized. The following excellent description is by Luther Burbank, of Santa Rosa: "This species is found growing wild in the mountains of northern Japan, and is, without doubt, as hardy as an oak. The leaves are of immense size, and a charming shade of green. The nuts, which are produced in extreme abundance, grow in clusters of fifteen or twenty, have a shell thicker than the English walnut, but not as thick as the black walnut, very much resembling pecan nuts. The meat is sweet, of the very best quality, flavor like butternut, but less oily, and much superior. The trees grow with great vigor, assume a very handsome form, need no pruning, mature early, bear young, and are more regular and productive than the English walnut." The nut has an exceedingly hard shell and does not rate commercially with the popular varieties of the English walnut.

Varieties Popular in California.-Since the walnut blight invaded the commercial orchards and resisted all remedies tried against it, great interest has arisen in particular trees which bore well in spite of the disease. As California has such a large acreage of seedling trees there was a full opportunity for the full manifestation of such resistance and a wide field in which to exercise the art of selection. The result is that many trees were found which were profitable even when the blight is worst, and such trees are being given distinct varietal names. The progress of this work should be watched in the publications of the University Experiment Station at Berkeley, for the experts at its branch laboratory and trial grounds in the walnut region of southern California, at Whittier, Los Angeles County, have been largely engaged in this work. Individual growers are alert at selection within their own orchards and the prospect is that a general escape from the blight and the establishment in our local pomology of a group of especially desirable varieties will ere long be attained. This proposition is fully discussed in Bulletin 231 by Prof. R. E. Smith, as already cited. Briefly it may be stated that these six are now most popular, according to reports of planters :

Eureka, Franquette, Mayette, Concord, Placentia Perfection, E1 Monte. 
Bulletin 231 says, "The Eureka comes very close to satisfying the requirements of an ideal walnut for California. It is a fine large nut of strikingly handsome appearance, extra full meat and weight, perfectly sealed, light colored meat, fine flavor, marked immunity to disease, a strong, vigorous grower and ultimately a heavy producer."

Of varieties named above as most popular the order of excellence, without regard to bearing habit of the tree, is as follows: 1. Eureka. 2. Franquette. 3. El Monte. 4. Disher's Prolific. 5. San Jose. 6. Chase. 7. Concord. The first three were about on a par and well ahead of the others. Placentia Perfection is an elegant nut, but is very subject to blight, though still in high favor with some planters. 


\section{PART EIGHT: FRUIT PRESERVATION}

\section{CHAPTER XXXVIII}

\section{FRUIT CANNING, CRYSTALLIZING, AND DRYING}

The preservation of fruit in various ways for home use and distant shipment, is one of the leading industries of California, employing a large amount of capital and labor, and distributing a vast amount of money among our people. These facts can be best emphasized by statements of the product of 1913, in the leading methods of preservation, by canning and drying:

Canned Fruit Próduct of 1913.

Cases.

Table fruits, $2 \mathrm{r} / 2$-1b. cans, 24 per case.................... 4,204,070

Pie fruits, 1 -gal. cans, 12 per case ......................... 629,830

Total equal to $122,571,5602 \mathrm{r} / 2-1 \mathrm{~b}$. cans, or ............. $4,833,900$

The relative use of different fruits is as follows:

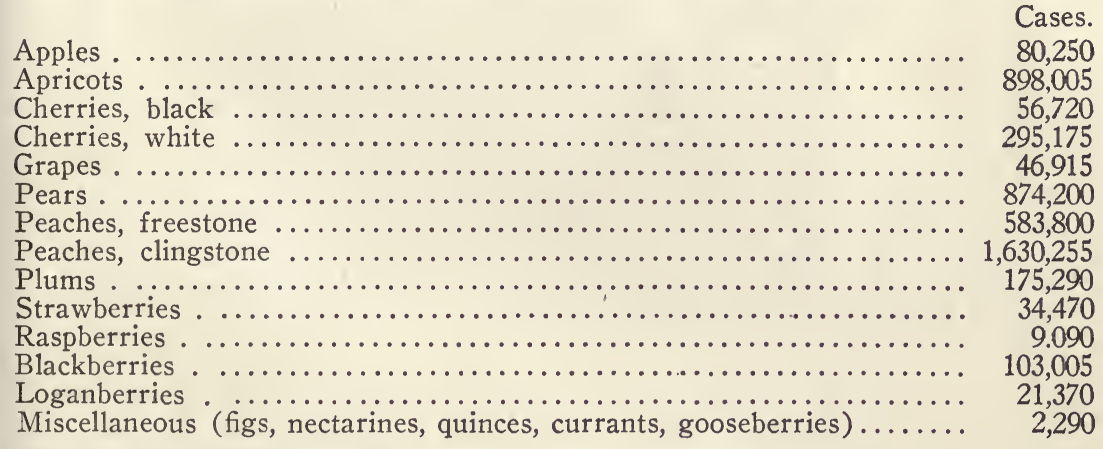

\section{THE CANNING INDUSTRY}

California stands first in the United States in the value of canned and dried fruits produced and in the amount of money as wages paid for labor in fruit canning and curing establishments. In point of capital invested therein California is credited by the United States census office in 1905 with a total of ten and one-quarter million dollars; thus standing second only to New York, which has ten and one-half millions. The style and capacity of the establishments is characteristically Californian, because California's ten millions are invested in 184 
concerns, while New York has 565 concerns; only a quarter of a million more value in three times as many outfits.

Fruit canning began in California over fifty years ago, but during the last three decades has attained its greatness, and is still promising much wider extension. The process is simple, and yet is attended at every point, from the purchase of the fruit, to the sale of the product, with operations which require experience, wisdom and good judgment. It will be obviously impossible to give in print a guide to the pursuit of such an industry. The principles involved in the process of commercial canning are, of course, the same as rule in the old kitchen processes, but to secure uniformity and cheapness of product a vast number of manipulations and labor; saving appliances have been devised. These begin with the manufacture of cans and attend the product to the end, and the realization of the commercial and uniform production which they assure involves the employment of large capital and the keenest business ability.* The canning interest has, therefore, segregated itself more and more widely from the growing interest. Orchard canning on a small scale, which was once thought feasible, has passed out of sight except as it is seen to lie in the foundations of a few of the smaller canneries which have been built upon it. It seems clear now that as a rule the fruit grower's duty to the canning interest ceases with the production of acceptable fruit unless individuals or associations can command capital enough to enter the field on equal vantage with the large commercial canneries. Capital is flowing toward the business; the field for the product seems to be constantly expanding, and canning centers are multiplying throughout the State wherever ample supplies of good fruits and vegetables are available.

Varieties for Canning.- The table previously given showing the fruits which enter in various amounts into the canned product gives a general idea of what fruits should be planted to minister to the canner's demand. As to varieties, it is not easy to make a general prescription, because the choice differs somewhat with different localities. It is a good idea for the new planter to consult with owners of adjacent bearing orchards and to secure from the nearest canneries lists of varieties which are acceptable to them.

\section{CRYSTALLIZED FRUITS}

Progress is being continually made in the production of candied, crystallized, or glace fruits, but the product is not a large one. Special establishments are now doing.this work in Los Angeles, San Jose and San Francisco. They have processes which are the result of considerable experimentation, and they do not make them public. To others the way lies open to similar experimentation. The general theory and an outline of practice as given by J. J. Pratt, an experienced processor, is as follows:

The theory is to extract the juice from the fruit, and replace it with sugar syrup, which, upon hardening, preserves the fruit from decay, and at the same

*A detailed account of the operations and outfit of a typical California cannery is given in the Pacific Rural Press of March 22 and 29, 1913. 
time retains the natural shape of the fruit. All kinds of fruit are capable of being preserved under this process. Though the method is very simple, there is a certain skill required that is acquired only by practice. The several successive steps in the process are about as follows:

First, the same care in selecting and grading the fruit should be taken as for canning; that is, the fruit should all be of one size, and as near the same ripeness as possible. The exact degree of ripeness is of great importance, which is at that stage when fruit is best for canning; peaches, pears, etc., are pared and cut in halves, as for canning; plums, cherries, etc., are pitted. The fruit, having thus been carefully prepared, is put into a basket, or a bucket with a perforated bottom, and immersed in boiling water. The object of this is to dilute and extract the juice of the fruit. The length of time the fruit is immerced is the most important part of the process. If left too long, it is overcooked, and becomes soft; if not immersed long enough, the juice is not sufficiently extracted, which prevents a perfect absorption of the sugar.

After the fruit has been thus scalded and allowed to cool, it can again be assorted as to softness. The next step is the syrup, which is made of white sugar and water. The softer the fruit, the heavier the syrup required. Ordinarily about seventy degree, Ballings' saccharometer, is about the proper weight for the syrup.

The fruit is then placed in earthen pans, and covered with the syrup, where it is left to remain about a week. 'The sugar enters the fruit and displaces what juice remained after the scalding process.

The fruit now requires careful watching, as fermentation will soon take place, and when this has reached a certain stage, the fruit and syrup are heated to a boiling degree, which checks the fermentation. This heating process should be repeated as often as necessary for about six weeks.

The fruit is then taken out of the syrup, and washed in clean water, and it is then ready to be either glaced or crystallized, as the operator may wish. If glaced, the fruit is dipped in thick sugar syrup and left to harden quickly in the open air. If it is to be crystallized, dip in the same kind of syrup, but allow to cool and harden slowly, thus causing the sugar which covers the fruit to crystallize. The fruit is now ready for boxing and shipping. Fruit thus prepared will keep in any climate and stand transportation.

Thus far the crystallized fruit produced in California has sold well. There is a considerable importation of French fruit to the United States, which may be displaced by the California product, and the business commends itself to those who have ingenuity, patience, and capital enough to enable them to experiment and wait for future success. The California producer has the advantage of an abundance of very fine fruit at a low price, but he carries a handicap in the high cost of transportation and of labor.

\section{CALIFORNIA DRIED FRUIT INDUSTRY}

A special census of the fruit-preserving manufactures of the United States in 1905 shows not only that California stands first in the dried fruit industry, but that the product (excluding raisins) was in that year valued at about fourteen million dollars and was over eighty-eight per cent of the whole national product. An adjacent tabulation compiled from the records of the California State Board of Trade gives interesting details of this product for a number of years.

As suggested on page 21 it is the function of the sunshine and dry air of California not only to bring vigorous growth to the tree and vine, and carry the fruit of both to fullness of size, beauty and quality, but to continue its beneficent action until the fruit, which is not required 
by the trade in fresh ripeness, is given imperishable form, in which its beauty, flavors, aromas and nutritive qualities remain available to delight and nourish mankind until the following year's sunshine wins from the earth another supply of fresh ripeness. There are many parts of the earth where good fruit is grown: there are few where conditions producing such fruit continue to accomplish its preservation, as they do in California, and this climatic endowment of the State yields an annual income of something like twenty millions of dollars, as the years run.

Product of California Cured Fruits in Tons-1900-1913.

\begin{tabular}{rrrrrrrrrrrr}
\hline Year & Peaches & Apricots Apples & Pears & Plums & $\begin{array}{c}\text { Nec- Grapes } \\
\text { tarines }\end{array}$ Figs & Prunes & Raisins \\
1900 & 17,170 & 14,000 & 3,150 & 7,275 & 1,950 & 435 & 240 & 2,000 & 87,000 & $47,16 i$ \\
1901 & 14,755 & 7,776 & 3,225 & 3,290 & 1,725 & 317 & 180 & 3,260 & 40,000 & 37,125 \\
1902 & 25,210 & 18,762 & 4,875 & 2,625 & 1,280 & 455 & 188 & 3,625 & 98,500 & 54,375 \\
1903 & 16,075 & 10,500 & 1,800 & 2,325 & 1,435 & 317 & 205 & 3,000 & 82,500 & 60,000 \\
1904 & 11,500 & 8,500 & 1,500 & 1,750 & 1,150 & 210 & 170 & 2,850 & 67,500 & 37,500 \\
1905 & 17,500 & 19,250 & 3,250 & 1,750 & 930 & 185 & 193 & 3,625 & 37,500 & 43,750 \\
1906 & 11,250 & 3,250 & 2,750 & 3,500 & 1,100 & 170 & 200 & 3,375 & 90,000 & 47,500 \\
1907 & 12,000 & 1,500 & 1,500 & 500 & 750 & 137 & 188 & 3,000 & 40,000 & 60,000 \\
1908 & 22,500 & 19,000 & 3,000 & 1,200 & 1,000 & 350 & 1,500 & 3,000 & 25,000 & 60,000 \\
1909 & 20,000 & 14,500 & 2,500 & 1,200 & 500 & 375 & 325 & 3,500 & 77,500 & 70,000 \\
1910 & 25,000 & 16,000 & 3,100 & 1,000 & 375 & 250 & 350 & 3,775 & 45,000 & 56,000 \\
1911 & 14,000 & 11,000 & 3,500 & 1,500 & 250 & 200 & 119 & 5,500 & 95,000 & 65,000 \\
1912 & 18,000 & 10,000 & 2,000 & 1,000 & 200 & 200 & 100 & 5,000 & 102,000 & 100,000 \\
1913 & 20,000 & 9,000 & 2,000 & 1,000 & 600 & 200 & 120 & 6,000 & 45,000 & 60,000 \\
\hline
\end{tabular}

In connection with this notable factor of our horticultural production, certain facts of its utilization and its significance should be clearly understood not only by those who actually employ it in their business, but by those who desire to properly appreciate the industrial resources of the State.

First. Cured fruits in California are a primary and not a secondary or by-product. It is true, of course, that curing fruit does, to a limited extent, save from loss fruit which shippers and canners are not at the time paying profitable prices for, and it is true also that the recourse to curing frees growers from helpless dependence upon fresh fruit buyers. But this does not mean that curing is a way of getting something from refuse fruit, not suited for other purposes. It should be taken as evidence that, for the most part, grades of fruit which are cured are the same which are also available for shipping and canning when prices are right. It is very important in many ways to have it clearly understood that, except to an insignificant extent, California fruit drying is not undertaken to save wastes or to get something from fruit which is not suited to higher uses.

Second. As our cured fruits are a primary and not a by-product, it becomes intelligible why such free investment is made in acres of well-made trays; in tramways and turntables for their movement from the shelter of convenient cutting or dipping and spreading houses; in capacious apartments and mechanical devises for giving the cut fruit its bath in sulphur fumes to preserve natural colors and to prevent fermentation and insect invasion; in the carefully prepared drying 


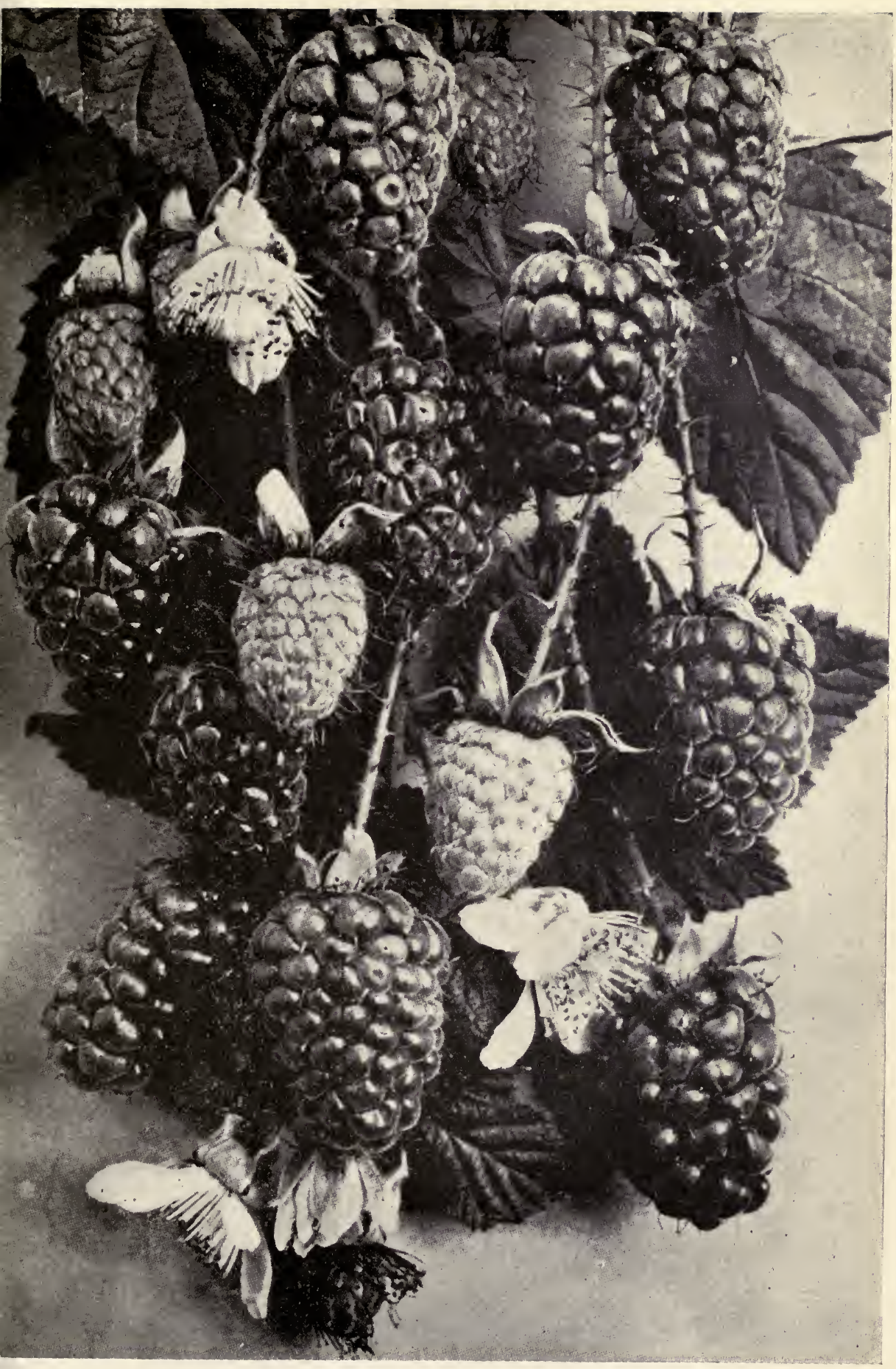

Plate XXI.-The Loganberry.-(See page 417.) 

floors; in well-fitted packing houses. Such investment has reached millions of dollars in the aggregate, and the standing of cured fruits as primary products is the justification of such outlay.

Third. The provision of such equipment is not alone evidence of the standing of the industry; it constitutes an obligation upon producers to put out a product which shall be true to its opportunity as a primary product, and not merely a makeshift to prevent loss or waste. Thirty years ago California dried fruit was a makeshift, and a disgracefully poor one. As enterprise and investment proceeded it was soon seen that style and quality alone could requite them. Next it was discerned that fruit for curing, to command profitable prices, must be as good as fruit for any other high purpose, as has been suggested. It was then believed that to secure handsome cured fruit which should only be relieved of its excess of water and still retain color, flavor and winning beauty, could only be produced in machine-evaporators with artificial heat, and a few years were given to invention, purchase and rejection of all such devices except as occasional refuges when the California climate forgets itself. When the demonstration came that with proper pre-treatment California sunshine and dry air would produce notably fine evaporated fruits without houses and furnaces, cured fruits entered upon their career as primary products, and planting to produce them began.

Fourth. The obligations upon producers, to make their output worthy of such standing, extended to the whole process of growing and curing. The fruit must be well grown, and fruit for curing should have size and quality which make it first class for other purposes, with the added excellence of being somewhat more mature, because it is not required to stand hauling and shipment. It should, however, be carefully handled to escape bruising, because discolorations are blemishes. It must be cleanly cut for removal of pit or core, because trimness, neatness and shapeliness are all essential to beauty. Before it reaches exposure to the protecting fumes of sulphur, it must be often saved from darkening by handling in water, when the nature of the fruit is such as to require it. It must be carefully and evenly spread upon the trays, especially if it be a cut fruit, so that no interference can prevent each piece from reaching its best estate. Sulphuring must be adequate, and yet not excessive, for sulphuring is a protecting and not a resurrecting process ; it is not to improve bad fruit, but to keep good fruit from becoming bad. The fruit must be sufficiently dried and yet not over-dried, and during the process must be protected from dust by the situation and character of the ground used, even if such protection costs trouble and outlay.

Although the sun drying of fruit may be a simple process, so many little arts, methods and appliances are continually being introduced to facilitate work or improve the product, that one can learn much by visiting the different fruit regions during the drying season. Such a course is commended to growers who contemplate large drying operations, for suggestions of great economic importance can be secured. The notes of practice which can be given in this connection must be brief and general. 
Trays for Drying.-The greater part of the fruit, including raisins, is placed upon trays for exposure to the sun. There is great variation in the size of the trays. The common small tray is made of one-half inch sugar-pine lumber two feet wide and three feet long, the boards forming it being held together by nailing to a cleat on each end, one by one and a quarter inches, and a lath or narrow piece of half-inch stuff is nailed over the ends of the boards, thus stiffening the tray and aiding to prevent warping. A cross-section of such a tray is shown at $A$.

A large tray which is used by some growers is four feet square, and is made of slats three-eighths of an inch thick, and one and a half inches wide, the slats being nailed to three cross slats three-eighths of an inch thick and three inches wide, and the ends nailed to a narrow strip one-half inch thick by three-quarters of an inch wide on the other side. A cross-section of this tray is shown at $B$.

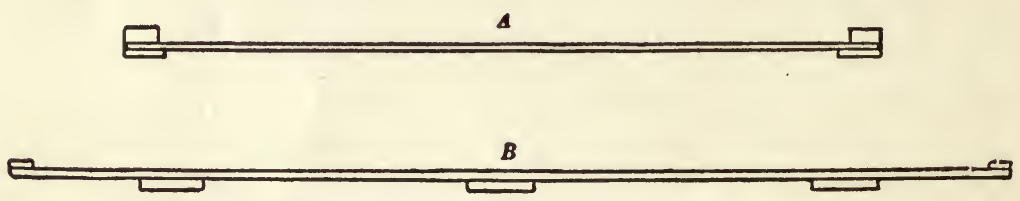

Cross-sections of drying trays.

Since large drying yards have been supplied with tramways and trucks for moving the fruit instead of hand carriage, larger trays, three feet by six or three feet by eight, have been largely employed. These tramways lead from the cutting sheds to the sulphur boxes and thence to various parts of the large drying grounds, making it possible to handle large amounts of fruit at a minimum cost.

Protecting Fruit from Dew.-In the interior there are seldom any deposit of dew in the drying season, but occasionally there are early rains before the drying season is over. The fruit is then protected by piling the trays one upon another, in which operation the thick cleats serve a good purpose. In dewy regions the trays are piled at night, or cloth or paper is sometimes stretched over the fruit, thus reducing the discoloration resulting from deposits of moisture upon it.

Drying Floors.-For the most part the trays are laid directly on the ground, but sometimes a staging of posts and rails is built to support them, about twenty inches from the ground. The drying trays are sometimes distributed through the orchard or vineyard, thus drying the fruit with as little carrying as possible. Others clear off a large space outside the plantation and spread the trays where full sunshine can be obtained. Drying spaces should be selected at a distance from traveled roads, to prevent the deposit of dust on the fruit.

Spaces used for drying are often idle the rest of the year and are weed-covered and unsightly during the rainy season, or are cultivated for grain-hay which loosens the surface and deepens the dust. When one has water for irrigation it is often practicable to reduce dust and 
secure an amount of desirable feed or hay by putting the piece down in alfalfa. Mr. P. T. Gannon of Yolo County proceeded in this way. He lowered the tramway tracks to the ground level, and turned up a furrow on the sides to hold the water from flooding the adjacent ground. In the fall, after the fruit-drying was over, he flooded the space, which is a little over half an acre (125 by 225 feet). Then it was disked both ways and leveled and harrowed and planted to alfalfa before the rains. The land was moist at the time and the seed came up and the plants grew more or less through the winter. In April he cut the first crop. Just before the space is needed for a drying yard, make another cutting, about June 20th, cutting it down close and raking it clean. The yard is then ready for the trays and fruit. When the drying season is over the yard is cleared, and the space then is as clean as a clay floor, from being used so much. In three weeks the top of the ground is green all over, and before the rains come there is another cutting crop, making three crops a year, a ton at each cutting. So something is made from the space and the dust problem is solved, which means clean fruit and better prices.

Grading.-It is of great advantage in drying to have all the fruit on a tray of approximately the same size, and grading before cutting is advisable. Machines are now made which accomplish this very cheaply and quickly.*

Cutting-Sheds.-Shelter of some kind is always provided for the fruit-cutters. Sometimes it is only a temporary bower made of poles and beams upon which tree branches are spread as a thatch; sometimes open-side sheds with boarded roof, and sometimes a finished fruit-house is built, two stories high, the lower story opening with large doors on the north side, and with a large loft above, where the dried fruit can be sweated, packed, and stored for sale. The climate is such that almost any shelter which suits the taste of the purse of the producer will answer the purpose.

Sulphuring.-The regulations promulgated under the pure food law enacted by Congress in 1906 established an arbitrary limit to the percentage of sulphur compounds in evaporated fruits, which was shown by producers to be destructive to their industry, and otherwise unwarranted and ureasonable. As a result of their protest the enforcement of such regulations was indefinitely postponed, pending the results of scientific investigation which began in 1898 .

From the point of view of the California producer it must be held that before the employment of the sulphur process, California cured fruits were suitable only to the lowest culinary uses. They were of undesirable color, devoid of natural flavor, offensive by content of insect life. They had no value which would induce production and discernible future. Placing the trays of freshly cut fruit in boxes or small "houses," with the fumes of burning sulphur, made it possible to preserve its natural color and flavor during the evaporation of its surplus moisture in the clear sunshine and dry air of the California summer. It also prevented souring, which with some fruits is otherwise not pre-

*See under plums and prunes," page 456. 
ventable in such open air drying, and it protected the fruit from insect attack during the drying process. By the use of sulphur and by no other agency has it been possible to lift the production of cured fruits of certain kinds from a low-value haphazard by-product to a primary product for which Californians have planted orchards, constructed packing houses and made a name in the world's markets.

The action of sulphuring is not alone to protect the fruit, it facilitates evaporation so that about one-half less time is required therefor. Not the least important bearing of this fact is the feasibility of curing fruits in larger pieces. The grand half-peaches, half-apricots, halfpears of the California cured fruits are the direct result of the sulphur process. Without it the fruit must be cut into small sections or ribbons, which in cooking break down into an uninviting mass, while, with the sulphuring, it is ordinary practice to produce the splendid halves with their natural color so preserved that they lie in cut glass dishes in suggestive semblance to the finest product of the canners, and are secured at a fraction of the cost.

There are various contrivances for the application of sulphur fumes to the freshly-cut fruit. Some are small for hand carriage of trays; some are large and the trays are wheeled into them upon trucks. The most common is a bottomless cabinet about five or six feet high, of a width equal to the length of the tray and a depth a little more than the width of the tray. The cabinet has a door the whole width of one side, and on the sides within cleats are nailed so that the trays of fruit slip in like drawers into a bureau. Some push in the trays so that the bottom one leaves a little space at the back, the next a little space at the front, and so on, that the fumes may be forced by the draft to pass between the trays back and forward. The essentials seem to be open holes or dampers in the bottom and top of the cabinet so that the fumes from the sulphur burning at the bottom may be thoroughly distributed through the interior, and then all openings are tightly closed. To secure a tight chamber the door has its edge felted and the cabinet is made of matched lumber. The sulphur is usually put on a shovel or iron pot, and it is ignited by a hot coal, or a hot iron, or it is thrown on paper of which the edges are set on fire, or a little alcohol is put on the sulphur and lighted, etc. The sulphur is usually burned in a pit in the ground under the cabinet. The application of sulphur must be watchfully and carefully made, and the exposure of the fruit should only be long enough to accomplish the end desired. The exposure required differs from different fruits, and with the same fruits in different conditions, and must be learned by experience.

Grading and Cleaning.-After the fruit is sufficiently dried (and it is impossible to describe how this point may be recognized except by the experienced touch), it is gathered from the trays into large boxes and taken to the fruit house. Some growers put it into a revolving drum of punctured sheet iron, which rubs the pieces together and separates it from dust, etc., which falls out through the apertures as the drum revolves. Others empty the fruit upon a large wire-cloth table and pick it over, grading it according to size and color, and at the same time the dust and small particles of foreign matter fall through the wire 
cloth. The fanning mill for cleaning grain may also be used for rapid separation of dirt, leaves, etc., with proper arrangement of metal screens.

Sweating.-All fruit, if stored in mass after drying, becomes moist. This action should take place before packing. To facilitate it, the fruit is put in piles on the floor of the fruit-house and turned occasionally with a scoop shovel; or, if allowed to sweat in boxes, the fruit is occasionally poured from one box to another. The sweating equalizes the moisture throughout the mass. Some large producers have sweat-rooms with tight walls, which preserve an even temperature. No fruit should be packed before "going through the sweat." If this is not done, discoloration and injury will result.

Dipping Before Packing.-All fruits except prunes can be packed in good condition without dipping, provided the fruit is not over-dried. Efforts should be made to take up the fruit when it is just sufficiently cured to prevent subsequent fermentation. If taken from the trays in the heat of the day and covered so that the fruit moth can not reach it there is little danger of worms. The highest grades of fruit are made in this way. If, however, the fruit has been over-dried or neglected, it can be dipped in boiling water to kill eggs of vermin and to make the fruit a little more pliable for the press. The dipping should be done quickly, and the fruit allowed to drain and then lie in a dark room, carefully covered, for twenty-four hours before packing.

Packing.-To open well, packages of dried fruit should be "faced." The many fine arts of paper lining, etc., must be learned by observation. Flatten some fair specimens of the fruit to be packed (and reference is especially made to such fruits as apricots, peaches and nectarines) by running them through a clothes wringer or similar pair of rollers set to flatten but not crush the fruit. Do not face with better fruit than the package is to contain. It is a fraud which will not in the end be profitable. Lay the flattened fruit (cup side down) neatly in the bottom of the box. Fill the box until it reaches the amount the box is to contain, and then apply the press until the bottom can be nailed on. Invert the box and put on the label or brand; the bottom then becomes the top.

Many different kinds of boxes are used. A very good size is made of seasoned pine, six inches deep by nine inches wide by fifteen inches long, inside measurements, and it will hold twenty-five pounds of fruit.

\section{METHODS WITH DIFFERENT FRUITS}

As already intimated, it will be impossible to enter minutely into the operations of drying and packing on a commercial scale, or even to notice all the small and ingenious arts by which the work is facilitated. Any one who contemplates production on a large scale should personally visit leading regions and inform himself by inquiry and observation. Such an education will save mistakes, which may cost many times more than the expense of getting it. California producers 
are usually quite willing to show visitors the methods they employ. Though this is the better way of proceeding, a few general hints will be given of methods with different fruits.

Apples.-There seems little use of drying apples unless a very light-colored, handsome product can be turned out. This can be done by sulphuring as soon as cut, and sun drying in a dry region, or by the use of a machine evaporator in regions of greater atmospheric humidity. Recently the product has largey increased in such large producing regions as the Pajaro Valley, and new labor saving devices are being continually introduced.

Apricots.-Apricots for drying should be fully ripe but not soft enough to be mushy. By the use of sulphur and sun heat, an ambercolored, semi-translucent fruit is obtained. The prevailing method of gathering is to shake down the fruit upon sheets, but the best product is hand picked. Pit the fruit by a clean cut completely around in the suture; do not cut part way around and then tear apart-a clean-cut edge is essential. Put on the trays with the skin down, or with the cut up, as it is sometimes described; sulphur, and then put in the sun. About three days of interior valley sunshine will finish the apricots. Apricots will yield on the average about one pound of dried fruit to five pounds of fresh.

Mr. Joseph T. Brooks, of San Jose, describes sulphuring of apricots as follows:

The fruit is cut in half and pitted, then spread upon the trays and put into an airtight suiphur house. These are made just large enough so that the trays may be slid in one over the other so that the sulphur fumes can permeate every part of the house. About one pound of powdered sulphur is poured into a hole in the ground and set fire, and as long as there is any oxygen left in the house, this sulphur will continue to burn and pour forth its volume of dense smoke. This process gives the apricots a very pretty golden appearance. The apricots, if dried without being sulphured, have a dark uninviting appearance, and are inclined to get wormy, although by dipping them in a strong solution of salt and hot water the possibility of infection might be overcome. Dried apricots are treated to a steam bath to make them pliable, and packed in boxes similar to prunes.

Berries and Cherries.-These fruits are only dried in the sun in small quantities for local sale, and ordinary farm-house methods are employed.

Figs*.-The fruit may be carefully picked from the tree so as to secure the whole of the stem, when the fruit is fully ripe, as is known by the seaming or slight shriveling of the skin. In drying the common black fig from large trees, however, the fruit is generally gathered from the ground, which is cleaned and smoothed before the crop ripens. In drying black figs the fruit is placed on trays and in most cases exposed to the sun, but some foothill growers maintain the advantage of drying in the shade. This is also practiced by some growers in the Fresno district, who stack the trays as soon as filled and thus cure by the natural movement of dry air instead of direct sunshine. The figs should not be allowed to dry hard. When sufficiently cured, put in sweat-boxes for

*A special illustrated account of handling dried figs in California is given in "The Smyrna Fig at Home and Abroad," by George C. Roeding, Fresno, Cal. 
several days, and when ready to pack dip in boiling salt water, or, as is the practice of some producers, dip in a thin syrup, boiling hot. In either method a good pliable condition and handsome color are obtained. In drying white figs many sulphur the fruit from fifteen minutes to an hour before putting out on the trays. Figs which dry slowly have to be turned several times during the drying, and those which are apt to run juice are placed so that the eye is raised a little until the juice is thickened. The white figs are also put in sweat-boxes and dipped in hot salt water before packing. In packing, the figs are often flattened and drawn out by the hand. Such manipulation gives the fig a lighter and more translucent appearance. The time required in drying figs is usually from five to eight or ten days, according to location and weather. The fruit does not cure evenly, and those which are finished (as determined by sight and touch-to be learned by experience) are picked from the trays, and others given more time.

Mr. Henry Markarian, of Fresno, a large producer of dried fig;, handles the fruit this way:

He has a cement tank, half filled with salt water and immerses by means of a wire basket a ton of figs at a time for from ten minutes to a half hour. The figs are hoisted and emptied through a feeder into trays, which are stacked in the drying yard till the fruit is of uniform quality. This takes about ten days, and is far superior and cheaper to the quicker sun drying. They are then poured into sweat-boxes like raisins and taken to the packer, culls being removed. The Adriatics are not dipped, but sulphured.

Culls are mostly splits. There are more some years than others; cool, damp weather being especially bad. Irregular weather, or very hot weather, will also develop culls, and rains will sour a good many, though Adriatics suffer worse than the others. In good years culls amount to only about two per cent. In bad years they will amount to eight and ten per cent.

Birds will do big damage. One year they destroyed eight tons of fruit. Afterwards a few boys with guns were kept busy, and one summer killed 4,000 birds, and there was hardly a bird picked fruit in the lot as a result.

The orchard on the best soil, and best attended to will give the finest fruit. However, the average Smyrna crop will run about 20 to 25 per cent extra fancy, about the same amount fancy, 45 per cent choice and 5 per cent cooking fruit, sold to bakers and pastry manufacturers. The choice fruit is split and packed in bricks as seen at fruit stands and grocers. The larger, finer figs can be sold whole, packed solid.

Pears.-The dried pear product is increasing, and, as with apples, only a light-colored product is profitable. These are made by sulphuring and sun drying, or by the use of the machine drier. Fosun drying the fruit of medium size is halved, the large fruit being quartered.

Peaches.-Peaches are sun dried in much the same way as apricots, already described. Take the fruit when it is fully ripe, but not mushy; cut cleanly all around to extract the pit and put on trays cup side up; get into the sulphur box as soon as possible after cutting. Peaches are dried both peeled and unpeeled, but drying without peeling is chiefly done. Peling is done with the small paring machines or with a knife. Peeling with lye has been generally abandoned because of discoloration of the fruit after packing, although it can be successfully done by frequently changing the lye and using ample quantities of fresh water for rinsing after dipping. 
Clingstone peaches are successfully handled with curved knives and spoon-shaped pitters in conjunction with ordinary fruit knives. Different styles are carried at the general stores in the fruit districts, and individuals differ widely in their preferences.

The weight of dried peaches which can be obtained from a certain weight of fresh fruit, depends upon the variety; some varieties yield at least a third more than others, and clings yield more than freestones as a rule. Dry-fleshed peaches, like the Muir, yield one pound dry from four or five pounds fresh, while other more juicy fruits may require six or seven pounds.

Nectarines.-Nectarines are handled like peaches; the production of translucent amber fruit in the sun depends upon the skillful use of sulphur.

Plums and Prunes.-Our pitted plums, which are an acid fruit, are meeting with more favor than formerly, and the product is increasing. Pitting is done by hand or by the use of foot-power "pitters." More rapid and capacious machines are being brought out by inventors.

Prunes are one of our greatest and most promising products. Several varieties which dry sweet with the pit in are used in making prunes, as already stated in Chapter XXIII, but the prevailing variety is the Prune D'Agen.

Prunes are gathered by shaking from the trees, usually upon sheets spread beneath. Several gatherings are made by light shakings which cause only the ripe specimens to fall. Some growers, however, hand-pick the fruit-going over the trees several times only shaking for the final gathering. A superior cured prune is secured by this extra effort to secure full and even ripening, but the cost is greater.

Prunes are usually graded before drying, and various homemade contrivances are employed. Some use inclined planes of adjustable slats, the grader being thus available for other fruits than prunes; the large fruit rolls along into receptacles at the bottom, while the small fruit falls through into other receptacles. Other grading devices are made with wire screens or riddles of different sizes of mesh. Some of them work on the principle of a fanning mill, three to four riddles, placed above one another, each with a slight incline and a spot on the side where each grade drops into a box. Some have a long riddle, say twelve feet long, with three different sizes of wire screen on it. This riddle is hung upon four ropes with an incline; the prunes are thrown in the higher end, and by shaking it they roll down and fall through the holes into boxes underneath. The first piece of screen should be small, to let only stems and dirt through, and no prunes. This long hanging screen is also used to grade prunes after drying. There are now several excellent manufactured fruit graders on sale in this State. Their work is very satisfactory, and they have largely displaced homemade contrivances.

The next step in the process is dipping in lye to thin and crack the skin, which facilitates the escape of moisture in the drying process. In the large caldron lye is made with one pound of concentrated lye to each thirty gallons of water, and kept boiling hot. The fruit is put into wire baskets or galvanized pails with perforated sides and bottoms, 

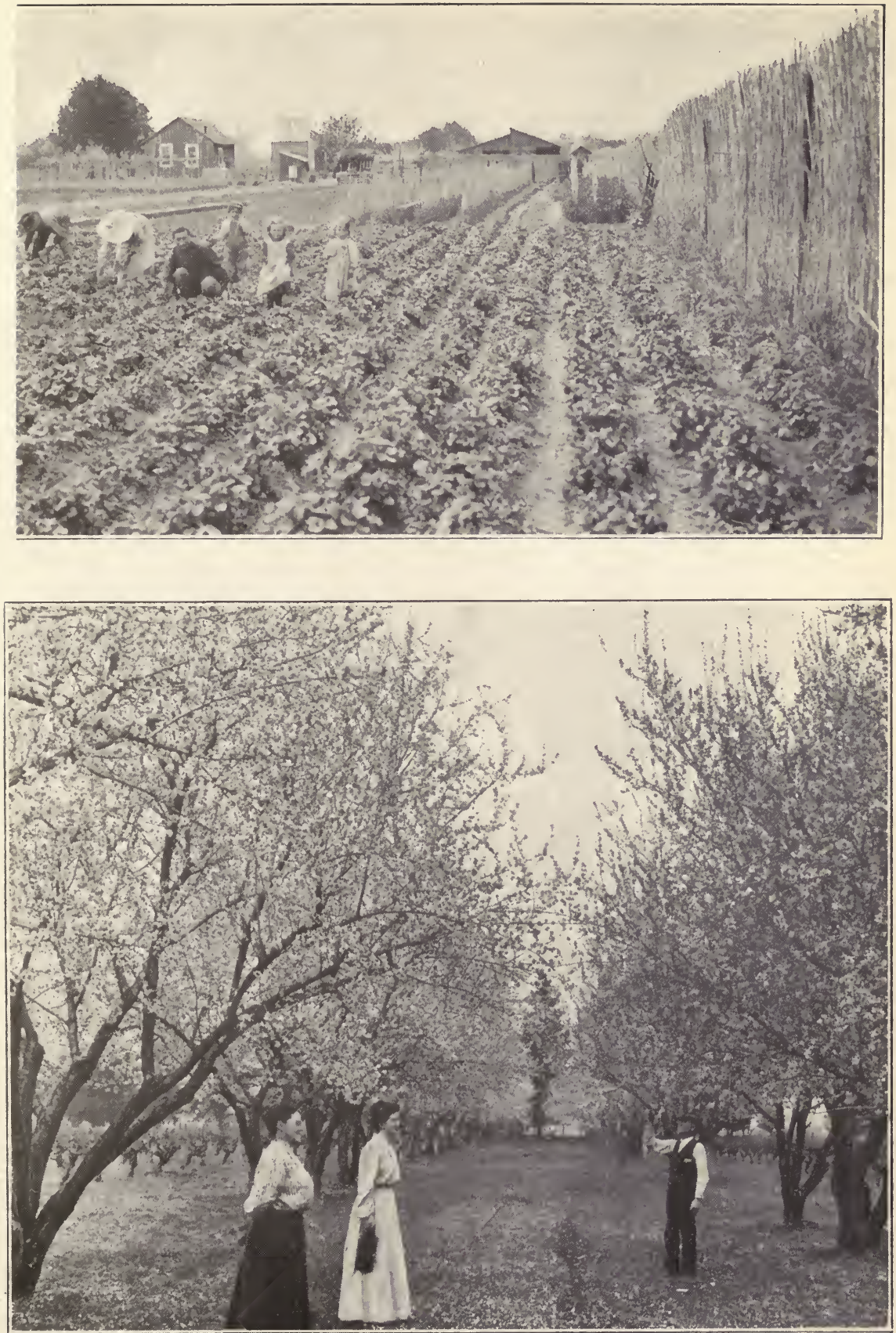

Plate XXII.-Strawberry patch with cane windbreak (see page 421); also almond trees in bloom (page 425). 

and dipped in the boiling lye for about a minute, or until the skin has a wrinkled appearance, then the basket is plunged into clean cold water to rinse off the lye. This rinsing water must be frequently changed, for it soon becomes very alkaline. Some begin with a stronger lye solution, one pound to ten gallons of water, claiming that a very short dip in stronger lye is better than long exposure in a weaker solution. After this dipping, the prunes are placed on trays. In the sun the prune dries sufficiently in from one to two weeks, according to the situation and weather.

A process of puncturing the skin of the prunes by causing them to roll over needle points has also been employed to some extent. There are now manufactured very capacious appliances for continuous dipping, rinsing, puncturing and spreading on the trays so that the fruit is handled in large quantities at a minimum cost. In no branch of our fruit industry, perhaps, has there been greater advance in labor-saving devices than in prune handling.

When sufficiently dried the prunes are put through the "sweat," which takes from several days to two or three weeks, and then are ready for grading, finishing, and packing. In grading, the prunes are separated by the use of a grader, as already described, into a number of grades, the largest, forty prunes to the pound, and so on, fifty, sixty, etc., to the smallest, which may run one hundred or more to the pound. Finishing consists in exposing to steam, in dipping in clear hot water, or hot sugar syrup, or in dipping in boiled juice of ripe prunes, or peaches or apples, etc. Although there is a great variety of materials used for "glossing" prunes by different producers, the prevailing practice is to rely upon hot water, to which purd glycerine is added at the rate of one pound to twenty gallons. Some growers also add a little brine (having first dissolved the salt and skinned off the impurities). This final hot dip kills insect eggs, and the fruit, after drying off away from the access of insects, should be packed tightly in boxes, usually holding twenty-five pounds, though thirty-eight to fifty-pound boxes are used in foreign trade.

The following explicit hints on the curing of prunes are based upon wide experience and observation in the Santa Clara Valley:

Be sure to allow the prunes to obtain all of the sugar they can from the trees by hanging until they drop of their own accord. Do not pick up until prunes are soft to the touch. These two rules are productive of nice black prunes. They may not be black when gathered in the bins but will color with age, without any foreign coloring matter.

Do not keep prunes in boxes over night. They go through a sweat, and do not make a first quality of dried fruit, and take much longer to dry. It is better to let the prunes lie on the ground under the tree for several days than to let the picked prunes lie in the boxes over one night.

The dipping fluid must be kept at the boiling point and no prunes put in unless it is boiling. It is not a matter of how strong! the lye is, but how hot is the water. On the trays prunes will either dry or ferment. Unless the dip is hot enough the prune will not immediately commence to dry, but will, in a few days, become a chocolate color and refuse to dry, sometimes a few on a tray, often half and sometimes nearly all. If the water is at the boiling point all through the dip, two pounds of lye to the 100 gallons of water, may be sufficient. If the water is not boiling, ten pounds of lye to the 100 gallons of water may be required. 
Weather conditions govern the time prunes should remain on the trays. Grasp a handful of prunes and give them a gentle squeeze and open the hand quickly, if the prunes separate they are ready to stack the trays and the fruit should be placed in the bin before it rattles on the trays.

When the prunes are sufficiently dry put them in a dry place where it will not rain on them, but do not prevent the air from getting to them. Let the wind have free access until the rains set in then close doors and make the house as snug as you can. In making bins, be sure the boards are dry and the bins well above ground, or you will have trouble. Do not let prunes get damaged by rain.

For dipping before packing, some use a brine dip-about five pounds of salt to 100 gallons of water is about right. This salt dip can be used more safely for prunes than for peaches or other pitted fruit, as in some instances it has attracted moisture and caused mold. As to the respective results of a sal solution and glycerine solution, the salt seems to be a cleansing process, which leaves the skin of the fruit in a bright, clear condition and brings out the blue bloom, which is desirable. The glycerine is more of a syrupy or glossy nature, and on prunes that are inclined to be reddish this solution can be used to advantage. A common method of treating prunes is to use the small prunes and suspend them in the steam-heated tarik of the Anderson dipper, where they can not come in contact with the steam (which causes the skin and meat of the prune to turn dark), dissolve out the juice, thus forming a dip liquor, and this is quite extensively used.

Raisins.-The varieties of grapes used for raisins are described in Chapter XXVIII. The production of raisins has reached such an extent, and employs so much skill and capital, that the processes employed to facilitate the curing and packing are so various that a description of them cannot be attempted. Besides an excellent special treatise has been written on this subject.* However, in beginning the commercial production of raisins, one should visit the raisin farms and packing-houses during the harvest. The following description by T. C. White, of Fresno, gives an outline of practice in the vineyard:

In Fresno picking commences about the first of September, although there have been seasons when it occurred as early as the 20th of August. The grapes under no circumstances should be picked for raisins until they are ripe. There are three ways by which to ascertain this: First, by the color, which should be a light amber; second, by the taste; and third, by the saccharometer, which is by far the most accurate. A grape may be ripe, and not have the proper color, when grown entirely in the shade. The juice of the grape should contain at least twenty-five per cent saccharine, to produce a good raisin.

The method of drying is with trays placed upon the ground. The almost entire absence of dew in our locality greatly facilitates this method. The trays are usually twenty-four by twenty-six inches, which hold about twenty pounds of fruit, and should produce from six to seven pounds of raisins. The product of a vineyard depends largely upon its age and favorable conditions, varying from two to nine tons of grapes per acre.

The trays are distributed along the sides of the roads, from which they are taken by the pickers as they are needed. As the grapes are picked from the vines, all imperfect berries, sticks, and dead leaves are removed from the bunches, which are then placed upon the trays, right side up. A cluster has what is called a right and a wrong side, the wrong side having more of the stems exposed than the right side. Great care should be used in picking, so as to handle the bunches only by the stem. If the berries come in contact with the hands, some of the bloom will be removed, which will injure the appearance of the raisins. The trays are placed, after filling, between the vines, one end being elevated so that the grapes may receive the more direct rays of the sun.

Too rapid drying is not desirable. The grapes are left upon the trays until about two-thirds dry, which, with us, will be from six to eight days. They are then turned. This is accomplished by placing an empty tray on top of the one

*The Raisin Industry by Gustav Eisen, San Francisco. 
filled with partially-dried raisins, and turning them both over. Then take off the upper or original tray, and you have the raisins turned without handling or damage. After turning, curing will proceed more rapidly, and frequently is completed in four or five days. During this time they should be carefully watched to prevent any from becoming too dry. When it is found they are dry enough, the trays are gathered and stacked one upon another as high as convenient for the sorting which follows. This protects them from the sun and prevents overdrying. Stacking should be attended to early in the morning, while the stems and berries are slightly moist and cool from the night air, as they will retain this moisture after being transferred to the sweat-boxes, and assist in quickening the sweating process.

As the raisins are taken off the trays, some of the berries on the bunch will be dry enough and a few will not be sufficiently cured. To remove the moist ones would destroy the appearance of the cluster, and to leave them out longer would shrivel the dry ones, hence the sweat-box. The moisture is diffused through the box, some being absorbed by the dry raisins, and the stems also taking their share are thus rendered tough and pliable and easily manipulated when ready for packing.

Sorting and grading require great care and judgment, and although a tedious process, it greatly facilitates rapid packing. The sweat-box is a little larger than the tray and about eight inches deep, and contains about one hundred and twenty-five pounds of raisins. Heavy manila paper is used in the sweat-boxes, one sheet being placed in the bottom, and three or four more at equal distance as the filling progresses. The object of the paper is to prevent the tangling of the stems and consequent breaking of the bunches when removed for packing.

The sorters have three sweat-boxes, one for the first, second and third qualities, as the grade will justify. The bunches should be handled by the stem and placed carefully in the sweat-boxes to avoid breaking the stems; thereby destroying the symmetry of the clusters. Any found to be too damp are returned to the trays and left a day or two longer in the sun. To ascertain if the fruit is perfectly cured, take a raisin between the thumb and forefinger and roll it gently until softened, when either jelly or water will exude from the stem endif water, it requires further drying. When the boxes are filled, they are taken to the equalizer. This should be built of brick or adobe, and as near air-tight as possible, but provided with windows to allow ventilation when necessary. The windows should have shutters to keep it dark. The filled boxes are placed one exactly above another to a convenient height, and should remain from ten to twenty days or more, when they will have passed through the sweating process. When the raisins are sufficiently equalized, the sweat-boxes are removed to the packing-room, which is provided with tables, presses, scales, etc.

The foregoing relates to the preparation of the standard clusters. Loose raisins are now being produced in increasing quantities. Loose Muscatels are prepared by being put through the stemmer and grader. The stemmer removes the berries from the stems, and the grader, by separating according to size, determines the grade. During the last few years the seeding of raisins has increased rapidly, and large establishments for this work, with every ingenious machinery, have been erected. Seeded raisins constitute a considerable portion of the product.

A considerable quantity of dipped raisins are also made of the Sultana and Thompson seedless grapes and of loose or inferior Muscatels. A lye dip of about one pound of potash to twelve gallons of water is used, and the solution is kept boiling hot. The ripe fruit is dipped for an instant, then plunged in fresh water for a thorough rinsing, and then placed on the trays. During warm, dry weather in the interior, the raisins are dried in the shade by leaving the trays in piles, but if cooler, moister weather prevails, the trays must be spread out. The product is a handsome amber color. 
An oil dip has been profitably used with Thompson's Seedless:

One quart of olive oil; $3 / 4$ pound Greenbank soda and 3 quarts water are made into an emulsion, and then reduced with 10 gallons water in the dipping tank, adding more soda to get lye-strength enough to cut the skins, and more soda has to be added from time to time to keep up the strength. The grapes are dipped in this solution and sulphured to the proper color.

Mr. Hecke's Way With Dipped Raisins.-Much experimentation has been undertaken by California growers to improve the process of making dipped raisins. The following is the method devised and largely used by Mr. G. H. Hecke of Woodland, Yolo County, in handling Thompson and Sultana raisins:

Before drying they are dipped in a solution of sodium bicarbonate, which just takes the bloom off, but does not cut the skin. This reduces the time of drying about half, so what it costs in one way it saves in part in another. A crew of seven men can keep two vats going and turn out 1,600 trays a day, 500 trays per ton of raisins.

The stock solution of bicarbonate is a pound to a gallon of water. It is diluted about 3 to 1 , the proportion varying with the effect on the grapes, more of the stock solution being added as desired. The grapes are dipped in wire trays in this warm solution and about four wire trays are needed to a vat. A thin layer of olive oil is kept on the surface of the solution, a trace of which touches the surface of the grapes as they come out and gives them the right color and quality. Only a tablespoonful of oil is added at a time and the actual amount of oil used is very small -about one gallon to 500 trays.

This method of bleaching is held to be vastly superior to sulphur drying. It gives as fine a looking raisin as the sulphured, the raisin has a natural flavor that is very attractive, and is better in other ways. The raisins are sun dried in eight to ten days, the trays being stacked if rain threatens, artificial drying not being necessary. The total cost of making raisins is about $\$ 25$ per ton, including harvesting.

\section{GRAPE SYRUP}

The manufacture of grape syrup, which was formerly of considerable prominence as a means of disposing of wine grapes, has recently received less attention because of low prices in competition with the vast amount of syrup available from the sugar refineries.

\section{MACHINE EVAPORATION}

Although California summer conditions of adequate heat and dry air favor open-air evaporation to such an extent that nearly all our product of cured fruit is secured in that way, there are some parts of the State where artificial heat would be a safer recourse and there are late fruits which sometimės collide with early rains in a way to cause losses even in our best sun-curing regions.

It is interesting, therefore, to describe a machine evaporator constructed upon true principles and having capacity sufficiently large to encourage its use. Mr. L. W. Parsons of Campbell, Santa Clara county, has given most of his life to the design and construction of fruit evaporators, and secured patents thereupon which have expired and are now public property. In the Pacific Rural Press of June 19 


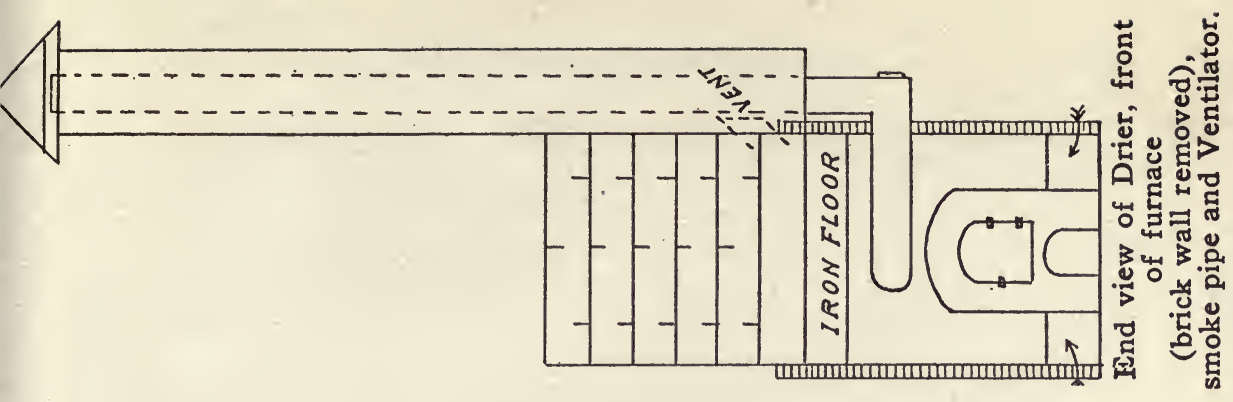


and 26,1909 , he gave his conclusions on the desirability of machine evaporation and a design for the construction of an evaporator which embodied all his best work in this line. The accompanying drawings and description are from Mr. Parson's writings :

A fruit drying chamber capable of holding about two tons is about as large as one heater can well handle. Wire trays two feet wide by five feet long are as large as one strong man can conveniently handle; he might prefer them shorter; in fact, if the smaller tray would give a drier of sufficient capacity to to meet the demands of the owner, he could make the trays two by four feet, with a narrower furnace, to correspond. Where two men are always available, trays three by six feet have been found to work well.

A drier five feet wide and twenty-four feet long, having ten trays in its length and twelve trays high, holds 120 trays 2 by 5 feet, or a total drying surface of 1,200 square feet, which at the rate of 3 pounds per foot give a total of 3,600 pounds at one time: that is, it would take that much gross weight of fruit before cutting to fill the trays. Large fruit would amount to more, and. small fruit or culls might not go above two pounds to the foot.

By making the drier one or two trays longer and higher, and a foot or so wider, the capacity may be materially increased. But if much more capacity is desired, it would be better to build another drier, or several of them, alongside of it, which would work satisfactorily.

The fruit drying chamber rests on top of the hot air chamber, which is surrounded by brick or cement walls about eight inches thick, and is as long and wide as the fruit chamber above. The height of these walls depends on the size and shape of the heater enclosed by them. The furnace is a very important factor: it must be strong, not easily burned out, smoke-tight in all its joints and flues, with caps or covers so placed to facilitate cleaning out soot and ashes. There must be room between the sides of the furnace and the side walls to allow for circulation of the air to be heated.

Fresh air enters this hot air chamber through openings about a foot square at the bottom in front between the furnace and the side walls; passes along on the sides and top of furnace and flues until it reaches the back end of the hot air chamber, where it passes up through an opening into the fruit chamber above,

The next, and perhaps the most critical, part in the construction, is the sheet iron floor which covers the furnace and flues and separates them from the fruit chamber above. This iron floor is made of either black or galvanized iron, not thinner than No. 24; thicker would be better. It rests on rods or bars of angle or $\mathrm{T}$ iron stretched from wall to wall, with ends built into the walls. These rods are spaced about two feet apart. The sheets of iron should be riveted or bolted together. The edges of this iron floor should project into and be imbedded in the brick walls in front and along the two side walls as far back as the floor goes to the other end of the drier. Cover this floor with an inch or so of sand or fine earth, so as to stop up all cracks. At the back end of drier this iron floor is cut short one foot, allowing an opening of one by about five feet to allow the hot air from the heater below the iron floor to pass up into the fruit chamber above. This floor must be made tight everywhere, so no hot air can possibly leak upward and scorch the fruit or the woodwork above.

The brick walls should be built about eighteen inches above the iron floor, so that the wooden frame which rests on top will be safe from burning.

The furnace may be about four feet high by three feet wide, with an ash pit in the bottom part, and six or eight or more feet long. This will handle four-foot cordwood conveniently and make a liberal combustion chamber for the flames. It may be a cylinder or shaped like a boiler, or have brick walls, with a cast iron arch on top. Or it may be a big wrought iron box made of quarter-inch boiler iron, braced with angle iron to prevent warping.

A smoke pipe one foot in diameter connects with the back end of the furnace, passes horizontally to near the back end of the drier, then by a short elbow doubles back, coming to the front over the top of the furnace, and then, by an elbow, to right or left through the side brick wall to connect with the pipe inside of the ventilator shaft or flue. 
If the furnace is four feet high and the pipe above it one foot thick, allow six inches space between the top of the furnace and the pipe and between the pipe and sheet-iron floor above it; then add the eighteen inches of brick wall above the iron floor; we get a total height of seven and a half feet from the ground to the top of wall.

But only the furnace needs to be as deep as this, and it can be placed in a pit at one end of the drier. The brick walls back of the furnace do not need to go so far down by perhaps two feet. The smoke pipe leaving the furnace close to its top can rest on a brick lying on top of the ground, so the part of the heating chamber holding the long flues may be shallower, thus saving some expense in masonry. The top of the brick wall should be level all the way around and even with the floor of the house, so the operator can easily handle the trays on the upper track. Do not insert woodwork of any kind into the brickwork.

Lay $2 \times 4$-inch sills on top of the brick walls all around the four sides. Cut your upright studding of $2 \times 4$ joists six and a half feet high, spaced two feet apart, and nail a $2 \times 4$ plate on top. This is for the two long sides. The ends of the drying chamber are to be supplied with close-fitting doors. Nail surfaced matched lumber on the inner face of the studding. These boards had better be of redwood, so as not to gum up the trays with pitch.

These two side walls should be perfectly straight, plumb and level and at equal distance apart at all points, so the trays will work true and not bind or slip off the tracks. Nail to these walls tracks or slides made of strips one and one-half inch square. Fasten to these tracks wheels, known to the hardware men as "sash rollers," one inch in diameter and spaced ten inches apart along the track. Cut a little notch in the edge of the track, so the wheel can turn freely, with the big side of the wheel upward. Put twelve of these tracks on each wall, spaced six inches apart, beginning six inches from the ceiling to the top of the first track, then six inches to the top of the next track, etc. One strong 16-penny wire nail driven into each stud will hold these tracks securely. Fasten the wheels with screws to the track on a work bench before nailing them to the walls.

Nail matched lumber on top of the plates from side to side for a ceiling. This wooden shell will have to be braced from the outside so it can stand up firmly with its load of fruit.

The ventilator or exhaust flue should be about two by three feet inside and extend about twenty feet above the top of the drier. Build it against the side of the drier, resting on top of the brick wall, close to the front end of the drier.

The smoke pipe must be carried by a suitable elbow from its position above the furnace through the side brick wall to a point under the center of the ventilator, thence up through it to the top. A sheet-iron weather cap on top should protect the ventilator and smoke pipe. Make the bottom of the ventilator where smoke pipe enters, air tight to ensure good draft. Provide caps or covers at the elbows to facilitate cleaning out the soot.

On the side of the drying chamber, at the bottom, cut a hole into the ventilator for the passage of the damp air from the fruit into the ventilator. This opening should be the shape of a right angled triangle, in such position as though the bottom cover of the side wall were being cut off. The bottom and perpendicular side of opening are to be three feet long. The lower tracks go past this opening to carry trays to the door. Some kind of a small guide rail should be placed to prevent the corners of the trays from striking against the edge of opening. The trays are to be one-half inch shorter than the drier is wide inside, so as to move freely without danger of being bound. Two or three rods of half-inch iron provided with screws and nuts should pass through from side to side of drying chamber about half way from top to bottom, to prevent the walls of the drier from warping or bulging, or else the trays will drop off the tracks.

Make four sides of the trays of stuff one and a half inches square, notched at the corners of trays. Tack on No. 3 mesh galvanized wire cloth. Over this nail strips one inch thick by one and a half inches wide for runners to roll over the wheels. Nail a similar strip across the middle of the tray to prevent the wire cloth from sagging. From twenty-five to fifty extra trays will be found convenient in operating the drier. The wire cloth is two feet wide. Make the tray frames two feet one inch wide. With average prices for labor and material this drier can be built in a substantial manner for about $\$ 500$. 
To Operate the Drier.-Get started right by taking all day in gradually filling the drier, so that by night time the first trays will be within a few hours of being finished. Start a good fire before putting in the fruit, so as to burn out the oily smells of the new ironwork. Use the eight lower tracks for the fresh fruit. Put eight trays in at a time, one tray on each of the eight lower tracks about every forty-five minutes, starting them at the front end over the furnace and pushing along. After ten lots have been so placed the eight lower tracks will be full. Then take out one tray from each track at the back end, and put them in on the upper four tracks, two trays on a track, to be gradually pushed along from time to time, as often as fresh trays are put in at the front, until the machine is full and the driest trays will be at the front end on the upper four tracks. The fresh, wet fruit will in this way have its vapor drawn out through the ventilator close by, without wetting the fruit which is partly dry.

As the fruit gets drier it is moved into drier and warmer air at the back end. But this fresh air right from the heater below is 180 degrees hot and unsafe for fruit to finish in, so it is started back on the upper tracks to finish in a milder temperature, with enough vapor in the air to allow the fruit to finish gradually without danger of scorching, turning out the fruit in a soft and flexible condition, evenly cured and right in color. This process is peculiar to this evaporator and yields the best results in quality of work done.

If fruit is rushed too fast through the drier it will get back to the front and top too soon and will be too wet to dry readily in the lower temperature.

If the beginner gets caught this way, it will be better, after waiting a little, to take it out, and, if still too wet, carry it back to the other end and put in on the upper four tracks again to gradually go forward again. After a little experience he will learn how and when to move the fruit.

As to variations of heat in different parts of the drier and the effects thereof, it may be added that the thermometer hanging in the current of air just as it strikes the fruit may register 190 degrees and do no harm, for the fruit at that end of the drier has enough moisture to save it. The air cools rapidly, and when it reaches the other end of the drier where the fruit on the top tracks is nearly dry, the temperature will be about 40 degrees cooler. This is one of the most valuable points in this evaporator, finishing the fruit in a lower temperature and yet with but little moisture in the air.

Wood or coal fires will fluctuate in heat, and a careless operator might allow the temperature to get to 200 or 210 degrees, but, if it does not last more than a few minutes, and as the fruit is not too near being finished at the exposed end, no harm is done. A thermometer resting on the tray at the back end lying between the fruit will show about 10 degrees lower temperature than when hanging clear in the draft at that point.

While drying the trays should not touch the ends of the drier, but be pushed back about a foot and a half from the doors to allow room for free circulation of air at the back end of drier and down at the front end. It takes sixteen to twenty-four hours to dry the fruit, according to kind and size. Apples are dried in six to eight hours.

The furnace may be made to use any fuel. An oil burner is the best, because the heat can be kept steadily at the maximum. 


\section{PART NINE: FRUIT PROTECTION}

\section{CHAPTER XXXIX}

\section{CALIFORNIA METHODS WITH INJURIOUS INSECTS}

The California climate, which so favors tree and vine by a long, mild, growing season also enables some insects to multiply much more rapidly than they do in wintry climes; some having several distinct broods, others carrying on the work of reproduction and destruction of plants nearly the year round. The difficulties of the problem of the control of injurious insects are constantly being increased because new pests, in spite of the most careful efforts to keep them outside our boundaries, occasionally find their way into our orchards and vineyards. Furthermore native species, feeding, unnoticed perhaps, upon wild growths have found, in certain instances, that cultivated plants offer to them most satisfactory food and then suddenly become a factor in the horticulturists' problem.

Undoubtedly parasitic and predaceous insects preying upon the injurious species found in the fruit plantations are of assistance, in greater or lesser measure, in reducing the pests, and this service is being promoted by the introduction of beneficial insects from other parts of the world. There are many of our native species of insects, also syrphus and ichneumon flies, ladybirds, etc., that are valuable in this regard. Other factors also, such as untoward weather-conditions at the time of hatching, bacterial and fungous diseases of insects, etc., assist the horticulturist in his warfare against injurious insects. It is also a fact that California conditions have demanded and favored the development of ways and means for the suppression of orchard and vineyard pests, and methods and appliances have been invented which have demonstrated notable efficiency and value.

While the literature upon the subject of insect pests in California is quite extensive, much of it is beyond the reach of the general reader. Nevertheless there are a number of publications which should be secured and studied by every fruit grower. These are the bulletins and reports of the Agricultural Experiment Station of the University of California, at Berkeley (from which many of the following statements are taken) ; of the State Board of Horticulture, at Sacramento; and of the Bureau of Entomology of the United States Department of Agriculture, at Washington. A most useful addition to the horticulturist's library will be found in Kellogg's "American Insects," published by Henry Holt and Company, New York. This excellent work of Professor Vernon Kellogg, of Stanford University, is particularly valuable because of its California observations and point of view. The latest and at the same time the most specific and practically valuable review of fruit pests and their control is "Injurious and Beneficial 
Insects of California," by E. O. Essig, State Board of Horticulture, Sacramento, 1913. Furthermore as the study of the pests and the invention of means for their destruction are continually progressing one can only keep himself up to date and enable himself to profit by improvemẹnts, by diligent reading of California's periodicals devoted to practical horticulture.

\section{CLASSES OF INSECTS}

In order to arrange injurious insects in classes in a popular way, the grouping here will be based upon the character of the work they do. This arrangement has been followed by other writers and is perhaps better than attempting to group the insects which prey upon any single tree or plant, because injurious insects seldom restrict themselves to a single food plant. Therefore the grouping will be as follows :

(1) Insects destroying foliage;

(2) Insects upon the bark or upon the surface of leaf and fruit;

(3) Insects boring into the twig, stem or root;

(4) Insects boring into the pulp of fruits.

\section{INSECTS DESTROYING FOLIAGE}

Cut Worms and Army Worms.-These are the larvae of Noctuid moths, which often become abundant over limited areas and do much damage to trees and plants. Cut worms and Army worms are terms applied to the same insects in California. In ordinary years they are not present in sufficient numbers to cause much concern, and in such years they are known simply as cut worms. When all conditions are favorable, however, certain species develop in enormous numbers and having exhausted the food supply where they breed, they begin to migrate or march, commonly in a definite direction, as an army in search of new food. When they thus appear in such large numbers and take on the migrating habit they are called army worms.

Some of the caterpillars have the habit of climbing up vines and trees and eating off the buds in the early spring. These are called climbing cut worms. Others remain at or near the surface of the ground and feed by cutting off the plants at this point. They are more commonly found in the grass lands, but very frequently attack cultivated crops, particularly on land that was in grass the previous year.

Of the methods used to protect trees and plants from cut worms, poisoned bait is probably the most common. This consists of bran and molasses or other sweet substance poisoned with arsenic and distributed in handfuls about the plants. The proportions are as follows: forty pounds of bran, two gallons of cheap molasses, and five pounds of arsenic. Cheap glycerine may be used to prevent the mixture from drying. This will be eaten by the worms in preference, usually, to the plants which it is desired to protect. 
Cut worms and army worms may also be captured by means of traps. Because of their habit of feeding at night and remaining concealed during the day, pieces of boards may be placed on the ground around the growth to be protected and these may be turned over during the day and the worms killed.

In case of outbreaks of army worms the most important and successful means of fighting them is to keep them out of the orchard or vineyard entirely. This can be successfully done if they are discovered in time, or if already in one portion, they can be kept from spreading over the rest of the property. They travel in immense numbers in a definite direction, coming generally from an adjoining or nearby grain field. If a furrow is plowed along the side of the place to be protected it will effectively stop their progress. This furrow should be plowed as deep as possible, with the vertical side next to the field to be protected. It can be further trimmed with a spade, preferably cutting under slightly, making a smooth surface, over which few if any, of the worms will make their way. Above this shoulder a fine pulverized earth should slope as abruptly upward as possible. If any of the worms succeed in climbing up over the smooth surface made by the spade they will be pretty sure to fall back as they reach this fine loose earth in an attempt to ascend over the projecting shoulder. Postholes should be dug on the straight edge of the furrow every fifteen or twenty feet. The worms in failing to scale the vertical side of the furrow will crawl along in the bottom and fall into these holes. Here they may be killed by pouring in a little crude oil, or by pouring in a little distillate and dropping in a match, thus burning them, or the holes filled in and others dug. They may also be killed in the furrow by sprinkling them with kerosene or by pouring a strip of crude oil along the furrow.

Canker Worms.-These are destructive leaf-feeding caterpillars, commonly known also as inchworms, loopers or measuring worms, because of the peculiar looping gait by which they move about. The male moths are slender bodied and have broad, thin wings, while the females are wingless, heavy-bodied creatures.

While these caterpillars can in large measure be controlled by spraying with lead arsenate or Paris green, it is by far the best to use the trap method of control and avoid having them upon the trees at all. The trap method depends for its success upon the fact that the wingless female moth upon emerging from her cocoon in the ground immediately crawls up the trunk of the nearby tree and places her' eggs upon the twigs. By trapping the females on their way up into the trees no eggs can be placed near the foliage and the caterpillars hatching from them can do no damage. The traps are made thus: Take No. 16 or No. 14 wire cloth in strips six inches wide, draw and tack the top edge close to the trunk of the tree over a bandage of cloth which is put on first to make the joint tight. The lower edge should flare out about an inch from the tree all around. This trap will need rather frequent cleaning while the moths are active.

Tent Caterpillars.-Several species of hairy caterpillars called "tent caterpillars," or "web worms," from their spinning covers of 
cobweb-like material, under which they take shelter in large colonies; but one, at least, of the group does not spin a web, though it lives in clusters on the tree. The worms can be killed by cutting off and burning the twig holding the cluster or by burning the colonies in place with a torch on the end of a pole, or by spraying the foliage with lead arsenate or Paris green. The pest can be reduced while pruning by carefully collecting and burning the egg clusters, which encircle the twig.

Red-Humped Caterpillar.-Striped caterpillars, not hairy, but having two rows of black spines along the back, also living in clusters; of reddish color with yellow and white lines; a short distance back of the red head of the caterpillar is a red hump on which are four black spines; black spines are also scattered over the body, but smaller than those on the back. Spray with lead arsenate or Paris green, or cut off and burn colonies.

Caterpillar of Tussock Moth.-A conspicuous caterpillar with four short, brush-like tufts on its back, and two long, black plumes at the front, and one at the rear of the body. This leaf-eater is found on apple, pear, plum and sometimes on other fruit trees, also on the walnut and oak. The caterpillars are peculiarly resistant to arsenical sprays and can not be successfully controlled by these. The larva spins a cocoon sometimes in the fold of a leaf, more commonly in crotches or rough places on the bark, or even on adjacent buildings or fences, and the female, after emerging from the cocoon, deposits her eggs upon the outside of it. The insect is fortunately very freely parasitized in the egg form and prevented from wide injury. It can be controlled by destroying the egg masses during pruning, as they are white and very conspicuous.

Pear and Cherry Slug.-A small, slimy, dark-colored worm, with the fore part of the body notably larger than the rear part, eating the upper surface of the leaves but not usually making holes through them. The insect can be checked by throwing fine road dust or airslacked lime over the tree, which cakes upon the slime of the worm and destroys it. On a large scale an arsenate of lead or Paris green spray is best.

Saw-Fly Worm.-There are several larvae of saw-flies which do much injury to pear trees, currants, etc., by eating the whole leaf substance except the larger ribs. The worms are small, not slimy like the pear slug, the one infesting the pear being about half an inch when fully grown. Its general appearance and work are shown by the engravings. The most available remedy is an arsenate of lead or Paris green spray.

Large Caterpillars on Grape-vines-The grape-vine is often seriously injured by the attacks of very large leaf-eating worms two inches and upwards in length, sometimes with a large horn, or spine, sometimes without. They are larvæ of several species of Sphinx moths or hawk-moths. The worms when new hatched can be killed by an arsenical spray or by hand-picking. The numbers of worms can be reduced by killing the large moths which are abundant at 
nightfall on beds of verbenas, or other garden flowers. These worms are related to the other large caterpillars which feed on tobacco, tomatoes, etc.

Leaf Eating Beetles.-There are many beetles, large and small, which infest grape leaves. They can all be reduced by the use of arsenate of lead or Paris green, or those which drop to the ground when disturbed may be collected in large numbers on sheets spread below.

The most notable of these because of its evil work in the central part of the State, and because the grub destroys the roots of the vine causing it to be called the "grape root worm," and the beetle riddles the leaves and young fruit. It is Adoxus vitis and a special study of it has been made by Professor H. J. Quayle, of the University Experiment Station, the results of which are published in Bulletin 195 of the station and remedies suggested. The beetle is about one-fifth of an inch in length and is black or brown.

\section{INSECTS UPON BARK OR SURFACE OF LEAVES OF FRUIT}

Leaf Lice.-Leaves of fruit trees, especially the apple and plum, are sometimes almost covered with lice or aphides of different colors, from light green to black, some individuals having wings and some wingless. Available remedies for all these leaf lice are the kerosene emulsions and other mixtures which will be given later as summer

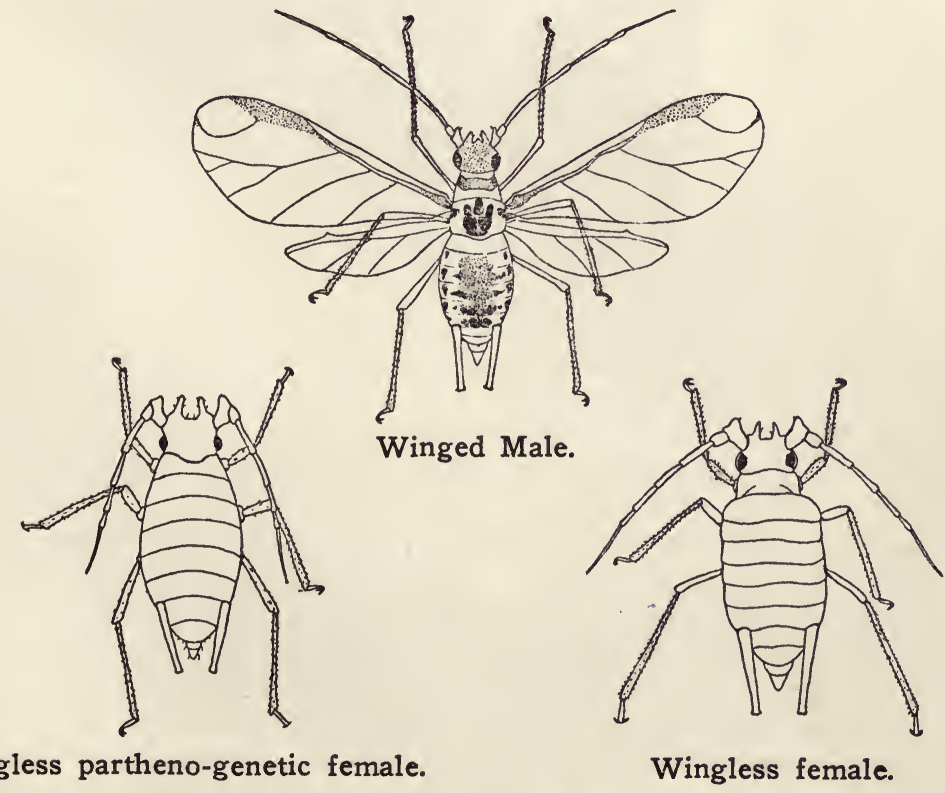

Forms of Aphids or Plant Lice. 
washes for scale insects, with a spray nozzle which sends spray upwards, so as to reach the under sides of the leaves. Very often these pests are apparently cleared out by lady-birds and other insects which devour them. The engraving shows the general form of the aphis tribe.

Thrips.-Very minute insects infesting buds, leaves and blossoms of pears, prunes, cherries, peaches and many other trees and plants. The attack on leaves and buds causes them to wither and fall off. Indeed complete defoliation may follow their attack. When thrips infest fruit blossoms the essential parts are eaten off by the insects and the attacked blossom sets no fruit. Much damage is done by the insect and thorough investigation has been pursued by several investigators of the Bureau of Entomology of the U. S. Department of Agriculture, and by the California Experiment Station. Early results indicate that the insect is capable of control by spring spraying with several insecticides. A United States Department of Agriculture bulletin and a bulletin by Earl Morris, County Commissioner of Santa Clara, by the Agricultural Experiment Station at Berkeley, both deal with this problem. The tobacco emulsion of the U. S. Department of Agriculture is considered the standard spray. It is as follows: Water, 12 gallons; whale oil soap, $30 \mathrm{lbs}$; distillate (32 to 34 degrees Baume), 20 lbs. For spraying, use one part to twenty parts of water and then add to each 200 gallons of the spray, one pint of tobacco extract containing 40 per cent nicotine or about three and one-half gallons of tobacco extract containing 23/4 per cent of nicotine.

Spraying can be done with safety to opening buds, but should not be sprayed on trees in full bloom, and its use can be resumed immediately after the blossoms have fallen, and later on the foliage for adults and larvæ. The first application should properly be made when the thrips are coming from the ground in maximum numbers and before the cluster buds are too far advanced. This period in the San Jose district is early in March, but it of course differs for the several varieties of fruits. An effort should be made to kill all adults in an orchard before March 15, when practically all thrips are out of the ground and when egg-laying on the stems of leaves or bloom begins.

The necessity for spraying will depend upon the number in a blossom. As the blossoms are barely opening the thrips should be shaken out of a few blossoms on white paper. If only two or three are in a blossom, it is probable that spraying may be omitted. If they are more numerous, it is quite certain that spraying will be required.

Vine Hoppers.-Very minute, yellowish, jumping insects infesting grape-vines very early in the season, and multiplying rapidly. The vine hopper (often called incorrectly the vine thrips) is the most widely distributed and most uniformly present of all the grape insects occurring in the State. It occurs in injurious numbers, however, chiefly in the Sacramento and San Joaquin Valleys. It is also present in the coast counties, but rarely in sufficient numbers to do much injury. Another large species (Tettigonia atropunctata) occurs in these localities and sometimes does considerable injury in the early part of the 
season. The principal injury caused by this insect is due to the extraction of the plant juices. These are sucked out by means of a sharp beak or proboscis, which is inserted into the plant tissues.

A method of control practiced by some growers is the use of a hopper cage in the early spring when the young shoots of the vine are about four or five inches long. The hopper cage consists of a framework of laths over which is tacked a double layer of mosquito wire netting or a single 20 -mesh wire screen. The bottom consists of a shallow pan or tray made by turning up about an inch of the edges of a sheet of light galvanized iron. One entire side of the cage is left open, and there is a V-shaped opening in the tray at the bottom which allows the cage to be pushed over the vine. The base of the V-shaped opening in the bottom is padded with leather and the vine is bumped and the hoppers jarred off, at the same time that the cage is being swung into position. The sides of the cage and the tray at the bottom are smeared with crude oil, and the hoppers as they are jarred off are caught in the oil.

If there is a breeze blowing the cage can be operated with the open side facing the wind and practically no hoppers will escape. If, however, the day is calm and warm and the hoppers are particularly active a curtain can readily be dropped over the open side as the cage is pushed on to the vine, and it will prevent any from escaping.

The V-shaped opening which might allow hoppers to drop to the ground in front of the vine can be covered with canvas, as follows: Take two pieces of canvas about the shape of the opening and a little wider. Double this once on itself and between the two layers sew in pieces of three-fourths inch rubber tubing transversely. These are then firmly tacked on the sides of the opening as shown in the engraving. This will allow the cage to be pushed in on the vine and the flexibility of the tubing will bring the canvas immediately into position again. This with the curtain in front, shuts off all possibility of escape.

If catching the over-wintering hoppers is not practicable, the next opportunity for effective work lies in killing the young insects, as they appear from eggs placed in the leaf-tissue by these over-wintering adults, before they get their wings. These young hoppers may be killed by means of a spray applied to the under side of the leaves, and this will be during May or the first part of June, depending upon the season and locality. The exact time may be determined by watching their development. When some of them have reached almost full size it is time to start the spraying. The spray to use is the Standard spray for thrips given on the preceding page. The spray should be thoroughly applied from below so as to strike the under side of the leaves, for the spray will kill only such hoppers as are hit.

False Chinch-Bugs.-Small, grayish-brown insects (about oneeighth of an inch long when fully grown), which injure the vine leaves. They drop to the ground when the vine is disturbed, and may be caught as just described for vine hoppers.

Grasshoppers.- These pests often invade orchard and vineyard, and sometimes kill the plants outright by completely defoliating them. This plague has been successfully met by the use of the arsenic and 
bran remedy, prepared as already described for cut-worms. A tablespoonful is thrown by the side of each vine or tree. If placed on shingles about the vineyard, much of the poison not eaten may be afterward gathered up and saved.*

Red Spider and Other Mites.-Very minute insects, usually discernible only with the aid of a magnifier, sometimes destroy the leaves, causing them to lose their color and health by their inroads upon the leaf surface. The red spider and yellow mite are conspicuous examples; they infest nearly all orchard trees, especially the almond, prune, and plum. The eggs of the red spider are ruby-red globules, as seen with the magnifier, and are deposited in vast numbers upon the bark of the tree, and leave a red color upon the finger if it is rubbed

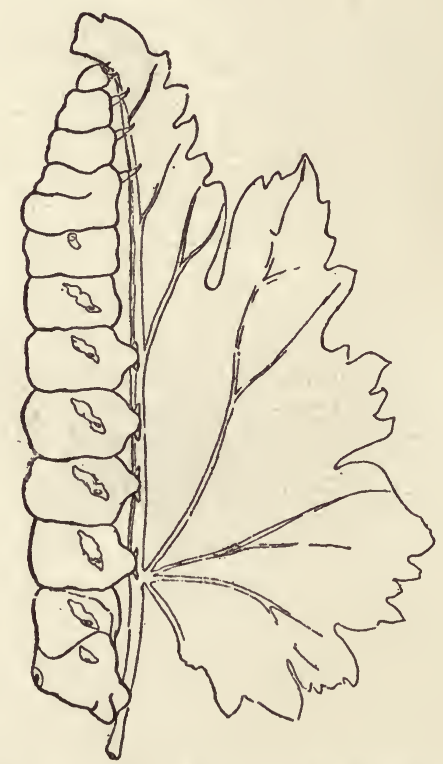

Hawk Moth larva. (Philampeles achemon Drury.)

over them. The eggs are very hard to kill, and treatment is most effective when applied in the spring and summer after the mites are hatched out. The popular remedy is a thorough dusting of the trees with sulphur. On a large scale the sulphur is applied in a cloud by means of a modification of the broad-cast barley sower or with the sulphur machines specially made for this purpose. On a small scale it may be applied with a bellows as for grape-vines, or shaken from a cheese-cloth bag at the end of a pole. Sulphur sprays have been found most effective in controlling the red spider. The ingredients of the sulphur sprays are prepared as follows:

*For the protection of nurseries, orchards, and vineyards it is often necessary to resort to various devices for excluding the grasshopper, or for destroying them upon adjoining fields. Publications describing such devices are Bulletins 142, 170 and 192, University Experiment Station, Berkeley. 


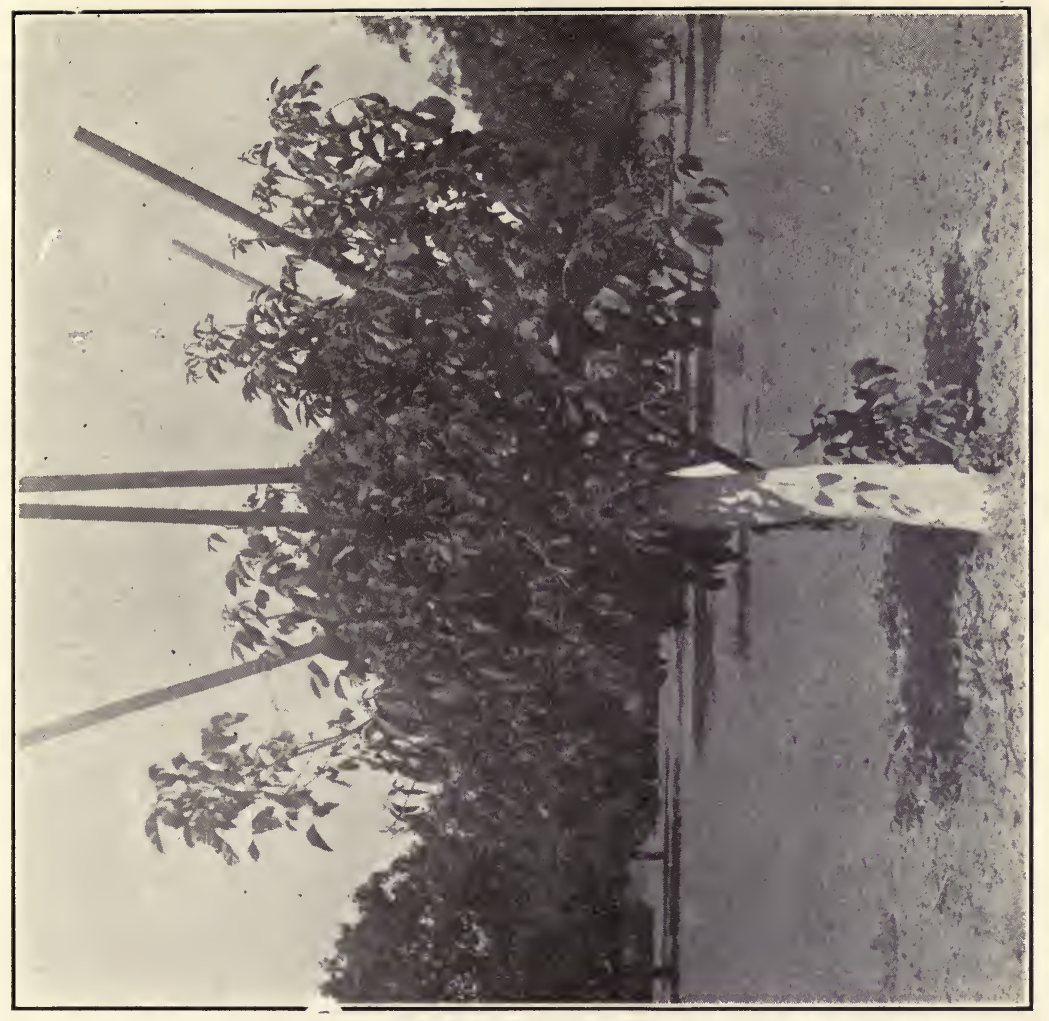

ำ

ช

8

$\mathscr{2}$

ए.

bo

들

훙

응

范

岁



疍

茨

苟

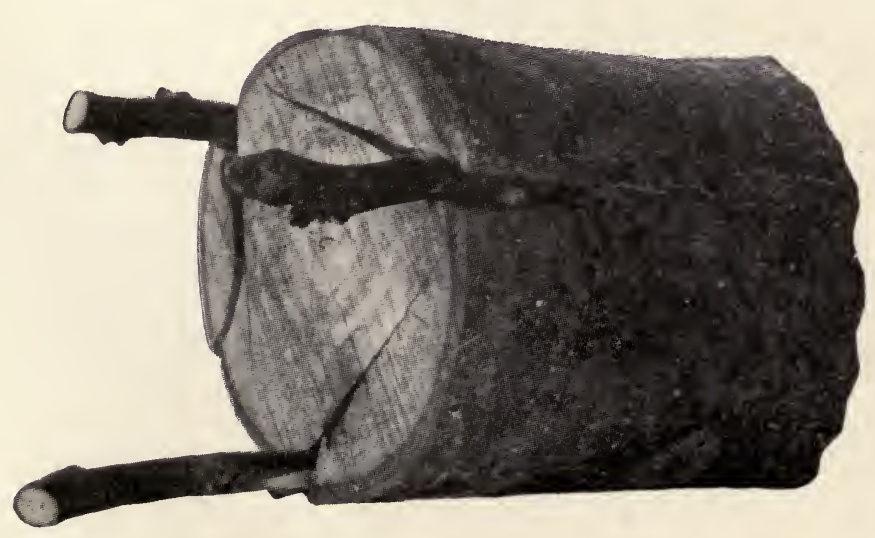

过 

Sulfid of Potash Stock Solution.-Granulated, powdered concentrated lye, 15 pounds; sulphur, 18 pounds; water to make 20 gallons. Stir the sulphur and lye together in a vessel which will allow plenty of room for boiling. When well mixed, add about one pint of water, placing it in a slight hollow in the mixture, and stir in slowly. The mixture will soon begin to melt and boil, forming a red fluid; stir until the boiling ceases, and then add water to make 20 gallons. This stock solution will keep for awhile, or indefinitely when protected from the air.

Preparation of the Spray Mixture with Sulfid of Potash.-Place 10 to 15 pounds of sublimed sulphur, or 14 to 20 pounds of ground sulphur in a spray tank with 4 gallons of flour paste and 1 to 2 gallons of the sulfid of potash stock solution; add water to make 100 gallons. For summer or spring spraying after the danger of rains is over, the minimum amount of sulphur is sufficient.

Flour-paste Spray for Red Spider.-Mr. W. B. Parker of the Bureau of Entomology of the U. S. Department of Agriculture has demonstrated the efficacy of a paste spray to be used during the summer while the insects are active:

Take 8 pounds of cheap flour and stir into a thin batter by adding a little cold water at a time until 8 gallons of water is used. Mash out all lumps.

Cook until paste is formed, stirring constantly to prevent caking or burning. Cooking slowly until the paste just begins to boil will usually be about right. If the paste is not sufficiently cooked, the resulting spray will not be effective, and if overcooked the paste will harden when thoroughly cool, and will not mix with water very readily.

Add cold water to the properly cooked paste, to make 100 gallons in the spray tank. Keep constantly stirred while spraying. Apply thoroughly to both the upper and under surfaces of the leaves. Use a nozzle making a coarse spray under $150 \mathrm{lbs}$. pressure, as the driving force is necessary to penetrate the webs and reach the spider. A fine mist spray would not be effective. This is a contact insecticide and the liquid must actually touch the spider. Watch carefully, and if newly hatched spiders are appearing, repeat the spraying in about seven days. Commercial paste, ready for diluting, can be purchased if desired.

Phylloxera.-This pest of the grape-vine is closely allied to the aphides, and lives both upon the root and leaf, though in this State the root type prevails and the leaf form is seldom seen. No remedy has yet been found effectual, but escape is had by using roots resisting the insects, as described in Chapter XXVI. The insects are recognized, by the aid of a magnifier, as minute yellow lice, chiefly on the rootlets. Full account of the insect is given in Bulletin 192, University of California Experiment Station.

The Woolly Aphis.-A louse of dark red color, occurring in groups, covered with a woolly substance which exudes from the bodies of the insects. The woolly aphis is an almost universal pest of the apple, though as shown by experience, some varieties are practically exempt from it. As the pest lives both upon root and top, its annihilation is impossible, but it may be reduced so that the fruitfulness and vigor of the tree are not impaired. The use of wood ashes around the tree close to the trunk has been beneficial. Removing the earth above the main roots, in a circle two to four feet in diameter and soaking the ground with kerosene emulsion, using from three to 


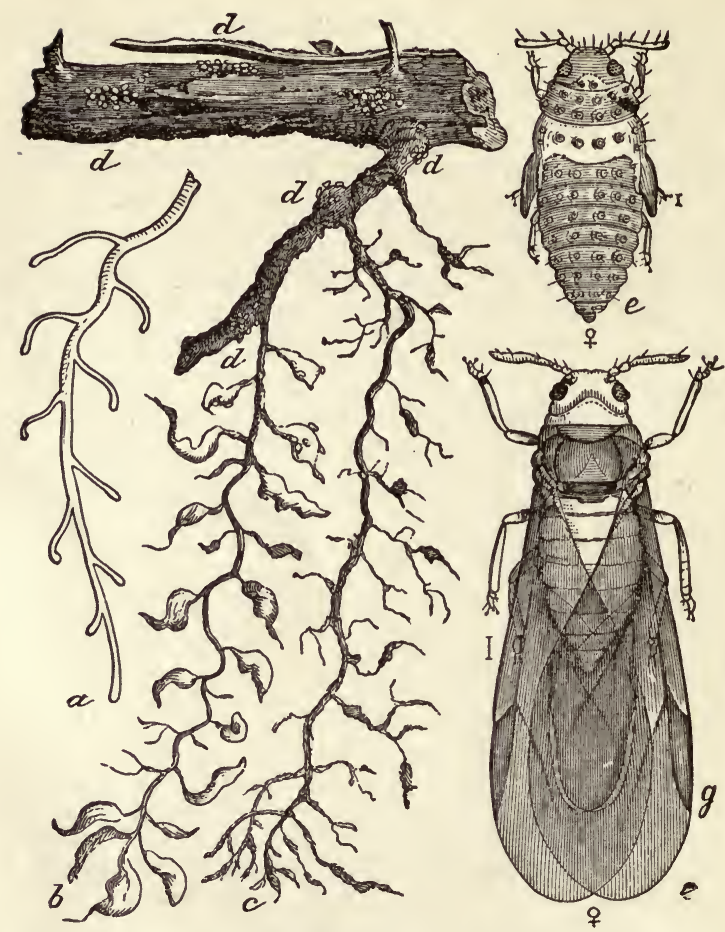

Root injury by Phylloxera and forms of insect.

A healthy root; b, root on which the lice are working, representing the knots and swell. ings caused by their punctures; $c$, root deserted by lice and beginning to decay; $d, d$, d, show how the lice appear on the larger roots; e, the nymph; g, winged female. After Riley.

five gallons to the tree, has been found very effective in killing the root form. The insect on the branches and twigs can be reduced by spraying with the summer washes soon to be given for scale insects. Ladybirds often clear away the woolly aphis, after reproduction has fallen below the normal, from the tree above ground. Some attention is being given to trial of resistant roots and it is likely that such roots will be generally used here as in Australia. How such trees are grown is described on page 197.

Scale Insects.-This is a large group of pests which occasion greater loss and trouble to our fruit growers than all other pests combined. There are many species, and no orchard tree is exempt from the attacks of one or more of them, though some trees are apparently more popular with the pests than others. The fruit grower should study their life history and classification as laid down in the works on entomology. It will only be possible in this connection to introduce a few engravings, by which some of the most prominent pests can be recognized, and to give some of the remedies which are now being most successfully employed against them. 
San Jose Scale (Aspidiotus perniciosus).-This was formerly one of the worst and most widespread of the species of scales preying on deciduous fruit trees in California, but at present, owing to the energetic war that fruit growers have had to wage against it, has become of minor importance, and, in fact, has practically disappeared from some regions where it was formerly most injurious. The work of this species is generally readily distinguished from other species of scale by the red blotches which are formed wherever it stings any part of the tree-either branch, leaf or fruit. These red blotches are more pronounced in some varieties than in others. When the scales are present in large numbers, it causes a complete discoloration of the bark clear to the sap-wood. This scale has its preference among the deciduous fruits. The apricot and certain varieties of cherries and plums are but little affected.

The Greedy Scale (Aspidiotus rapax.).-This species affects many kinds of trees, deciduous as well as evergreens. Scale, about onesixteenth of an inch in length; form, ovoid; color, drab ; female, bright yellow. This insect is found in many places along the coast. It is distinguishable easily from the Aspidiotus periciosus by its whitish yellow color, contrasting with the dark color of the latter. Generally this scale has only one brood in the season, and, as compared with the San Jose scale, it is of little danger, owing to its slow-breeding propensities.

Oyster Shell Scale of Apple (Lepidosaphes ulmi).-This scale affects the apple chiefly, although sometimes the pear also. Owing to the thickness of the armor, it is one of the most difficult of the scales to exterminate. It is easily recognized by its long curved form.

Rose and Berry Scale (Aulacaspis rosae).-This scale has such striking forms that it can be readily recognized. The round white scale is that of the female, the elongated one with ridges is the male. The rose scale infests, besides roses, various fruit bushes, especially blackberries and raspberries. Remedy: For raspberries and blackberries the cutting down of the canes to the ground should be adopted, and the stumps sprayed or washed with kerosene emulsion, recommended under the head of general remedies for scale insects.

Oleander Scale (Aspidiotus hederac).-This scale is small, flat, yellowish-white. It affects a great many trees, especially evergreens. Lemon trees become badly affected, and the fruit is sometimes completely covered. The olive is also subject, and the fruit of the olive when infested does not mature well, and wherever a scale is found, a green blotch makes its appearance.

Red Scale of Orange and Lemon.-(Chrysomphales aurantii).This scale affects citrus trees in both the coast and interior regions. The scale fully grown is one-twelfth of an inch or a little more in diameter, center yellow, margin light brown. The appearance of trees infested with this pest is very striking, very much resembling those diseased from other causes, such as bad drainage, the leaf presenting a mottled appearance, a light blotch around the scale contrast- 
ing with the natural green of the leaf. The branches are but little troubled, but the fruit, like the leaf, becomes completely covered with the insects. An orange tree infested with this scale gradually becomes sickly and languishes.

Other Citrus Tree Scales.-Two scales more recently brought into this State from Florida are the "purple scale," Lepidosaphes beskii, and the "long scale," Lepidosaphes gloverii. The red and purple scales of citrus trees are only treated successfully by fumigation with hydrocyanic acid gas. This treatment is an elaborate one, requiring special appliances which are fully illustrated and described in publications by the Agricultural Experiment Station at Berkeley:

The Black Scale (Saissetia oleae).-This scale is almost a universal pest, especially in regions adjacent to the coast, though it has recently demonstrated its ability to endure interior valley conditions. It affects citrus fruit trees and some deciduous trees as well, and a fungus growing on its exudation causes the black smut, which renders tree and fruit unsightly; but this smut accompanies other scale insects as well as this one. It is especially troublesome on the olive, and will quickly spread to ornamental plants and vines in the garden. It is a very difficult scale to subdue. On citrus trees the fumigation method is the only practical recourse. On deciduous fruits it requires both winter and summer spraying to hold it in check. It spite of the fact that immense numbers are killed by parasites, and perhaps by fungi as well, it is still a grievous pest, and should be fought unceasingly.

Soft Orange Scale (Coccus Hesperidum).-This scale is a pest of citrus trees the world over. The scale is ovoid, a little wider at one end than the other; length, from one-twelfth to one-seventh of an inch; color, dark brown on convex part, and a lighter brown surrounding margin; it has two indentations on each side, and one on posterior end. This scale prefers to collect on the under sides of the leaves along the midrib, the upper sides being covered with smut. It fortunately is usually held in check by natural agencies.

Brown Apricot Scale.-The apricot tree, though defying the most ruinous scales of some other trees, is beset by certain scales. The black scale is one and the brown apricot scale another. The brown apricot scale (Eulecanium Armeniacum) is boat-shaped; when reaching maturity, wrinkled; the color is a shiny brown, darker in the center, lighter at the edges. A full-sized scale has a length of a quarter of an inch, and a width of one-eighth of an inch. This scale attacks nearly all kinds of deciduous fruits, but especially the prune and apricot. It is a very hardy scale, and the remarks about the black scale apply to it also.

Other Lecaniums.-There are several other scales on fruit trees; The filbert scale (hemisphericum), which is common in greenhouses and occurs to limited extent on citrus trees; the frosted scale (pruinosum), very large oval and convex, covered with dense, whitish bloom, occurs on deciduous fruit trees. 
Cottony Cushion Scale or Fluted Scale (Icerya purchasi).-This promised at one time to be the most grievous of all scales in its rapid increase and wide range of food plants, but it was speedily reduced by an Australian ladybird, Novius (Vedalia) cardinalis, introduced by Albert Koebele, with such success that specimens were for a number of years rarely seen, but have recently become abundant in some localities.

Mealy Bugs.-Closely allied to the scales are the mealy bugs (species of dactylopius), soft and of a pale pink color, generally covered with a whitish mealy powder, hence the name. The common species is found in nearly every greenhouse in the world, and in California climate lives in the open air on many kinds of plants, and has at various times proved quite troublesome. Unless checked by natural enemies, the mealy bugs multiply very rapidly, and mass themselves in the corners of the leaves. The plants turn black from the fungus growth growing on the honeydew, and the bush presents the same appearance as a scale-infested plant. With the aid of a magnifier the appearance of the mealy bugs, as different from scales can be readily recognized.

Remedies for Scale Insects.-Though most of the scale insects are attacked by parasitic and predaceous insects, as already stated, these natural agencies have generally not proved rapid enough to cope with the increase of the scales, and insecticides have to be employed to save the fruit and trees. There is a vast number of these washes, many of which will do good work if thoroughly applied, which is usually the secret of success. A few which have proved of special value will be given at the close of this chapter.

\section{INSECTS BORING IN TWIG, STEM, OR ROOT}

Peach Twig-Borer or Peach Worm.-This larva is probably the most serious insect pest that the California peach grower has had to contend with. The creature hibernates as a young larva in burrows in the crotches of the tree.

As soon as the tree begins to grow in the spring the larva becomes active, eats its way out of its winter home and bores into the new growing twigs causing them to wither and die. Later generations of the worm attack the fruit and from the two forms of attack much loss may accrue to the orchardist.

Destruction of the larvæ in their winter burrows overcomes all damage from these pests. This can be accomplished by spraying with the lime-sulphur compound in the late winter or very early spring when the buds are expanding.

The Common Borer.-An insect which has done vast injury in this State is the "flat-headed apple borer" (chrysobothris femorata). It affects chiefly apples, peaches and plums, etc., which have been injured by sunburn. It is a pale-colored grub with a brown head, the forepart of the body being greatly flattened. The matured beetle 
is greenish black or bronze colored, copper colored on the under side. If any tree receives any damage to the bark, either by sunburn or other causes, the borer is sure to find it, and it works itself into the tree, its castings being the only guide to its presence. The best remedy is prevention by protection from sunburn, as described in Chapter XI. Whenever a borer is removed, the debris and dead wood should be entirely cleaned out and the smooth surface left, taking care to preserve the bark as much as possible. Then the wound should be smeared over with grafting wax, and a rag tied about it. In this manner young trees have been saved, but if seriously attacked, it is better to put in a sound tree and protect it.

Sun-Scald Borer.-Another borer which delights in sunburned trees is a minute beetle, making a burrow hardly larger than a pinhole. It is known as the sun-scald beetle (Xyloborus xylographus). The remedy, as in the former case, is to prevent injury to the bark, for this precedes the attack of the beetle.

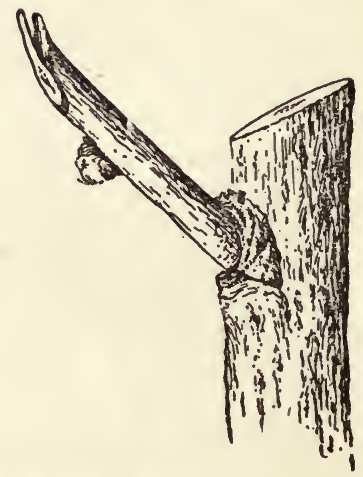

Peach twig showing winter burrow, natural size.

The Olive Twig-Borer.-A reddish brown beetle boring into twigs of olive and other orchard trees, and grape canes, at the axils of the leaves. It is Polycaon confertus, and it breeds in decaying logs and stumps and old grape-vines, apparently visiting the fruit trees merely to gratify its appetite. Its work is not fatal to the tree, but unless proper pruning and attention be afterwards given, it may spoil the shape of the young tree. Remove the affected branches below the burrows of the beetle, or if it would be difficult to replace a branch, see that the beetle is destroyed and the entrance to the hole stopped up - this to prevent decay and a weak branch following. Spraying with ill-smelling solutions may prevent their attack, but the insect has not been sufficiently abundant to invite serious effort thus far.

Peach Crown-Borer.-A grub boring into peach trees just below the ground surface, its presence being shown by copious gumming. The insect, which has become quite troublesome in Santa Clara County, resembles the Eastern crown-borer of the peach, but is a distinct species (Sanninoidea opalescens). The best methods of suppressing this insect 
are three. A preventive treatment consists in coating the base of the tree a few inches below the surface and a foot above with a whitewash, with a pint of coal tar to each five pounds of quicklime, put in while the lime is slacking. More recently the application of asphaltum has been demonstrated by Earl Morris, horticultural commissioner of Santa Clara County, to be very efficient. Hard asphaltum (grades " $\mathrm{C}$ " and

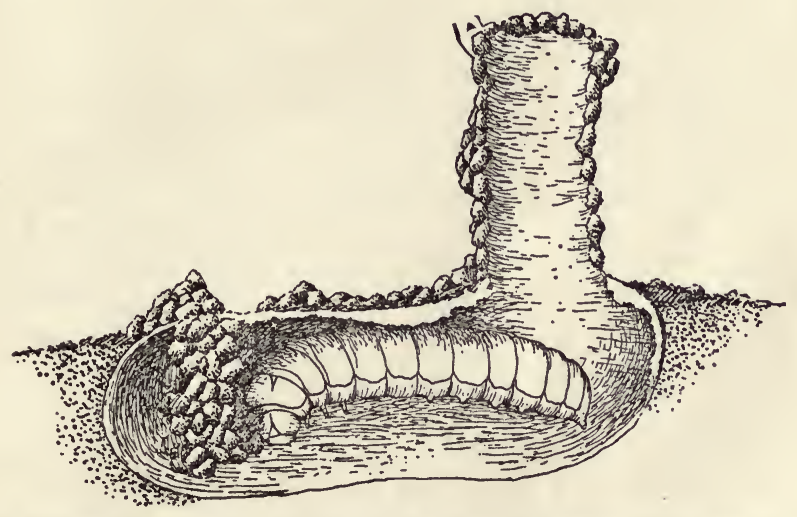

Peach Worm Burrow laid open, the worm beginning spring workgreatly enlarged.

"D") was applied early in the spring to badly infested trees from which the borers had not been dug. A thick, heavy coating prevents both the issuance and the entrance of about 95 to 98 per cent of the insects, the degree of efficiency depending upon the thoroughness of the application. Asphaltum does not penetrate, crack, or deteriorate, or bind the tree, since it yields to the slightest pressure.

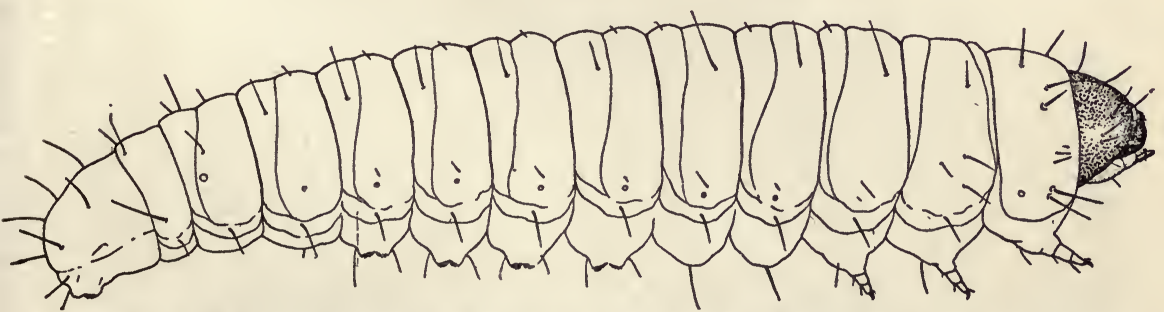

A nearly full-grown larva of the Peach-tree Borer, Magnified four diameters.

The material is applied warm with a brush from five inches below to five inches above the ground. It is easier to apply two or more coatings than to try to put on more at one time than will adhere firmly. The first coating will harden very quickly, and the second can be applied without loss of time. Two coatings are generally sufficient unless the bark is very rough. But in any case, a thick uniform covering is absolutely necessary for the best results. 
The method in most general use is known as the "worming process." This consists essentially in carefully cutting out and killing the individual borers. Special tools have been devised for this work. A full description of these processes can be found in Bulletin 143, University of California, College of Agriculture.

Strawberry Root-Borer.-The larva of another clear-winged moth (Aegeria impropria), boring into the root of the strawberry plants, found in various portions of the State, and doing considerable damage, forcing the growers to resort to replanting much earlier than otherwise would be necessary. Flooding the vines has a great tendency to kill out the worms, and if the water was retained, say four or five days during the winter, all over the plants, doubtless all the larvae would be killed.

Currant and Gooseberry Borer.-A white worm eating out the central pith of currant and gooseberry plants-the larva of another clear-winged moth (Aegeria tipuliformis). Spraying with whale-oil soap after the crop is gathered, pruning out and burning in the fall of all old wood which can be spared, will reduce the evil.

The Raspberry Cane Borer.-This intruder is one of the family Tenthredinideae, or saw flies. The very small maggot or larva will be found by peeling the bark carefully. This larva can not crawl, therefore if the tips be cut and the worm is taken out he will die. A better way is to cut all tips, as fast as they show signs of wilting, and burn them. Thorough work in topping is alsolutely necessary, so as to be sure that none of the worms remain to produce flies. Topping causes the canes to throw out laterals, thus producing more fruit. The canes are not affected after July.

Another cane-borer, occurring in the foothills, has been identified by E. O. Essig, as the larva of a horn-tail. It is fully described in Bulletin 12 of the California State Board of Horticulture. Its treatment is like that just described.

\section{INSECTS DEVOURING THE PULP OF FRUITS}

The Apple Worm.-The codlin moth (Carpocapsa pomonella) is one of the great pests of the State. It preys chiefly upon the apple and pear, but the quince and other large fruits are sometimes invaded by it. The first moths appear at some time after the blossoming of the apple, and deposit their eggs on the young fruit, or on adjacent leaves. The young worm hatches in from seven to ten days, generally seeks the eye or calyx, and eats its way into the fruit, and in twenty days its full growth is attained, and it goes out through the side of the apple, and, by means of its spinnaret, reaches the ground or some large branch. If landed on the ground, it usually seeks the trunk, which it ascends and soon finds a hiding-place under the loose bark, where it spins its cocoon, and in eight or ten days comes forth a moth, ready to lay eggs anew. The egg is laid all over the fruit and especially at a point where two fruits touch. Usually we have in this State two broods, at least, but sometimes three, and, naturally, if unchecked, the 


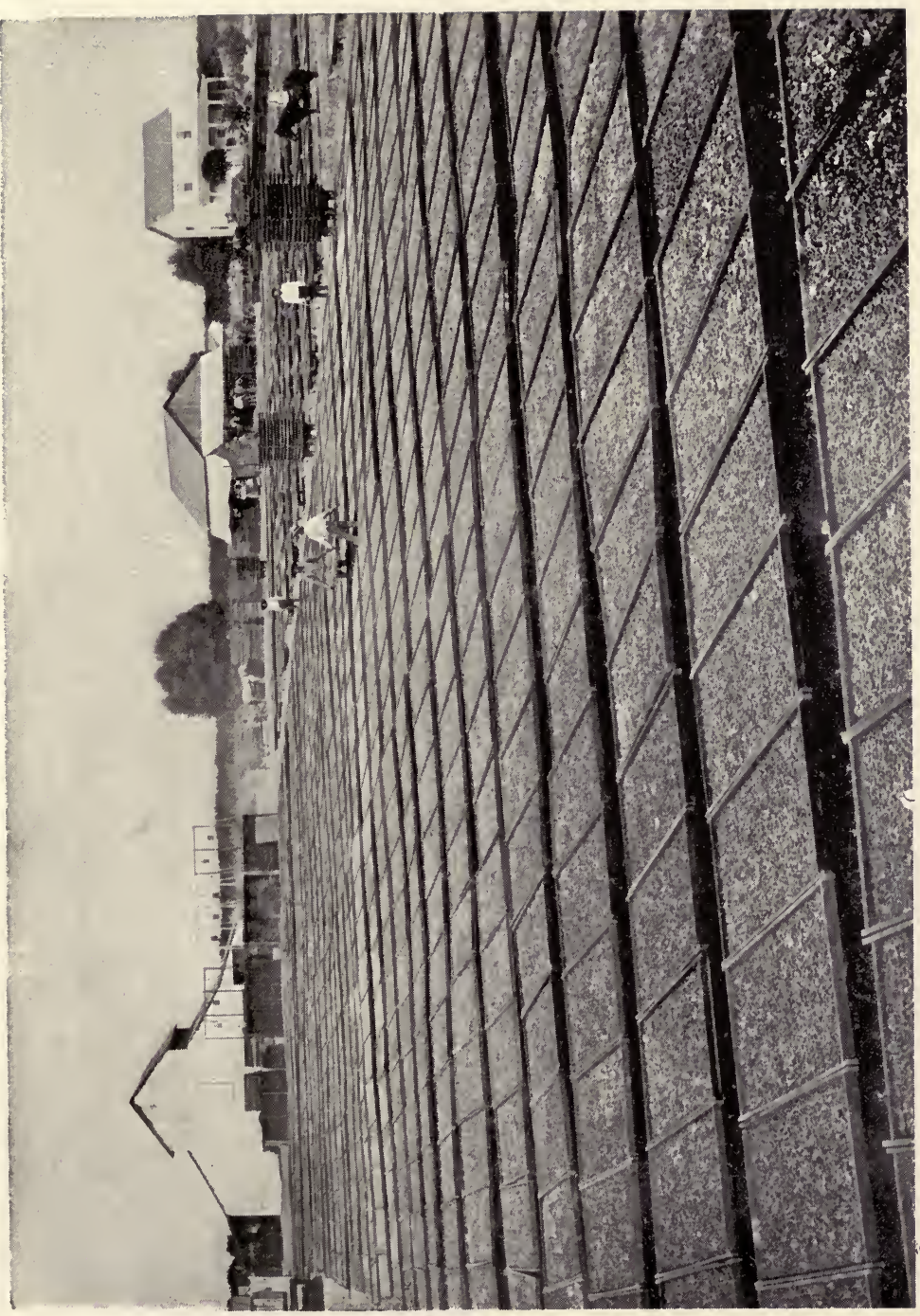

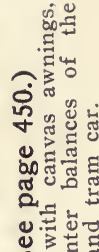

थ त्र

एक



큭.룬

है

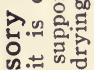

oै

U⿺辶

作

击

药。

눙

훈.

은

胥

या कर

넝

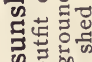

ข

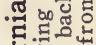

년현돈

ชี

1 । यें

$>$.

政

$x$.

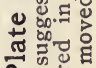

ค1 के 

increase from the first to the last is enormous. The worms escaping from the fruit in the fall hibernate as larvae under the loose bark of the tree, or in store-houses, or in any available dry place.

On the basis of long experience in the Watsonville district $\mathrm{Mr}$. W. H. Volck, county entomologist, gives the following outline of operations against the apple worm:

There are two broods in a season. The first develops from worms that have wintered over in protected places both above and in the ground. The moths of the first generation begin emerging in April, but very few eggs are laid before the middle of May. In this locality, egg-laying is then continuous until the last of June.

The full-grown worms of the first generation after emerging from the apples seek some hiding place where they may rest for a few days, transforming into pupa and then into full-grown moths.

The moths of the second generation begin laying eggs early in August and may continue to do so as late as October or November. It is the worms of the second generation that do the greatest amount of damage.

The codlin moth lays its eggs on the surface of the leaves and fruit. The eggs are about the size of a small pin-head, circular and flat. The color varies from white to pink and red. These eggs are easily seen when the observer knows what to look for.

The young worms hatch from the eggs about ten days after laying, and then begin crawling about in search of fruit to burrow into. This short period between the hatching of the egg and the entrance into the apple is the only time that spraying operations can prove effective. It is clearly impossible to accomplish anything by the use of sprays that will only kill by contact, for such sprays would have to be applied nearly every day. The spraying operation must then leave a deposit of some substance on the fruit that will kill the worms when they attempt to burrow through the rind. The only substance now known that answers these requirements are the compounds of arsenic.

Arsenic is, however, poisonous to plants and must be applied with caution. The arsenic compound used must be entirely insoluble in water, and not subject to weathering, in order that it shall be perfectly safe. The wet fogs of the Pajaro valley summers give arsenical spray compounds a very severe test, and it has been found that only the best quality of arsenate of lead is able to stand it. Arsenate of lead may do very severe burning, however, if it is not of the proper quality.

It is a well-known fact that a large percentage of the worms of the first generation enter at the blossom ends of the apples. This part is protected or more or less closed over by the leaves of the calyx.

The calyx cups close very shortly after the petals fall, and so it is advised by the best authorities to spray as soon after the falling of the blossoms as possible in order to leave a deposit of poison there. In the Pajaro valley and adjoining sections, weather conditions often prevent spraying at this time, but where possible to apply, the calyx cup spraying is advised.

Good results have been obtained by spraying in the early part of May, which is considerably after the calyx cups have closed. Spraying at this time gives much the same effeet as the calyx cup application, especially where it is applied with proper thoroughness. This early spraying is applied before any worms have appeared and is intended merely to have the poison in readiness for the insects when they do come.

The first codlin moth spraying should contain Bordeaux Mixture as a precaution against the scab:

Bordeaux Mixture-Copper sulphate 3 pounds, lime 4 pounds, water 50 gallons.

Arsenate of Lead-Use $2 \frac{1}{2}$ to 3 pounds of arsenate of lead to each 50 gallons of Bordeaux.

The Bordeaux Mixture should be prepared first, and enough space left in the tank to allow for the addition of the arsenate of lead and the water required to mix it. 
The second spraying should be applied from the last of May to the first ten days in June, and is intended to augment the effect of the first spraying by further covering the rapidly expanding fruit.

For this spraying use 2 pounds of arsenate of lead to 50 gallons of water. No Bordeaux should be used with this spraying, for the previous sprayings containing Bordeaux will have controlled the scab, and the further use of the fungicide is likely to russet the apples.

Very few eggs are laid during July, so if the second spraying has been applied about the first of June it should suffice to control the first generation attack. The second generation worms begin to appear about the first of August, so a spraying is due at this time.

For the third spraying, use 2 pounds of arsenate of lead to 50 gallons of water.

If the apples are to remain on the trees through September they should receive another spraying about the first of this month.

For the fourth spraying, if the other three have been applied according to directions, use $1 \mathrm{t} / 2$ pounds of arsenate of lead to 50 gallons of water.

The schedule of sprayings above laid out should control the codlin moth under average conditions, and even where the insect is extremely abundant should yield 95 per cent clean fruit.

The codlin moth is much affected by weather conditions, and if there is a large amount of cold fog during the summer the damage will be much reduced.

The Peach Worm.-As already stated, the larva of the peach moth, which early in the spring bores into the twigs, is sometimes found later in the season in the flesh of the peach. Hence the importance of saving the fruit by proper treatment of the hibernating worms which emerge as the blossom buds are opening.

The Diabrotica.-A light green beetle with twelve spots on his back (Diabrotica soror), is sometimes very injurious to early fruit, by eating into it when ripe. The insect also eats leaves and blossoms. As the insect attacks the fruit just as. it is ready to pick, it is impossible to apply any disagreeable or poisonous spray. Sometimes the insects are driven away by dense smoke from fires in and around the orchard.

The Dried Fruit Worm.-Dried fruit is often seriously injured after packing, by a small worm, larva of a moth not yet determined. The eggs are deposited on the fruit either while drying or while in the packing-house, or through the cloth of the sacks, or seams of the package. The eggs may be killed on the fruit before packing, by dipping in boiling water, or by heating in an oven and after that preventing the access of the moth. Infested fruit can also be treated by bisulphide vapor, the method being the same as described for nursery stock below.

\section{ANTS AND YELLOW JACKETS}

These insects are often of serious trouble during fruit drying. Ants are most effectually disposed of by slightly opening their holes in the ground by thrusting down a crowbar and pouring in a couple of ounces of carbon bisulphide and closing again with earth. Yellow jackets also nest in the ground in old squirrel or gopher holes, and they too can be suffocated with carbon bisulphide or by pouring in gasoline or kerosene and firing it. Hornets which nest in trees are troublesome, but are much less numerous than the cave-dwelling species. 
To destroy yellow jackets by trapping and poison is also feasible. W. F. Moyer, of Napa, proceeds in this way:

Make a thin fruit syrup by mashing the boiling ripe fruit, strain it and add a little sugar. Place the soup dishes on the drying ground where the "jackets" are thickest. When the top of the syrup is covered with drowned and drowning "jackets," scoop them out with the hand and crush them with the foot. They won't sting unless you pinch ihem. As the syrup evaporates fill up the dishes with water. If a day or two should elapse when no fruit is cut, be sure the traps are well cared for, as they will swarm around them thicker than ever, especially if the weather is hot. For dishes to place the syrup in, cut kerosene cans so as to make two cans, each about six and one-half inches deep.

Poisoning to carry destruction to the young brood is also practicable. Dr. J. H. Miller, of San Leandro, saved his fruit in this way:

I bought half a dozen beef livers, five pounds of arsenious acid and several pounds of baling wire. Cutting the liver into pieces as large as a man's fist, I put them into a hot solution of arsenious acid, and, bending the wire into a hook at each end, I suspended the pieces from the lower limbs of trees all around my drying-ground. The fruit was soon deserted, and the little insects busily working at the fragrant liver. The insects carried pieces of the liver up to their nests, and besides causing the death of those that had been destroying my fruit, the rext generation of yellow jackets was also destroyed, and so complete was the destruction that there were not enough of the little pests in that neighborhood the following year to require a repetition of the treatment. There is no risk in so using the poison, for the yellow jackets will not return to the fruit, and bees will not go near the meat.

\section{DISINFECTING NURSERY STOCK}

Cuttings, scions, young trees and vines, etc., can be freed from insects by inclosing in a tight box or cask and placing a saucerful of carbon bisulphide on the top of them, covering it with canvas or any tight-fitting cover. The bisulphide vapor will destroy all insect life in forty minutes.

Disinfecting such materials on a larger scale may be done in this way:

Use square canvas sheets, sixteen to twenty feet in diameter, made of the best ducking, double stitched and then painted with boiled linseed oil to make it gas proof. The canvas must be perfectly dry before it is rolled up, or it is liable to be destroyed by spontaneous combustion. To fumigate evergreen stock use one ounce of cyanide of potassium (in lumps, not pulverized), one fluid ounce of commercial sulphuric acid, and two fluid ounces of water to one hundred cubic feet of enclosed space. For deciduous and hardy trees, when dormant, use one-fourth more of each of the above. When the canvas has been placed over the stock to be fumigated, prepare the charge. Take a three or four-gallon glazed earthenware jar, into which pour the necessary quantity of water, then the sulphuric acid, and place it well under the canvas, the edges of which are secured with soil or in some way so as to prevent the gas escaping, with the exception of the edge immediately in front of the jar. The proper amount of cyanide of potassium is then dropped into the jar from a long scoop, and the tent is immediately closed, and remains so for one hour.

\section{INSECTICIDES}

It is hoped that this chapter will convey useful hints in the warfare against insects. Whenever questions arise which are not met thereby, appeal should be made to the University Experiment Station at Berke- 
ley. A condensed statement of useful insecticides is issued by the Station, prepared by Professor H. J. Quayle, as follows :

\section{Lime Sulphur:}

Quicklime, 33 pounds; sulphur, 66 pounds; water, 200 gallons.

Sift sulphur through box with screen bottom into boiling tank with 50 gallons of water. Add the lime and boil 45 minutes to one hour. Stir frequently. Strain through cheese cloth or burlap and dilute to make 200 gallons. If extra lime is desired strain in milk of lime when spray is ready for use.

\section{Commercial Lime Sulphur:}

If of $32^{\circ}$ Baumé dilute 1 to 9 . If $36^{\circ}$ dilute 1 to 11 .

Either of the above for San Jose and other armored scales to be applied during dormant season, preferably in early winter or early spring. For Peach Moth, as the buds are expanding in the spring.

3. Distillate $28^{\circ}$ to $34^{\circ}$ Baumé :

Distillate, 10 to 20 gallons; water, 200 gallons.

For use only with power sprayer with good agitator, which is necessary to make a mechanical mixture of the oil and water.

For the Brown Apricot, Black, and other unarmored scales, and for Woolly Aphis, to be applied during dormant season, preferably in early winter.

\section{Distillate Potash:}

Distillate, 10 gallons; lye or caustic soda, 5 pounds; water, 200 gallons.

Preparation and uses same as under 3. Has the advantage of freeing tree from moss.

\section{Distilllate Emulsion :}

Stock emulsion: Hot water, 12 gallons; fish oil (see 10) or whale-oil soap, 30 pounds; distillate $30^{\circ}$ to $34^{\circ}, 20$ gallons.

Add soap to hot water in spray tank with agitator going. After soap is dissolved add oil slowly, keeping mixture agitated. Pump out through nozzle at 175 pounds pressure in storage tank.

For use take: Stock emulsion, 11 gallons; blackleaf 40, 1 pint; water, 200 gallons.

Place oil emulsion in spray tank, start agitator and add the water. When diluted add the Blackleaf.

For thrips, Black Peach Aphis, and other plant lice.

\section{Kerosene Emulsion :}

Dissolve $1 / 2$ pound soap in 1 gallon hot water; add 1 gallon kerosene.

Mix thoroughly with spray pump by turning nozzle back into mixture.

a. For plant lice and other sucking insects during growing season, dilute with 15 to 25 gallons water.

$b$. For scale insects, Woolly Aphis and other sucking insects during dormant season, dilute with 5 to 10 gallons water.

For use on small scale with hand sprayer. 
6a. Kerosene Emulsion. Kitchen formula:

Dissolve 1 inch cube soap in 1 pint hot water; add 1 pint kerosene. Churn with egg beater.

For growing plants, dilute to 2 or 3 gallons water. For dormant plants, 1 gallon.

7. Miscbible Oils:

Commercial preparations of oil so treated as to mix directly with water. Follow directions on container. Uses same as 3, 4, 5, 6, 8, 9, and 10.

8. Straight Kerosene or Water White Oil:

Water white oil ( $42^{\circ}$ Baumé) or kerosene, 20 gallons; water, 200 gallons.

In use for scale insects of citrus trees. Application by power outfit.

9. Soap Solution :

Soap, 1 pound; water, 5 to 15 gallons.

Whale-oil or fish-oil soap preferable, but for small amounts any yellow laundry soap will answer.

For plant lice and other sucking insects during summer.

$9 a$. Soap Solution. Kitchen formula :

11/4 inch cube soap; 1 gallon warm water.

10. Homemade Soap:

Water, 6 gallons; lye (98\%), 2 pounds; fish oil, 11/2 gallons.

Add lye to water in boiler. When dissolved and water boiling, pour in the fish oil, stirring in mean time, and boil slowly for two hours. This will give about 40 pounds soap.

For use, dilute with 5 to 15 gallons water for each pound.

For same pests as No. 9.

11. Tobacco or Nicotine:

a. Blackleaf 40 (40\% nicotine), 1 pint; water, 200 gallons.

$b$. Tobacco stems, 1 pound; water, 4 gallons.

Steep stems in 1 gallon warm water and dilute to 4 gallons.

For plant lice and other sucking insects during growing season. For Woolly Aphis and Peach Aphis underground. A liberal solution, or the dry dust in early winter, about the base of the tree, the surface soil first being removed.

12. Tobacco Soap:

Blackleaf 40, 1 pound (1-10 gal.) ; cresol soap, 1 gallon-or

Whale-oil soap, 10 pounds; water, 200 gallons.

The cresol soap requires no heating.

Uses same as 11. Penetrating and wetting power better.

13. Lime Sulphur Tobacco:

Commercial lime sulphur $36^{\circ}, 2.1$ gallons; blackleaf 40, 14 fluid ounces; water, 200 gallons.

For Orange Thrips.

14. Carbolic Lime:

Quicklime, 150 pounds; crude carbolic acid, 2 gallons; water, 200 gallons.

For Pear Thrips. 


\section{Sulphur:}

a. Dry. Thoroughly dust over foliage, preferably when moist with dew. Hydrated lime about equal parts with sulphur will increase adhesiveness.

b. Spray. Sulphur, 30 pounds; lime; (as milk of lime), 15 pounds; water, 200 gallons.

c. Spray. Commercial lime sulphur, 4 to 5 gallons; water, 200 gallons.

For Red Spiders and Silver Mite.

\section{Lead Arsenate:}

Lead arsenate, 6 to 12 pounds; water, 200 gallons.

First mix arsenate with 2 or 3 gallons of water.

For Codlin Moth and most defoliating insects.

16a. Lead Arsenate. Kitchen formula:

Lead arsenate, 1 tablespoonful (1 oz.); water, 1 gallon.

\section{Paris GREen :}

Paris green, 11-3 to 2 pounds; water, 200 gallons.

For Codlin Moth and most defoliating insects.

Not to be used along coast or moist situations where injury is likely to result to foliage.

17a. Paris Green. Kitchen formula:

Paris green, 1 teaspoonful ( $1 / 4$ oz.) ; lime, 3 teaspoonfuls; water, 2 gallons.

18. Zinc Arsenite:

Zinc arsenite, 2 to 6 pounds; water, 200 gallons.

A powerful poison for resistant insects, as the Tussock Moth, or for early spraying for Canker Worm and Codling Moth in the dry interior climates.

19a. Porsoned Bart. Kitchen formula:

Bran, 10 pounds; Paris green, 1 pound-or

White arsenic, $1 / 2$ pound; molasses, $1 / 2$ gallon; water, 2 gallons.

Mix paris green with the bran dry. Add the molasses to the water and mix into the bran, making a moist paste.

For Cut Worms and Grasshoppers, distribute a small handful about the base of the vine or tree, or scatter about plants in the garden. May be distributed broadcast for Grasshoppers and Army Worms.

20. Carbon Bisulphide:

For treatment of stored products and underground insects.

Usual dosage, 1 pint to 1,000 cubic feet space.

Place liquid in saucers or shallow vessels above material to be treated. Inflammable; avoid lights.

For underground insects, a tablespoonful in holes 3 or 4 feet apart.

21. Resin Dipping Solution :

Resin, 20 pounds; caustic soda or lye, 8 pounds; fish oil, 3 pints; water, 100 gallons.

Boil resin and caustic soda in 50 gallons of water for 1 hour. Dilute to 100 gallons.

In use for dipping citrus nursery stock for scale insects and Red Spiders.

Kerosene emulsion and lime sulphur solution also used for dipping deciduous nursery stock. 
22. Hydrocyanic Acid Gas :

Potassium cyanide $\ldots \ldots \ldots \ldots \ldots \ldots \ldots \ldots \ldots \ldots, 1$ ounce

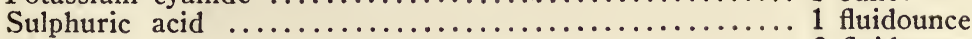

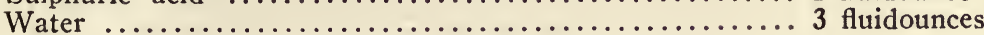
or

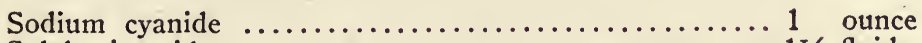

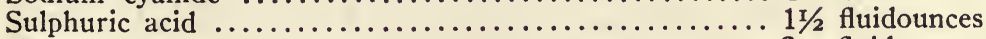

Water ..................................... 2 fluidounces

Place water and acid in earthenware vessel and add cyanide. To be used under tents or tight rooms or boxes.

For fumigating buildings or nursery stock, 1 ounce of cyanide to each 100 cubic feet for 1 hour.

For scale insects on citrus trees, maximum or Purple Scale dosage found by mutiplying distance around by distance over top of tented tree and pointing off two places. Example:

Distance around 40 feet
Distance over

$$
\times \quad 20 \text { feet }=8 \text { ounces cyanide }
$$

For Red or Black Scale, reduce one quarter.

For details of fumigation processes for scale insects on citrus trees, apply for publications to the University Experiment Station and to Horticultural Commissioners in citrus fruit growing counties. 


\section{CHAPTER XL}

\section{DISEASES OF TREES AND VINES}

A few suggestions concerning pathological conditions which arise in trees and vines and prescription of treatment and remedies may be helpful: First, diseases demonstrated to be caused by fungi and bacteria; second, abnormal conditions, of which the causes are not yet clear.

Powdery Mildews.-Fungi which bring upon the leaf surface the appearance of a whitish powder and afterwards cause the leaf to curl and dry without producing marked swelling, perforation or discoloration, can be checked by the use of sulphur. The chief of these is the mildew of the grape, the mildew of the apple, appearing chiefly on the young growth, etc. The way to use sulphur for vine mildew is to freely apply finely ground or sublimed sulphur on the young foliage at the first sign of the trouble, or before it appears in regions where it may always be expected, either by hand or with suitable machinery which is fully discussed in Bulletin 186 of the California Experiment Station. Apple mildew is treated by spraying with iron sulphide added to the poison used for the codlin moth, as described in the preceding chapter.

Leaf-Spotting, Puncturing or Deforming Fungi.-These classes are usually distinguishable by the results they produce. The mildew of the peach produces dense, whitish patches on the leaves and growing fruit; the curl-leaf fungus of the peach produces swellings and contortions of the leaf; the scab of the apple and pear produces first a smoky appearance on the leaf and afterwards causes black scabby patches on the fruit and on the young twigs; the slot-hole fungus of the apricot, plum, cherry, and almond cuts roundish holes in the leaves as though a shotgun had been discharged through the foliage, and then, in the case of the apricot, produces roundish, dark red pustules on the fruit; the brown rot which attacks both twigs and fruit of apricots, prune, blackberry, etc., produced eruptions on plums and peaches; the rust fungi of the under sides of the leaves, first of a yellowish or orange color, changing to dark brown or black, and causing the leaf to fall. These fungi are only slightly, if at all, checked by the dry sulphur treatment, and are best subdued by the use of copper solutions:

The Bordeaux Mixture.-Lime, four pounds; bluestone (sulphate of copper), four pounds; water, forty gallons. Use part of the water to slake the lime and dissolve the bluestone, which should be done in separate vessels. The bluestone should not be put in a metal vessel. If put into a bag and suspended near the surface of the water, it will dissolve more readily, or hot water may be used in making the solution. Both should be cold when mixed, and the resultant mixture will be a beautiful blue wash. If mixed hot, a black compound (copper oxide) is produced, which reduces the value of the wash. After thorough mixing of the solution, water should be added to bring the bulk up to forty gallons. 
This is safe to use on foliage. It may be used much stronger when trees are dormant-as strong as ten pounds of lime and ten pounds of bluestone to forty gallons of water to kill spores of fungi on the bark, but the chief advantage of the stronger mixture is not directly in sporekilling but in the longer resistance to removal by rains. This winter treatment is a successful preventive of curl-leaf on the peach, shot-hole on the apricot, scab on the apple and pear, rust on the prune, etc. In the case of the peach blight, which is an invasion of the young bark by the shot-hole fungus, an autumn spraying is imperative to protect the dormant twigs.* When the fungus survives winter treatment or when it attacks the fruit, as in case of the apricot particularly, or the leaf in the peach, there should follow the weaker Bordeaux in the spring or summer, as early as indications of the diseases may appear. In spraying for apple and pear scab, the addition of five pounds of lead arsenate to each one hundred gallons of the Bordeaux Mixture makes the application answer also for the codlin moth, as described in the preceding chapter.

When it is desirable to use a fungicide on fruit near the picking season, or on ornamental plants, which would be disfigured with the lime wash, the ammonical copper carbonate may be submitted for the Bordeaux Mixture, viz.: Copper carbonate, four ounces; ammonia, forty ounces; water forty gallons.

The usual way of making this wash is to dissolve copper carbonate in ammonia, and then dilute. If the carbonate is not fully dissolved before the water is added, it can not be further dissolved, and not only is the carbonate wasted, but the fluid will not be up to standard strength. It is well, therefore, to give the ammonia ample time to act, say over night, before adding the water.

The lime, salt and sulphur mixture, as already prescribed for scale insects in the preceding chapter, is an active fungicide for winter use. It is sometimes a satisfactory curl-leaf preventive in the interior valleys especially.

Toadstool Destruction.-Trees are often destroyed through invasion by toadstool fungi from the decaying roots or wood with which their roots come in contact. The injury is often not detected until the tree is ruined and it is too late for treatment. If only part is affected, the disease may sometimes be arrested by cutting away the diseased parts and disinfection of the exposed tissue with Bordeaux Mixture. Stone fruits are most susceptible to this trouble, the cherry being least affected. Young apples are sometimes attacked, but mature trees resist it. Pears and figs are also largely resistant.

Moss, Lichens, etc., on the Bark.-It has been clearly shown by investigation at the University Experiment Station that the growth of moss, etc., upon the bark of fruit trees is a decided injury. All trees should be assisted to maintain clean, healthy bark. This is accomplished by the use of the lime, salt and sulphur mixture already prescribed for scale insects. It can also be done by winter spraying with caustic soda or potash, one pound to six gallons of water.

\footnotetext{
*Consult Bulletin 191 of the University Experiment Station on "California Peach Blight."
} 
Blights and Decays. - There are several blights which are traceable to bacteria, parasitic growths which are not discernible as are the fungi, and not usually amenable to spray treatment, because they exist wholly within the tissues of the plant and are not reached by applications. The blights of the pear, the black heart of the apricot, the olive tuberculosis, etc., are instances. Cutting back to healthy wood (with tools dipped after each cut in corrosive sublimate, one part to one thousand parts of water), and burning all removed parts, is the best treatment which can at present be prescribed.

The walnut blight, demonstrated by Newton B. Pierce, of Santa Ana, to be caused by a bacterium, has so far resisted treatment, though there seems to be promise of good results from spraying in February or March, before growth begins, with the lime-sulphur wash. This serves also for the reduction of walnut aphis, which is sometimes very injurious to the tree and the crop.* The disease also affects the leaves and young wood. The recourse seems walnut; generally worst at the blossom end and usually first seen there early in the season; later the spots run together and encompass considerable areas of the surface. As the disease progresses the nut is transformed into a hateful black mass and is utterly destroyed. The disease also affects the leaves and young wood, but does not attack the old wood and the roots as the pear blight does. It is therefore more easily controlled. The recourse seems to be toward resistant varieties, as stated in the chapter on the walnut.

\section{DISEASES NOT TRACEABLE TO PARASITIC GROWTHS}

There are a number of prominent troubles which are not traceable to parasitic invasion of any kind, and yet may be in some cases promoted by bacterial growth invited by preceding conditions.

Sour Sap.- There is a fermentation of the sap, quite noticeable by its odor, which may be found in all parts of the tree, from the root to the topmost twigs; sometimes in one part and not in another. Sour sap in the root is generally due to standing water in the soil, and the remedy is drainage. Trees thus affected make an effort to grow and then the young growth shrivels. Severe cutting back of the top to reduce evaporation until the roots can restore their feeding fibers is the only treatment of the tree, and its success depends upon the extent of the root injury. Sour sap may also be caused in the branches by the occurrence of frost after the sap flow has actually started. Cutting back the diseased parts, as soon as discovered, to sound wood, is the proper treatment.

Die-Back.-Dying back of twigs or branches may occur without parasitic invasion through root-weakness or partial failure. It may be due to standing water or to lack of soil moisture, either of which will destroy the root-hairs and bring the tree into distress. The treatment is cutting back to sound wood and correcting the soil conditions, either by irrigation or drainage, as one or the other may be needed to advance vigorous growth in the tree.

${ }^{*}$ See Pacific Rural Press, March 7, 1914. 
Gummosis.-This is a convenient term to designate the gumming which is seen on many kinds of trees. As has been said of die-back, gumming may result from excess of water or of drouth in the soil. Gumming is, therefore, not always to be considered in itself a disease, but rather an indication of conditions unfavorable to the thrifty growth of the tree. It has been usually found by investigation that trees in perfect condition of health, with the moisture just enough and not excessive, are not troubled with gumming; but there are cases in which this statement does not wholly apply. Prof. H. S. Fawcett has demonstrated that in some cases it is communicable because of a fungus cause. Full accounts of this may be expected in University publications.

When there is an outbreak of gum where it can be treated it is desirable to cleanly remove all the unhealthy bark-cutting clean to sound bark and covering the wound with paint or wax to exclude the air. Prof. Fawcett gives these detailed suggestions:

The treatment for the gummosis caused by fungi is still somewhat in the experimental stage. All that can be said at present is that in several lemon groves where the Bordeaux paste was used over a year ago the results appear very promising.

Before applying the paste on lemon trees the bark that is dead to the wood should be removed to allow the Bordeaux to get in to kill out the infection. In case the outer bark is infected over a large area and the inner or cambium is still alive, the outer layer may be scraped off with a curved sharp tool made on purpose for this work, leaving the cambium covered by inner bark still attached. In this way the infection is killed and a new bark is built up under the Bordeaux. One must expect the gum to run out freely for several weeks or months after treatment. One should watch for the formation of new, healthy tissue, rather than the amount of gum that may run out after treatment. The gum that has formed under the bark beyond where any fungus has gone will continue to drain out. The gum itself does not as a rule have any infectious principle in it. The fungus is in the diseased bark at the junction of the live and dead tissue. The fungus does not manufacture the gum, but causes the tree to produce it from its own products.

The formula for the Bordeaux paste is as follows:

One pound of bluestone dissolved in 1 gallon of water in a wooden or earthen vessel by hanging it in the top in a sack. Two pounds of unslaked lime, slaked in about one-half gallon of water. Stir together when cool, making a light blue mixture about the consistency of whitewash. Apply with a brush. This may also be applied to healthy bark as a preventive against new infections.

Root Knots.-These are excrescences upon the roots or at the root crown of various trees and of grape-vines, and they have been a serious trouble in this State for a good many years. Some of the knots have been studied by experts in plant pathology and the cause of the trouble demonstrated to be a fungus and the disease infectious. A satisfactory treatment has, however, been discovered. If the knot has not increased in size sufficiently to seriously interfere with the growth of the tree it can be smoothly removed, the wound treated with the Bordeaux Mixture, and the knot will not reappear at the same place. Success has also been had with boring a hole into the knot and filling the hole with bluestone solttion, but some trees have been killed in this way. Bluestone can be used with least danger when the tree is dormant. 
As for treatment, however, it seems to be clear that if the trees are not too large, the knots can be removed with a chisel and the wound painted with the Bordeaux Mixture to the advantage of the tree; but if the trees are old and the knots so large that their removal would almost girdle the tree, it is a question whether it is not better, on the whole, to take out the trees and plant new ones. It really depends upon the condition of the trees whether it is worth while to remove the knots or not. If the tree has the start of the knot, it will be helped by removal; if the knot has the start of the tree so that it is unthrifty, there is small chance of reinvigorating the knots. This is apparently true, both with young and old trees. Old trees are sometimes badly knotted and still productive and profitable. 


\section{CHAPTER XLI}

\section{SUPPRESSION OF INJURIOUS ANIMALS AND BIRDS}

The beasts of the field and the fowls of the air are sometimes such grievous trespassers upon the fruit plantation that protection has to be sought against them. The animals which figure in this evil work are mainly species of rodentia, some of them burrowers; as, for example, the ground-squirrel and gopher; others, surface dwellers, like the hare or jackass rabbit. Occasionally there is injury done by deer in the orchard and vineyard, and coons in the melon patch, but these larger animals may usually be left to the hunters and the dogs.

\section{RABBITS}

Though there are three species prevalent, none are burrowers. This fact has led to united efforts at their suppression by driving them, with mounted horsemen, from a wide stretch of country into a narrow, fenced inclosure, where they are killed with clubs. During the last few years tens of thousands have been killed in this way, and comparatively few are now found in the localities where the method has been adopted. Still, however, there are plenty at large to vex the fruit planter, and he must protect himself against them.

Rabbit Fences.-The surest protection against rabbits is a fence which prevents their entrance, and many miles of such fence have been built in this State. Several styles prevail. The ordinary board fence, with the boards running horizontally, is made rabbit-proof by placing the lower boards close together, with openings of but about two inches

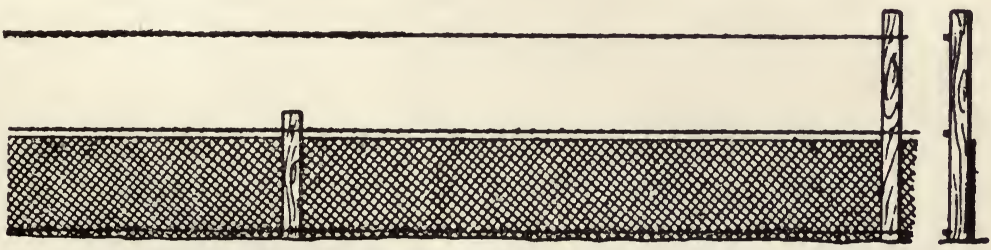

A rabbit-proof fence in successful use in the San Joaquin Valley

between them. A barbed wire, with barbs about two and one-half inches apart, can be used to advantage by running it along at or a little below the surface of the ground to prevent scratching under.

The cost of board fences has led to the use of barbed wire and wire netting, or of perpendicular slats interwoven with wire. Such materials are sold in large quantities. A very effective combination of barbed wire and netting, which is used in the upper San Joaquin Valley, is described as follows: 
The tall posts are regular split redwood posts. The intermediate small ones are made by sawing in two the regular posts and splitting them into eight small posts, or rather, large stakes. The netting is of galvanized wire, No. 19 gauge, and one and one-half inch mesh. This netting is stapled to the posts and stakes on the inside, or toward the field. This is of prime importance, as it will not serve the purpose if it is placed on the outside. The bottom of the netting is to come down to the ground, and the ground must be left hard, and not plowed to prevent burrowing, or scratching the dirt from underneath, which can be easily done if the dirt is softened up. It is not at all necessary to set the netting below the ground. In the sketch are shown three barbed wires, with barbs two and a half inches apart. These wires must be placed on the outside of the posts. This position is also a prime necessity. The lower wire is stretched just clear of the surface of the ground. The middle wire is one inch higher than the top of the netting, and the top wire, which is intended only as against cattle, is at a height suitable for the purpose. The rabbit-proof portion is comprised in the netting and the two lower wires. Hence, if cattle are not feared, and rabbits are the only foe, the top wire can be dispensed with, and the posts can be all short with a greater proportion of stakes, having only enough stout posts to stand the strain of the wires. The theory of this construction is that a rabbit can only pass the fence over the top or under the bottom of the netting, and this is effectually prevented by the barbed wires, which tear the animal if it attempts either to leap or climb over or to scratch under.

Smears Distasteful to Rabbits.-Where the expense of a fence can not be assumed, measurable protection can be had by sprinkling the leaves or smearing the stems of plants with substances distasteful to the animals, which are quite dainty in this respect. Commercial aloes, one pound to four gallons of water, both sprinkled on leaves and painted on the bark, gives a bitter taste, which repels rabbits. A tea made of steeping quassia chips is said to produce the same effect. Rancid grease, liquid manure, putrescent flesh or blood, have been approved as a daub for tree trunks, but the efficacy is only of limited duration.

Rabbit Poison.-Pieces of watermelon rind, cantaloupe, or other vegetable of which they are very fond, may be poisoned with strychnine and then scattered around the orchard. Rabbits will not touch the bark as long as they can find this bait, and one meal is effective, for the rabbit never gets far away from it. The same results can be obtained by the following mixture: To one hundred pounds of wheat take nine gallons of water and one pound of phosphorus, one pound of sugar, and one ounce of oil of rhodium. Heat the water to boiling point and let it stand all night. Next morning stir in flour sufficient to make a sort of paste. The rabbits eat it with avidity if scattered about.

Another preparation is half a teaspoonful of powdered strychnine, two teaspoonfuls of fine salt, and four of granulated sugar. Put all in a tin box and shake well. Pour in small heaps on a board. It hardens into a solid mass. They lick it for the salt, and the sugar disguises the poison, which kills great numbers.

\section{GROUND-SQUIRRELS}

Ground-squirrels are poisoned by the use of the poisoned wheats which are sold in the markets, or by use of bisulphide of carbon, or "smokers," which are arranged to force smoke or poisonous vapors 
into the holes. A small quantity of bisulphide of carbon poured into the hole, and the hole closed with dirt, is probably the most effective squirrel killer, when the ground is wet, so that the vapor is held in the burrow. Smokers are also most effective when the soil is moist. When the ground is dry, poison is the best means of reducing squirrels. The following is an exceedingly effective preparation, of which a few grains should be placed in or near each hole:

Take strychnine, one ounce; cyanide of potassium, one and one-half ounces; eggs, one dozen; honey, one pint; vinegar, one and one-half pints; wheat or barley, thirty pounds. Dissolve strychnine in the vinegar; and you will have to pulverize it in the vinegar, or it will gather into a lump. See that it is all dissolved. Dissolve the cyanide of potassium in a little water. Beat the eggs. Mix all the ingredients together thoroughly before adding to the barley. Let it stand twenty-four hours, mixing often. - Spread to dry before using, as it will mold if put away wet.

What is known as the "government formula," being used by various departments of the United States government in the effort to eradicate ground squirrels from California, is as follows:

Strychnine (pulverized alkaloid), 3 ounces.

Starch, $1 / 2$ pint.

Barley, 13 gallons.

Water, 3 pints.

The water is placed over a fire and heated to boiling; the starch is dissolved in a half-pint of cold water and added to the boiling water, which is stirred carefully to insure uniform consistence. When the starch has become clear, remove it from the fire and add the strychnine. Mix this poisoned paste with the barley until every kernel is coated.

To keep squirrels from gnawing fruit trees, or climbing and getting the fruit, tying a newspaper around the trunk of the tree, letting the paper extend out four inches at the upper edges, is said to be effective by some growers, though this is denied by others. The rattle of the paper when the squirrels attempt to get over it will frighten them.

\section{GOPHERS}

Gophers can often be destroyed by the use of $n$; soned wheat, especially prepared with a little oil of rhodium, which seems to be very attractive to all rodents. Pieces of fruits or vegetable, or the succulent stems of alfalfa, into which a few grains of strychnine have been inserted by making a cut with a knife-blade and then squeezing it together again, are also handy conveyors of death to gophers. There are two ways to put poisoned materials into a gopher runway. One is to look for fresh open holes and put in the poison as far as possible with a long-handled spoon ; another is to take a round, pointed stick and shove it into the ground near the gopher mounds until it strikes their runway, then drop in the poisoned bait. Close up the hole with some grass; level down mounds, so that if the poison does not kill all the gophers, you will soon discover their new mounds. If there are many mounds, put the poison in a number of places. 
Bisulphide of carbon is also successfully used in killing gophers, while the ground is wet, using an injector which is furnished with the poison to force the vapor through the long burrows.

Trapping Gophers.--Some are very successful in using gopher traps, of which there are several styles sold. Gophers come to the surface in the night, and generally close their holes soon after daybreak. They frequently emerge again about noon, and a third time late in the afternoon. It is best to set the trap in an open hole; still the holes may be opened if the dirt is still fresh, with a good prospect of the gopher's return. Therefore, the trapper may make his rounds three times a day, as above indicated. Care should be exercised in preparing the hole for the insertion of the trap, a straight hole for a distance of at least ten inches, with no lateral branches, otherwise the gopher in pushing out the dirt will likely enough thrust the trap to one side, cover it up, or spring it without being exposed to its grasp. The trapper should be supplied with at least two varieties of traps-one for the larger gophers, and the other for the smaller ones. The common iron gopher trap, which springs downward, is excellent for the former, and the small wire trap, which springs upward, is generally successful with the latter. The size of the hole is indicative of the size of the gopher. Either trap should be inserted nearly its full length into the hole, pressed down firmly, and a little dirt piled at the outer end to prevent its being easily pushed out. After the trap is set, it is well to cover the opening with some grass or weeds. Sometimes the holes require a little enlarging, but care should be taken to make the fit as close as possible, that the body of the gopher may be kept near the center, and thus more exposed to the prongs of the trap. In the fourth place, the trapper should have a small spade and a little gouge-shaped instrument for trimming the hole.

Gopher Pitfalls.-If gophers are abundant, large numbers can be captured in this way: Dig a trench around the orchard or vineyard about the width of a spade and from fourteen to sixteen inches deep. In the bottom of the ditches, about a hundred feet apart, sink fivegallon oil cans, leaving the tops level with the ditch bottom. The gophers migrate in the night, and in attempting to come into the inclosure will fall into the ditch and then run along the bottom until they drop into the cans. Of course the ditch must not be wider than the cans. As many as fifteen live gophers have been found in one can. The cats soon learn to help themselves out of the cans. The ditch must be kept clean, and if any roads cross the tract, set up a board at night, to compel the gophers to tumble in the ditch. This ditch should be constructed about the first of June, when the oustide feed begins to dry up, and the pests rush for the cultivated ground. With such protection from the outside, and the use of poison and traps inside, the trees and vines can be saved.

\section{DESTRUCTIVE BIRDS}

Fruit growers generally appreciate the value of insectivorous birds, but there are feathered pests which do such ruinous work in disbudding the trees in spring-time, and in destroying ripe fruit that protective 
measures have to be adopted against them. The so-called "California linnet," which is not a linnet, but a finch (Carpodacus frontalis), a persistent destroyer of buds, and the English sparrow, infamous the world over, are probably the most grievous pests, though there are other destructive birds, including the beautiful California quail, which is protected by law, and yet must be destroyed in some parts of the State or the grape crop must be abandoned.

For the killing of the smaller birds poison is usually employed, and it is the best administered in water. Poisoned water made of one-eighth ounce of strychnine to three gallons of water and placed in shallow tin pans in the trees, has been widely approved. Cutting oranges in halves, spreading strychnine over the cut surface and empaling the half oranges on twigs high up in the apricot trees, has destroyed hundreds of linnets. Some advocate the use of the shotgun, No. 30 caliber, with a small charge of good powder and No. 10 shot. As many as five hundred linnets have been killed in two days. The advantage of this plan is that one kills linnets and not other birds, while poison kills both friends and foes. 


\section{PROTECTION FROM WIND AND FROSTS}

Though the climate of California renders unnecessary the protection against rigorous weather which fruit growers in some other parts of the world have to provide, there is often advantage in securing shelter from winds and protection from late frosts.

The general subject of forest planting in California, and the effect of preservation and extension of our forest area upon our fruit industries, has received the attention of our best-informed growers. The planting of shelter belts at intervals across our broad valleys at right angles to the courses of prevailing or most violent winds, has also been urged with great force. These greater enterprises and projects are beyond the scope of this treatise. It is rather concerning the planting of trees to shelter individual possessions that a few suggestions will be offered.

It has been already remarked that on the immediate coast the successful growth of fruit will sometimes be wholly dependent upon proper shelter from prevailing winds, and in regions farther from the ocean the topography may induce strong currents of air which will illy affect trees and vines. In all such places the fruit grower should plant windbreaks, and will find himself well repaid for the ground they occupy, by the successful production on the protected area.

In the interior valleys there is also need of shelter from occasional high winds which may visit the orchards either in summer or winter, and prove destructive both to trees and fruit. In some cases long lines of the sheltering trees have been cut down because they affected the fruiting of orchard trees planted too near them, and afterwards the losses through lack of protection were far greater than would have been incurred by retaining them.

What Kind of Trees to Plant.-This is a question concerning which there is much to be learned. Data is accumulating in the growth of trees planted to test their suitability, and the future planter will have more certain ground to proceed upon than is now available. Mention will be made, however, of a few trees, which are now most widely grown.

The most widely planted shelter tree is the Eucalyptus globulus, or Australian blue gum. It is a rapid grower and voracious feeder, and wonderful for root extension, for which it has been roundly abused. It is doubtful, however, whether we have a better tree for high growth, and consequent large area over which its shelter will be felt. It is deficient in undergrowth, and if a close screen is desired, the planting of eucalyptus and Monterey cypress (Cupressus macrocarpa) is a common practice. The latter also attains a good height, but its broad, thick base fills the gaps between the bare stems of the gum trees. Another tree which has often been planted with the blue gum, to supply a thick, 
low growth, is the pepper tree (Schinus molle). It is also grown in rows by itself. It makes a dense head, grows rapidly, and flourishes without much care. Trees planted eighteen feet apart will soon come together and make a dense wall of very beautiful, bright, light-green foliage. The pepper is not only a good windbreak, but also an excellent dust-catcher. Unlike most trees which are used for this purpose, it does not become laden with dust. The leaves are smooth and glossy, and therefore repel the dust particles, which, stopped in their flight by the dense foliage of the tree, instead of clinging to it drop to the ground. The growth of the pepper tree near the coast is much slower than that of the Monterey cypress. The eucalyptus and the cypress for the coast, and the eucalyptus and pepper for the interior valleys, make, probably, as perfect a wall of foliage all the year round as can be had. The blue gum is, however, somewhat subject to frost killing, especially when young, and in very frosty places is objected to on that account. A number of other species of eucalyptus are now being planted, and are being found more hardy than the blue gum. The rostrata, rudis, tereticornis, polyanthema, amygdalina, viminalis and others are of this character.

The Monterey pine (Pinus insignis) is a rapid, high-growing tree, and, though a native of the coast, has proved itself well adapted to the -interior valleys of the central portion of the State. Its foliage is dense for a pine, and its shelter, therefore, the more complete. A native white cedar (Libocedrus decurrens) has also been employed as a shelter tree in the San Joaquin Valley, and is commended as a rapid grower in the interior as on the coast. Its ability to stand drouth, heat and frost is said to exceed that of any of the conifers of the seacoast. It stands well in the most exposed situations, as its roots run very deep into the earth and it is claimed that it does not sap the fertility from the soil around its base, as with the blue gum. It is also said to be less subject to frost injury than the Monterey cypress and pine.

All the foregoing are evergreen trees, and therefore afford protection summer and winter alike. Of deciduous trees there are many which may be well employed. The California black walnut makes a very satisfactory growth both in the interior and upon the coast, and is largely used for roadside planting. The California broad-leaved maple (Acer macrophylla) is very beautiful, rapid in growth, and dense in foliage, and the same is true of the box elder (Acer negrundo), but probably both trees are especially suited to the coast regions. Of the poplars, the Carolina (Populus monilifera) is best, because of its breadth, density of foliage, and comparative freedom from suckering. The locust (Robinia pseudacacia) is used to some extent, but its suckering is very objectionable.

Quite a number of the larger-growing deciduous fruit trees are used to some extent along the exterior lines of orchards for the protection of the inclosure. The fig, the walnut, the chestnut, seedling almonds, and apricots are especially commended for such use.

Growing Trees from Seed.-Much that has been said in Chapter VIII will be suggestive to one who desires to grow his own shelter trees from seed. Trees from small seeds are best grown in boxes, and 
in many cases, as with eucalyptus and cypress especially, do best when put in permanent place when quite small. Whether put at once in permanent place, or in nursery, the land should be deeply worked and the young plant well planted and cared for.

Cultivation of Shelter Trees.-If one desires rapid growth of shelter trees, they should be cultivated the first few years as thoroughly as an orchard. Much disappointment results from allowing roadside trees to shift for themselves in a hard, dry soil. With such treatment the root extension is naturally most rapid into cultivated orchard ground, which is undesirable. Cultivate and enrich the roadside, and the tree will grow chiefly on the waste land. At the same time the roadside will be prevented from producing vast quantities of weed seed, to be blown over the fence, and the place will have a name for neatness, which is too rare even in California.

\section{PROTECTION FROM FROSTS}

Much attention has been given during recent years to the protection of citrus fruits as they approach maturity, and of deciduous fruits as they are starting on their growth, from occasional fall of the mercury a few degrees below the freezing point. It has been shown by ample experience that fruits may escape injury by a temperature of 28 degrees if the ground surface is wet and the exposure be but of short duration. Fruit has, therefore, been saved by irrigation, while that over dry ground has been nipped by the same temperature. About the same result has been secured by checking radiation of heat by covering the orchard or vineyard with a cloud of smoke. Both these protective measures fail when the temperature falls a few degrees below 28 degrees or when such freezing temperature is continued for several hours.

During recent years much progress has been made in preventing frost by numerous small fires distributed among the trees to be protected and many devices to secure such distributed heat easily and economically are being enterprisingly promoted by inventors and manufacturers. The extreme low temperatures of January, 1913, gave full opportunities for testing orchard heating appliances and very striking success was had with them. Comparative tests and observations have been made by the experts of the University Experiment Station, but conclusions are not available at this date. They must be looked for in Experiment Station bulletins, in the publications of the U. S. Weather Bureau and in the horticultural journals. The subject is clearly seen to be too complex to admit of a brief generalization except to say that, when orchard heating is thoroughly and economically done, it is a most profitable investment and should be studied by all growers.

\section{SUSCEPTIBILITY OF DECIDUOUS FRUITS}

No systematic observations of danger points in deciduous fruits have been made in this State, but work elsewhere is suggestive. Professor W. L. Howard, of the Missouri Experiment Station, after arti- 
ficially freezing thousands of peach buds, summarized the critical temperatures as follows:

"Fully dormant peach buds can stand 8 or $9^{\circ}$ below zero, F. When they are appreciably swollen, zero is the danger point. When the buds are showing pink, they can stand $15^{\circ}$ above zero. When the buds are almost open, $25^{\circ}$ is the danger point. When they are newly opened, about $26^{\circ}$ would be the point of danger. When the petals are beginning to fall, $28^{\circ}$ above zero is dangerous. When the petals are off they can stand $30^{\circ}$ above zero. When the 'shucks' (calyx lobes) are beginning to fall off, $32^{\circ}$ is the danger point." Presumably these limits would apply also to the apricot and almond.

Apple buds, when the petals begin to show, can stand from 10 to 12 degrees of freezing. When the petals are well emerged, but have not opened, they can stand from 4 to 6 degrees of freezing, but when open, but not yet fertilized, there would be great danger at two or three degrees below the freezing point. When the flowers have been fertilized, the petals dropped, and the young fruit increasing in size, the slender stems which support the apples are unable to resist a temperature lower than the freezing point. The apples seem to be more hardy than their stems, but if the latter are injured of course the fruit also suffers. Similar behavior may be expected from the pear, cherry and plum.

Orchard Frost Studies.-In connection with the foregoing it is interesting to note conclusions which Prof. Fabian Garcia draws from several years' observation of frost effects in the orchard of the New Mexico Experiment Station and of which full details are given in Bulletin 89 of that station, under date of February, 1914:

The data recorded at the Station show that the fruit-buds, particularly those of the peach, at their different stages of development are somewhat more resistant to cold than has been reported by observers from others parts of the United States. The data further show that the degree of resistibility to frost varies with different stages of growth.

The peach is least resistant when it is about the size of a pea, when the calices are falling off. Contrary to the findings of other observers, the bloom is not the most tender stage of growth. In other words, the data indicate that the newly set peach is more delicate than the newly opened blossom.

In the majority of cases a temperature of 26 degrees, lasting only a short time, did little or no injury to the opening bud, newly opened blossom or newly set fruit of the peach, native plum, pear and apple; while one-half a degree below this, or $251 / 2$ degrees, although lasting only a few minutes, killed a large percentage of the opening buds, newly set blossoms and young fruits of the peach, in a number of cases. In other words, 26 degrees was the danger point, and any temperature below this is liable to do more or less injury; depending on how low it gets and how long it remains at that temperature. In some cases, however, a temperature of 24 degrees lasting only a short time left about $25 \%$ of the blooms and $9 \%$ of the newly set fruits uninjured.

Three important factors that influence the amount of damage done by spring frosts are: the degree of killing temperature, the time of day at which it occurs, and the length of time the temperature remains below the danger point. The longer the time the killing temperature remains and the nearer it is to sunrise, the greater the amount of damage that should be expected.

As a rule, the killing temperature recorded in these experiments occurred in the morning, from one to two hours before sunrise. Whenever the killing temperature occurred this late in the morning, and remained below the danger point until sunrise, the injury was great. On the other hand, if the minimum occurred at midnight, or a little later, and then gradually rose to the freezing point, so that the frozen buds, blooms and fruits had time to thaw out slowly before sunrise, the injury was insignificant. This was the case April 5, 1910, when at $2: 15$ A. M. the temperature was $243 / 4$ degrees. By $5: 30$ it had risen to 31 degrees, and the injurv done to the Alexander peaches-which were in full bloom-was estimated at $21 / 2 \%$.

As the young fruit develops it seems to get more tender, until it grows beyond a certain size, when it seems to get hardier again; so that the later frosts 
which come after the young fruits have made quite a growth are liable to do more injury than the same degree of cold in the earlier part of the season, when the fruits are less developed. At this stage of growth, $261 / 2$ to 27 degrees would probably be dangerous to peaches. The very late frost of 25 degrees on May 4, 1912 , killed practically all the peaches in the experimental orchard, while $243 / 4$ degrees on April 17, 1910, left about $30 \%$ of the Elbertas.

On the whole, apples and pears seem to be more resistant than peaches and plums. This was strongly indicated in 1912, when practically all the peach crop was destroyed on May 4, by a temperature of 25 degrees; while there was a very heavy crop of pears and apples harvested the following fall.

Euro ean and native plums seem to be slightly hardier than Japanese plums and apricots, which are the least resistant of all the fruits included in the test.

\section{DELAYING BLOOM BY WHITEWASH}

Another suggestion comes from Missouri. It is concerning delaying activity in deciduous trees by spraying with whitewash to reflect heat - the absorption of which by dark bark causes the activity of the tree to begin. Prof. J. C. Whitton of the Missouri Experiment Station says :

Purple coloring matter on untreated peach trees often absorbs heat enough on a sunny cold day in winter to raise the temperature of the trees 25 degrees or more above the temperature of the atmospehere. Whitened trees remain at atmospheric temperature or usually a degree or so below.

For ten consecutive years we whitewashed diagonal rows of peach trees across the Experiment Station orchard just before midwinter. In this section we had five general peach crops during that ten years on untreated trees; on whitewashed trees of the same varieties we had eight crops. The five crops on untreated trees were not all full crops; the eight crops on treated trees were all but two full crops. More than double the amount of fruit was secured in ten years on the whitewashed trees. The treated trees were anywhere from a few days up to ten days later in blooming. In years when normal weather prevailed until sudden warm spells brought the trees into bloom hurriedly, there was only a little difference in time of blooming, and under normal conditions there was a week or more difference in their blooming. This is less important, however, than the fact that the whitewashed trees began their first starting into slight growth on sunny days in late winter fully six weeks later than the naked trees. 


\section{PART TEN: MISCELLANEOUS}

\section{CHAPTER XLIII}

\section{UTILIZING FRUIT WASTES}

Some progress has been recently attained in the securing of horticultural by-products from various kinds of fruit wastes. There is a considerable product of cream of tartar from the pomace and lees of the wineries in central California. In southern California citric acid factories have to some extent used lemons rejected in packing, and some other by-products of citrus fruits have been secured in small quantities. Vinegar from wine and cider are, of course, made here as everywhere in fruit countries.

There has arisen also a profitable export demand for fruit pits and apricot and peach pits, which formerly were burned, are now selling profitably-machinery for cheap extraction of the kernels have been contrived by California inventors. The kernels are bought by agents of European manufacturers of oils and essences. Apricot kernels are used by confectioners in place of almonds.

Comparative value of fruits, and hay, grains, meals, etc.

\begin{tabular}{|c|c|c|c|c|c|c|c|c|c|c|c|c|}
\hline FRESH FRUITS. & 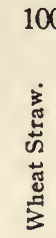 & 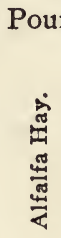 & 密 & 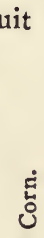 & कृष & 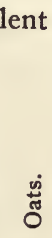 & 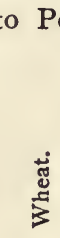 & 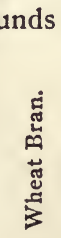 & 点 & 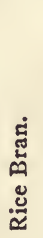 & 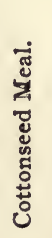 & 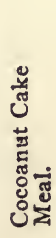 \\
\hline 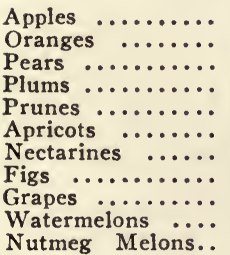 & $\begin{array}{l}34 \\
33 \\
40 \\
50 \\
46 \\
40 \\
43 \\
50 \\
50 \\
22 \\
19\end{array}$ & $\begin{array}{l}20 \\
19 \\
23 \\
30 \\
27 \\
23 \\
26 \\
30 \\
30 \\
13 \\
11\end{array}$ & $\begin{array}{l}24 \\
23 \\
30 \\
36 \\
33 \\
29 \\
30 \\
37 \\
37 \\
16 \\
13\end{array}$ & $\begin{array}{r}15 \\
14 \\
17 \\
22 \\
20 \\
17 \\
19 \\
23 \\
23 \\
10 \\
8\end{array}$ & $\begin{array}{r}15 \\
14 \\
18 \\
24 \\
22 \\
18 \\
20 \\
24 \\
24 \\
10 \\
9\end{array}$ & $\begin{array}{r}17 \\
16 \\
20 \\
25 \\
23 \\
20 \\
22 \\
26 \\
26 \\
11 \\
9\end{array}$ & $\begin{array}{r}16 \\
15 \\
19 \\
24 \\
22 \\
19 \\
21 \\
25 \\
25 \\
11 \\
9\end{array}$ & $\begin{array}{l}18 \\
17 \\
20 \\
26 \\
24 \\
20 \\
23 \\
27 \\
27 \\
12 \\
10\end{array}$ & $\begin{array}{r}16 \\
15 \\
19 \\
24 \\
22 \\
19 \\
21 \\
25 \\
25 \\
11 \\
9\end{array}$ & $\begin{array}{r}13 \\
12 \\
15 \\
20 \\
18 \\
15 \\
17 \\
20 \\
20 \\
8 \\
7\end{array}$ & $\begin{array}{r}9 \\
8 \\
11 \\
14 \\
13 \\
11 \\
12 \\
14 \\
14 \\
6 \\
5\end{array}$ & $\begin{array}{r}13 \\
12 \\
15 \\
20 \\
18 \\
15 \\
17 \\
20 \\
20 \\
8 \\
7\end{array}$ \\
\hline \multicolumn{13}{|l|}{ DRIED FRUITS. } \\
\hline $\begin{array}{l}\text { Dried prunes } . . . \\
\text { Dried apricots } \ldots \\
\text { Dried peaches } \ldots \\
\text { Dried figs } \ldots \ldots \ldots \\
\text { Raisins } \ldots \ldots \ldots \ldots\end{array}$ & $\begin{array}{l}175 \\
194 \\
190 \\
186 \\
216\end{array}$ & $\begin{array}{l}104 \\
115 \\
113 \\
110 \\
128\end{array}$ & $\begin{array}{l}125 \\
138 \\
135 \\
132 \\
153\end{array}$ & $\begin{array}{l}78 \\
86 \\
85 \\
83 \\
97\end{array}$ & $\begin{array}{r}82 \\
90 \\
88 \\
85 \\
100\end{array}$ & $\begin{array}{r}88 \\
97 \\
95 \\
93 \\
108\end{array}$ & $\begin{array}{r}84 \\
93 \\
91 \\
89 \\
103\end{array}$ & $\begin{array}{r}92 \\
102 \\
100 \\
97 \\
111\end{array}$ & $\begin{array}{r}84 \\
93 \\
91 \\
89 \\
103\end{array}$ & $\begin{array}{l}67 \\
74 \\
72 \\
71 \\
82\end{array}$ & $\begin{array}{l}48 \\
53 \\
51 \\
50 \\
59\end{array}$ & $\begin{array}{l}68 \\
76 \\
74 \\
72 \\
84\end{array}$ \\
\hline
\end{tabular}

The disposition of waste fruit by growers must, however, always lie chiefly in the line of feeding animals unless denatured alcohol enterprises should arise to consume it at prices to pay something more than 
cost of handling. Refuse fresh fruits of all kinds, and especially refuse dried fruits have nutritive value which should not be lost. A statement of the value of various fruits as compared with various cattle foods has been prepared by Prof. M. C. Jaffa, of the University Experiment Station, in the adjacent table.

A good average of the pitted fresh fruits is represented by prunes. Using the equivalents in the table above for computation, it appears that if wheat bran costs $\$ 15$ per ton, fresh prunes would be worth as a substitute $\$ 3$ per ton; likewise, if cottonseed meal is selling for $\$ 21$ per ton, the prune value would be about $\$ 2.75$. At the market price of oat hay, the figures for fresh prunes should be nearly $\$ 3$ per ton.

The dried fruits naturally rank far above the fresh material as stock feed. Of the dried fruits represented in the table, raisins lead in food value; containing one and one-fourth to one and one-half times the nutritive ingredients of alfalfa and oat hays, respectively; 100 pounds of the fruit being practically equal to the same quantity of grain, but to only eighty-two and fifty-nine pounds respectively of rice bran and cottonseed meal.

Dried apricots rank slightly lower than raisins, because they contain more water. Apricots are, however, of equal value as a feeding stuff with wheat bran and almond hulls about half as much as alfalfa hay, bran or middlings.

Concerning the feeding of raisins to hogs, the following statements are made: "With raisins selling at $21 / 2$ cents per pound they are much cheaper food for hogs than corn or barley. Raisins give the hog a hard, sweet meat and are much sought after by the butchers. Care must, however, be exercised in feeding raisins to hogs, as they are full of sugar, and consequently too rich when fed alone. They heat the animal up so that the hogs lose flesh and will kill the little pigs of a farrowing sow. For fall and winter feeding, pumpkins, citrons and alfalfa are the best feed to give the hogs in conjunction with the raisins, but in the spring a change is found very beneficial.

Many people feeding raisins to hogs are not having the success they should because they overfeed their porkers. A pound of raisins a day is ample to start in with. After the system of the hog has become accustomed to the raisins the amount can be increased so that the last three weeks each hog should get about four pounds of raisins a day. The last ten days, when the finishing touches are being put on, the hogs should be allowed all they can get."

Wine grapes rich in sugar have been cheaply dried on the ground and used to advantage for hog feed. One grower says that in 1908 failing to get $\$ 6$ per ton for his grapes, he dried them and fed them to hogs. Having more than the hogs required, grapes were fed to the horses: "The horses soon got a taste for them and seemed to thrive well on the new diet and in a short time became fat and sleek, while they were being worked as hard as ever, and we continued to feed them dried grapes and have kept it up for a whole year. The effect seems to have made the old horses five years younger, both in looks and in ability to work. The hogs fattened up so quick that we thought the 
pork would be soft and sloppy, but to our surprise, we never had better bacon and ham than was produced from these grape-fed porkers. It was not only solid, but sweet and tender."

Prune-fed and raisin-fed pork is indeed an accomplished fact in California. As to the acceptability of the fruit diet to the hog, what could be more pertinent and more fitting appendix to this treatise than this little tale? It is stated that Mr. Balaam, of Farmersville, used to have a pet pig that ran under the fig trees near the house. When the fruit began to drop, he ate figs and rested in the shade until he finally grew too fat to move about to gather the sweet morsels. By this time his owner became so much interested in the case as to carry him his figs regularly three times daily. Gradually he grew so fat that his eyes closcd entirely, but still he ate figs in contentment and delight. 


\section{PLATE ILLUSTRATIONS}

OPPOSITE

PAGE NO.

Plate I. California homes submerged in a sea of winter blossoms.... 3

II. Apricot trees with and without summer cultivation........ 105

III. Ground view of good job of fruit-thinning............. 121

IV. Cement ditches and irrigation by check system........... 169

V. Orchard irrigation by furrow system ............... 185

VI. A good start toward an apple orchard............... 201

VII. 'Typical aspect of a bearing apricot orchard............ 217

VIII. Young cherry orchard in good form................ 225

IX. Bearing peach orchard in an irrigated district........... 257

X. Development of strong-branching form in the French prune.. 265

XI. Orchard of shipping plums in a foothill district.......... 281

XII. Shaping the grape clusters for shipping .............. 289

XIII. Profuse bearing of vine by long pruning ............. 321

XIV. Bearing of the young date palm in California ........... 329

XV. The Sevillano olive as grown in Tehama County......... 345

XVI. Olives popular in California ...................... 353

XVII. Partial view of Riverside citrus district............... 385

XVIII. Orange trees with sucker growth and good growth........ 393

XIX. Bearing of young budded orange tree and orchard view with cover crop ...............................409

XX. The Mammoth Blackberry ......................... 417

XXI. The Loganberry . ........................... 449

XXII. Strawberry patch with cane windbreak and almond trees in bloom . ................................. 457

XXIII. Cleft graft of walnut and method of supporting grafts..... 473

XXIV. California sunshine evaporator with accessory buildings..... 481 


\section{INDEX}

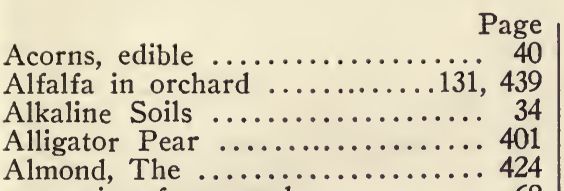

growing from seed............. 68

hulling and bleaching ..........4 426

pollination ................ 428

propagation $\ldots \ldots \ldots \ldots \ldots \ldots .68,425$

pruning ...................4 426

situations and soils ............. 425

wild ..................... 40

varieties $\ldots . \ldots \ldots \ldots \ldots \ldots . . .194,429$

Animals, injurious ............ 493

Ants, killing ................. 482

Apple in California............... 195

aphis, resistant ............. 197

aphis, woolly .............. 473

drying $. . . \ldots \ldots \ldots \ldots \ldots \ldots . . . . .454$

exposures for .............. 196

gathering .................. 200

irrigation $\ldots \ldots \ldots \ldots \ldots \ldots \ldots 200$

localities for ............... 196

mildew ..................... 488

mission $\ldots \ldots \ldots \ldots \ldots \ldots \ldots \ldots . . . .42$

native crab ................... 36

planting distance ............. 198

picking and packing ............ 202

pollination .................... 204

propagation ................. 197

pruning .................... 198

scab or smut ................ 488

second crop ................... 196

seedlings, growing $\ldots \ldots \ldots \ldots 66,67$

shipping $\ldots \ldots \ldots \ldots \ldots \ldots \ldots \ldots 203$

soils for ................... 197

storehouse for $\ldots \ldots \ldots \ldots \ldots \ldots . .201$

summer and fall............. 203

table of varieties.............. 209

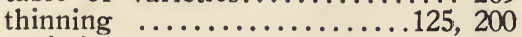

varieties, most popular.......... 193

when to pick.............. 200

winter .................... 203

worm .................... 480

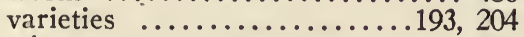

Apricot ................... 210

climatic requirements .......... 211

diseases of $\ldots \ldots \ldots \ldots \ldots \ldots \ldots .217$

distances for $\ldots \ldots \ldots \ldots \ldots \ldots \ldots . \ldots 213$

drying $\ldots \ldots \ldots \ldots \ldots \ldots \ldots . \ldots 454$

exposures for $\ldots \ldots \ldots \ldots \ldots \ldots . \ldots 211$

growing seedlings ........... 68

irrigation $\ldots \ldots \ldots \ldots \ldots \ldots \ldots . .216$

localities for $\ldots \ldots \ldots \ldots \ldots \ldots \ldots . . .211$

mission

Apricot-continued

Page

old trees $\ldots \ldots \ldots \ldots \ldots \ldots \ldots . . .210$

on almond root $\ldots \ldots \ldots \ldots \ldots \ldots . \ldots 213$

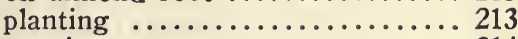

pruning .................. 214

shot-hole fungus ............ 218

stocks and soils for.......... 212

table of varieties............ 218

thinning ................125, 216

varieties ..................193, 219

Army Worms ................ 466

Atmospheric humidity ........... 21

Banana, The ................. 393

Barberry, native ............. 39

Bear berry ................. 39

Bergamot .................. 392

Berries and currants ........... 404

Berries, various wild .......... 38

Birds, poisoning ............. 496

Blackberry, The ............. 405

cultivation ................4 406

hybrids .................. 417

distances for .............. 405

irrigating .................. 408

longevity of ............... 408

propagation .................4 405

pruning ...............406, 410

wild $\ldots \ldots \ldots \ldots \ldots \ldots \ldots \ldots, 38$

varieties .................. 408

Blasting for planting ........... 94

Borers ...............104, 477, 480

Bones, treatment of ............ 143

Bordeaux Mixture ..........488, 491

Brush, cutting to kill........... 62

Budding, common method........ 73

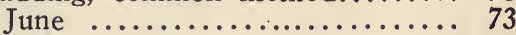

over old trees................ 82

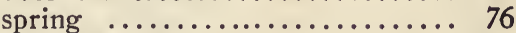

Bud, cutting to a............ 123

Buds, dormant .............. 81

Buffalo berry ................ 39

Cactus fruits ................ 40

Canned fruit product ........... 445

Canning industry ............. 445

Canker worms .............. 467

Caterpillars ................ 467

Chain for laying out........... 93

Chamisal and chanarral ......... 57

Charcoal making .................. 61

Cherimoyer .................... 394

Cherry ................... 221

delayed fruiting of $\ldots \ldots \ldots \ldots \ldots .223$

distances for $\ldots \ldots \ldots \ldots \ldots \ldots .225$

exposures for ............... 224

gum disease $\ldots \ldots \ldots \ldots \ldots \ldots \ldots . \ldots 228$ 


\begin{tabular}{|c|c|}
\hline Cherry-continued & Cuttings, fruit trees from... \\
\hline grafting the ... & $\begin{array}{l}\text { Cuttıngs, fruit trees from... } \\
\text { Dates } \ldots \ldots \ldots \ldots \ldots \ldots \ldots \ldots\end{array}$ \\
\hline localities for ... & at the missions \\
\hline moisture requirements ... & bearing age of \\
\hline old trees & bearing in Solano County. \\
\hline pests and diseases $\ldots \ldots \ldots \ldots \ldots 228$ & blooming of $\ldots \ldots \ldots \ldots \ldots$ \\
\hline $\begin{array}{l}\text { uning the } \ldots \ldots \ldots \ldots \ldots \ldots \ldots \ldots \\
\text { edlings growing } \ldots \ldots \ldots \ldots \ldots\end{array}$ & first fruit ... \\
\hline $\begin{array}{l}\text { eedlings growing } \ldots \ldots \ldots \ldots \ldots \ldots \\
\text { lug } \ldots \ldots \ldots \ldots \ldots \ldots \ldots \ldots \ldots \ldots\end{array}$ & from seed ... \\
\hline 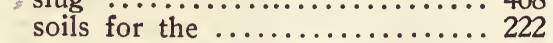 & propagation of \\
\hline stocks for the & requirements of \\
\hline table of varieties $\ldots \ldots \ldots \ldots \ldots \ldots 230$ & transplanting. \\
\hline wild $\ldots \ldots \ldots \ldots \ldots \ldots \ldots \ldots \ldots \ldots \ldots$ & Dewberry $\ldots . .$. \\
\hline 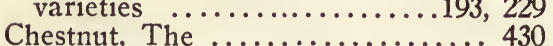 & Diabroticas ..... \\
\hline 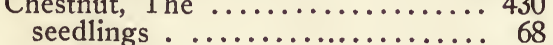 & $\begin{array}{l}\text { Die-back } \dddot{\text { Do..... }} \\
\text { Dormant buds }\end{array}$ \\
\hline$\ldots \ldots \ldots \ldots \ldots \ldots \ldots, 40$ & Drainage desirable \\
\hline Choco or Chayota ............ 394 & Dried fruits (see fruits) ........ 447 \\
\hline Cipoletti Weir ............... 183 & boxes for ...... \\
\hline Citron, The $\ldots \ldots \ldots \ldots \ldots \ldots \ldots \ldots 392$ & covering $\ldots \ldots \ldots \ldots \ldots \ldots \ldots \ldots \ldots$ \\
\hline Clearing land for fruit $\ldots \ldots \ldots \ldots . \quad 57$ & cutting sheds .. \\
\hline 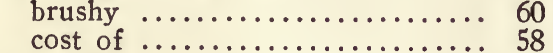 & $\begin{array}{l}\ldots \ldots \ldots \ldots \\
\ldots \ldots \ldots \ldots\end{array}$ \\
\hline $\begin{array}{l}\text { cost of } \ldots \ldots \ldots \ldots \ldots \cdots \cdots \\
\text { grading } \ldots \ldots \ldots \ldots \cdots \cdots\end{array}$ & $\begin{array}{l}\text { drying floors } \ldots \ldots \ldots \ldots \ldots . . .451,450 \\
\text { grading and cleaning } \ldots \ldots \ldots .451,45\end{array}$ \\
\hline steam puller $\ldots \ldots \ldots \ldots \ldots \ldots \ldots$ & packing $\ldots \ldots$..... \\
\hline stump puller ........ & product of $\ldots$... \\
\hline with powder..$;: \ldots \ldots$ & sulphuring $\ldots .$. \\
\hline time to cut to kill............ 62 & sweating $\ldots \ldots$ \\
\hline Climate, divisions of California.... & $\ldots \ldots \ldots \ldots{ }^{450}$ \\
\hline of California, characteristics of. 9, 18 & $\begin{array}{l}\ldots \ldots \ldots \\
\ldots \ldots \ldots\end{array}$ \\
\hline 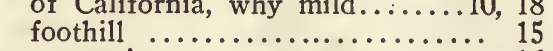 & Evaporated Fruits ... \\
\hline mountain ... & rator, sunshine \\
\hline coast..$\ldots \ldots \ldots$. & Fertilizers in California ..... \\
\hline alley $\ldots \ldots \ldots \ldots \ldots \ldots \ldots \ldots \ldots \ldots$ & in use of $\ldots \ldots \ldots \ldots 141,148$ \\
\hline 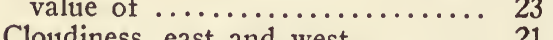 & $\cdots \cdots \cdots \cdots$ \\
\hline $\begin{array}{l}\text { udiness, east and west........... } \\
\text { st pests and diseases........... }\end{array}$ & plying ............ 149 \\
\hline 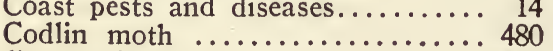 & value of green $\ldots \ldots \ldots \ldots \ldots \ldots \ldots \ldots \ldots \ldots$ \\
\hline osting $\ldots$ & 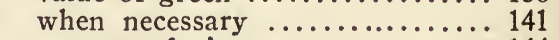 \\
\hline to find true .... & sources of $n$ \\
\hline rops $\ldots \ldots \ldots \ldots \ldots \ldots \ldots \ldots \ldots$ & sources of phosphoric acid. \\
\hline Cranberries...$\ldots \ldots \ldots$ & sources of potash \\
\hline . & ducts for \\
\hline ees or vines. & Feijoa Sellowiana .... \\
\hline allizing fruit.... & Fig $\ldots \ldots \ldots \ldots \ldots$ \\
\hline vation $\ldots \ldots \ldots$ & bearing age .... \\
\hline $\begin{array}{l}\text { equate } \\
\text { side }\end{array}$ s... & 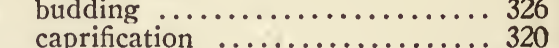 \\
\hline lods of & drying....... \\
\hline oses of & foes of . \\
\hline , resu & from cuttings \\
\hline$\ldots 135,1$ & from seeds $\ldots \ldots \ldots$. \\
\hline 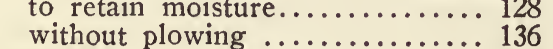 & 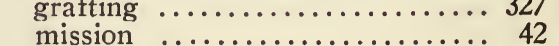 \\
\hline 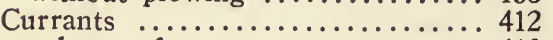 & planting and pruning ...... \\
\hline$\cdot 4$ & regions for \\
\hline for . & size of old trees $\ldots . .$. \\
\hline rieties grown & soils for..$\ldots \ldots \ldots$ \\
\hline ard apple $\ldots \ldots \ldots \ldots \ldots \ldots \ldots$ & $\begin{array}{l}\text { varieties } \ldots \ldots \ldots \ldots \ldots \ldots 194,350 \text {, } 431 \\
\text { Filbert growing } \ldots \ldots \ldots \ldots \ldots \ldots \ldots \\
431\end{array}$ \\
\hline 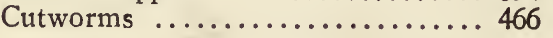 & wild $\ldots \ldots \ldots \ldots$ \\
\hline
\end{tabular}


Page

Frosts, susceptibility of fruits..... 500 protection from ............ 500

Fruit cultivation .............. 128

Fruit gardens, early .......... 46

Fruit industries, influence of..... 53

Fruit industries, outlook of...... 54

Fruit interest, extent of ........ 52

Fruit shipments ............ 51

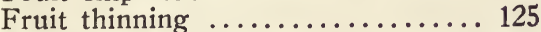

Fruit tree acreage ..........52, 448

Fruit products, value of ........ 53

Fruits, crystallizing ...........446

drying .................. 447

drying floors .............. 450

graders .................. 451

grafted, first in California ...... 46

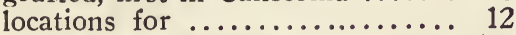

value as stock food ........... 503

Fruit shipments, eastern........50, 51

Fruit trees, dwarf ............. 46

Fruits, commercial varieties ....... 191

Fruits, locations for ............ 12

Fruits, mission ............. 42

Fruits, native ............... 36

Fruits, Russian ............ 45

Fruit, soil ingredients of ........ 142

Goat nut, or jajoba .......... 40

Gooseberry, The .............. 414

culture of ................ 414

requirements of $\ldots \ldots \ldots \ldots \ldots . . .414$

varieties ................ 415

wild ................... 38

Gophers, killing ............. 495

pitfall for ................ 496

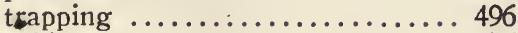

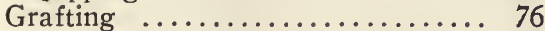

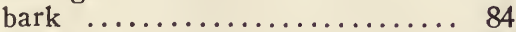

cleft .................. 79

old trees $\ldots \ldots \ldots \ldots \ldots \ldots \ldots \ldots \ldots$

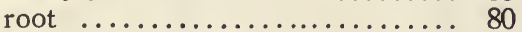

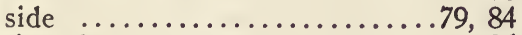

time for ................ 85

top .................. 83

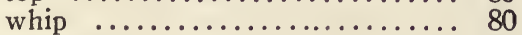

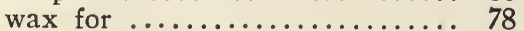

waxed bands ............ 78

Graft, time to ............. 85

Grafts, planting out ........... 80

Granadilla ................. 396

Grape, area of ............... 285

budding ................... 292

conditions of ripening ......... 19

cutting grafts ............... 296

dibbles for planting ........... 299

diseases of $\ldots \ldots \ldots \ldots \ldots \ldots \ldots . \ldots . \ldots 312$

distance ................... 298

from cuttings ............... 289

from layers ............... 288

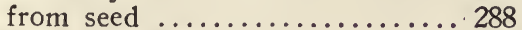

frost injuries .............. 311

grafting ................292, 295

insects

470,474
Grape-continued

Page

industry ................. 285

length of season ............ 286

mildew ................. 488

mission . .................42, 44

number per acre ............ 298

planting devices ............ 298

planting in rows ............. 297

products .................... 285

pruning ..................302, 311

pruning, long .............. 308

pruning, short .............. 285

resistant .................. 296

rooting in nursery ........... 292

shipping $\ldots \ldots \ldots \ldots \ldots \ldots \ldots 285,286$

soils for ..................... 286

stakes, twine, etc ........... 302

suckering ................... 310

sulphuring $\ldots \ldots \ldots \ldots \ldots \ldots \ldots, 488$

summer pruning ...........305, 310

syrup ..................... 460

topping .................. 306

trellising ................. 309

varieties .................. 313

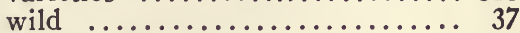

wine, varieties of .......... 318

Grasshoppers, killing .......... 471

Growing season, long.......... 23

Guava, The .................. 395

Gummosis .................. 491

Gypsum, uses of ............. 145

Hardpan, breaking up .......... 94

Heat, deficient on coast ......... 13

importance of .............. 18

summer, records of .......... 19

Heeling in young trees .......... 99

Hexagonal planting ...........91, 92

Hillside, rows on ............. 90

use of triangle on ........... 93

Holes for trees .................94, 95

Huckleberries, wild ............ 39

Humidity, atmospheric ......... 21

deficient .................... 24

east and west .................. 22

excessive ................... 24

Insects, injurious ........... 465

Insects, remedies ............... 483

Irrigation $\ldots \ldots \ldots \ldots \ldots \ldots \ldots \ldots \ldots . \ldots . \ldots 153$

distributing manure by......... 149

ditches ...................... 178

drainage and ................. 188

evils of excessive............ 154

flooding ................... 163

for citrus fruits ...............157

for deciduous fruits .......... 157

flume building for .......... 176

hillside ................... 173

how much ..............155, 157

implements for ............. 165

in early days .............. 49

in basins .................. 168

in checks $\ldots \ldots \ldots \ldots \ldots \ldots \ldots \ldots . \ldots 163$ 


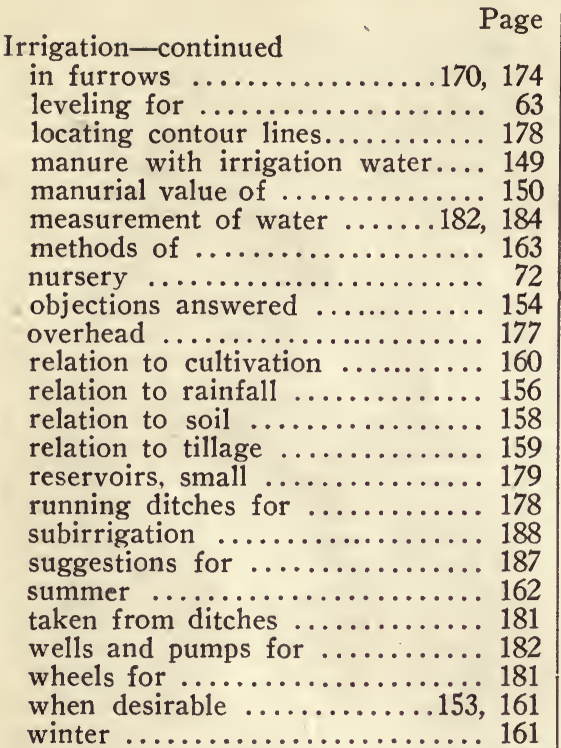

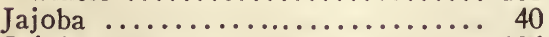

Jujube of commerce ............. 396

Jujube, native .............. 40

June buds .................73, 81

Kai Annle .................. 403

Laying out land for fruit ........ 87

Leaf lice .................. 469

Legumes in orchard ........... 151

Lemon berry ................ 40

Lemon, The ................ 354

curing ...................388, 390

picking $. . . \ldots \ldots \ldots \ldots \ldots \ldots . \ldots . \ldots 388$

planting and pruning ......... 387

propagation $\ldots \ldots \ldots \ldots \ldots \ldots .70,387$

situations and soils for ........ 355

varieties ..................... 391

Lemon berry ................. 40

Lice, leaf .................. 469

Lime, The ................. 391

Lime, uses of .............. 144

Loganberry ................. 417

Loquat, The ................ 397

Manure, care of ................ 145

green .................... 150

poultry ...................... 147

sheep, use of ............. 147

Manuring at planting ............ 103

Manzanita berries ............ 39

Map of orchard and vineyard...... 104

Marls ........................ 145

Mealy bugs .................. 477

Measuring wire $. . . \ldots \ldots \ldots \ldots \ldots . . .68$

Melon shrub ................. 400

Melon tree ..................4 400

Mildew ..................... 488

Miner's inch ................... 182

Mission fruits .............. 42
Page

Moisture lost by weed growth..... 130

retained by cultivation ......... 128

Morning Glory, killing .......... 138

Moss, removing ............... 489

Mulberry, The .............. 415

Mulching, after planting ......... 104

Mulching as substitute for cultivation 139

Nectarine .................. 252 compared with peach .......... 252 dried .................253, 456 future of $\ldots \ldots \ldots \ldots \ldots \ldots \ldots .254$ varieties .................. 253

Nitrogen for fruits ..........144, 150

Nursery ..................64, 365 budding and grafting ......... 73 classes of nursery stock........ 81 growing seedlings ............ 66 imported seedlings ........... 69

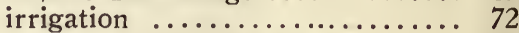
laying out and planting ........ 70 pruning in ................. 81 selection of site for ............ 64 soil, preparation of .......... 66 soil, proper for ............. 64 trees, ages of $\ldots \ldots \ldots \ldots \ldots \ldots .80,81$ trees, digging ............. 98 trees, disinfecting ........... 483 trees, selecting ............. 97 when to plant $\ldots \ldots \ldots \ldots \ldots \ldots \ldots .60$ Nuts growing in California....... 424 growing from seed ........... 68 wild ....................... 40

Olives ..................... 335

at old missions $\ldots \ldots \ldots \ldots \ldots \ldots . .43$

budding ..................... 338

canning ...................... 348

climate for ............... 335

from cuttings ............... 337

from seed ................ 336

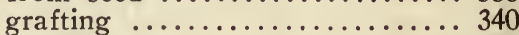

localities for ............... 336

oil making ...............344, 345

planting ................... 341

preferred varieties ........... 349

pruning .................... 342

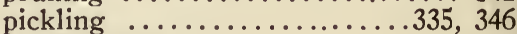

small cuttings ............. 337

soils for ................ 336

truncheons .................. 338

twig borer ..................... 478 wild .................... 40 varieties ................194, 348

Orchard land, preparation of ..... 86 laying out in squares ......... 87 alternating squares ........... 88 equilateral triangles ............ 91 measure and sight $\ldots \ldots \ldots \ldots \ldots, 88$ measuring wire ............ 88 quincunx planting ............ 91 time for planting $. . \ldots \ldots \ldots \ldots . .100$

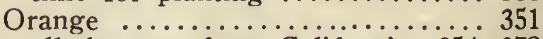
all the year from California..354, 378 
Orange-continued
Oudding and grafting $\ldots \ldots \ldots \ldots \ldots$

in central California ........... 357

in southern California.......... 359

mission ................... 42

nursery $\ldots \ldots \ldots \ldots \ldots \ldots \ldots \ldots \ldots . \ldots . \ldots . \ldots 365$

outlook ....................361

packing .................... 380

planting in orchard $\ldots \ldots \ldots \ldots 368$

product ............53, 351, 353, 355

propagation $\ldots \ldots \ldots \ldots \ldots \ldots \ldots . \ldots 363$

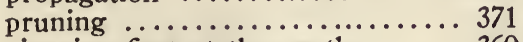

ripening first at the north....... 360

seedlings, care of ............. 365

situation and soils for......... 362

superiority of semi-tropical...... 354

transplanting ................. 368

world's industry ............. 351

varieties ................194, 378

Orchard planting ............. 94

Oregon grape ............... 39

Oso berry .................. 37

Popular fruit varieties .......... 191

Palm nuts ................. 40

Peach ........................ 233

age at planting $\ldots \ldots \ldots \ldots \ldots \ldots . .238$

approved lists of ............ 247

blight ................... 489

blooming of varieties.......... 246

curl-leaf .................... 244

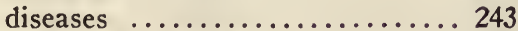

distance in planting.......... 238

dormant buds ............... 238

drying $\ldots . \ldots \ldots \ldots \ldots \ldots \ldots . \ldots 455$

early bearing .............. 234

grafting $\ldots \ldots \ldots \ldots \ldots \ldots \ldots \ldots \ldots \ldots \ldots \ldots$

growing season of $\ldots \ldots \ldots \ldots \ldots 23$

irrigation ................ 242

localities for ................ 235

longevity of $\ldots \ldots \ldots \ldots \ldots \ldots .233$

mildew .................... 244

mission ................. 42

moth ................477, 482

"peach almond" ............... 238

pitting clings .............. 455

propagation . ............... 237

pruning $\ldots \ldots \ldots \ldots \ldots 116,234,239$

ripening of varieties .......... 246

root borer ................ 478

seedlings $\ldots \ldots \ldots \ldots \ldots \ldots \ldots \ldots 66$

soils for ................. 236

stocks for ................. 238

table of varieties.............. 245

thinning $\ldots \ldots \ldots \ldots \ldots \ldots \ldots \ldots \ldots \ldots \ldots \ldots \ldots, 241$

varieties $\ldots \ldots \ldots \ldots \ldots \ldots \ldots . \ldots \ldots \ldots .193,246$
Page

Peanut growing .............4 431

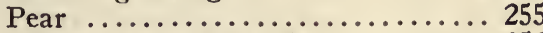

Bartlett, why popular ........ 256

blight .................258, 261

characteristics in California .... 256

diseases .................. 261

distances for the............ 259

drying $. . . \ldots \ldots \ldots \ldots \ldots \ldots . \ldots 45$

dwarf .....................258

for alkali soil ............. 258

irrigation ................. 260

largest on record ........... 255

localities for ................ 256

mission ................. 42

on quince stock ............ 258

pollination .................. 260

propagation of ............. 258

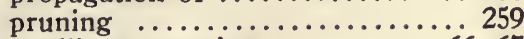

seedlings, growing .........66, 67

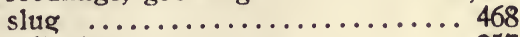

soils for ................ 257

storing and ripening .......... 262

tables of varieties ........... 267

thinning .................. 260

varieties ................193, 264

Pear, Alligator ............. 401

Pecan, The ................ 432

Persimmon, Japanese ........... 397

Persimmon, Virginian .......... 397

Persimmons, curing ........... 398

Phenomenal berry ........... 417

Phosphates .................. 143

Phyloxera .................. 474

Pioneers, planting by ........... 46

Pineapple ................... 398

Pine nuts $\ldots \ldots \ldots \ldots \ldots \ldots \ldots \ldots . . . .40$

Pistachio, The ................ 433

Planting, conditions favoring...... 100

bar for setting .............. 96

cutting back after .........105, 112

depth of .................. 103

digging holes for ........... 94

laying off for $\ldots \ldots \ldots \ldots .87,88,89$

mulching $\ldots \ldots \ldots \ldots \ldots \ldots \ldots \ldots \ldots$

operation of $\ldots \ldots \ldots \ldots \ldots \ldots \ldots . . .101$

preparing land for .......... 86

speed in ................. 103

time for ................... 100

triangular tree setter .......... 96

use of manure ............... 103

use of water ................ 102

Plowing, devices for ........... 132

orchard and vineyard .......... 133

on hillside ................. 134

to break hardpan ..........8. 133

Plow, laying off with........... 88

Plums and prunes ............. 268

California false ............ 37

confusion in names ............ 274

definition of a prune........... 268

drying ..................4 456

from the root .............. 271 


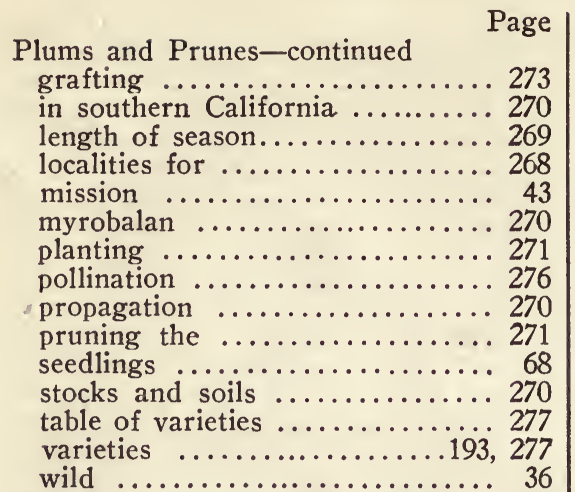

Plumcot, The ..................... 274

Pomegranate, The ..........43, 399

Pomelo . .................... 383

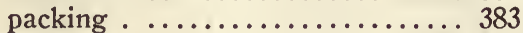

varieties . ..............194, 384

Potash . ................... 143

Prickly Pear ..............41, 400

Prune curing ...............4456

Pruning . ................... 106

bearing trees ............... 115

California style ............. 109

effects of .................. 109

gathering brush ............ 124

influenced by location .......... 109

low, advantages of ........... 109

nursery . ................. 81

prunings as fertilizer ........124, 148

purposes of .............. 107

times for ................... 119

tools ..................... 123

to renew old trees ........... 122

vase form, securing ........... 109

wounds, covering ............ 124

Quince ...................... 282

demand for ............... 282

propagation . ................ 282

pruning . ................. 283

soils for $\ldots \ldots \ldots \ldots \ldots \ldots \ldots \ldots . \ldots 283$

varieties . ................ 283

Quincunx planting ............. 91

Rabbit, fences ................ 493

Rabbits, destroying .............. 494

poisons for ............... 494

smears for .................. 494

Rainfall, records of ............. 12

Rainfall or irrigation ...........153, 156

Raisin making ..........193, 318, 458

Raspberry, The .............4 416

black-caps . ................... 417

hybrids . ................. 417

pruning . .................. 416

varieties, popular ..........194, 417

wild ..................... 38

Red Spider ................ 472

Root $\operatorname{rot} . . . \ldots \ldots \ldots \ldots . \ldots . \ldots 49$

Root-knots
Russian introduction of fruits..... Page 45

Salal . ..................... 39

Salmon berry ................ 38

Scale Insects ................ 474

black . ................... 476

brown apricot ............ 476

cottony cushion ............ 477

pear .................... 475

orange, red .............. 475

orange, soft .............446

oyster shell ............... 475

rose and berry ............ 475

San Jose .................. 475

remedies for ................ 484

Sapota, white ............... 402

Scions, care of ................ 76

selection of $\ldots \ldots \ldots \ldots \ldots \ldots \ldots, 77$

Sea Fig .................. 40

Seed, growing trees from........ 68

Seedlings, imported ............ 69

Septuple, laying off ............ 92

Service Berry ............... 39

Soils for fruits .............. 25

adobe . .................... 33

alkali ................... 34

alluvial . ................. 31

bed-rock or hard-pan ......... 34

blasting . .................. 94

characteristics of California .... 26

classification of ............. 28

clay ................... 33

defective ................. 33

desert .................... 30

examination of $\ldots \ldots \ldots \ldots \ldots \ldots 35$

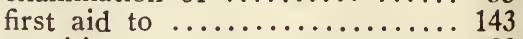

granitic . ................. 32

loams . ............. 29, 32

mesa . .................. 30

organic matter ............... 151

plains . .................. 29

red ........................ 32

river bottom .............. 31

sedimentary or silty .......... 31

shallow. blasting ............. 94

Sour sap .................. 490

Snider, red ............... 472

Stock, fruit as food for......... 503

Sruares, laying off in ......... 87

Squirrels, destroying ........... 494

Strawberry . ............... 418

care of ..................... 421

continuous bearing ............. 421

laying out for ............ 420

planting .................... 421

propagation ................. 419

situations and soils ..........4 419

varieties, popular .............194. 421

wild .........................38, 421

Strawberry tree ............ 400

Summer pruning ............. 120

Sunhurn, protection from ......104, 478

Sunlight, value of direct ....... 20 


\begin{tabular}{|c|c|}
\hline \multirow{2}{*}{\multicolumn{2}{|c|}{ Jalnut-continued }} \\
\hline & \\
\hline shine, records of $\ldots \ldots \ldots \ldots \ldots$ & gathering and drying \\
\hline Temperature, lowest ............. & grafting ............ \\
\hline Temperature, records of $\ldots . . . . .$. & growing seedlings. \\
\hline Thinning fruit $\ldots \ldots \ldots \ldots \ldots \ldots$ & hybrids...... \\
\hline Thrips...$\ldots \ldots$. & propagation and planting \\
\hline Tomato, tree $\ldots \ldots \ldots \ldots \ldots \ldots \ldots \ldots 40$ & pruning...... \\
\hline$\ldots \ldots \ldots \ldots \ldots$ & soils \\
\hline Trees, activity and rest of & eties \\
\hline heeling in ........ & i \\
\hline . $\ldots \ldots \ldots \ldots \ldots \ldots$, & $r$ measurements \\
\hline$\ldots \ldots \ldots \ldots \ldots 99,96$ & dltivation. \\
\hline Triangle for laying out $\ldots \ldots \ldots \ldots$ 92 & Weeds, evaporation by \\
\hline$\ldots \ldots \ldots \ldots \ldots, 41$ & ir measu \\
\hline$\ldots \ldots \ldots \ldots 468$ & sunburn \\
\hline efly planted ......... & y bloom. . \\
\hline ppers.$\ldots \ldots \ldots \ldots \ldots \ldots 470$ & fruits of California .. \\
\hline 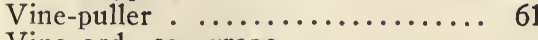 & Windbreaks . ........... \\
\hline d-see grape & \\
\hline lnut, black & rap \\
\hline ut, English & Winery refuse as manure. \\
\hline osis or blight .......443, 49 & r-killing, unknown \\
\hline ling. & Wire, measuring \\
\hline blossoms of & Woolly aphis ...... \\
\hline ..... & nds, c c r a \\
\hline & ckets, killing \\
\hline
\end{tabular}




\title{
California Vegetables
}

IN

\section{GARDEN AND FIELD}

\author{
A MANUAL OF PRACTICE WITH AND WITHOUT IRRIGATION
}

BY EDWARD J. WICKSON, A. M.

\section{CONTENTS}

Chapter.

I. Vegetable Growing in California.

II. Farmers' Gardens in California.

III. California Climate as Related to Vegetable Growing.

IV. Vegetable Soils of California.

V. Garden Irrigation.

VI. Garden Drainage in California.

VII. Cultivation.

VIII. Fertilization.

1X. Garden Location and Arrange-

$X$. The Planting Season.

XI. Propagation.

XII. Asparagus.

XIII. Artichokes.

XIV. Beans.

XV. Beet.

XVI. Cabbage Family.

XVII. Carrot, Parsnip and Salsify.

XVIII. Celery.

XIX. Chicory.

\section{Chapter.}

XX. Corn.

XXI. Cucumber.

XXII. Egg Plant.

XXIII. Lettuce.

XXIV. Melons.

XXV. Onion Family.

XXVI. Peas.

XXVII. Peppers.

XXVIII. Potatoes.

XXIX. Radishes.

XXX. Rhubarb.

XXXI. Spinach.

XXXII. Squashes.

XXXIII. Tomato.

XXXIV. Turnip.

XXXV. Vegetable Sundries.

XXXVI. Vegetables for Canning and Drying.

XXXVII. Seed Growing in California. XXXVIII. Garden Protection.

XXXIX. Weeds in California.

\section{Third Edition-Revised and Extended}

\section{COMMENDATION OF "CALIFORNIA VEGETABLES"}

The work will be found of inestimable assistance to those who are interested in vegetable growing in California, not only on a large scale, but to the large number who cultivate these products for their own consumption.-San Francisco Call.

We take no risk in advising every Californian who has a garden spot to procure a copy.San Francisco Chronicle.

Not only interesting but valuable to every one in this State who cultivates ever so small a lot of ground. The author is eminently qualified for the work which he has just completed. -San Francisco Bulletin.

It treats of the proper culture of all leading vegetables in California. It is full of information and instruction. It is so clear that whoever uses it as a guide book can not go astray in vegetable culture. The work is freely illustrated and handsomely bound.-Sacramento Record-Union.

Covers every part of the State in technical analysis and physical demonstration.-Petaluma Courier.

It treats of every feature of vegetable production in plain, common-sense terms, and gives reasons for its assertions.-Pomona Times.

\section{Price \$2.00, Postpaid Anywhere}





\section{UNIVERSITY OF CALIFORNIA LIBRARY BERKELEY}

Return to desk from which borrowed.

This book is DUE on the last date stamped below.

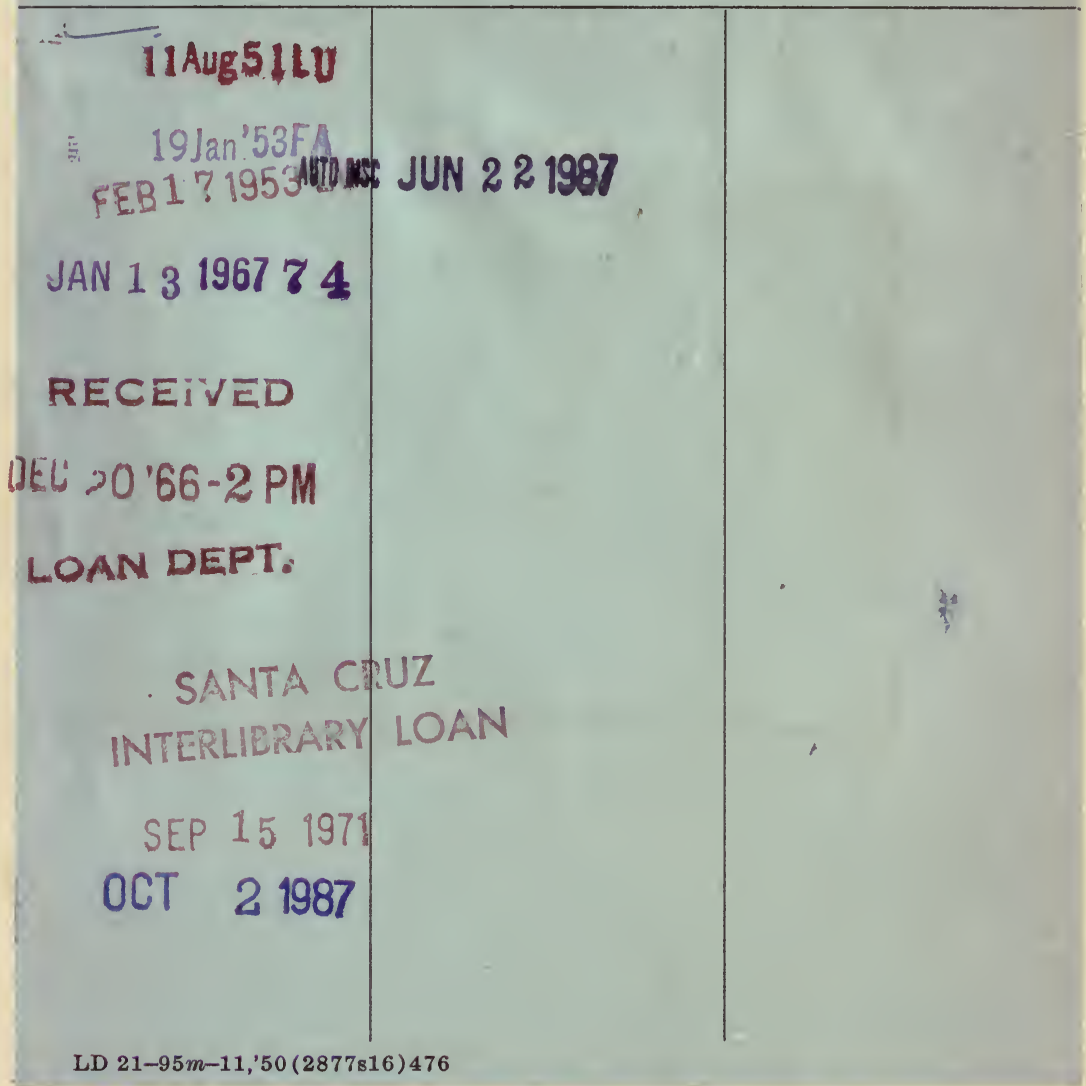




\section{YC 6206́3}

GENERAL LIBRARY - U.C. BERKELEY

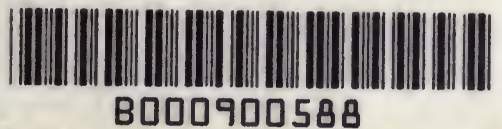

B०00900588

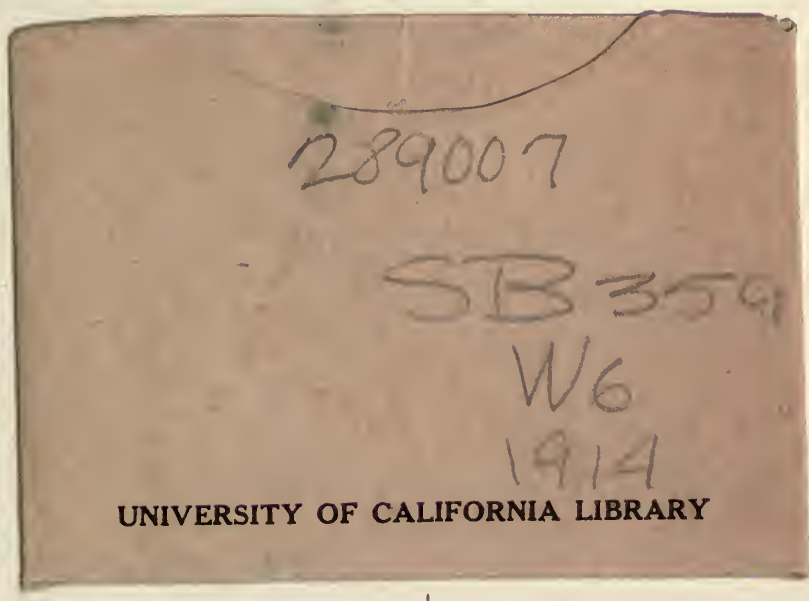


sis 